\title{
$39^{\text {th }}$ Willing Contribution
}

to

Flora of Greece

June-July 2017

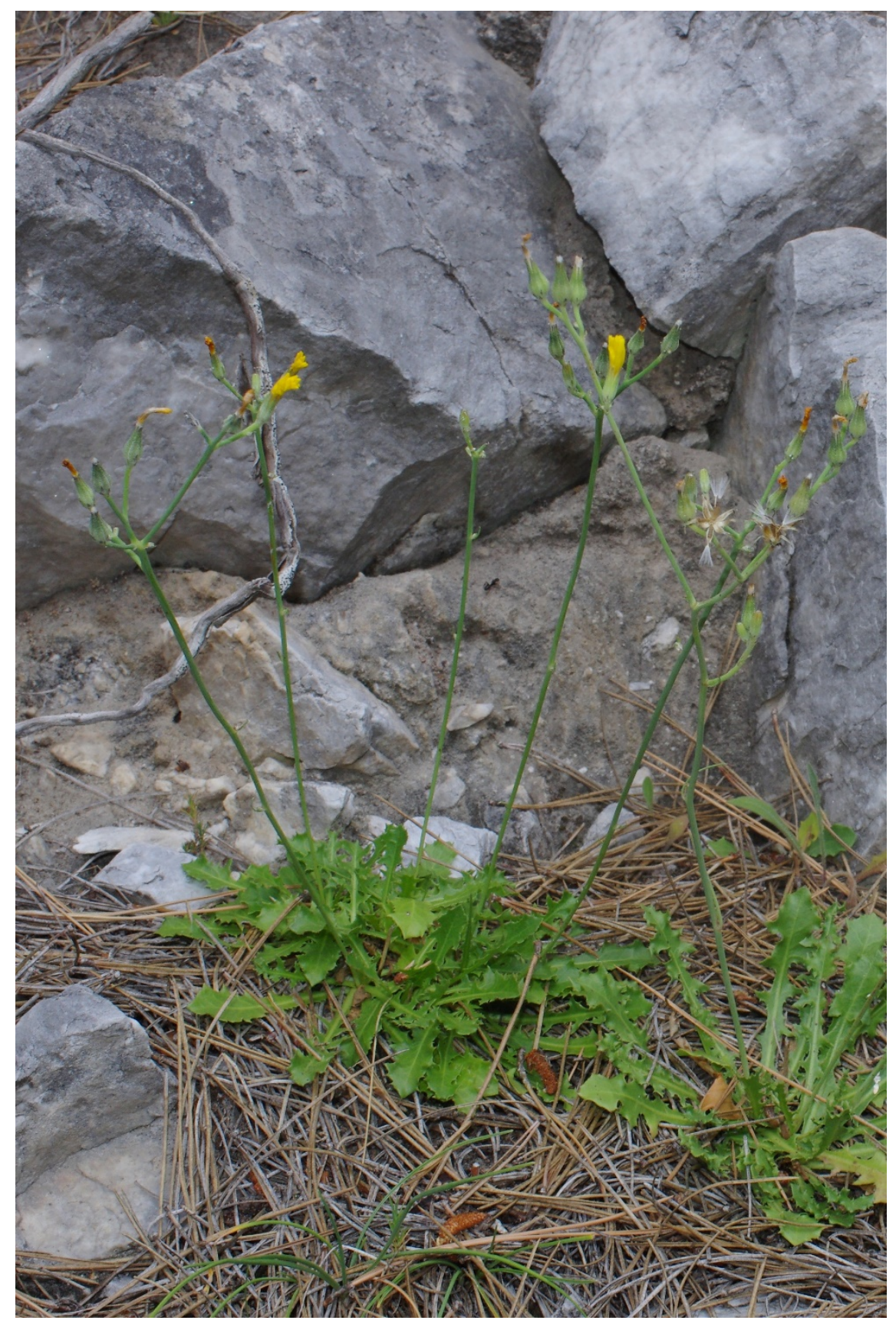

by Rita \& Eckhard Willing

Compiled March 2018 

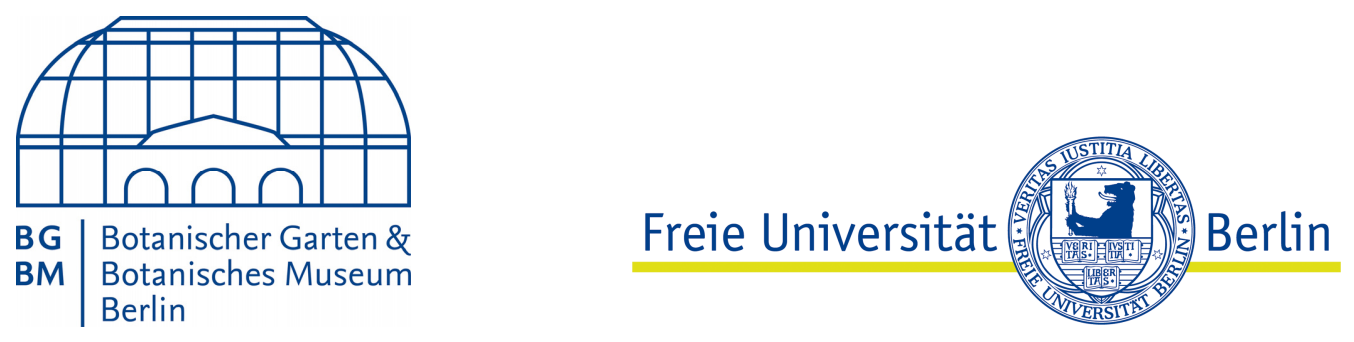

Published by BGBM Press

Botanic Garden and Botanical Museum Berlin

Freie Universität Berlin

(c) 2018 Eckhard \& Rita Willing

ISBN 978-3-946292-25-8

doi: https://doi.org/10.3372/wfr2017.2

Published online on 11 April 2018 by the Botanic Garden and Botanical Museum Berlin, Freie Universität Berlin - www.bgbm.org

The Botanic Garden and Botanical Museum Berlin as publisher reserves the right not to be responsible for the topicality, correctness, completeness or quality of the information provided.

The information provided is based on material identified by the authors. The entire collections are preserved in the Herbarium of the Botanic Garden and Botanical Museum Berlin, where the determinations can be reassessed.

Citation:

Willing R. \& Willing E. 2018: $39^{\text {th }}$ Willing contribution to Flora of Greece. June-July 2017. Berlin: Botanic Garden and Botanical Museum Berlin. doi: https://doi.org/10.3372/wfr2017.2

Address of the authors:

Rita and Eckhard Willing

Augustenhof 14

D-06842 Dessau-Roßlau

Germany

eisenwill@gmx.net

www.willing-botanik.de

Cover image: Chondrilla urumoffii, Greece, Falakron, 1140 m, 3 July 2017, photograph by E. Willing. 


\section{Summary}

From May 12th to July 20th 2017 we stayed in northern Greece in the prefectures loannina, Grevena, Kozanis, Kilkis, Imathia, Pella, Serres, Drama and Kavalla. We visited 345 localities, 71 of them between 1 and 499 m, 118 between 500 and 999 m, 105 between 1000 and 1499 m and 51 above $1500 \mathrm{~m}$.

Our primary plan to concentrate on regions between seeside and $1000 \mathrm{~m}$ mainly in prefectures Kavala, Drama and Serres was unrealistic because of very hot weather conditions in the weeks before.

We collected 8045 specimen of at least 1370 different species and sub-species and made 76 additional observations. Of at least 820 species we have taken photographs.

All localities with their geographical data and the herbarium numbers of collected specimen are listed in chapter 3 Localities. Collected and observed species with selected photographs are listed in chapter 4 Species.

All specimen and the photographs belonging to them will be published in the virtual herbarium Jacq. A selection of phographs may be seen in www.willing-botanik.de.

We kindly ask all readers and users of this report to name wrong determinations and send us corrections.

\section{Einführung}

Vom 12.Juni bis zum 20.Juli 2017 haben wir unsere floristischen Feldstudien in NordGriechenland fortgesetzt. Die Ziele dieser Reise waren zweigeteilt.

Erstens wollten wir das Flach- und Hügelland in den Präfekturen Serres, Drama und Kavala besuchen, das uns bei der Durchfahrt im Jahr 2014 als ausgesprochen blumenreich erschien und das in der Vergangenheit insgesamt unzureichend bis schlecht besammelt worden ist.

Zweitens wollten wir die Gebirgsmassive besuchen, in denen ich (Eckhard) zu meinen „Orchideenzeiten“ mehrfach war, aber neben den Orchideen nur wenige andere Arten gesammelt oder photographiert habe. In diesen Gebirgsmassiven ging es uns also weniger darum, das Verbreitungsgebiet der Arten zu erweitern, als die speziell in höheren Lagen vorkommenden Arten zu photographieren. Dabei folgten wir dem Grundsatz, dass jede photographierte Art auch gesammelt wird.

Das Sammeln in den niedrigen und mittleren Lagen, bis in Höhen von $1300 \mathrm{~m}$ an südexponierten Hängen, wurde dadurch erschwert bzw. fast unmöglich gemacht, dass in den Monaten zuvor extrem heiße Luft afrikanischer Herkunft in das Landesinnere geströmt war und die gesamte Flora in Mitleidenschaft gezogen hat. Einheimische berichteten uns, dass selbst Garten- und Ackerpflanzen „verbrannt“ seien und selbst durch starkes Bewässern nicht gerettet werden konnten. Aus diesem Grund haben wir uns stärker dem zweiten Ziel gewidmet. In den höheren Lagen des Nord-Pindus, des Voras, des Vermion, des Paikon, der Ori Vrondous, des Falakron, der Rodopen und des Pangaion zeigte sich die Pflanzenwelt von ihrer besten Seite. In einzelnen Massiven hätten wir uns deutlich länger aufhalten können. Zum Schluss fehlten uns 1 bis 2 Wochen, um das Vermion und die Berge von Kastoria und loannina zu besuchen.

Wie in den vergangenen Jahren hat es sich als überaus erfolgreich und angenehm erwiesen, zu zweit ins Gelände zu gehen. Wir schauen und sammeln so unterschiedlich, dass wir in der Regel mit sehr unterschiedlichen Arten zum Auto zurückkommen. Eckhard bereitet die Pflanzen vor und versieht sie mit den bewährten Pflanzennummern. Rita erweist sich nach wie vor als die beste denkbare „Pflanzeneinlegerin“ und schafft es, auch stark angetrocknete Pflanzen, vor 
allem einjährige, in der Presse wie gebügelt aussehen zu lassen. Während sie die Pflanzen einlegt, nutzt Eckhard die Zeit für weitere Pflanzenphotos.

Die weiteren Arbeiten wie Montage, Sortieren, Bestimmen, Eingabe in die Datenbank, Vorbereiten der Daten und der Photographien für das Virtuelle Herbar und für die Datenbank der Flora of Greece erledigt Eckhard, so dass auch alle Bestimmungsfehler von ihm verursacht werden.

Er hat wiederum versucht alle Herbarbelege bis zur Art zu bestimmen, musste aber feststellen, dass für einige Gattungen die notwendige Literatur und vor allem die Bestimmungserfahrungen fehlen. In einigen Fällen hat er deshalb Belege nur bis zur Gattung bestimmt, so bei Festuca, Galium, Pilosella, Hieracium und Rosa. Alle Belege sind im Herbarium des Botanischen Museums Berlin als Dauerleihgabe hinterlegt. Sie können dort eingesehen oder zur Bearbeitung nachgefragt werden. Umbestimmungen werden im BGBM in die Virtuellen Herbare eingetragen.

Die Mehrzahl der Photos ist mit Angabe der Fundstellen und den dazu gehörenden Herbarnummern auf www.willing-botanik.de auf der Seite "Pflanzen Griechenlands" einzusehen.

Alle Herbarbelege mit den dazugehörigen Photos werden kurzfristig im Virtual Herbarium BGBM "www.bgbm.org/de/herbarium" und verfügbar sein.

im Virtual Herbarium Jacq "http://herbarium.univie.ac.at/database/search.php“.

Wir bitten ausdrücklich darum, uns und das BGBM auf Bestimmungsfehler hinzuweisen. Die korrigierten Daten werden in jedem Fall umgehend unter Angabe des Umbestimmers in die Dokumente eingefügt.

Unsere Reiseroute und die besuchten Fundstellen sind der Fundortliste in Kapitel 3 zu entnehmen und auf folgender aus unser privaten Zentraldatenbank abgefragten Karte zu erkennen. Die gegenüber den Reisen der letzten Jahre erheblich größeren Entfernungen der einzelnen Sammelgebiete konnten wir dank der Autobahn Egnatia ohne große Zeitverluste bewältigen. Trotz der zahlreichen Warnschilder konnten wir hier keine Bären sehen.

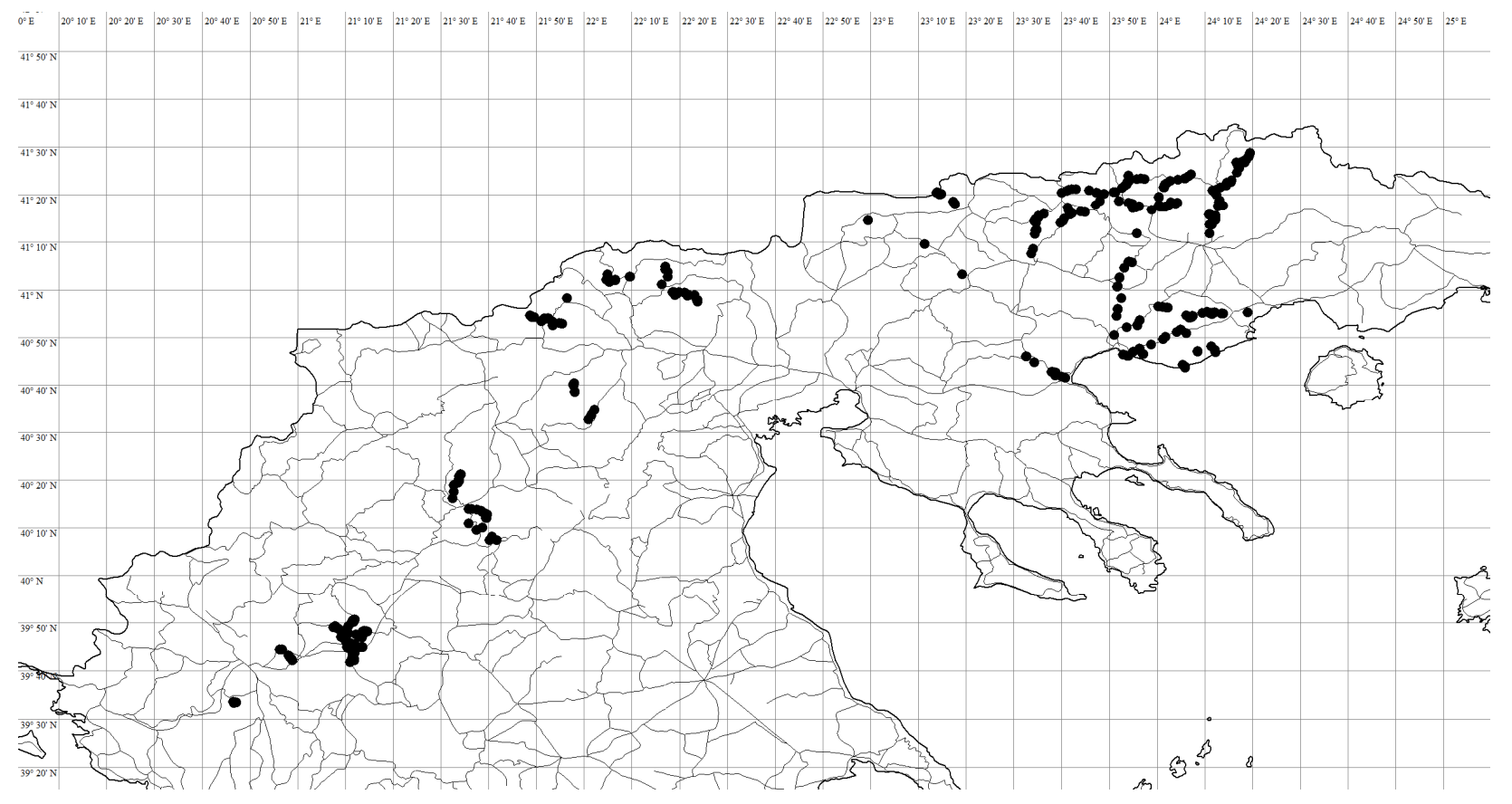




\section{Interessante Funde}

Für uns waren Pflanzenfunde immer dann besonders interessant, wenn wir die jeweilige Art zum ersten Mal oder während unser vielen Griechenlandreisen weniger als 5 Mal gefunden hatten. Dies war während dieser Reise relativ häufig der Fall, da wir in den besuchten Gebirgsmassiven selten oder nie über 1300 m Höhe gesammelt hatten.

Weiterhin waren die Arten interessant, die wir noch nie photographiert hatten. Dies ist zwar wenig wissenschaftlich, wird aber immer dann interessant, wenn es für die betreffenden Arten in der frei zugängigen Literatur oder im Internet keine oder nur sehr wenige Abbildungen gibt. Bei der Auswertung des Bildmaterials mussten wir leider feststellen, dass wir einige interessantere Arten nicht photographiert haben. Dies lage zum einen daran, dass wir die Arten im Gelände nicht erkannt haben, zum anderen daran, dass wir uns in Gebieten mit Photographierverbot befanden (z.B. auf den Höhen des Kerkini) oder die Umstände (Hitze, starker Wind) das Photographieren erschwerten.

Für die Checklist Vascular Plants of Greece (CVPG) waren die Funde interessant, wenn sie in neuen floristischen Regionen lagen. In diesem Zusammenhang fanden wir es ein wenig unbefriedigend, dass die summarische Zuordnung von Arten zu den floristischen Regionen nichts darüber aussagt, ob die Art hier häufig oder nur ein einziges Mal gefunden worden ist.

Am interessantesten sind natürlich Funde, die neu für Griechenland sind oder die keiner der in der Literatur angegebenen Arten zugeordnet werden können. Typisch hierfür sind die Arten der Gattung Taraxacum, die ich nicht bestimmen konnte, da sie noch nicht beschrieben waren.

Ein paar Beispiele sollen hier erwähnt werden:

Matricaria discoidea: Eine wenig spektakuläre Art, die einem aus der deutschen Flora so geläufig ist, dass man sie auch in Griechenland kaum als etwas Besonderes wahrnimmt. In CVPG wird sie nur für Nordost angegeben. Wir fanden sie in Nordzentral im Bereich der Skistation des Kajmakcalan in schönen Beständen. Pella, Kajmaktsalan, 1975 m, 4054'12"N, $21^{\circ} 49^{\prime} 04 " E, 14.07 .2017$

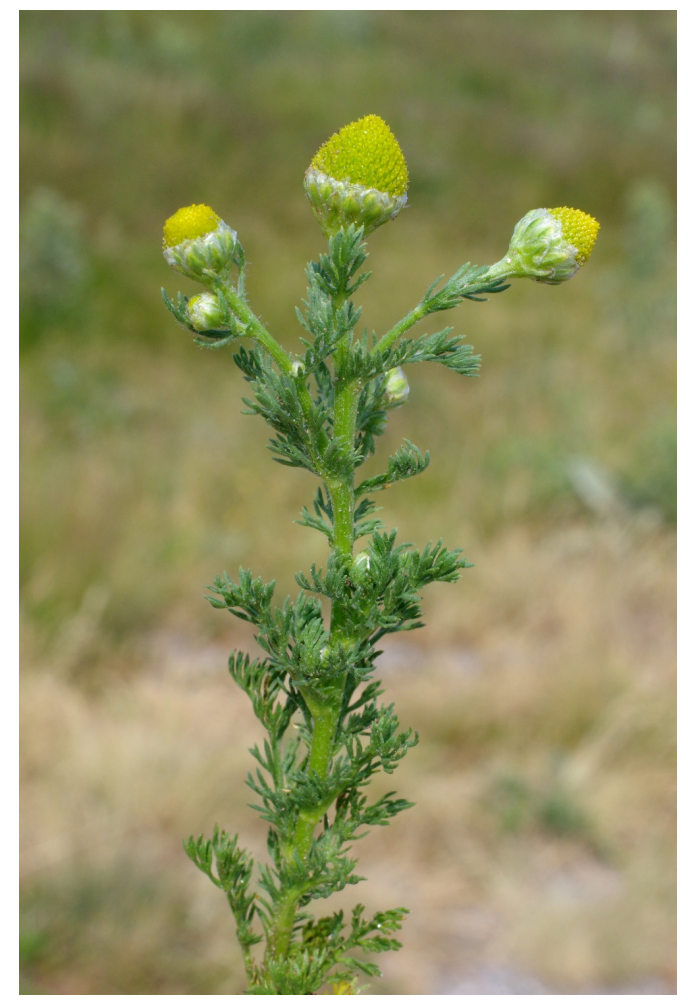


Chondrilla urumoffii: Die Art wird in Mountain Flora of Greece Vol.2 pg.573 für Nordost Pangaion, Menikion und Orvilos angegeben. Wegen der Nähe verwundert es nicht, dass wir sie auch am Falakron finden konnten: Drama, Falakron, felsiges Gelände in Buchenwald, $1140 \mathrm{~m}, 41^{\circ} 17^{\prime} 48^{\prime \prime} \mathrm{N}, 24^{\circ} 00^{\prime 2} 27^{\prime \prime E}, 03.07 .2017 ; 280.972$
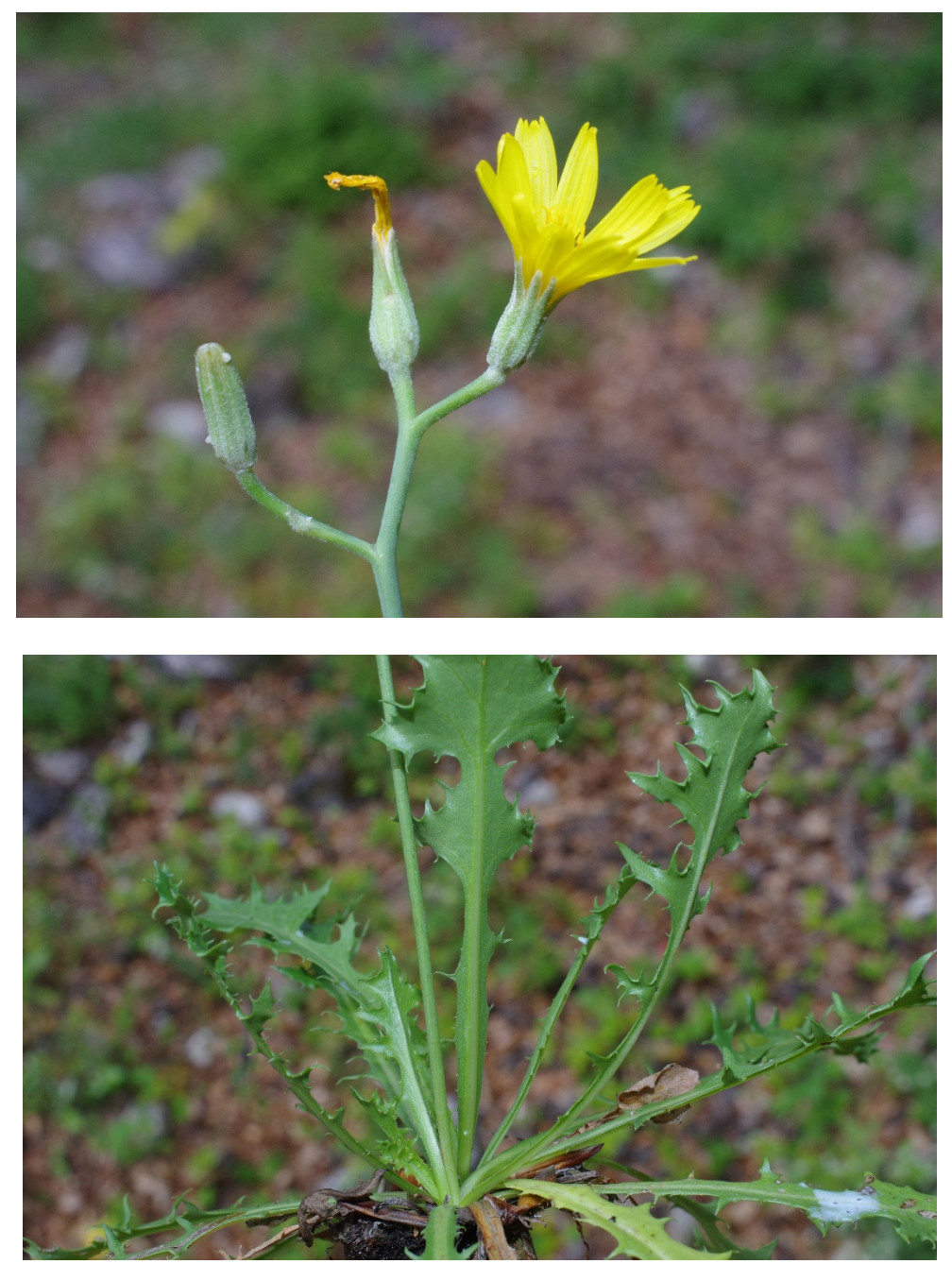

Medicago minima?: Südwestlich von Kavala sammelten wir ein Medicago, dessen Besonderheit wir im Gelände nicht erkannt haben: Kavala, NO Paralia Myrtofytou, $60 \mathrm{~m}$, $40^{\circ} 46^{\prime} 49^{\prime \prime} \mathrm{N}, 24^{\circ} 12^{\prime} 18^{\prime \prime} \mathrm{E}, 24.06 .2017 ; 278.781$

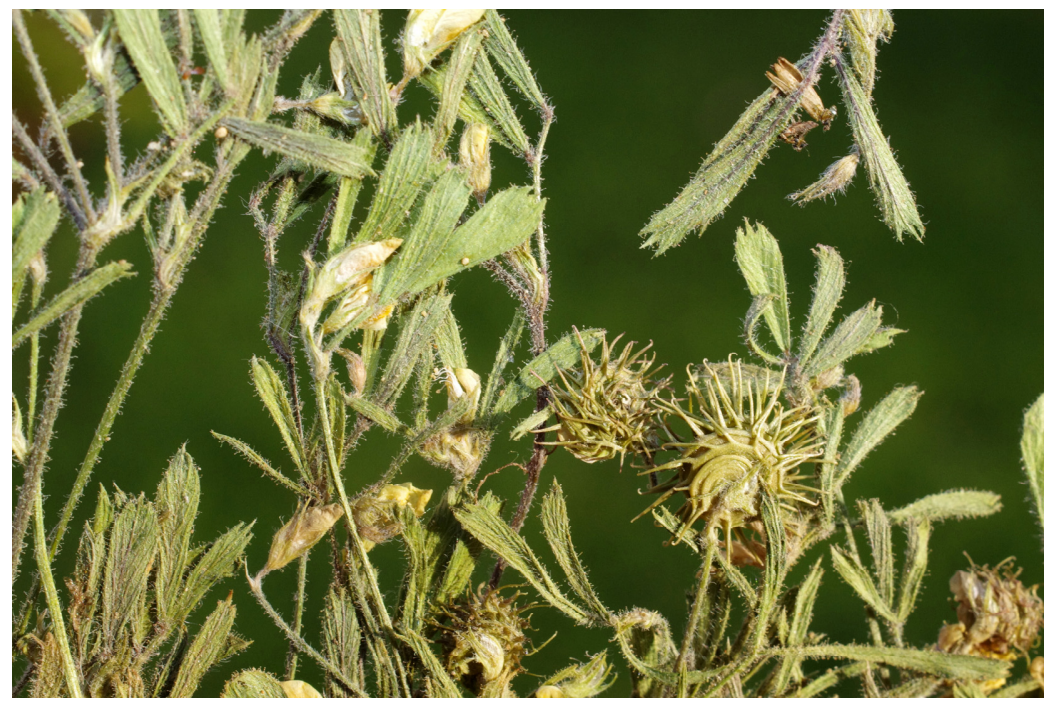




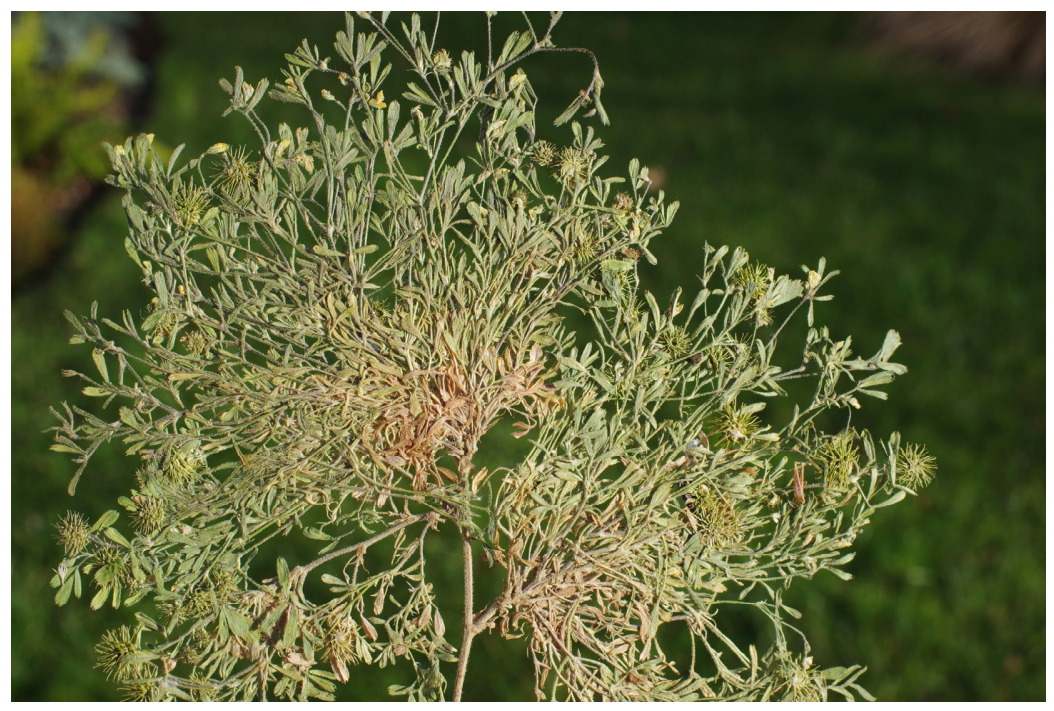

Die gesamte Pflanze ist dicht drüsenhaarig. Blättchen 6-7 mm x 1,1-2,1 mm, schmal keilförmig, Apex scharfdreizähnig, die äußeren Zähne nach innen geneigt (0,5-0,8 mm lang). Nebenblättchen lanzettlich, nicht gezähnt. Blüten 1-2, Kronblätter 5-6,5 mm, Kelch 3,5-5,5, Kelchzähne 2,5-3,5 mm, Früchte 12-15 mm-gestielt.

In keiner der uns zur Verfügung stehenden Veröffentlichungen fanden wir Hinweise auf eine Art, die auch nur näherungsweise der gefundenen Pflanze entsprechen. Lediglich die Früchte erinnern stark an Medicago minima. Da wir die Besonderheit im Gelände nicht erkannt haben, haben wir nicht darauf geachtet, wieviele Pflanze dieses Typus am Fundort existierten, und haben leider nur diese eine Pflanze entnommen. 


\section{Fundpunkte}

Die geographischen Daten wurden mit Hilfe eines Garmin GPS 72 ermittelt, die Höhenangaben mit Hilfe von Geoplaner überprüft und gegebenenfalls korrigiert. Die UTM-Daten wurden in der Form (z.B. Fundort 4) 34 S 05152654405181 notiert, werden von uns aber in der Form EK 10.55.22 verwendet. Wir haben vor vielen Jahren diese Form gewählt; mit ihr ist das gesamte Land auf einfache Art in $10 \times 10 \mathrm{~km}$-Quadranten aufgeteilt; das ermöglicht uns eine optimale Planung und Auswertung der Pflanzensammlung. Wenn wir in bereits bereiste Regionen fahren, haben wir für diese Region Listen der in jedem $10 \times 10$ km- bzw. $5 \times 5$ km-Quadranten bereits erfassten Arten vorbereitet. Damit können wir soweit wie möglich Sammel-Dubletten vermeiden.

\begin{tabular}{|c|c|c|c|}
\hline 1 & $\begin{array}{l}\text { GR, loannina, NO Dodoni, } 12.06 .2017 \\
\text { Felsige Krautfluren, } 675 \mathrm{~m} \\
\text { loa } 1125,39^{\circ} 33^{\prime} 37^{\prime \prime N}, 20^{\circ} 46^{\prime} 36^{\prime \prime E} 1\end{array}$ & DJ 87.09.80 & $276.290-276.332$ \\
\hline 2 & $\begin{array}{l}\text { GR, loannina, NO Dodoni, } 12.06 .2017 \\
\text { Laubgebüsch, } 645 \mathrm{~m} \\
\text { loa } 1126,39^{\circ} 33^{\prime} 19^{\prime \prime N}, 20^{\circ} 46^{\prime} 39^{\prime \prime} \mathrm{E}\end{array}$ & DJ 87.08.94 & $276.333-276.340$ \\
\hline 3 & $\begin{array}{l}\text { GR, loannina, Dodonoupoli, } 12.06 .2017 \\
\text { Krautfluren, Laubgebüsch, } 685 \mathrm{~m} \\
\text { loa } 1127,39^{\circ} 33^{\prime} 27^{\prime \prime} \mathrm{N}, 20^{\circ} 47^{\prime} 10^{\prime \prime} \mathrm{E}\end{array}$ & DJ 87.18.67 & $276.341-276.346$ \\
\hline 4 & $\begin{array}{l}\text { GR, loannina, N Metsovo, } 13.06 .2017 \\
\text { Bergwiesen, Juniperus, } 1415 \mathrm{~m} \\
\text { loa } 1055,39^{\circ} 47^{\prime} 47^{\prime \prime N}, 21^{\circ} 10^{\prime} 41^{\prime \prime E}\end{array}$ & EK 10.55.22 & $276.347-276.381$ \\
\hline 5 & $\begin{array}{l}\text { GR, loannina, NO Metsovo, } 13.06 .2017 \\
\text { Bergwiesen, Juniperus, } 1595 \mathrm{~m} \\
\text { loa } 1128,39^{\circ} 47^{\prime} 31^{\prime \prime N}, 21^{\circ} 12^{\prime} 17^{\prime \prime E}\end{array}$ & EK 10.74.57 & $276.382-276.401$ \\
\hline 6 & $\begin{array}{l}\text { GR, loannina, Katara-Pass, } 13.06 .2017 \\
\text { Buchenwald, Krautfluren, Felsfluren, } 1715 \mathrm{~m} \\
\text { loa } 1058,39^{\circ} 47^{\prime} 21^{\prime \prime N}, 21^{\circ} 13^{\prime} 38^{\prime \prime E}\end{array}$ & EK 10.94.54 & $276.402-276.427$ \\
\hline 7 & $\begin{array}{l}\text { GR, loannina, NO Metsovo, } 13.06 .2017 \\
\text { Quelle, nasser Graben unter Buchenwald, } 1655 \mathrm{~m} \\
\text { loa } 1057,39^{\circ} 47^{\prime} 29^{\prime \prime N}, 21^{\circ} 12^{\prime} 38^{\prime \prime E}\end{array}$ & EK 10.84.06 & $276.428-276.441$ \\
\hline 8 & $\begin{array}{l}\text { GR, Trikala, N Katara-Pass, } 13.06 .2017 \\
\text { Wasserüberlaufener Felshang, Krautfluren, } 1635 \mathrm{~m} \\
\text { Tri 456, 394} 48^{\prime} 13^{\prime \prime N}, 21^{\circ} 13^{\prime} 42^{\prime \prime} \mathrm{E}\end{array}$ & EK 10.96.50 & $276.442-276.450$ \\
\hline 9 & $\begin{array}{l}\text { GR, Trikala, NO Katara-Pass, } 13.06 .2017 \\
\text { Buchenwald, Krautfluren, } 1595 \mathrm{~m} \\
\text { Tri } 457,39^{\circ} 48^{\prime} 17^{\prime \prime N}, 21^{\circ} 14^{\prime} 00^{\prime E}\end{array}$ & EK 20.06.01 & $276.451-276.460$ \\
\hline 10 & $\begin{array}{l}\text { GR, Trikala, NO Katara-Pass, } 13.06 .2017 \\
\text { Steiniger Hang unter Nadelwald, } 1525 \mathrm{~m} \\
\text { Tri } 458,39^{\circ} 48^{\prime} 11^{\prime \prime N}, 21^{\circ} 14^{\prime} 34^{\prime \prime E}\end{array}$ & EK 20.05.89 & $276.461-276.472$ \\
\hline 11 & $\begin{array}{l}\text { GR, loannina, Ag. Nikolaos, } 14.06 .2017 \\
\text { Laubgebüsch, Krautfluren, } 544 \mathrm{~m} \\
\text { loa } 1059,39^{\circ} 42^{\prime} 111^{\prime \prime N}, 20^{\circ} 58^{\prime} 57 " \mathrm{E}\end{array}$ & DJ 99.84 .58 & $276.473-276.544$ \\
\hline 12 & $\begin{array}{l}\text { GR, loannina, SSO Potamia, } 14.06 .2017 \\
\text { Felshang unter Laubgebüsch, } 560 \mathrm{~m} \\
\text { loa } 1060,39^{\circ} 42^{\prime} 23^{\prime \prime N}, 20^{\circ} 58^{\prime} 47^{\prime \prime E}\end{array}$ & DJ 99.85.32 & $276.545-276.588$ \\
\hline
\end{tabular}




\begin{tabular}{|c|c|c|c|}
\hline 13 & $\begin{array}{l}\text { GR, loannina, SSO Potamia, } 14.06 .2017 \\
\text { Felshang unter Laubgebüsch, } 560 \mathrm{~m} \\
\text { loa } 1061,39^{\circ} 42^{\prime} 36^{\prime \prime N}, 20^{\circ} 58^{\prime} 40^{\prime \prime E}\end{array}$ & DJ 99.85.16 & $276.589-276.608$ \\
\hline 14 & $\begin{array}{l}\text { GR, loannina, SO Potamia, } 14.06 .2017 \\
\text { Laubwald, Quelle, } 550 \mathrm{~m} \\
\text { loa } 1062,39^{\circ} 42^{\prime} 51^{\prime \prime N}, 20^{\circ} 58^{\prime} 25^{\prime \prime E}\end{array}$ & DJ 99.76 .70 & $276.609-276.630$ \\
\hline 15 & $\begin{array}{l}\text { GR, loannina, W Potamia, } 14.06 .2017 \\
\text { Laubwald, nasser Graben, } 525 \mathrm{~m} \\
\text { loa } 1063,39^{\circ} 43^{\prime} 08^{\prime \prime} \mathrm{N}, 20^{\circ} 58^{\prime} 09^{\prime \prime} \mathrm{E}\end{array}$ & DJ 99.76.46 & $276.631-276.660$ \\
\hline 16 & $\begin{array}{l}\text { GR, loannina, ONO Karyes, } 14.06 .2017 \\
\text { Kies-Felshänge, Hartlaubgebüsch, } 565 \text { m } \\
\text { loa } 1064,39^{\circ} 44^{\prime} 25^{\prime \prime N}, 20^{\circ} 56^{\prime} 46^{\prime \prime E}\end{array}$ & DJ 99.58.49 & $276.661-276.674$ \\
\hline 17 & $\begin{array}{l}\text { GR, Ioannina, NO Karyes, } 14.06 .2017 \\
\text { Eichenwald, } 540 \mathrm{~m} \\
\text { loa } 1065,39^{\circ} 44^{\prime} 25^{\prime \prime N}, 20^{\circ} 56^{\prime} 19^{\prime \prime E}\end{array}$ & DJ 99.48.79 & $276.675-276.716$ \\
\hline 18 & $\begin{array}{l}\text { GR, loannina, NO Karyes, } 14.06 .2017 \\
\text { Felsiger Hang, Kieshaufen, } 600 \mathrm{~m} \\
\text { loa } 1066,39^{\circ} 44^{\prime} 111^{\prime \prime N}, 29^{\circ} 56^{\prime} 08^{\prime \prime E}\end{array}$ & QE.50.12.66 & $276.717-276.730$ \\
\hline 19 & $\begin{array}{l}\text { GR, loannina, NW Metsovo, } 15.06 .2017 \\
\text { Felskante, Buchenwald, } 1390 \mathrm{~m} \\
\text { loa } 1067,39^{\circ} 46^{\prime} 46^{\prime \prime N}, 21^{\circ} 10^{\prime} 07^{\prime \prime E}\end{array}$ & EK 10.43.43 & $276.731-276.737$ \\
\hline 20 & $\begin{array}{l}\text { GR, loannina, NW Metsovo, } 15.06 .2017 \\
\text { Bergwiesen, Quellbäche, } 1380 \mathrm{~m} \\
\text { loa 1068, 394 } 47^{\prime} 044^{\prime N}, 21^{\circ} 09^{\prime} 33^{\prime E} \mathrm{E}\end{array}$ & EK 10.33.68 & $276.738-276.757$ \\
\hline 21 & $\begin{array}{l}\text { GR, loannina, NW Metsovo, } 15.06 .2017 \\
\text { Bergwiesen, Quellbäche, Buchenwald, } 1430 \mathrm{~m} \\
\text { loa } 1069,39^{\circ} 47^{\prime} 00^{\prime N}, 21^{\circ} 09^{\prime} 12^{\prime \prime E}\end{array}$ & EK 10.33.17 & $276.758-276.784$ \\
\hline 22 & $\begin{array}{l}\text { GR, Ioannina, NNW Metsovo, } 15.06 .2017 \\
\text { Wiesen, Sumpfwiesen, } 1360 \mathrm{~m} \\
\text { loa } 1070,39^{\circ} 47^{\prime} 58^{\prime \prime} \mathrm{N}, 21^{\circ} 09^{\prime} 35^{\prime \prime} \mathrm{E}\end{array}$ & EK 10.35.75 & $276.785-276.840$ \\
\hline 23 & 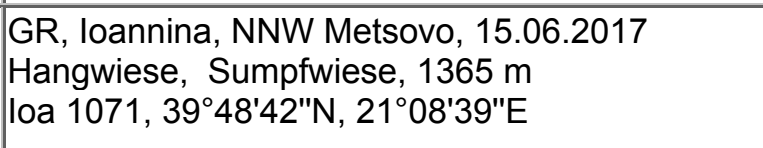 & EK 10.26.39 & $276.841-276.865$ \\
\hline 24 & $\begin{array}{l}\text { GR, loannina, NNW Metsovo, } 15.06 .2017 \\
\text { Buchenwald, Hangwiese, Bäche, } 1370 \text { m } \\
\text { loa } 1072,39^{\circ} 48^{\prime} 51^{\prime \prime N}, 21^{\circ} 08^{\prime} 27^{\prime E} E\end{array}$ & EK 10.27.11 & $276.866-276.896$ \\
\hline 25 & $\begin{array}{l}\text { GR, loannina, NNW Metsovo, } 15.06 .2017 \\
\text { Buchenwald, Hangwiese, Bäche, } 1370 \mathrm{~m} \\
\text { loa } 1073,39^{\circ} 49^{\prime} 15^{\prime \prime N}, 21^{\circ} 07^{\prime} 57^{\prime \prime E}\end{array}$ & EK 10.17.39 & $276.897-276.918$ \\
\hline 26 & $\begin{array}{l}\text { GR, loannina, NNW Metsovo, } 15.06 .2017 \\
\text { Felsige Krautfluren unter Buchenwald, } 1385 \mathrm{~m} \\
\text { loa } 1074,39^{\circ} 49^{\prime} 03^{\prime \prime N}, 21^{\circ} 07^{\prime} 29^{\prime \prime E}\end{array}$ & EK 10.07.75 & $276.919-276.935$ \\
\hline 27 & $\begin{array}{l}\text { GR, loannina, NW Anilio, } 16.06 .2017 \\
\text { Felshang, Laubgebüsch, } 925 \mathrm{~m} \\
\text { loa } 1075,39^{\circ} 45^{\prime} 42^{\prime \prime N}, 21^{\circ} 10^{\prime} 59^{\prime \prime E}\end{array}$ & EK 10.51.73 & $276.936-276.945$ \\
\hline 28 & $\begin{array}{l}\text { GR, loannina, ONO Anilio, } 16.06 .2017 \\
\text { Straßenrand, Tannenwald, } 940 \mathrm{~m} \\
\text { loa } 1076,39^{\circ} 45^{\prime} 25^{\prime \prime N}, 21^{\circ} 11^{\prime} 13^{\prime \prime E}\end{array}$ & EK 10.60.08 & $276.946-276.955$ \\
\hline
\end{tabular}




\begin{tabular}{|c|c|c|c|}
\hline & $\begin{array}{l}\text { GR, loannina, S Anilio, } 16.06 .2017 \\
\text { Laubgebüsch, } 1100 \mathrm{~m} \\
\text { loa } 1077,39^{\circ} 45^{\prime} 45^{\prime \prime N}, 21^{\circ} 10^{\prime} 58^{\prime \prime} \mathrm{E}\end{array}$ & EK 10.51.74 & $276.956-276.957$ \\
\hline 30 & $\begin{array}{l}\text { GR, loannina, O Anilio, } 16.06 .2017 \\
\text { Tannenwald, } 1370 \mathrm{~m} \\
\text { loa } 1078,39^{\circ} 45^{\prime} 25^{\prime \prime} \mathrm{N}, 21^{\circ} 11^{\prime} 55^{\prime \prime} \mathrm{E}\end{array}$ & EK 10.70.08 & $276.958-276.969$ \\
\hline 31 & $\begin{array}{l}\text { GR, loannina, SO Anilio, } 16.06 .2017 \\
\text { Felsanriss unter Kiefern, } 1620 \mathrm{~m} \\
\text { loa } 1079,39^{\circ} 44^{\prime} 52^{\prime \prime N}, 21^{\circ} 13^{\prime} 2 \text { "E }\end{array}$ & EJ 19.99.18 & 276.970 - 277.007 \\
\hline 32 & $\begin{array}{l}\text { GR, loannina, SO Anilio, } 16.06 .2017 \\
\text { Bergwiesen, kuhbeweidet, } 1660 \mathrm{~m} \\
\text { loa } 1080,9,39^{\circ} 44^{\prime} 52^{\prime \prime N}, 21^{\circ} 13^{\prime} 35^{\prime E} \mathrm{E}\end{array}$ & EJ 19.99.48 & $277.008-277.074$ \\
\hline 33 & $\begin{array}{l}\text { GR, loannina, W Metsovo, } 16.06 .2017 \\
\text { Felsanriss, Krautfluren, } 1165 \mathrm{~m} \\
\text { loa } 1081,39^{\circ} 46^{\prime} 03^{\prime \prime N}, 21^{\circ} 10^{\prime} 06^{\prime \prime E}\end{array}$ & EK 10.42.40 & $277.075-277.090$ \\
\hline 34 & $\begin{array}{l}\text { GR, loannina, Metsovo, 17.06.2017 } \\
\text { Kiefernwald, nasser Hang, } 1355 \mathrm{~m} \\
\text { loa 1082, 39²46'38"N, } 21^{\circ} 10^{\prime} 20^{\prime \prime} \mathrm{E}\end{array}$ & EK 10.43.70 & \\
\hline 35 & $\begin{array}{l}\text { GR, loannina, NW Metsovo, } 17.06 .2017 \\
\text { Bergwiesen, Wacholder, } 1420 \mathrm{~m} \\
\text { loa } 1083,39^{\circ} 48^{\prime} 01^{\prime \prime N}, 21^{\circ} 10^{\prime} 31^{\prime \prime E}\end{array}$ & EK 10.55.06 & $277.091-277.095$ \\
\hline 36 & $\begin{array}{l}\text { GR, loannina, N Metsovo, } 17.06 .2017 \\
\text { Sumpfwiesen, } 1380 \mathrm{~m} \\
\text { loa } 1084,39^{\circ} 48^{\prime} 29^{\prime \prime} \mathrm{N}, 21^{\circ} 10^{\prime} 13^{\prime \prime} \mathrm{E}\end{array}$ & EK 10.46.65 & $277.096-277.145$ \\
\hline 37 & $\begin{array}{l}\text { GR, loannina, N Metsovo, } 17.06 .2017 \\
\text { Bergwiesen, Sumpfwiesen, } 1380 \mathrm{~m} \\
\text { loa } 1085,39^{\circ} 49^{\prime} 18^{\prime \prime N}, 21^{\circ} 10^{\prime} 41^{\prime \prime E}\end{array}$ & EK 10.58.20 & $277.146-277.154$ \\
\hline 38 & $\begin{array}{l}\text { GR, loannina, NNO Metsovo, } 17.06 .2017 \\
\text { Steinige Krautfluren, Buchenwald, } 1485 \mathrm{~m} \\
\text { loa } 1086,39^{\circ} 50^{\prime} 05 " \mathrm{~N}, 21^{\circ} 11^{\prime} 26^{\prime \prime E}\end{array}$ & EK 10.69.34 & $277.155-277.189$ \\
\hline 39 & $\begin{array}{l}\text { GR, loannina, NNO Metsovo, 17.06.2017 } \\
\text { Buchenwald, } 1530 \mathrm{~m} \\
\text { loa } 1087,39^{\circ} 50^{\prime} 15^{\prime \prime N}, 21^{\circ} 11^{\prime} 45^{\prime \prime E}\end{array}$ & EK 10.69.87 & $277.190-277.204$ \\
\hline 40 & $\begin{array}{l}\text { GR, loannina, NNO Metsovo, 17.06.2017 } \\
\text { Buchenwald, } 1560 \mathrm{~m} \\
\text { loa } 1088,39^{\circ} 50^{\prime} 26^{\prime \prime} \mathrm{N}, 21^{\circ} 11^{\prime} 34^{\prime E} \mathrm{E}\end{array}$ & EK 11.60.51 & $277.205-277.223$ \\
\hline 41 & $\begin{array}{l}\text { GR, loannina, NNO Metsovo, } 17.06 .2017 \\
\text { Buchenwald, } 1540 \mathrm{~m} \\
\text { loa } 1089,39^{\circ} 50^{\prime} 30^{\prime \prime} \mathrm{N}, 21^{\circ} 11^{\prime} 37^{\prime \prime E}\end{array}$ & EK 11.60.62 & $277.224-277.258$ \\
\hline 42 & $\begin{array}{l}\text { GR, loannina, NNO Metsovo, } 17.06 .2017 \\
\text { Buchen-Kiefernwald, } 1535 \mathrm{~m} \\
\text { loa } 1090,39^{\circ} 50^{\prime} 34^{\prime \prime N}, 21^{\circ} 11^{\prime} 40^{\prime E}\end{array}$ & EK 11.60.63 & $277.259-277.284$ \\
\hline 43 & $\begin{array}{l}\text { GR, loannina, SW Milia, 17.06.2017 } \\
\text { Bergwiesen, } 1490 \mathrm{~m} \\
\text { loa 1091, 3950'37"N, } 21^{\circ} 12^{\prime} 02^{\prime \prime E}\end{array}$ & EK 11.70.24 & $277.285-277.311$ \\
\hline 44 & $\begin{array}{l}\text { GR, loannina, NO Metsovo, } 17.06 .2017 \\
\text { Buchen-Tannenwald, felsige Böschung, } 1480 \mathrm{~m} \\
\text { loa } 1092,39^{\circ} 50^{\prime} 24 " \mathrm{~N}, 21^{\circ} 11^{\prime} 41^{\prime \prime E}\end{array}$ & EK 11.60.70 & $277.312-277.323$ \\
\hline
\end{tabular}




\begin{tabular}{|c|c|c|c|}
\hline 45 & $\begin{array}{l}\text { GR, loannina, S Anilio, } 18.06 .2017 \\
\text { Felswand, Hartlaubgebüsch, } 995 \mathrm{~m} \\
\text { loa } 1093,39^{\circ} 44^{\prime} 48^{\prime \prime N}, 21^{\circ} 10^{\prime} 35^{\prime \prime E}\end{array}$ & EJ 19.59.17 & $277.324-277.389$ \\
\hline 46 & $\begin{array}{l}\text { GR, loannina, S Anilio, 18.06.2017 } \\
\text { Felswand, Hartlaubgebüsch, Wassergraben, } 980 \text { m } \\
\text { loa 1094, } 39^{\circ} 44^{\prime} 42^{\prime \prime N}, 21^{\circ} 10^{\prime} 46^{\prime \prime E}\end{array}$ & EJ 19.59.45 & $277.390-277.421$ \\
\hline 47 & $\begin{array}{l}\text { GR, loannina, S Anilio, } 18.06 .2017 \\
\text { Feuchte Fels-Kieshänge, } 995 \mathrm{~m} \\
\text { loa } 1095,39^{\circ} 44^{\prime} 37^{\prime \prime N}, 21^{\circ} 11^{\prime} 00^{\prime E}\end{array}$ & EJ 19.59.73 & $277.422-277.455$ \\
\hline 48 & 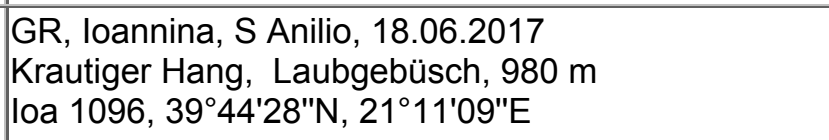 & EJ 19.59.90 & $277.456-277.474$ \\
\hline 49 & $\begin{array}{l}\text { GR, loannina, S Anilio, } 18.06 .2017 \\
\text { Tannenwald, Bachlauf, } 1050 \mathrm{~m} \\
\text { loa } 1097,39^{\circ} 44^{\prime} 21^{\prime \prime N}, 21^{\circ} 11^{\prime} 48^{\prime \prime E}\end{array}$ & EJ 19.68.98 & $277.475-277.539$ \\
\hline 50 & $\begin{array}{l}\text { GR, loannina, S Anilio, } 18.06 .2017 \\
\text { Buchen-Tannenwald, } 1165 \mathrm{~m} \\
\text { Ioa } 1098,39^{\circ} 43^{\prime} 56^{\prime \prime N}, 21^{\circ} 11^{\prime} 58^{\prime \prime E}\end{array}$ & EJ 19.78.11 & $277.540-277.553$ \\
\hline 51 & 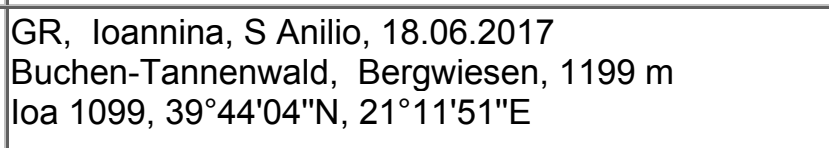 & EJ 19.68.93 & $277.554-277.570$ \\
\hline 53 & $\begin{array}{l}\text { GR, loannina, S Anilio, } 18.06 .2017 \\
\text { Buchenwald, } 1150 \mathrm{~m} \\
\text { loa } 1100,39^{\circ} 43^{\prime} 53^{\prime \prime} \mathrm{N}, 21^{\circ} 11^{\prime} 51^{\prime \prime} \mathrm{E}\end{array}$ & EJ 19.68.90 & $277.571-277.589$ \\
\hline 53 & $\begin{array}{l}\text { GR, loannina, S Anilio, } 19.06 .2017 \\
\text { Buchenwald, } 1295 \mathrm{~m} \\
\text { loa } 1101,39^{\circ} 43^{\prime} 48^{\prime \prime} \mathrm{N}, 21^{\circ} 11^{\prime} 50^{\prime \prime} \mathrm{E}\end{array}$ & EJ 19.67.98 & $277.590-277.594$ \\
\hline 54 & $\begin{array}{l}\text { GR, loannina, S Anilio, } 19.06 .2017 \\
\text { Felshang, Juniperus, } 1350 \mathrm{~m} \\
\text { loa 1102, 394 } 43^{\prime} 566^{\prime \prime N}, 21^{\circ} 11^{\prime} 35^{\prime \prime E}\end{array}$ & EJ 19.68.51 & $277.595-277.604$ \\
\hline 55 & $\begin{array}{l}\text { GR, loannina, S Anilio, } 19.06 .2017 \\
\text { Bergwiesen, Buchenwald, } 1460 \mathrm{~m} \\
\text { Ioa } 1103,39^{\circ} 43^{\prime} 29^{\prime \prime N}, 21^{\circ} 11^{\prime} 38^{\prime \prime E}\end{array}$ & EJ 19.67.62 & $277.605-277.619$ \\
\hline 56 & $\begin{array}{l}\text { GR, loannina, S Anilio, } 19.06 .2017 \\
\text { Bergwiesen, Teich, } 1630 \mathrm{~m} \\
\text { Ioa } 1104,39^{\circ} 42^{\prime} 56^{\prime \prime N}, 21^{\circ} 11^{\prime} 40^{\prime E} \mathrm{E}\end{array}$ & EJ 19.66.72 & $277.620-277.642$ \\
\hline
\end{tabular}

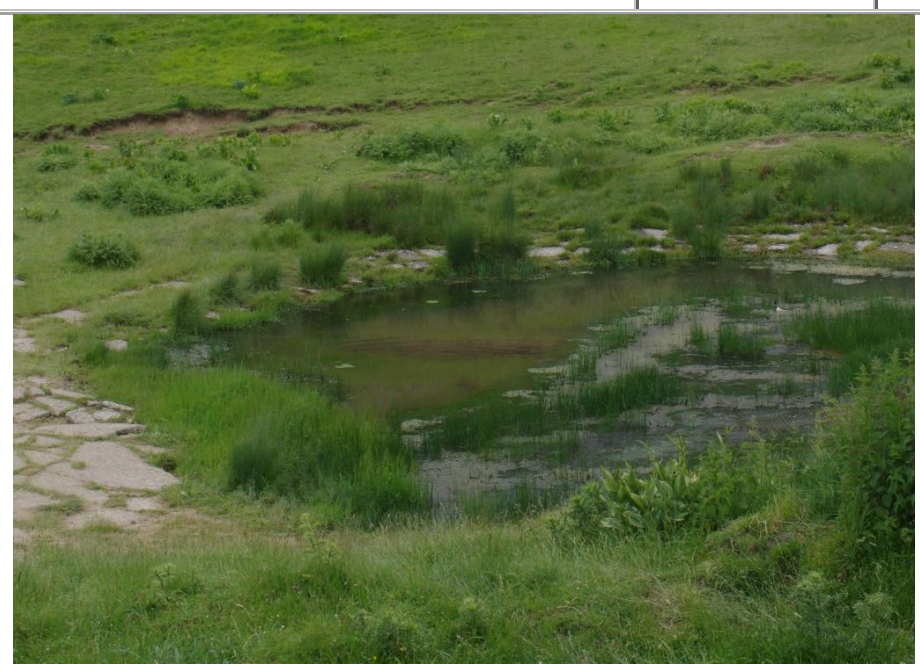




\begin{tabular}{|c|c|c|c|}
\hline 57 & $\begin{array}{l}\text { GR, loannina, Anilio, 19.06.2017 } \\
\text { Bergwiesen, } 1635 \mathrm{~m} \\
\text { Ioa } 1105,39^{\circ} 42^{\prime} 49^{\prime \prime N}, 21^{\circ} 11^{\prime} 32^{\prime E} \mathrm{E}\end{array}$ & EJ 19.66.50 & $277.643-277.650$ \\
\hline 58 & $\begin{array}{l}\text { GR, loannina, Anilio, } 19.06 .2017 \\
\text { Bergwiesen, } 1620 \mathrm{~m} \\
\text { Ioa } 1106,39^{\circ} 42^{\prime} 37^{\prime \prime N}, 21^{\circ} 11^{\prime} 39^{\prime \prime E}\end{array}$ & EJ 19.65.66 & $277.651-277.661$ \\
\hline 59 & $\begin{array}{l}\text { GR, Trikala, N Chaliki, 19.06.2017 } \\
\text { Bergwiesen, } 1565 \mathrm{~m} \\
\text { Tri 459, 3942'24"N, } 21^{\circ} 11^{\prime} 53^{\prime \prime E}\end{array}$ & EJ 19.75.02 & 277.662 - 277.668 \\
\hline 60 & $\begin{array}{l}\text { GR, Trikala, N Chaliki, } 19.06 .2017 \\
\text { Krautig-felsige Hänge, Adlerfarn, } 1420 \mathrm{~m} \\
\text { Tri } 460,39^{\circ} 42^{\prime} 11 " \mathrm{~N}, 21^{\circ} 11^{\prime} 53^{\prime \prime E}\end{array}$ & EJ 19.74.08 & $277.669-277.738$ \\
\hline 61 & $\begin{array}{l}\text { GR, Trikala, N Chaliki, 19.06.2017 } \\
\text { Felsige Hänge, sehr nass, Juniperus, } 1380 \mathrm{~m} \\
\text { Tri } 461,39^{\circ} 42^{\prime} 11^{\prime \prime N}, 21^{\circ} 11^{\prime} 41^{\prime \prime E}\end{array}$ & EJ 19.64.78 & $277.739-277.761$ \\
\hline 62 & $\begin{array}{l}\text { GR, Trikala, N Chaliki, } 19.06 .2017 \\
\text { Hangwiesen, Juniperus, } 1240 \mathrm{~m} \\
\text { Tri } 462,39^{\circ} 41^{\prime} 50^{\prime \prime N}, 21^{\circ} 11^{\prime} 03^{\prime \prime E}\end{array}$ & EJ 19.54.82 & $277.762-277.778$ \\
\hline 63 & $\begin{array}{l}\text { GR, Kozanis, SO Siatista, 20.06.2017 } \\
\text { Ackerrand, Acker, Hartlaubgebüsch, } 760 \mathrm{~m} \\
\text { Koz 385, } 40^{\circ} 13^{\prime} 54^{\prime \prime N}, 21^{\circ} 35^{\prime} 53^{\prime \prime E}\end{array}$ & EK 540994 & $277.779-277.805$ \\
\hline 64 & $\begin{array}{l}\text { GR, Kozanis, SO Siatista, } 20.06 .2017 \\
\text { Blumenreiche Felsfluren, } 800 \mathrm{~m} \\
\text { Koz 386, } 40^{\circ} 13^{\prime} 53^{\prime \prime} \mathrm{N}, 21^{\circ} 36^{\prime} 28^{\prime \prime} \mathrm{E}\end{array}$ & EK 551376 & $277.806-277.811$ \\
\hline 65 & $\begin{array}{l}\text { GR, Kozanis, SO Siatista, Vourinos, 20.06.2017 } \\
\text { Blumenreiche Felsfluren, } 950 \mathrm{~m} \\
\text { Koz } 387,40^{\circ} 13^{\prime} 42^{\prime \prime N}, 21^{\circ} 37^{\prime} 34^{\prime \prime E}\end{array}$ & EK 553333 & $277.812-277.859$ \\
\hline 66 & $\begin{array}{l}\text { GR, Kozanis, SO Siatista, Vourinos, } 20.06 .2017 \\
\text { Laubgebüsch, Bergwiesen, } 1065 \mathrm{~m} \\
\text { Koz } 388,40^{\circ} 13^{\prime} 28^{\prime \prime N}, 21^{\circ} 38^{\prime} 26^{\prime \prime E}\end{array}$ & EK 554259 & $277.860-277.887$ \\
\hline 67 & $\begin{array}{l}\text { GR, Kozanis, SO Siatista, Vourinos, 20.06.2017 } \\
\text { Wiesen, kuhbeweidet, } 1105 \mathrm{~m} \\
\text { Koz } 389,40^{\circ} 13^{\prime} 17^{\prime \prime N}, 21^{\circ} 38^{\prime} 42^{\prime \prime E}\end{array}$ & EK 554295 & $277.888-277.902$ \\
\hline
\end{tabular}

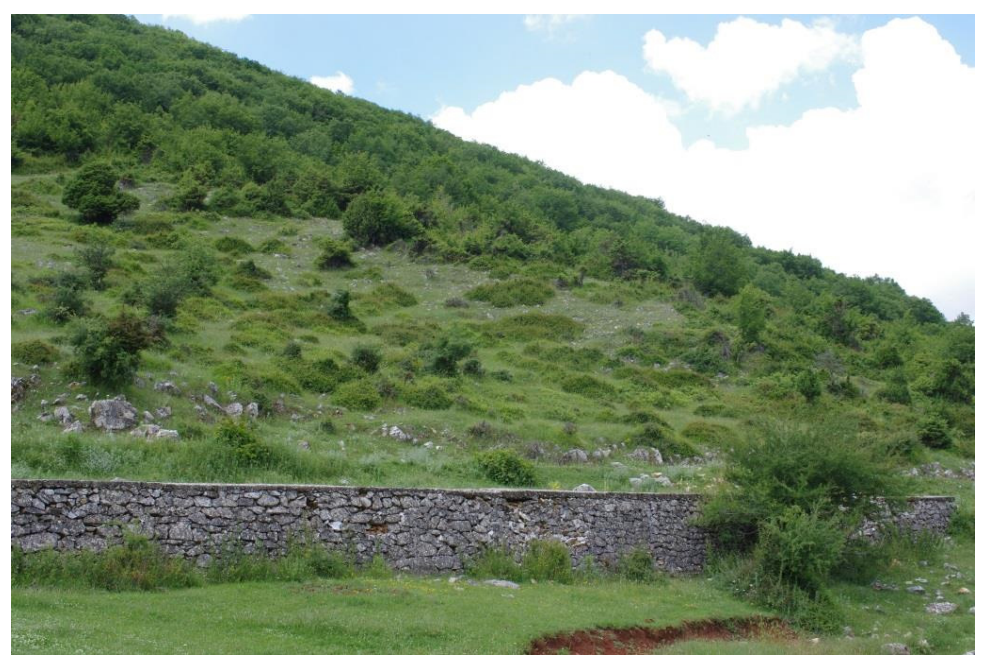




\begin{tabular}{|c|c|c|c|}
\hline 68 & $\begin{array}{l}\text { GR, Kozanis, SO Siatista, Vourinos, 20.06.2017 } \\
\text { Acker, } 1125 \mathrm{~m} \\
\text { Koz } 390,40^{\circ} 13^{\prime} 04 " \mathrm{~N}, 21^{\circ} 39^{\prime} 14^{\prime \prime E}\end{array}$ & EK 555261 & $277.903-277.915$ \\
\hline 69 & $\begin{array}{l}\text { GR, Kozanis, SO Siatista, Vourinos, 20.06.2017 } \\
\text { Eichengebüsch, Wiesen, } 1135 \mathrm{~m} \\
\text { Koz } 391,40^{\circ} 1^{\prime} \text { '"N, } 21^{\circ} 39^{\prime} 44^{\prime \prime E}\end{array}$ & EK 556145 & $277.916-277.922$ \\
\hline 70 & $\begin{array}{l}\text { GR, Kozanis, SO Siatista, Vourinos, 20.06.2017 } \\
\text { Laubgebüsch, Felsfluren, } 1330 \mathrm{~m} \\
\text { Koz } 392,40^{\circ} 11^{\prime} 58 " \mathrm{~N}, 21^{\circ} 39^{\prime} 29^{\prime \prime} \mathrm{E}\end{array}$ & EK 556001 & $\begin{array}{l}277.923-277.947 \\
277.981-277.992\end{array}$ \\
\hline 71 & $\begin{array}{l}\text { GR, Kozanis, Vourinos, } 20.06 .2017 \\
\text { Laubwald, Wiesen, } 1265 \mathrm{~m} \\
\text { Koz } 374,40^{\circ} 11^{\prime} 58^{\prime \prime N}, 21^{\circ} 39^{\prime} 38^{\prime \prime E}\end{array}$ & EK 556021 & 277.948 - 277.980 \\
\hline 72 & $\begin{array}{l}\text { GR, Kozanis, O Dafnero, 21.06.2017 } \\
\text { Fast bewuchslose Felshänge, Juniperus, } 655 \text { m } \\
\text { Koz } 375,40^{\circ} 11^{\prime} 05^{\prime \prime N}, 21^{\circ} 35^{\prime} 48^{\prime \prime E}\end{array}$ & EK 54.07.79 & $277.981-278.014$ \\
\hline
\end{tabular}

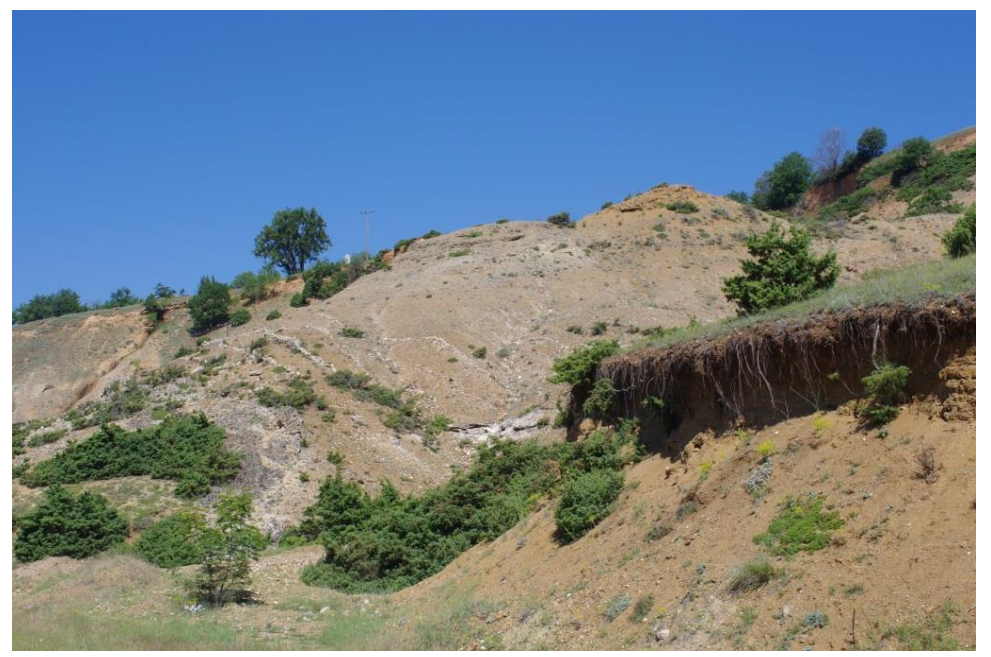

\begin{tabular}{|c|c|c|c|}
\hline 73 & $\begin{array}{l}\text { GR, Kozanis, W Exarcho, 21.06.2017 } \\
\text { Krautiger Felsanriss, } 705 \mathrm{~m} \\
\text { Koz } 376,40^{\circ} 09^{\prime} 32^{\prime \prime} \mathrm{N}, 21^{\circ} 37^{\prime} 30^{\prime \prime E}\end{array}$ & EK 54.35.26 & $278.015-278.035$ \\
\hline 74 & $\begin{array}{l}\text { GR, Grevena, NO Exarcho, } 21.06 .2017 \\
\text { Hangsumpf, Bergwiesen, } 870 \mathrm{~m} \\
\text { Gre } 491,40^{\circ} 10^{\prime} 04^{\prime \prime N}, 21^{\circ} 38^{\prime} 43^{\prime \prime E}\end{array}$ & EK 54.46.96 & $278.036-278.056$ \\
\hline
\end{tabular}




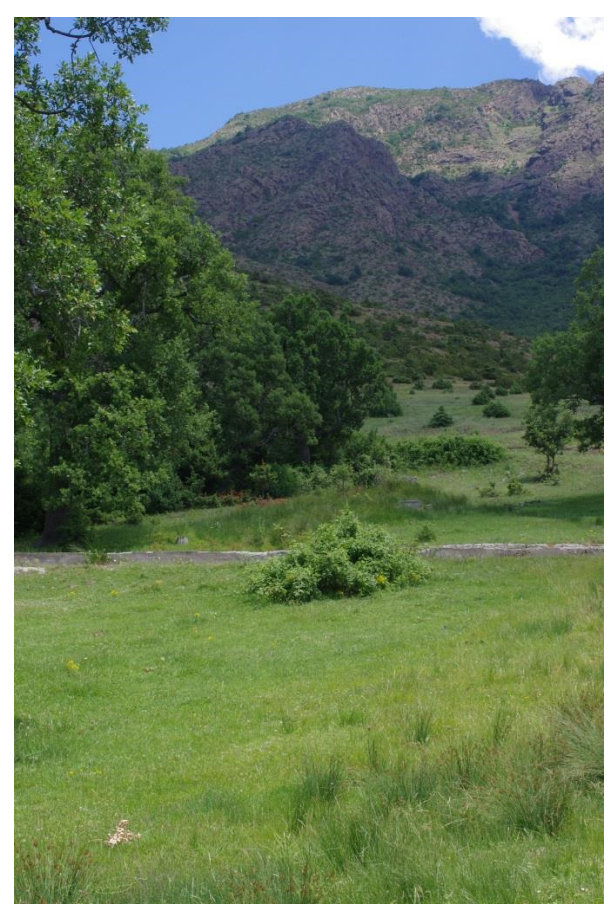

\begin{tabular}{|c|c|c|c|}
\hline 75 & $\begin{array}{l}\text { GR, Grevena, NO Varis, } 21.06 .2017 \\
\text { Steppenheide, } 750 \mathrm{~m} \\
\text { Gre } 492,46^{\circ} 07^{\prime} 43^{\prime \prime N}, 21^{\circ} 40^{\prime} 18^{\prime \prime E}\end{array}$ & ES.50.18.96 & $\begin{array}{l}278.057-278.119 \\
278.132-278.151\end{array}$ \\
\hline 76 & $\begin{array}{l}\text { GR, Grevena, NO Varis, } 21.06 .2017 \\
\text { Wiesen, Juniperus, } 815 \mathrm{~m} \\
\text { Gre } 493,40^{\circ} 08^{\prime} 10^{\prime \prime} \mathrm{N}, 21^{\circ} 40^{\prime} 45^{\prime \prime} \mathrm{E}\end{array}$ & EK 54.73.91 & $278.120-278.131$ \\
\hline 77 & $\begin{array}{l}\text { GR, Grevena, SO Varis, } 21.06 .2017 \\
\text { Ackerrand, Straßenrand, } 765 \mathrm{~m} \\
\text { Gre } 494,40^{\circ} 07^{\prime} 21^{\prime \prime N}, 21^{\circ} 40^{\prime} 17^{\prime \prime} \mathrm{E}\end{array}$ & EK 54.71.26 & $278.132-278.175$ \\
\hline 78 & $\begin{array}{l}\text { GR, Grevena, Varis, } 21.06 .2017 \\
\text { Alter Acker, Straßenbaukies, } 718 \mathrm{~m} \\
\text { Gre } 495,40^{\circ} 07^{\prime} 20^{\prime \prime} \mathrm{N}, 21^{\circ} 41^{\prime} 41^{\prime \prime} \mathrm{E}\end{array}$ & EK 54.91.26 & $278.176-278.193$ \\
\hline 79 & $\begin{array}{l}\text { GR, Kozanis, N Siatista, } 22.06 .2017 \\
\text { Felsfluren, } 995 \mathrm{~m} \\
\text { Koz } 377,40^{\circ} 16^{\prime} 05^{\prime \prime N}, 21^{\circ} 32^{\prime} 31^{\prime \prime E}\end{array}$ & EK 45.67.07 & $278.194-278.214$ \\
\hline 80 & $\begin{array}{l}\text { GR, Kozanis, N Siatista, 22.06.2017 } \\
\text { Felsfluren, } 1130 \mathrm{~m} \\
\text { Koz } 378,40^{\circ} 17^{\prime} 28^{\prime \prime N}, 21^{\circ} 32^{\prime} 47^{\prime \prime E}\end{array}$ & EK 46.60 .42 & $278.215-278.246$ \\
\hline 81 & $\begin{array}{l}\text { GR, Kozanis, Galatini, 22.06.2017 } \\
\text { Krautfluren, } 1005 \mathrm{~m} \\
\text { Koz } 379,40^{\circ} 19^{\prime} 23^{\prime \prime N}, 21^{\circ} 33^{\prime} 34^{\prime \prime E}\end{array}$ & EK 46.73.57 & 278.247 - 278.284 \\
\hline 82 & $\begin{array}{l}\text { GR, Kozanis, Galatini, 22.06.2017 } \\
\text { Ackerrand, Krautflurenn, } 1009 \mathrm{~m} \\
\text { Koz 380, 40⒈'46"N, } 21^{\circ} 33^{\prime} 48^{\prime \prime E}\end{array}$ & EK 46.74.34 & $278.285-278.309$ \\
\hline 83 & $\begin{array}{l}\text { GR, Kozanis, Galatini, } 22.06 .2017 \\
\text { Steinige Krautfluren, } 1030 \mathrm{~m} \\
\text { Koz } 381,40^{\circ} 20^{\prime} 16^{\prime \prime} \mathrm{N}, 21^{\circ} 33^{\prime} 54^{\prime \prime} \mathrm{E}\end{array}$ & EK 46.85.04 & $278.310-278.354$ \\
\hline 84 & $\begin{array}{l}\text { GR, Kozanis, Galatini, 22.06.2017 } \\
\text { Ackerrand, } 990 \mathrm{~m} \\
\text { Koz 382, } 40^{\circ} 20^{\prime} 47^{\prime \prime N}, 21^{\circ} 13^{\prime} 35^{\prime \prime} \mathrm{E}\end{array}$ & EK 46.76.93 & $278.355-278.388$ \\
\hline
\end{tabular}




\begin{tabular}{|c|c|c|c|}
\hline & $\begin{array}{l}\text { GR, Kozanis, Galatini, } 22.06 .2017 \\
\text { Eichengebüsch, Krautfluren, } 1025 \mathrm{~m} \\
\text { Koz 383, } 40^{\circ} 21^{\prime} 11^{\prime \prime N}, 21^{\circ} 34^{\prime} 10^{\prime \prime E}\end{array}$ & EK 46.87.31 & $278.389-278.414$ \\
\hline 86 & $\begin{array}{l}\text { GR, Kozanis, Galatini, } 22.06 .2017 \\
\text { Kraut- und Felsfluren, } 1035 \mathrm{~m} \\
\text { Koz } 384,40^{\circ} 18^{\prime} 52^{\prime \prime N}, 21^{\circ} 32^{\prime} 47^{\prime \prime} \mathrm{E}\end{array}$ & EK 46.62 .47 & $278.415-278.432$ \\
\hline 87 & $\begin{array}{l}\text { GR, Thessaloniki, Paralia Vrasna, 23.06.2017 } \\
\text { Ölbaumhain, Sandhaufen, } 10 \mathrm{~m} \\
\text { Thes } 214,40^{\circ} 41^{\prime 2} 26^{\prime \prime N}, 23^{\circ} 40^{\prime} 46^{\prime \prime} \mathrm{E}\end{array}$ & GL 20.67.49 & $278.433-278.466$ \\
\hline 88 & $\begin{array}{l}\text { GR, Thessaloniki, Paralia Vrasna, } 23.06 .2017 \\
\text { Laubgebüsch, alter Acker, Rubus, } 30 \mathrm{~m} \\
\text { Thes } 215,40^{\circ} 41^{\prime} 41^{\prime \prime N}, 23^{\circ} 40^{\prime} 03^{\prime \prime E}\end{array}$ & GL 20.58.43 & $278.467-278.491$ \\
\hline 89 & $\begin{array}{l}\text { GR, Thessaloniki, Vrasna, } 23.06 .2017 \\
\text { Felsfluren, } 114 \mathrm{~m} \\
\text { Thes } 216,20^{\circ} 42^{\prime} 00^{\prime \prime} \mathrm{N}, 23^{\circ} 38^{\prime} 38^{\prime \prime} \mathrm{E}\end{array}$ & GH.79.51.42 & $278.492-278.504$ \\
\hline 90 & $\begin{array}{l}\text { GR, Thessaloniki, Vrasna, } 23.06 .2017 \\
\text { Felsanriss, Krautfluren, } 152 \mathrm{~m} \\
\text { Thes } 217,40^{\circ} 42^{\prime} 39^{\prime \prime} \mathrm{N}, 23^{\circ} 38^{\prime} 49^{\prime \prime} \mathrm{E}\end{array}$ & GL 21.30.60 & $278.505-278.526$ \\
\hline 91 & $\begin{array}{l}\text { GR, Thessaloniki, Vrasna, 23.06.2017 } \\
\text { Felshänge, Hartlaubgebüsch, Platanen, } 230 \mathrm{~m} \\
\text { Thes } 218,40^{\circ} 42^{\prime} 43^{\prime \prime} \mathrm{N}, 23^{\circ} 38^{\prime} 12^{\prime \prime E}\end{array}$ & GL 21.20.71 & $278.527-278.538$ \\
\hline 92 & $\begin{array}{l}\text { GR, Thessaloniki, Vrasna, 23.06.2017 } \\
\text { Felshänge, Hartlaubgebüsch, Platanen, Quelle, } 255 \mathrm{~m} \\
\text { Thes } 219,40^{\circ} 42^{\prime} 43^{\prime \prime N}, 23^{\circ} 37^{\prime} 58^{\prime \prime E}\end{array}$ & GL 21.20.41 & $278.539-278.567$ \\
\hline 93 & $\begin{array}{l}\text { GR, Thessaloniki, Arethousa, 23.06.2017 } \\
\text { Acker, Ackerrand, } 363 \mathrm{~m} \\
\text { Thes } 220,40^{\circ} 44^{\prime} 40^{\prime \prime} \mathrm{N}, 23^{\circ} 34^{\prime} 20^{\prime \prime} \mathrm{E}\end{array}$ & GL 11.73.26 & 278.568 - 278.599 \\
\hline 94 & $\begin{array}{l}\text { GR, Thessaloniki, Arethousa, } 23.06 .2017 \\
\text { Acker, Laubgebüsch, } 355 \mathrm{~m} \\
\text { Thes } 221,40^{\circ} 46^{\prime} 00^{\prime \prime} \mathrm{N}, 23^{\circ} 32^{\prime} 39^{\prime \prime} \mathrm{E}\end{array}$ & GL 11.46.70 & $278.600-278.632$ \\
\hline 95 & $\begin{array}{l}\text { GR, Kavala, Paralia Ofryniou, } 24.06 .2017 \\
\text { Krautfluren, alter Acker, } 5 \mathrm{~m} \\
\text { Kav } 106,40^{\circ} 46^{\prime} 02^{\prime \prime N}, 23^{\circ} 54^{\prime} 21^{\prime \prime E}\end{array}$ & GL 41.47.80 & $278.633-278.666$ \\
\hline 96 & $\begin{array}{l}\text { GR, Kavala, Paralia Ofryniou, } 24.06 .2017 \\
\text { Sandige Krautfluren, alte Sumpfwiese, } 5 \text { m } \\
\text { Kav } 107,40^{\circ} 46^{\prime} 02^{\prime \prime N}, 23^{\circ} 53^{\prime} 45^{\prime \prime E}\end{array}$ & GL 41.47.40 & $278.667-278.713$ \\
\hline 97 & $\begin{array}{l}\text { GR, Kavala, Paralia Ofryniou, } 24.06 .2017 \\
\text { Sandstrand, strandnahe Krautfluren, } 5 \text { m } \\
\text { Kav } 108,40^{\circ} 46^{\prime} 23^{\prime \prime N}, 23^{\circ} 52^{\prime} 59^{\prime E}\end{array}$ & GL 41.37.36 & $278.714-278.737$ \\
\hline 98 & $\begin{array}{l}\text { GR, Kavala, Loutra Eleftherion, 24.06.2017 } \\
\text { Laubgebüsch, } 27 \mathrm{~m} \\
\text { Kav } 109,40^{\circ} 43^{\prime} 53^{\prime \prime} \mathrm{N}, 24^{\circ} 05^{\prime} 50^{\prime \prime} \mathrm{E}\end{array}$ & KF 51.43.90 & $278.738-278.758$ \\
\hline 99 & $\begin{array}{l}\text { GR, Kavala, Loutra Eleftherion, } 24.06 .2017 \\
\text { Kiefern-Laubwald, Schwefelquelle, } 20 \mathrm{~m} \\
\text { Kav } 110,40^{\circ} 44^{\prime} 13^{\prime \prime N}, 24^{\circ} 05^{\prime} 24^{\prime \prime E}\end{array}$ & KF 51.43.36 & $278.759-278.768$ \\
\hline 100 & $\begin{array}{l}\text { GR, Kavala, Loutra Eleftherion, } 24.06 .2017 \\
\text { Felsanriss, } 10 \mathrm{~m} \\
\text { Kav } 111,40^{\circ} 43^{\prime} 37^{\prime \prime N}, 24^{\circ} 05^{\prime} 54^{\prime \prime E}\end{array}$ & KF 51.42.95 & $278.769-278.771$ \\
\hline
\end{tabular}




\begin{tabular}{|c|c|c|c|}
\hline 101 & $\begin{array}{l}\text { GR, Kavala, NO Paralia Myrtofytou, 24.06.2017 } \\
\text { Felsanriss, steinige Krautfluren, } 60 \mathrm{~m} \\
\text { Kav } 112,40^{\circ} 46^{\prime} 49^{\prime \prime} \mathrm{N}, 24^{\circ} 12^{\prime} 18^{\prime \prime E}\end{array}$ & KF 61.48 .11 & $278.772-278.791$ \\
\hline 102 & $\begin{array}{l}\text { GR, Kavala, NO Paralia Myrtofytou, 24.06.2017 } \\
\text { Ölbaumhain, Krautfluren, } 95 \mathrm{~m} \\
\text { Kav } 113,40^{\circ} 47^{\prime} 16^{\prime \prime N}, 24^{\circ} 12^{\prime} 11^{\prime \prime E}\end{array}$ & KF 61.49 .00 & $278.792-278.807$ \\
\hline 103 & $\begin{array}{l}\text { GR, Kavala, SO Folia, } 24.06 .2017 \\
\text { Weinkultur, Nussbäume, } 193 \mathrm{~m} \\
\text { Kav } 114,40^{\circ} 48^{\prime} 08^{\prime \prime N}, 24^{\circ} 11^{\prime} 25^{\prime \prime E}\end{array}$ & KF 62.30.06 & $278.808-278.842$ \\
\hline 104 & $\begin{array}{l}\text { GR, Kavala, SW Folia, } 24.06 .2017 \\
\text { Laubgebüsch, } 250 \mathrm{~m} \\
\text { Kav } 115,40^{\circ} 47^{\prime} 01 " \mathrm{~N}, 24^{\circ} 08^{\prime} 30 " \mathrm{E}\end{array}$ & KF 51.88.87 & $278.843-278.876$ \\
\hline 105 & $\begin{array}{l}\text { GR, Kavala, S Ofrynia, } 25.06 .2017 \\
\text { Krautfluren, Ölbaumhain, } 60 \mathrm{~m} \\
\text { Kav } 116,40^{\circ} 46^{\prime} 57^{\prime \prime N}, 23^{\circ} 54^{\prime} 59^{\prime \prime E}\end{array}$ & GL 41.68 .17 & $278.877-278.912$ \\
\hline 106 & $\begin{array}{l}\text { GR, Kavala, Galipsos, } 25.06 .2017 \\
\text { Junger Ölbaumhain, Straßenrand, } 115 \mathrm{~m} \\
\text { Kav } 117,40^{\circ} 47^{\prime} 33^{\prime \prime N}, 23^{\circ} 566^{\prime} 21^{\prime \prime E}\end{array}$ & GL 41.89.09 & $278.913-278.930$ \\
\hline 107 & $\begin{array}{l}\text { GR, Kavala, Galipsos, } 25.06 .2017 \\
\text { Phrygana, Hartlaubgebüsch, } 202 \mathrm{~m} \\
\text { Kav 118, } 40^{\circ} 48^{\prime} 27^{\prime \prime N}, 23^{\circ} 58^{\prime} 49^{\prime \prime E}\end{array}$ & GL 52.11.47 & $278.931-278.953$ \\
\hline 108 & $\begin{array}{l}\text { GR, Kavala, Podochori, } 25.06 .2017 \\
\text { Alter Acker, } 265 \mathrm{~m} \\
\text { Kav } 119,40^{\circ} 49^{\prime} 32^{\prime \prime} \mathrm{N}, 24^{\circ} 01^{\prime} 17^{\prime \prime E}\end{array}$ & KF 42.83.87 & $278.954-278.024$ \\
\hline 109 & $\begin{array}{l}\text { GR, Kavala, Podochori, 25.06.2017 } \\
\text { Felsiger Hang, Ölbäume, Hartlaubgebüsch, } 257 \text { m } \\
\text { Kav } 120,40^{\circ} 50^{\prime} 02^{\prime \prime N}, 24^{\circ} 01^{\prime} 45^{\prime \prime E}\end{array}$ & KF 42.94.56 & $279.025-279.048$ \\
\hline 110 & $\begin{array}{l}\text { GR, Kavala, Platanotopos, 25.06.2017 } \\
\text { Hartlaubgebüsch, } 272 \mathrm{~m} \\
\text { Kav } 121,40^{\circ} 51^{\prime} 03 \text { "N, } 24^{\circ} 04^{\prime} 07^{\prime \prime E}\end{array}$ & KF 52.26.93 & $279.049-279.076$ \\
\hline 111 & $\begin{array}{l}\text { GR, Kavala, Misoropi, } 25.06 .2017 \\
\text { Laubgebüsch, Wasserrinne, } 265 \mathrm{~m} \\
\text { Kav } 89,40^{\circ} 51^{\prime} 36^{\prime \prime N}, 24^{\circ} 04^{\prime} 59^{\prime \prime E}\end{array}$ & KF 52.47.13 & $279.077-279.096$ \\
\hline 112 & $\begin{array}{l}\text { GR, Kavala, SW Moustheni, 25.06.2017 } \\
\text { Ackerrand, } 120 \mathrm{~m} \\
\text { Kav } 90,40^{\circ} 50^{\prime} 51^{\prime \prime N}, 24^{\circ} 06^{\prime} 08^{\prime \prime} \mathrm{E}\end{array}$ & KF 52.55.79 & $279.097-279.115$ \\
\hline 113 & $\begin{array}{l}\text { GR, Kavala, Orfani, } 25.06 .2017 \\
\text { Platanen, nasse Mulde, } 38 \mathrm{~m} \\
\text { Kav } 91,40^{\circ} 46^{\prime} 29^{\prime \prime N}, 23^{\circ} 57^{\prime} 08^{\prime \prime E}\end{array}$ & GL 41.97.19 & $279.116-279.137$ \\
\hline 114 & $\begin{array}{l}\text { GR, Serres, S Nea Mesolakkia, 26.06.2017 } \\
\text { Krautfluren, felskante, } 10 \mathrm{~m} \\
\text { Ser } 255,40^{\circ} 50^{\prime} 28^{\prime \prime N}, 23^{\circ} 51^{\prime} 03^{\prime \prime E}\end{array}$ & GL 42.05.30 & $279.138-279.170$ \\
\hline 115 & $\begin{array}{l}\text { GR, Serres, Paleokomi, } 26.06 .2017 \\
\text { Ackerrand, } 115 \mathrm{~m} \\
\text { Ser } 256,40^{\circ} 52^{\prime} 08^{\prime \prime N}, 23^{\circ} 53^{\prime} 38^{\prime \prime E}\end{array}$ & GL 42.38.92 & $279.171-279.195$ \\
\hline 116 & $\begin{array}{l}\text { GR, Serres, Nea Fili, 26.06.2017 } \\
\text { Krautfluren, Hartlaubgebüsch, } 250 \mathrm{~m} \\
\text { Ser } 257,40^{\circ} 52^{\prime} 31^{\prime \prime N}, 23^{\circ} 55^{\prime} 57^{\prime \prime E}\end{array}$ & GL 42.79.11 & $279.196-279.231$ \\
\hline
\end{tabular}




\begin{tabular}{|c|c|c|c|}
\hline 117 & $\begin{array}{l}\text { GR, Serres, Mikro Souli, } 26.06 .2017 \\
\text { Felskante, Hartlaubgebüsch, } 275 \mathrm{~m} \\
\text { Ser } 258,40^{\circ} 53^{\prime} 35^{\prime \prime N}, 23^{\circ} 56^{\prime} 25^{\prime E}\end{array}$ & GL 43.71.70 & $279.232-279.280$ \\
\hline 118 & $\begin{array}{l}\text { GR, Serres, Proti, } 26.06 .2017 \\
\text { Krautfluren, alter Acker, } 313 \mathrm{~m} \\
\text { Ser } 259,40^{\circ} 56^{\prime} 29^{\prime \prime} \mathrm{N}, 24^{\circ} 00^{\prime} 20^{\prime \prime} \mathrm{E}\end{array}$ & KF 43.76.96 & $279.281-279.312$ \\
\hline 119 & $\begin{array}{l}\text { GR, Serres, Proti, } 26.06 .2017 \\
\text { Felsige Krautfluren, Hartlaubgebüsch, } 415 \text { m } \\
\text { Ser } 260,40^{\circ} 56^{\prime} 22^{\prime \prime N}, 24^{\circ} 01^{\prime} 18^{\prime E}\end{array}$ & KF 43.96.33 & $279.313-279.336$ \\
\hline 120 & $\begin{array}{l}\text { GR, Serres, Proti, } 26.06 .2017 \\
\text { Felskante, Laubgebüsch, } 520 \mathrm{~m} \\
\text { Ser } 261,40^{\circ} 56^{\prime} 12^{\prime \prime} \mathrm{N}, 24^{\circ} 02^{\prime} 04^{\prime \prime} \mathrm{E}\end{array}$ & KF 53.06.30 & $279.337-279.373$ \\
\hline 121 & $\begin{array}{l}\text { GR, Serres, Proti, } 26.06 .2017 \\
\text { Felskante, Laubgebüsch, } 565 \mathrm{~m} \\
\text { Ser } 262,40^{\circ} 56^{\prime} 09^{\prime \prime N}, 24^{\circ} 02^{\prime} 18^{\prime \prime} \mathrm{E}\end{array}$ & KF 53.05.79 & $279.374-279.393$ \\
\hline 122 & $\begin{array}{l}\text { GR, Kavala, Pangaion, } 27.06 .2017 \\
\text { Quercus coccifera_Gebüsch, } 255 \mathrm{~m} \\
\text { Kav } 92,40^{\circ} 54^{\prime} 54^{\prime \prime} \mathrm{N}, 24^{\circ} 13^{\prime} 55^{\prime \prime} \mathrm{E}\end{array}$ & KF 63.63.90 & $279.394-279.413$ \\
\hline 123 & $\begin{array}{l}\text { GR, Kavala, Pangaion, } 27.06 .2017 \\
\text { Quercus coccifera_Gebüsch, } 350 \mathrm{~m} \\
\text { Kav } 93,40^{\circ} 54^{\prime} 55^{\prime \prime} \mathrm{N}, 24^{\circ} 13^{\prime} 35^{\prime \prime} \mathrm{E}\end{array}$ & KF 63.63.41 & 279.414- 279.423 \\
\hline 124 & $\begin{array}{l}\text { GR, Kavala, Pangaion, } 27.06 .2017 \\
\text { Eichen-Mischwald, } 515 \mathrm{~m} \\
\text { Kav } 94,40^{\circ} 55^{\prime} 15^{\prime \prime} \mathrm{N}, 24^{\circ} 19^{\prime} 01^{\prime \prime} \mathrm{E}\end{array}$ & KF 73.43.14 & $279.424-279.436$ \\
\hline 125 & $\begin{array}{l}\text { GR, Kavala, Pangaion, 27.06.2017 } \\
\text { Eichenwald, } 650 \mathrm{~m} \\
\text { Kav } 95,40^{\circ} 55^{\prime} 11^{\prime \prime N}, 24^{\circ} 12^{\prime} 08^{\prime E}\end{array}$ & KF 63.43 .46 & $279.437-279.464$ \\
\hline 126 & $\begin{array}{l}\text { GR, Kavala, Pangaion, 27.06.2017 } \\
\text { Kastanien-Eichen-Buchenwald, } 800 \text { m } \\
\text { Kav 96, 4055'05"N, 24¹1'41"E }\end{array}$ & KF 63.33.84 & $279.465-279.476$ \\
\hline 127 & $\begin{array}{l}\text { GR, Kavala, Pangaion, } 27.06 .2017 \\
\text { Eichen-Buchenwald, } 875 \mathrm{~m} \\
\text { Kav } 97,40^{\circ} 54^{\prime} 53^{\prime \prime N}, 24^{\circ} 11^{\prime} 29^{\prime E}\end{array}$ & KF 63.33.51 & $279.477-279.497$ \\
\hline 128 & 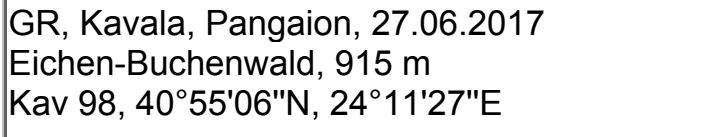 & KF 63.33.45 & $279.498-279.510$ \\
\hline 129 & $\begin{array}{l}\text { GR, Kavala, Pangaion, 27.06.2017 } \\
\text { Buchenwald, kiesige Lichtung, } 1020 \text { m } \\
\text { Kav 99, 4055'06"N, 2410'58"E }\end{array}$ & KF 63.23.85 & $\begin{array}{l}279.511-279.531 \\
279.545-279.547\end{array}$ \\
\hline 130 & $\begin{array}{l}\text { GR, Kavala, Pangaion, 27.06.2017 } \\
\text { Buchenwald, } 1097 \mathrm{~m} \\
\text { Kav } 100,40^{\circ} 55^{\prime} 19^{\prime \prime} \mathrm{N}, 24^{\circ} 10^{\prime} 33^{\prime \prime} \mathrm{E}\end{array}$ & KF 63.23.29 & $279.532-279.538$ \\
\hline 131 & $\begin{array}{l}\text { GR, Kavala, Pangaion, 27.06.2017 } \\
\text { Buchenwald, } 1196 \mathrm{~m} \\
\text { Kav 101, } 40^{\circ} 55^{\prime} 02^{\prime \prime} \mathrm{N}, 24^{\circ} 09^{\prime} 31^{\prime} \mathrm{E}\end{array}$ & KF 63.03.75 & $\begin{array}{l}279.539-279.544 \\
279.548-279.551\end{array}$ \\
\hline 132 & $\begin{array}{l}\text { GR, Kavala, Pangaion, 27.06.2017 } \\
\text { Buchenwald, Felshänge, } 1162 \text { m } \\
\text { Kav } 102,40^{\circ} 54^{\prime} 28^{\prime \prime N}, 24^{\circ} 07^{\prime} 28^{\prime \prime E}\end{array}$ & KF 53.72.85 & $279.552-279.566$ \\
\hline
\end{tabular}




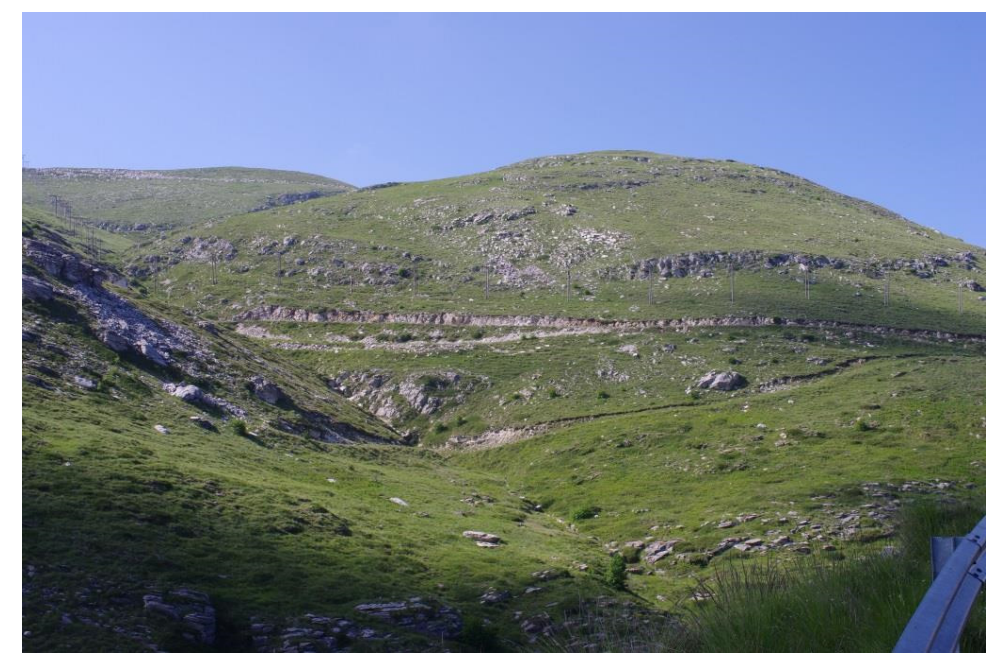

\begin{tabular}{|c|c|c|c|}
\hline 133 & $\begin{array}{l}\text { GR, Kavala, Pangaion, } 27.06 .2017 \\
\text { Felsfluren, } 1490 \mathrm{~m} \\
\text { Kav } 103,40^{\circ} 54^{\prime} 14^{\prime \prime N}, 24^{\circ} 07^{\prime} 15^{\prime \prime} \mathrm{E}\end{array}$ & KF 53.72.51 & $279.567-279.581$ \\
\hline 134 & $\begin{array}{l}\text { GR, Kavala, Pangaion, 27.06.2017 } \\
\text { Felsfluren, } 1540 \mathrm{~m} \\
\text { Kav } 104,40^{\circ} 54^{\prime} 08^{\prime \prime} \mathrm{N}, 24^{\circ} 06^{\prime} 51^{\prime \prime} \mathrm{E}\end{array}$ & KF 53.61.99 & $279.582-279.597$ \\
\hline 135 & $\begin{array}{l}\text { GR, Kavala, Pangaion, } 27.06 .2017 \\
\text { Felsfluren, } 1620 \mathrm{~m} \\
\text { Kav } 105,40^{\circ} 54^{\prime} 32^{\prime \prime} \mathrm{N}, 24^{\circ} 06^{\prime} 06^{\prime \prime} \mathrm{E}\end{array}$ & KF 53.52.97 & $279.598-279.609$ \\
\hline 136 & $\begin{array}{l}\text { GR, Serres, Dravoskas, 28.06.2017 } \\
\text { Krautfluren, Straßenböschung, } 67 \mathrm{~m} \\
\text { Ser } 263,40^{\circ} 54^{\prime} 31^{\prime \prime N}, 23^{\circ} 51^{\prime} 33^{\prime E}\end{array}$ & GL 43.02.85 & $279.610-279.643$ \\
\hline 137 & $\begin{array}{l}\text { GR, Serres, Dravoskas, 28.06.2017 } \\
\text { Krautfluren, Straßenböschung, } 87 \text { m } \\
\text { Ser } 264,40^{\circ} 55^{\prime} 53^{\prime \prime N}, 23^{\circ} 51^{\prime} 45 " \mathrm{E}\end{array}$ & GL 43.15.01 & $279.644-279.654$ \\
\hline 138 & $\begin{array}{l}\text { GR, Serres, Myrrini, } 28.06 .2017 \\
\text { Krautfluren, Laubgebüsch, Schilf, } 20 \text { m } \\
\text { Ser } 265,40^{\circ} 58^{\prime} 15^{\prime \prime N}, 23^{\circ} 52^{\prime} 32^{\prime E}\end{array}$ & GL 43.29.05 & $279.655-279.682$ \\
\hline 139 & $\begin{array}{l}\text { GR, Serres, SO Nea Zichni, 28.06.2017 } \\
\text { Ackerrand, Straßenböschung, } 210 \mathrm{~m} \\
\text { Ser } 266,41^{\circ} 00^{\prime} 38^{\prime \prime N}, 23^{\circ} 51^{\prime} 43^{\prime E}\end{array}$ & GL 44.03.79 & $279.683-279.716$ \\
\hline 140 & $\begin{array}{l}\text { GR, Serres, SW Skopia, 28.06.2017 } \\
\text { Ackerrand, Straßenböschung, } 335 \mathrm{~m} \\
\text { Ser } 267,41^{\circ} 02^{\prime} 30^{\prime \prime N}, 23^{\circ} 52^{\prime} 11^{\prime E}\end{array}$ & GL 44.17.24 & $279.717-279.737$ \\
\hline 141 & $\begin{array}{l}\text { GR, Serres, SW Skopia, 28.06.2017 } \\
\text { Krautfluren, Straßenböschung, } 560 \text { m } \\
\text { Ser } 268,41^{\circ} 04^{\prime} 32^{\prime \prime N}, 23^{\circ} 53^{\prime} 09^{\prime E}\end{array}$ & GL 45.21.42 & $279.738-279.793$ \\
\hline 142 & $\begin{array}{l}\text { GR, Serres, NO Skopia, } 28.06 .2017 \\
\text { Krautfluren, Kiefernwald, } 526 \mathrm{~m} \\
\text { Ser } 269,41^{\circ} 05^{\prime} 48^{\prime \prime N}, 23^{\circ} 54^{\prime} 08^{\prime \prime E}\end{array}$ & GL 45.33.75 & $279.794-279.825$ \\
\hline 143 & $\begin{array}{l}\text { GR, Drama, NO Skopia, } 28.06 .2017 \\
\text { Felsige Krautfluren, alter Acker, } 470 \mathrm{~m} \\
\text { Dra } 207,41^{\circ} 05^{\prime} 50^{\prime \prime} \mathrm{N}, 23^{\circ} 54^{\prime} 21^{\prime \prime} \mathrm{E}\end{array}$ & GL 45.43.06 & $279.826-279.841$ \\
\hline
\end{tabular}




\begin{tabular}{|c|c|c|c|}
\hline 144 & $\begin{array}{l}\text { GR, Drama, NO Skopia, } 28.06 .2017 \\
\text { Felsige Krautfluren, Acker, } 415 \mathrm{~m} \\
\text { Dra } 208,41^{\circ} 05^{\prime} 43^{\prime \prime} \mathrm{N}, 23^{\circ} 54^{\prime} 50^{\prime \prime} \mathrm{E}\end{array}$ & GL 45.43.74 & $279.842-279.850$ \\
\hline 145 & $\begin{array}{l}\text { GR, Drama, Livadero, } 29.06 .2017 \\
\text { Felskante, Krautfluren, Laubgebüsch, } 785 \text { m } \\
\text { Dra 209, 41 } 17^{\prime} 35^{\prime \prime N}, 24^{\circ} 12^{\prime} 50^{\prime \prime E}\end{array}$ & KF 67.65.70 & $279.851-279.898$ \\
\hline 146 & $\begin{array}{l}\text { GR, Drama, Livadero, 29.06.2017 } \\
\text { Felskante, Krautfluren, Laubgebüsch, } 810 \text { m } \\
\text { Dra } 210,41^{\circ} 17^{\prime} 41^{\prime \prime N}, 24^{\circ} 13^{\prime} 50^{\prime E} \mathrm{E}\end{array}$ & KF 67.85.12 & $279.899-279.917$ \\
\hline 147 & $\begin{array}{l}\text { GR, Drama, Livadero, } 29.06 .2017 \\
\text { Laubgebüsch, Quelle, } 840 \mathrm{~m} \\
\text { Dra } 211,41^{\circ} 17^{\prime} 58^{\prime \prime} \mathrm{N}, 24^{\circ} 13^{\prime} 16^{\prime \prime} \mathrm{E}\end{array}$ & KF 67.75.37 & $279.918-279.938$ \\
\hline 148 & $\begin{array}{l}\text { GR, Drama, Livadero, 29.06.2017 } \\
\text { Felsanriss, Laubgebüsch, } 856 \mathrm{~m} \\
\text { Dra } 212,41^{\circ} 18^{\prime} 04^{\prime \prime} \mathrm{N}, 24^{\circ} 13^{\prime} 21^{\prime \prime} \mathrm{E}\end{array}$ & KF 67.75 .59 & $279.939-279.957$ \\
\hline 149 & $\begin{array}{l}\text { GR, Drama, Livadero, } 29.06 .2017 \\
\text { Felsanriss, Laubgebüsch, } 878 \mathrm{~m} \\
\text { Dra } 213,41^{\circ} 18^{\prime} 26^{\prime \prime} \mathrm{N}, 24^{\circ} 13^{\prime} 01^{\prime \prime} \mathrm{E}\end{array}$ & KF 67.76.06 & $279.958-279.977$ \\
\hline 150 & $\begin{array}{l}\text { GR, Drama, Livadero, 29.06.2017 } \\
\text { Laubwald, } 868 \mathrm{~m} \\
\text { Dra } 214,41^{\circ} 18^{\prime} 45^{\prime \prime} \mathrm{N}, 24^{\circ} 13^{\prime} 12^{\prime \prime E}\end{array}$ & KF 67.77.32 & 279.978 - 279.992 \\
\hline 151 & $\begin{array}{l}\text { GR, Drama, Livadero, } 29.06 .2017 \\
\text { Krautfluren, Laubgebüsch, } 657 \mathrm{~m} \\
\text { Dra 215, 41 }{ }^{\circ} 19^{\prime} 52^{\prime \prime N}, 24^{\circ} 12^{\prime} 31^{\prime \prime} \mathrm{E}\end{array}$ & KF 67.69.43 & $279.993-280.003$ \\
\hline 152 & $\begin{array}{l}\text { GR, Drama, Sidironero, } 29.06 .2017 \\
\text { Felskante, Laubgebüsch, } 547 \mathrm{~m} \\
\text { Dra } 216,4^{\circ} 20^{\prime} 20^{\prime \prime} \mathrm{N}, 24^{\circ} 12^{\prime} 14^{\prime \prime} \mathrm{E}\end{array}$ & KF 68.60.02 & $280.004-280.041$ \\
\hline 153 & $\begin{array}{l}\text { GR, Drama, Sidironero, } 29.06 .2017 \\
\text { Laubwald, } 493 \mathrm{~m} \\
\text { Dra } 217,41^{\circ} 20^{\prime} 54^{\prime \prime N}, 24^{\circ} 11^{\prime} 38^{\prime \prime} \mathrm{E}\end{array}$ & KF 68.51.22 & $280.042-280.058$ \\
\hline 154 & $\begin{array}{l}\text { GR, Drama, Sidironero, 29.06.2017 } \\
\text { Laubwald, } 477 \mathrm{~m} \\
\text { Dra } 218,41^{\circ} 21^{\prime} 09^{\prime \prime} \mathrm{N}, 24^{\circ} 12^{\prime} 36^{\prime \prime} \mathrm{E}\end{array}$ & KF 68.61.66 & $280.059-280.072$ \\
\hline 155 & $\begin{array}{l}\text { GR, Drama, Sidironero, } 29.06 .2017 \\
\text { Felsfluren, Krautfluren, } 574 \mathrm{~m} \\
\text { Dra } 219,41^{\circ} 21^{\prime} 35^{\prime \prime N}, 24^{\circ} 13^{\prime} 18^{\prime \prime E}\end{array}$ & KF 68.72.64 & $280.073-280.095$ \\
\hline 156 & $\begin{array}{l}\text { GR, Drama, Sidironero, 29.06.2017 } \\
\text { Krautfluren, Laubgebüsch, Wacholder, } 698 \text { m } \\
\text { Dra } 220,41^{\circ} 21^{\prime} 58^{\prime \prime N}, 24^{\circ} 14^{\prime} 32^{\prime E}\end{array}$ & KF 68.93.31 & $280.096-280.122$ \\
\hline 157 & $\begin{array}{l}\text { GR, Drama, NO Drama, } 30.06 .2017 \\
\text { Felskante, Krautfluren, } 430 \mathrm{~m} \\
\text { Dra } 221,41^{\circ} 14^{\prime} 43^{\prime \prime N}, 24^{\circ} 12^{\prime} 17^{\prime \prime} \mathrm{E}\end{array}$ & KF 66.59.88 & $280.123-280.168$ \\
\hline 158 & $\begin{array}{l}\text { GR, Drama, NO Drama, } 30.06 .2017 \\
\text { Felskante, Krautfluren, } 420 \mathrm{~m} \\
\text { Dra } 222,41^{\circ} 14^{\prime} 32^{\prime \prime N}, 24^{\circ} 12^{\prime} 09^{\prime \prime} \mathrm{E}\end{array}$ & KF 66.59.64 & $280.169-280.175$ \\
\hline 159 & $\begin{array}{l}\text { GR, Drama, NO Drama, } 30.06 .2017 \\
\text { Felskante, Krautfluren, } 395 \mathrm{~m} \\
\text { Dra } 223,41^{\circ} 13^{\prime} 41^{\prime \prime N}, 24^{\circ} 11^{\prime} 30^{\prime \prime E}\end{array}$ & KF 66.47.69 & $280.176-280.196$ \\
\hline
\end{tabular}




\begin{tabular}{|c|c|c|c|}
\hline 160 & $\begin{array}{l}\text { GR, Drama, NO Drama, } 30.06 .2017 \\
\text { Krautfluren, Laubgebüsch, } 385 \mathrm{~m} \\
\text { Dra } 224,41^{\circ} 13^{\prime} 45^{\prime \prime} \mathrm{N}, 24^{\circ} 11^{\prime} 26^{\prime \prime} \mathrm{E}\end{array}$ & KF 66.48 .50 & $280.197-280.212$ \\
\hline 161 & $\begin{array}{l}\text { GR, Drama, NO Drama, } 30.06 .2017 \\
\text { Krautfluren, Hartlaubgebüsch, } 330 \mathrm{~m} \\
\text { Dra } 225,41^{\circ} 11^{\prime} 47^{\prime \prime} \mathrm{N}, 24^{\circ} 10^{\prime} 59^{\prime E}\end{array}$ & KF 66.34.84 & $280.213-280.229$ \\
\hline 162 & $\begin{array}{l}\text { GR, Drama, N Taxiarches, } 30.06 .2017 \\
\text { Steinige Krautfluren, } 450 \mathrm{~m} \\
\text { Dra } 226,41^{\circ} 13^{\prime} 41^{\prime \prime} \mathrm{N}, 24^{\circ} 11^{\prime} 04^{\prime \prime} \mathrm{E}\end{array}$ & KF 66.47.09 & $280.230-280.241$ \\
\hline 163 & $\begin{array}{l}\text { GR, Drama, Livadero, } 30.06 .2017 \\
\text { Laubwald, Laubgebüsch, } 460 \mathrm{~m} \\
\text { Dra } 227,41^{\circ} 15^{\prime} 31^{\prime \prime} \mathrm{N}, 24^{\circ} 12^{\prime} 16^{\prime \prime} \mathrm{E}\end{array}$ & KF 67.51 .82 & $280.242-280.262$ \\
\hline 164 & $\begin{array}{l}\text { GR, Drama, Livadero, 30.06.2017 } \\
\text { Eichenwald, } 675 \mathrm{~m} \\
\text { Dra } 228,41^{\circ} 15^{\prime} 37^{\prime \prime} \mathrm{N}, 24^{\circ} 11^{\prime} 01^{\prime \prime} \mathrm{E}\end{array}$ & KF 67.41 .15 & $280.263-280.296$ \\
\hline 165 & $\begin{array}{l}\text { GR, Drama, SO Dendrakia, 30.06.2017 } \\
\text { Eichenwald, } 760 \mathrm{~m} \\
\text { Dra } 229,41^{\circ} 15^{\prime} 49^{\prime \prime} \mathrm{N}, 24^{\circ} 11^{\prime} 07^{\prime \prime} \mathrm{E}\end{array}$ & KF 67.41.28 & $280.297-280.317$ \\
\hline 166 & $\begin{array}{l}\text { GR, Drama, Livadero, } 30.06 .2017 \\
\text { Eichenwald, } 795 \mathrm{~m} \\
\text { Dra } 230,41^{\circ} 15^{\prime} 49^{\prime \prime} \mathrm{N}, 24^{\circ} 10^{\prime} 54^{\prime \prime} \mathrm{E}\end{array}$ & KF 67.31.99 & $280.318-280.347$ \\
\hline 167 & $\begin{array}{l}\text { GR, Drama, Sidironero, } 01.07 .2017 \\
\text { Felsige Krautfluren, Laubgebüsch, } 775 \text { m } \\
\text { Dra } 231,41^{\circ} 22^{\prime} 31^{\prime \prime N}, 24^{\circ} 14^{\prime} 37^{\prime E}\end{array}$ & KF 68.94.51 & $280.348-280.357$ \\
\hline 168 & $\begin{array}{l}\text { GR, Drama, Sidironero, } 01.07 .2017 \\
\text { Eichen-Wacholderwald, } 830 \mathrm{~m} \\
\text { Dra } 232,41^{\circ} 22^{\prime} 44^{\prime \prime} \mathrm{N}, 24^{\circ} 15^{\prime} 34^{\prime \prime} \mathrm{E}\end{array}$ & KF 78.04.84 & $280.358-280.399$ \\
\hline 169 & $\begin{array}{l}\text { GR, Drama, Sidironero, } 01.07 .2017 \\
\text { Krautige Stellen in Laubwald, } 870 \mathrm{~m} \\
\text { Dra } 233,41^{\circ} 23^{\prime} 00^{\prime \prime} \mathrm{N}, 24^{\circ} 15^{\prime} 40^{\prime \prime} \mathrm{E}\end{array}$ & KF 78.14.09 & $280.400-280.430$ \\
\hline 170 & $\begin{array}{l}\text { GR, Drama, Skaloti, 01.07.2017 } \\
\text { Laub-Kiefernwald, Krautfluren am Ortsrand, } 955 \text { m } \\
\text { Dra } 234,41^{\circ} 24^{\prime} 38^{\prime \prime} \mathrm{N}, 24^{\circ} 16^{\prime} 46^{\prime E}\end{array}$ & KF 78.27.69 & $280.431-280.484$ \\
\hline 171 & $\begin{array}{l}\text { GR, Drama, Skaloti, 01.07.2017 } \\
\text { Kiefernwald, } 1165 \mathrm{~m} \\
\text { Dra } 235,41^{\circ} 25^{\prime} 42^{\prime \prime N}, 24^{\circ} 17^{\prime} 11^{\prime \prime E}\end{array}$ & KF 78.39.39 & $280.485-280.501$ \\
\hline 172 & $\begin{array}{l}\text { GR, Drama, Skaloti, 01.07.2017 } \\
\text { Mischwald, } 1240 \mathrm{~m} \\
\text { Dra 236, } 41^{\circ} 26^{\prime} 16^{\prime \prime N}, 24^{\circ} 16^{\prime} 51^{\prime \prime E}\end{array}$ & KF 79.20.89 & $280.502-280.543$ \\
\hline 173 & $\begin{array}{l}\text { GR, Drama, Skaloti, 01.07.2017 } \\
\text { Kiefernwald, } 1315 \mathrm{~m} \\
\text { Dra } 237,41^{\circ} 26^{\prime} 44^{\prime \prime N}, 24^{\circ} 16^{\prime} 37^{\prime \prime E}\end{array}$ & KF 79.21.58 & $280.544-280.563$ \\
\hline 174 & $\begin{array}{l}\text { GR, Drama, Skaloti, 01.07.2017 } \\
\text { Lichtung in Kiefernwald, } 1410 \mathrm{~m} \\
\text { Dra } 238,41^{\circ} 26^{\prime} 32^{\prime \prime N}, 24^{\circ} 17^{\prime} 00^{\prime E} \mathrm{E}\end{array}$ & KF 79.31.04 & $280.564-280.618$ \\
\hline 175 & $\begin{array}{l}\text { GR, Drama, Skaloti, } 02.07 .2017 \\
\text { Lichtung in Kiefernwald, } 1450 \mathrm{~m} \\
\text { Dra } 239,41^{\circ} 26^{\prime} 52^{\prime \prime N}, 24^{\circ} 17^{\prime} 43^{\prime \prime E}\end{array}$ & KF 79.42.10 & $280.619-280.624$ \\
\hline
\end{tabular}




\begin{tabular}{|l|l|l|l|}
\hline 176 & $\begin{array}{l}\text { GR, Drama, Elatias, 02.07.2017 } \\
\text { Lichtung in Kiefernwald, } 1415 \mathrm{~m} \\
\text { Dra 240, } 41^{\circ} 26^{\prime} 45^{\prime \prime} \mathrm{N}, 24^{\circ} 18^{\prime} 21^{\prime \prime} \mathrm{E}\end{array}$ & KF 79.41.98 & $280.625-280.634$ \\
\hline 177 & $\begin{array}{l}\text { GR, Drama, Elatias, 02.07.2017 } \\
\text { Wiesen in Kiefernwald, } 1440 \mathrm{~m} \\
\text { Dra 241, } 41^{\circ} 27^{\prime} 02 " \mathrm{~N}, 24^{\circ} 18^{\prime} 20^{\prime \prime} \mathrm{E}\end{array}$ & KF 79.42.93 & $280.635-280.647$ \\
\hline 178 & $\begin{array}{l}\text { GR, Drama, SW Walddorf Elatias, 02.07.2017 } \\
\text { Buchenwald, Wiesen, } 1530 \mathrm{~m} \\
\text { Dra 242, } 41^{\circ} 27^{\prime} 19^{\prime \prime N}, 24^{\circ} 18^{\prime} 29 " \mathrm{E}\end{array}$ & KF 79.52.28 & $280.648-280.701$ \\
\hline
\end{tabular}

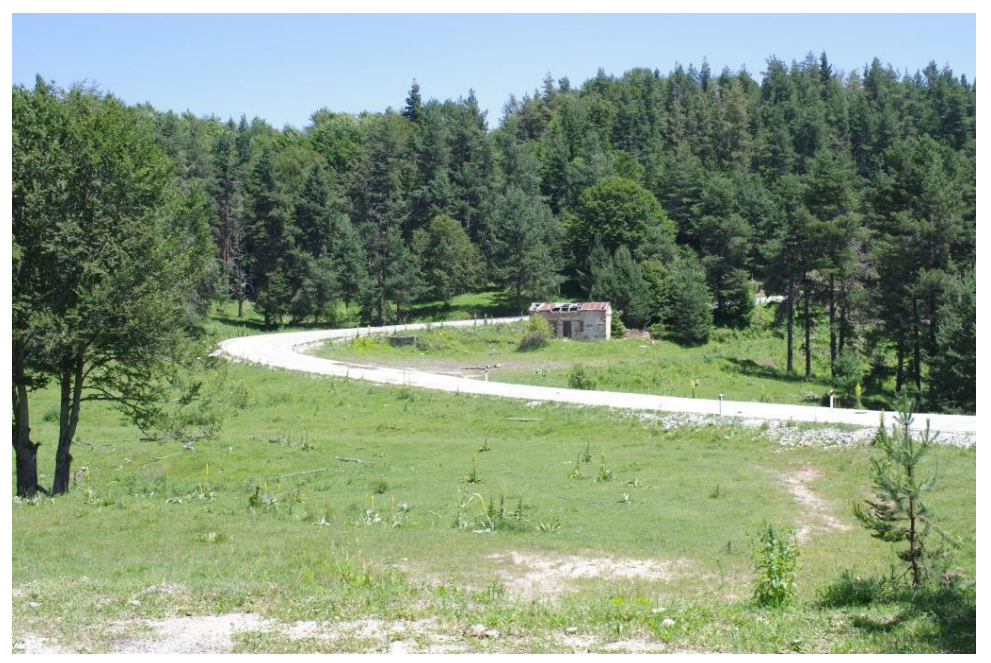

\begin{tabular}{|c|c|c|c|}
\hline & $\begin{array}{l}\text { GR, Drama, SW Walddorf Elatias, 02.07.2017 } \\
\text { Kiefernwald, } 1550 \mathrm{~m} \\
\text { Dra } 243,41^{\circ} 27^{\prime} 24^{\prime \prime N}, 24^{\circ} 18^{\prime} 45^{\prime \prime E}\end{array}$ & KF 79.52.59 & $280.702-280.724$ \\
\hline 180 & $\begin{array}{l}\text { GR, Drama, SW Walddorf Elatias, 02.07.2017 } \\
\text { Buchen-Fichtenwald, } 1570 \mathrm{~m} \\
\text { Dra } 244,41^{\circ} 27^{\prime} 49^{\prime \prime N}, 24^{\circ} 19^{\prime} 03^{\prime \prime E}\end{array}$ & KF 79.63.07 & $280.725-280.737$ \\
\hline 181 & $\begin{array}{l}\text { GR, Drama, SW Walddorf Elatias, 02.07.2017 } \\
\text { Schuttreiße in Fichtenwald, } 1560 \mathrm{mv6} \\
\text { Dra } 245,41^{\circ} 28^{\prime} 01^{\prime \prime N}, 24^{\circ} 19^{\prime} 15^{\prime \prime E}\end{array}$ & KF 79.64.31 & $280.738-280.756$ \\
\hline 182 & $\begin{array}{l}\text { GR, Drama, SW Walddorf Elatias, 02.07.2017 } \\
\text { Fichtenwald, Hangsumpf, } 1560 \mathrm{~m} \\
\text { Dra 246, } 241^{\circ} 28^{\prime} 222^{\prime \prime N}, 24^{\circ} 19^{\prime} 25^{\prime \prime E}\end{array}$ & KF 79.64.57 & $280.757-280.773$ \\
\hline 183 & $\begin{array}{l}\text { GR, Drama, Walddorf Elatias, } 02.07 .2017 \\
\text { Fichtenwald, Krautfluren, } 1545 \mathrm{~m} \\
\text { Dra } 247,41^{\circ} 28^{\prime} 49^{\prime \prime N}, 24^{\circ} 19^{\prime} 30^{\prime \prime E}\end{array}$ & KF 79.65.75 & $280.774-280.811$ \\
\hline 184 & $\begin{array}{l}\text { GR, Drama, Falakron, 03.07.2017 } \\
\text { Felsfluren, } 1365 \mathrm{~m} \\
\text { Dra } 248,41^{\circ} 17^{\prime} 25^{\prime \prime} \mathrm{N}, 24^{\circ} 01^{\prime} 42^{\prime \prime} \mathrm{E}\end{array}$ & KF 57.15.22 & $280.812-280.858$ \\
\hline 185 & $\begin{array}{l}\text { GR, Drama, Falakron, } 03.07 .2017 \\
\text { Felsfluren, } 1750 \mathrm{~m} \\
\text { Dra } 249,41^{\circ} 18^{\prime} 16^{\prime \prime} \mathrm{N}, 24^{\circ} 04^{\prime} 14^{\prime \prime E}\end{array}$ & KF 57.46.87 & $280.859-280.879$ \\
\hline 186 & $\begin{array}{l}\text { GR, Drama, Falakron, 03.07.2017 } \\
\text { Felskante, Bergwiesen, } 1772 \mathrm{~m} \\
\text { Dra } 250,41^{\circ} 17^{\prime} 59^{\prime \prime N}, 24^{\circ} 03^{\prime} 56^{\prime \prime E}\end{array}$ & KF 57.46.32 & $280.880-280.905$ \\
\hline
\end{tabular}




\begin{tabular}{|c|c|c|}
\hline 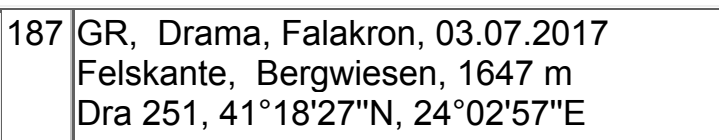 & KF 57.37.01 & $280.906-280.919$ \\
\hline \begin{tabular}{|l|l|}
188 & GR, Drama, Falakron, 03.07.2017 \\
& Felskante, Bergwiesen, Kiefern, $1528 \mathrm{~m}$ \\
& Dra 252, 41 $1^{\circ} 17^{\prime} 40^{\prime \prime N}, 24^{\circ} 02^{\prime} 33^{\prime \prime E}$
\end{tabular} & KF 57.25.47 & $280.920-280.925$ \\
\hline
\end{tabular}

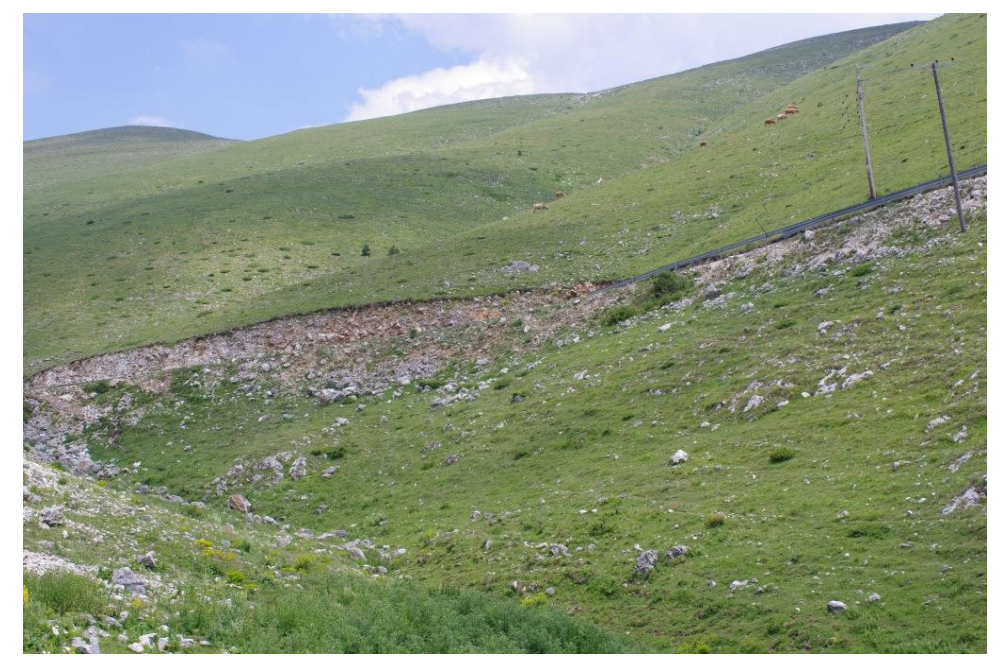

\begin{tabular}{|c|c|c|c|}
\hline & $\begin{array}{l}\text { GR, Drama, Falakron, 03.07.2017 } \\
\text { Felshänge, Wacholder, } 1315 \mathrm{~m} \\
\text { Dra } 253,41^{\circ} 17^{\prime} 25^{\prime \prime N}, 24^{\circ} 01^{\prime} 25^{\prime \prime E}\end{array}$ & KF 57.05.83 & $280.926-280.939$ \\
\hline 190 & $\begin{array}{l}\text { GR, Drama, Falakron, 03.07.2017 } \\
\text { Felshänge, Wacholder, } 1228 \mathrm{~m} \\
\text { Dra } 254,41^{\circ} 17^{\prime} 27^{\prime \prime N}, 24^{\circ} 00^{\prime} 38^{\prime \prime} \mathrm{E}\end{array}$ & KF 47.95.74 & $280.940-280.947$ \\
\hline 191 & $\begin{array}{l}\text { GR, Drama, Falakron, 03.07.2017 } \\
\text { Krautige Lichtung in Buchenwald, } 1180 \mathrm{~m} \\
\text { Dra } 255,41^{\circ} 17^{\prime} 35^{\prime \prime N}, 24^{\circ} 00^{\prime} 25^{\prime \prime E}\end{array}$ & KF 47.95.46 & $280.948-280.967$ \\
\hline 192 & $\begin{array}{l}\text { GR, Drama, Falakron, 03.07.2017 } \\
\text { Felsen in Kiefern-Buchenwald, } 1140 \mathrm{~m} \\
\text { Dra } 256,41^{\circ} 17^{\prime} 48^{\prime \prime N}, 24^{\circ} 00^{\prime} 27^{\prime \prime E}\end{array}$ & KF 47.96 .40 & $280.968-280.979$ \\
\hline 193 & $\begin{array}{l}\text { GR, Drama, Granitis, 04.07.2017 } \\
\text { Krautfluren, Straßenböschung im Ort, } 820 \mathrm{~m} \\
\text { Dra 257, } 41^{\circ} 17^{\prime} 24^{\prime \prime N}, 23^{\circ} 55^{\prime} 41^{\prime \prime E}\end{array}$ & GL 47.55.21 & $280.980-281.018$ \\
\hline 194 & $\begin{array}{l}\text { GR, Drama, Granitis, } 04.07 .2017 \\
\text { Laubwald, } 810 \mathrm{~m} \\
\text { Dra } 258,41^{\circ} 17^{\prime} 23^{\prime \prime N}, 23^{\circ} 55^{\prime} 32^{\prime \prime E}\end{array}$ & GL 47.55.00 & $281.019-281.041$ \\
\hline 195 & $\begin{array}{l}\text { GR, Drama, Granitis, } 04.07 .2017 \\
\text { Laubwald, Straßenböschung, } 825 \mathrm{~m} \\
\text { Dra 259, } 41^{\circ} 17^{\prime} 23^{\prime \prime N}, 23^{\circ} 55^{\prime} 23^{\prime \prime E}\end{array}$ & GL 47.45.80 & $281.042-281.064$ \\
\hline 196 & $\begin{array}{l}\text { GR, Drama, Granitis, 04.07.2017 } \\
\text { Laubwald Laubgebüsch, } 830 \mathrm{~m} \\
\text { Dra } 260,41^{\circ} 17^{\prime} 19^{\prime \prime N}, 23^{\circ} 55^{\prime} 11^{\prime \prime E}\end{array}$ & GL 47.44 .59 & 281.065 - 281.094 \\
\hline 197 & $\begin{array}{l}\text { GR, Drama, Granitis, } 04.07 .2017 \\
\text { Krautfluren, Buchenwald, } 885 \mathrm{~m} \\
\text { Dra } 261,41^{\circ} 17^{\prime} 12^{\prime \prime N}, 23^{\circ} 54^{\prime} 53^{\prime \prime E}\end{array}$ & GL 47.44.17 & $281.095-281.128$ \\
\hline
\end{tabular}




\begin{tabular}{|c|c|c|c|}
\hline & $\begin{array}{l}\text { GR, Drama, NW Granitis, 04.07.2017 } \\
\text { Felsige Krautfluren, Kiefernwald, } 760 \mathrm{~m} \\
\text { Dra } 262,41^{\circ} 18^{\prime} 02^{\prime \prime N}, 23^{\circ} 54^{\prime} 50^{\prime E} \mathrm{E}\end{array}$ & GL 47.46.02 & $281.129-281.160$ \\
\hline 199 & $\begin{array}{l}\text { GR, Drama, NW Granitis, 04.07.2017 } \\
\text { Krautfluren, Laubgebüsch, } 700 \mathrm{~m} \\
\text { Dra } 263,41^{\circ} 18^{\prime} 18^{\prime \prime N}, 23^{\circ} 54^{\prime} 04 " \mathrm{E}\end{array}$ & GL 47.26.97 & $281.161-281.167$ \\
\hline 200 & $\begin{array}{l}\text { GR, Drama, NO Ochyro, 04.07.2017 } \\
\text { Acker, Ackerrand, } 565 \mathrm{~m} \\
\text { Dra } 264,41^{\circ} 18^{\prime} 38^{\prime \prime} \mathrm{N}, 23^{\circ} 52^{\prime} 04^{\prime \prime E}\end{array}$ & GL 47.07.12 & $281.168-281.204$ \\
\hline
\end{tabular}

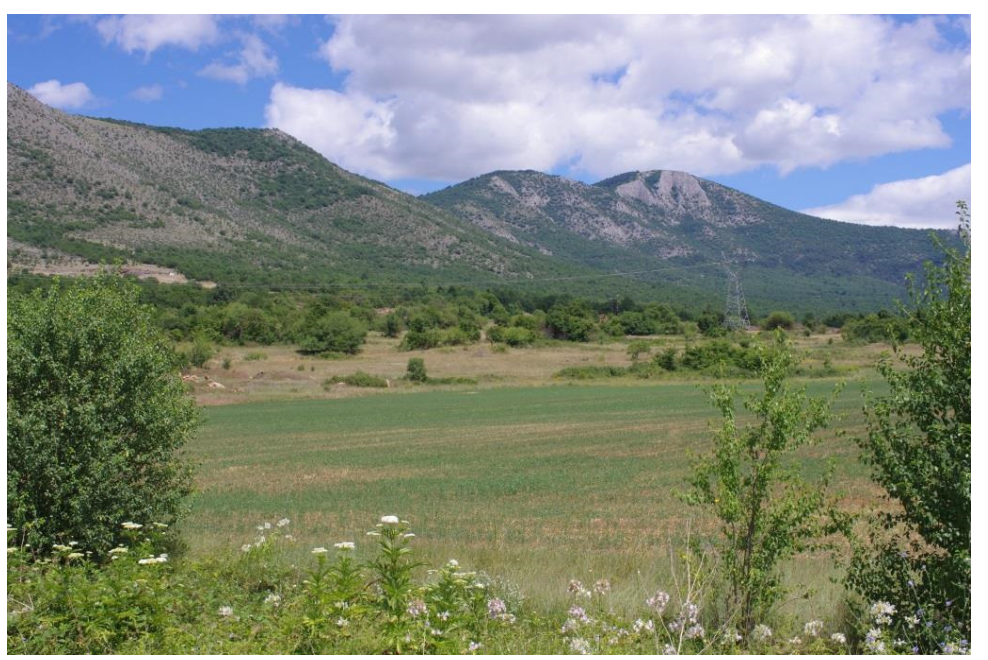

\begin{tabular}{|c|c|c|c|}
\hline 201 & $\begin{array}{l}\text { GR, Drama, NO Granitis, } 04.07 .2017 \\
\text { Felsige Krautfluren, } 800 \mathrm{~m} \\
\text { Dra } 265,41^{\circ} 17^{\prime} 33^{\prime \prime N}, 23^{\circ} 56^{\prime} 16^{\prime \prime E}\end{array}$ & GL 47.65.04 & $281.205-281.245$ \\
\hline 202 & $\begin{array}{l}\text { GR, Drama, W Nevrokopi, 05.07.2017 } \\
\text { Straßenrand, Krautfluren, } 580 \mathrm{~m} \\
\text { Dra } 266,41^{\circ} 20^{\prime} 33^{\prime \prime N}, 23^{\circ} 51^{\prime} 19^{\prime \prime E}\end{array}$ & GL 38.80.97 & $281.246-281.279$ \\
\hline 203 & 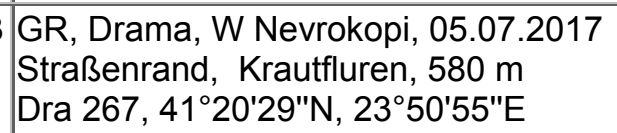 & GL 38.80.36 & $281.280-281.306$ \\
\hline 204 & $\begin{array}{l}\text { GR, Drama, W Nevrokopi, 05.07.2017 } \\
\text { Acker, Ackerrand, } 575 \mathrm{~m} \\
\text { Dra } 268,41^{\circ} 20^{\prime} 13^{\prime \prime N}, 23^{\circ} 48^{\prime} 57 " \mathrm{E}\end{array}$ & GL 38.50.60 & $281.307-281.325$ \\
\hline 205 & $\begin{array}{l}\text { GR, Drama, W Nevrokopi, 05.07.2017 } \\
\text { Ackerrand, Laubgebüsch, } 575 \mathrm{~m} \\
\text { Dra } 269,41^{\circ} 20^{\prime} 25^{\prime \prime} \mathrm{N}, 23^{\circ} 47^{\prime} 20^{\prime \prime E}\end{array}$ & GL 38.30.43 & $281.326-281.335$ \\
\hline 206 & $\begin{array}{l}\text { GR, Drama, W Nevrokopi, } 05.07 .2017 \\
\text { Laubgebüsch, Kiefernwald, } 600 \mathrm{~m} \\
\text { Dra } 270,41^{\circ} 20^{\prime} 58^{\prime \prime} \mathrm{N}, 23^{\circ} 45^{\prime} 50^{\prime \prime E}\end{array}$ & GL 38.11.22 & $281.336-281.359$ \\
\hline 207 & $\begin{array}{l}\text { GR, Drama, Vathytopo, } 05.07 .2017 \\
\text { Laubgebüsch, Bach, } 600 \mathrm{~m} \\
\text { Dra } 271,41^{\circ} 21^{\prime} 09^{\prime \prime N}, 23^{\circ} 43^{\prime} 03^{\prime \prime E}\end{array}$ & GL 28.71.35 & $281.360-281.379$ \\
\hline 208 & $\begin{array}{l}\text { GR, Drama, Vathytopo, 05.07.2017 } \\
\text { Krautige Straßenböschung, } 711 \mathrm{~m} \\
\text { Dra } 272,41^{\circ} 21^{\prime} 07 " \mathrm{~N}, 23^{\circ} 42^{\prime} 08^{\prime \prime E}\end{array}$ & GL 28.61.14 & $281.380-281.402$ \\
\hline
\end{tabular}




\begin{tabular}{|l|l|l|l|}
\hline 209 & $\begin{array}{l}\text { GR, Drama, Vathytopo, 05.07.2017 } \\
\text { Kraut- und Ruderalfluren am Ort, 748 m } \\
\text { Dra 273, } 41^{\circ} 21^{\prime} 05^{\prime \prime}, 23^{\circ} 41^{\prime} 32 " \mathrm{E}\end{array}$ & GL 28.51.23 & $281.403-281.427$ \\
\hline 210 & $\begin{array}{l}\text { GR, Drama, Vathytopo, 05.07.2017 } \\
\text { Felsige Krautfluren, 800 m } \\
\text { Dra 274, } 41^{\circ} 20^{\prime} 48^{\prime \prime} \mathrm{N}, 23^{\circ} 41^{\prime} 00 " \mathrm{E}\end{array}$ & GL 28.40.57 & $281.428-281.467$ \\
\hline
\end{tabular}

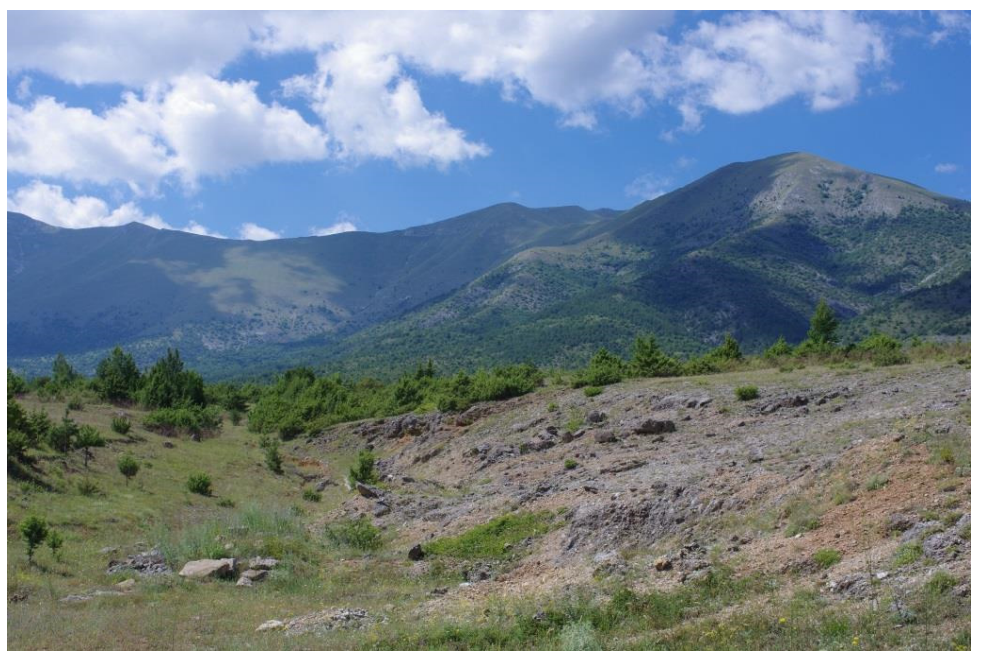

\begin{tabular}{|c|c|c|c|}
\hline & $\begin{array}{l}\text { GR, Drama, Vathytopo, } 05.07 .2017 \\
\text { Krautfluren, Buchenwald am Stausee, } 895 \mathrm{~m} \\
\text { Dra } 275,41^{\circ} 20^{\prime} 25^{\prime \prime} \mathrm{N}, 23^{\circ} 40^{\prime} 03^{\prime \prime} \mathrm{E}\end{array}$ & GL 28.30.20 & $281.468-281.492$ \\
\hline 212 & $\begin{array}{l}\text { GR, Drama, Perithori, 06.07.2017 } \\
\text { Ackerrand, } 575 \mathrm{~m} \\
\text { Dra } 276,41^{\circ} 18^{\prime} 42^{\prime \prime} \mathrm{N}, 23^{\circ} 48^{\prime} 01^{\prime \prime} \mathrm{E}\end{array}$ & GL 37.47.41 & $281.493-281.545$ \\
\hline 213 & $\begin{array}{l}\text { GR, Drama, Perithori, 06.07.2017 } \\
\text { Ackerrand, steinige Krautfluren, } 575 \mathrm{~m} \\
\text { Dra } 277,41^{\circ} 17^{\prime} 46^{\prime \prime} \mathrm{N}, 23^{\circ} 47^{\prime} 12^{\prime \prime E}\end{array}$ & GL 37.35.34 & $281.546-281.584$ \\
\hline 214 & $\begin{array}{l}\text { GR, Drama, Kato Vrondous, 06.07.2017 } \\
\text { Krautfluren, Laubgebüsch, Buchenwald, } 720 \mathrm{~m} \\
\text { Dra 278, } 41^{\circ} 16^{\prime} 20^{\prime \prime N}, 23^{\circ} 44^{\prime} 56^{\prime \prime E}\end{array}$ & GL 37.02.26 & $281.585-281.621$ \\
\hline 215 & $\begin{array}{l}\text { GR, Drama, Kato Vrondous, 06.07.2017 } \\
\text { Felshang, Eichen, Wacholder, Buchenwald, } 875 \text { m } \\
\text { Dra } 279,41^{\circ} 16^{\prime} 26^{\prime \prime N}, 23^{\circ} 44^{\prime} 02^{\prime \prime E}\end{array}$ & GL 27.92.08 & $281.622-281.653$ \\
\hline 216 & $\begin{array}{l}\text { GR, Serres, Kato Vrondous, } 06.07 .2017 \\
\text { Laubgebüsch, } 995 \mathrm{~m} \\
\text { Ser } 270,41^{\circ} 16^{\prime} 00^{\prime \prime} \mathrm{N}, 23^{\circ} 42^{\prime} 14^{\prime \prime E}\end{array}$ & GL 27.61.59 & $281.654-281.670$ \\
\hline 217 & $\begin{array}{l}\text { GR, Serres, Kato Vrondous, 06.07.2017 } \\
\text { Alter Acker, Krautfluren, } 1005 \mathrm{~m} \\
\text { Ser } 271,41^{\circ} 15^{\prime} 58^{\prime \prime N}, 23^{\circ} 41^{\prime} 58^{\prime \prime} \mathrm{E}\end{array}$ & GL 27.61.18 & $281.671-281.693$ \\
\hline 218 & $\begin{array}{l}\text { GR, Drama, Nevrokopi, } 06.07 .2017 \\
\text { Felsanriss, Krautfluren, } 645 \mathrm{~m} \\
\text { Dra } 280,41^{\circ} 21^{\prime} 25^{\prime \prime} \mathrm{N}, 23^{\circ} 52^{\prime} 37^{\prime \prime E}\end{array}$ & GL 48.02.74 & $281.694-281.725$ \\
\hline 219 & $\begin{array}{l}\text { GR, Drama, OSO Granitis, } 07.07 .2017 \\
\text { Felskante, steinige Krautfluren, } 660 \mathrm{~m} \\
\text { Dra } 281,41^{\circ} 16^{\prime} 51^{\prime \prime N}, 23^{\circ} 58^{\prime} 53^{\prime \prime E}\end{array}$ & GL 47.94 .72 & $281.726-281.766$ \\
\hline
\end{tabular}




\begin{tabular}{|c|c|c|c|}
\hline & $\begin{array}{l}\text { GR, Drama, NNO Volakas, 07.07.2017 } \\
\text { Krautige Hänge, nasser Straßengraben, } 785 \text { m } \\
\text { Dra 282, } 41^{\circ} 19^{\prime} 29^{\prime \prime N}, 24^{\circ} 00^{\prime} 24^{\prime \prime E}\end{array}$ & KF 47.99.51 & $281.767-281.803$ \\
\hline 221 & $\begin{array}{l}\text { GR, Drama, NNO Volakas, } 07.07 .2017 \\
\text { Krautige Hänge, Laubgebüsch, } 720 \mathrm{~m} \\
\text { Dra 283, } 41^{\circ} 21^{\prime} 30^{\prime \prime N}, 24^{\circ} 01^{\prime} 30^{\prime E} \mathrm{E}\end{array}$ & KF 58.12.18 & $281.804-281.832$ \\
\hline 222 & $\begin{array}{l}\text { GR, Drama, NNO Volakas, } 07.07 .2017 \\
\text { Felskante, Laubgebüsch, } 683 \mathrm{~m} \\
\text { Dra } 284,41^{\circ} 22^{\prime} 09^{\prime \prime} \mathrm{N}, 24^{\circ} 01^{\prime} 41^{\prime \prime E}\end{array}$ & KF 58.14.40 & $281.833-281.853$ \\
\hline 223 & $\begin{array}{l}\text { GR, Drama, NNO Volakas, } 07.07 .2017 \\
\text { Felskante, Laubgebüsch, } 530 \mathrm{~m} \\
\text { Dra } 285,41^{\circ} 22^{\prime} 26^{\prime \prime N}, 24^{\circ} 02^{\prime} 00^{\prime E}\end{array}$ & KF 58.14.95 & $281.854-281.863$ \\
\hline 224 & $\begin{array}{l}\text { GR, Drama, SW Mikroklisoura, } 07.07 .2017 \\
\text { Krautige Böschung, Laubgebüsch, } 442 \mathrm{~m} \\
\text { Dra 286, } 41^{\circ} 22^{\prime} 54^{\prime \prime N}, 24^{\circ} 02^{\prime} 48^{\prime \prime E}\end{array}$ & KF 58.35.03 & $281.864-281.875$ \\
\hline 225 & $\begin{array}{l}\text { GR, Drama, O Mikroklisoura, 07.07.2017 } \\
\text { Krautige Böschung, Laubgebüsch, } 405 \text { m } \\
\text { Dra 287, } 41^{\circ} 23^{\prime} 10^{\prime N}, 24^{\circ} 04^{\prime} 23 " E\end{array}$ & KF 58.55.38 & $281.876-281.920$ \\
\hline 226 & $\begin{array}{l}\text { GR, Drama, SW Potamia, 07.07.2017 } \\
\text { Maisfeld, nasser Graben, } 395 \mathrm{~m} \\
\text { Dra } 288,41^{\circ} 23^{\prime} 23^{\prime \prime N}, 24^{\circ} 05^{\prime} 52^{\prime \prime E}\end{array}$ & KF 58.76.31 & $281.921-281.945$ \\
\hline 227 & $\begin{array}{l}\text { GR, Drama, NO Potamia, 07.07.2017 } \\
\text { Krautfluren, } 440 \mathrm{~m} \\
\text { Dra } 289,41^{\circ} 23^{\prime} 55^{\prime \prime} \mathrm{N}, 24^{\circ} 06^{\prime} 36^{\prime \prime} \mathrm{E}\end{array}$ & KF 58.87.40 & $281.946-281.968$ \\
\hline 228 & $\begin{array}{l}\text { GR, Drama, NO Potamia, 07.07.2017 } \\
\text { Krautfluren, Laubgebüsch, } 565 \mathrm{~m} \\
\text { Dra } 290,41^{\circ} 24^{\prime} 15^{\prime \prime N}, 24^{\circ} 07^{\prime} 11^{\prime \prime E}\end{array}$ & KF 58.97.26 & $281.969-281.984$ \\
\hline 229 & $\begin{array}{l}\text { GR, Drama, W Prosotsani, 08.07.2017 } \\
\text { Maisfeld, Ackerrand, } 145 \mathrm{~m} \\
\text { Dra 291, } 41^{\circ} 11^{\prime} 46^{\prime \prime N}, 23^{\circ} 55^{\prime} 45^{\prime \prime} \mathrm{E}\end{array}$ & GL 46.54.67 & $281.985-282.000$ \\
\hline 230 & $\begin{array}{l}\text { GR, Drama, Nevrokopi, 08.07.2017 } \\
\text { Krautfluren, Laubgebüsch, } 630 \mathrm{~m} \\
\text { Dra 292, } 41^{\circ} 21^{\prime} 57^{\prime \prime N}, 23^{\circ} 53^{\prime} 21^{\prime \prime E}\end{array}$ & GL 48.13.64 & $282.001-282.034$ \\
\hline 231 & $\begin{array}{l}\text { GR, Drama, Nevrokopi, 08.07.2017 } \\
\text { Krautfluren, Laubgebüsch, } 612 \mathrm{~m} \\
\text { Dra 293, } 41^{\circ} 22^{\prime} 12^{\prime \prime N}, 23^{\circ} 53^{\prime} 36^{\prime \prime E}\end{array}$ & GL 48.23.09 & $282.035-282.056$ \\
\hline 232 & $\begin{array}{l}\text { GR, Drama, NO Nevrokopi, 08.07.2017 } \\
\text { Laubgebüsch, nasser Straßengraben, } 595 \mathrm{~m} \\
\text { Dra 294, } 41^{\circ} 22^{\prime} 41^{\prime \prime N}, 23^{\circ} 53^{\prime} 46 " \mathrm{E}\end{array}$ & GL 48.24.28 & $282.057-282.078$ \\
\hline 233 & $\begin{array}{l}\text { GR, Drama, NO Nevrokopi, 08.07.2017 } \\
\text { Laubgebüsch, Laubwald, einzelne Kiefern, } 620 \mathrm{~m} \\
\text { Dra 295, } 41^{\circ} 22^{\prime} 55^{\prime \prime N}, 23^{\circ} 54^{\prime} 13^{\prime \prime E}\end{array}$ & GL 48.25.82 & $282.079-282.106$ \\
\hline 234 & $\begin{array}{l}\text { GR, Drama, NO Nevrokopi, 08.07.2017 } \\
\text { Eichengebüsch, } 670 \mathrm{~m} \\
\text { Dra 296, } 41^{\circ} 23^{\prime} 24^{\prime \prime N}, 23^{\circ} 54^{\prime} 18^{\prime \prime} \mathrm{E}\end{array}$ & GL 48.26.91 & $282.107-282.130$ \\
\hline 235 & $\begin{array}{l}\text { GR, Drama, Lefkogia, } 08.07 .2017 \\
\text { Kraut- und Ruderalfluren, } 610 \mathrm{~m} \\
\text { Dra } 297,41^{\circ} 23^{\prime} 58^{\prime \prime} \mathrm{N}, 23^{\circ} 54^{\prime} 03^{\prime \prime} \mathrm{E}\end{array}$ & GL 48.27.52 & $282.131-282.153$ \\
\hline
\end{tabular}




\begin{tabular}{|c|c|c|c|}
\hline & $\begin{array}{l}\text { GR, Drama, SO Lefkogia, 08.07.2017 } \\
\text { Krautfluren, Kiefern-Mischwald, } 650 \mathrm{~m} \\
\text { Dra } 298,41^{\circ} 23^{\prime} 19^{\prime \prime N}, 23^{\circ} 55^{\prime} 44^{\prime E} \mathrm{E}\end{array}$ & GL 48.46.90 & $282.154-282.168$ \\
\hline 237 & $\begin{array}{l}\text { GR, Drama, SO Lefkogia, 08.07.2017 } \\
\text { Krautfluren, Kiefern-Mischwald, Krautfluren, } 697 \text { m } \\
\text { Dra } 299,41^{\circ} 23^{\prime} 26^{\prime \prime N}, 23^{\circ} 56^{\prime} 37^{\prime \prime E}\end{array}$ & GL 48.66.13 & $282.169-282.201$ \\
\hline
\end{tabular}

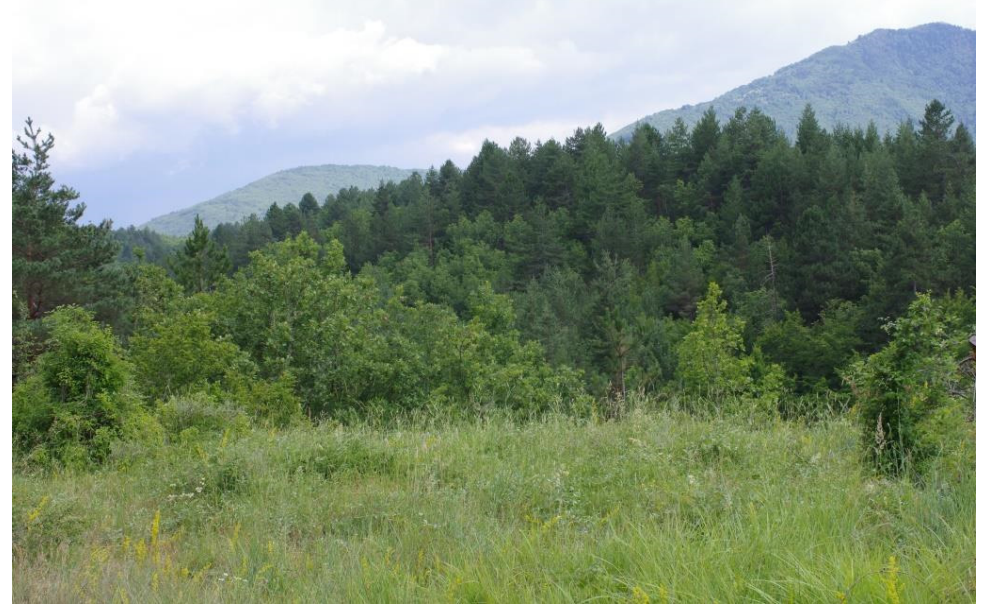

\begin{tabular}{|c|c|c|c|}
\hline & $\begin{array}{l}\text { GR, Drama, SO Lefkogia, 08.07.2017 } \\
\text { Kiefern-Mischwald, } 768 \mathrm{~m} \\
\text { Dra } 300,41^{\circ} 23^{\prime} 14^{\prime \prime N}, 23^{\circ} 57^{\prime} 23^{\prime \prime E}\end{array}$ & GL 48.76.20 & $282.202-282.233$ \\
\hline 239 & $\begin{array}{l}\text { GR, Drama, Ano Vrondous, 09.07.2017 } \\
\text { Kiefern-Buchenwald, } 973 \mathrm{~m} \\
\text { Dra } 301,41^{\circ} 15^{\prime} 50^{\prime \prime N}, 23^{\circ} 41^{\prime} 45^{\prime \prime E}\end{array}$ & GL 27.51.86 & $282.234-282.265$ \\
\hline 240 & $\begin{array}{l}\text { GR, Serres, Ano Vrondous, 09.07.2017 } \\
\text { Buchenwald, Laubgebüsch, Kartoffelacker, } 968 \text { m } \\
\text { Ser } 272,41^{\circ} 16^{\prime} 31^{\prime \prime N}, 23^{\circ} 41^{\prime} 34^{\prime \prime E}\end{array}$ & GL 27.52.58 & $282.266-282.293$ \\
\hline 241 & $\begin{array}{l}\text { GR, Serres, Ano Vrondous, 09.07.2017 } \\
\text { Laubgebüsch, } 1042 \mathrm{~m} \\
\text { Ser } 273,41^{\circ} 17^{\prime} 19^{\prime \prime N}, 23^{\circ} 41^{\prime} 18^{\prime \prime E}\end{array}$ & GL 27.54.13 & $282.294-282.309$ \\
\hline 242 & $\begin{array}{l}\text { GR, Serres, SSW Ano Vrondous, } 09.07 .2017 \\
\text { Krautfluren, Laubwald, Kartoffelacker, } 935 \mathrm{~m} \\
\text { Ser } 274,41^{\circ} 14^{\prime} 58^{\prime \prime N}, 23^{\circ} 40^{\prime} 36^{\prime \prime E}\end{array}$ & GL 26.49.39 & $282.310-282.343$ \\
\hline 243 & $\begin{array}{l}\text { GR, Serres, SSW Ano Vrondous, 09.07.2017 } \\
\text { Buchenwald, Ackerrand, } 902 \mathrm{~m} \\
\text { Ser } 275,41^{\circ} 14^{\prime} 22^{\prime \prime N}, 23^{\circ} 40^{\prime} 18^{\prime \prime E}\end{array}$ & GL 26.38.98 & $282.344-282.355$ \\
\hline 244 & $\begin{array}{l}\text { GR, Serres, SSW Ano Vrondous, 09.07.2017 } \\
\text { Laubgebüsch, Krautfluren, } 905 \mathrm{~m} \\
\text { Ser } 276,41^{\circ} 14^{\prime} 02^{\prime \prime N}, 23^{\circ} 39^{\prime} 48^{\prime \prime E}\end{array}$ & GL 26.38.21 & $282.356-282.384$ \\
\hline 245 & $\begin{array}{l}\text { GR, Serres, ONO Serres, 09.07.2017 } \\
\text { Steinige Krautfluren, } 340 \mathrm{~m} \\
\text { Ser } 277,41^{\circ} 08^{\prime} 30^{\prime \prime N}, 23^{\circ} 34^{\prime} 06 " \mathrm{E}\end{array}$ & GL 15.57.57 & $282.385-282.392$ \\
\hline 246 & $\begin{array}{l}\text { GR, Serres, N Serres, 09.07.2017 } \\
\text { Kiefernwald, } 295 \mathrm{~m} \\
\text { Ser } 278,41^{\circ} 07^{\prime} 35^{\prime \prime N}, 23^{\circ} 33^{\prime} 39^{\prime E} \mathrm{E}\end{array}$ & GL 15.55 .09 & $282.393-282.408$ \\
\hline
\end{tabular}




\begin{tabular}{|c|c|c|c|}
\hline & $\begin{array}{l}\text { GR, Serres, Orini, } 10.07 .2017 \\
\text { Felsige Krautfluren, } 925 \mathrm{~m} \\
\text { Ser } 279,41^{\circ} 12^{\prime} 30^{\prime \prime} \mathrm{N}, 23^{\circ} 34^{\prime} 46^{\prime \prime} \mathrm{E}\end{array}$ & GL 16.65.31 & $282.409-282.435$ \\
\hline 248 & $\begin{array}{l}\text { GR, Serres, Orini, 10.07.2017 } \\
\text { Felsige Krautfluren, Kiefern, Wacholder, } 1022 \mathrm{~m} \\
\text { Ser } 280,41^{\circ} 12^{\prime} 25^{\prime \prime N}, 23^{\circ} 34^{\prime} 36 " \mathrm{E}\end{array}$ & GL 16.64.09 & $282.436-282.462$ \\
\hline 249 & $\begin{array}{l}\text { GR, Serres, Ori Vrondous, } 10.07 .2017 \\
\text { Felsiger Kiefernwald, } 1236 \mathrm{~m} \\
\text { Ser } 281,41^{\circ} 14^{\prime} 01^{\prime \prime N}, 23^{\circ} 34^{\prime} 39^{\prime E} \mathrm{E}\end{array}$ & GL 16.67.09 & $282.463-282.482$ \\
\hline
\end{tabular}

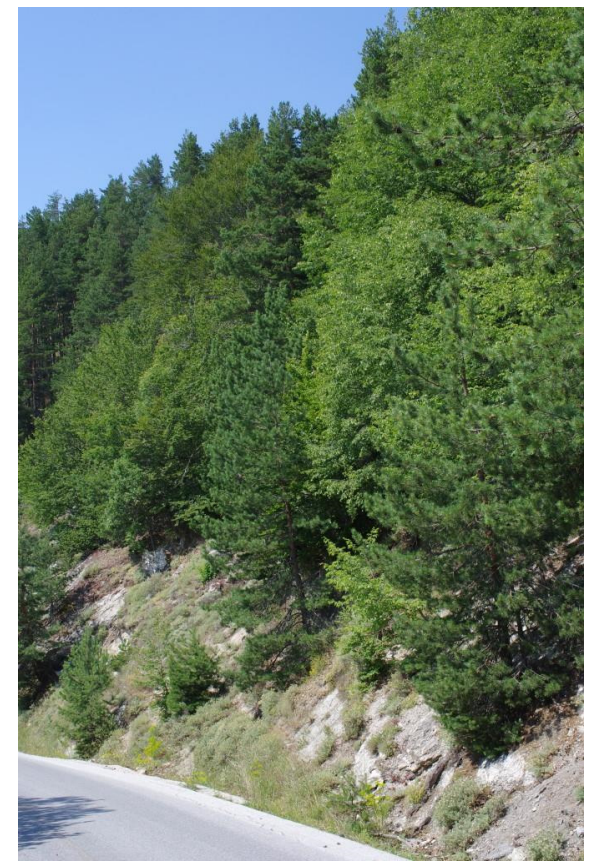

\begin{tabular}{|c|c|c|c|}
\hline 250 & $\begin{array}{l}\text { GR, Serres, Ori Vrondous, } 10.07 .2017 \\
\text { Kiefern-Buchenwald, } 1395 \mathrm{~m} \\
\text { Ser } 282,41^{\circ} 11^{\prime} 41^{\prime \prime N}, 23^{\circ} 34^{\prime} 30 " \mathrm{E}\end{array}$ & GL 16.53.96 & $282.483-282.513$ \\
\hline 251 & $\begin{array}{l}\text { GR, Serres, Ori Vrondous, } 10.07 .2017 \\
\text { Kiefern-Buchenwald, } 1438 \mathrm{~m} \\
\text { Ser } 283,41^{\circ} 14^{\prime} 28^{\prime \prime N}, 23^{\circ} 34^{\prime} 23^{\prime \prime E}\end{array}$ & GL 16.58.67 & $282.514-282.529$ \\
\hline 252 & $\begin{array}{l}\text { GR, Serres, Ori Vrondous, } 10.07 .2017 \\
\text { Krautreicher Kiefernwald, } 1544 \mathrm{~m} \\
\text { Ser } 284,41^{\circ} 14^{\prime} 50^{\prime \prime N}, 23^{\circ} 34^{\prime} 34^{\prime E}\end{array}$ & GL 16.59.94 & $282.530-282.545$ \\
\hline 253 & $\begin{array}{l}\text { GR, Serres, Ori Vrondous, } 10.07 .2017 \\
\text { Krautreicher Kiefernwald, } 1504 \text { m Ser } 286 \\
\text { Ser } 285,41^{\circ} 15^{\prime} 11^{\prime \prime N}, 23^{\circ} 35^{\prime} 11^{\prime \prime E}\end{array}$ & GL 17.60.71 & $282.546-282.567$ \\
\hline 254 & $\begin{array}{l}\text { GR, Serres, Ori Vrondous, } 10.07 .2017 \\
\text { Buchenwald, } 1496 \mathrm{~m} \\
\text { Ser } 286,41^{\circ} 15^{\prime} 21^{\prime \prime N}, 23^{\circ} 35^{\prime} 07^{\prime E}\end{array}$ & GL 17.60 .64 & $282.568-282.580$ \\
\hline 255 & $\begin{array}{l}\text { GR, Serres, Ori Vrondous, 10.07.2017 } \\
\text { Buchenwald, Wassergraben, } 1588 \mathrm{~m} \\
\text { Ser } 287,41^{\circ} 15^{\prime} 32^{\prime \prime N}, 23^{\circ} 35^{\prime} 11^{\prime \prime E}\end{array}$ & GL 17.60 .77 & $282.581-282.599$ \\
\hline 256 & $\begin{array}{l}\text { GR, Serres, Ori Vrondous, } 10.07 .2017 \\
\text { Grasige Skipiste in Buchenwald, } 1571 \mathrm{~m} \\
\text { Ser } 288,41^{\circ} 15^{\prime} 53^{\prime \prime N}, 23^{\circ} 36^{\prime} 16^{\prime E}\end{array}$ & GL 17.81.24 & $282.600-282.625$ \\
\hline
\end{tabular}




\begin{tabular}{|c|c|c|c|}
\hline & $\begin{array}{l}\text { GR, Serres, Ori Vrondous, } 10.07 .2017 \\
\text { Kiefernwald, } 1515 \mathrm{~m} \\
\text { Ser } 289,41^{\circ} 15^{\prime} 19^{\prime \prime} \mathrm{N}, 23^{\circ} 35^{\prime} 10^{\prime \prime} \mathrm{E}\end{array}$ & GL 17.60 .73 & 282.626- 282.631 \\
\hline 258 & $\begin{array}{l}\text { GR, Serres, N Neo Petritsi, } 11.07 .2017 \\
\text { Krautfluren, Laubwald, } 1330 \mathrm{~m} \\
\text { Ser } 290,41^{\circ} 20^{\prime} 26^{\prime \prime N}, 23^{\circ} 13^{\prime} 53^{\prime \prime E}\end{array}$ & FL 87.69.70 & $282.632-282.721$ \\
\hline 259 & $\begin{array}{l}\text { GR, Serres, N Neo Petritsi, } 11.07 .2017 \\
\text { Buchenwald, Laubgebüsch, } 1251 \mathrm{~m} \\
\text { Ser } 291,41^{\circ} 20^{\prime} 34^{\prime \prime N}, 23^{\circ} 14^{\prime} 02^{\prime E} \mathrm{E}\end{array}$ & FL 87.69.92 & $282.722-282.738$ \\
\hline 260 & $\begin{array}{l}\text { GR, Serres, N Neo Petritsi, } 11.07 .2017 \\
\text { Laubwald, } 1124 \mathrm{~m} \\
\text { Ser } 292,41^{\circ} 20^{\prime} 05^{\prime \prime N}, 23^{\circ} 14^{\prime} 33^{\prime \prime E}\end{array}$ & FL 87.78.63 & $282.739-282.757$ \\
\hline 261 & $\begin{array}{l}\text { GR, Serres, N Neo Petritsi, 11.07.2017 } \\
\text { Laubwald, } 1025 \mathrm{~m} \\
\text { Ser } 293,41^{\circ} 20^{\prime} 08^{\prime \prime N}, 23^{\circ} 14^{\prime} 52^{\prime \prime} \mathrm{E}\end{array}$ & FL 87.88.14 & $282.758-282.780$ \\
\hline 262 & 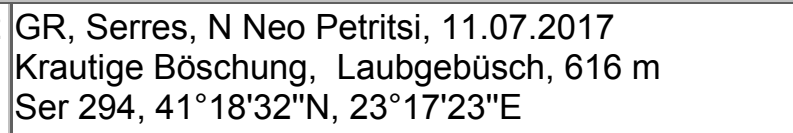 & FL 97.15.76 & $282.781-282.785$ \\
\hline 263 & 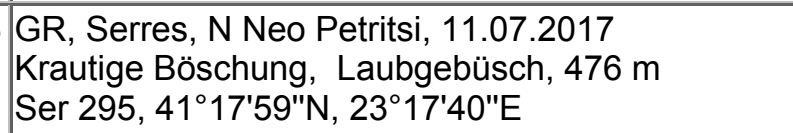 & GA.48.72.51 & $282.786-282.793$ \\
\hline 264 & $\begin{array}{l}\text { GR, Serres, NO Strymoniko, } 12.07 .2017 \\
\text { Straßenrand, Ackerrand, } 36 \text { m } \\
\text { Ser } 296,41^{\circ} 03^{\prime} 10^{\prime \prime N}, 23^{\circ} 19^{\prime} 15^{\prime \prime E}\end{array}$ & FL 94.57.02 & $282.794-282.822$ \\
\hline 265 & $\begin{array}{l}\text { GR, Serres, W Kerkini-See, } 12.07 .2017 \\
\text { Krautfluren am See, } 38 \mathrm{~m} \\
\text { Ser } 297,41^{\circ} 09^{\prime} 36^{\prime \prime N}, 23^{\circ} 11^{\prime} 222^{\prime E}\end{array}$ & FL 85.38.78 & 282.823 \\
\hline 266 & $\begin{array}{l}\text { GR, Serres, Parapotamos, } 12.07 .2017 \\
\text { Sumpfwiese, Laubgebüsch, } 71 \mathrm{~m} \\
\text { Ser } 298,41^{\circ} 14^{\prime} 33^{\prime \prime N}, 22^{\circ} 59^{\prime} 27^{\prime \prime E}\end{array}$ & FL 66.67.86 & $282.824-282.845$ \\
\hline 267 & $\begin{array}{l}\text { GR, Kilkis, NW Griva, } 12.07 .2017 \\
\text { Felswand, Platanen, } 583 \mathrm{~m} \\
\text { Kil } 294,40^{\circ} 57^{\prime} 27^{\prime \prime N}, 22^{\circ} 23^{\prime} 46^{\prime \prime} \mathrm{E}\end{array}$ & FL 13.75.50 & $282.846-282.857$ \\
\hline 268 & $\begin{array}{l}\text { GR, Kilkis, NW Griva, } 12.07 .2017 \\
\text { Laubgebüsch, Kirschbäume, } 640 \mathrm{~m} \\
\text { Kil 295, } 40^{\circ} 57^{\prime} 47^{\prime \prime N}, 22^{\circ} 23^{\prime} 44^{\prime \prime E}\end{array}$ & FL 13.75.46 & $282.858-282.893$ \\
\hline 269 & $\begin{array}{l}\text { GR, Kilkis, N Kastaneri, } 12.07 .2017 \\
\text { Krautige Straßenböschung, Obstbäume, } 820 \text { m } \\
\text { Kil } 296,940^{\circ} 58^{\prime} 52^{\prime \prime N}, 22^{\circ} 23^{\prime} 10^{\prime \prime E}\end{array}$ & FL 13.67.66 & $282.894-282.903$ \\
\hline 270 & $\begin{array}{l}\text { GR, Kilkis, WNW Kastaneri, 12.07.2017 } \\
\text { Krautige Straßenböschung, Buchenwald, } 1095 \mathrm{~m} \\
\text { Kil } 297,40^{\circ} 59^{\prime} 18^{\prime \prime N}, 22^{\circ} 21^{\prime} 07^{\prime \prime E}\end{array}$ & FL 13.38.73 & $282.904-282.932$ \\
\hline 271 & $\begin{array}{l}\text { GR, Kilkis, WNW Kastaneri, } 12.07 .2017 \\
\text { Krautige Straßenböschung, Buchenwald, } 1140 \mathrm{~m} \\
\text { Kil } 298,40^{\circ} 59^{\prime} 16^{\prime \prime N}, 22^{\circ} 19^{\prime} 18^{\prime \prime E}\end{array}$ & FL 13.18.22 & $282.933-282.953$ \\
\hline 272 & $\begin{array}{l}\text { GR, Kilkis, Kastaneri, 13.07.2017 } \\
\text { Felsiger Buchenwald, Krautfluren, } 1064 \mathrm{~m} \\
\text { Kil } 299,40^{\circ} 58^{\prime} 51^{\prime \prime N}, 22^{\circ} 22^{\prime} 00^{\prime E} \mathrm{E}\end{array}$ & FL 13.57.05 & $282.954-282.978$ \\
\hline & $\begin{array}{l}\text { GR, Kilkis, Kastaneri, } 13.07 .2017 \\
\text { Buchenwald, } 1062 \mathrm{~m} \\
\text { Kil } 300,40^{\circ} 58^{\prime} 45^{\prime \prime N}, 22^{\circ} 21^{\prime} 39^{\prime \prime E}\end{array}$ & FL 13.47.53 & $282.979-282.992$ \\
\hline
\end{tabular}




\begin{tabular}{|c|c|c|c|}
\hline & $\begin{array}{l}\text { GR, Kilkis, Kastaneri, } 13.07 .2017 \\
\text { Buchenwald, } 1156 \mathrm{~m} \\
\text { Kil } 301,40^{\circ} 59^{\prime} 27^{\prime \prime N}, 22^{\circ} 19^{\prime} 59^{\prime \prime E}\end{array}$ & FL 13.28.16 & $282.993-283.004$ \\
\hline 275 & $\begin{array}{l}\text { GR, Kilkis, Kastaneri, } 13.07 .2017 \\
\text { Buchenwald, feuchter Graben, } 1135 \mathrm{~m} \\
\text { Kil } 302,40^{\circ} 59^{\prime} 05^{\prime \prime N}, 22^{\circ} 19^{\prime} 21^{\prime E} \mathrm{E}\end{array}$ & FL 13.17.39 & $283.005-283.024$ \\
\hline 276 & $\begin{array}{l}\text { GR, Kilkis, SO Livadia, } 13.07 .2017 \\
\text { Wiesen in Buchenwald, } 1155 \mathrm{~m} \\
\text { Kil } 303,40^{\circ} 58^{\prime} 50^{\prime \prime} \mathrm{N}, 22^{\circ} 19^{\prime} 05^{\prime \prime} \mathrm{E}\end{array}$ & FL 13.07.94 & $283.025-283.040$ \\
\hline 277 & $\begin{array}{l}\text { GR, Kilkis, SO Livadia, 13.07.2017 } \\
\text { Hochstaudenfluren an Bach, Buchenwald, } 1118 \mathrm{~m} \\
\text { Kil } 304,40^{\circ} 59^{\prime} 14 " \mathrm{~N}, 22^{\circ} 19^{\prime} 25^{\prime \prime E}\end{array}$ & FL 13.18.32 & $283.041-283.049$ \\
\hline 278 & $\begin{array}{l}\text { GR, Kilkis, SO Livadia, } 13.07 .2017 \\
\text { Buchenwald, Quelle, } 1190 \mathrm{~m} \\
\text { Kil } 305,40^{\circ} 59^{\prime} 25^{\prime \prime N}, 22^{\circ} 18^{\prime} 59^{\prime \prime E}\end{array}$ & FL 13.08.75 & $283.050-283.054$ \\
\hline 279 & $\begin{array}{l}\text { GR, Kilkis, SO Livadia, } 13.07 .2017 \\
\text { Buchenwald, } 1180 \mathrm{~m} \\
\text { Kil } 306,40^{\circ} 59^{\prime} 26^{\prime \prime} \mathrm{N}, 22^{\circ} 18^{\prime} 38^{\prime \prime} \mathrm{E}\end{array}$ & FL 13.08.25 & $283.055-283.061$ \\
\hline 280 & $\begin{array}{l}\text { GR, Kilkis, NW Livadia, } 13.07 .2017 \\
\text { Felsige Krautfluren, } 1185 \mathrm{~m} \\
\text { Kil } 307,41^{\circ} 01^{\prime} 04^{\prime \prime N}, 22^{\circ} 16^{\prime} 20^{\prime E}\end{array}$ & FL 04.71.05 & $283.062-283.086$ \\
\hline 281 & $\begin{array}{l}\text { GR, Pella, S Archangelo, } 13.07 .2017 \\
\text { Buchenwald, Krautfluren, } 1155 \mathrm{~m} \\
\text { Pel } 339,41^{\circ} 02^{\prime} 39^{\prime \prime N}, 22^{\circ} 17^{\prime} 34^{\prime E}\end{array}$ & FL 04.84.75 & $283.087-283.113$ \\
\hline 282 & $\begin{array}{l}\text { GR, Pella, SSO Archangelo, } 13.07 .2017 \\
\text { Felsige Krautfluren, Buchenwald, } 1075 \mathrm{~m} \\
\text { Pel } 340,41^{\circ} 03^{\prime} 39^{\prime \prime N}, 22^{\circ} 17^{\prime} 31^{\prime \prime E}\end{array}$ & FL 04.86.63 & $283.114-283.131$ \\
\hline 283 & $\begin{array}{l}\text { GR, Pella, S Archangelo, } 13.07 .2017 \\
\text { Felsige Krautfluren, Buchenwald, } 970 \mathrm{~m} \\
\text { Pel } 341,41^{\circ} 04^{\prime} 13^{\prime \prime N}, 22^{\circ} 17^{\prime} 06 " \mathrm{E}\end{array}$ & FL 04.87.04 & $283.132-283.144$ \\
\hline 284 & $\begin{array}{l}\text { GR, Pella, S Archangelo, } 13.07 .2017 \\
\text { Felsige Böschung, Laubwald, } 855 \mathrm{~m} \\
\text { Pel } 342,41^{\circ} 04^{\prime} 48^{\prime \prime N}, 22^{\circ} 17^{\prime} 03^{\prime \prime E}\end{array}$ & FL 04.78.94 & $283.145-283.158$ \\
\hline 285 & $\begin{array}{l}\text { GR, Pella, Kerasia, } 14.07 .2017 \\
\text { Laubwald, } 1164 \mathrm{~m} \\
\text { Pel } 343,40^{\circ} 52^{\prime} 50^{\prime \prime}, 21^{\circ} 55^{\prime} 23^{\prime \prime} \mathrm{E}\end{array}$ & EL 72.75.89 & $283.159-283.201$ \\
\hline 286 & $\begin{array}{l}\text { GR, Pella, Kerasia, } 14.07 .2017 \\
\text { Laubwald, krautreicher Hang, } 1209 \mathrm{~m} \\
\text { Pel } 344,40^{\circ} 52^{\prime} 58^{\prime \prime} \mathrm{N}, 21^{\circ} 54^{\prime} 49^{\prime \prime} \mathrm{E}\end{array}$ & EL 72.76.01 & $283.202-283.214$ \\
\hline 287 & $\begin{array}{l}\text { GR, Pella, Kerasia, 14.07.2017 } \\
\text { Laubwald, krautreicher Hang, } 1391 \mathrm{~m} \\
\text { Pel 345, } 40^{\circ} 52^{\prime} 30^{\prime \prime N}, 21^{\circ} 53^{\prime} 29^{\prime \prime} \mathrm{E}\end{array}$ & EL 72.55.13 & $283.215-283.234$ \\
\hline 288 & $\begin{array}{l}\text { GR, Pella, Kajmaktsalan, } 14.07 .2017 \\
\text { Buchenwald, felsige Böschung, } 1540 \mathrm{~m} \\
\text { Pel } 346,40^{\circ} 53^{\prime} 23^{\prime \prime N}, 21^{\circ} 53^{\prime} 15^{\prime \prime E}\end{array}$ & EL 72.46.89 & 283.235- 283.255 \\
\hline 289 & $\begin{array}{l}\text { GR, Pella, Kajmaktsalan, } 14.07 .2017 \\
\text { Buchenwald, felsige Böschung Kiefern, } 1594 \text { m } \\
\text { Pel } 347,40^{\circ} 53^{\prime} 59^{\prime \prime N}, 21^{\circ} 52^{\prime} 32^{\prime E} \text { E }\end{array}$ & EL 72.38.70 & $283.256-283.268$ \\
\hline 290 & $\begin{array}{l}\text { GR, Pella, Kajmaktsalan, } 14.07 .2017 \\
\text { Buchenwald, } 1564 \mathrm{~m} \\
\text { Pel } 348,40^{\circ} 53^{\prime} 54 " \mathrm{~N}, 21^{\circ} 51^{\prime} 46 " \mathrm{E}\end{array}$ & EL 72.27.78 & $283.269-283.282$ \\
\hline
\end{tabular}




\begin{tabular}{|c|c|c|c|}
\hline & $\begin{array}{l}\text { GR, Pella, Kajmaktsalan, } 14.07 .2017 \\
\text { Felsigie Krautfluren, Kiefern, Sumpfwiese, } 1615 \mathrm{~m} \\
\text { Pel } 349,40^{\circ} 53^{\prime} 40^{\prime \prime N}, 21^{\circ} 51^{\prime} 31^{\prime \prime E}\end{array}$ & EL 72.27.34 & $283.2830-283.299$ \\
\hline 292 & $\begin{array}{l}\text { GR, Pella, Kajmaktsalan, } 14.07 .2017 \\
\text { Bergwiesen, nasse Mulde, } 1702 \mathrm{~m} \\
\text { Pel } 350,40^{\circ} 53^{\prime} 23^{\prime \prime N}, 21^{\circ} 51^{\prime} 09^{\prime \prime E}\end{array}$ & EL 72.16.89 & $283.300-283.318$ \\
\hline 293 & $\begin{array}{l}\text { GR, Pella, Kajmaktsalan, } 14.07 .2017 \\
\text { Felsige Bergwiesen, Sumpfwiese, } 1873 \mathrm{~m} \\
\text { Pel } 351,40^{\circ} 54^{\prime} 09^{\prime \prime N}, 21^{\circ} 49^{\prime} 32^{\prime \prime E}\end{array}$ & EL 62.98.53 & $283.319-283.342$ \\
\hline 294 & $\begin{array}{l}\text { GR, Pella, Kajmaktsalan, } 14.07 .2017 \\
\text { Felsige Bergwiesen, } 1975 \mathrm{~m} \\
\text { Pel } 352,40^{\circ} 54^{\prime} 12^{\prime \prime N}, 21^{\circ} 49^{\prime} 04 " \mathrm{E}\end{array}$ & EL 62.88.93 & $283.343-283.364$ \\
\hline
\end{tabular}
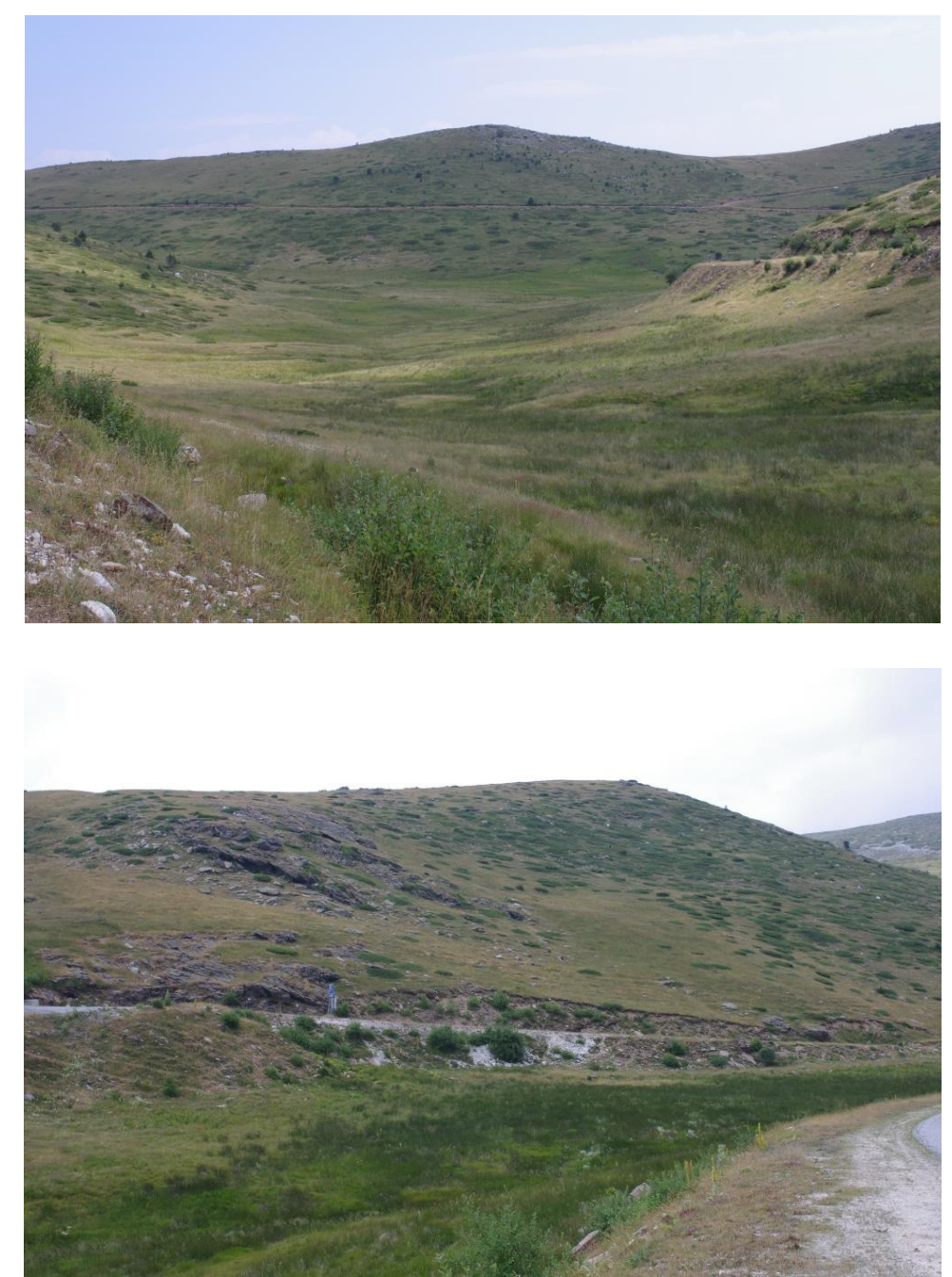

\begin{tabular}{|c|c|c|c|}
\hline & $\begin{array}{l}\text { GR, Pella, Kajmaktsalan, } 14.07 .2017 \\
\text { Felsige Bergwiesen, } 2025 \mathrm{~m} \\
\text { Pel } 353 \text {, EL 62.89.41, } 40^{\circ} 54^{\prime} 36^{\prime \prime} \mathrm{N}, 21^{\circ} 48^{\prime} 46^{\prime \prime E}\end{array}$ & EL 62.89.41 & $283.365-283.379$ \\
\hline 296 & $\begin{array}{l}\text { GR, Pella, NW Loutraki, } 15.07 .2007 \\
\text { Felshänge, Laubgebüsch, } 435 \mathrm{~m} \\
\text { Pel } 354 \text {, EL } 73.75 .89,40^{\circ} 58^{\prime} 14^{\prime \prime N}, 21^{\circ} 55^{\prime} 29^{\prime E} \mathrm{E}\end{array}$ & EL 73.75.89 & $283.380-283.406$ \\
\hline 297 & $\begin{array}{l}\text { GR, Pella, NW Loutraki, } 15.07 .2007 \\
\text { Felshänge, Laubgebüsch, Platanen, } 613 \mathrm{~m} \\
\text { Pel } 355,40^{\circ} 58^{\prime} 39^{\prime \prime N}, 21^{\circ} 54^{\prime} 45^{\prime E}\end{array}$ & EL 73.66.87 & $283.407-283.436$ \\
\hline
\end{tabular}




\begin{tabular}{|c|c|c|c|}
\hline 298 & $\begin{array}{l}\text { GR, Pella, NW Loutraki, } 15.07 .2007 \\
\text { Laubgebüsch, nasse Krautfluren, Acker, } 740 \text { m } \\
\text { Pel } 356,40^{\circ} 58^{\prime} 59^{\prime \prime N}, 21^{\circ} 54^{\prime} 04^{\prime \prime E}\end{array}$ & EL 73.57.83 & $283.437-283.449$ \\
\hline 299 & $\begin{array}{l}\text { GR, Pella, NW Loutraki, } 15.07 .2007 \\
\text { Felshänge, Laubgebüsch, } 760 \mathrm{~m} \\
\text { Pel } 357,40^{\circ} 58^{\prime} 53^{\prime \prime N}, 21^{\circ} 54^{\prime} 42^{\prime \prime E}\end{array}$ & EL 73.67.71 & $283.450-283.456$ \\
\hline 300 & $\begin{array}{l}\text { GR, Pella, N Loutraki, } 15.07 .2017 \\
\text { Felshang, Wacholder, Buchenwald, } 882 \mathrm{~m} \\
\text { Pel } 358,40^{\circ} 58^{\prime} 52^{\prime \prime N}, 21^{\circ} 55^{\prime} 56^{\prime \prime E}\end{array}$ & EL 73.87.41 & $283.457-283.481$ \\
\hline 301 & $\begin{array}{l}\text { GR, Pella, NW Loutraki, } 15.07 .2017 \\
\text { Wiesen, Wacholder, Buchen, } 1234 \mathrm{~m} \\
\text { Pel } 359,40^{\circ} 59^{\prime} 48^{\prime \prime N}, 21^{\circ} 54^{\prime} 55 " \mathrm{E}\end{array}$ & EL 73.78.08 & $283.482-283.501$ \\
\hline 302 & $\begin{array}{l}\text { GR, Pella, SW Promachi, } 15.07 .2017 \\
\text { Laubwald, } 538 \mathrm{~m} \\
\text { Pel } 360,41^{\circ} 00^{\prime} 56^{\prime \prime} \mathrm{N}, 21^{\circ} 58^{\prime} 56^{\prime \prime} \mathrm{E}\end{array}$ & EL 84.20.69 & $283.502-283.518$ \\
\hline 303 & $\begin{array}{l}\text { GR, Pella, SW Promachi, } 15.07 .2017 \\
\text { Laubwald, } 590 \mathrm{~m} \\
\text { Pel } 361,41^{\circ} 01^{\prime} 10^{\prime \prime N}, 21^{\circ} 59^{\prime} 00^{\prime E} \mathrm{E}\end{array}$ & EL 84.21.74 & $283.519-283.536$ \\
\hline 304 & $\begin{array}{l}\text { GR, Pella, NW Promachi, } 15.07 .2017 \\
\text { Buchenwald, Bach, } 770 \mathrm{~m} \\
\text { Pel } 362,41^{\circ} 02^{\prime} 09^{\prime \prime N}, 21^{\circ} 59^{\prime} 16^{\prime \prime} \mathrm{E}\end{array}$ & EL 84.33.02 & $283.537-283.563$ \\
\hline 305 & $\begin{array}{l}\text { GR, Pella, NW Promachi, } 15.07 .2017 \\
\text { Felsiger Laubwald, } 893 \mathrm{~m} \\
\text { Pel } 363,41^{\circ} 02^{\prime} 07^{\prime \prime N}, 21^{\circ} 59^{\prime} 13^{\prime \prime} \mathrm{E}\end{array}$ & EL 84.33.01 & $283.564-283.582$ \\
\hline 306 & $\begin{array}{l}\text { GR, Pella, Loutraki, } 16.07 .2017 \\
\text { Straßenrand Im Ort, } 300 \mathrm{~m} \\
\text { Pel } 364,40^{\circ} 58^{\prime} 10^{\prime \prime N}, 21^{\circ} 56^{\prime} 26 " \mathrm{E}\end{array}$ & EL 73.95.18 & $283.583-283.600$ \\
\hline 307 & $\begin{array}{l}\text { GR, Pella, N Vorino, 16.07.2017 } \\
\text { Nadelwald, } 372 \mathrm{~m} \\
\text { Pel } 365,41^{\circ} 03^{\prime} 11^{\prime \prime N}, 22^{\circ} 04^{\prime} 59^{\prime E} \mathrm{E}\end{array}$ & EL 94.15.02 & $283.601-283.618$ \\
\hline 308 & $\begin{array}{l}\text { GR, Pella, N Vorino, } 16.07 .2017 \\
\text { Alter Acker, } 175 \mathrm{~m} \\
\text { Pel } 366,41^{\circ} 01^{\prime} 57^{\prime \prime N}, 22^{\circ} 05^{\prime} 00^{\prime \prime E}\end{array}$ & EL 94.12.19 & $283.619-283.637$ \\
\hline 309 & $\begin{array}{l}\text { GR, Pella, Vorino, } 16.07 .2017 \\
\text { Platanen, Obstbaumkultur, } 175 \mathrm{~m} \\
\text { Pel } 367,41^{\circ} 02^{\prime} 03^{\prime \prime} \mathrm{N}, 22^{\circ} 04^{\prime} 42^{\prime \prime} \mathrm{E}\end{array}$ & EL 94.03.61 & $283.638-283.659$ \\
\hline 310 & $\begin{array}{l}\text { GR, Pella, OSO Vorino, } 16.07 .2017 \\
\text { Obstbaumkultur, Ackerrand, } 172 \mathrm{~m} \\
\text { Pel } 368,41^{\circ} 01^{\prime} 37^{\prime \prime N}, 22^{\circ} 05^{\prime} 19^{\prime E}\end{array}$ & EL 94.12.53 & $283.660-283.679$ \\
\hline 311 & $\begin{array}{l}\text { GR, Pella, O Vorino, } 16.07 .2017 \\
\text { Obstbaumkultur, Ackerrand, alter Acker, } 165 \mathrm{~m} \\
\text { Pel } 369,41^{\circ} 02^{\prime} 00^{\prime N}, 22^{\circ} 06^{\prime} 36^{\prime \prime E}\end{array}$ & EL 94.33.31 & $283.680-283.708$ \\
\hline 312 & $\begin{array}{l}\text { GR, Pella, SW Foustani, } 16.07 .2017 \\
\text { Acker, } 220 \mathrm{~m} \\
\text { Pel } 370,41^{\circ} 02^{\prime} 40^{\prime \prime} \mathrm{N}, 22^{\circ} 09^{\prime} 38^{\prime \prime} \mathrm{E}\end{array}$ & EL 94.74.53 & $283.709-283.730$ \\
\hline 313 & $\begin{array}{l}\text { GR, Imathia, S Ag. Pavlos, } 18.07 .2017 \\
\text { Krautfluren, Wassergraben, } 1310 \mathrm{~m} \\
\text { Ima } 172,40^{\circ} 39^{\prime} 51^{\prime \prime N}, 21^{\circ} 57^{\prime} 51^{\prime E} \mathrm{E}\end{array}$ & EL 80.11.59 & $283.731-283.770$ \\
\hline 314 & $\begin{array}{l}\text { GR, Imathia, S Ag. Pavlos, } 18.07 .2017 \\
\text { Buchenwald, } 1275 \mathrm{~m} \\
\text { Ima } 173,40^{\circ} 40^{\prime} 05^{\prime \prime} \mathrm{N}, 21^{\circ} 57^{\prime} 47^{\prime E}\end{array}$ & EL 80.12.44 & $283.771-283.792$ \\
\hline
\end{tabular}




\begin{tabular}{|c|c|c|c|}
\hline & $\begin{array}{l}\text { GR, Imathia, S Ag. Pavlos, 18.07.2017 } \\
\text { Buchenwald, } 1304 \text { m } \\
\text { Ima } 174,40^{\circ} 40^{\prime} 20^{\prime \prime N}, 21^{\circ} 57^{\prime} 55^{\prime \prime E}\end{array}$ & EL 80.12.68 & $283.793-283.804$ \\
\hline 316 & $\begin{array}{l}\text { GR, Imathia, S Ag. Pavlos, } 18.07 .2017 \\
\text { Alte Kiesgrube in Buchenwald, } 1335 \mathrm{~m} \\
\text { Ima } 175,40^{\circ} 40^{\prime} 13^{\prime \prime} \mathrm{N}, 21^{\circ} 57^{\prime} 53^{\prime \prime} \mathrm{E}\end{array}$ & EL 80.12.56 & $283.805-283.808$ \\
\hline 317 & $\begin{array}{l}\text { GR, Imathia, Tria-Pende Pigadia, 18.07.2017 } \\
\text { Buchenwald, } 1385 \mathrm{~m} \\
\text { Ima } 176,40^{\circ} 38^{\prime} 36^{\prime \prime N}, 21^{\circ} 58^{\prime} 07 " \mathrm{E}\end{array}$ & EK 89.19.96 & $283.809-283.832$ \\
\hline 318 & $\begin{array}{l}\text { GR, Imathia, Tria-Pende Pigadia, 18.07.2017 } \\
\text { Skipiste in Buchenwald, } 1445 \mathrm{~m} \\
\text { Ima } 177,40^{\circ} 38^{\prime} 25^{\prime \prime N}, 21^{\circ} 58^{\prime} 03^{\prime \prime E}\end{array}$ & EK 89.19.83 & $283.833-283.842$ \\
\hline 319 & $\begin{array}{l}\text { GR, Imathia, Seli, } 18.07 .2017 \\
\text { Buchenwald, } 1245 \mathrm{~m} \\
\text { Ima } 178,40^{\circ} 34^{\prime} 33^{\prime \prime N}, 22^{\circ} 02^{\prime} 11 " \mathrm{E}\end{array}$ & EK 89.72.72 & $283.843-283.871$ \\
\hline 320 & $\begin{array}{l}\text { GR, Imathia, Seli, 18.07.2017 } \\
\text { Krautfluren, Wacholder, } 1450 \mathrm{~m} \\
\text { Ima } 179,40^{\circ} 33^{\prime} 33^{\prime \prime N}, 22^{\circ} 01^{\prime} 31^{\prime \prime E}\end{array}$ & EK 89.60.83 & $283.872-283.896$ \\
\hline 321 & $\begin{array}{l}\text { GR, loannina, Seli, } 18.07 .2017 \\
\text { Bergwiesen, Wacholder, } 1512 \mathrm{~m} \\
\text { Ima } 180,40^{\circ} 32^{\prime} 36^{\prime \prime N}, 22^{\circ} 01^{\prime} 00^{\prime E}\end{array}$ & EK 88.68.16 & $283.897-283.925$ \\
\hline 322 & $\begin{array}{l}\text { GR, loannina, N Metsovo, 19.07.2017 } \\
\text { Felshang, Buchenwald, Kiefern, } 1390 \mathrm{~m} \\
\text { loa } 1107,39^{\circ} 46^{\prime} 46^{\prime \prime N}, 21^{\circ} 10^{\prime} 06^{\prime \prime} \mathrm{E}\end{array}$ & EK 10.43.43 & $283.926-283.933$ \\
\hline 323 & $\begin{array}{l}\text { GR, loannina, N Metsovo, 19.07.2017 } \\
\text { Buchenwald, Wiese, Hangsumpf,1402 m } \\
\text { loa } 1108,39^{\circ} 46^{\prime} 53^{\prime \prime N}, 21^{\circ} 09^{\prime} 36^{\prime \prime E}\end{array}$ & EK 10.33.75 & $283.934-283.942$ \\
\hline
\end{tabular}

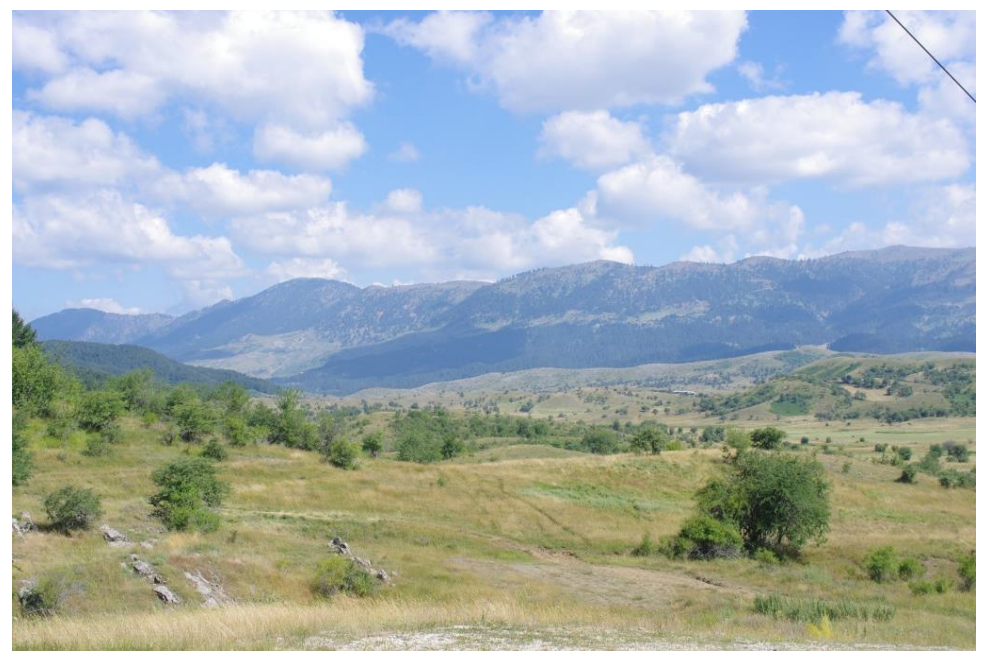

\begin{tabular}{|c|c|c|c|}
\hline 324 & $\begin{array}{l}\text { GR, loannina, NW Metsovo, 19.07.2017 } \\
\text { Krautiger Hang, Buchenwald, } 1425 \mathrm{~m} \\
\text { loa } 1109,39^{\circ} 47^{\prime} 00^{\prime \prime N}, 21^{\circ} 09^{\prime} 19^{\prime \prime E}\end{array}$ & EK 10.33.37 & $283.943-283.953$ \\
\hline 325 & $\begin{array}{l}\text { GR, loannina, N Metsovo, } 19.07 .2017 \\
\text { Felsige Krautfluren, Wacholder, Kiefern, } 1430 \mathrm{~m} \\
\text { loa } 1110,39^{\circ} 47^{\prime} 52^{\prime \prime N}, 21^{\circ} 10^{\prime} 177^{\prime E}\end{array}$ & EK 10.45.73 & $283.954-283.964$ \\
\hline 5 & $\begin{array}{l}\text { GR, loannina, NO Metsovo, } 19.07 .2017 \\
\text { Bergwiesen, Juniperus, } 1559 \mathrm{~m} \\
\text { loa } 1056 \mathrm{~b}, 39^{\circ} 47^{\prime} 31^{\prime \prime N}, 21^{\circ} 12^{\prime} 17^{\prime \prime E}\end{array}$ & EK 10.74.57 & $283.965-283.976$ \\
\hline
\end{tabular}




\begin{tabular}{|c|c|c|c|}
\hline & $\begin{array}{l}\text { GR, loannina, NO Metsovo, 19.07.2017 } \\
\text { Felsige Böschung, Buchenwald, } 1645 \mathrm{~m} \\
\text { loa } 1111,39^{\circ} 47^{\prime} 19^{\prime \prime N}, 21^{\circ} 12^{\prime} 57^{\prime \prime E}\end{array}$ & EK 10.84.53 & $283.977-283.989$ \\
\hline 327 & $\begin{array}{l}\text { GR, loannina, Katara-Paß, 19.07.2017 } \\
\text { Felsige Böschung, Buchenwald, } 1663 \mathrm{~m} \\
\text { loa 1112, 3947'09"N, } 21^{\circ} 13^{\prime} 16^{\prime \prime E}\end{array}$ & EK 10.84.90 & $283.990-283.998$ \\
\hline 328 & $\begin{array}{l}\text { GR, loannina, Katara-Paß, } 19.07 .2017 \\
\text { Felsige Böschung, Buchenwald, } 1680 \mathrm{~m} \\
\text { loa } 1113,39^{\circ} 47^{\prime} 22^{\prime \prime N}, 21^{\circ} 13^{\prime} 32^{\prime \prime E}\end{array}$ & EK 10.94.34 & $283.999-284.010$ \\
\hline 329 & $\begin{array}{l}\text { GR, loannina, Katara-Paß, } 19.07 .2017 \\
\text { Felsige Böschung, Buchenwald, kiefern, } 1775 \mathrm{~m} \\
\text { loa } 1114,39^{\circ} 46^{\prime} 55^{\prime \prime N}, 21^{\circ} 13^{\prime} 30^{\prime \prime E}\end{array}$ & EK 10.93.36 & $284.011-284.015$ \\
\hline 330 & $\begin{array}{l}\text { GR, loannina, N Metsovo, 19.07.2017 } \\
\text { Krautiger Hang, Wacholder, Kiefern, } 1416 \mathrm{~m} \\
\text { loa } 1115,39^{\circ} 48^{\prime} 01^{\prime \prime N}, 21^{\circ} 10^{\prime} 30^{\prime \prime E}\end{array}$ & EK 10.55.06 & $284.016-284.020$ \\
\hline 331 & $\begin{array}{l}\text { GR, loannina, SW Milia, 19.07.2017 } \\
\text { Buchenwald, Laubgebüsch, Krautfluren, } 1480 \mathrm{~m} \\
\text { loa } 1116,39^{\circ} 50^{\prime} 05^{\prime \prime N}, 21^{\circ} 11^{\prime} 27^{\prime \prime E}\end{array}$ & EK 10.69.34 & $284.021-284.045$ \\
\hline 332 & $\begin{array}{l}\text { GR, loannina, Anilio, } 20.07 .2017 \\
\text { Felshang, Laubgebüsch, } 943 \mathrm{~m} \\
\text { loa } 1117,39^{\circ} 45^{\prime} 41^{\prime \prime N}, 21^{\circ} 11^{\prime} 00^{\prime \prime E}\end{array}$ & EK 10.51.73 & $284.046-284.059$ \\
\hline 333 & $\begin{array}{l}\text { GR, loannina, Anilio, 20.07.2017 } \\
\text { Felsige Krautfluren, Laubwald, Tannen, } 1233 \mathrm{~m} \\
\text { loa } 1118,39^{\circ} 45^{\prime} 34^{\prime \prime N}, 21^{\circ} 11^{\prime} 58^{\prime \prime E}\end{array}$ & EK 10.71.11 & $284.060-284.077$ \\
\hline 334 & $\begin{array}{l}\text { GR, Ioannina, Anilio, 20.07.2017 } \\
\text { Tannen-Kiefern-Buchenwald, } 1430 \mathrm{~m} \\
\text { loa } 1119,39^{\circ} 45^{\prime} 05^{\prime \prime} \mathrm{N}, 21^{\circ} 12^{\prime} 16^{\prime \prime} \mathrm{E}\end{array}$ & EK 10.70.52 & $284.078-284.084$ \\
\hline 335 & $\begin{array}{l}\text { GR, loannina, Anilio, } 20.07 .2017 \\
\text { Felsige Bergwiesen, Bach, } 1627 \mathrm{~m} \\
\text { loa } 1120,39^{\circ} 44^{\prime} 52^{\prime \prime N}, 21^{\circ} 13^{\prime} 35^{\prime \prime E}\end{array}$ & EJ 19.99.48 & $284.085-284.098$ \\
\hline 336 & $\begin{array}{l}\text { GR, loannina, Anilio, 20.07.2017 } \\
\text { Felshang, Hartlaubgebüsch, } 1045 \mathrm{~m} \\
\text { loa } 1121,39^{\circ} 44^{\prime} 53^{\prime \prime N}, 21^{\circ} 10^{\prime} 28^{\prime \prime E}\end{array}$ & EJ 19.49.98 & $284.099-284.119$ \\
\hline $\begin{array}{c}46 \\
\mathrm{~b}\end{array}$ & $\begin{array}{l}\text { GR, loannina, S Anilio, } 20.07 .2017 \\
\text { Felswand, Hartlaubgebüsch, Wassergraben, } 980 \mathrm{~m} \\
\text { loa } 1094,39^{\circ} 44^{\prime} 42^{\prime \prime N}, 21^{\circ} 10^{\prime} 46^{\prime E}\end{array}$ & EJ 19.59.45 & $284.120-284.128$ \\
\hline $\begin{array}{c}47 \\
\mathrm{~b}\end{array}$ & $\begin{array}{l}\text { GR, loannina, S Anilio, } 20.07 .2017 \\
\text { Feuchte Fels-Kieshänge, } 995 \mathrm{~m} \\
\text { loa } 1095,39^{\circ} 44^{\prime} 37^{\prime \prime N}, 21^{\circ} 11^{\prime} 00^{\prime \prime E}\end{array}$ & EJ 19.59.73 & $284.129-284.133$ \\
\hline $\begin{array}{c}48 \\
\mathrm{~b}\end{array}$ & $\begin{array}{l}\text { GR, loannina, S Anilio, 18.06.2017 } \\
\text { Krautiger Hang, Laubgebüsch, } 980 \mathrm{~m} \\
\text { loa 1096, 394 } 44^{\prime} 28^{\prime \prime N}, 21^{\circ} 11^{\prime} 09^{\prime \prime E}\end{array}$ & EJ 19.59.90 & $284.134-284.148$ \\
\hline 337 & $\begin{array}{l}\text { GR, loannina, Anilio, } 20.07 .2017 \\
\text { Felshang, Buchenwald, } 1229 \mathrm{~m} \\
\text { loa } 1122,39^{\circ} 43^{\prime} 50^{\prime \prime N}, 21^{\circ} 11^{\prime} 57^{\prime \prime E}\end{array}$ & EJ 19.77.19 & $284.149-284.154$ \\
\hline $\begin{array}{c}32 \\
\mathrm{~b}\end{array}$ & $\begin{array}{l}\text { GR, loannina, SO Anilio, } 20.07 .2017 \\
\text { Bergwiesen, kuhbeweidet, } 1660 \mathrm{~m} \\
\text { loa } 1080,9,39^{\circ} 44^{\prime} 52^{\prime \prime} \mathrm{N}, 21^{\circ} 13^{\prime} 35^{\prime \prime} \mathrm{E}\end{array}$ & EJ 19.99.48 & $284.155-284.159$ \\
\hline \begin{tabular}{c|c}
55 \\
$b$
\end{tabular} & $\begin{array}{l}\text { GR, loannina, S Anilio, } 20.07 .2017 \\
\text { Bergwiesen, Buchenwald, } 1460 \mathrm{~m} \\
\text { loa } 1103,39^{\circ} 43^{\prime} 29^{\prime \prime} \mathrm{N}, 21^{\circ} 11^{\prime} 38^{\prime \prime E}\end{array}$ & EJ 19.67.62 & $284.155-284.159$ \\
\hline
\end{tabular}




\section{Species}

Acanthus balcanicus

277.406, loa 1094, S Anilio

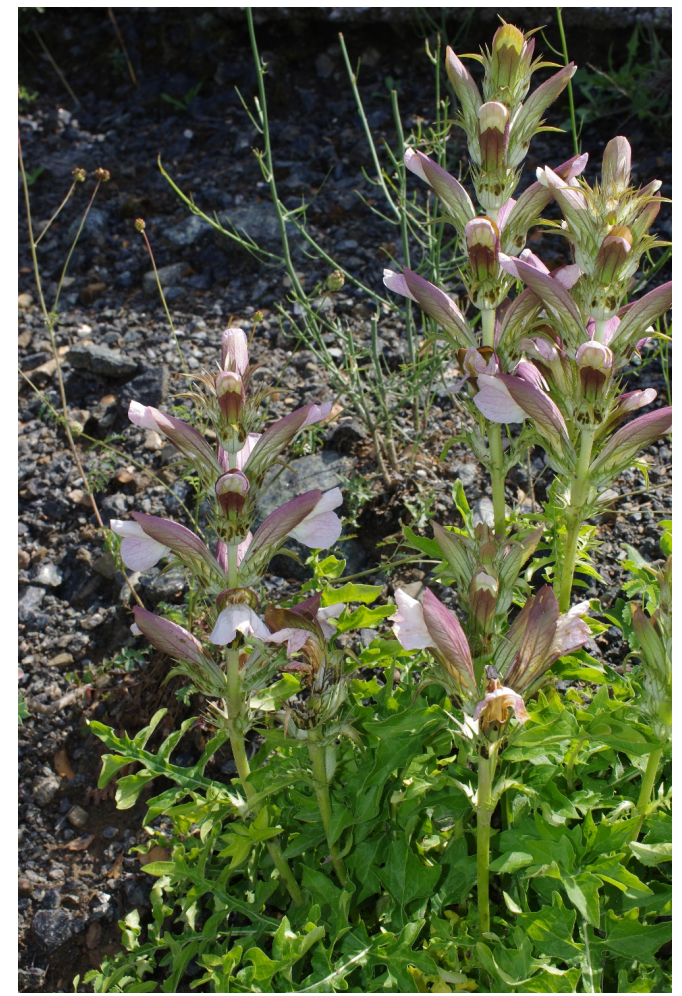

\section{Acer campestre}

276.633, loa 1063, W Potamia; 277.902, Koz 389, SO Siatista, Vourinos; 282.026, Dra 292, Nevrokopi; 283.526, Pel 361, SW Promachi

\section{Acer monspessulanum}

277.803, Koz 385, SO Siatista

\section{Acer obtusatum}

277.543, loa 1098, S Anilio

\section{Acer platanoides}

282.631, Ser 289, Ori Vrondous

\section{Acer pseudoplatanus}

282.731, Ser 291, N Neo Petritsi; 283.029, Kil 303, SO Livadia

Achillea ageratifolia subsp. ageratifolia

280.902, Dra 250, Falakron

Achillea chrysocoma

283.908, Ima 180, Seli

\section{Achillea clusiana}

283.368, Pel 353, Kajmaktsalan

\section{Achillea clypeolata}

280.926, Dra 253, Falakron; 281.129, Dra 262, NW Granitis; 281.763, Dra 281, OSO Granitis

\section{Achillea coarctata}

277.798, Koz 385, SO Siatista; 278.010, Koz 375, O Dafnero; 278.515, Thes 217, Vrasna;

278.630, Thes 221, Arethousa; 279.049, Kav 121, Platanotopos; 280.001, Dra 215, Livadero; 
280.221, Dra 225, NO Drama; 280.493, Dra 235, Skaloti; 281.340, Dra 270, W Nevrokopi; 281.610, Dra 278, Kato Vrondous; 281.849, Dra 284, NNO Volakas; 281.981, Dra 290, NO Potamia; 282.130, Dra 296, NO Nevrokopi; 282.171, Dra 299, SO Lefkogia; 282.429, Ser 279, Orini; 282.744, Ser 292, N Neo Petritsi; 282.765, Ser 293, N Neo Petritsi; 282.962, Kil 299, Kastaneri; 283.230, Pel 345, Kerasia; 283.326, Pel 351, Kajmaktsalan; 283.420, Pel 355, NW Loutraki; 283.529, Pel 361, SW Promachi; 283.616, Pel 365, N Vorino; 283.628, Pel 366, N Vorino

\section{Achillea collina}

280.022, Dra 216, Sidironero; 280.456, Dra 234, Skaloti; 280.747, Dra 245, SW Walddorf Elatias; 280.785, Dra 247, Walddorf Elatias; 281.186, Dra 264, NO Ochyro; 281.384, Dra 272, Vathytopo; 281.510, Dra 276, Perithori; 282.024, Dra 292, Nevrokopi; 282.311, Ser 274, SSW Ano Vrondous; 282.602, Ser 288, Ori Vrondous; 283.072, Kil 307, NW Livadia; 283.941, loa 1108, N Metsovo

\section{Achillea crithmifolia}

276.509, loa 1059, Ag. Nikolaos; 276.969, loa 1078, O Anilio; 279.421, Kav 93, Pangaion; 279.484, Kav 97, Pangaion; 280.254, Dra 227, Livadero; 281.765, Dra 281, OSO Granitis; 282.297, Ser 273, Ano Vrondous; 283.832, Ima 176, Tria-Pende Pigadia

\section{Achillea grandifolia}

282.523, Ser 283, Ori Vrondous; 282.739, Ser 292, N Neo Petritsi

\section{Achillea holosericea}

277.664, Tri 459, N Chaliki; 277.948, Koz 374, Vourinos; 284.011, loa 1114, Katara-Paß

\section{Achillea millefolium}

276.461, Tri 458, NO Katara-Pass; 277.465 , loa 1096, S Anilio; 277.676 , Tri 460, N Chaliki; 278.008, Koz 375, O Dafnero; 278.144, Gre 492, NO Varis; 278.298, Koz 380, Galatini; 278.311, Koz 381, Galatini; 279.329, Ser 260, Proti; 279.886, Dra 209, Livadero; 280.133, Dra 221, NO Drama; 280.258, Dra 227, Livadero; 280.274, Dra 228, Livadero; 280.365, Dra 232, Sidironero; 280.554, Dra 237, Skaloti; 280.682, Dra 242, SW Walddorf Elatias; 280.784, Dra 247, Walddorf Elatias; 280.836, Dra 248, Falakron; 281.269, Dra 266, W Nevrokopi; 281.601, Dra 278, Kato Vrondous; 281.689, Ser 271, Kato Vrondous; 281.718 , Dra 280, Nevrokopi; 281.772, Dra 282, NNO Volakas; 281.886, Dra 287, O Mikroklisoura; 282.121, Dra 296, NO Nevrokopi; 282.192, Dra 299, SO Lefkogia; 282.430, Ser 279, Orini; 282.543, Ser 284, Ori Vrondous; 282.666, Ser 290, N Neo Petritsi; 282.875, Kil 295, NW Griva; 283.008, Kil 302, Kastaneri; 283.162, Pel 343, Kerasia; 283.258, Pel 347, Kajmaktsalan; 283.487, Pel 359, NW Loutraki; 283.756, Ima 172, S Ag. Pavlos; 283.842, Ima 178, Seli; 283.857, Ima 178, Seli; 283.979, loa 1111, NO Metsovo; 284.041, loa 1116, SW Milia; 284.060, loa 1118, Anilio; 284.155, loa 1103 b, S Anilio

\section{Achillea nobilis}

277.877, Koz 388, SO Siatista, Vourinos; 279.552, Kav 102, Pangaion; 279.588, Kav 104, Pangaion; 280.160, Dra 221, NO Drama; 280.997, Dra 257, Granitis; 282.229 , Dra 300, SO Lefkogia; 283.898, Ima 180, Seli

\section{Achillea stricta}

282.904, Kil 297, WNW Kastaneri

\section{Acinos alpinus subsp. alpinus}

276.824, loa 1070, NNW Metsovo; 277.356, loa 1093, S Anilio; 278.151, Gre 492, NO Varis; 278.233, Koz 378, N Siatista; 278.785, Kav 112, NO Paralia Myrtofytou; 279.717, Ser 267, SW Skopia; 281.149, Dra 262, NW Granitis; 281.231, Dra 265, NO Granitis; 281.726, Dra 281, OSO Granitis

\section{Acinos alpinus subsp. majoranifolius}

277.859, Koz 387, SO Siatista, Vourinos; 277.871, Koz 388, SO Siatista, Vourinos; 277.975 , Koz 374, Vourinos; 280.879, Dra 249, Falakron

Acinos alpinus subsp. meridionalis 
276.357, loa 1128, N Metsovo; 276.792, loa 1070, NNW Metsovo; 276.972, loa 1079, SO Anilio; 277.272, loa 1090, NNO Metsovo; 277.604, loa 1102, S Anilio; 282.125, Dra 296, NO Nevrokopi; 283.248, Pel 346, Kajmaktsalan; 283.340, Pel 351, Kajmaktsalan; 283.419, Pel 355, NW Loutraki; 283.925, Ima 180, Seli

\section{Acinos arvensis}

277.816, Koz 387, SO Siatista, Vourinos; 278.946, Kav 118, Galipsos; 279.069, Kav 121, Platanotopos; 280.099, Dra 220, Sidironero; 280.402, Dra 233, Sidironero; 280.558, Dra 237, Skaloti; 281.143, Dra 262, NW Granitis; 281.786, Dra 282, NNO Volakas; 281.830, Dra 283, NNO Volakas

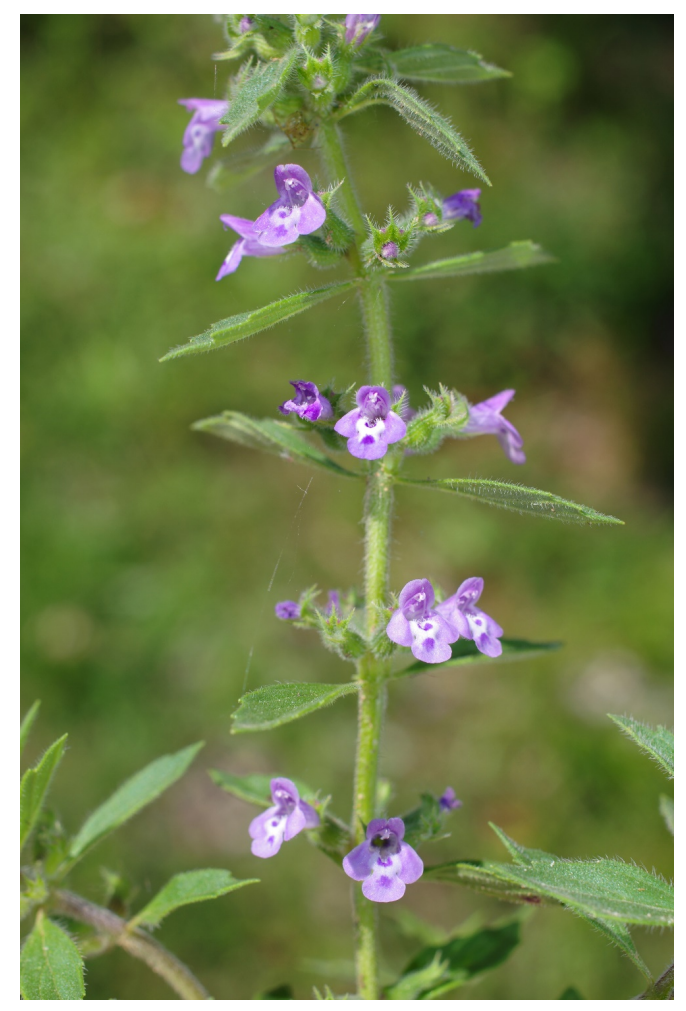

\section{Acinos graveolens}

280.094, Dra 219, Sidironero; 282.197, Dra 299, SO Lefkogia

\section{Acinos suaveolens}

278.980, Kav 119, Podochori; 279.051, Kav 121, Platanotopos; 279.276, Ser 258, Mikro Souli; 279.317, Ser 260, Proti; 279.401, Kav 92, Pangaion; 280.109, Dra 220, Sidironero; 280.134, Dra 221, NO Drama; 281.450, Dra 274, Vathytopo; 281.975, Dra 290, NO Potamia; 282.387, Ser 277, ONO Serres

\section{Adiantum capillus-veneris}

283.431, Pel 355, NW Loutraki

Adonis flammea

278.255, Koz 379, Galatini; 278.372, Koz 382, Galatini

\section{Aegilops geniculata}

276.490, loa 1059, Ag. Nikolaos; 276.511, loa 1059, Ag. Nikolaos; 279.493, Kav 97, Pangaion; 280.118, Dra 220, Sidironero

\section{Aegilops neglecta subsp. neglecta}

276.486, loa 1059, Ag. Nikolaos; 276.640, loa 1063, W Potamia; 277.147, loa 1085, N Metsovo; 277.333, loa 1093, S Anilio; 277.918, Koz 391, SO Siatista, Vourinos; 278.312, Koz 381, Galatini; 278.543, Thes 219, Vrasna; 278.611, Thes 221, Arethousa; 278.933, Kav 118, Galipsos; 278.964, Kav 119, Podochori; 279.223, Ser 257, Nea Fili; 279.409, Kav 92, Pangaion; 279.471, Kav 96, Pangaion; 280.237, Dra 226, N Taxiarches; 281.490, Dra 275, Vathytopo; 281.647, Dra 279, Kato Vrondous; 282.441, Ser 280, Orini; -20.482, loa 1066, NO Karyes 


\section{Aegilops triuncialis}

276.303, loa 1125, NO Dodoni; 276.307, loa 1125, NO Dodoni; 276.514, loa 1059, Ag. Nikolaos; 276.687, loa 1065, NO Karyes; 277.834, Koz 387, SO Siatista, Vourinos; 278.101, Gre 492, NO Varis; 278.186, Gre 495, Varis; 278.389, Koz 383, Galatini; 278.822 , Kav 114, SO Folia; 278.995, Kav 119, Podochori; 279.064, Kav 121, Platanotopos; 279.231, Ser 257, Nea Fili; 279.272, Ser 258, Mikro Souli; 279.290, Ser 259, Proti; 279.494, Kav 97, Pangaion; 279.827, Dra 207, NO Skopia; 279.858, Dra 209, Livadero; 280.092, Dra 219, Sidironero; 281.061, Dra 259, Granitis; 281.212, Dra 265, NO Granitis; 281.355, Dra 270, W Nevrokopi; 281.582, Dra 277, Perithori; 281.803, Dra 282, NNO Volakas; 282.158, Dra 298, SO Lefkogia; 282.370, Ser 276, SSW Ano Vrondous; 282.410, Ser 279, Orini; 282.793, Ser 295, N Neo Petritsi

\section{Aegopodium podagraria}

276.466, Tri 458, NO Katara-Pass; 277.541, loa 1098, S Anilio; 280.740, Dra 245, SW Walddorf Elatias; 281.087, Dra 260, Granitis; 281.630, Dra 279, Kato Vrondous; 282.346 , Ser 275, SSW Ano Vrondous; 282.597, Ser 287, Ori Vrondous

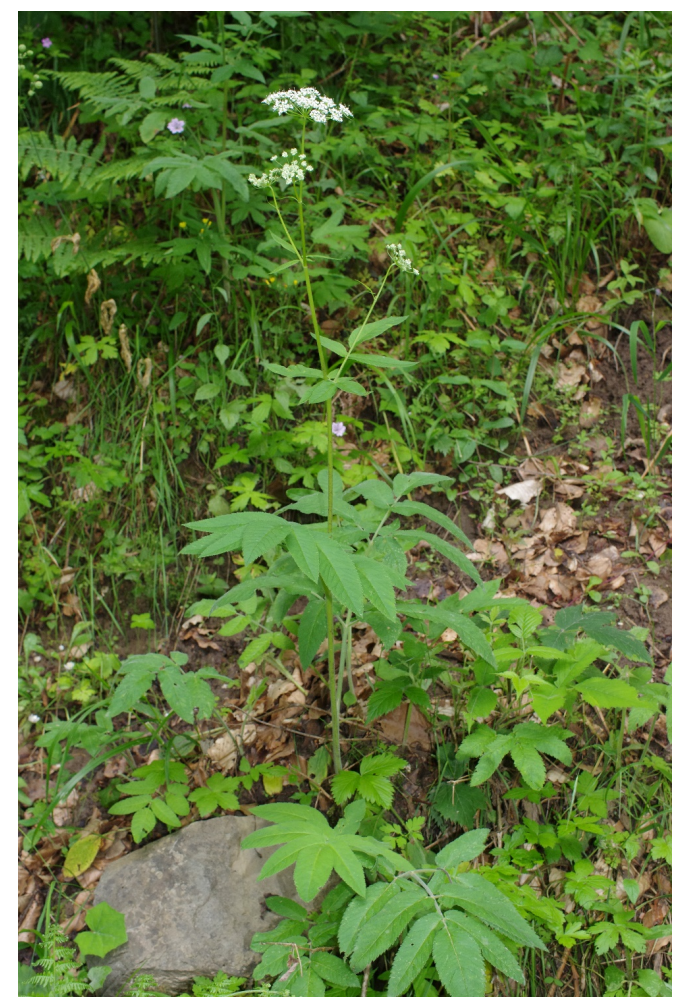

\section{Aethionema saxatile subsp. graecum}

276.432, loa 1058, Katara-Pass; 277.090, loa 1081, W Metsovo; 277.324, loa 1093, S Anilio; 278.135, Gre 492, NO Varis; 279.601, Kav 105, Pangaion; 280.938, Dra 253, Falakron

\section{Agrimonia eupatoria}

276.645, loa 1063, W Potamia; 277.793, Koz 385, SO Siatista; 278.161, Gre 494, SO Varis; 278.551, Thes 219, Vrasna; 278.856, Kav 115, SW Folia; 279.363, Ser 261, Proti; 279.428 , Kav 94, Pangaion; 279.772, Ser 268, SW Skopia; 279.819, Ser 269, NO Skopia; 280.013 , Dra 216, Sidironero; 280.292, Dra 228, Livadero; 280.376, Dra 232, Sidironero; 281.283, Dra 267, W Nevrokopi; 281.576, Dra 277, Perithori; 281.705, Dra 280, Nevrokopi; 281.731, Dra 281, OSO Granitis; 281.845, Dra 284, NNO Volakas; 282.019, Dra 292, Nevrokopi; 282.185, Dra 299, SO Lefkogia; 282.268, Ser 272, Ano Vrondous; 282.375, Ser 276, SSW Ano Vrondous; 282.957, Kil 299, Kastaneri; 283.439, Pel 356, NW Loutraki; 283.686, Pel 369, O Vorino; 283.745, Ima 172, S Ag. Pavlos

\section{Agrimonia procera}

277.566, loa 1099, S Anilio; 281.083, Dra 260, Granitis 


\section{Agrostis}

282.857, Kil 294, NW Griva

Agrostis canina

283.310, Pel 350, Kajmaktsalan; 283.376, Pel 353, Kajmaktsalan

Agrostis capillaris

283.412, Pel 355, NW Loutraki

\section{Agrostis gigantea}

283.041, Kil 304, SO Livadia; 283.178, Pel 343, Kerasia

\section{Agrostis stolonifera}

278.122, Gre 493, NO Varis; 280.105, Dra 220, Sidironero; 280.222, Dra 225, NO Drama; 280.423, Dra 233, Sidironero; 280.983, Dra 257, Granitis; 281.554, Dra 277, Perithori; 281.891, Dra 287, O Mikroklisoura; 282.058, Dra 294, NO Nevrokopi; 282.273, Ser 272, Ano Vrondous; 282.455, Ser 280, Orini; 282.617, Ser 288, Ori Vrondous; 282.903, Kil 296, N Kastaneri; 282.956, Kil 299, Kastaneri; 282.968, Kil 299, Kastaneri; 283.109, Pel 339, S Archangelo; 283.201, Pel 343, Kerasia; 283.393, Pel 354, NW Loutraki; 283.527, Pel 361, SW Promachi; 283.807, Ima 175, S Ag. Pavlos; 283.894, Ima 179, Seli; 284.017, loa 1115, N Metsovo; 284.059, loa 1117, Anilio

\section{Aira caryophyllea}

279.943, Dra 212, Livadero; 280.591, Dra 238, Skaloti; 282.542, Ser 284, Ori Vrondous

\section{Aira elegantissima}

277.596, loa 1102, S Anilio; 279.918, Dra 211, Livadero; 280.410, Dra 233, Sidironero

\section{Ajuga chamaepitys}

276.598, loa 1061, SSO Potamia; 278.337, Koz 381, Galatini; 278.996, Kav 119, Podochori; 279.729, Ser 267, SW Skopia; 279.763, Ser 268, SW Skopia; 280.234, Dra 226, N

Taxiarches; 281.142, Dra 262, NW Granitis

\section{Ajuga genevensis}

277.861, Koz 388, SO Siatista, Vourinos; 280.602, Dra 238, Skaloti; 280.712, Dra 243, SW Walddorf Elatias; 282.596, Ser 287, Ori Vrondous

\section{Ajuga pyramidalis}

279.502, Kav 98, Pangaion

\section{Ajuga reptans}

277.028, loa 1080, SO Anilio; 277.638, loa 1104, S Anilio

\section{Alcea rosea}

277.802, Koz 385, SO Siatista; 279.115, Kav 90, SW Moustheni; 282.787 , Ser 295, N Neo Petritsi

\section{Alchemilla glabra}

283.327, Pel 351, Kajmaktsalan

\section{Alchemilla monticola}

280.630, Dra 240, Elatias; 280.637, Dra 241, Elatias; 280.875, Dra 249, Falakron

\section{Alchemilla xanthochlora}

276.768, loa 1069, NW Metsovo; 276.875, loa 1072, NNW Metsovo; 277.054, loa 1080, SO Anilio; 277.618, loa 1103, S Anilio

\section{Alisma lanceolatum}

281.934, Dra 288, SW Potamia

\section{Alkanna pindicola}




\title{
Alliaria petiolata
}

277.511, loa 1097, S Anilio; 282.247, Dra 301, Ano Vrondous; 282.579, Ser 286, Ori Vrondous

\section{Allium}

283.961, loa 1110, N Metsovo

\section{Allium ampeloprasum}

278.441, Thes 214, Paralia Vrasna; 278.571, Thes 220, Arethousa; 278.643, Kav 106, Paralia Ofryniou; 279.042, Kav 120, Podochori; 279.096, Kav 89, Misoropi; 279.304, Ser 259, Proti; 279.656, Ser 265, Myrrini; 281.088, Dra 260, Granitis; 281.316, Dra 268, W Nevrokopi

\section{Allium chamaespathum}

\author{
279.702, Ser 266, SO Nea Zichni
}

\section{Allium flavum subsp. flavum}

279.048, Kav 120, Podochori; 279.274, Ser 258, Mikro Souli; 283.783, Ima 173, S Ag. Pavlos

\section{Allium flavum subsp. tauricum}

278.029, Koz 376, W Exarcho; 278.093, Gre 492, NO Varis; 278.629, Thes 221, Arethousa; 278.934, Kav 118, Galipsos; 279.026, Kav 120, Podochori; 279.196, Ser 257, Nea Fili; 279.273, Ser 258, Mikro Souli; 279.327, Ser 260, Proti; 279.722, Ser 267, SW Skopia; 279.829, Dra 207, NO Skopia; 280.156, Dra 221, NO Drama; 281.130, Dra 262, NW Granitis; 281.211, Dra 265, NO Granitis; 281.429, Dra 274, Vathytopo; 281.875, Dra 286, SW Mikroklisoura; 281.979, Dra 290, NO Potamia; 282.002, Dra 292, Nevrokopi; 283.443, Pel 356, NW Loutraki; 283.486, Pel 359, NW Loutraki; 283.960, loa 1110, N Metsovo; 284.007, Ioa 1113, Katara-Paß

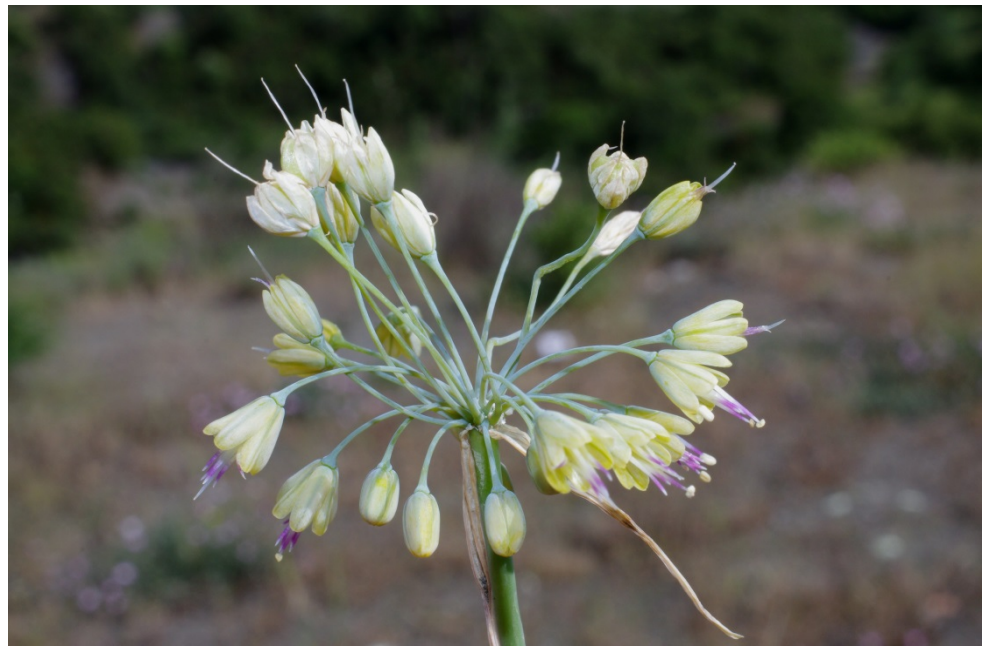

\section{Allium guttatum subsp. sardoum}

278.726, Kav 108, Paralia Ofryniou; 279.613, Ser 263, Dravoskas; 282.395, Ser 278, N Serres; 282.781, Ser 294, N Neo Petritsi; 283.457, Pel 358, N Loutraki; 283.966, loa 1056 b, NO Metsovo

\section{Allium scorodoprasum}

278.355, Koz 382, Galatini; 278.420, Koz 384, Galatini; 281.958, Dra 289, NO Potamia; 282.077, Dra 294, NO Nevrokopi; 282.078, Dra 294, NO Nevrokopi; 283.427, Pel 355, NW Loutraki

\section{Allium sphaerocephalon}

278.035, Koz 376, W Exarcho; 278.215, Koz 378, N Siatista; 278.601, Thes 221, Arethousa; 279.043, Kav 120, Podochori; 279.848, Dra 208, NO Skopia; 282.168, Dra 298, SO Lefkogia; 
283.403, Pel 354, NW Loutraki; 283.450, Pel 357, NW Loutraki; 283.606, Pel 365, N Vorino; 284.119, loa 1121, Anilio

\section{Allium trifoliatum}

277.032, loa 1080, SO Anilio

\section{Allium ursinum subsp. ucrainicum}

277.219, loa 1088, NNO Metsovo

\section{Allium vineale}

284.056, loa 1117, Anilio

\section{Alopecurus myosuroides}

278.257, Koz 379, Galatini; 278.663, Kav 106, Paralia Ofryniou

\section{Alopecurus pratensis}

277.251, loa 1089, NNO Metsovo; 280.091, Dra 219, Sidironero

\section{Alopecurus rendlei}

276.911, loa 1073, NNW Metsovo

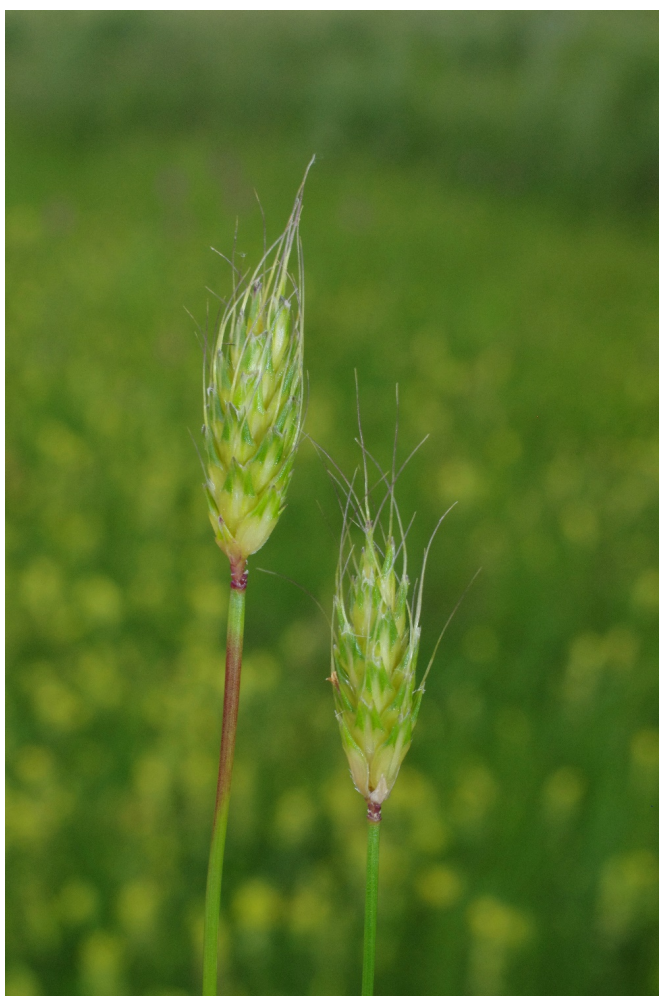

\section{Althaea cannabina}

278.637, Kav 106, Paralia Ofryniou; 279.645, Ser 264, Dravoskas; 283.636, Pel 366, N Vorino

\section{Alyssum}

276.561, loa 1060, SSO Potamia

\section{Alyssum alyssoides}

276.832, loa 1070, NNW Metsovo; 277.481, loa 1097, S Anilio; 277.673, Tri 460, N Chaliki; 280.114, Dra 220, Sidironero

\section{Alyssum chalcidicum}

277.153, loa 1085, N Metsovo; 278.009, Koz 375, O Dafnero; 278.069, Gre 492, NO Varis; 279.199, Ser 257, Nea Fili; 279.233, Ser 258, Mikro Souli; 279.318, Ser 260, Proti; 279.634 , Ser 263, Dravoskas; 279.726, Ser 267, SW Skopia; 280.062, Dra 218, Sidironero; 280.145 , Dra 221, NO Drama; 280.262, Dra 227, Livadero; 280.360, Dra 232, Sidironero; 280.925 , 
Dra 252, Falakron; 281.064, Dra 259, Granitis; 281.232, Dra 265, NO Granitis; 281.589, Dra 278, Kato Vrondous; 281.742, Dra 281, OSO Granitis; 281.982, Dra 290, NO Potamia;

282.363, Ser 276, SSW Ano Vrondous; 283.489, Pel 359, NW Loutraki

Alyssum corymbosoides

282.783, Ser 294, N Neo Petritsi

Alyssum heldreichii

276.435, loa 1058, Katara-Pass

Alyssum montanum subsp. montanum

276.453, Tri 457, NO Katara-Pass; 277.360 , loa 1093, S Anilio; 278.424, Koz 384, Galatini; 280.123, Dra 221, NO Drama; 280.827, Dra 248, Falakron

Alyssum montanum subsp. repens

276.591, loa 1061, SSO Potamia; 277.747, Tri 461, N Chaliki; 278.432, Koz 384, Galatini; 281.235, Dra 265, NO Granitis

\section{Alyssum simplex}

276.971, loa 1079, SO Anilio; 277.112, loa 1084, N Metsovo; 277.925, Koz 392, SO Siatista, Vourinos; 278.305, Koz 380, Galatini; 278.360, Koz 382, Galatini

\section{Alyssum smolikanum}

283.806, Ima 175, S Ag. Pavlos

\section{Alyssum strigosum}

278.744, Kav 109, Loutra Eleftherion; 278.806, Kav 113, NO Paralia Myrtofytou; 278.959,

Kav 119, Podochori

\section{Alyssum turkestanicum}

277.828, Koz 387, SO Siatista, Vourinos

\section{Amaranthus albus}

278.689, Kav 107, Paralia Ofryniou; 278.799, Kav 113, NO Paralia Myrtofytou; 278.922, Kav 117, Galipsos; 279.015, Kav 119, Podochori; 279.213, Ser 257, Nea Fili; 279.271, Ser 258, Mikro Souli; 282.817, Ser 296, NO Strymoniko; 283.714, Pel 370, SW Foustani

\section{Amaranthus blitoides}

278.677, Kav 107, Paralia Ofryniou

\section{Amaranthus deflexus}

279.095, Kav 89, Misoropi; 279.132, Kav 91, Orfani

\section{Amaranthus hybridus}

282.278, Ser 272, Ano Vrondous; 282.819, Ser 296, NO Strymoniko

\section{Amaranthus powellii}

281.420, Dra 273, Vathytopo

\section{Amaranthus retroflexus}

278.929, Kav 117, Galipsos; 279.130, Kav 91, Orfani; 279.187, Ser 256, Paleokomi;

279.749, Ser 268, SW Skopia; 280.988, Dra 257, Granitis; 281.204, Dra 264, NO Ochyro;

281.255, Dra 266, W Nevrokopi; 281.505, Dra 276, Perithori; 283.584, Pel 364, Loutraki;

283.662, Pel 368, OSO Vorino

\section{Anacamptis coriophora}

276.781, loa 1069, NW Metsovo; 276.896, loa 1072, NNW Metsovo; 277.095, loa 1083, NW Metsovo; 277.297, loa 1091, SW Milia

\section{Anacamptis morio}

-20.480, loa 1056, NO Metsovo; -20.484, loa 1078, O Anilio 


\section{Anacamptis pyramidalis}

277.488, loa 1097, S Anilio; 277.668, Tri 459, N Chaliki

\section{Anagallis arvensis}

276.479, loa 1059, Ag. Nikolaos; 277.477, loa 1097, S Anilio; 278.085, Gre 492, NO Varis; 278.260, Koz 379, Galatini; 278.368, Koz 382, Galatini; 278.683, Kav 107, Paralia Ofryniou; 278.975, Kav 119, Podochori; 279.767, Ser 268, SW Skopia; 280.107, Dra 220, Sidironero

\section{Anchusa azurea}

276.669, loa 1064, ONO Karyes

\section{Anchusa officinalis subsp. intacta}

278.020, Koz 376, W Exarcho; 278.459, Thes 214, Paralia Vrasna; 278.614, Thes 221, Arethousa; 278.669, Kav 107, Paralia Ofryniou; 279.100, Kav 90, SW Moustheni; 279.670, Ser 265, Myrrini; 280.209, Dra 224, NO Drama; 281.247, Dra 266, W Nevrokopi; 281.460, Dra 274, Vathytopo; 281.688, Ser 271, Kato Vrondous; 281.826, Dra 283, NNO Volakas; 281.868, Dra 286, SW Mikroklisoura; 281.971, Dra 290, NO Potamia; 282.610, Ser 288, Ori Vrondous; 282.772, Ser 293, N Neo Petritsi; 282.826, Ser 298, Parapotamos

\section{Anchusa officinalis subsp. officinalis}

276.662, loa 1064, ONO Karyes; 277.931, Koz 392, SO Siatista, Vourinos; 278.362, Koz 382, Galatini; 278.762, Kav 110, Loutra Eleftherion; 279.796, Ser 269, NO Skopia; 280.148, Dra 221, NO Drama; 280.807, Dra 247, Walddorf Elatias; 280.992, Dra 257, Granitis; 281.174, Dra 264, NO Ochyro; 281.339, Dra 270, W Nevrokopi; 281.493, Dra 276, Perithori; 282.378, Ser 276, SSW Ano Vrondous

\section{Anchusa undulata subsp. hybrida}

276.308, loa 1125, NO Dodoni; 276.601, loa 1061, SSO Potamia

\section{Anethum graveolens}

280.982, Dra 257, Granitis

\section{Anogramma leptophylla}

279.559, Kav 102, Pangaion; 280.976, Dra 256, Falakron

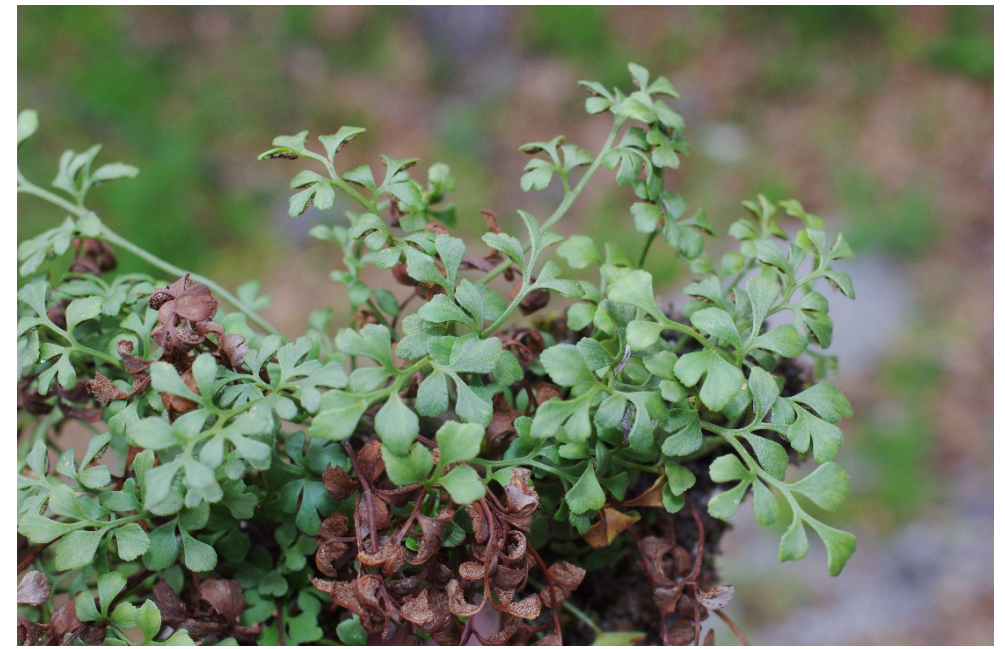

\section{Anthemis}

277.211, loa 1088, NNO Metsovo; 279.909, Dra 210, Livadero; 279.955, Dra 212, Livadero; 281.477, Dra 275, Vathytopo; 282.162, Dra 298, SO Lefkogia

\section{Anthemis altissima}

276.810, loa 1070, NNW Metsovo; 277.474, loa 1096, S Anilio; 278.100, Gre 492, NO Varis; 278.388, Koz 382, Galatini; 278.584, Thes 220, Arethousa; 278.728, Kav 108, Paralia 
Ofryniou; 279.191, Ser 256, Paleokomi; 279.350, Ser 261, Proti; 279.840, Dra 207, NO Skopia; 280.084, Dra 219, Sidironero

Anthemis arvensis subsp. arvensis

276.487, loa 1059, Ag. Nikolaos; 277.116, loa 1084, N Metsovo; 277.826, Koz 387, SO Siatista, Vourinos; 281.518, Dra 276, Perithori

\section{Anthemis arvensis subsp. cyllenea}

276.585, loa 1060, SSO Potamia; 276.837, loa 1070, NNW Metsovo; 277.687, Tri 460, N Chaliki; 278.011, Koz 375, O Dafnero; 278.087, Gre 492, NO Varis; 278.341, Koz 381, Galatini; 278.446, Thes 214, Paralia Vrasna; 279.004, Kav 119, Podochori; 279.065, Kav 121, Platanotopos; 279.207, Ser 257, Nea Fili; 281.322, Dra 268, W Nevrokopi; 283.169, Pel 343, Kerasia

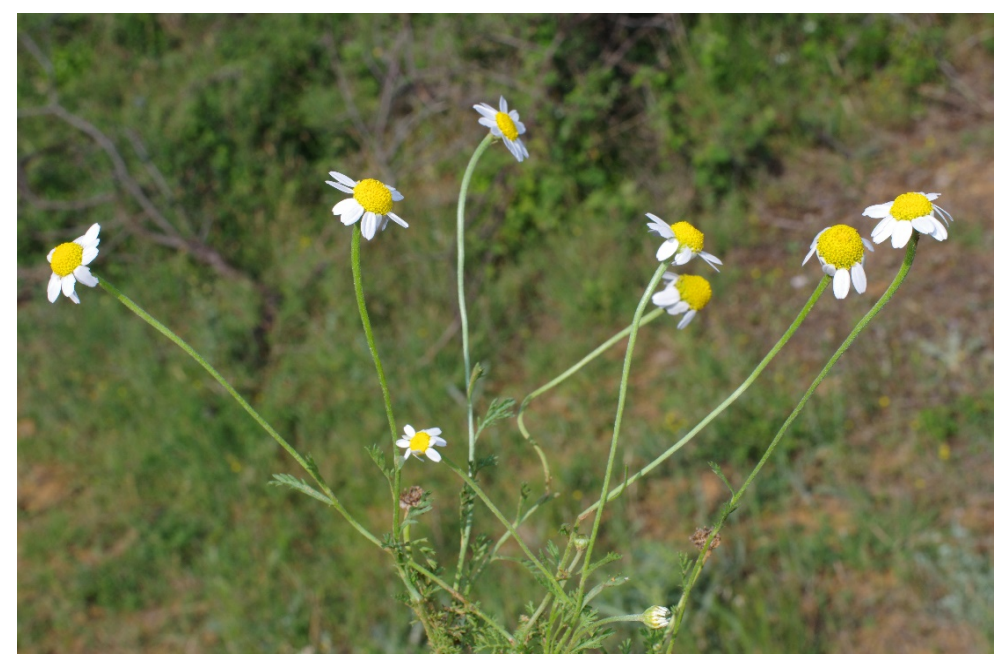

Anthemis arvensis subsp. incrassata

283.359, Pel 352, Kajmaktsalan

Anthemis auriculata

279.334, Ser 260, Proti

\section{Anthemis carpatica}

280.781, Dra 247, Walddorf Elatias; 282.270, Ser 272, Ano Vrondous; 282.328, Ser 274, SSW Ano Vrondous

\section{Anthemis cotula}

278.267, Koz 379, Galatini; 278.581, Thes 220, Arethousa; 279.496, Kav 97, Pangaion; 280.096, Dra 220, Sidironero; 280.415, Dra 233, Sidironero; 280.981, Dra 257, Granitis; 281.080, Dra 260, Granitis; 281.253, Dra 266, W Nevrokopi; 281.334, Dra 269, W Nevrokopi; 281.361, Dra 271, Vathytopo; 281.623, Dra 279, Kato Vrondous; 281.790, Dra 282, NNO Volakas; 281.954, Dra 289, NO Potamia; 282.206, Dra 300, SO Lefkogia; 282.371, Ser 276, SSW Ano Vrondous; 283.659, Pel 367, Vorino

Anthemis cretica subsp. carpatica

277.021, loa 1080 , SO Anilio

Anthemis orbelica

282.231, Dra 300, SO Lefkogia

\section{Anthemis parnassica}

277.372, loa 1093, S Anilio; 277.378, loa 1093, S Anilio; 278.168, Gre 494, SO Varis;

278.302, Koz 380, Galatini; 278.598, Thes 220, Arethousa; 281.911, Dra 287, O

Mikroklisoura

\section{Anthemis pindicola}


276.665, loa 1064, ONO Karyes; 277.519, loa 1097, S Anilio; 280.878, Dra 249, Falakron; 280.910, Dra 251, Falakron; 280.941, Dra 254, Falakron; 281.163, Dra 263, NW Granitis; 281.692, Ser 271, Kato Vrondous; 283.369, Pel 353, Kajmaktsalan

\section{Anthemis tinctoria subsp. tinctoria}

277.786, Koz 385, SO Siatista; 278.402, Koz 383, Galatini; 278.508, Thes 217, Vrasna; 278.858, Kav 115, SW Folia; 279.112, Kav 90, SW Moustheni; 279.355, Ser 261, Proti; 279.458, Kav 95, Pangaion; 279.716, Ser 266, SO Nea Zichni; 279.765, Ser 268, SW Skopia; 279.879, Dra 209, Livadero; 280.228, Dra 225, NO Drama; 280.273, Dra 228, Livadero; 280.370, Dra 232, Sidironero; 280.457, Dra 234, Skaloti; 280.809, Dra 247, Walddorf Elatias; 281.052, Dra 259, Granitis; 281.282, Dra 267, W Nevrokopi; 281.432, Dra 274, Vathytopo; 281.563, Dra 277, Perithori; 281.612, Dra 278, Kato Vrondous; 281.700, Dra 280, Nevrokopi; 281.741, Dra 281, OSO Granitis; 281.839, Dra 284, NNO Volakas; 281.898, Dra 287, O Mikroklisoura; 282.110, Dra 296, NO Nevrokopi; 282.174, Dra 299, SO Lefkogia; 282.372, Ser 276, SSW Ano Vrondous; 282.459, Ser 280, Orini; 282.869, Kil 295, NW Griva; 283.002, Kil 301, Kastaneri; 283.086, Kil 307, NW Livadia; 283.801, Ima 174, S Ag. Pavlos

\section{Anthemis tomentosa}

278.731, Kav 108, Paralia Ofryniou; 284.212, Kav 108, Paralia Ofryniou

\section{Anthemis triumfettii}

276.463, Tri 458, NO Katara-Pass; 279.306, Ser 259, Proti; 282.641, Ser 290, N Neo Petritsi; 282.757, Ser 292, N Neo Petritsi; 283.460, Pel 358, N Loutraki; 284.148, loa 1096 b, S Anilio

\section{Anthemis werneri}

278.688, Kav 107, Paralia Ofryniou

\section{Anthoxanthum odoratum}

277.560, loa 1099, S Anilio; 280.608, Dra 238, Skaloti; -20.474, loa 1055, N Metsovo

\section{Anthyllis aurea}

280.896, Dra 250, Falakron

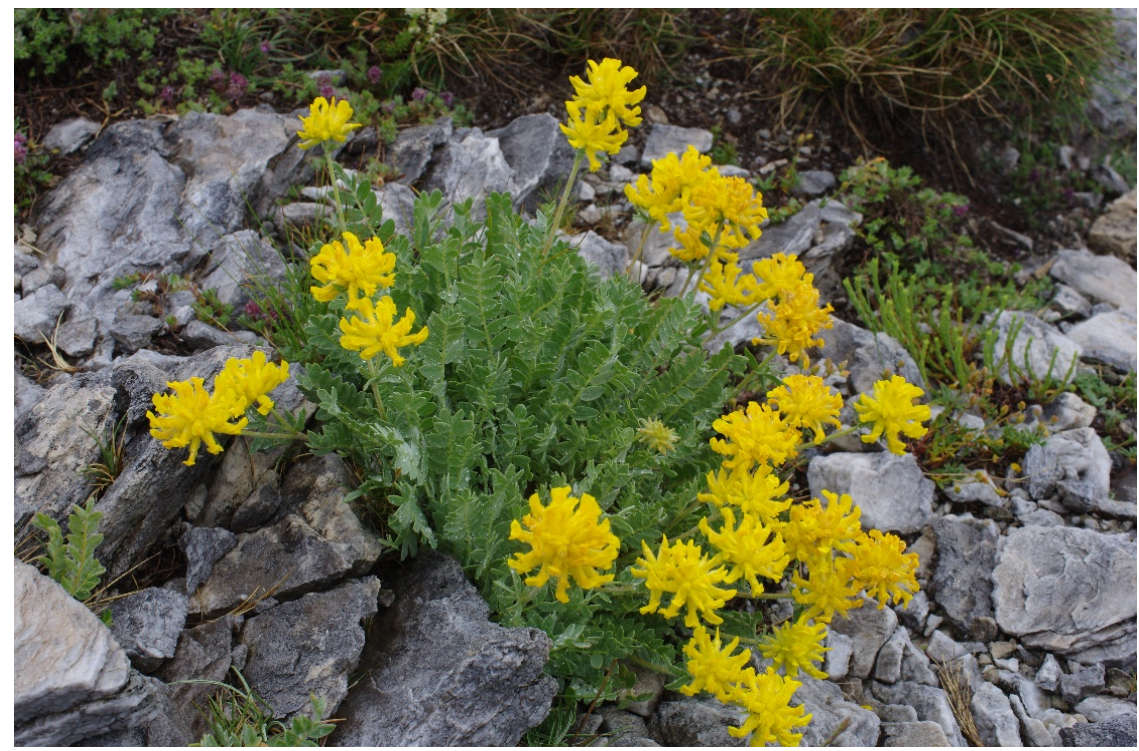

Anthyllis montana subsp. jacquinii 280.894, Dra 250, Falakron

Anthyllis vulneraria subsp. alpestris

276.468, Tri 458, NO Katara-Pass; 277.711, Tri 460, N Chaliki 


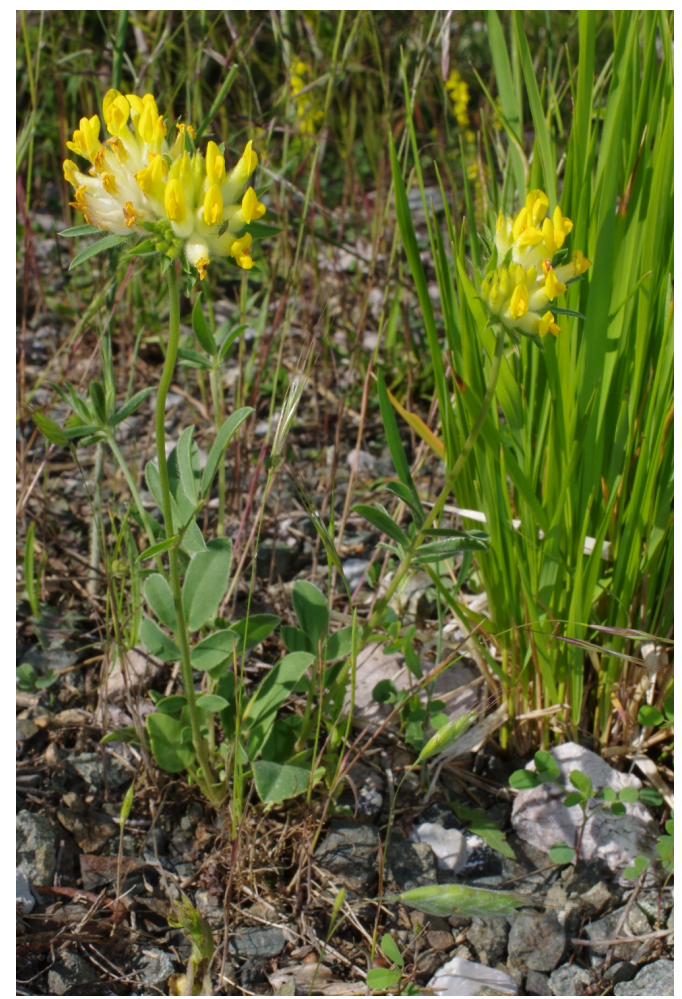

Anthyllis vulneraria subsp. bulgarica

277.349, loa 1093, S Anilio; 278.127, Gre 493, NO Varis

Anthyllis vulneraria subsp. pindicola

276.588, loa 1060, SSO Potamia

\section{Anthyllis vulneraria subsp. rubriflora}

276.720, loa 1066, NO Karyes; 277.868, Koz 388, SO Siatista, Vourinos; 278.242, Koz 378, N Siatista; 280.835, Dra 248, Falakron; 280.961, Dra 255, Falakron; 283.038, Kil 303, SO Livadia

\section{Apera spica-venti}

278.193, Gre 495, Varis; 278.262, Koz 379, Galatini; 278.452, Thes 214, Paralia Vrasna; 279.339, Ser 261, Proti; 279.939, Dra 212, Livadero; 279.965, Dra 213, Livadero; 281.040 , Dra 258, Granitis; 281.213, Dra 265, NO Granitis; 281.310, Dra 268, W Nevrokopi; 281.528 , Dra 276, Perithori; 281.673, Ser 271, Kato Vrondous; 282.167, Dra 298, SO Lefkogia; 282.255, Dra 301, Ano Vrondous; 282.335, Ser 274, SSW Ano Vrondous; 283.090, Pel 339, S Archangelo; 284.228, Dra 301, Ano Vrondous

\section{Arabis glabra}

276.323, loa 1125, NO Dodoni; 277.751, Tri 461, N Chaliki; 279.567, Kav 103, Pangaion; 280.638, Dra 241, Elatias; 280.811, Dra 247, Walddorf Elatias; 282.533 , Ser 284, Ori Vrondous; 282.607, Ser 288, Ori Vrondous

\section{Arabis sagittata}

276.469, Tri 458, NO Katara-Pass; 276.581, loa 1060, SSO Potamia; 276.885, loa 1072, NNW Metsovo; 277.275, loa 1090, NNO Metsovo; 277.530, loa 1097, S Anilio; 277.707, Tri 460, N Chaliki; 277.991, Koz 375, O Dafnero; 279.778, Ser 268, SW Skopia; 280.522, Dra 236, Skaloti; 280.745, Dra 245, SW Walddorf Elatias; 281.717, Dra 280, Nevrokopi

\section{Arabis sudetica}

276.348, loa 1128, N Metsovo; 277.592, loa 1101, S Anilio; 281.751, Dra 281, OSO Granitis; 283.278, Pel 348, Kajmaktsalan

\section{Arabis turrita}

276.656, loa 1063, W Potamia; 277.505, loa 1097, S Anilio; 284.144, loa 1096 b, S Anilio 
Arctium lappa

282.742, Ser 292, N Neo Petritsi

\section{Arctium tomentosum}

281.379, Dra 271, Vathytopo

\section{Aremonia agrimonoides}

276.879, loa 1072, NNW Metsovo; 277.190, loa 1087, NNO Metsovo; 277.614, loa 1103, S Anilio

\section{Arenaria leptoclados}

276.689, loa 1065, NO Karyes; 277.709, Tri 460, N Chaliki; 277.953, Koz 374, Vourinos; 284.001, loa 1113, Katara-Paß

\section{Arenaria rotundifolia}

280.770, Dra 246, SW Walddorf Elatias

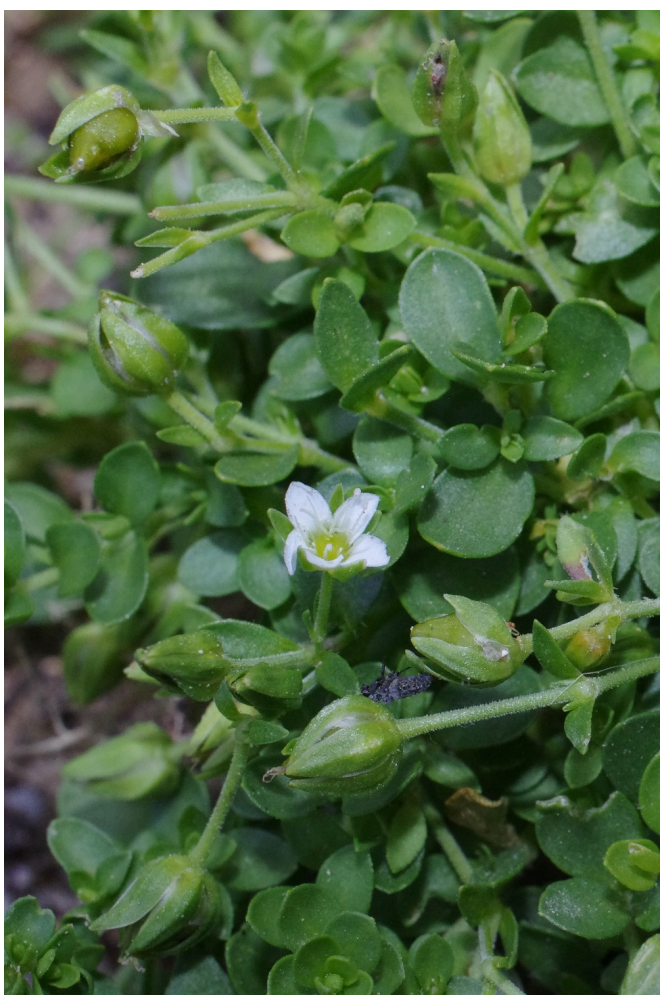

\section{Arenaria serpyllifolia}

276.429, loa 1058, Katara-Pass; 276.449, Tri 456, N Katara-Pass; 276.863 , loa 1071, NNW Metsovo; 276.889, loa 1072, NNW Metsovo; 276.994, loa 1079, SO Anilio; 277.005, loa 1079, SO Anilio; 277.066, loa 1080, SO Anilio; 277.117, loa 1084, N Metsovo; 277.229, loa 1089, NNO Metsovo; 277.478, loa 1097, S Anilio; 278.472, Thes 215, Paralia Vrasna; 279.523, Kav 99, Pangaion; 279.570, Kav 103, Pangaion; 280.357, Dra 231, Sidironero; 280.575, Dra 238, Skaloti; 281.032, Dra 258, Granitis; 282.220, Dra 300, SO Lefkogia; 283.362, Pel 352, Kajmaktsalan; 284.185, loa 1097, S Anilio

\section{Aristolochia clematitis}

278.642, Kav 106, Paralia Ofryniou

\section{Armeria canescens}

276.752, loa 1068, NW Metsovo; 276.795, loa 1070, NNW Metsovo; 277.030, loa 1080, SO Anilio; 277.291, loa 1091, SW Milia; 277.606, loa 1103, S Anilio; 277.717, Tri 460, N Chaliki; 277.951, Koz 374, Vourinos; 278.041, Gre 491, NO Exarcho; 283.973, loa 1056 b, NO Metsovo; 284.090, loa 1120, Anilio; 284.164, loa 1124, 


\section{Armeria rumelica}

283.298, Pel 349, Kajmaktsalan; 283.498, Pel 359, NW Loutraki

\section{Arrhenatherum elatius}

276.919, loa 1074, NNW Metsovo; 277.507, loa 1097, S Anilio; 277.924, Koz 392, SO Siatista, Vourinos; 282.508, Ser 282, Ori Vrondous; 282.516, Ser 283, Ori Vrondous; 282.638, Ser 290, N Neo Petritsi; 283.118, Pel 340, SSO Archangelo

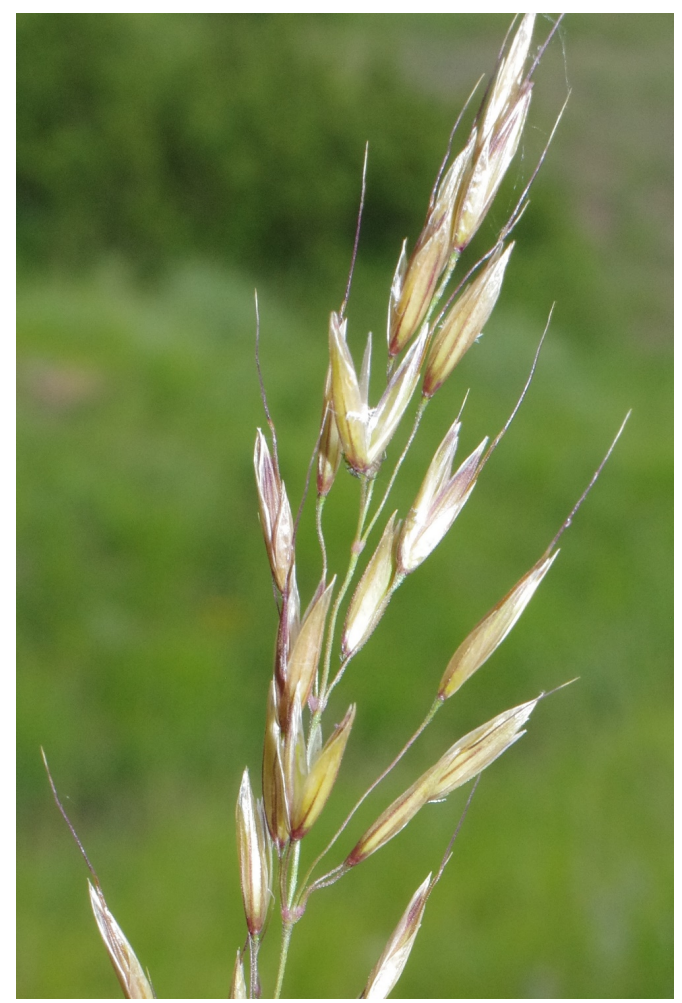

\section{Artemisia absinthium}

277.775, Tri 462, N Chaliki; 282.663, Ser 290, N Neo Petritsi; 283.978, loa 1111, NO Metsovo

\section{Artemisia vulgaris}

282.799, Ser 296, NO Strymoniko; 284.129, ioa 1095 b, S Anilio

\section{Asparagus acutifolius}

278.931, Kav 118, Galipsos

\section{Asperula aristata subsp. condensata}

276.888, loa 1072, NNW Metsovo; 277.111, loa 1084, N Metsovo; 280.147, Dra 221, NO Drama; 283.901, Ima 180, Seli

\section{Asperula aristata subsp. longiflora}

279.013, Kav 119, Podochori; 279.701, Ser 266, SO Nea Zichni; 282.425, Ser 279, Orini

\section{Asperula aristata subsp. nestia}

278.723, Kav 108, Paralia Ofryniou; 278.910, Kav 116, S Ofrynia; 278.945, Kav 118, Galipsos; 279.166, Ser 255, S Nea Mesolakkia; 279.217, Ser 257, Nea Fili; 279.630, Ser 263, Dravoskas; 279.740, Ser 268, SW Skopia; 279.847, Dra 208, NO Skopia; 281.242, Dra 265, NO Granitis; 281.286, Dra 267, W Nevrokopi; 282.011, Dra 292, Nevrokopi; 282.196, Dra 299, SO Lefkogia; 282.385, Ser 277, ONO Serres; 283.137, Pel 341, S Archangelo; 283.329, Pel 351, Kajmaktsalan; 283.399, Pel 354, NW Loutraki; 283.611, Pel 365, N Vorino; 284.182, Ima 180, Seli

\section{Asperula aristata subsp. thessala}

279.315, Ser 260, Proti; 280.831, Dra 248, Falakron; 280.888, Dra 250, Falakron; 281.153, Dra 262, NW Granitis; 281.711, Dra 280, Nevrokopi; 281.820, Dra 283, NNO Volakas; 
281.972, Dra 290, NO Potamia; 283.921, Ima 180, Seli; 283.965, loa 1056 b, NO Metsovo; 283.980, loa 1111, NO Metsovo; 284.089, loa 1120, Anilio

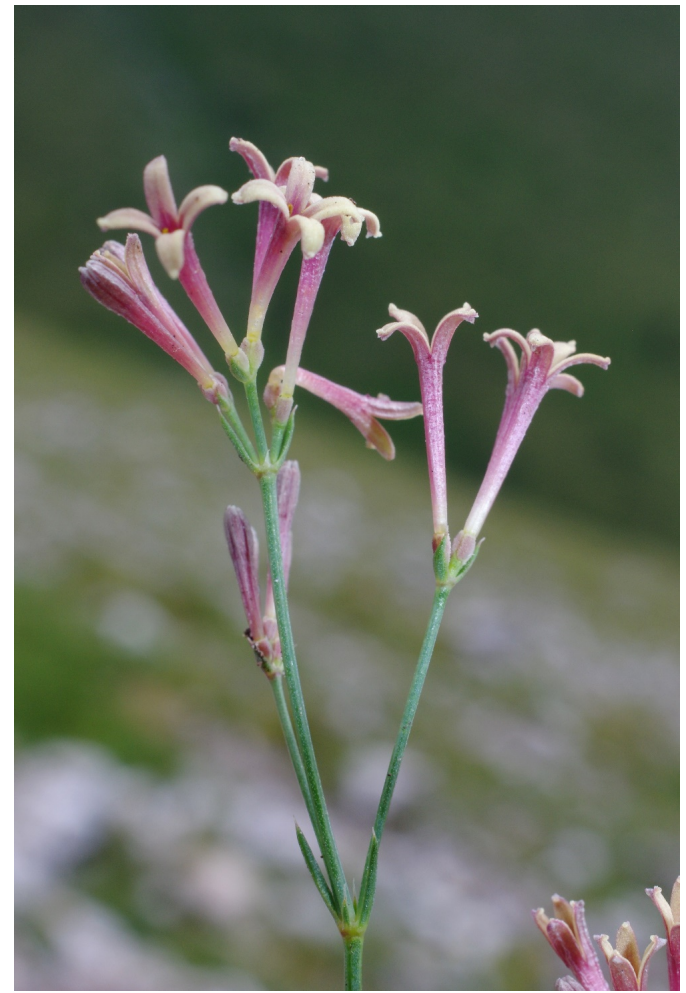

\section{Asperula laevigata}

276.713, loa 1065, NO Karyes; 277.534, loa 1097, S Anilio; 277.735, Tri 460, N Chaliki

Asperula purpurea subsp. purpurea

280.373, Dra 232, Sidironero

Asphodeline liburnica

279.394, Kav 92, Pangaion

\section{Asphodelus fistulosus}

278.705, Kav 107, Paralia Ofryniou

Asplenium trichomanes subsp. quadrivalens

284.146, loa 1096 b, S Anilio

Asplenium trichomanes subsp. trichomanes

276.657, loa 1063, W Potamia; 277.515, loa 1097, S Anilio; 279.560, Kav 102, Pangaion; 279.968, Dra 213, Livadero; 281.748, Dra 281, OSO Granitis

\section{Astragalus angustifolius}

279.592, Kav 104, Pangaion

Astragalus glycyphyllos subsp. glycyphylloides

277.680, Tri 460, N Chaliki; 281.277, Dra 266, W Nevrokopi; 281.669, Ser 270, Kato Vrondous

\section{Astragalus glycyphyllos subsp. glycyphyllos}

277.522, loa 1097, S Anilio; 279.864, Dra 209, Livadero; 280.467, Dra 234, Skaloti; 280.957, Dra 255, Falakron; 282.313, Ser 274, SSW Ano Vrondous; 282.489, Ser 282, Ori Vrondous; 282.982, Kil 300, Kastaneri

Astragalus mayeri

278.423, Koz 384, Galatini 


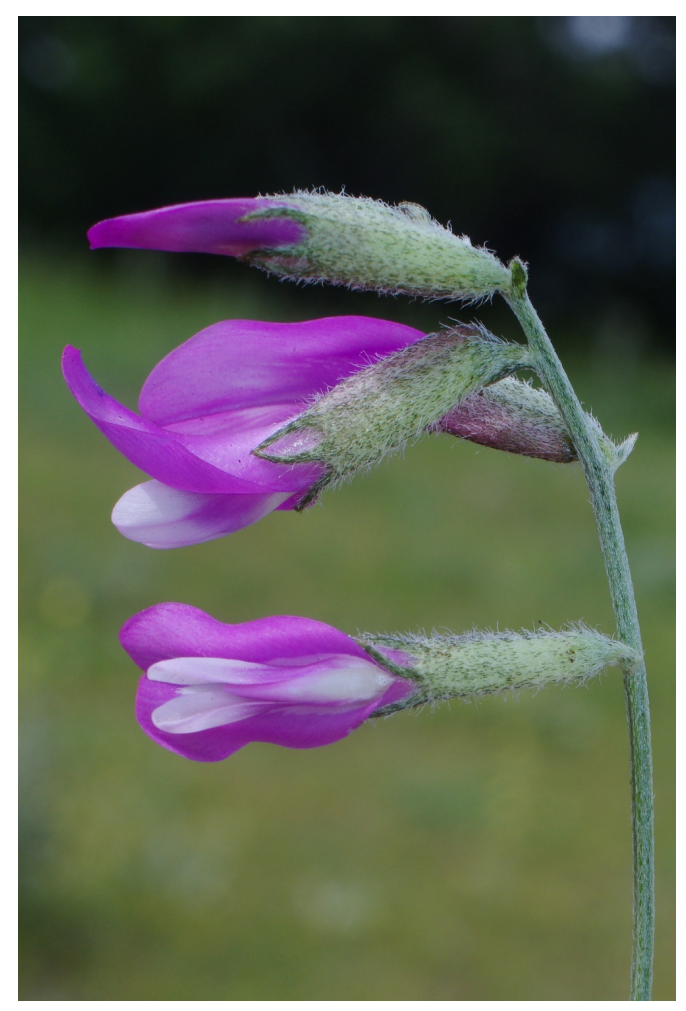

Astragalus onobrychis

278.031, Koz 376, W Exarcho; 280.204, Dra 224, NO Drama; 281.984, Dra 290, NO

Potamia; 282.374, Ser 276, SSW Ano Vrondous; 282.454, Ser 280, Orini

Astragalus thracicus subsp. parnassi

277.654, loa 1106, Anilio

\section{Asyneuma canescens}

277.656, loa 1106, Anilio; 281.434, Dra 274, Vathytopo; 283.003, Kil 301, Kastaneri; 283.279, Pel 348, Kajmaktsalan

\section{Asyneuma limonifolium}

278.076, Gre 492, NO Varis; 278.345, Koz 381, Galatini; 278.415, Koz 384, Galatini; 280.849, Dra 248, Falakron; 281.761, Dra 281, OSO Granitis; 282.361 , Ser 276, SSW Ano Vrondous; 283.995, loa 1112, Katara-Paß

\section{Athyrium filix-femina}

277.571, loa 1100, S Anilio; 282.882, Kil 295, NW Griva; 283.056, Kil 306, SO Livadia; 284.158, loa 1103 b, S Anilio

\section{Atropa bella-donna}

283.798, Ima 174, S Ag. Pavlos

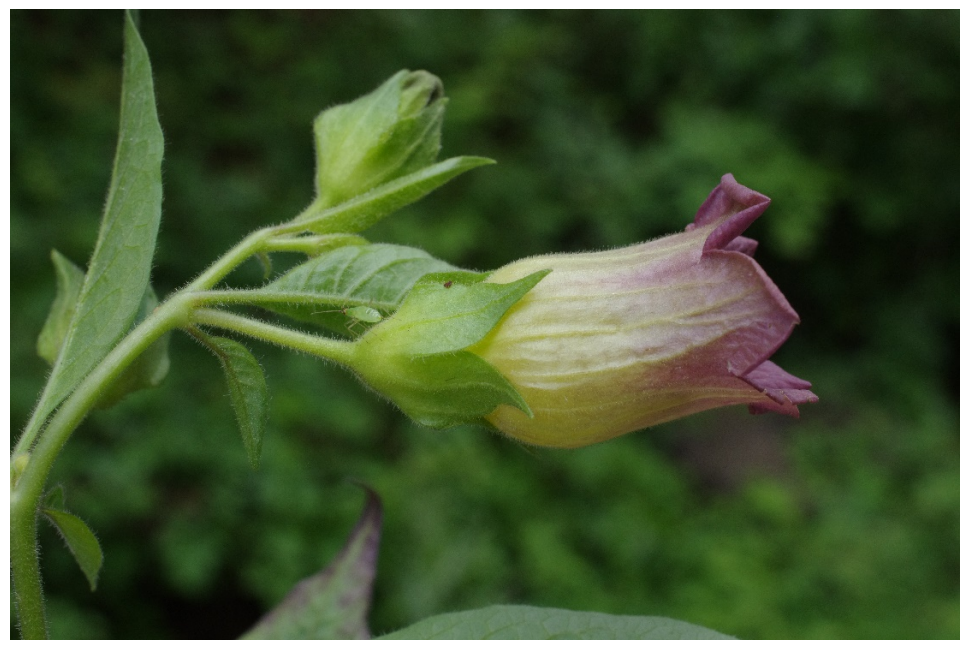




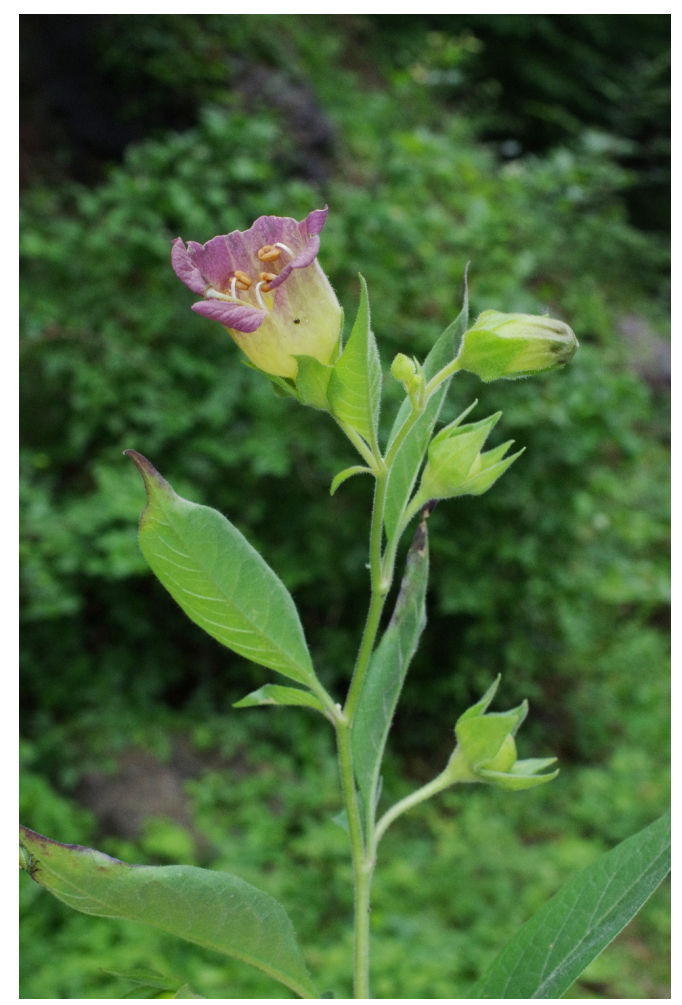

\section{Aurinia corymbosa}

278.427, Koz 384, Galatini

\section{Avena barbata}

276.533, loa 1059, Ag. Nikolaos; 277.432, loa 1095, S Anilio; 278.445, Thes 214, Paralia Vrasna; 278.646, Kav 106, Paralia Ofryniou; 278.810, Kav 114, SO Folia; 279.109, Kav 90, SW Moustheni; 279.171, Ser 256, Paleokomi; 279.259, Ser 258, Mikro Souli; 279.288, Ser 259, Proti; 279.698, Ser 266, SO Nea Zichni; 280.185, Dra 223, NO Drama

\section{Avena sterilis}

277.332, loa 1093, S Anilio; 278.113, Gre 492, NO Varis; 279.189, Ser 256, Paleokomi; 279.758, Ser 268, SW Skopia; 281.987, Dra 291, W Prosotsani

\section{Ballota nigra subsp. meridionalis}

278.553, Thes 219, Vrasna; 278.569, Thes 220, Arethousa; 278.765, Kav 110, Loutra Eleftherion; 279.077, Kav 89, Misoropi; 279.177, Ser 256, Paleokomi; 279.293, Ser 259, Proti; 279.663, Ser 265, Myrrini; 279.700, Ser 266, SO Nea Zichni; 280.432, Dra 234, Skaloti; 281.015, Dra 257, Granitis; 281.274, Dra 266, W Nevrokopi; 281.360, Dra 271, Vathytopo; 281.543, Dra 276, Perithori; 281.678, Ser 271, Kato Vrondous; 281.998, Dra 291, W Prosotsani; 282.754, Ser 292, N Neo Petritsi; 283.634, Pel 366, N Vorino

\section{Ballota nigra subsp. sericea}

278.300, Koz 380, Galatini

\section{Ballota nigra subsp. uncinata}

278.015, Koz 376, W Exarcho; 278.490, Thes 215, Paralia Vrasna; 278.828, Kav 114, SO

Folia; 279.253, Ser 258, Mikro Souli; 281.196, Dra 264, NO Ochyro; 281.914, Dra 287, O Mikroklisoura; 282.136, Dra 297, Lefkogia; 282.307, Ser 273, Ano Vrondous; 282.985, Kil 300, Kastaneri; 283.691, Pel 369, O Vorino

\section{Barbarea vulgaris}

277.220, loa 1088, NNO Metsovo; 280.625, Dra 240, Elatias; 280.656, Dra 242, SW Walddorf Elatias

\section{Bellardia trixago}




\section{Bellis perennis}

276.715, loa 1065, NO Karyes; 276.904, loa 1073, NNW Metsovo; 277.057, loa 1080, SO Anilio; 277.098, loa 1084, N Metsovo; 277.238, loa 1089, NNO Metsovo; 277.536, loa 1097, S Anilio; 279.352, Ser 261, Proti; 283.840, Ima 177, Tria-Pende Pigadia; -20.490, loa 1078, O Anilio; -20.476, loa 1055, N Metsovo; -20.496, loa 1083, N Metsovo

\section{Berteroa incana}

279.935, Dra 211, Livadero; 280.796, Dra 247, Walddorf Elatias; 281.017, Dra 257, Granitis; 281.672, Ser 271, Kato Vrondous; 282.294, Ser 273, Ano Vrondous; 282.355 , Ser 275, SSW Ano Vrondous; 283.540, Pel 362, NW Promachi

\section{Berteroa obliqua subsp. pindicola}

278.529, Thes 218, Vrasna; 278.623, Thes 221, Arethousa; 279.087, Kav 89, Misoropi; 281.257, Dra 266, W Nevrokopi; 282.768, Ser 293, N Neo Petritsi

\section{Berula erecta}

278.561, Thes 219, Vrasna

\section{Betonica alopecurus}

283.993, loa 1112, Katara-Paß

\section{Betonica officinalis subsp. officinalis}

282.080, Dra 295, NO Nevrokopi

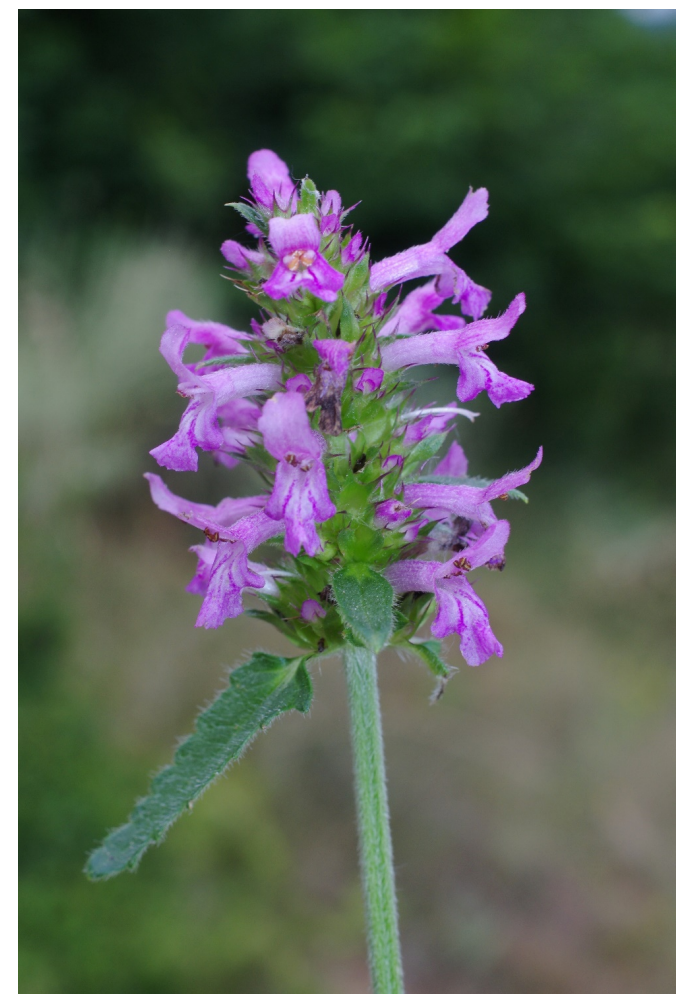

\section{Betula pendula}

282.676, Ser 290, N Neo Petritsi; 283.264, Pel 347, Kajmaktsalan

\section{Bifora radians}

278.188, Gre 495, Varis; 278.259, Koz 379, Galatini; 278.371, Koz 382, Galatini; 279.748, Ser 268, SW Skopia

\section{Bituminaria bituminosa}

276.324, Ioa 1125, NO Dodoni; 278.740, Kav 109, Loutra Eleftherion; 278.788, Kav 112, NO Paralia Myrtofytou; 278.891, Kav 116, S Ofrynia; 279.294, Ser 259, Proti; 279.611, Ser 263, 
Dravoskas; 279.683, Ser 266, SO Nea Zichni; 279.770, Ser 268, SW Skopia; 280.074, Dra 219, Sidironero; 280.184, Dra 223, NO Drama; 281.965, Dra 289, NO Potamia

Blackstonia perfoliata subsp. perfoliata

277.344, loa 1093, S Anilio; 278.750, Kav 109, Loutra Eleftherion; 283.441, Pel 356, NW Loutraki; 284.136, loa 1096 b, S Anilio

Bolanthus chelmicus subsp. meteoricus

278.001, Koz 375, O Dafnero

Bolboschoenus maritimus

278.693, Kav 107, Paralia Ofryniou

\section{Bornmuellera tymphaea}

276.382, loa 1056, NO Metsovo; 276.440, loa 1058, Katara-Pass; 277.306, loa 1091, SW Milia; 278.461, Thes 214, Paralia Vrasna; 278.686, Kav 107, Paralia Ofryniou

\section{Bothriochloa ischaemum}

277.796, Koz 385, SO Siatista; 278.106, Gre 492, NO Varis; 278.547, Thes 219, Vrasna; 278.907, Kav 116, S Ofrynia; 278.953, Kav 118, Galipsos; 278.993, Kav 119, Podochori; 279.198, Ser 257, Nea Fili; 279.257, Ser 258, Mikro Souli; 279.325, Ser 260, Proti; 279.408, Kav 92, Pangaion; 279.684, Ser 266, SO Nea Zichni; 279.866, Dra 209, Livadero; 280.149 , Dra 221, NO Drama; 280.394, Dra 232, Sidironero; 281.136, Dra 262, NW Granitis;

281.285, Dra 267, W Nevrokopi; 281.607, Dra 278, Kato Vrondous; 281.804, Dra 283, NNO Volakas; 281.937, Dra 288, SW Potamia; 282.022, Dra 292, Nevrokopi; 282.124, Dra 296, NO Nevrokopi; 282.359, Ser 276, SSW Ano Vrondous; 282.390, Ser 277, ONO Serres; 282.434, Ser 279, Orini; 283.388, Pel 354, NW Loutraki

\section{Botrychium lunaria}

284.217, loa 1080 , SO Anilio

\section{Brachypodium distachyon \\ 279.228, Ser 257, Nea Fili}

\section{Brachypodium pinnatum}

276.337, loa 1126, NO Dodoni; 276.362, loa 1128, N Metsovo; 276.929, loa 1074, NNW Metsovo; 276.997, loa 1079, SO Anilio; 277.694, Tri 460, N Chaliki; 277.848, Koz 387, SO Siatista, Vourinos; 279.710, Ser 266, SO Nea Zichni; 280.178, Dra 223, NO Drama; 280.438, Dra 234, Skaloti; 281.160, Dra 262, NW Granitis; 281.961, Dra 289, NO Potamia; 282.404, Ser 278, N Serres; 283.063, Kil 307, NW Livadia; 283.673, Pel 368, OSO Vorino; 283.821, Ima 176, Tria-Pende Pigadia; 284.016, loa 1115, N Metsovo

\section{Brachypodium sylvaticum subsp. sylvaticum}

276.527, loa 1059, Ag. Nikolaos; 277.199, loa 1087, NNO Metsovo; 277.321, loa 1092, NO Metsovo; 277.499, loa 1097, S Anilio; 279.348, Ser 261, Proti; 279.431, Kav 94, Pangaion; 279.466, Kav 96, Pangaion; 279.818, Ser 269, NO Skopia; 280.260, Dra 227, Livadero; 280.264, Dra 228, Livadero; 280.368, Dra 232, Sidironero; 280.749, Dra 245, SW Walddorf Elatias; 281.353, Dra 270, W Nevrokopi; 281.599, Dra 278, Kato Vrondous; 281.631, Dra 279, Kato Vrondous; 281.805, Dra 283, NNO Volakas; 282.074, Dra 294, NO Nevrokopi; 282.184, Dra 299, SO Lefkogia; 282.353, Ser 275, SSW Ano Vrondous; 282.738, Ser 291, N Neo Petritsi; 282.850, Kil 294, NW Griva; 282.920, Kil 297, WNW Kastaneri; 283.188, Pel 343, Kerasia; 283.434, Pel 355, NW Loutraki; 283.544, Pel 362, NW Promachi; 283.855, Ima 178, Seli

\section{Brassica oleracea}

276.626, loa 1062, SO Potamia

\section{Briza maxima}

276.666, loa 1064, ONO Karyes 


\section{Briza media subsp. elatior}

276.370, loa 1128, N Metsovo; 276.899, loa 1073, NNW Metsovo; 277.085, loa 1081, W Metsovo; 277.278, loa 1090, NNO Metsovo; 279.377, Ser 262, Proti; 279.488, Kav 97, Pangaion; 279.583, Kav 104, Pangaion; 279.907, Dra 210, Livadero; 280.420, Dra 233, Sidironero; 280.539, Dra 236, Skaloti; 280.949, Dra 255, Falakron; 282.070, Dra 294, NO Nevrokopi; 282.258, Dra 301, Ano Vrondous; 282.997, Kil 301, Kastaneri; 283.069, Kil 307, NW Livadia; 283.860, Ima 178, Seli

\section{Briza media subsp. media}

277.100, loa 1084, N Metsovo; 277.698, Tri 460, N Chaliki; 280.251, Dra 227, Livadero; 280.286, Dra 228, Livadero; 280.473, Dra 234, Skaloti; 280.718, Dra 243, SW Walddorf Elatias; 281.483, Dra 275, Vathytopo; 283.226, Pel 345, Kerasia

\section{Bromus arvensis}

276.411, loa 1057, NO Metsovo; 276.532, loa 1059, Ag. Nikolaos; 277.427, loa 1095, S Anilio; 277.527, loa 1097, S Anilio; 278.264, Koz 379, Galatini; 279.834, Dra 207, NO Skopia; 281.264, Dra 266, W Nevrokopi; 281.323, Dra 268, W Nevrokopi; 281.324, Dra 268, W Nevrokopi; 281.562, Dra 277, Perithori; 281.665, Ser 270, Kato Vrondous; 281.788, Dra 282, NNO Volakas; 281.893, Dra 287, O Mikroklisoura; 281.964, Dra 289, NO Potamia; 282.250, Dra 301, Ano Vrondous; 283.064, Kil 307, NW Livadia; 283.094, Pel 339, S Archangelo; 283.173, Pel 343, Kerasia

\section{Bromus cappadocicus subsp. lacmonicus}

277.675, Tri 460, N Chaliki; 280.935, Dra 253, Falakron

\section{Bromus commutatus}

276.462, Tri 458, NO Katara-Pass; 276.654, loa 1063, W Potamia; 276.886, loa 1072, NNW Metsovo; 276.897, loa 1073, NNW Metsovo; 277.631, loa 1104, S Anilio; 279.427, Kav 94, Pangaion; 279.859, Dra 209, Livadero; 279.971, Dra 213, Livadero; 282.964, Kil 299, Kastaneri; 283.102, Pel 339, S Archangelo; 283.740, Ima 172, S Ag. Pavlos

\section{Bromus diandrus}

278.838, Kav 114, SO Folia

\section{Bromus hordeaceus subsp. hordeaceus}

276.976, loa 1079, SO Anilio; 277.004, loa 1079, SO Anilio; 277.160, loa 1086, NNO Metsovo; 279.344, Ser 261, Proti; 279.453, Kav 95, Pangaion; 279.960, Dra 213, Livadero; 279.988, Dra 214, Livadero; 281.516, Dra 276, Perithori; -20.461, loa 1052, NO Dodoni

\section{Bromus hordeaceus subsp. mediterraneus}

276.565, loa 1060, SSO Potamia; 276.688, loa 1065, NO Karyes; 276.803 , loa 1070, NNW Metsovo; 277.363, loa 1093, S Anilio

\section{Bromus inermis}

276.356, loa 1128, N Metsovo; 277.013, loa 1080, So Anilio; 277.110, loa 1084, N Metsovo; 280.541, Dra 236, Skaloti; 280.698, Dra 242, SW Walddorf Elatias; 282.253, Dra 301, Ano Vrondous; 282.336, Ser 274, SSW Ano Vrondous

\section{Bromus intermedius}

276.315, loa 1125, NO Dodoni; 276.989, loa 1079, SO Anilio; 277.120, loa 1084, N Metsovo; 277.242, loa 1089, NNO Metsovo; 277.371, loa 1093, S Anilio; 278.058, Gre 492, NO Varis; 278.098, Gre 492, NO Varis; 278.119, Gre 492, NO Varis; 278.258, Koz 379, Galatini; 278.333, Koz 381, Galatini; 280.102, Dra 220, Sidironero; 280.175, Dra 222, NO Drama; 280.344, Dra 230, Livadero; 280.361, Dra 232, Sidironero; 280.389, Dra 232, Sidironero; 280.450, Dra 234, Skaloti; 280.939, Dra 253, Falakron; 281.182, Dra 264, NO Ochyro; 281.502, Dra 276, Perithori; 282.161, Dra 298, SO Lefkogia; 282.317, Ser 274, SSW Ano Vrondous; 282.538, Ser 284, Ori Vrondous; 282.709, Ser 290, N Neo Petritsi; 283.276, Pel 348, Kajmaktsalan; 284.175, loa 1060, SSO Potamia; 284.176, Dra 253, Falakron

\section{Bromus japonicus}




\section{Bromus scoparius}

277.777, Tri 462, N Chaliki

\section{Bromus squarrosus}

276.314, loa 1125, NO Dodoni; 276.460, Tri 457, NO Katara-Pass; 276.567 , loa 1060, SSO

Potamia; 276.966, loa 1078, O Anilio; 277.414, loa 1094, S Anilio; 277.734 , Tri 460, N

Chaliki; 277.835, Koz 387, SO Siatista, Vourinos; 277.973, Koz 374, Vourinos; 279.489,

Kav 97, Pangaion; 281.778, Dra 282, NNO Volakas; 281.978, Dra 290, NO Potamia;

282.207, Dra 300, SO Lefkogia; 284.012, loa 1114, Katara-Paß

\section{Bromus sterilis}

276.638, loa 1063, W Potamia; 277.165, loa 1086, NNO Metsovo; 277.334, loa 1093, S Anilio; 277.946, Koz 392, SO Siatista, Vourinos; 277.989, Koz 375, O Dafnero; 278.295, Koz 380, Galatini; 279.492, Kav 97, Pangaion; 279.509, Kav 98, Pangaion; 280.309, Dra 229, SO Dendrakia; 281.103, Dra 261, Granitis; 281.417, Dra 273, Vathytopo; 281.501, Dra 276, Perithori; 283.338, Pel 351, Kajmaktsalan

\section{Bromus tectorum}

276.430, loa 1058, Katara-Pass; 277.840, Koz 387, SO Siatista, Vourinos; 279.574, Kav 103, Pangaion; 279.986, Dra 214, Livadero; 280.078, Dra 219, Sidironero; 281.685, Ser 271, Kato Vrondous; 281.963, Dra 289, NO Potamia

\section{Bruckenthalia spiculifolia}

280.706, Dra 243, SW Walddorf Elatias; 283.304, Pel 350, Kajmaktsalan

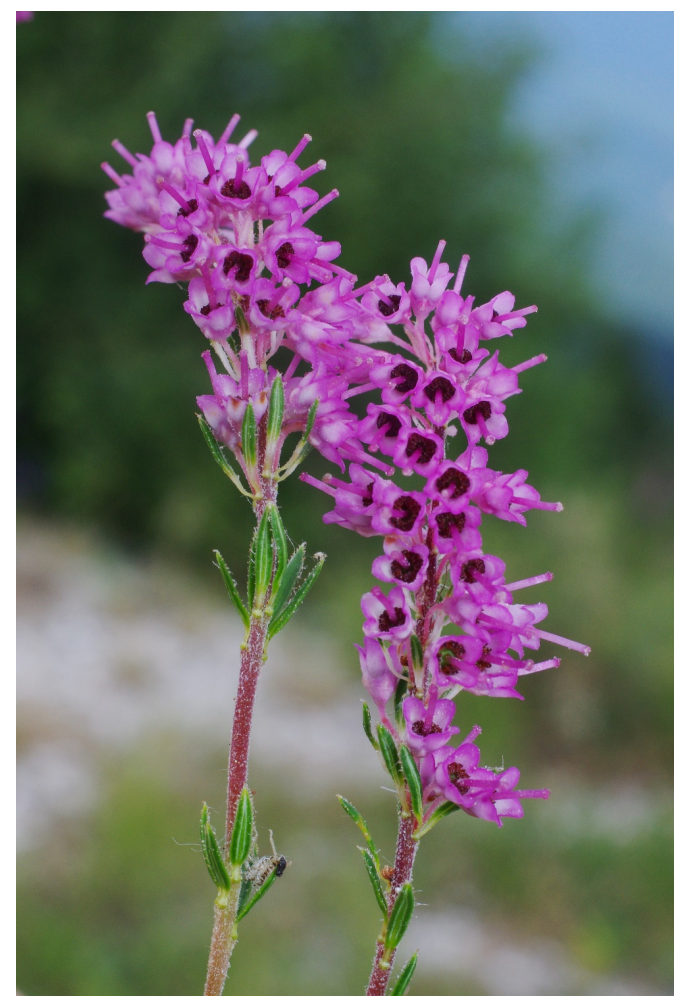

\section{Bryonia dioica}

276.659, loa 1063, W Potamia

\section{Bubon macedonicum}

276.570, loa 1060, SSO Potamia 


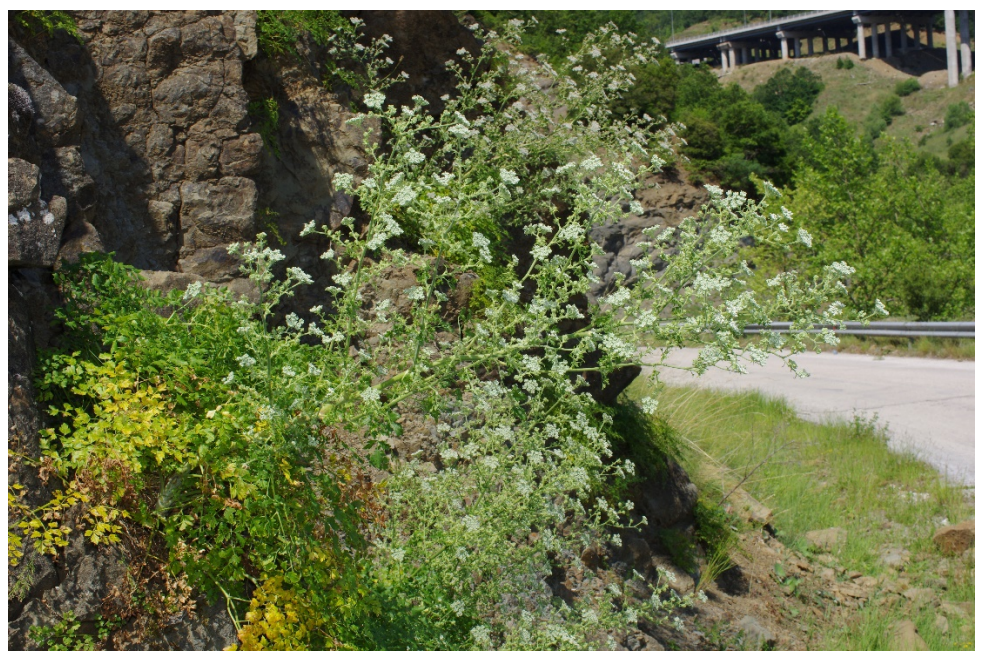

Buglossoides incrassata subsp. splitgerberi 277.942, Koz 392, SO Siatista, Vourinos; 278.308, Koz 380, Galatini

\section{Bunias erucago}

277.888, Koz 389, SO Siatista, Vourinos; 278.364, Koz 382, Galatini

\section{Bunium ferulaceum}

276.854, loa 1071, NNW Metsovo; 276.958, loa 1078, O Anilio; 277.182, loa 1086, NNO Metsovo; 277.277, loa 1090, NNO Metsovo; 277.290, loa 1091, SW Milia; 277.508, loa 1097, S Anilio; 280.971, Dra 256, Falakron

\section{Bupleurum affine}

283.957, loa 1110, N Metsovo; 284.029, loa 1116, SW Milia; 284.062, loa 1118, Anilio; 284.114, loa 1121, Anilio

\section{Bupleurum apiculatum}

277.838, Koz 387, SO Siatista, Vourinos; 277.966, Koz 374, Vourinos

\section{Bupleurum asperuloides}

278.230, Koz 378, N Siatista

\section{Bupleurum flavicans}

278.227, Koz 378, N Siatista; 284.199, Koz 378, N Siatista

\section{Bupleurum gerardi}

278.181, Gre 495, Varis; 284.200, Gre 495, Varis

\section{Bupleurum glumaceum}

276.508, loa 1059, Ag. Nikolaos; 276.728, loa 1066, NO Karyes

\section{Bupleurum odontites}

278.146, Gre 492, NO Varis; 278.314, Koz 381, Galatini; 278.404, Koz 383, Galatini; 278.425, Koz 384, Galatini; 278.603, Thes 221, Arethousa; 279.032, Kav 120, Podochori; 279.197, Ser 257, Nea Fili; 279.715, Ser 266, SO Nea Zichni; 279.843, Dra 208, NO Skopia; 280.232, Dra 226, N Taxiarches; 281.131, Dra 262, NW Granitis; 281.207, Dra 265, NO Granitis; 281.296, Dra 267, W Nevrokopi; 281.451, Dra 274, Vathytopo; 282.386, Ser 277, ONO Serres; 284.110, loa 1121, Anilio; 284.195, Dra 226, N Taxiarches; 284.196, Dra 262, NW Granitis; 284.197, Ser 277, ONO Serres; 284.198, loa 1121, Anilio 


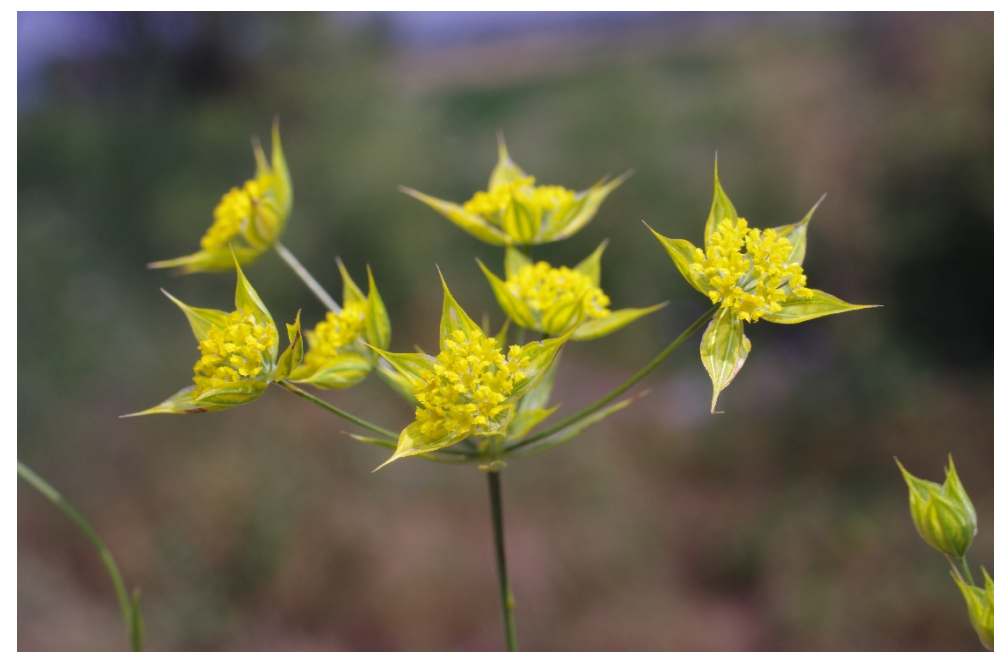

Bupleurum rotundifolium

278.301, Koz 380, Galatini; 278.356, Koz 382, Galatini

\section{Bupleurum trichopodum}

278.047, Gre 491, NO Exarcho; 282.751, Ser 292, N Neo Petritsi

\section{Cakile maritima}

278.735, Kav 108, Paralia Ofryniou

\section{Calamagrostis arundinacea}

279.571, Kav 103, Pangaion; 280.489, Dra 235, Skaloti; 280.517, Dra 236, Skaloti; 280.555 , Dra 237, Skaloti; 283.313, Pel 350, Kajmaktsalan

\section{Calamagrostis epigejos}

277.146, loa 1085, N Metsovo; 278.859, Kav 115, SW Folia; 279.388, Ser 262, Proti; 279.461, Kav 95, Pangaion; 279.906, Dra 210, Livadero; 281.059, Dra 259, Granitis; 281.155, Dra 262, NW Granitis; 281.676, Ser 271, Kato Vrondous; 282.256, Dra 301, Ano Vrondous; 282.501, Ser 282, Ori Vrondous; 282.650, Ser 290, N Neo Petritsi; 282.973, Kil 299, Kastaneri; 282.996, Kil 301, Kastaneri; 283.252, Pel 346, Kajmaktsalan; 283.809, Ima 176, Tria-Pende Pigadia

\section{Calamagrostis pseudophragmites}

283.223, Pel 345, Kerasia

\section{Calamintha grandiflora}

277.531, loa 1097, S Anilio; 282.934, Kil 298, WNW Kastaneri; 283.834, Ima 177, Tria-Pende Pigadia; 283.943, loa 1109, NW Metsovo; 284.081, loa 1119, Anilio; 284.143, loa 1096 b, S Anilio

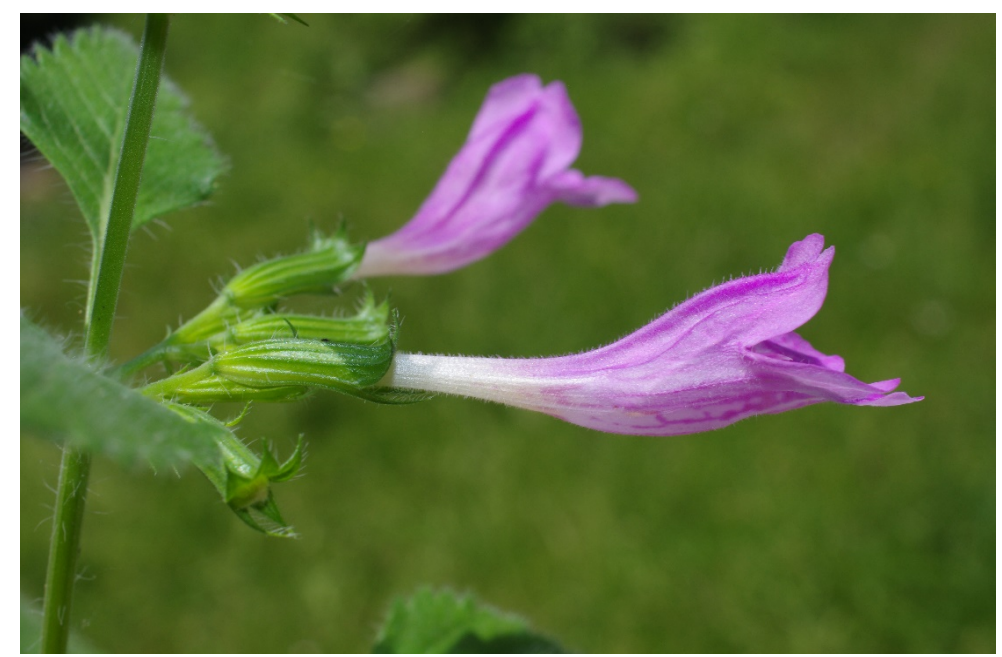


Calamintha nepeta subsp. nepeta

280.174, Dra 222, NO Drama; 283.151, Pel 342, S Archangelo; 283.417, Pel 355, NW

Loutraki; 283.509, Pel 360, SW Promachi; 283.602, Pel 365, N Vorino; 283.632, Pel 366, N

Vorino; 283.707, Pel 369, O Vorino

\section{Calendula arvensis}

278.987, Kav 119, Podochori

\section{Caltha palustris}

276.913, loa 1073, NNW Metsovo

\section{Calystegia sepium}

281.542, Dra 276, Perithori; 282.836, Ser 298, Parapotamos; 283.589, Pel 364, Loutraki

\section{Calystegia silvatica}

277.582, loa 1100, S Anilio; 278.759, Kav 110, Loutra Eleftherion; 279.510, Kav 98,

Pangaion; 280.270, Dra 228, Livadero

\section{Camelina microcarpa}

278.359, Koz 382, Galatini; 278.575, Thes 220, Arethousa

\section{Campanula}

280.873, Dra 249, Falakron

\section{Campanula bononiensis}

280.176, Dra 223, NO Drama; 282.048, Dra 293, Nevrokopi; 283.163, Pel 343, Kerasia

\section{Campanula cervicaria}

277.091, loa 1083, NW Metsovo; 280.304, Dra 229, SO Dendrakia; 282.491, Ser 282, Ori Vrondous; 282.493, Ser 282, Ori Vrondous; 282.585, Ser 287, Ori Vrondous

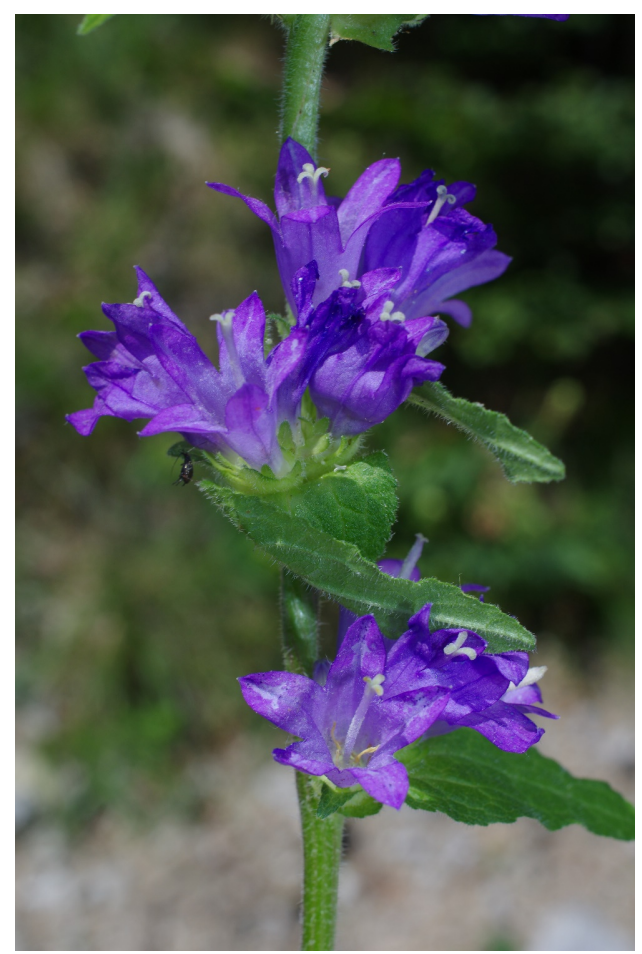

\section{Campanula foliosa}

276.734, loa 1067, NW Metsovo; 277.514, loa 1097, S Anilio

\section{Campanula glomerata}

277.550, loa 1098, S Anilio; 279.538, Kav 100, Pangaion

\section{Campanula hawkinsiana}


276.406, loa 1057, NO Metsovo; 277.000, loa 1079, SO Anilio; 284.102, loa 1121, Anilio

\section{Campanula lingulata}

277.837, Koz 387, SO Siatista, Vourinos; 277.936, Koz 392, SO Siatista, Vourinos; 277.996, Koz 375, O Dafnero; 278.495, Thes 216, Vrasna; 278.524, Thes 217, Vrasna; 278.784, Kav 112, NO Paralia Myrtofytou; 278.876, Kav 115, SW Folia; 279.033, Kav 120, Podochori; 279.053, Kav 121, Platanotopos; 279.165, Ser 255, S Nea Mesolakkia; 279.340, Ser 261, Proti; 279.482, Kav 97, Pangaion; 279.564, Kav 102, Pangaion; 279.734, Ser 267, SW Skopia; 279.787, Ser 268, SW Skopia; 279.825, Ser 269, NO Skopia; 279.861, Dra 209, Livadero; 279.946, Dra 212, Livadero; 280.163, Dra 221, NO Drama; 280.385, Dra 232, Sidironero; 280.543, Dra 236, Skaloti; 280.845, Dra 248, Falakron; 280.968, Dra 256, Falakron; 281.063, Dra 259, Granitis; 281.435, Dra 274, Vathytopo; 282.426, Ser 279, Orini; 284.190, Dra 216, Sidironero; -20.499, Ser 261, Proti; -20.506, Dra 236, Skaloti

\section{Campanula orphanidea}

279.609, Kav 105, Pangaion

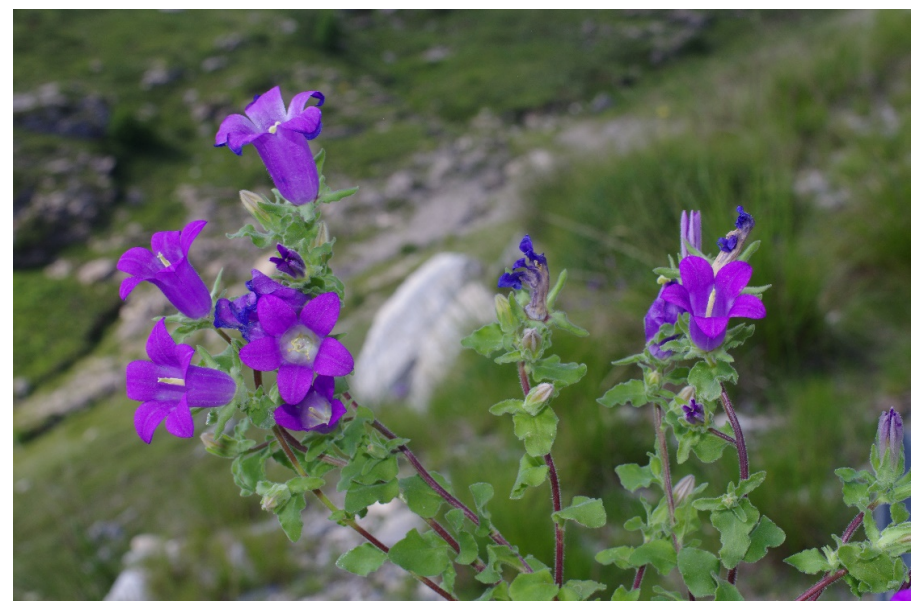

\section{Campanula patula subsp. patula}

277.209, loa 1088, NNO Metsovo; 277.312, loa 1092, NO Metsovo; 277.847, Koz 387, SO Siatista, Vourinos; 277.852, Koz 387, SO Siatista, Vourinos; 277.897, Koz 389, SO Siatista, Vourinos; 279.952, Dra 212, Livadero; 280.004, Dra 216, Sidironero; 280.043, Dra 217, Sidironero; 280.319, Dra 230, Livadero; 280.414, Dra 233, Sidironero; 280.581, Dra 238, Skaloti; 280.655, Dra 242, SW Walddorf Elatias; 280.777, Dra 247, Walddorf Elatias; 280.803, Dra 247, Walddorf Elatias; 281.033, Dra 258, Granitis; 281.306, Dra 267, W Nevrokopi; 281.674, Ser 271, Kato Vrondous; 282.241, Dra 301, Ano Vrondous; 282.518, Ser 283, Ori Vrondous; 282.936, Kil 298, WNW Kastaneri; 284.074, loa 1118, Anilio

\section{Campanula persicifolia}

276.956, loa 1077, S Anilio; 279.500, Kav 98, Pangaion; 279.958, Dra 213, Livadero; 282.494, Ser 282, Ori Vrondous; 282.648, Ser 290, N Neo Petritsi; 284.189, Dra 216, Sidironero

\section{Campanula phrygia}

278.475, Thes 215, Paralia Vrasna; 280.177, Dra 223, NO Drama; 280.196, Dra 223, NO Drama; 282.779, Ser 293, N Neo Petritsi

\section{Campanula ramosissima}

276.300, loa 1125, NO Dodoni; 276.496, loa 1059, Ag. Nikolaos; 276.690, loa 1065, NO Karyes; 277.461 , loa 1096, S Anilio

\section{Campanula rapunculoides}

280.032, Dra 216, Sidironero; 280.337, Dra 230, Livadero; 280.444, Dra 234, Skaloti; 280.553, Dra 237, Skaloti; 280.954, Dra 255, Falakron; 281.120, Dra 261, Granitis; 281.841 , Dra 284, NNO Volakas

\section{Campanula rapunculus}


277.733, Tri 460, N Chaliki; 278.043, Gre 491, NO Exarcho

\section{Campanula rotundifolia}

283.314, Pel 350, Kajmaktsalan

Campanula sparsa subsp. sparsa

277.601, loa 1102, S Anilio

Campanula sparsa subsp. sphaerothrix

277.562, loa 1099, S Anilio; 278.840, Kav 114, SO Folia; 283.009, Kil 302, Kastaneri; 283.548, Pel 362, NW Promachi

\section{Campanula spatulata subsp. spatulata}

276.381, loa 1128, N Metsovo; 276.840, loa 1070, NNW Metsovo; 283.914, Ima 180, Seli

\section{Campanula spatulata subsp. spruneriana}

276.557, loa 1060, SSO Potamia; 276.959, loa 1078, O Anilio; 277.483, loa 1097, S Anilio; 277.713, Tri 460, N Chaliki; 282.084, Dra 295, NO Nevrokopi; 282.670, Ser 290, N Neo

\section{Petritsi}

\section{Campanula trachelium subsp. athoa}

281.618, Dra 278, Kato Vrondous; 282.740, Ser 292, N Neo Petritsi; 282.890, Kil 295, NW Griva; 283.145, Pel 342, S Archangelo; 283.768, Ima 172, S Ag. Pavlos; 283.877, Ima 179, Seli; 283.944, loa 1109, NW Metsovo; 284.135, loa 1096 b, S Anilio

\section{Campanula trachelium subsp. trachelium}

282.929, Kil 297, WNW Kastaneri; 283.538, Pel 362, NW Promachi

\section{Campanula tymphaea}

276.376, loa 1128, N Metsovo; 276.742, loa 1068, NW Metsovo; 276.804, loa 1070, NNW Metsovo; 277.027, loa 1080, SO Anilio; 277.288, loa 1091, SW Milia; 277.605, loa 1103, S Anilio

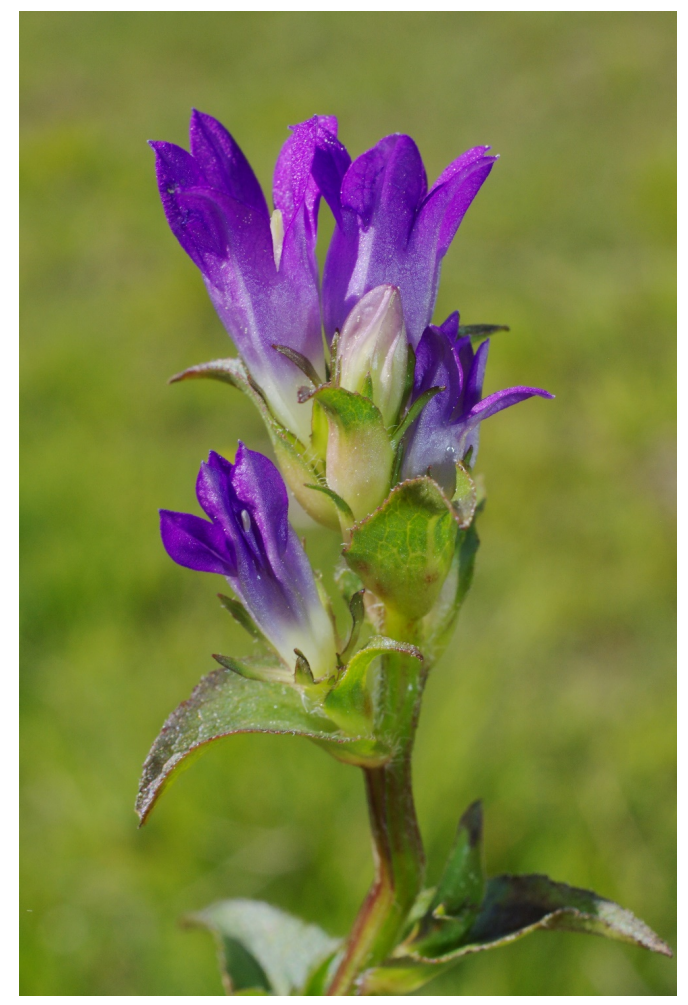

Capsella bursa-pastoris

276.648, loa 1063, W Potamia; 276.891, loa 1072, NNW Metsovo; 277.015, loa 1080, SO Anilio; 277.130, loa 1084, N Metsovo; 277.454, loa 1095, S Anilio; 278.837, Kav 114, SO 
Folia; 279.136, Kav 91, Orfani; 280.622, Dra 239, Skaloti; 281.313, Dra 268, W Nevrokopi; 281.504, Dra 276, Perithori; 281.683, Ser 271, Kato Vrondous; 282.274, Ser 272, Ano Vrondous; 282.324, Ser 274, SSW Ano Vrondous

Cardamine barbareoides

277.055, loa 1080, SO Anilio

\section{Cardamine bulbifera}

276.456, Tri 457, NO Katara-Pass; 277.180, loa 1086, NNO Metsovo; 277.577, loa 1100, S Anilio; 280.728, Dra 244, SW Walddorf Elatias; 282.594, Ser 287, Ori Vrondous

Cardamine glauca

276.402, loa 1057, NO Metsovo

Cardamine graeca

277.588, loa 1100, S Anilio

\section{Cardamine hirsuta}

276.409, loa 1057, NO Metsovo; 277.022, loa 1080, SO Anilio; 277.246, loa 1089, NNO Metsovo; 277.526, loa 1097, S Anilio; 280.579, Dra 238, Skaloti

Cardamine impatiens

280.714, Dra 243, SW Walddorf Elatias

\section{Cardamine pectinata}

276.771, loa 1069, NW Metsovo

\section{Carduus}

283.024, Kil 302, Kastaneri

\section{Carduus candicans}

276.501, loa 1059, Ag. Nikolaos; 278.807, Kav 113, NO Paralia Myrtofytou; 279.694, Ser 266, SO Nea Zichni

\section{Carduus kerneri subsp. scardicus}

281.260, Dra 266, W Nevrokopi; 282.513, Ser 282, Ori Vrondous; 283.970, loa 1056 b, NO Metsovo

\section{Carduus pycnocephalus}

276.484, loa 1059, Ag. Nikolaos

\section{Carduus thoermeri}

276.596, loa 1061, SSO Potamia; 277.370, loa 1093, S Anilio; 278.133, Gre 492, NO Varis; 278.627, Thes 221, Arethousa; 278.917, Kav 117, Galipsos; 279.242, Ser 258, Mikro Souli; 279.616, Ser 263, Dravoskas; 280.129, Dra 221, NO Drama; 280.934, Dra 253, Falakron; 281.428, Dra 274, Vathytopo; 281.850, Dra 284, NNO Volakas; 283.911, Ima 180, Seli

\section{Carduus tmoleus}

277.841, Koz 387, SO Siatista, Vourinos; 278.288, Koz 380, Galatini; 278.383, Koz 382, Galatini; 278.401, Koz 383, Galatini; 278.462, Thes 214, Paralia Vrasna; 278.514, Thes 217, Vrasna; 278.540, Thes 219, Vrasna; 278.604, Thes 221, Arethousa; 280.568, Dra 238, Skaloti; 280.917, Dra 251, Falakron; 281.192, Dra 264, NO Ochyro; 281.373, Dra 271, Vathytopo; 281.534, Dra 276, Perithori; 282.068, Dra 294, NO Nevrokopi; 282.135, Dra 297, Lefkogia; 282.368, Ser 276, SSW Ano Vrondous; 282.558, Ser 285, Ori Vrondous; 282.987 , Kil 300, Kastaneri; 283.027, Kil 303, SO Livadia; 283.270, Pel 348, Kajmaktsalan; 283.825, Ima 176, Tria-Pende Pigadia; 283.896, Ima 179, Seli; 284.023, Ioa 1116, SW Milia; 284.076, loa 1118, Anilio

\section{Carex acuta}

276.916, loa 1073, NNW Metsovo 


\section{Carex davalliana}

276.422, loa 1057, NO Metsovo; 276.783, loa 1069, NW Metsovo; 277.154, loa 1085, N Metsovo

\section{Carex digitata}

276.931, loa 1074, NNW Metsovo

\section{Carex distans}

276.379, loa 1128, N Metsovo; 276.417, loa 1057, NO Metsovo; 276.864, loa 1071, NNW Metsovo; 276.909, loa 1073, NNW Metsovo; 277.058, loa 1080, SO Anilio; 277.106, loa 1084, N Metsovo; 277.294, loa 1091, SW Milia; 277.298, loa 1091, SW Milia; 277.398, loa 1094, S Anilio; 277.731, Tri 460, N Chaliki; 278.557, Thes 219, Vrasna

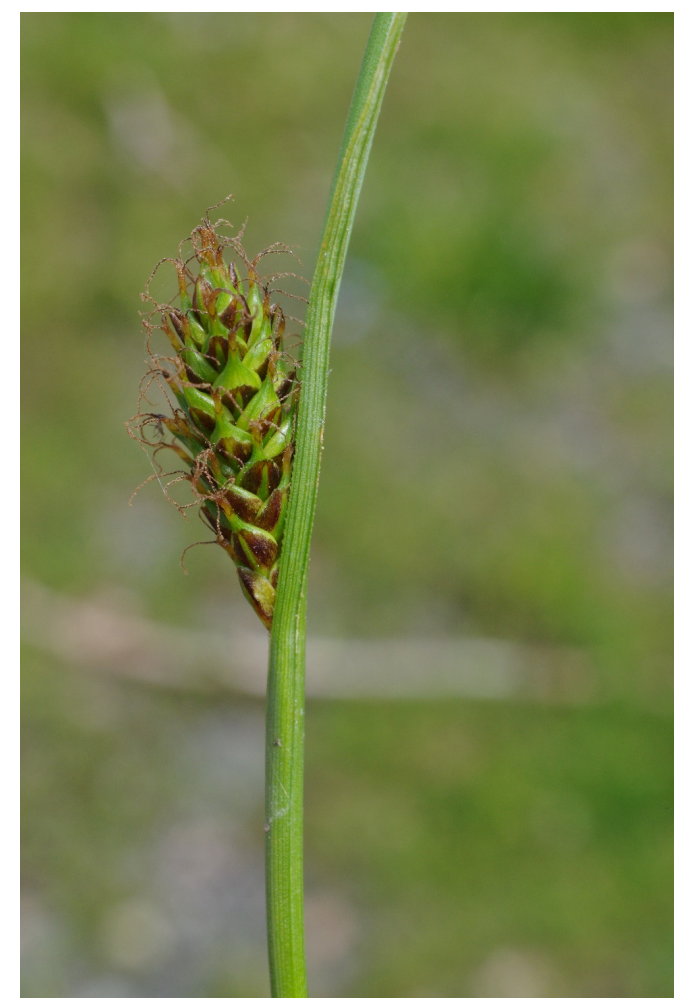

\section{Carex divisa}

277.061, loa 1080, SO Anilio

\section{Carex divulsa}

276.714, loa 1065, NO Karyes; 279.372, Ser 261, Proti; 279.450, Kav 95, Pangaion

\section{Carex flacca}

279.878, Dra 209, Livadero; 280.481, Dra 234, Skaloti; 282.873, Kil 295, NW Griva

\section{Carex hirta}

276.782, loa 1069, NW Metsovo; 277.621, loa 1104, S Anilio

\section{Carex leersii}

276.334, loa 1126, NO Dodoni; 276.647, loa 1063, W Potamia; 277.200, loa 1087, NNO Metsovo; 277.241, loa 1089, NNO Metsovo; 277.736, Tri 460, N Chaliki; 280.710, Dra 243, SW Walddorf Elatias; 282.562, Ser 285, Ori Vrondous; 282.649, Ser 290, N Neo Petritsi; 283.569, Pel 363, NW Promachi

\section{Carex lepidocarpa}

276.410, loa 1057, NO Metsovo

\section{Carex leporina}


277.062, loa 1080, SO Anilio; 277.641, loa 1104, S Anilio; 280.549, Dra 237, Skaloti; 282.564, Ser 285, Ori Vrondous; 282.584, Ser 287, Ori Vrondous; 283.331, Pel 351, Kajmaktsalan

\section{Carex liparocarpos}

276.664, loa 1064, ONO Karyes

\section{Carex muricata}

276.408, loa 1057, NO Metsovo; 276.413, loa 1057, NO Metsovo; 276.914, loa 1073, NNW Metsovo; 277.060, loa 1080, SO Anilio; 284.224, loa 1080, SO Anilio

\section{Carex otrubae}

276.673, loa 1064, ONO Karyes; 277.025, loa 1080, SO Anilio; 277.104, loa 1084, N Metsovo; 277.402, loa 1094, S Anilio

\section{Carex pallescens}

276.373, loa 1128, N Metsovo; 276.865, loa 1071, NNW Metsovo; 277.037, loa 1080, SO Anilio; 280.552, Dra 237, Skaloti

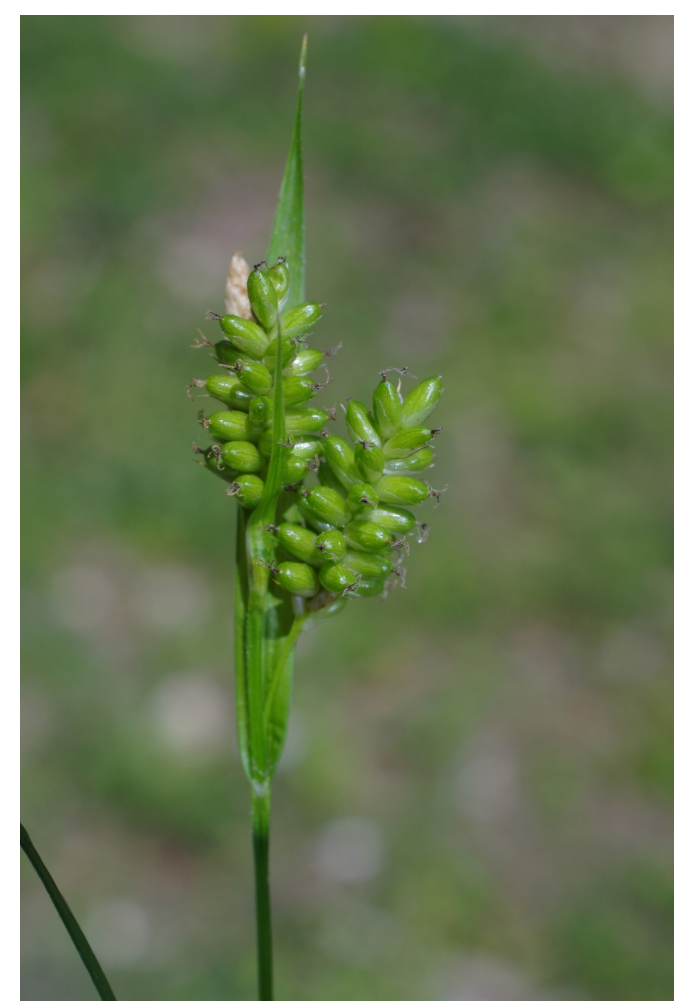

\section{Carex remota}

277.207, loa 1088, NNO Metsovo; 283.577, Pel 363, NW Promachi

\section{Carex rostrata}

280.773, Dra 246, SW Walddorf Elatias

\section{Carex spicata}

276.821, loa 1070, NNW Metsovo; 276.921, loa 1074, NNW Metsovo; 277.262, loa 1090, NNO Metsovo; 277.761, Tri 461, N Chaliki; 277.928, Koz 392, SO Siatista, Vourinos; 280.741, Dra 245, SW Walddorf Elatias; 283.113, Pel 339, S Archangelo

\section{Carex sylvatica}

277.050, loa 1080, SO Anilio; 277.206, loa 1088, NNO Metsovo; 277.276, loa 1090, NNO Metsovo; 277.501, loa 1097, S Anilio; 280.510, Dra 236, Skaloti

\section{Carlina vulgaris}


283.219, Pel 345, Kerasia; 283.799, Ima 174, S Ag. Pavlos; 283.983, loa 1111, NO Metsovo; 284.042, loa 1116, SW Milia; 284.054, loa 1117, Anilio; 284.161, loa 1123, N Charikli

\section{Carpinus orientalis}

276.942, loa 1075, NW Anilio; 278.241, Koz 378, N Siatista; 281.220, Dra 265, NO Granitis; 282.009, Dra 292, Nevrokopi; 282.874, Kil 295, NW Griva; 283.404, Pel 354, NW Loutraki; 283.536, Pel 361, SW Promachi

\section{Carthamus lanatus}

278.162, Gre 494, SO Varis; 279.252, Ser 258, Mikro Souli; 279.313, Ser 260, Proti; 279.668, Ser 265, Myrrini; 281.238, Dra 265, NO Granitis; 281.289, Dra 267, W Nevrokopi; 281.769, Dra 282, NNO Volakas; 281.967, Dra 289, NO Potamia; 283.504, Pel 360, SW Promachi; 283.612, Pel 365, N Vorino; 283.619, Pel 366, N Vorino; 283.625, Pel 366, N Vorino; 283.705, Pel 369, O Vorino

\section{Carum graecum subsp. graecum}

283.266, Pel 347, Kajmaktsalan

\section{Carum graecum subsp. serpentinicum}

276.399, loa 1056, NO Metsovo; 277.644, loa 1105, Anilio; 277.977, Koz 374, Vourinos; 280.892, Dra 250, Falakron; 280.893, Dra 250, Falakron

\section{Carum multiflorum}

276.676, loa 1065, NO Karyes

\section{Carum strictum}

279.937, Dra 211, Livadero; 282.310, Ser 274, SSW Ano Vrondous

\section{Castanea sativa}

279.462, Kav 95, Pangaion; 282.736, Ser 291, N Neo Petritsi; 282.900, Kil 296, N Kastaneri

\section{Catapodium rigidum}

277.366, loa 1093, S Anilio

\section{Caucalis platycarpos}

277.832, Koz 387, SO Siatista, Vourinos; 277.903, Koz 390, SO Siatista, Vourinos; 278.092, Gre 492, NO Varis; 278.391, Koz 383, Galatini; 278.985, Kav 119, Podochori; 279.472, Kav 96, Pangaion; 279.753, Ser 268, SW Skopia; 280.928, Dra 253, Falakron

\section{Centaurea affinis subsp. affinis}

279.851, Dra 209, Livadero; 280.975, Dra 256, Falakron; 281.221, Dra 265, NO Granitis; 281.476, Dra 275, Vathytopo; 281.517, Dra 276, Perithori; 281.795, Dra 282, NNO Volakas; 281.900, Dra 287, O Mikroklisoura; 281.908, Dra 287, O Mikroklisoura; 282.023, Dra 292, Nevrokopi; 282.295, Ser 273, Ano Vrondous; 282.474, Ser 281, Ori Vrondous; 282.546, Ser 285, Ori Vrondous; 282.664, Ser 290, N Neo Petritsi; 282.684, Ser 290, N Neo Petritsi; 282.856, Kil 294, NW Griva; 283.111, Pel 339, S Archangelo; 283.422, Pel 355, NW Loutraki; 283.497, Pel 359, NW Loutraki; 283.511, Pel 360, SW Promachi; 283.815, Ima 176, TriaPende Pigadia; 283.887, Ima 179, Seli; 284.053, loa 1117, Anilio; 284.087, loa 1120, Anilio; 284.107, loa 1121, Anilio

Centaurea affinis subsp. pallidior 281.870, Dra 286, SW Mikroklisoura; 282.777, Ser 293, N Neo Petritsi; 283.280, Pel 348, Kajmaktsalan

Centaurea benedicta

278.591, Thes 220, Arethousa

Centaurea cuneifolia 281.739, Dra 281, OSO Granitis

\section{Centaurea cyanus}


277.781, Koz 385, SO Siatista; 278.256, Koz 379, Galatini; 278.572 , Thes 220, Arethousa; 283.639, Pel 367, Vorino

Centaurea depressa

278.373, Koz 382, Galatini

\section{Centaurea deustiformis}

283.958, loa 1110, N Metsovo

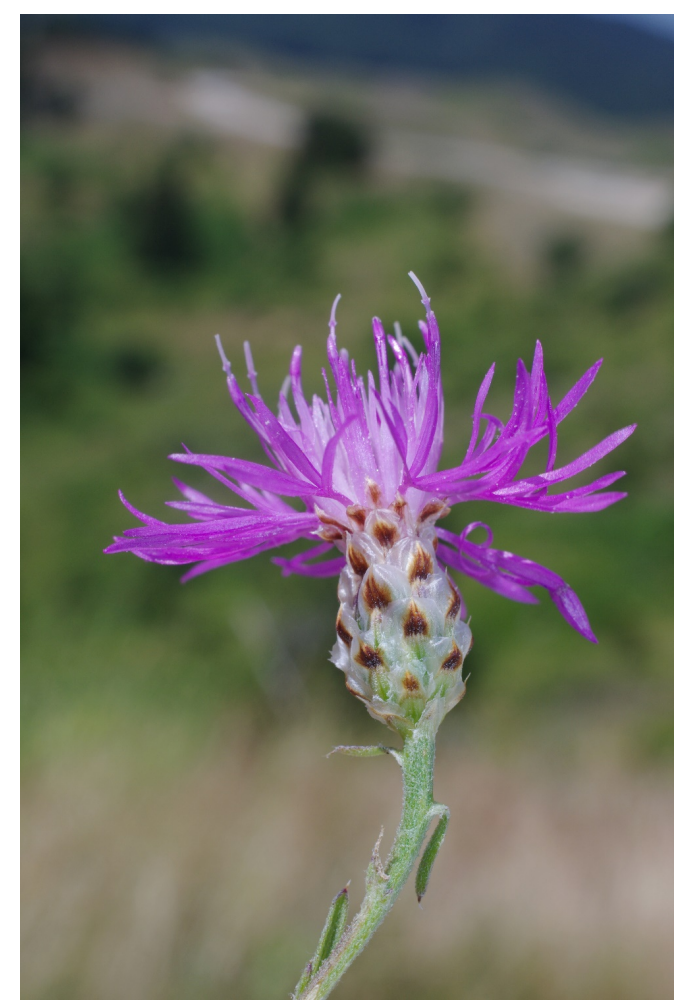

\section{Centaurea diffusa}

278.889, Kav 116, S Ofrynia; 279.610, Ser 263, Dravoskas; 279.676, Ser 265, Myrrini; 279.803, Ser 269, NO Skopia; 281.735, Dra 281, OSO Granitis; 281.994, Dra 291, W Prosotsani; 282.794, Ser 296, NO Strymoniko; 283.605, Pel 365, N Vorino; 283.615, Pel 365, N Vorino; 283.622, Pel 366, N Vorino; 283.697, Pel 369, O Vorino; 283.720, Pel 370, SW Foustani

\section{Centaurea graeca}

278.152, Gre 494, SO Varis

\section{Centaurea grisebachii}

278.194, Koz 377, N Siatista; 278.732, Kav 108, Paralia Ofryniou; 278.898, Kav 116, S Ofrynia; 280.049, Dra 217, Sidironero; 281.049, Dra 259, Granitis; 281.168, Dra 264, NO Ochyro; 281.315, Dra 268, W Nevrokopi; 281.592, Dra 278, Kato Vrondous; 281.807, Dra 283, NNO Volakas; 282.114, Dra 296, NO Nevrokopi; 282.190, Dra 299, SO Lefkogia; 282.428, Ser 279, Orini; 282.795, Ser 296, NO Strymoniko; 282.906, Kil 297, WNW Kastaneri; 283.040, Kil 303, SO Livadia; 283.065, Kil 307, NW Livadia; 283.160, Pel 343, Kerasia; 283.380, Pel 354, NW Loutraki; 283.610, Pel 365, N Vorino; 283.746, Ima 172, S Ag. Pavlos

\section{Centaurea jacea}

281.712, Dra 280, Nevrokopi; 284.066, loa 1118, Anilio

\section{Centaurea jacea subsp. weldeniana}

280.899, Dra 250, Falakron; 283.787, Ima 173, S Ag. Pavlos

\section{Centaurea lacerata}


277.757, Tri 461, N Chaliki; 282.381, Ser 276, SSW Ano Vrondous; 283.947, loa 1109, NW Metsovo; 284.005, loa 1113, Katara-Paß; 284.167, loa 1124, N Charikli

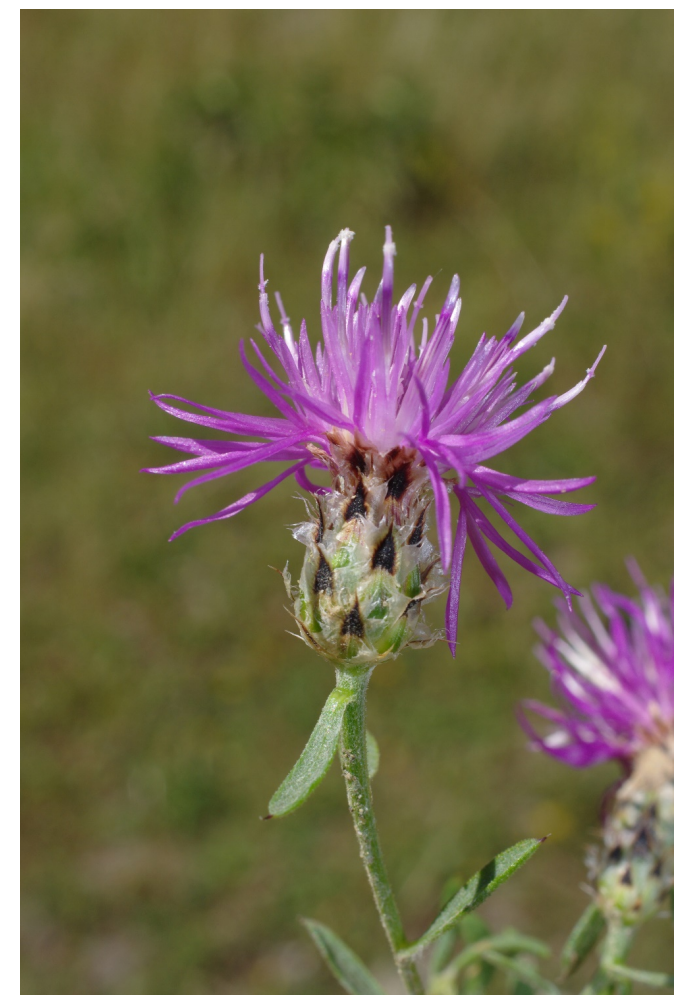

Centaurea macedonica

277.997, Koz 375, O Dafnero; 281.169, Dra 264, NO Ochyro; 282.771, Ser 293, N Neo Petritsi; 282.848, Kil 294, NW Griva; 283.510, Pel 360, SW Promachi

\section{Centaurea nervosa subsp. nervosa}

283.319, Pel 351, Kajmaktsalan

\section{Centaurea salonitana}

277.790, Koz 385, SO Siatista; 278.624, Thes 221, Arethousa; 278.940, Kav 118, Galipsos; 279.014, Kav 119, Podochori; 279.707, Ser 266, SO Nea Zichni; 279.771, Ser 268, SW Skopia; 280.122, Dra 220, Sidironero; 280.216, Dra 225, NO Drama; 281.060, Dra 259, Granitis; 281.233, Dra 265, NO Granitis; 281.290, Dra 267, W Nevrokopi; 281.443, Dra 274, Vathytopo; 281.640, Dra 279, Kato Vrondous; 281.713, Dra 280, Nevrokopi; 281.817, Dra 283, NNO Volakas; 281.983, Dra 290, NO Potamia; 282.013, Dra 292, Nevrokopi

Centaurea solstitialis subsp. adamii

278.017, Koz 376, W Exarcho; 278.064, Gre 492, NO Varis; 278.177, Gre 495, Varis

\section{Centaurea solstitialis subsp. solstitialis}

278.718, Kav 108, Paralia Ofryniou; 279.039, Kav 120, Podochori; 279.234, Ser 258, Mikro Souli; 279.650, Ser 264, Dravoskas; 279.687, Ser 266, SO Nea Zichni; 279.816, Ser 269, NO Skopia; 281.995, Dra 291, W Prosotsani; 282.789, Ser 295, N Neo Petritsi

\section{Centaurea stenolepis}

280.605, Dra 238, Skaloti; 281.065, Dra 260, Granitis; 281.625, Dra 279, Kato Vrondous; 282.088, Dra 295, NO Nevrokopi; 282.208, Dra 300, SO Lefkogia; 283.936, loa 1108, N Metsovo

\section{Centaurea triumfettii}

283.363, Pel 352, Kajmaktsalan

\section{Centaurea zuccariniana}

276.717, loa 1066, NO Karyes 
Centaurium erythraea subsp. erythraea

278.714, Kav 108, Paralia Ofryniou; 278.850, Kav 115, SW Folia; 279.056, Kav 121,

Platanotopos; 279.385, Ser 262, Proti; 279.407, Kav 92, Pangaion; 279.788, Ser 268, SW Skopia; 280.205, Dra 224, NO Drama; 281.883, Dra 287, O Mikroklisoura; 282.714, Ser 290, N Neo Petritsi; 282.868, Kil 295, NW Griva; 282.981, Kil 300, Kastaneri; 283.161, Pel 343, Kerasia; 283.424, Pel 355, NW Loutraki; 283.859, Ima 178, Seli; 284.046, loa 1117, Anilio; 284.152, loa 1122, Anilio

Centaurium erythraea subsp. rumelicum

278.067, Gre 492, NO Varis; 279.852, Dra 209, Livadero; 281.479, Dra 275, Vathytopo; 282.061, Dra 294, NO Nevrokopi

\section{Centaurium tenuiflorum}

279.029, Kav 120, Podochori; 279.142, Ser 255, S Nea Mesolakkia; 279.621, Ser 263,

Dravoskas; 280.188, Dra 223, NO Drama; 281.706, Dra 280, Nevrokopi; 281.930, Dra 288, SW Potamia

Cephalanthera damasonium

277.179, loa 1086, NNO Metsovo; 277.250, loa 1089, NNO Metsovo

\section{Cephalanthera longifolia}

276.452, Tri 457, NO Katara-Pass

\section{Cephalanthera rubra}

276.702, loa 1065, NO Karyes; 277.216, loa 1088, NNO Metsovo; 277.547, loa 1098, S

Anilio; 280.547, Dra 237, Skaloti; 283.217, Pel 345, Kerasia; -20.529, Ima 173, S Ag. Pavlos

\section{Cephalaria transsylvanica}

283.679, Pel 368, OSO Vorino

\section{Cerastium banaticum subsp. banaticum}

284.015, loa 1114, Katara-Paß

Cerastium banaticum subsp. speciosum

280.863, Dra 249, Falakron; 280.882, Dra 250, Falakron

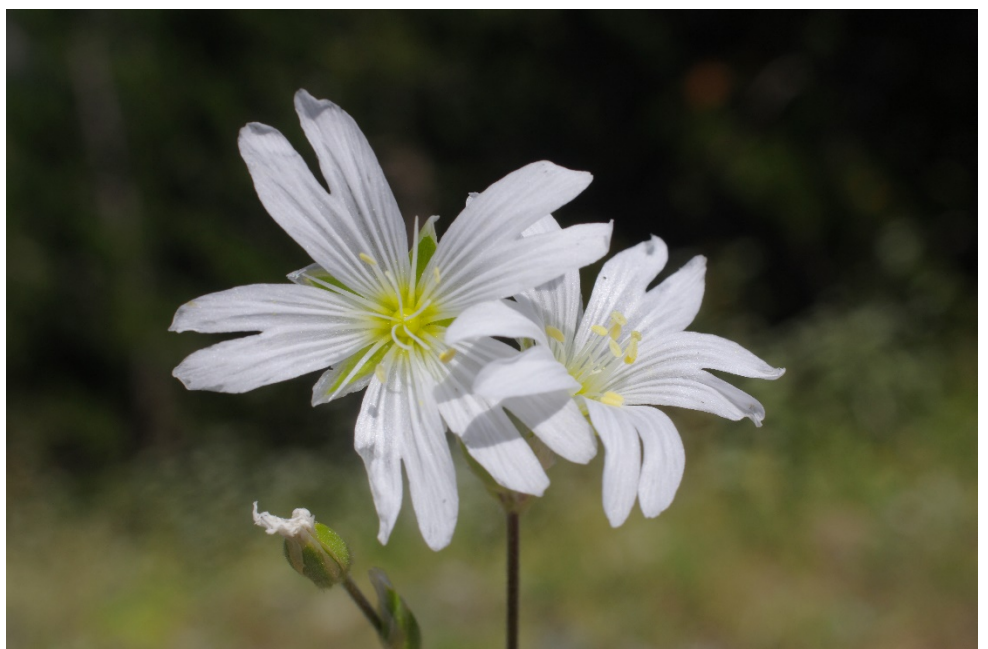

\section{Cerastium brachypetalum subsp. roeseri}

276.790, loa 1070, NNW Metsovo; 277.247, loa 1089, NNO Metsovo; 277.480, loa 1097, S Anilio; -20.492, loa 1078, O Anilio

Cerastium brachypetalum subsp. tenoreanum

276.336, loa 1126, NO Dodoni; 276.369, loa 1128, N Metsovo; 277.686, Tri 460, N Chaliki 


\section{Cerastium decalvans}

277.034, loa 1080, SO Anilio

Cerastium glomeratum

276.395, loa 1056, NO Metsovo; 278.833, Kav 114, SO Folia

\section{Cerastium glutinosum}

277.129, loa 1084, N Metsovo

\section{Cerastium holosteoides}

276.448, Tri 456, N Katara-Pass; 276.774, loa 1069, NW Metsovo; 276.982, loa 1079, SO Anilio; 277.222, loa 1088, NNO Metsovo; 277.576, loa 1100, S Anilio; 279.544, Kav 101, Pangaion; 279.573, Kav 103, Pangaion; 279.973, Dra 213, Livadero; 280.338, Dra 230, Livadero; 280.454, Dra 234, Skaloti; 280.504, Dra 236, Skaloti; 280.610, Dra 238, Skaloti; 280.623, Dra 239, Skaloti; 280.708, Dra 243, SW Walddorf Elatias; 280.909, Dra 251,

Falakron; 282.598, Ser 287, Ori Vrondous; 283.057, Kil 306, SO Livadia; 284.188, loa 1128, $\mathrm{N}$ Metsovo

\section{Cerastium semidecandrum}

277.038, loa 1080, SO Anilio; 277.070, loa 1080, SO Anilio; 277.965, Koz 374, Vourinos; 283.375, Pel 353, Kajmaktsalan

Cerinthe minor subsp. auriculata

282.043, Dra 293, Nevrokopi

\section{Chaenorhinum minus}

276.320, loa 1125, NO Dodoni; 276.477, loa 1059, Ag. Nikolaos; 277.479, loa 1097, S Anilio; 278.184, Gre 495, Varis; 280.901, Dra 250, Falakron; 282.480, Ser 281, Ori Vrondous; 283.372, Pel 353, Kajmaktsalan; 283.876, Ima 179, Seli; 283.962, loa 1110, N Metsovo; 284.057, loa 1117, Anilio

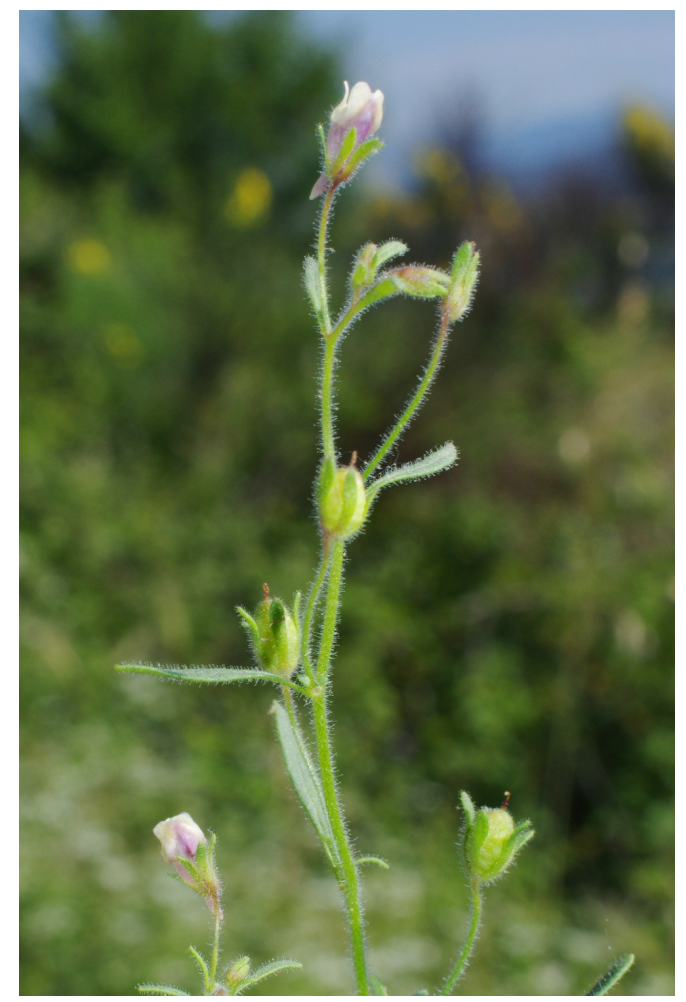

\section{Chaerophyllum aromaticum}

283.928, loa 1107, N Metsovo; 284.030, loa 1116, SW Milia

\section{Chaerophyllum aureum}

277.463, loa 1096, S Anilio; 280.513, Dra 236, Skaloti; 280.687, Dra 242, SW Walddorf Elatias; 280.808, Dra 247, Walddorf Elatias; 282.510, Ser 282, Ori Vrondous; 282.557 , Ser 285, Ori Vrondous; 282.729, Ser 291, N Neo Petritsi; 282.924, Kil 297, WNW Kastaneri; 
283.023, Kil 302, Kastaneri; 283.744, Ima 172, S Ag. Pavlos; 283.823, Ima 176, Tria-Pende Pigadia

\section{Chaerophyllum bulbosum}

276.765, loa 1069, NW Metsovo; 281.003, Dra 257, Granitis; 281.067, Dra 260, Granitis; 281.189, Dra 264, NO Ochyro; 281.333, Dra 269, W Nevrokopi; 281.365, Dra 271, Vathytopo; 281.500, Dra 276, Perithori; 282.249, Dra 301, Ano Vrondous

\section{Chaerophyllum nodosum}

276.592, loa 1061, SSO Potamia

\section{Chaerophyllum temulum}

276.697, loa 1065, NO Karyes; 277.929, Koz 392, SO Siatista, Vourinos; 282.239, Dra 301, Ano Vrondous; 283.205, Pel 344, Kerasia; 283.864, Ima 178, Seli

\section{Chamaecytisus}

283.582, Pel 363, NW Promachi

\section{Chamaecytisus austriacus}

276.744, loa 1068, NW Metsovo

\section{Chamaecytisus austriacus subsp. austriacus}

279.521, Kav 99, Pangaion; 279.599, Kav 105, Pangaion; 280.058, Dra 217, Sidironero; 280.314, Dra 229, SO Dendrakia; 280.430, Dra 233, Sidironero; 281.585, Dra 278, Kato Vrondous; 282.718, Ser 290, N Neo Petritsi

\section{Chamaecytisus austriacus subsp. heuffelii}

281.027, Dra 258, Granitis; 283.778, Ima 173, S Ag. Pavlos

\section{Chamaecytisus eriocarpus}

277.700, Tri 460, N Chaliki; 279.862, Dra 209, Livadero; 280.490, Dra 235, Skaloti; 280.654, Dra 242, SW Walddorf Elatias; 280.791, Dra 247, Walddorf Elatias; 281.482, Dra 275, Vathytopo; 281.570, Dra 277, Perithori; 281.861, Dra 285, NNO Volakas; 282.157, Dra 298, SO Lefkogia; 282.299, Ser 273, Ano Vrondous; 282.464, Ser 281, Ori Vrondous

\section{Chelidonium majus}

281.424, Dra 273, Vathytopo; 282.725, Ser 291, N Neo Petritsi; 283.735, Ima 172, S Ag.

Pavlos

\section{Chenopodium album}

278.467, Thes 215, Paralia Vrasna; 278.550, Thes 219, Vrasna; 278.578, Thes 220, Arethousa; 278.709, Kav 107, Paralia Ofryniou; 278.923, Kav 117, Galipsos; 279.010, Kav 119, Podochori; 279.674, Ser 265, Myrrini; 280.108, Dra 220, Sidironero; 281.056, Dra 259, Granitis; 281.184, Dra 264, NO Ochyro; 281.327, Dra 269, W Nevrokopi; 281.558, Dra 277, Perithori; 281.621, Dra 278, Kato Vrondous; 281.840, Dra 284, NNO Volakas; 281.929, Dra 288, SW Potamia; 282.269, Ser 272, Ano Vrondous; 282.896, Kil 296, N Kastaneri; 283.108, Pel 339, S Archangelo; 283.449, Pel 356, NW Loutraki; 283.595, Pel 364, Loutraki; 283.665, Pel 368, OSO Vorino

\section{Chenopodium opulifolium}

278.097, Gre 492, NO Varis; 278.296, Koz 380, Galatini; 278.698, Kav 107, Paralia Ofryniou; 279.212, Ser 257, Nea Fili; 279.278, Ser 258, Mikro Souli; 279.768, Ser 268, SW Skopia; 281.263, Dra 266, W Nevrokopi; 281.408, Dra 273, Vathytopo; 282.688, Ser 290, N Neo Petritsi

\section{Chenopodium polyspermum}

283.559, Pel 362, NW Promachi

\section{Chenopodium vulvaria}

278.670, Kav 107, Paralia Ofryniou; 278.969, Kav 119, Podochori; 279.194, Ser 256,

Paleokomi; 279.195, Ser 256, Paleokomi; 281.426, Dra 273, Vathytopo 


\section{Chondrilla juncea}

278.692, Kav 107, Paralia Ofryniou; 278.885, Kav 116, S Ofrynia; 279.040, Kav 120,

Podochori; 279.646, Ser 264, Dravoskas; 281.007, Dra 257, Granitis; 281.193, Dra 264, NO Ochyro; 281.270, Dra 266, W Nevrokopi; 281.646, Dra 279, Kato Vrondous; 281.865, Dra 286, SW Mikroklisoura; 282.066, Dra 294, NO Nevrokopi; 282.412, Ser 279, Orini; 282.669, Ser 290, N Neo Petritsi; 282.827, Ser 298, Parapotamos; 283.453, Pel 357, NW Loutraki; 283.603, Pel 365, N Vorino; 283.660, Pel 368, OSO Vorino; 284.106, loa 1121, Anilio

\section{Chondrilla urumoffii}

280.972, Dra 256, Falakron; 284.184, Dra 256, Falakron

\section{Chrozophora tinctoria}

278.678, Kav 107, Paralia Ofryniou; 278.919, Kav 117, Galipsos; 279.211, Ser 257, Nea Fili; 279.275, Ser 258, Mikro Souli

\section{Chrysopogon gryllus}

276.634, loa 1063, W Potamia; 278.002, Koz 375, O Dafnero; 278.079, Gre 492, NO Varis; 278.310, Koz 381, Galatini; 279.432, Kav 94, Pangaion; 279.742, Ser 268, SW Skopia; 279.922, Dra 211, Livadero; 280.069, Dra 218, Sidironero; 280.257, Dra 227, Livadero; 280.404, Dra 233, Sidironero; 281.133, Dra 262, NW Granitis; 281.442, Dra 274, Vathytopo; 281.644, Dra 279, Kato Vrondous; 281.806, Dra 283, NNO Volakas; 282.106, Dra 295, NO Nevrokopi

\section{Cicer arietinum}

278.174, Gre 494, SO Varis

\section{Cichorium intybus}

276.485, loa 1059, Ag. Nikolaos; 277.327, loa 1093, S Anilio; 277.473, loa 1096, S Anilio; 278.650, Kav 106, Paralia Ofryniou; 278.911, Kav 116, S Ofrynia; 279.876, Dra 209, Livadero; 280.159, Dra 221, NO Drama; 280.980, Dra 257, Granitis; 281.511, Dra 276, Perithori; 282.260, Dra 301, Ano Vrondous; 282.465, Ser 281, Ori Vrondous; 282.824 , Ser 298, Parapotamos; 283.001, Kil 301, Kastaneri; 283.186, Pel 343, Kerasia; 283.382, Pel 354, NW Loutraki; 283.702, Pel 369, O Vorino

\section{Cichorium pumilum}

278.764, Kav 110, Loutra Eleftherion

\section{Circaea lutetiana}

282.234, Dra 301, Ano Vrondous; 282.884, Kil 295, NW Griva

\section{Cirsium appendiculatum}

280.566, Dra 238, Skaloti; 282.589, Ser 287, Ori Vrondous; 283.474 , Pel 358, N Loutraki; 283.553, Pel 362, NW Promachi

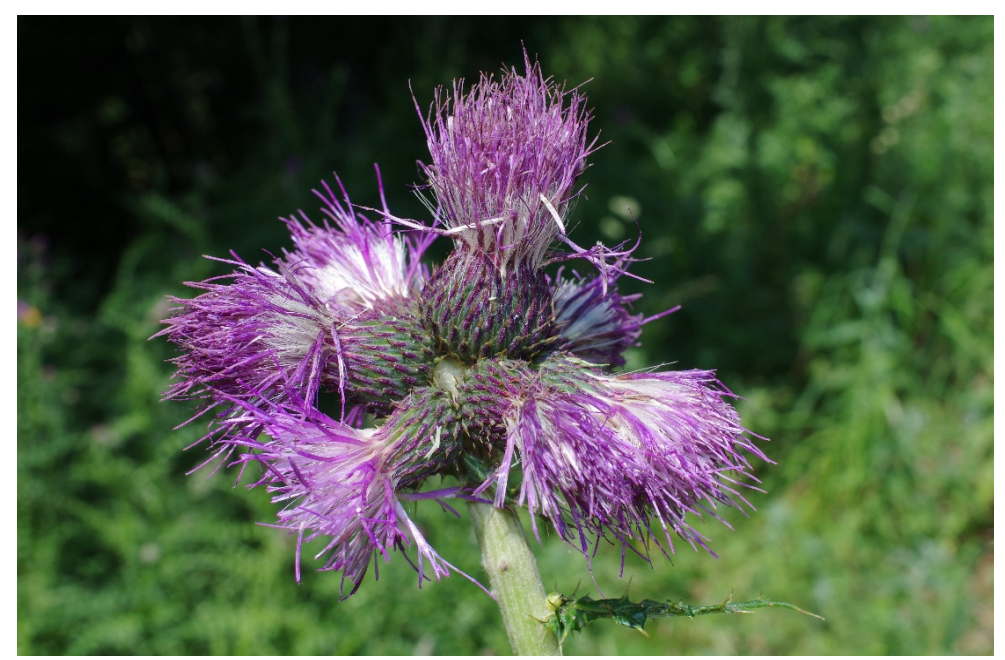

\section{Cirsium arvense}

278.155, Gre 494, SO Varis; 279.831, Dra 207, NO Skopia; 281.127, Dra 261, Granitis; 281.321, Dra 268, W Nevrokopi; 281.523, Dra 276, Perithori; 281.936, Dra 288, SW Potamia; 
282.057, Dra 294, NO Nevrokopi; 282.296, Ser 273, Ano Vrondous; 282.687, Ser 290, N Neo Petritsi; 282.977, Kil 299, Kastaneri; 283.081, Kil 307, NW Livadia; 283.180, Pel 343,

Kerasia; 283.447, Pel 356, NW Loutraki; 283.671, Pel 368, OSO Vorino; 283.754, Ima 172, S Ag. Pavlos; 283.847, Ima 178, Seli; 284.022, loa 1116, SW Milia; 284.072, loa 1118, Anilio

\section{Cirsium candelabrum}

284.044, loa 1116, SW Milia; 284.084, loa 1119, Anilio

\section{Cirsium creticum}

283.523, Pel 361, SW Promachi

\section{Cirsium tymphaeum}

280.678, Dra 242, SW Walddorf Elatias; 283.791, Ima 173, S Ag. Pavlos; 283.977, loa 1111, NO Metsovo; 284.045, loa 1116, SW Milia

\section{Cistus creticus}

277.412, loa 1094, S Anilio; 279.430, Kav 94, Pangaion; 283.481, Pel 359, NW Loutraki

\section{Clematis flammula}

276.316, loa 1125, NO Dodoni; 278.613, Thes 221, Arethousa; 278.738, Kav 109, Loutra Eleftherion; 278.817, Kav 114, SO Folia; 278.968, Kav 119, Podochori; 279.080, Kav 89, Misoropi; 279.298, Ser 259, Proti; 279.416, Kav 93, Pangaion; 283.395, Pel 354, NW Loutraki; 283.455, Pel 357, NW Loutraki; 283.700, Pel 369, O Vorino

\section{Clematis vitalba}

276.341, loa 1127, Dodonoupoli; 277.331, loa 1093, S Anilio; 278.512, Thes 217, Vrasna; 278.595, Thes 220, Arethousa; 278.743, Kav 109, Loutra Eleftherion; 278.811, Kav 114, SO Folia; 279.081, Kav 89, Misoropi; 279.473, Kav 96, Pangaion; 279.832, Dra 207, NO Skopia; 279.868, Dra 209, Livadero; 280.011, Dra 216, Sidironero; 280.167, Dra 221, NO Drama; 280.998, Dra 257, Granitis; 281.303, Dra 267, W Nevrokopi; 281.391, Dra 272, Vathytopo; 281.627, Dra 279, Kato Vrondous; 281.851, Dra 284, NNO Volakas; 281.876 , Dra 287, O Mikroklisoura; 282.182, Dra 299, SO Lefkogia; 282.337, Ser 274, SSW Ano Vrondous; 282.753, Ser 292, N Neo Petritsi; 282.876, Kil 295, NW Griva; 283.156, Pel 342, S Archangelo; 283.184, Pel 343, Kerasia; 283.505, Pel 360, SW Promachi; 283.699, Pel 369, O Vorino; 283.782, Ima 173, S Ag. Pavlos

\section{Clinopodium dalmaticum subsp. bulgaricum}

Dra 277, Drama, Perithori,

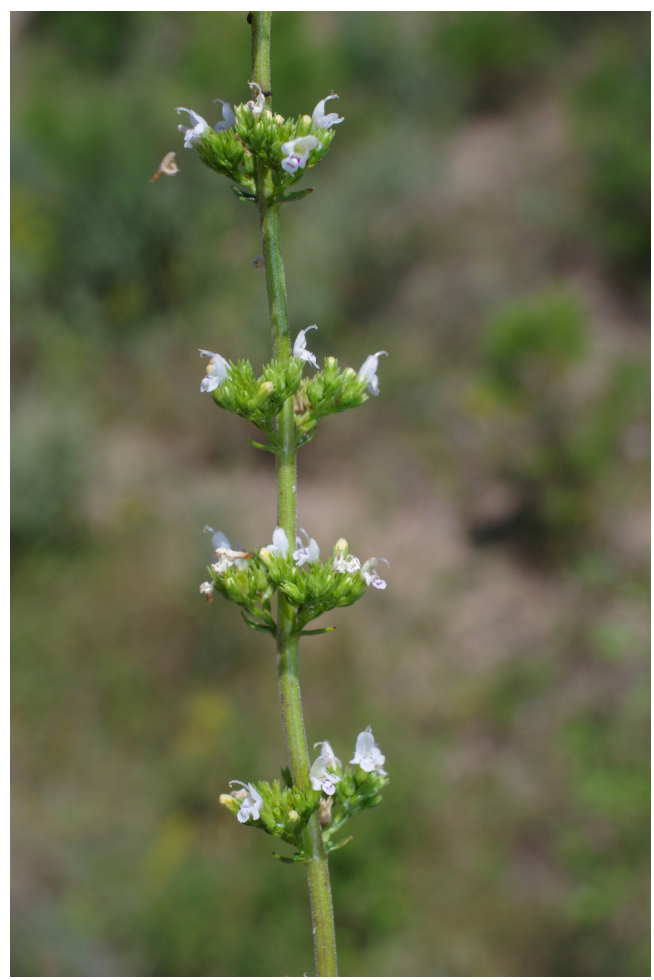




\section{Clinopodium vulgare subsp. orientale}

277.814, Koz 387, SO Siatista, Vourinos; 278.484, Thes 215, Paralia Vrasna; 278.521, Thes 217, Vrasna; 278.870, Kav 115, SW Folia; 279.226, Ser 257, Nea Fili; 280.288, Dra 228, Livadero; 280.952, Dra 255, Falakron; 281.901, Dra 287, O Mikroklisoura; 282.525, Ser 283, Ori Vrondous; 282.853, Kil 294, NW Griva; 283.541, Pel 362, NW Promachi

\section{Clinopodium vulgare subsp. vulgare}

276.291, loa 1125, NO Dodoni; 276.443, Tri 456, N Katara-Pass; 276.729 , loa 1066, NO Karyes; 276.748, loa 1068, NW Metsovo; 276.927, loa 1074, NNW Metsovo; 277.184, loa 1086, NNO Metsovo; 277.411, loa 1094, S Anilio; 277.688, Tri 460, N Chaliki; 278.082, Gre 492, NO Varis; 279.055, Kav 121, Platanotopos; 279.369, Ser 261, Proti; 279.397, Kav 92, Pangaion; 279.783, Ser 268, SW Skopia; 279.893, Dra 209, Livadero; 280.018, Dra 216, Sidironero; 280.378, Dra 232, Sidironero; 280.515, Dra 236, Skaloti; 280.661, Dra 242, SW Walddorf Elatias; 280.722, Dra 243, SW Walddorf Elatias; 281.401, Dra 272, Vathytopo; 281.615, Dra 278, Kato Vrondous; 281.831, Dra 283, NNO Volakas; 282.081, Dra 295, NO Nevrokopi; 282.180, Dra 299, SO Lefkogia; 282.399, Ser 278, N Serres; 282.573, Ser 286, Ori Vrondous; 282.668, Ser 290, N Neo Petritsi; 283.071, Kil 307, NW Livadia; 283.200, Pel 343, Kerasia; 283.462, Pel 358, N Loutraki; 283.737, Ima 172, S Ag. Pavlos; 283.810, Ima 176, Tria-Pende Pigadia; 283.846, Ima 178, Seli; 284.040, loa 1116, SW Milia

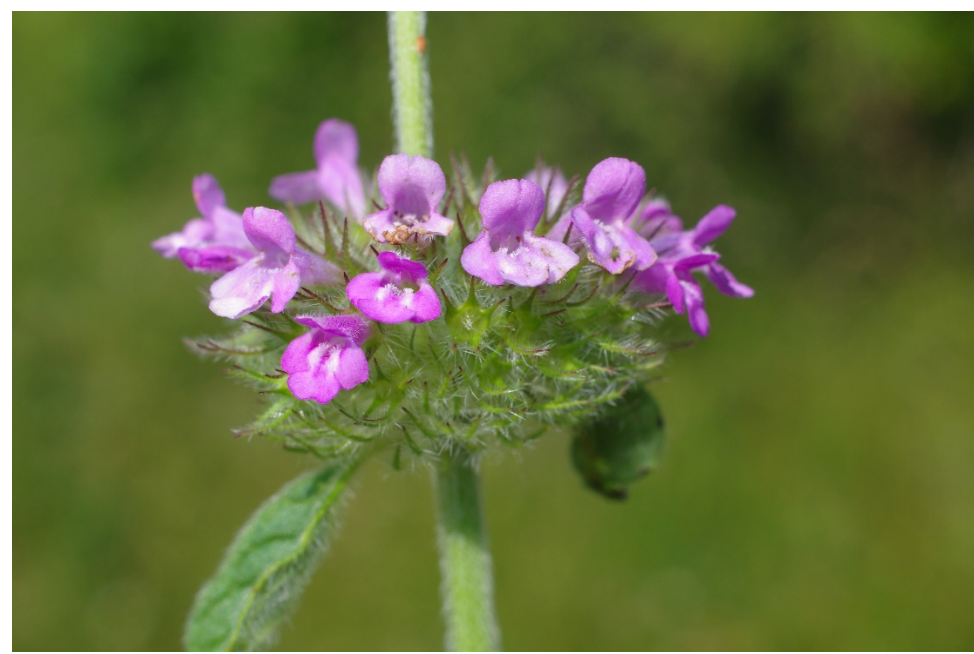

\section{Colutea arborescens}

276.569, loa 1060, SSO Potamia; 278.523, Thes 217, Vrasna; 283.212, Pel 344, Kerasia

\section{Conium maculatum}

276.936, loa 1075, NW Anilio; 278.620, Thes 221, Arethousa; 279.655, Ser 265, Myrrini; 281.328, Dra 269, W Nevrokopi; 281.525, Dra 276, Perithori; 282.132, Dra 297, Lefkogia

\section{Consolida ajacis}

279.241, Ser 258, Mikro Souli

\section{Consolida phrygia}

279.098, Kav 90, SW Moustheni

\section{Consolida regalis}

277.789, Koz 385, SO Siatista; 278.263, Koz 379, Galatini; 278.374, Koz 382, Galatini; 278.588, Thes 220, Arethousa; 278.954, Kav 119, Podochori; 279.108, Kav 90, SW Moustheni; 279.162, Ser 255, S Nea Mesolakkia; 279.202, Ser 257, Nea Fili; 279.614, Ser 263, Dravoskas; 279.667, Ser 265, Myrrini; 279.757, Ser 268, SW Skopia; 281.170, Dra 264, NO Ochyro; 281.254, Dra 266, W Nevrokopi; 281.512, Dra 276, Perithori; 281.698, Dra 280, Nevrokopi; 281.962, Dra 289, NO Potamia; 281.989, Dra 291, W Prosotsani; 282.204, Dra 300, SO Lefkogia; 283.641, Pel 367, Vorino

\section{Convolvulus arvensis}

276.529, loa 1059, Ag. Nikolaos; 276.681, loa 1065, NO Karyes; 277.067 , loa 1080, SO Anilio; 277.109, loa 1084, N Metsovo; 277.447, loa 1095, S Anilio; 277.667, Tri 459, N 
Chaliki; 277.883, Koz 388, SO Siatista, Vourinos; 278.114, Gre 492, NO Varis; 278.268 , Koz 379, Galatini; 278.382, Koz 382, Galatini; 278.437, Thes 214, Paralia Vrasna; 278.596, Thes 220, Arethousa; 278.645, Kav 106, Paralia Ofryniou; 278.930, Kav 117, Galipsos; 279.176, Ser 256, Paleokomi; 279.249, Ser 258, Mikro Souli; 279.641, Ser 263, Dravoskas; 279.835, Dra 207, NO Skopia; 279.913, Dra 210, Livadero; 280.104, Dra 220, Sidironero; 280.211, Dra 224, NO Drama; 280.421, Dra 233, Sidironero; 280.447, Dra 234, Skaloti; 281.016, Dra 257, Granitis; 281.197, Dra 264, NO Ochyro; 281.278, Dra 266, W Nevrokopi; 281.530, Dra 276, Perithori; 281.737, Dra 281, OSO Granitis; 281.985, Dra 291, W Prosotsani; 282.118, Dra 296, NO Nevrokopi; 282.267, Ser 272, Ano Vrondous; 282.457, Ser 280, Orini; 282.616, Ser 288, Ori Vrondous; 282.855, Kil 294, NW Griva; 283.168, Pel 343, Kerasia; 283.469, Pel 358, N Loutraki; 283.600, Pel 364, Loutraki; 283.668, Pel 368, OSO Vorino

Convolvulus boissieri subsp. parnassicus

278.027, Koz 376, W Exarcho; 278.139, Gre 492, NO Varis

\section{Convolvulus cantabrica}

276.586, loa 1060, SSO Potamia; 277.358, loa 1093, S Anilio; 278.086, Gre 492, NO Varis; 278.943, Kav 118, Galipsos; 279.424, Kav 94, Pangaion; 280.101, Dra 220, Sidironero; 280.126, Dra 221, NO Drama; 280.356, Dra 231, Sidironero; 281.145, Dra 262, NW Granitis; 281.230, Dra 265, NO Granitis; 284.124, loa 1094 b, S Anilio

\section{Convolvulus elegantissimus}

276.535, loa 1059, Ag. Nikolaos; 278.517, Thes 217, Vrasna; 278.694, Kav 107, Paralia Ofryniou; 278.757, Kav 109, Loutra Eleftherion; 278.899, Kav 116, S Ofrynia; 279.066, Kav 121, Platanotopos; 279.143, Ser 255, S Nea Mesolakkia; 279.222, Ser 257, Nea Fili; 279.255, Ser 258, Mikro Souli

\section{Cornus sanguinea subsp. australis}

276.655, loa 1063, W Potamia; 279.079, Kav 89, Misoropi; 280.038, Dra 216, Sidironero; 281.392, Dra 272, Vathytopo; 281.604, Dra 278, Kato Vrondous; 281.949, Dra 289, NO Potamia

\section{Coronilla coronata}

276.385, loa 1056, NO Metsovo

\section{Coronilla scorpioides}

276.949, loa 1076, ONO Anilio; 277.362, loa 1093, S Anilio; 277.983, Koz 375, O Dafnero

\section{Corylus avellana}

279.966, Dra 213, Livadero; 280.041, Dra 216, Sidironero; 280.313, Dra 229, SO Dendrakia; 281.619, Dra 278, Kato Vrondous; 282.029, Dra 292, Nevrokopi; 282.340, Ser 274, SSW Ano Vrondous; 282.352, Ser 275, SSW Ano Vrondous; 282.522, Ser 283, Ori Vrondous

\section{Crataegus monogyna}

283.530, Pel 361, SW Promachi

\section{Crepis conyzifolia}

280.797, Dra 247, Walddorf Elatias

\section{Crepis dioscoridis}

276.311, loa 1125, NO Dodoni; 276.604, loa 1061, SSO Potamia; 276.730, loa 1066, NO Karyes

\section{Crepis foetida subsp. foetida}

279.057, Kav 121, Platanotopos

\section{Crepis foetida subsp. rhoeadifolia}

276.516, loa 1059, Ag. Nikolaos; 277.374, loa 1093, S Anilio; 278.145, Gre 492, NO Varis; 278.439, Thes 214, Paralia Vrasna; 278.531, Thes 218, Vrasna; 278.704, Kav 107, Paralia 
Ofryniou; 278.753, Kav 109, Loutra Eleftherion; 278.805, Kav 113, NO Paralia Myrtofytou; 278.847, Kav 115, SW Folia; 278.908, Kav 116, S Ofrynia; 279.147, Ser 255, S Nea Mesolakkia; 279.219, Ser 257, Nea Fili; 279.250, Ser 258, Mikro Souli; 279.310, Ser 259, Proti; 279.403, Kav 92, Pangaion; 279.625, Ser 263, Dravoskas; 279.736, Ser 267, SW Skopia; 279.800, Ser 269, NO Skopia; 279.812, Ser 269, NO Skopia; 279.856, Dra 209, Livadero; 280.003, Dra 215, Livadero; 280.166, Dra 221, NO Drama; 280.495, Dra 235, Skaloti; 281.005, Dra 257, Granitis; 281.041, Dra 258, Granitis; 281.245, Dra 265, NO Granitis; 281.350, Dra 270, W Nevrokopi; 281.431, Dra 274, Vathytopo; 281.744, Dra 281, OSO Granitis; 281.869, Dra 286, SW Mikroklisoura; 281.887, Dra 287, O Mikroklisoura; 282.164, Dra 298, SO Lefkogia; 282.402, Ser 278, N Serres; 282.796, Ser 296, NO Strymoniko; 283.231, Pel 345, Kerasia; 283.398, Pel 354, NW Loutraki; 283.593, Pel 364, Loutraki; 283.728, Pel 370, SW Foustani; 283.790, Ima 173, S Ag. Pavlos; 283.875, Ima 179, Seli

\section{Crepis neglecta subsp. neglecta}

276.338, loa 1126, NO Dodoni; 276.502, loa 1059, Ag. Nikolaos; 277.379, loa 1093, S Anilio; 277.767, Tri 462, N Chaliki; 277.823, Koz 387, SO Siatista, Vourinos; 278.075, Gre 492, NO Varis; 278.190, Gre 495, Varis; 278.270, Koz 379, Galatini; 278.334, Koz 381, Galatini

\section{Crepis pulchra}

278.096, Gre 492, NO Varis

\section{Crepis rubra}

276.609, loa 1062, SO Potamia

\section{Crepis sancta}

276.955, loa 1076, ONO Anilio; 276.975, loa 1079, SO Anilio; 277.118, loa 1084, N Metsovo; 277.428, loa 1095, S Anilio; 277.773, Tri 462, N Chaliki

\section{Crepis setosa}

276.560, loa 1060, SSO Potamia; 276.898, loa 1073, NNW Metsovo; 277.910, Koz 390, SO Siatista, Vourinos; 278.278, Koz 379, Galatini; 278.385, Koz 382, Galatini; 278.473, Thes 215, Paralia Vrasna; 278.597, Thes 220, Arethousa; 278.808, Kav 114, SO Folia; 278.830, Kav 114, SO Folia; 280.098, Dra 220, Sidironero; 281.108, Dra 261, Granitis; 281.551, Dra 277, Perithori; 282.839, Ser 298, Parapotamos; 283.352, Pel 352, Kajmaktsalan

\section{Crepis vesicaria subsp. taraxacifolia}

280.441, Dra 234, Skaloti

\section{Crucianella angustifolia}

277.764, Tri 462, N Chaliki; 281.217, Dra 265, NO Granitis

\section{Crucianella graeca}

278.003, Koz 375, O Dafnero; 279.784, Ser 268, SW Skopia

\section{Crucianella latifolia}

278.546, Thes 219, Vrasna; 278.789, Kav 112, NO Paralia Myrtofytou; 279.018, Kav 119, Podochori

\section{Cruciata laevipes}

276.378, loa 1128, N Metsovo; 276.797, loa 1070, NNW Metsovo; 277.053, loa 1080, SO Anilio; 277.105, loa 1084, N Metsovo; 277.267, loa 1090, NNO Metsovo; 277.947, Koz 392, SO Siatista, Vourinos; 280.443, Dra 234, Skaloti; 280.711, Dra 243, SW Walddorf Elatias; 280.867, Dra 249, Falakron; 282.540, Ser 284, Ori Vrondous; 283.016, Kil 302, Kastaneri; 283.291, Pel 349, Kajmaktsalan; 283.913, Ima 180, Seli; -20.505, Dra 236, Skaloti

\section{Cruciata pedemontana}

276.801, loa 1070, NNW Metsovo; 277.008, loa 1080, SO Anilio; 277.151, loa 1085, N Metsovo; 277.690, Tri 460, N Chaliki; 277.919, Koz 391, SO Siatista, Vourinos; 278.289 , Koz 380, Galatini; -20.495, loa 1083, N Metsovo 


\section{Cruciata verna}

282.560, Ser 285, Ori Vrondous

\section{Crupina crupinastrum}

276.726, loa 1066, NO Karyes; 277.086, loa 1081, W Metsovo; 277.657, loa 1106, Anilio; 283.475, Pel 358, N Loutraki

\section{Crupina vulgaris}

276.568, loa 1060, SSO Potamia; 277.339, loa 1093, S Anilio; 277.692, Tri 460, N Chaliki; 277.982, Koz 375, O Dafnero; 278.049, Gre 491, NO Exarcho; 278.081, Gre 492, NO Varis; 278.237, Koz 378, N Siatista; 278.352, Koz 381, Galatini; 279.429, Kav 94, Pangaion;

280.155, Dra 221, NO Drama; 280.353, Dra 231, Sidironero; 281.132, Dra 262, NW Granitis

\section{Cuscuta approximata}

277.804, Koz 385, SO Siatista

\section{Cuscuta campestris}

279.666, Ser 265, Myrrini

\section{Cuscuta epithymum subsp. kotschyi}

278.094, Gre 492, NO Varis; 283.772, Ima 173, S Ag. Pavlos

\section{Cynanchum acutum}

278.668, Kav 107, Paralia Ofryniou; 278.751, Kav 109, Loutra Eleftherion; 278.883, Kav 116, S Ofrynia; 279.145, Ser 255, S Nea Mesolakkia; 279.637, Ser 263, Dravoskas; 279.664, Ser 265, Myrrini

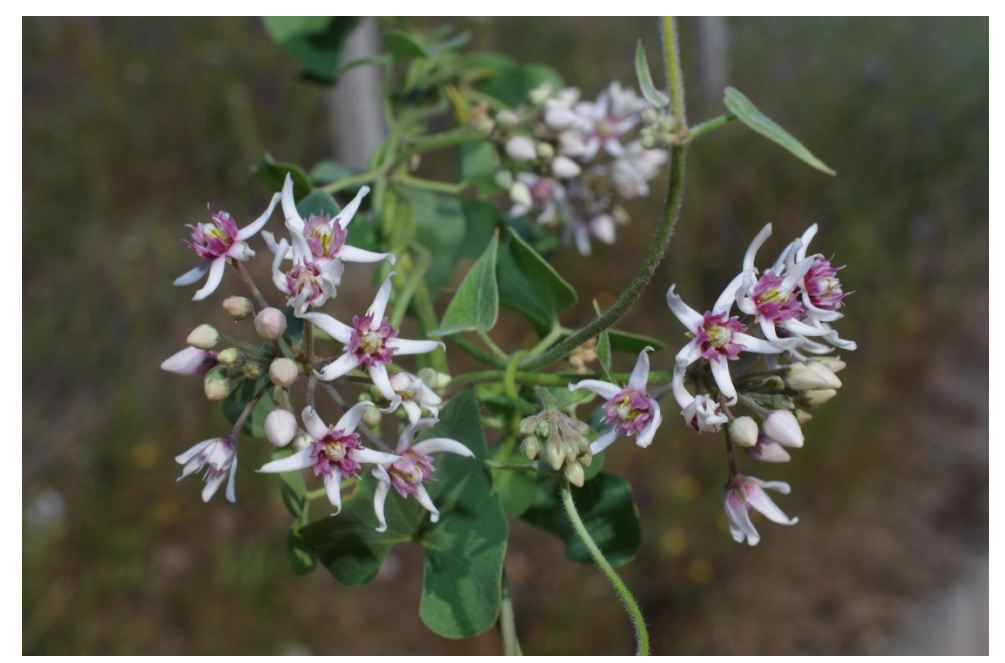

\section{Cynodon dactylon}

278.548, Thes 219, Vrasna; 278.701, Kav 107, Paralia Ofryniou; 278.770, Kav 111, Loutra Eleftherion; 278.979, Kav 119, Podochori; 279.188, Ser 256, Paleokomi; 279.215, Ser 257, Nea Fili; 279.425, Kav 94, Pangaion; 279.678, Ser 265, Myrrini; 280.208, Dra 224, NO Drama; 280.364, Dra 232, Sidironero; 281.008, Dra 257, Granitis; 281.265, Dra 266, W Nevrokopi; 282.392, Ser 277, ONO Serres; 282.833, Ser 298, Parapotamos; 283.591 , Pel 364, Loutraki; 283.638, Pel 367, Vorino

\section{Cynoglossum creticum}

276.400, loa 1056, NO Metsovo; 276.767, loa 1069, NW Metsovo; 276.818, loa 1070, NNW Metsovo; 277.164, loa 1086, NNO Metsovo; 277.540, loa 1098, S Anilio; 278.635, Kav 106, Paralia Ofryniou; 280.203, Dra 224, NO Drama; 280.571, Dra 238, Skaloti; 280.885, Dra 250, Falakron; 284.215, loa 1065, NO Karyes

\section{Cynoglossum montanum}

280.690, Dra 242, SW Walddorf Elatias

\section{Cynoglossum officinale}

277.595, loa 1102, S Anilio; 277.681, Tri 460, N Chaliki 


\section{Cynosurus cristatus}

276.363, loa 1128, N Metsovo; 276.554, loa 1060, SSO Potamia; 276.706, loa 1065, NO Karyes; 276.856, loa 1071, NNW Metsovo; 276.998, loa 1079, SO Anilio; 277.134, loa 1084, N Metsovo; 277.244, loa 1089, NNO Metsovo; 277.300, loa 1091, SW Milia; 277.503, loa 1097, S Anilio; 277.587, loa 1100, S Anilio; 280.266, Dra 228, Livadero; 283.334, Pel 351, Kajmaktsalan; 283.484, Pel 359, NW Loutraki

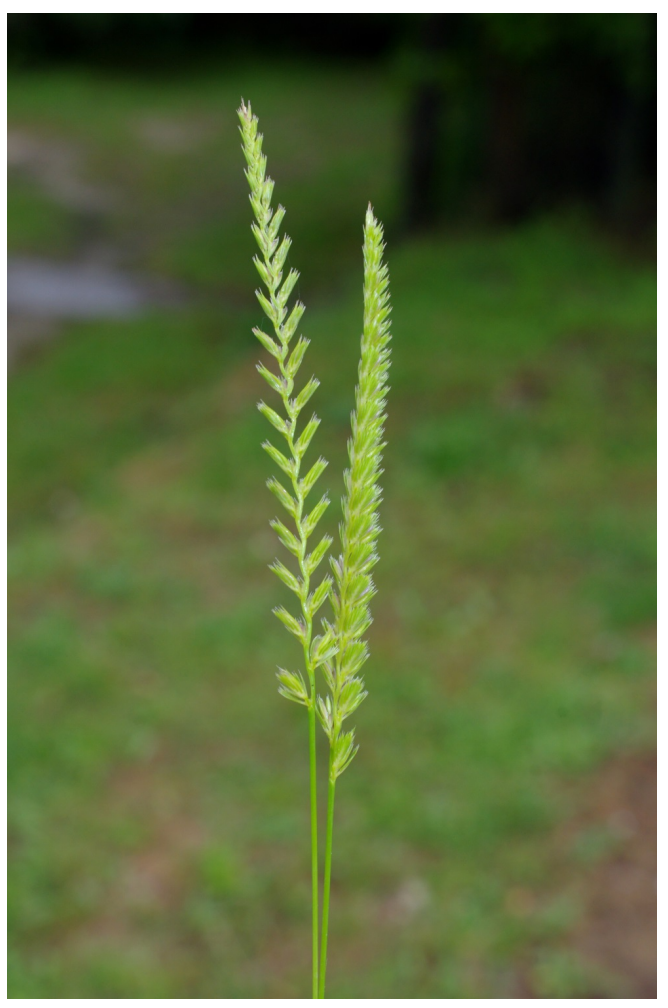

\section{Cynosurus echinatus}

276.472, Tri 458, NO Katara-Pass; 276.595, loa 1061, SSO Potamia; 276.830, loa 1070, NNW Metsovo; 276.873, loa 1072, NNW Metsovo; 277.023, loa 1080, SO Anilio; 277.049, loa 1080, SO Anilio; 277.203, loa 1087, NNO Metsovo; 277.236, loa 1089, NNO Metsovo; 277.357, loa 1093, S Anilio; 277.699, Tri 460, N Chaliki; 277.856, Koz 387, SO Siatista, Vourinos; 278.149, Gre 492, NO Varis; 278.400, Koz 383, Galatini; 279.349, Ser 261, Proti; 279.464, Kav 95, Pangaion; 279.912, Dra 210, Livadero; 280.100, Dra 220, Sidironero; 280.398, Dra 232, Sidironero; 280.922, Dra 252, Falakron; 281.206, Dra 265, NO Granitis; 281.756, Dra 281, OSO Granitis; -20.469, loa 1053, NO Dodoni

\section{Cynosurus effusus}

276.705, loa 1065, NO Karyes; 282.537, Ser 284, Ori Vrondous

\section{Cyperus esculentus}

278.590, Thes 220, Arethousa; 279.126, Kav 91, Orfani

\section{Cyperus longus}

278.438, Thes 214, Paralia Vrasna; 278.556, Thes 219, Vrasna; 278.599, Thes 220, Arethousa; 278.633, Kav 106, Paralia Ofryniou; 278.826, Kav 114, SO Folia; 278.928, Kav 117, Galipsos; 279.119, Kav 91, Orfani; 279.836, Dra 207, NO Skopia; 282.841, Ser 298, Parapotamos

\section{Cyperus rotundus}

278.981, Kav 119, Podochori; 279.114, Kav 90, SW Moustheni; 279.128, Kav 91, Orfani; 279.268, Ser 258, Mikro Souli; 283.716, Pel 370, SW Foustani

\section{Cytisus scoparius}

276.386, loa 1056, NO Metsovo 


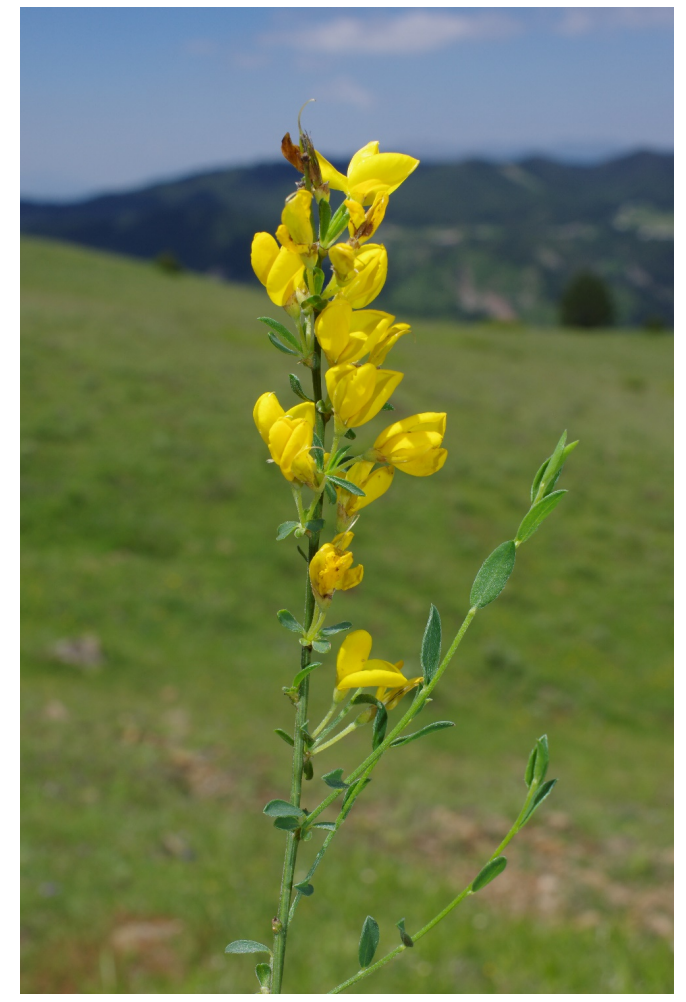

\section{Cytisus villosus}

280.059, Dra 217, Sidironero

\section{Dactylis glomerata subsp. glomerata}

280.991, Dra 257, Granitis; 282.252, Dra 301, Ano Vrondous; 282.339 , Ser 274, SSW Ano

Vrondous; 282.945, Kil 298, WNW Kastaneri; -20.524, Ser 286, SW Mikroklisoura

\section{Dactylis glomerata subsp. hispanica}

276.478, loa 1059, Ag. Nikolaos; 276.521, loa 1059, Ag. Nikolaos; 276.700, loa 1065, NO Karyes; 277.011, loa 1080, SO Anilio; 277.145, loa 1084, N Metsovo; 277.337, loa 1093, S Anilio; 277.938, Koz 392, SO Siatista, Vourinos; 278.549, Thes 219, Vrasna; 279.356, Ser 261, Proti; 279.434, Kav 94, Pangaion; 279.443, Kav 95, Pangaion; 279.575, Kav 103, Pangaion; 279.898, Dra 209, Livadero; 280.375, Dra 232, Sidironero; 280.458, Dra 234, Skaloti; 280.669, Dra 242, SW Walddorf Elatias; 280.783, Dra 247, Walddorf Elatias; 281.632, Dra 279, Kato Vrondous; 282.021, Dra 292, Nevrokopi; 282.170, Dra 299, SO Lefkogia; 282.347, Ser 275, SSW Ano Vrondous; 282.406, Ser 278, N Serres; 282.445, Ser 280, Orini; 282.655, Ser 290, N Neo Petritsi; 282.899, Kil 296, N Kastaneri; 283.255, Pel 346, Kajmaktsalan; 283.483, Pel 359, NW Loutraki

\section{Dactylorhiza cordigera}

280.765, Dra 246, SW Walddorf Elatias

\section{Dactylorhiza iberica}

278.036, Gre 491, NO Exarcho 


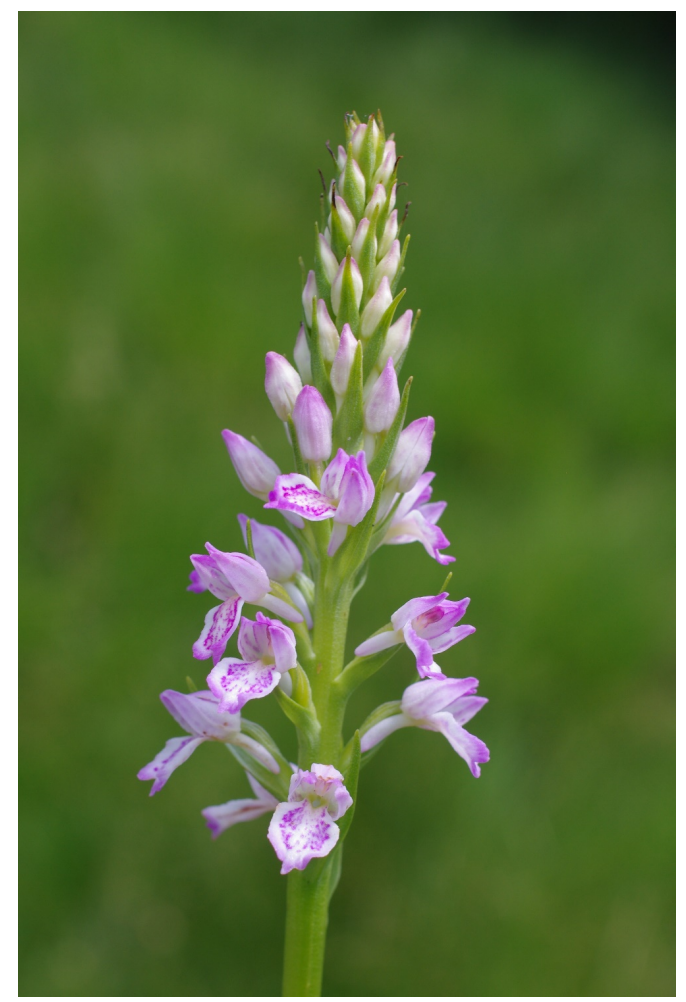

Dactylorhiza kalopissii

276.770, loa 1069, NW Metsovo

\section{Dactylorhiza saccifera}

277.279, loa 1090, NNO Metsovo; 277.487, loa 1097, S Anilio; 280.550, Dra 237, Skaloti; 282.911, Kil 297, WNW Kastaneri; 283.045, Kil 304, SO Livadia; 283.293, Pel 349,

Kajmaktsalan

\section{Dactylorhiza sambucina}

-20.479 , loa 1056, NO Metsovo

\section{Daphne oleoides}

276.390, loa 1056, NO Metsovo; 276.965, loa 1078, O Anilio; 277.033, loa 1080, SO Anilio; 277.602, loa 1102, S Anilio; 277.765, Tri 462, N Chaliki; 283.882, Ima 179, Seli

\section{Dasypyrum villosum}

277.830, Koz 387, SO Siatista, Vourinos; 278.110, Gre 492, NO Varis; 278.297, Koz 380, Galatini; 278.331, Koz 381, Galatini; 278.625, Thes 221, Arethousa; 278.973, Kav 119, Podochori; 279.063, Kav 121, Platanotopos; 279.204, Ser 257, Nea Fili; 279.235, Ser 258, Mikro Souli; 279.279, Ser 258, Mikro Souli; 279.297, Ser 259, Proti; 279.420, Kav 93, Pangaion; 279.448, Kav 95, Pangaion; 279.642, Ser 263, Dravoskas; 279.692, Ser 266, SO Nea Zichni; 279.807, Ser 269, NO Skopia; 280.146, Dra 221, NO Drama; 281.413, Dra 273, Vathytopo; 281.728, Dra 281, OSO Granitis; 282.411, Ser 279, Orini; 283.105, Pel 339, S Archangelo; -20.456, loa 1052, NO Dodoni

\section{Datura stramonium}

281.412, Dra 273, Vathytopo; 281.544, Dra 276, Perithori; 282.809, Ser 296, NO Strymoniko; 283.642, Pel 367, Vorino

\section{Daucus carota}

278.849, Kav 115, SW Folia; 278.892, Kav 116, S Ofrynia; 279.031, Kav 120, Podochori; 279.084, Kav 89, Misoropi; 279.158, Ser 255, S Nea Mesolakkia; 279.657, Ser 265, Myrrini; 280.226, Dra 225, NO Drama; 280.986, Dra 257, Granitis; 281.331, Dra 269, W Nevrokopi; 281.535, Dra 276, Perithori; 281.996, Dra 291, W Prosotsani; 282.791, Ser 295, N Neo Petritsi; 282.806, Ser 296, NO Strymoniko; 283.153, Pel 342, S Archangelo; 283.197, Pel 343, Kerasia; 283.390, Pel 354, NW Loutraki; 283.655, Pel 367, Vorino 
Daucus guttatus subsp. guttatus

278.448, Thes 214, Paralia Vrasna; 278.708, Kav 107, Paralia Ofryniou; 279.626, Ser 263, Dravoskas; 280.189, Dra 223, NO Drama; 281.980, Dra 290, NO Potamia; 282.398, Ser 278, N Serres; 283.571, Pel 363, NW Promachi

\section{Delphinium balcanicum}

278.458, Thes 214, Paralia Vrasna; 278.622, Thes 221, Arethousa; 282.780, Ser 293, N Neo

Petritsi; 284.132, ioa 1095 b, S Anilio

Delphinium fissum

280.921, Dra 252, Falakron

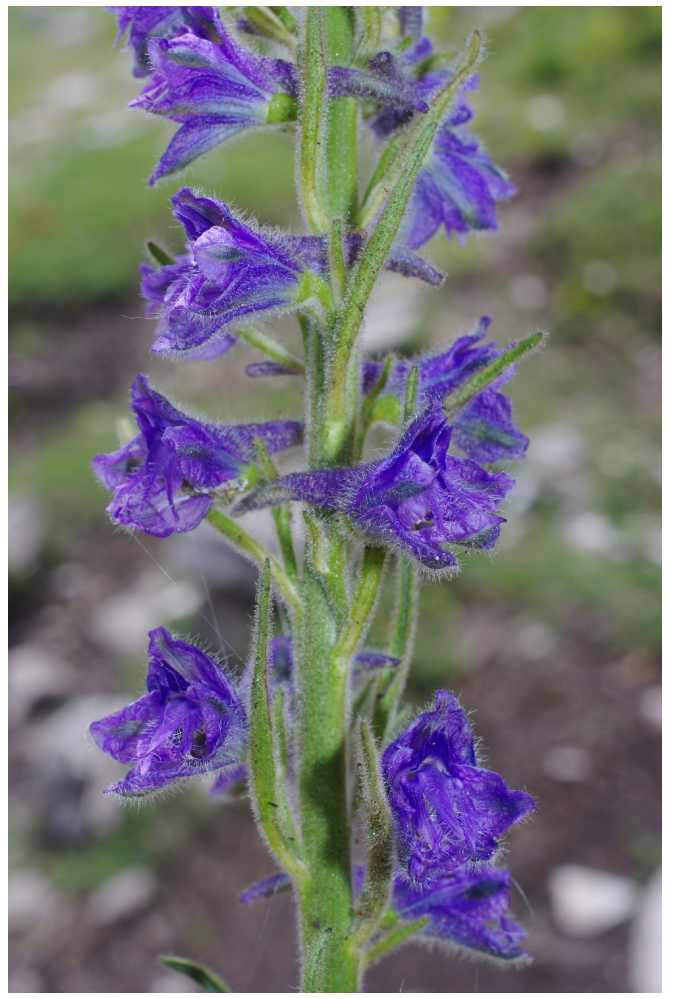

Deschampsia cespitosa

283.247, Pel 346, Kajmaktsalan

\section{Dianthus armeria}

281.100, Dra 261, Granitis; 282.095, Dra 295, NO Nevrokopi; 283.211, Pel 344, Kerasia

\section{Dianthus cruentus}

277.597, loa 1102, S Anilio; 279.903, Dra 210, Livadero; 280.060, Dra 218, Sidironero; 280.408, Dra 233, Sidironero; 280.448, Dra 234, Skaloti; 281.047, Dra 259, Granitis;

281.655, Ser 270, Kato Vrondous; 282.285, Ser 272, Ano Vrondous

Dianthus deltoides subsp. degenii

278.022, Koz 376, W Exarcho

\section{Dianthus giganteus}

277.567, loa 1099, S Anilio; 278.299, Koz 380, Galatini; 278.335, Koz 381, Galatini; 281.191, Dra 264, NO Ochyro; 283.035, Kil 303, SO Livadia; 283.566, Pel 363, NW Promachi

Dianthus gracilis subsp. drenowskianus

281.764, Dra 281, OSO Granitis

Dianthus gracilis subsp. gracilis

277.263, loa 1090, NNO Metsovo; 277.303, loa 1091, SW Milia; 279.360, Ser 261, Proti; 279.779, Ser 268, SW Skopia; 279.882, Dra 209, Livadero; 280.143, Dra 221, NO Drama; 
280.841, Dra 248, Falakron; 281.243, Dra 265, NO Granitis; 281.297, Dra 267, W Nevrokopi; 281.722, Dra 280, Nevrokopi; 282.382, Ser 276, SSW Ano Vrondous; 282.388, Ser 277 , ONO Serres; 282.609, Ser 288, Ori Vrondous; 282.993, Kil 301, Kastaneri

Dianthus haematocalyx subsp. pindicola

283.992, loa 1112, Katara-Paß; 284.095, loa 1120, Anilio

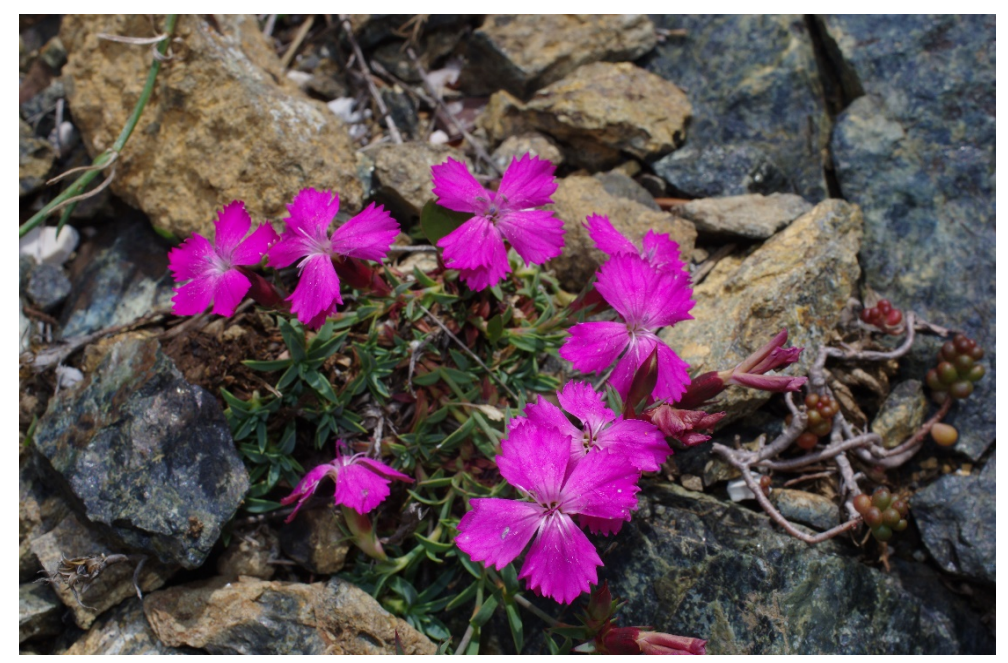

Dianthus monadelphus subsp. pallens

278.967, Kav 119, Podochori; 279.041, Kav 120, Podochori

\section{Dianthus petraeus subsp. orbelicus}

279.577, Kav 103, Pangaion; 279.608, Kav 105, Pangaion; 280.862, Dra 249, Falakron

\section{Dianthus pinifolius}

277.797, Koz 385, SO Siatista; 278.063, Gre 492, NO Varis; 278.236, Koz 378, N Siatista; 281.341, Dra 270, W Nevrokopi; 281.658, Ser 270, Kato Vrondous; 282.111, Dra 296, NO Nevrokopi; 282.450 , Ser 280, Orini

\section{Dianthus strymonis}

280.217, Dra 225, NO Drama; 281.550, Dra 277, Perithori; 282.067, Dra 294, NO Nevrokopi; 284.169, loa 1124,

\section{Dianthus sylvestris}

276.940, loa 1075, NW Anilio; 277.355, loa 1093, S Anilio; 283.956, loa 1110, N Metsovo; 284.051, loa 1117, Anilio; 284.117, loa 1121, Anilio; 284.166, loa 1124, ; 284.186, loa 1110, N Metsovo

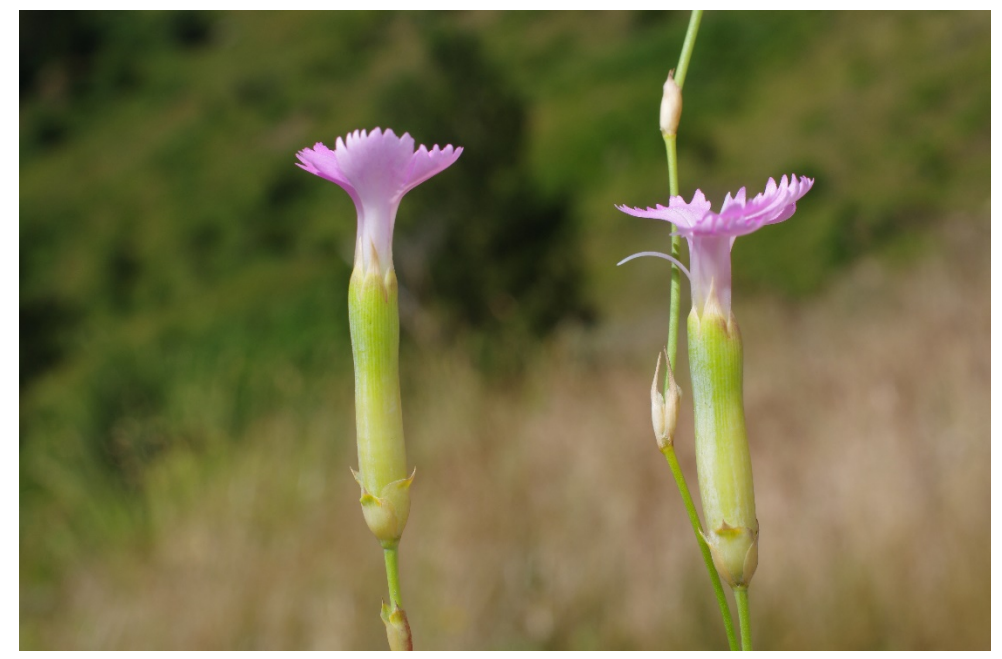

\section{Dianthus tenuiflorus}

277.304, loa 1091, SW Milia; 277.611, loa 1103, S Anilio; 279.433, Kav 94, Pangaion; 280.632, Dra 240, Elatias; 283.330, Pel 351, Kajmaktsalan 


\section{Dianthus viscidus}

276.329, loa 1125, NO Dodoni; 276.611, loa 1062, SO Potamia; 276.789, loa 1070, NNW Metsovo; 276.796, loa 1070, NNW Metsovo; 277.046, loa 1080, SO Anilio; 277.559, loa 1099, S Anilio; 277.911, Koz 390, SO Siatista, Vourinos; 278.048, Gre 491, NO Exarcho; 278.129, Gre 493, NO Varis; 278.538, Thes 218, Vrasna; 279.438, Kav 95, Pangaion; 281.489, Dra 275, Vathytopo; 281.579, Dra 277, Perithori; 281.639, Dra 279, Kato Vrondous; 281.917, Dra 287, O Mikroklisoura; 281.918, Dra 287, O Mikroklisoura; 282.082, Dra 295, NO Nevrokopi; 283.466, Pel 358, N Loutraki; 283.490, Pel 359, NW Loutraki

\section{Digitalis ferruginea}

283.164, Pel 343, Kerasia; 283.777, Ima 173, S Ag. Pavlos; 283.861, Ima 178, Seli; 283.904, Ima 180, Seli; 283.954, loa 1110, N Metsovo; 284.039, loa 1116, SW Milia

\section{Digitalis grandiflora}

276.957, loa 1077, S Anilio; 277.539, loa 1097, S Anilio; 283.187, Pel 343, Kerasia

\section{Digitalis laevigata subsp. graeca}

283.786, Ima 173, S Ag. Pavlos; 284.116, loa 1121, Anilio

\section{Digitalis lanata}

276.686, loa 1065, NO Karyes; 278.865, Kav 115, SW Folia; 279.139, Ser 255, S Nea Mesolakkia; 279.457, Kav 95, Pangaion; 279.499, Kav 98, Pangaion; 279.732, Ser 267, SW Skopia; 279.799, Ser 269, NO Skopia; 279.887, Dra 209, Livadero; 280.009, Dra 216, Sidironero; 280.172, Dra 222, NO Drama; 280.285, Dra 228, Livadero; 280.387, Dra 232, Sidironero; 280.843, Dra 248, Falakron; 281.603, Dra 278, Kato Vrondous; 281.716 , Dra 280, Nevrokopi; 281.856, Dra 285, NNO Volakas; 282.033, Dra 292, Nevrokopi; 282.199, Dra 299, SO Lefkogia; 282.331, Ser 274, SSW Ano Vrondous; 282.449, Ser 280, Orini; 282.721, Ser 290, N Neo Petritsi

\section{Digitalis viridiflora}

277.583, loa 1100, S Anilio; 279.549, Kav 101, Pangaion; 279.933, Dra 211, Livadero; 280.076, Dra 219, Sidironero; 280.551, Dra 237, Skaloti; 280.683, Dra 242, SW Walddorf Elatias; 280.798, Dra 247, Walddorf Elatias; 280.973, Dra 256, Falakron; 281.116, Dra 261, Granitis; 281.468, Dra 275, Vathytopo; 281.650, Dra 279, Kato Vrondous; 282.275, Ser 272, Ano Vrondous; 282.488, Ser 282, Ori Vrondous; 282.660, Ser 290, N Neo Petritsi; 282.995 , Kil 301, Kastaneri; -20.521, Ser 285, NNO Volakas

\section{Digitaria sanguinalis}

279.184, Ser 256, Paleokomi; 281.410, Dra 273, Vathytopo; 283.588, Pel 364, Loutraki

\section{Dipsacus laciniatus}

283.770, Ima 172, S Ag. Pavlos

\section{Doronicum austriacum}

283.320, Pel 351, Kajmaktsalan 


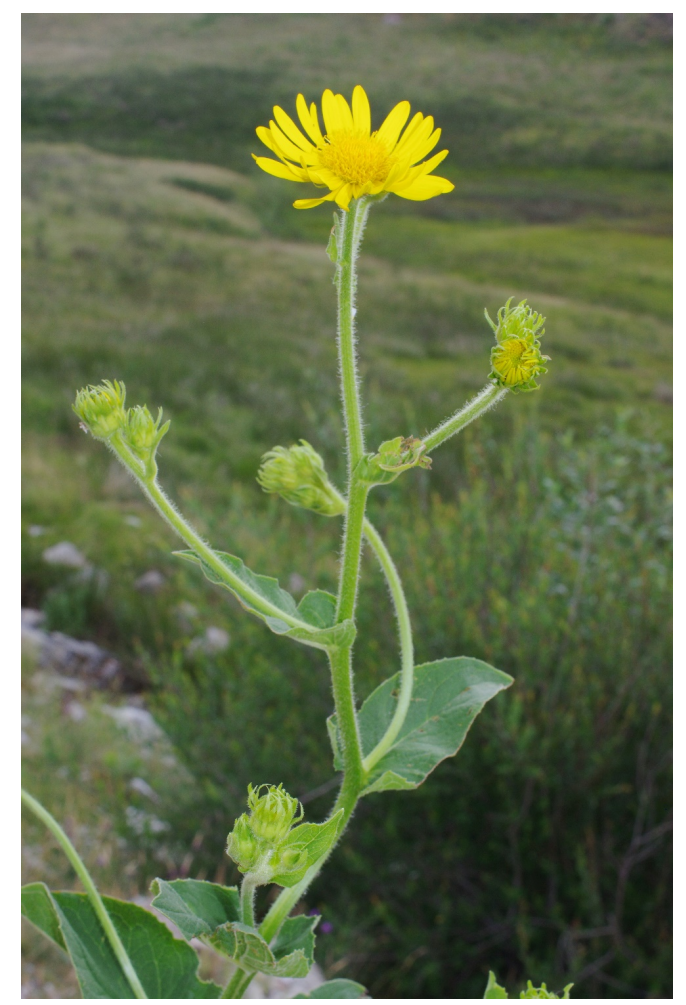

Doronicum hungaricum

282.595, Ser 287, Ori Vrondous

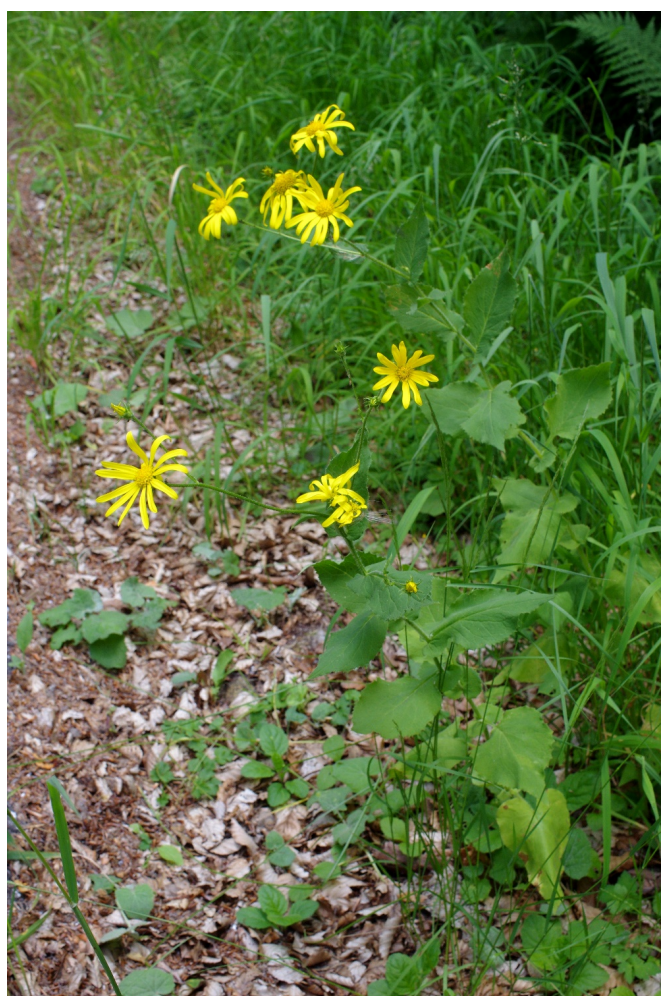

\section{Doronicum columnae}

276.414, loa 1057, NO Metsovo

\section{Dorycnium germanicum}

278.080, Gre 492, NO Varis

\section{Dorycnium herbaceum}

276.290, loa 1125, NO Dodoni; 276.571, loa 1060, SSO Potamia; 276.663, loa 1064, ONO Karyes; 276.707 , loa 1065, NO Karyes; 276.903, loa 1073, NNW Metsovo; 277.213, loa 1088, NNO Metsovo; 277.284, loa 1090, NNO Metsovo; 277.345, loa 1093, S Anilio; 
277.666, Tri 459, N Chaliki; 277.844, Koz 387, SO Siatista, Vourinos; 278.852, Kav 115, SW Folia; 279.365, Ser 261, Proti; 279.445, Kav 95, Pangaion; 279.764, Ser 268, SW Skopia; 279.863, Dra 209, Livadero; 280.021, Dra 216, Sidironero; 280.181, Dra 223, NO Drama; 280.207, Dra 224, NO Drama; 280.259, Dra 227, Livadero; 280.374, Dra 232, Sidironero; 280.942, Dra 254, Falakron; 281.234, Dra 265, NO Granitis; 281.287, Dra 267, W Nevrokopi; 281.571, Dra 277, Perithori; 281.714, Dra 280, Nevrokopi; 281.736, Dra 281, OSO Granitis; 281.800, Dra 282, NNO Volakas; 281.882, Dra 287, O Mikroklisoura; 282.004, Dra 292, Nevrokopi; 282.864, Kil 295, NW Griva; 283.068, Kil 307, NW Livadia; 283.133, Pel 341, S Archangelo; 283.189, Pel 343, Kerasia; 283.415, Pel 355, NW Loutraki; 283.753, Ima 172, S Ag. Pavlos; 283.863, Ima 178, Seli; 284.020, loa 1115, N Metsovo

\section{Dorycnium hirsutum}

276.296, loa 1125, NO Dodoni; 276.558, loa 1060, SSO Potamia; 277.376, loa 1093, S Anilio; 280.210, Dra 224, NO Drama; 283.413, Pel 355, NW Loutraki

\section{Draba verna}

277.063, loa 1080 , SO Anilio

\section{Dryopteris}

277.585, loa 1100 , S Anilio

\section{Dryopteris filix-mas}

276.766, loa 1069, NW Metsovo; 277.194, loa 1087, NNO Metsovo; 277.492, loa 1097, S Anilio; 277.594, loa 1101, S Anilio; 280.589, Dra 238, Skaloti; 280.733, Dra 244, SW Walddorf Elatias; 284.147, loa 1096 b, S Anilio

\section{Dysphania botrys}

278.454, Thes 214, Paralia Vrasna; 278.522, Thes 217, Vrasna; 278.682, Kav 107, Paralia Ofryniou; 278.971, Kav 119, Podochori; 279.483, Kav 97, Pangaion; 280.190, Dra 223, NO Drama; 280.341, Dra 230, Livadero; 283.473, Pel 358, N Loutraki; 283.531, Pel 361, SW Promachi

\section{Ecballium elaterium}

279.182, Ser 256, Paleokomi

\section{Echinochloa crus-galli}

278.582, Thes 220, Arethousa; 279.122, Kav 91, Orfani; 279.183, Ser 256, Paleokomi; 281.107, Dra 261, Granitis; 281.188, Dra 264, NO Ochyro; 281.924, Dra 288, SW Potamia; 282.821, Ser 296, NO Strymoniko; 282.831, Ser 298, Parapotamos; 282.976, Kil 299, Kastaneri; 283.533, Pel 361, SW Promachi; 283.663, Pel 368, OSO Vorino

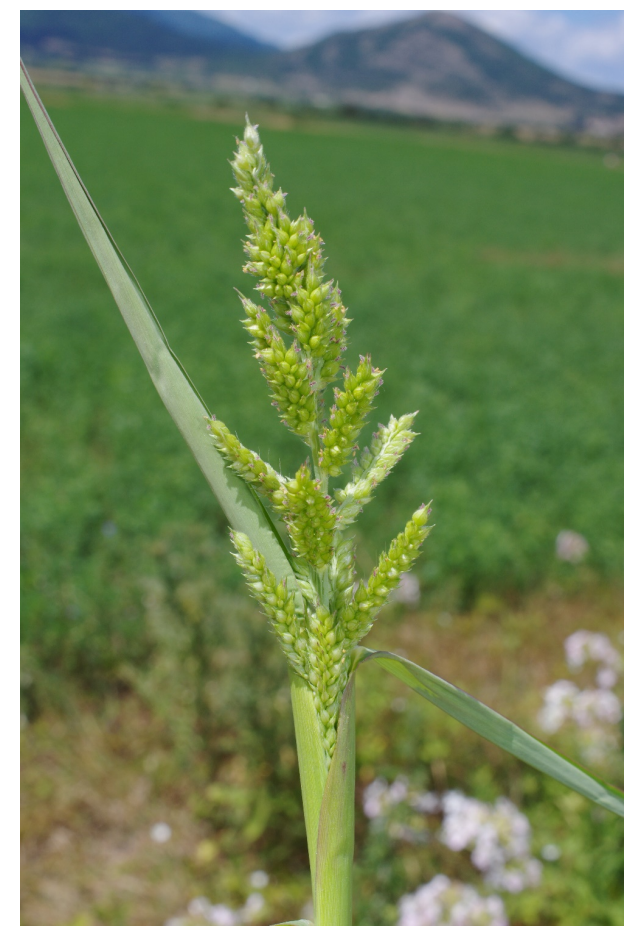


Echinops ritro subsp. ritro

283.959, loa 1110, N Metsovo

Echinops ritro subsp. ruthenicus

284.112, loa 1121, Anilio

\section{Echinops sphaerocephalus}

281.299, Dra 267, W Nevrokopi; 282.782, Ser 294, N Neo Petritsi; 282.785, Ser 294, N Neo Petritsi; 283.618, Pel 365, N Vorino

Echium italicum subsp. italicum

277.075, loa 1081, W Metsovo; 278.567, Thes 220, Arethousa; 280.191, Dra 223, NO

Drama; 283.517, Pel 360, SW Promachi; 283.626, Pel 366, N Vorino

\section{Echium plantagineum}

278.451, Thes 214, Paralia Vrasna; 278.779, Kav 112, NO Paralia Myrtofytou; 278.824, Kav 114, SO Folia

\section{Echium vulgare}

277.175, loa 1086, NNO Metsovo; 278.157, Gre 494, SO Varis; 279.967, Dra 213, Livadero; 280.015, Dra 216, Sidironero; 280.068, Dra 218, Sidironero; 280.323, Dra 230, Livadero; 280.401, Dra 233, Sidironero; 280.433, Dra 234, Skaloti; 280.679, Dra 242, SW Walddorf Elatias; 280.793, Dra 247, Walddorf Elatias; 280.993, Dra 257, Granitis; 281.176, Dra 264, NO Ochyro; 281.660, Ser 270, Kato Vrondous; 281.754, Dra 281, OSO Granitis; 281.822 , Dra 283, NNO Volakas; 282.012, Dra 292, Nevrokopi; 282.151, Dra 297, Lefkogia; 282.187, Dra 299, SO Lefkogia; 282.280, Ser 272, Ano Vrondous; 282.334 , Ser 274, SSW Ano Vrondous; 282.478, Ser 281, Ori Vrondous; 282.734, Ser 291, N Neo Petritsi; 283.074, Kil 307, NW Livadia; 283.685, Pel 369, O Vorino

\section{Eleocharis palustris}

277.065, loa 1080, SO Anilio; 278.054, Gre 491, NO Exarcho; 278.055, Gre 491, NO

Exarcho; 284.214, loa 1073, NNW Metsovo

\section{Eleocharis uniglumis}

276.915, loa 1073, NNW Metsovo; 277.138, loa 1084, N Metsovo; 277.624, loa 1104, S Anilio

\section{Eleusine indica}

279.135, Kav 91, Orfani; 283.586, Pel 364, Loutraki

\section{Elymus caninus}

282.943, Kil 298, WNW Kastaneri

\section{Elymus panormitanus}

278.716, Kav 108, Paralia Ofryniou; 282.356, Ser 276, SSW Ano Vrondous

\section{Elytrigia elongata}

282.010, Dra 292, Nevrokopi

\section{Elytrigia obtusiflora}

277.821, Koz 387, SO Siatista, Vourinos; 281.284, Dra 267, W Nevrokopi

\section{Elytrigia repens}

276.938, loa 1075, NW Anilio; 279.978, Dra 214, Livadero; 280.557, Dra 237, Skaloti; 281.105, Dra 261, Granitis; 281.526, Dra 276, Perithori; 281.553, Dra 277, Perithori; 281.699, Dra 280, Nevrokopi; 282.069, Dra 294, NO Nevrokopi; 282.553, Ser 285, Ori Vrondous; 282.881, Kil 295, NW Griva; 282.963, Kil 299, Kastaneri; 283.101, Pel 339, S Archangelo; 283.895, Ima 179, Seli 


\section{Epilobium}

282.972, Kil 299, Kastaneri

\section{Epilobium angustifolium}

279.594, Kav 104, Pangaion; 279.957, Dra 212, Livadero; 281.397, Dra 272, Vathytopo; 281.894, Dra 287, O Mikroklisoura; 282.060, Dra 294, NO Nevrokopi; 282.266, Ser 272, Ano Vrondous; 282.466, Ser 281, Ori Vrondous; 282.611, Ser 288, Ori Vrondous; 282.690, Ser 290, N Neo Petritsi; 282.928, Kil 297, WNW Kastaneri; 283.119, Pel 340, SSO Archangelo; 283.209, Pel 344, Kerasia; 283.910, Ima 180, Seli

\section{Epilobium collinum}

276.961, loa 1078, O Anilio; 277.260, loa 1090, NNO Metsovo; 277.319, loa 1092, NO Metsovo; 279.390, Ser 262, Proti; 279.512, Kav 99, Pangaion; 280.508, Dra 236, Skaloti; 280.604, Dra 238, Skaloti; 281.400, Dra 272, Vathytopo; 281.472, Dra 275, Vathytopo; 282.726, Ser 291, N Neo Petritsi; 282.930, Kil 297, WNW Kastaneri; 282.948, Kil 298, WNW Kastaneri; 283.059, Kil 306, SO Livadia; 283.763, Ima 172, S Ag. Pavlos; 284.149, loa 1122, Anilio

\section{Epilobium dodonaei}

283.256, Pel 347, Kajmaktsalan

\section{Epilobium hirsutum}

281.326, Dra 269, W Nevrokopi; 281.402, Dra 272, Vathytopo; 282.147, Dra 297, Lefkogia; 282.286, Ser 272, Ano Vrondous; 282.902, Kil 296, N Kastaneri; 283.054, Kil 305, SO Livadia; 283.562, Pel 362, NW Promachi; 283.738, Ima 172, S Ag. Pavlos; 284.052, loa 1117, Anilio

\section{Epilobium lanceolatum}

277.542, loa 1098, S Anilio; 279.504, Kav 98, Pangaion; 279.576, Kav 103, Pangaion; 279.949, Dra 212, Livadero; 280.600, Dra 238, Skaloti; 283.563, Pel 362, NW Promachi; 284.008, loa 1113, Katara-Paß

\section{Epilobium montanum}

276.780, loa 1069, NW Metsovo; 277.202, loa 1087, NNO Metsovo; 277.226, loa 1089, NNO Metsovo; 277.315, loa 1092, NO Metsovo; 277.581, loa 1100, S Anilio; 277.591, loa 1101, S Anilio; 279.092, Kav 89, Misoropi; 279.563, Kav 102, Pangaion; 279.941, Dra 212 , Livadero; 280.037, Dra 216, Sidironero; 280.333, Dra 230, Livadero; 280.652, Dra 242, SW Walddorf Elatias; 280.731, Dra 244, SW Walddorf Elatias; 280.732, Dra 244, SW Walddorf Elatias; 280.876, Dra 249, Falakron; 281.122, Dra 261, Granitis; 282.245, Dra 301, Ano Vrondous; 282.497, Ser 282, Ori Vrondous; 282.532, Ser 284, Ori Vrondous; 282.576, Ser 286, Ori Vrondous; 282.604, Ser 288, Ori Vrondous; 282.722, Ser 291, N Neo Petritsi; 282.728, Ser 291, N Neo Petritsi; 282.762, Ser 293, N Neo Petritsi; 283.087, Pel 339, S Archangelo; 283.244, Pel 346, Kajmaktsalan; 284.150, loa 1122, Anilio

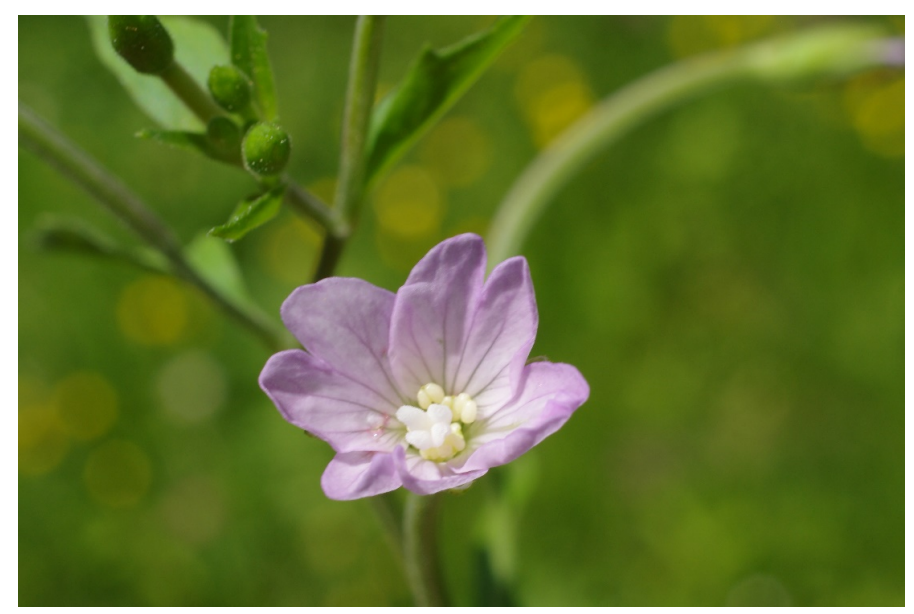




\section{Epilobium parviflorum}

276.631, loa 1063, W Potamia; 279.086, Kav 89, Misoropi; 280.518, Dra 236, Skaloti; 282.941, Kil 298, WNW Kastaneri; 283.284, Pel 349, Kajmaktsalan; 283.285, Pel 349, Kajmaktsalan; 283.440, Pel 356, NW Loutraki; 283.522, Pel 361, SW Promachi; 283.766, Ima 172, S Ag. Pavlos; 283.938, loa 1108, N Metsovo; 284.120, loa 1094 b, S Anilio

\section{Epilobium roseum}

280.324, Dra 230, Livadero; 283.290, Pel 349, Kajmaktsalan; 283.324, Pel 351, Kajmaktsalan; 283.574, Pel 363, NW Promachi

\section{Epilobium tetragonum}

279.391, Ser 262, Proti; 281.923, Dra 288, SW Potamia; 282.888, Kil 295, NW Griva; 283.532, Pel 361, SW Promachi; 283.764, Ima 172, S Ag. Pavlos; 284.121, loa 1094 b, S Anilio

\section{Epilobium vernonicum}

283.287, Pel 349, Kajmaktsalan

\section{Epipactis helleborine}

280.380, Dra 232, Sidironero; 280.562, Dra 237, Skaloti; 281.094, Dra 260, Granitis; 282.958, Kil 299, Kastaneri; 283.804, Ima 174, S Ag. Pavlos; 283.871, Ima 178, Seli; 20.498, Ioa 1095, Anilio

\section{Epipactis palustris}

283.940, loa 1108, N Metsovo; -20.497, loa 1094, S Anilio

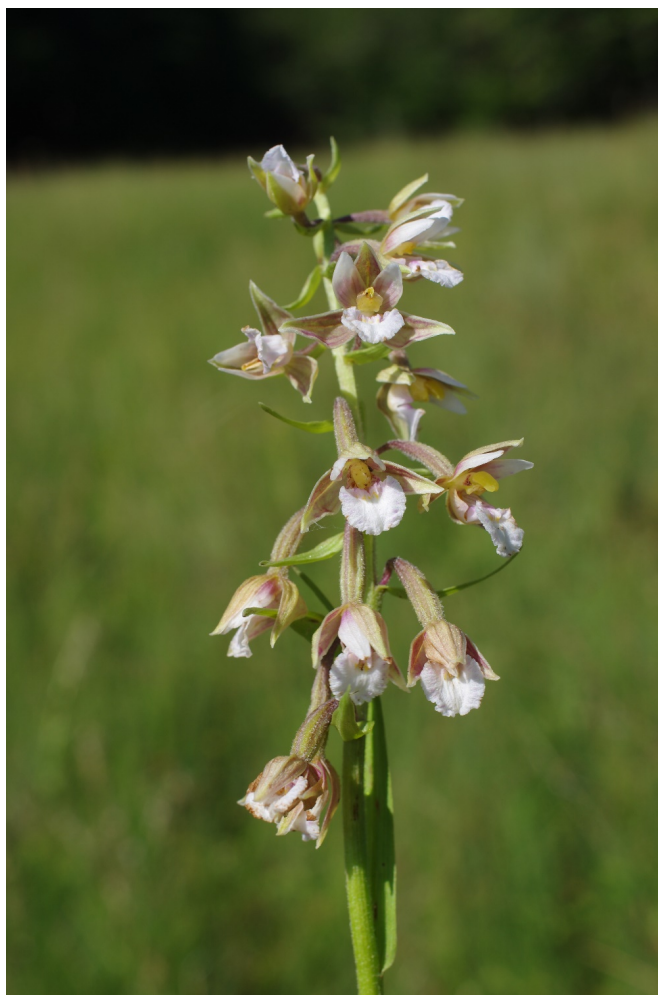

\section{Epipactis persica}

283.282, Pel 348, Kajmaktsalan

\section{Epipactis subclausa}

283.236, Pel 346, Kajmaktsalan; 283.803, Ima 174, S Ag. Pavlos 


\section{Equisetum arvense}

276.755, loa 1068, NW Metsovo; 277.101, loa 1084, N Metsovo; 277.205, loa 1088, NNO Metsovo; 277.502, loa 1097, S Anilio; 277.744, Tri 461, N Chaliki; 279.934, Dra 211, Livadero; 280.514, Dra 236, Skaloti; 281.305, Dra 267, W Nevrokopi; 281.524, Dra 276, Perithori; 281.634, Dra 279, Kato Vrondous; 281.889, Dra 287, O Mikroklisoura; 282.289, Ser 272, Ano Vrondous; 283.047, Kil 304, SO Livadia; 283.430, Pel 355, NW Loutraki; 283.542, Pel 362, NW Promachi

\section{Equisetum palustre}

276.890, loa 1072, NNW Metsovo; 277.059, loa 1080, SO Anilio; 281.066, Dra 260, Granitis

\section{Equisetum ramosissimum}

278.745, Kav 109, Loutra Eleftherion; 279.679, Ser 265, Myrrini

\section{Equisetum sylvaticum}

282.883, Kil 295, NW Griva

\section{Equisetum telmateia}

278.562, Thes 219, Vrasna; 279.938, Dra 211, Livadero; 283.521, Pel 361, SW Promachi

\section{Eragrostis minor}

278.710, Kav 107, Paralia Ofryniou; 278.815, Kav 114, SO Folia; 279.209, Ser 257, Nea Fili; 279.265, Ser 258, Mikro Souli; 279.756, Ser 268, SW Skopia; 281.180, Dra 264, NO Ochyro; 282.221, Dra 300, SO Lefkogia; 282.815, Ser 296, NO Strymoniko; 283.715, Pel 370, SW Foustani

\section{Erigeron acris}

280.306, Dra 229, SO Dendrakia; 280.366, Dra 232, Sidironero; 280.688, Dra 242, SW Walddorf Elatias; 280.768 , Dra 246, SW Walddorf Elatias; 280.947, Dra 254, Falakron; 281.210, Dra 265, NO Granitis; 281.492, Dra 275, Vathytopo; 281.643, Dra 279, Kato Vrondous; 281.855, Dra 285, NNO Volakas; 282.154, Dra 298, SO Lefkogia; 282.710, Ser 290, N Neo Petritsi; 282.950, Kil 298, WNW Kastaneri; 282.954, Kil 299, Kastaneri; 283.141, Pel 341, S Archangelo; 283.228, Pel 345, Kerasia; 283.238, Pel 346, Kajmaktsalan; 283.741, Ima 172, S Ag. Pavlos; 283.873, Ima 179, Seli; 283.907, Ima 180, Seli; 283.933, loa 1107, N Metsovo; 283.984, loa 1111, NO Metsovo

\section{Erigeron annuus}

276.551, loa 1060, SSO Potamia; 279.815, Ser 269, NO Skopia; 279.994, Dra 215, Livadero; 280.179, Dra 223, NO Drama; 281.070, Dra 260, Granitis; 281.248, Dra 266, W Nevrokopi; 281.381, Dra 272, Vathytopo; 281.509, Dra 276, Perithori; 281.854, Dra 284, NNO Volakas; 281.884, Dra 287, O Mikroklisoura; 282.840, Ser 298, Parapotamos; 282.859, Kil 295, NW Griva; 283.007, Kil 302, Kastaneri; 283.438, Pel 356, NW Loutraki; 283.597, Pel 364, Loutraki; 283.650, Pel 367, Vorino; 283.698, Pel 369, O Vorino; 283.709, Pel 370, SW Foustani

\section{Erigeron bonariensis}

278.906, Kav 116, S Ofrynia; 278.965, Kav 119, Podochori; 279.190, Ser 256, Paleokomi; 279.677, Ser 265, Myrrini; 279.745, Ser 268, SW Skopia; 282.814, Ser 296, NO Strymoniko; 283.730, Pel 370, SW Foustani

\section{Erigeron canadensis}

278.498, Thes 216, Vrasna; 278.775, Kav 112, NO Paralia Myrtofytou; 278.801, Kav 113, NO Paralia Myrtofytou; 278.809, Kav 114, SO Folia; 279.636, Ser 263, Dravoskas; 282.803, Ser 296, NO Strymoniko; 282.832, Ser 298, Parapotamos; 282.879, Kil 295, NW Griva;

283.166, Pel 343, Kerasia; 283.647, Pel 367, Vorino; 283.657, Pel 367, Vorino; 283.704, Pel 369, O Vorino; 283.713, Pel 370, SW Foustani

\section{Erigeron sumatrensis}

278.652, Kav 106, Paralia Ofryniou; 279.137, Kav 91, Orfani 
Eriophorum angustifolium

283.322, Pel 351, Kajmaktsalan

Eriophorum latifolium

280.759, Dra 246, SW Walddorf Elatias

Erodium botrys

281.031, Dra 258, Granitis

Erodium ciconium

278.671, Kav 107, Paralia Ofryniou

\section{Erodium cicutarium}

276.480, loa 1059, Ag. Nikolaos; 277.445, loa 1095, S Anilio; 278.307, Koz 380, Galatini; 278.361, Koz 382, Galatini

\section{Eryngium amethystinum}

283.085, Kil 307, NW Livadia; 283.401, Pel 354, NW Loutraki; 283.500, Pel 359, NW

Loutraki; 283.789, Ima 173, S Ag. Pavlos; 283.814, Ima 176, Tria-Pende Pigadia; 283.883, Ima 179, Seli; 283.935, loa 1108, N Metsovo; 283.969, loa 1056 b, NO Metsovo; 284.031, Ioa 1116, SW Milia; 284.033, loa 1116, SW Milia; 284.163, loa 1123, N Charikli

\section{Eryngium campestre}

278.024, Koz 376, W Exarcho; 278.239, Koz 378, N Siatista; 279.230, Ser 257, Nea Fili; 279.654, Ser 264, Dravoskas; 280.822, Dra 248, Falakron; 281.280, Dra 267, W Nevrokopi; 281.642, Dra 279, Kato Vrondous; 281.860, Dra 285, NNO Volakas; 282.112, Dra 296, NO Nevrokopi; 282.373, Ser 276, SSW Ano Vrondous; 282.422, Ser 279, Orini; 282.733, Ser 291, N Neo Petritsi; 282.960, Kil 299, Kastaneri; 283.601, Pel 365, N Vorino; 283.604, Pel 365, N Vorino; 283.624, Pel 366, N Vorino; 284.049, loa 1117, Anilio

\section{Eryngium creticum}

279.648, Ser 264, Dravoskas

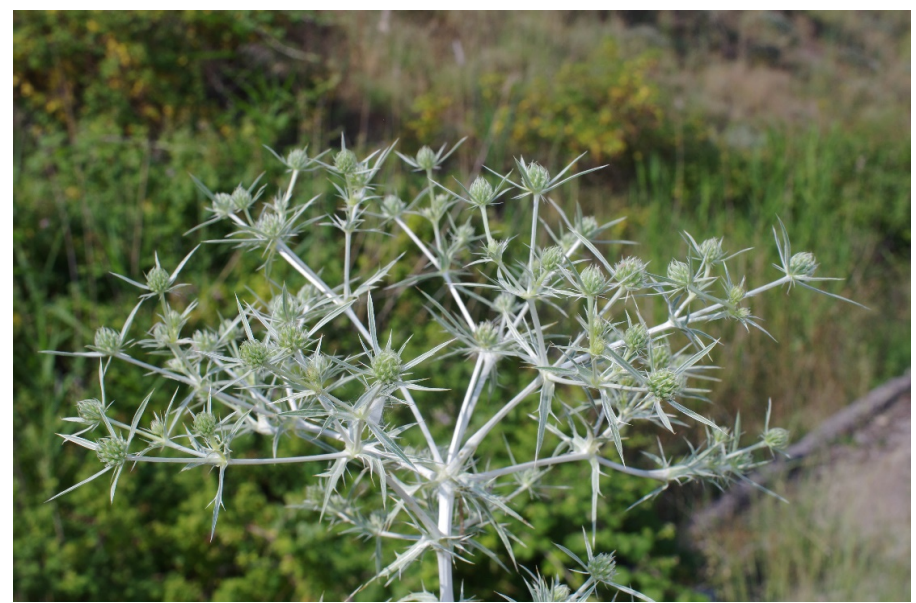

\section{Eryngium maritimum}

278.733, Kav 108, Paralia Ofryniou

\section{Erysimum calycinum}

277.818, Koz 387, SO Siatista, Vourinos; 278.499, Thes 216, Vrasna; 278.519, Thes 217, Vrasna; 278.719, Kav 108, Paralia Ofryniou; 278.944, Kav 118, Galipsos; 279.164, Ser 255, S Nea Mesolakkia; 279.258, Ser 258, Mikro Souli; 279.809, Ser 269, NO Skopia; 280.064 , Dra 218, Sidironero; 280.417, Dra 233, Sidironero; 281.244, Dra 265, NO Granitis; 281.583 , Dra 277, Perithori; 282.163, Dra 298, SO Lefkogia

\section{Erysimum comatum}

276.489, loa 1059, Ag. Nikolaos; 279.595, Kav 104, Pangaion; 280.857, Dra 248, Falakron 


\section{Erysimum crassistylum}

278.607, Thes 221, Arethousa; 279.059, Kav 121, Platanotopos; 279.102, Kav 90, SW Moustheni; 279.399, Kav 92, Pangaion; 279.615, Ser 263, Dravoskas; 279.873, Dra 209, Livadero; 280.140, Dra 221, NO Drama; 281.021, Dra 258, Granitis; 281.272, Dra 266, W Nevrokopi; 281.637, Dra 279, Kato Vrondous; 281.734, Dra 281, OSO Granitis; 281.838, Dra 284, NNO Volakas; 282.052, Dra 293, Nevrokopi; 282.358, Ser 276, SSW Ano

Vrondous; 282.436, Ser 280, Orini

\section{Erysimum cuspidatum}

277.968, Koz 374, Vourinos; 282.701, Ser 290, N Neo Petritsi; 282.719, Ser 290, N Neo Petritsi; 283.136, Pel 341, S Archangelo; 283.773, Ima 173, S Ag. Pavlos

\section{Erysimum microstylum}

276.445, Tri 456, N Katara-Pass; 276.951, loa 1076, ONO Anilio; 276.978 , loa 1079, SO Anilio; 276.993, loa 1079, SO Anilio; 277.335, loa 1093, S Anilio; 277.472, loa 1096, S Anilio; 277.658, loa 1106, Anilio; 277.748, Tri 461, N Chaliki; 278.136, Gre 492, NO Varis; 278.406, Koz 383, Galatini; 281.709, Dra 280, Nevrokopi; 281.781, Dra 282, NNO Volakas; 283.897, Ima 180, Seli

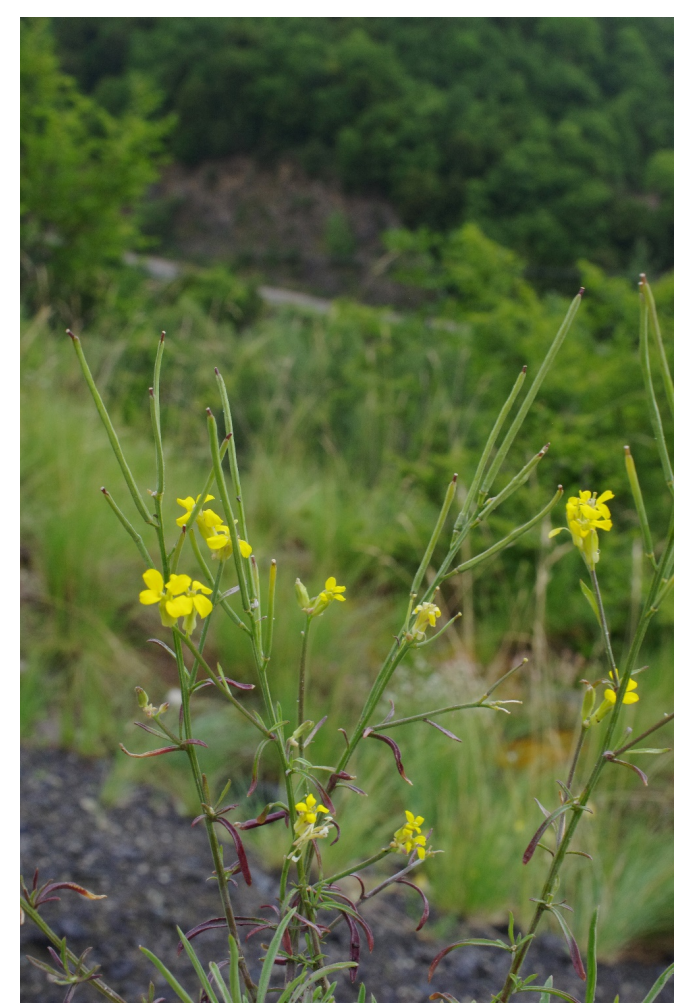

\section{Euonymus verrucosus}

281.329, Dra 269, W Nevrokopi

\section{Eupatorium cannabinum}

282.891, Kil 295, NW Griva; 283.150, Pel 342, S Archangelo; 283.394, Pel 354, NW Loutraki; 283.561, Pel 362, NW Promachi; 284.122, loa 1094 b, S Anilio

\section{Euphorbia amygdaloides subsp. amygdaloides}

277.593, loa 1101, S Anilio; 280.546, Dra 237, Skaloti; 280.657, Dra 242, SW Walddorf Elatias; 280.738, Dra 245, SW Walddorf Elatias; 281.651, Dra 279, Kato Vrondous; 282.759, Ser 293, N Neo Petritsi; 283.185, Pel 343, Kerasia; 283.575, Pel 363, NW Promachi; 283.835, Ima 177, Tria-Pende Pigadia; 283.844, Ima 178, Seli; 283.918, Ima 180, Seli

\section{Euphorbia baselices}

280.898, Dra 250, Falakron 


\section{Euphorbia chamaesyce}

278.902, Kav 116, S Ofrynia; 278.960, Kav 119, Podochori; 279.237, Ser 258, Mikro Souli; 279.741, Ser 268, SW Skopia; 280.240, Dra 226, N Taxiarches; 281.991, Dra 291, W

Prosotsani; 283.640, Pel 367, Vorino; 283.683, Pel 369, O Vorino

\section{Euphorbia cyparissias}

277.960, Koz 374, Vourinos; 279.170, Ser 255, S Nea Mesolakkia; 280.905, Dra 250,

Falakron; 282.420, Ser 279, Orini; 282.467, Ser 281, Ori Vrondous

\section{Euphorbia epithymoides}

276.762, loa 1069, NW Metsovo

\section{Euphorbia falcata}

276.718, loa 1066, NO Karyes; 277.475, loa 1097, S Anilio; 277.783, Koz 385, SO Siatista; 278.088, Gre 492, NO Varis; 279.005, Kav 119, Podochori; 279.711, Ser 266, SO Nea Zichni

\section{Euphorbia glabriflora}

278.118, Gre 492, NO Varis

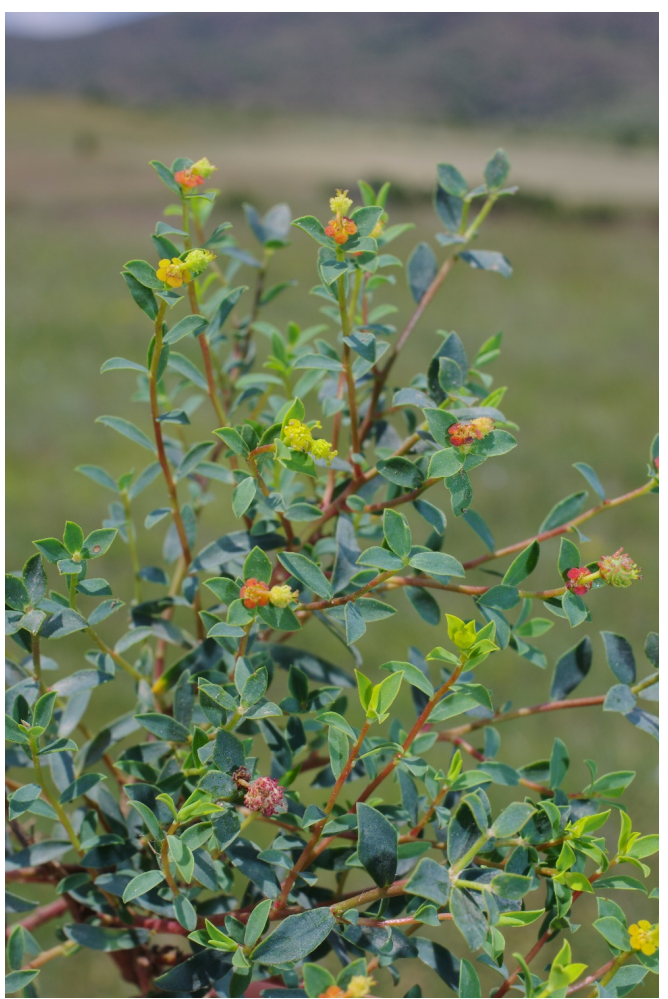

\section{Euphorbia helioscopia}

277.431, loa 1095, S Anilio; 277.771, Tri 462, N Chaliki

\section{Euphorbia maculata}

278.707, Kav 107, Paralia Ofryniou; 278.804, Kav 113, NO Paralia Myrtofytou; 281.266, Dra 266, W Nevrokopi; 282.820, Ser 296, NO Strymoniko; 283.726, Pel 370, SW Foustani

\section{Euphorbia myrsinites}

276.985, loa 1079, SO Anilio; 277.645, loa 1105, Anilio

\section{Euphorbia platyphyllos}

279.524, Kav 99, Pangaion; 279.556, Kav 102, Pangaion; 280.028, Dra 216, Sidironero; 280.500, Dra 235, Skaloti; 280.538, Dra 236, Skaloti; 280.693, Dra 242, SW Walddorf Elatias; 280.743 , Dra 245, SW Walddorf Elatias; 281.077, Dra 260, Granitis; 282.284, Ser 272, Ano Vrondous; 282.550, Ser 285, Ori Vrondous; 283.112, Pel 339, S Archangelo

\section{Euphorbia salicifolia}

282.506, Ser 282, Ori Vrondous 


\section{Euphorbia seguierana subsp. niciciana}

278.436, Thes 214, Paralia Vrasna; 278.511, Thes 217, Vrasna; 278.621, Thes 221, Arethousa; 278.697, Kav 107, Paralia Ofryniou; 278.882, Kav 116, S Ofrynia; 279.045, Kav 120, Podochori; 279.417, Kav 93, Pangaion; 279.491, Kav 97, Pangaion; 279.639, Ser 263, Dravoskas; 279.661, Ser 265, Myrrini; 279.804, Ser 269, NO Skopia; 280.201, Dra 224, NO Drama; 280.316, Dra 229, SO Dendrakia; 280.671, Dra 242, SW Walddorf Elatias; 280.906, Dra 251, Falakron; 281.345, Dra 270, W Nevrokopi; 281.387, Dra 272, Vathytopo; 281.750, Dra 281, OSO Granitis; 282.301, Ser 273, Ano Vrondous; 282.413, Ser 279, Orini; 282.656, Ser 290, N Neo Petritsi; 283.701, Pel 369, O Vorino

\section{Euphorbia seguierana subsp. seguierana}

278.739, Kav 109, Loutra Eleftherion; 279.260, Ser 258, Mikro Souli; 279.374, Ser 262, Proti; 280.371, Dra 232, Sidironero; 280.463, Dra 234, Skaloti; 281.046, Dra 259, Granitis; 281.578, Dra 277, Perithori; 281.588, Dra 278, Kato Vrondous; 282.108, Dra 296, NO Nevrokopi; 283.099, Pel 339, S Archangelo; 283.400, Pel 354, NW Loutraki

\section{Euphorbia serrulata}

280.302, Dra 229, SO Dendrakia

\section{Euphorbia taurinensis}

278.005, Koz 375, O Dafnero; 278.246, Koz 378, N Siatista; 280.363, Dra 232, Sidironero; 280.931, Dra 253, Falakron

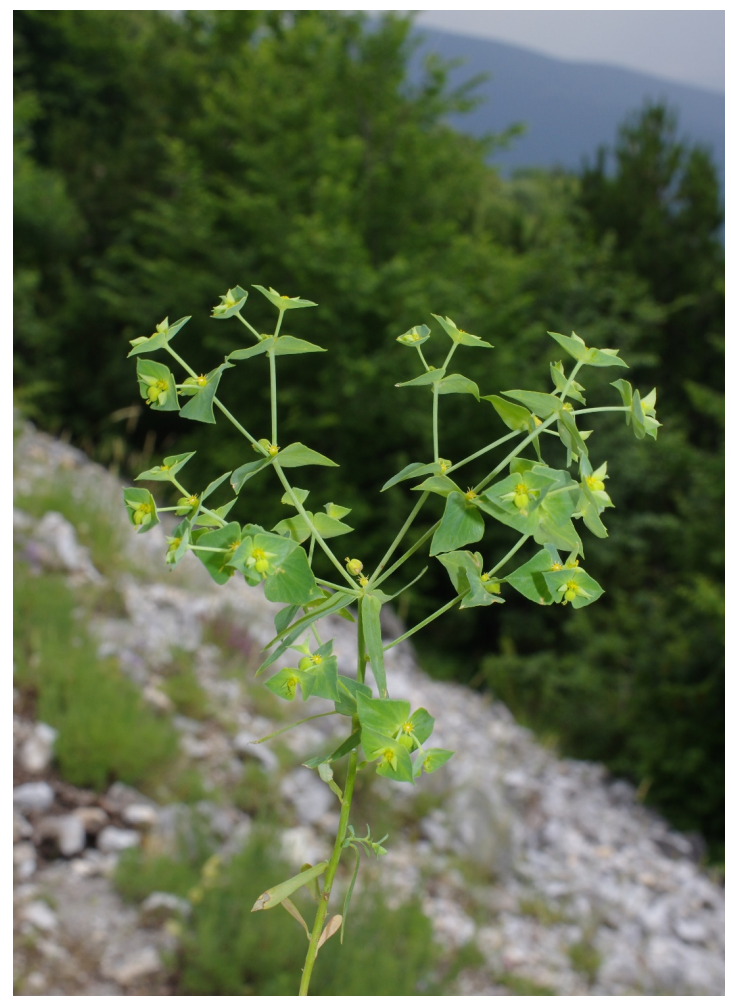

\section{Euphorbia villosa}

279.553, Kav 102, Pangaion

\section{Euphrasia liburnica}

276.850, loa 1071, NNW Metsovo; 277.608, loa 1103, S Anilio; 277.685, Tri 460, N Chaliki; 277.873, Koz 388, SO Siatista, Vourinos; 278.121, Gre 493, NO Varis; 279.790, Ser 268, SW Skopia; 280.821, Dra 248, Falakron; 283.458, Pel 358, N Loutraki; 283.501, Pel 359, NW Loutraki; 283.975, loa 1056 b, NO Metsovo; 284.065, loa 1118, Anilio; 284.172, loa 1124, N Charikli; 284.194, loa 1128, N Metsovo 


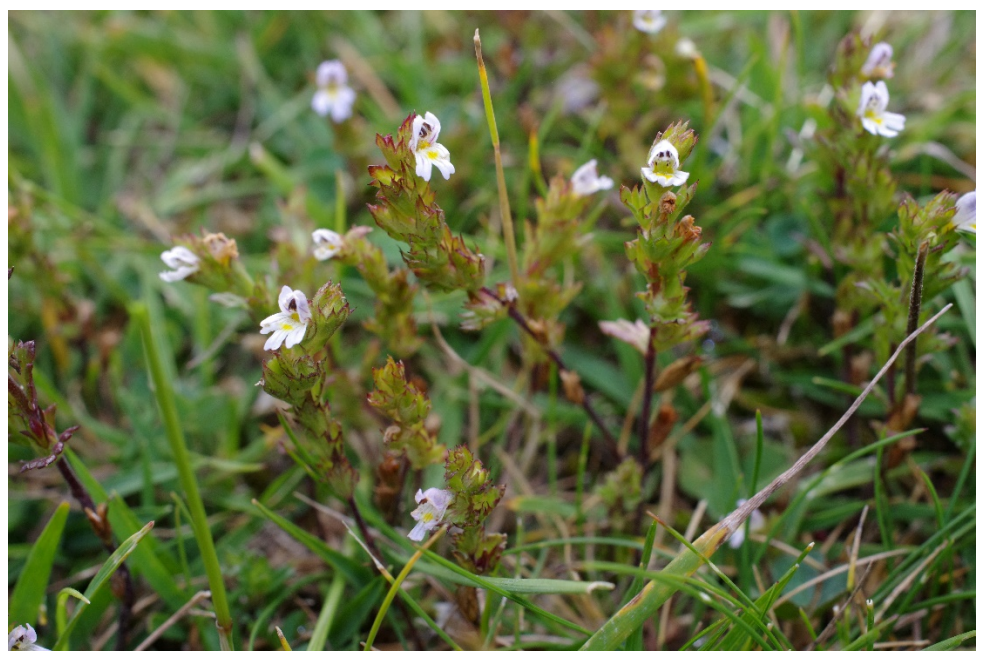

\section{Euphrasia minima}

276.368, loa 1128, N Metsovo; 277.295, loa 1091, SW Milia

\section{Euphrasia pectinata}

277.081, loa 1081, W Metsovo; 280.093, Dra 219, Sidironero; 281.123, Dra 261, Granitis; 281.441, Dra 274, Vathytopo; 283.214, Pel 344, Kerasia; 283.899, Ima 180, Seli

\section{Fagus sylvatica subsp. sylvatica}

277.196, loa 1087, NNO Metsovo; 277.253, loa 1089, NNO Metsovo; 279.542, Kav 101, Pangaion; 282.764, Ser 293, N Neo Petritsi; 282.925, Kil 297, WNW Kastaneri

\section{Fallopia convolvulus}

278.060, Gre 492, NO Varis; 278.249, Koz 379, Galatini; 279.691, Ser 266, SO Nea Zichni; 280.573, Dra 238, Skaloti; 281.181, Dra 264, NO Ochyro; 281.259, Dra 266, W Nevrokopi; 281.418, Dra 273, Vathytopo; 281.497, Dra 276, Perithori; 281.789, Dra 282, NNO Volakas; 281.927, Dra 288, SW Potamia; 281.988, Dra 291, W Prosotsani; 282.155, Dra 298, SO Lefkogia; 282.227, Dra 300, SO Lefkogia; 282.306, Ser 273, Ano Vrondous; 282.614, Ser 288, Ori Vrondous; 282.686, Ser 290, N Neo Petritsi; 282.990, Kil 300, Kastaneri

\section{Farn}

276.427, loa 1057, NO Metsovo; 276.764, loa 1069, NW Metsovo; 276.883, loa 1072, NNW Metsovo; 279.969, Dra 213, Livadero; 280.590, Dra 238, Skaloti; 280.727, Dra 244, SW Walddorf Elatias; 283.479, Pel 358, N Loutraki; 283.565, Pel 363, NW Promachi

\section{Ferulago sylvatica subsp. sylvatica}

280.946, Dra 254, Falakron; 283.031, Kil 303, SO Livadia

\section{Festuca}

276.471, Tri 458, NO Katara-Pass; 276.566, loa 1060, SSO Potamia; 276.630, loa 1062, SO Potamia; 276.710, loa 1065, NO Karyes; 276.798, loa 1070, NNW Metsovo; 276.933, loa 1074, NNW Metsovo; 276.983, loa 1079, SO Anilio; 276.995, loa 1079, SO Anilio; 277.119 , loa 1084, N Metsovo; 277.296, loa 1091, SW Milia; 277.369, loa 1093, S Anilio; 277.628, Ioa 1104, S Anilio; 277.695, Tri 460, N Chaliki; 277.715, Tri 460, N Chaliki; 278.327, Koz 381, Galatini; 279.540, Kav 101, Pangaion; 280.469, Dra 234, Skaloti; 280.644, Dra 241, Elatias; 280.715 , Dra 243, SW Walddorf Elatias; 280.774, Dra 247, Walddorf Elatias; 283.013, Kil 302, Kastaneri; 283.250, Pel 346, Kajmaktsalan; 283.347, Pel 352, Kajmaktsalan

\section{Festuca arundinacea}

276.355, loa 1128, N Metsovo; 276.671, loa 1064, ONO Karyes; 277.136, loa 1084, N Metsovo; 277.732, Tri 460, N Chaliki; 277.743, Tri 461, N Chaliki; 282.073, Dra 294, NO Nevrokopi; 284.077, loa 1118, Anilio

\section{Festuca horvatiana}

283.379, Pel 353, Kajmaktsalan 


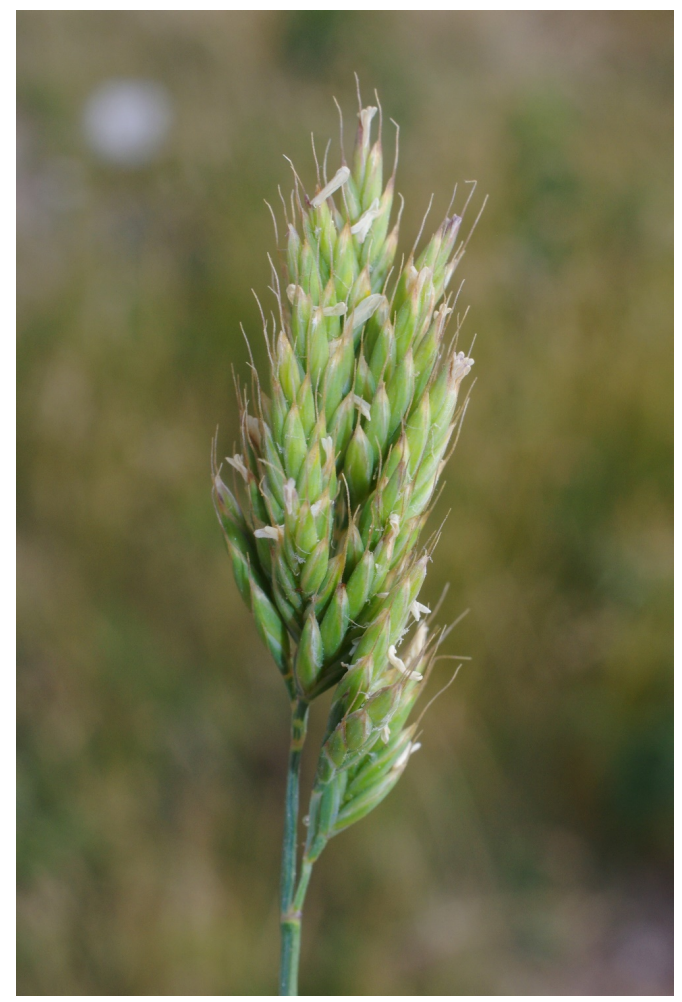

\section{Festuca pratensis}

276.694, loa 1065, NO Karyes; 276.739, loa 1068, NW Metsovo; 277.248, loa 1089, NNO Metsovo; 281.797, Dra 282, NNO Volakas; 283.760, Ima 172, S Ag. Pavlos

\section{Fibigia clypeata}

277.932, Koz 392, SO Siatista, Vourinos

\section{Filago arvensis}

277.721, Tri 460, N Chaliki; 278.191, Gre 495, Varis; 278.294, Koz 380, Galatini; 278.500, Thes 216, Vrasna; 278.875, Kav 115, SW Folia; 279.384, Ser 262, Proti; 279.987, Dra 214, Livadero; 281.474, Dra 275, Vathytopo; 282.099, Dra 295, NO Nevrokopi; 282.173, Dra 299, SO Lefkogia; 282.447, Ser 280, Orini

\section{Filago eriocephala}

277.999, Koz 375, O Dafnero; 278.120, Gre 493, NO Varis; 278.329, Koz 381, Galatini; 278.478, Thes 215, Paralia Vrasna; 278.609, Thes 221, Arethousa; 278.813, Kav 114, SO Folia; 278.958, Kav 119, Podochori; 279.332, Ser 260, Proti; 279.720, Ser 267, SW Skopia; 281.574, Dra 277, Perithori; 282.747, Ser 292, N Neo Petritsi; 282.867, Kil 295, NW Griva

\section{Filago minima}

278.502, Thes 216, Vrasna; 278.872, Kav 115, SW Folia

\section{Filago pyramidata}

276.301, loa 1125, NO Dodoni; 276.483, loa 1059, Ag. Nikolaos; 276.721, loa 1066, NO Karyes; 277.959, Koz 374, Vourinos; 278.315, Koz 381, Galatini; 278.936, Kav 118, Galipsos; 279.067, Kav 121, Platanotopos; 279.200, Ser 257, Nea Fili; 280.110, Dra 220, Sidironero; 280.233, Dra 226, N Taxiarches; 280.348, Dra 231, Sidironero; 282.400, Ser 278, N Serres

\section{Filipendula vulgaris}

276.751, loa 1068, NW Metsovo; 276.806, loa 1070, NNW Metsovo; 277.739, Tri 461, N Chaliki; 277.894, Koz 389, SO Siatista, Vourinos; 278.042, Gre 491, NO Exarcho; 278.148, Gre 492, NO Varis; 280.427, Dra 233, Sidironero; 280.487, Dra 235, Skaloti; 280.948, Dra 255, Falakron 


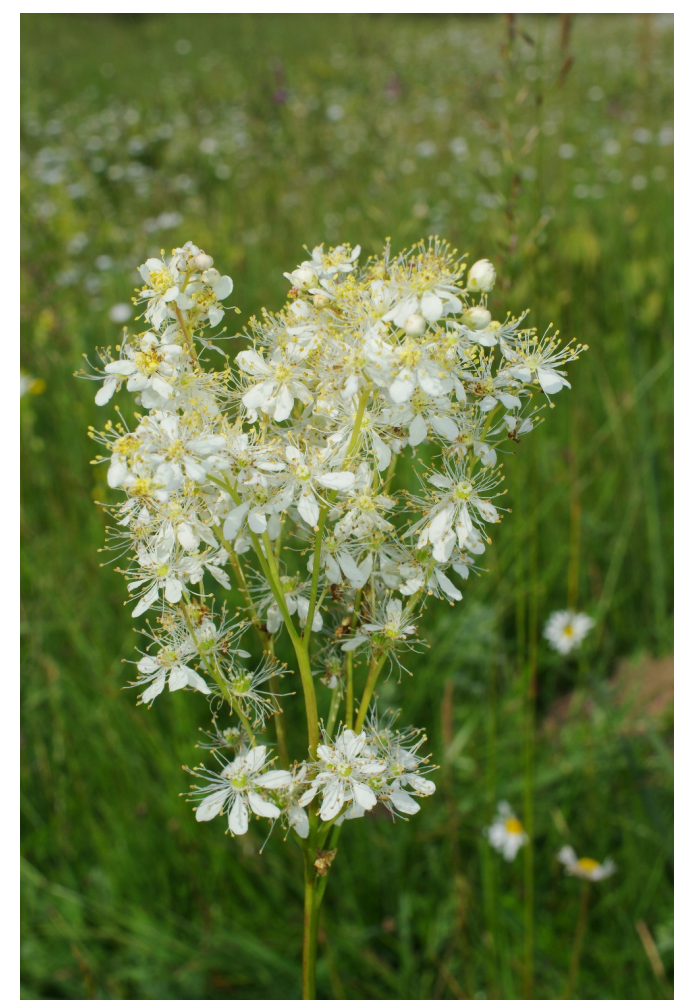

\section{Fragaria vesca}

277.545, loa 1098, S Anilio; 277.923, Koz 392, SO Siatista, Vourinos; 280.648, Dra 242, SW Walddorf Elatias; 282.619, Ser 288, Ori Vrondous; 283.010, Kil 302, Kastaneri; 283.240, Pel 346, Kajmaktsalan

\section{Fragaria viridis}

277.163, loa 1086, NNO Metsovo

\section{Frangula alnus}

280.040, Dra 216, Sidironero; 283.147, Pel 342, S Archangelo; 283.263, Pel 347, Kajmaktsalan

\section{Fraxinus ornus}

280.295, Dra 228, Livadero

\section{Fumana procumbens}

277.994, Koz 375, O Dafnero; 278.197, Koz 377, N Siatista; 280.173, Dra 222, NO Drama

\section{Fumana thymifolia}

278.932, Kav 118, Galipsos

\section{Fumaria densiflora}

279.024, Kav 119, Podochori

\section{Fumaria officinalis}

279.106, Kav 90, SW Moustheni

\section{Fumaria rostellata}

278.303, Koz 380, Galatini

\section{Galega officinalis}

278.795, Kav 113, NO Paralia Myrtofytou; 279.672, Ser 265, Myrrini; 279.925, Dra 211, Livadero; 281.079, Dra 260, Granitis

\section{Galeopsis bifida}

281.126, Dra 261, Granitis; 282.567, Ser 285, Ori Vrondous; 282.625, Ser 288, Ori Vrondous 


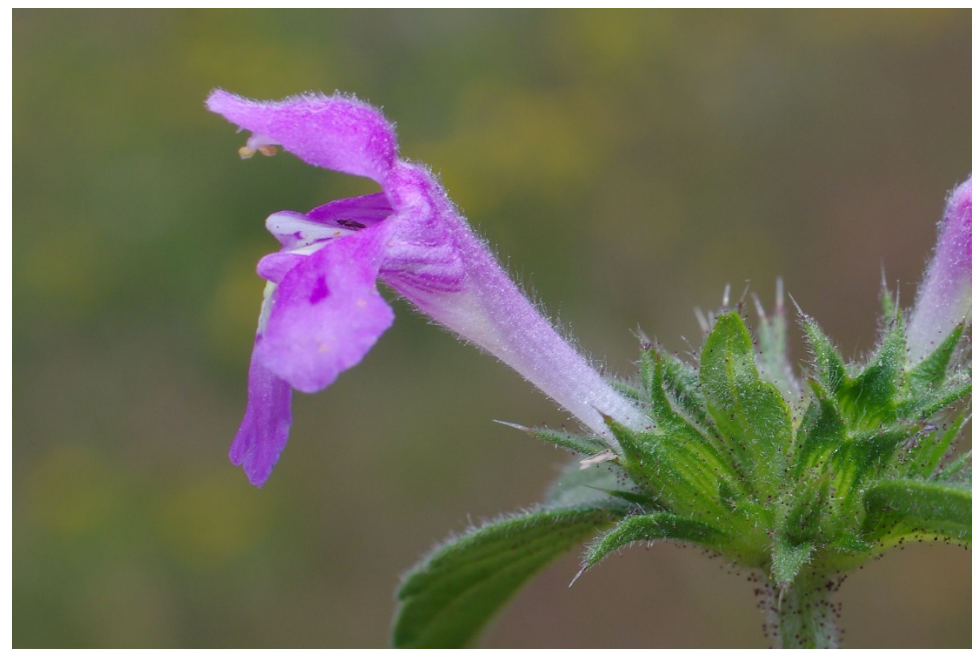

\section{Galinsoga parviflora}

280.461, Dra 234, Skaloti; 281.001, Dra 257, Granitis; 281.308, Dra 268, W Nevrokopi; 281.407, Dra 273, Vathytopo; 281.541, Dra 276, Perithori; 282.350, Ser 275, SSW Ano Vrondous; 282.849, Kil 294, NW Griva

\section{Galium}

276.340, loa 1126, NO Dodoni; 276.556, loa 1060, SSO Potamia; 277.365, loa 1093, S Anilio; 277.538, loa 1097, S Anilio; 277.728, Tri 460, N Chaliki; 277.866, Koz 388, SO Siatista, Vourinos; 279.480, Kav 97, Pangaion; 279.548, Kav 101, Pangaion; 279.956, Dra 212, Livadero; 280.386, Dra 232, Sidironero; 280.763, Dra 246, SW Walddorf Elatias; 281.203, Dra 264, NO Ochyro; 281.304, Dra 267, W Nevrokopi; 281.438, Dra 274, Vathytopo; 281.522, Dra 276, Perithori; 281.942, Dra 288, SW Potamia; 282.101, Dra 295, NO Nevrokopi; 282.490, Ser 282, Ori Vrondous; 282.640, Ser 290, N Neo Petritsi; 283.242, Pel 346, Kajmaktsalan; 283.545, Pel 362, NW Promachi; 283.675, Pel 368, OSO Vorino; 283.991, loa 1112, Katara-Paß; 284.101, loa 1121, Anilio

\section{Galium aegaeum}

280.818, Dra 248, Falakron

\section{Galium anisophyllon}

280.874, Dra 249, Falakron

\section{Galium aparine}

276.643, loa 1063, W Potamia; 276.848, loa 1071, NNW Metsovo; 277.280, loa 1090, NNO Metsovo; 277.486, loa 1097, S Anilio; 280.334, Dra 230, Livadero; 281.521, Dra 276, Perithori; 282.248, Dra 301, Ano Vrondous; 282.630, Ser 289, Ori Vrondous; -20.516, Dra 238, Skaloti

\section{Galium asparagifolium}

276.709, loa 1065, NO Karyes; 276.943, loa 1075, NW Anilio; 281.147, Dra 262, NW Granitis; 282.545, Ser 284, Ori Vrondous

\section{Galium capitatum}

276.312, loa 1125, NO Dodoni; 284.219, loa 1125, NO Dodoni

\section{Galium debile}

279.998, Dra 215, Livadero; 280.090, Dra 219, Sidironero; 283.301, Pel 350, Kajmaktsalan

\section{Galium degenii}

284.118, loa 1121, Anilio 


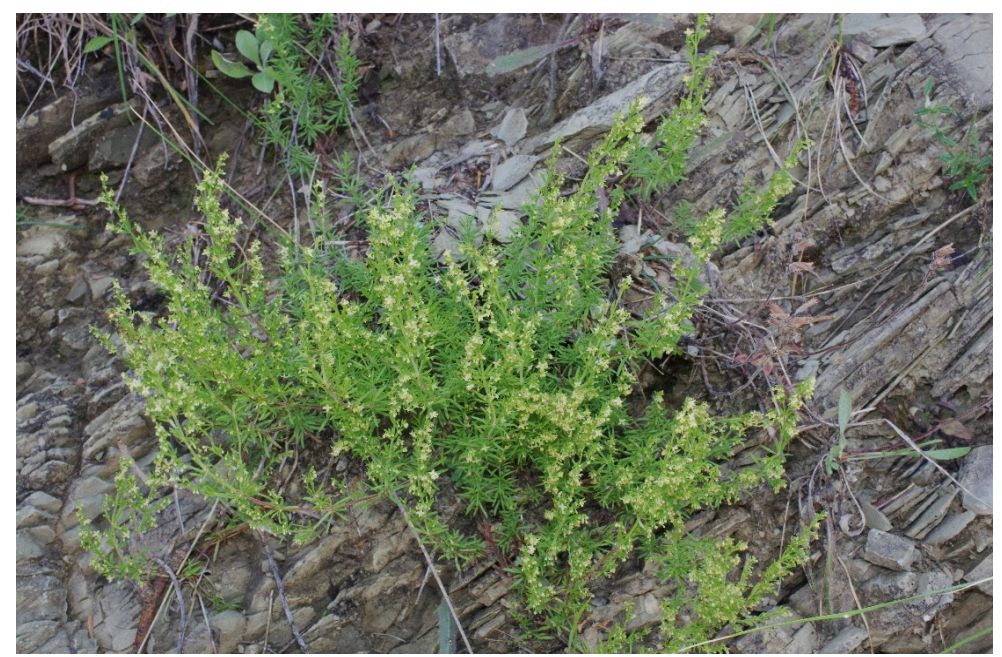

\section{Galium divaricatum}

278.123, Gre 493, NO Varis; 278.888, Kav 116, S Ofrynia; 279.022, Kav 119, Podochori

\section{Galium hellenicum}

277.084, loa 1081, W Metsovo; 277.661, loa 1106, Anilio; 277.980, Koz 374, Vourinos; 280.503, Dra 236, Skaloti; 280.606, Dra 238, Skaloti

Galium incrassatum

278.032, Koz 376, W Exarcho

\section{Galium laconicum}

282.872, Kil 295, NW Griva; 282.951, Kil 298, WNW Kastaneri; 283.053, Kil 305, SO Livadia; 283.157, Pel 342, S Archangelo; 283.930, loa 1107, N Metsovo

\section{Galium mirum}

280.195, Dra 223, NO Drama; 281.125, Dra 261, Granitis

\section{Galium odoratum}

277.177, loa 1086, NNO Metsovo; 280.725, Dra 244, SW Walddorf Elatias; 282.580 , Ser 286, Ori Vrondous; 282.723, Ser 291, N Neo Petritsi

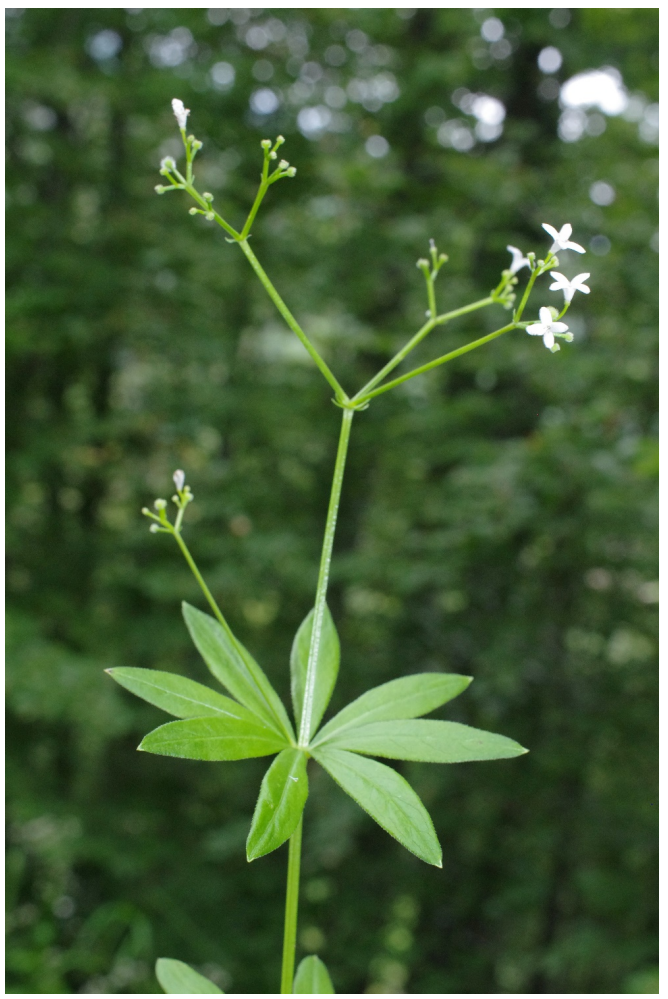




\section{Galium oreophilum}

279.358, Ser 261, Proti

\section{Galium palustre}

277.103, loa 1084, N Metsovo; 280.761, Dra 246, SW Walddorf Elatias

\section{Galium pisoderium ?}

277.083, loa 1081, W Metsovo; 277.726, Tri 460, N Chaliki; 277.727, Tri 460, N Chaliki; 277.922, Koz 391, SO Siatista, Vourinos; 278.218, Koz 378, N Siatista; 279.597, Kav 104, Pangaion; 280.846, Dra 248, Falakron; 282.620, Ser 288, Ori Vrondous; 283.328, Pel 351, Kajmaktsalan; 283.335, Pel 351, Kajmaktsalan; 283.373, Pel 353, Kajmaktsalan

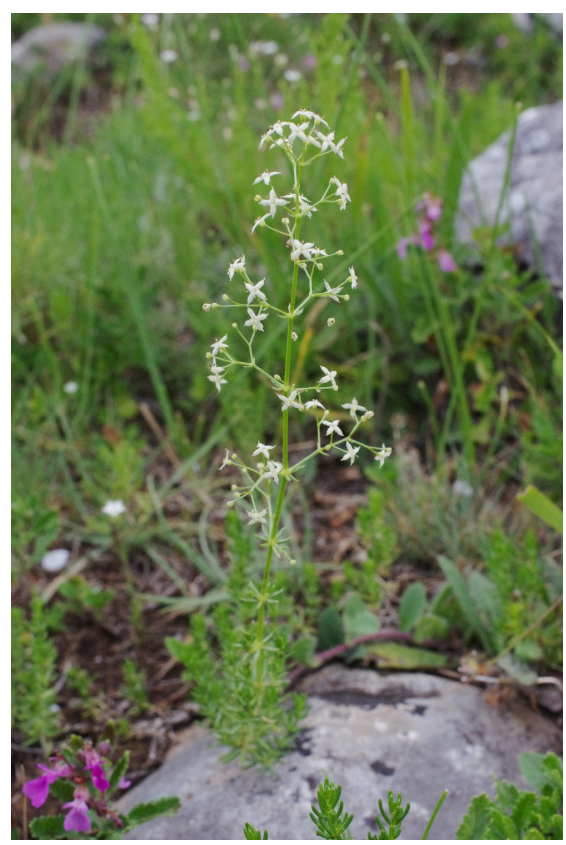

\section{Galium pseudaristatum}

277.860, Koz 388, SO Siatista, Vourinos; 282.741, Ser 292, N Neo Petritsi; 282.760, Ser 293, N Neo Petritsi; 283.459, Pel 358, N Loutraki; 283.539, Pel 362, NW Promachi; 283.796, Ima 174, S Ag. Pavlos; 284.151, loa 1122, Anilio

\section{Galium rivale}

281.076, Dra 260, Granitis; 281.371, Dra 271, Vathytopo; 281.677, Ser 271, Kato Vrondous; 282.259, Dra 301, Ano Vrondous

\section{Galium rotundifolium}

277.506, loa 1097, S Anilio; 284.159, loa 1103 b, S Anilio

\section{Galium samothracicum ?}

279.529, Kav 99, Pangaion; 279.920, Dra 211, Livadero; 282.176, Dra 299, SO Lefkogia; 282.571, Ser 286, Ori Vrondous; 282.679, Ser 290, N Neo Petritsi

\section{Galium speciosum}

282.548, Ser 285, Ori Vrondous

\section{Galium tricornutum}

277.908, Koz 390, SO Siatista, Vourinos; 278.084, Gre 492, NO Varis; 278.284, Koz 379, Galatini

\section{Galium verum}

278.103, Gre 492, NO Varis; 278.280, Koz 379, Galatini; 278.328, Koz 381, Galatini; 278.483, Thes 215, Paralia Vrasna; 278.530, Thes 218, Vrasna; 278.583, Thes 220, Arethousa; 278.644, Kav 106, Paralia Ofryniou; 278.819, Kav 114, SO Folia; 278.862, Kav 115, SW Folia; 279.104, Kav 90, SW Moustheni; 279.168, Ser 255, S Nea Mesolakkia; 279.357, Ser 261, Proti; 279.423, Kav 93, Pangaion; 279.671, Ser 265, Myrrini; 279.723, Ser 267, SW Skopia; 279.785, Ser 268, SW Skopia; 279.877, Dra 209, Livadero; 280.065, 
Dra 218, Sidironero; 280.157, Dra 221, NO Drama; 280.269, Dra 228, Livadero; 280.397, Dra 232, Sidironero; 280.449, Dra 234, Skaloti; 280.990, Dra 257, Granitis; 281.252, Dra 266, W Nevrokopi; 281.383, Dra 272, Vathytopo; 281.498, Dra 276, Perithori; 281.602, Dra 278, Kato Vrondous; 281.733, Dra 281, OSO Granitis; 281.867, Dra 286, SW Mikroklisoura; 281.920, Dra 287, O Mikroklisoura; 281.997, Dra 291, W Prosotsani; 282.122, Dra 296, NO Nevrokopi; 282.360 , Ser 276, SSW Ano Vrondous; 282.536, Ser 284, Ori Vrondous; 282.735, Ser 291, N Neo Petritsi; 282.852, Kil 294, NW Griva; 283.028, Kil 303, SO Livadia; 283.079, Kil 307, NW Livadia; 283.179, Pel 343, Kerasia; 283.448, Pel 356, NW Loutraki; 283.676, Pel 368, OSO Vorino; 283.749, Ima 172, S Ag. Pavlos; 283.880, Ima 179, Seli; 284.183, Dra 257, Granitis

\section{Gaudinia fragilis}

276.538, loa 1059, Ag. Nikolaos; 277.397, loa 1094, S Anilio

\section{Genista carinalis}

279.581, Kav 103, Pangaion; 279.874, Dra 209, Livadero; 279.954, Dra 212, Livadero; 280.305, Dra 229, SO Dendrakia; 280.383, Dra 232, Sidironero; 280.428, Dra 233,

Sidironero; 280.560, Dra 237, Skaloti; 280.833, Dra 248, Falakron; 280.891, Dra 250, Falakron; 282.209, Dra 300, SO Lefkogia; 282.418, Ser 279, Orini

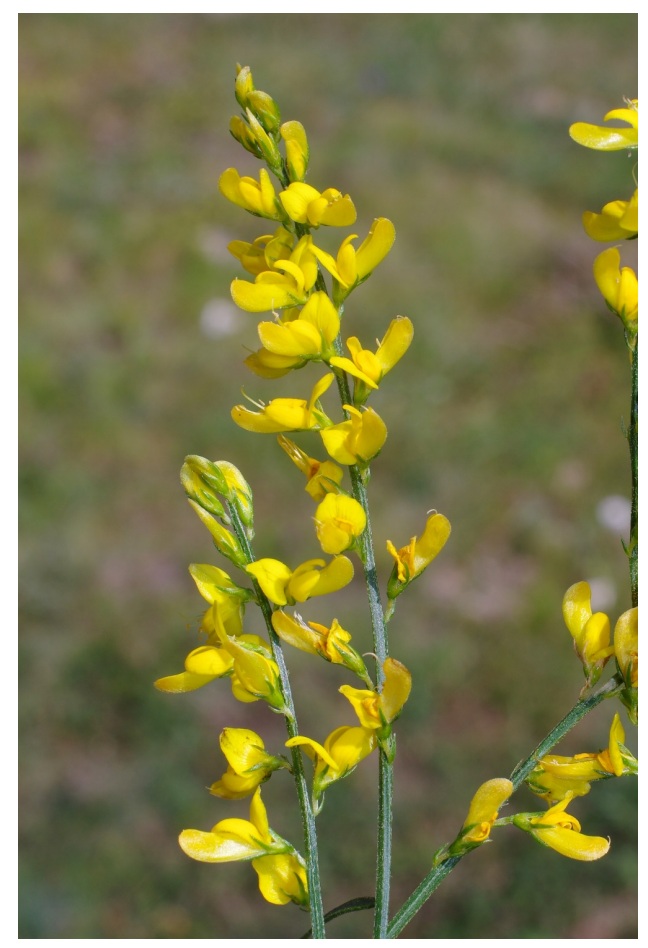

\section{Genista radiata}

277.189, loa 1086, NNO Metsovo

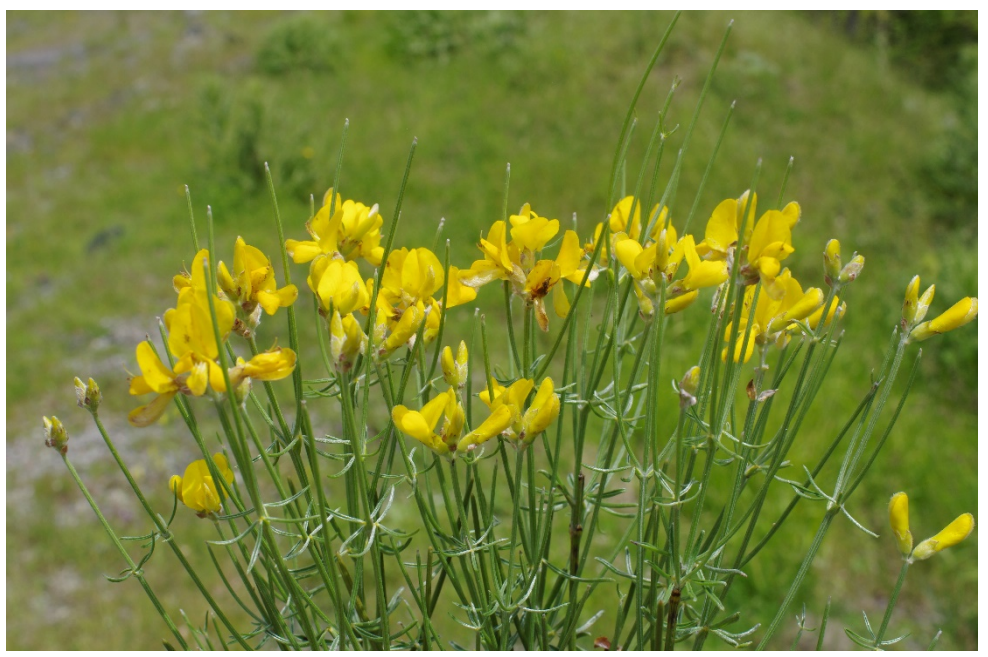




\section{Genista sagittalis}

280.594, Dra 238, Skaloti; 280.707, Dra 243, SW Walddorf Elatias

\section{Genista tinctoria}

280.007, Dra 216, Sidironero; 280.213, Dra 225, NO Drama; 282.921, Kil 297, WNW

Kastaneri

\section{Gentiana cruciata subsp. cruciata}

279.338, Ser 261, Proti; 283.036, Kil 303, SO Livadia; 283.100, Pel 339, S Archangelo; 283.939, loa 1108, N Metsovo; -20.527, Ima 173, S Ag. Pavlos

\section{Geranium asphodeloides}

276.862, loa 1071, NNW Metsovo; 277.035, loa 1080, SO Anilio; 277.224, loa 1089, NNO Metsovo; 277.672, Tri 460, N Chaliki

\section{Geranium columbinum}

277.342, loa 1093, S Anilio; 279.354, Ser 261, Proti; 279.447, Kav 95, Pangaion; 279.747, Ser 268, SW Skopia; 280.046, Dra 217, Sidironero; 280.079, Dra 219, Sidironero; 280.336, Dra 230, Livadero; 280.359, Dra 232, Sidironero; 280.528, Dra 236, Skaloti; 281.038, Dra 258, Granitis; 281.222, Dra 265, NO Granitis; 281.668, Ser 270, Kato Vrondous; -20.460, loa 1052, NO Dodoni

\section{Geranium lucidum}

276.621, loa 1062, SO Potamia; 277.330, loa 1093, S Anilio; 277.766, Tri 462, N Chaliki; 277.952, Koz 374, Vourinos; 278.429, Koz 384, Galatini; 279.269, Ser 258, Mikro Souli; 279.341, Ser 261, Proti; -20.486, loa 1078, O Anilio

\section{Geranium macrorrhizum}

280.570, Dra 238, Skaloti; 280.726, Dra 244, SW Walddorf Elatias; 282.592, Ser 287, Ori Vrondous; 283.051, Kil 305, SO Livadia

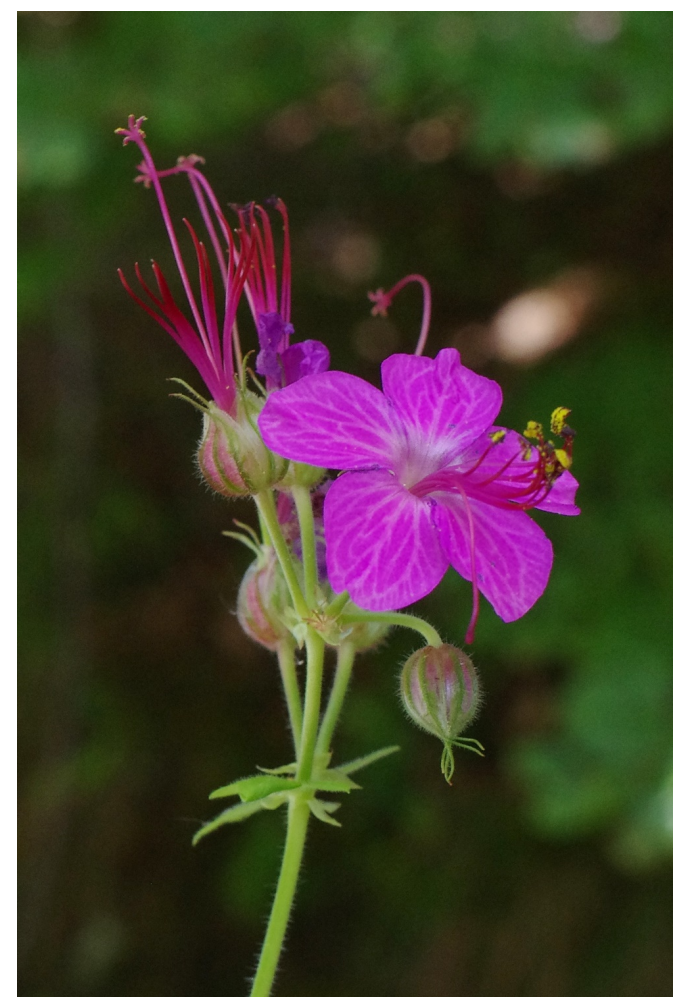

\section{Geranium molle}

277.833, Koz 387, SO Siatista, Vourinos; 277.970, Koz 374, Vourinos; 283.021, Kil 302, Kastaneri 
278.421, Koz 384, Galatini; 279.675, Ser 265, Myrrini; 282.946, Kil 298, WNW Kastaneri; 20.488, loa 1078, O Anilio

\section{Geranium pusillum}

276.530, loa 1059, Ag. Nikolaos; 276.845, loa 1071, NNW Metsovo; 277.193, loa 1087, NNO Metsovo; 277.489, loa 1097, S Anilio; 277.760, Tri 461, N Chaliki; 280.614, Dra 238,

Skaloti; 280.709, Dra 243, SW Walddorf Elatias; 282.292, Ser 272, Ano Vrondous

\section{Geranium reflexum}

276.773, loa 1069, NW Metsovo; 277.573, loa 1100, S Anilio

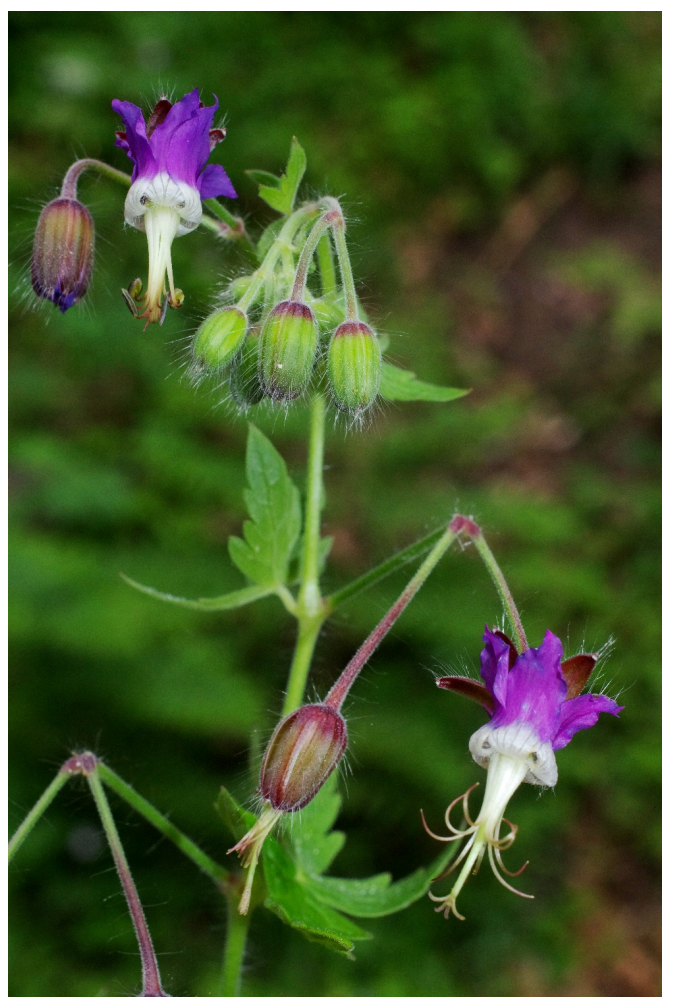

\section{Geranium robertianum}

276.759, loa 1069, NW Metsovo; 276.877, loa 1072, NNW Metsovo; 277.159, loa 1086, NNO Metsovo; 277.195, loa 1087, NNO Metsovo; 277.264, loa 1090, NNO Metsovo; 277.537, loa 1097, S Anilio; 280.311, Dra 229, SO Dendrakia; 280.519, Dra 236, Skaloti; 280.724, Dra 243, SW Walddorf Elatias; 280.810, Dra 247, Walddorf Elatias; 282.574 , Ser 286, Ori

Vrondous; 283.853, Ima 178, Seli; -20.487, Ioa 1078, O Anilio

\section{Geranium rotundifolium}

279.023, Kav 119, Podochori; 279.342, Ser 261, Proti; 279.681, Ser 265, Myrrini

\section{Geranium sanguineum}

277.418, loa 1094, S Anilio; 279.468, Kav 96, Pangaion; 279.550, Kav 101, Pangaion; 280.117, Dra 220, Sidironero; 280.220, Dra 225, NO Drama; 280.245, Dra 227, Livadero; 280.377, Dra 232, Sidironero; 280.484, Dra 234, Skaloti; 280.542, Dra 236, Skaloti; 280.816, Dra 248, Falakron; 281.834, Dra 284, NNO Volakas; 282.062, Dra 294, NO Nevrokopi; 282.230, Dra 300, SO Lefkogia; 282.486, Ser 282, Ori Vrondous

\section{Geranium subcaulescens}

283.316, Pel 350, Kajmaktsalan

\section{Geranium versicolor}

276.736, loa 1067, NW Metsovo; 276.872, loa 1072, NNW Metsovo; 277.520, loa 1097, S Anilio; 281.626, Dra 279, Kato Vrondous; 282.344, Ser 275, SSW Ano Vrondous; 284.080, Ioa 1119, Anilio; 284.137, loa 1096 b, S Anilio 


\section{Geum urbanum}

276.380, loa 1128, N Metsovo; 276.451, Tri 457, NO Katara-Pass; 276.597, loa 1061, SSO Potamia; 276.881, loa 1072, NNW Metsovo; 277.185, loa 1086, NNO Metsovo; 277.259, Ioa 1090, NNO Metsovo; 277.513, loa 1097, S Anilio; 277.944, Koz 392, SO Siatista,

Vourinos; 280.329, Dra 230, Livadero; 280.672, Dra 242, SW Walddorf Elatias; 282.244 , Dra 301, Ano Vrondous; 282.484, Ser 282, Ori Vrondous; 282.566, Ser 285, Ori Vrondous; 282.748, Ser 292, N Neo Petritsi; 283.251, Pel 346, Kajmaktsalan; 283.567, Pel 363, NW Promachi; 283.747, Ima 172, S Ag. Pavlos; 283.837, Ima 177, Tria-Pende Pigadia; - 20.504, Dra 236, Skaloti

\section{Gladiolus imbricatus}

276.749, loa 1068, NW Metsovo; 276.852, loa 1071, NNW Metsovo

\section{Glaucium flavum}

278.690, Kav 107, Paralia Ofryniou

\section{Globularia bisnagarica}

280.856, Dra 248, Falakron; 281.447, Dra 274, Vathytopo

\section{Globularia cordifolia}

280.895, Dra 250, Falakron

\section{Glyceria notata}

277.623, loa 1104, S Anilio; 281.372, Dra 271, Vathytopo

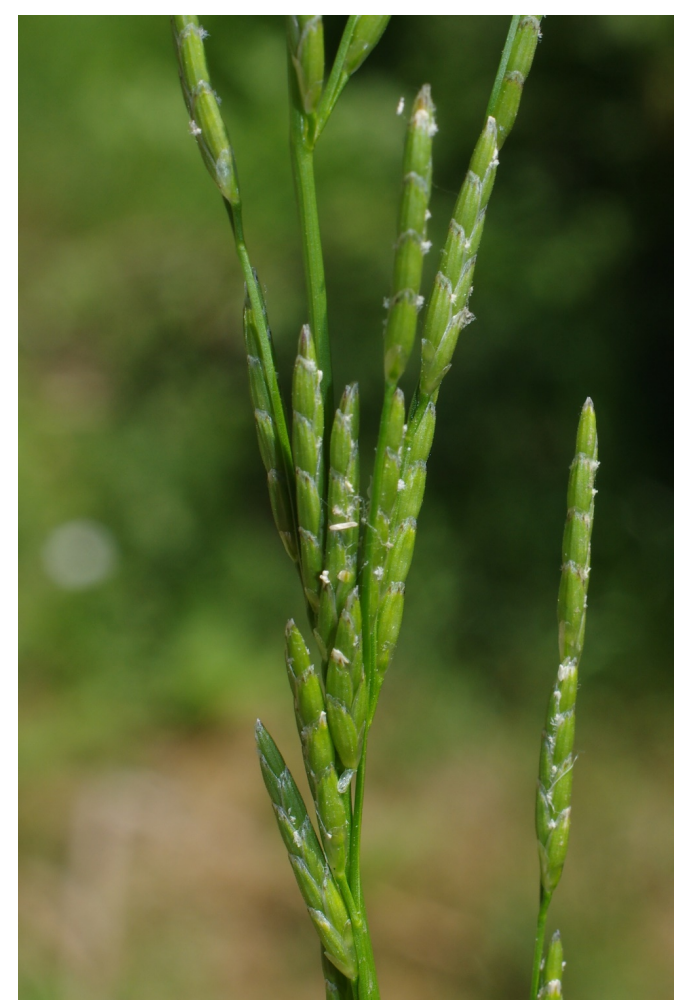

\section{Gnaphalium sylvaticum}

283.953, loa 1109, NW Metsovo

\section{Goniolimon tataricum}

278.722, Kav 108, Paralia Ofryniou; 279.696, Ser 266, SO Nea Zichni; 281.164, Dra 263, NW Granitis; 281.446, Dra 274, Vathytopo

\section{Gymnadenia conopsea}

276.963, loa 1078, O Anilio; 277.652, loa 1106, Anilio; 280.545, Dra 237, Skaloti; 280.744, Dra 245, SW Walddorf Elatias; 280.884, Dra 250, Falakron; 283.033, Kil 303, SO Livadia; -20.493, loa 1083, N Metsovo 
Haplophyllum suaveolens

277.850, Koz 387, SO Siatista, Vourinos; 278.877, Kav 116, S Ofrynia; 279.629, Ser 263, Dravoskas

Hedypnois rhagadioloides subsp. rhagadioloides

276.504, loa 1059, Ag. Nikolaos

Helianthemum nummularium subsp. nummularium

276.344, loa 1127, Dodonoupoli; 276.779, loa 1069, NW Metsovo; 277.186, loa 1086, NNO Metsovo; 277.382, loa 1093, S Anilio; 277.653, loa 1106, Anilio; 277.710, Tri 460, N Chaliki; 277.874, Koz 388, SO Siatista, Vourinos; 279.479, Kav 97, Pangaion; 280.826, Dra 248, Falakron; 281.661, Ser 270, Kato Vrondous; 281.745, Dra 281, OSO Granitis; 283.006, Kil 302, Kastaneri; -20.471, loa 1053, NO Dodoni

Helianthemum nummularium subsp. tomentosum 280.593, Dra 238, Skaloti

Helianthemum oelandicum subsp. canum

279.607, Kav 105, Pangaion; 280.889, Dra 250, Falakron

Helictochloa aetolica

276.360, loa 1128, N Metsovo

Heliotropium dolosum

283.645, Pel 367, Vorino

Heliotropium europaeum

278.180, Gre 495, Varis; 278.457, Thes 214, Paralia Vrasna; 278.921, Kav 117, Galipsos; 278.976, Kav 119, Podochori; 279.210, Ser 257, Nea Fili; 279.240, Ser 258, Mikro Souli; 279.782, Ser 268, SW Skopia; 281.201, Dra 264, NO Ochyro; 283.687, Pel 369, O Vorino; 283.711, Pel 370, SW Foustani

Heliotropium suaveolens

278.612, Thes 221, Arethousa; 278.638, Kav 106, Paralia Ofryniou; 278.656, Kav 106, Paralia Ofryniou; 278.793, Kav 113, NO Paralia Myrtofytou; 282.822, Ser 296, NO

Strymoniko

Helleborus odorus subsp. cyclophyllus

277.073, loa 1080, SO Anilio; 277.646, loa 1105, Anilio

Helminthotheca echioides

278.634, Kav 106, Paralia Ofryniou; 279.652, Ser 264, Dravoskas; 280.206, Dra 224, NO Drama

Helosciadium nodiflorum

276.620, loa 1062, SO Potamia; 277.390, loa 1094, S Anilio; 277.753 , Tri 461, N Chaliki

Heracleum sphondylium subsp. sphondylium

279.932, Dra 211, Livadero; 280.308, Dra 229, SO Dendrakia; 282.927, Kil 297, WNW

Kastaneri; 283.233, Pel 345, Kerasia; 283.780, Ima 173, S Ag. Pavlos; 283.927, loa 1107, N Metsovo

Heracleum sphondylium subsp. ternatum

280.572, Dra 238, Skaloti; 281.335, Dra 269, W Nevrokopi; 282.240, Dra 301, Ano Vrondous; 282.524, Ser 283, Ori Vrondous

Herniaria glabra 
276.404, loa 1057, NO Metsovo; 280.577, Dra 238, Skaloti; 280.609, Dra 238, Skaloti; 281.687, Ser 271, Kato Vrondous; 282.645, Ser 290, N Neo Petritsi; 282.705, Ser 290, N Neo Petritsi; 283.354, Pel 352, Kajmaktsalan

\section{Herniaria hirsuta}

278.453, Thes 214, Paralia Vrasna; 278.665, Kav 106, Paralia Ofryniou; 279.036, Kav 120 , Podochori; 281.317, Dra 268, W Nevrokopi; 283.721, Pel 370, SW Foustani

\section{Herniaria incana}

277.892, Koz 389, SO Siatista, Vourinos; 278.170, Gre 494, SO Varis; 278.212 , Koz 377, N Siatista; 278.330, Koz 381, Galatini; 278.605, Thes 221, Arethousa; 278.778, Kav 112, NO Paralia Myrtofytou; 279.830, Dra 207, NO Skopia; 280.151, Dra 221, NO Drama; 280.837 , Dra 248, Falakron; 281.205, Dra 265, NO Granitis; 281.697, Dra 280, Nevrokopi; 281.746, Dra 281, OSO Granitis; 282.159, Dra 298, SO Lefkogia; 282.472, Ser 281, Ori Vrondous; 282.752, Ser 292, N Neo Petritsi; 283.465, Pel 358, N Loutraki; 283.878, Ima 179, Seli

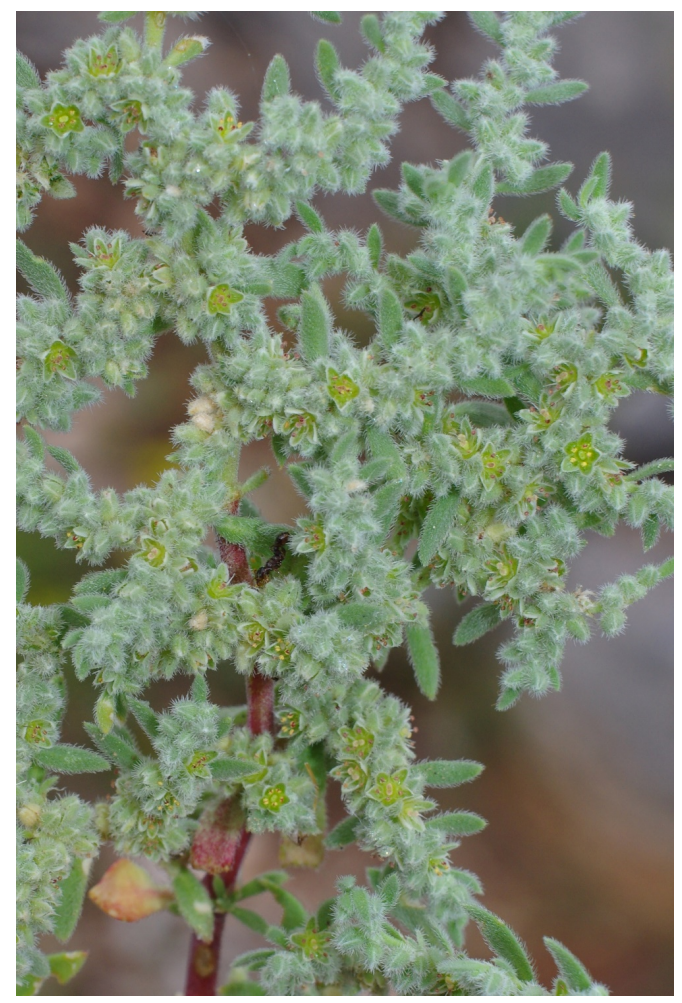

Herniaria parnassica subsp. parnassica

279.547, Kav 99, Pangaion; 281.106, Dra 261, Granitis; 282.222, Dra 300, SO Lefkogia

Hesperis theophrasti subsp. rechingeri

278.007, Koz 375, O Dafnero

Hesperis theophrasti subsp. theophrasti

279.586, Kav 104, Pangaion; 280.880, Dra 250, Falakron

\section{Hieracium}

276.550, loa 1060, SSO Potamia; 276.629, loa 1062, SO Potamia; 276.732, loa 1067, NW Metsovo; 277.231, loa 1089, NNO Metsovo; 277.495, loa 1097, S Anilio; 277.590, loa 1101, S Anilio; 279.821, Ser 269, NO Skopia; 279.822, Ser 269, NO Skopia; 279.950, Dra 212, Livadero; 279.961, Dra 213, Livadero; 279.977, Dra 213, Livadero; 280.424, Dra 233, Sidironero; 280.429, Dra 233, Sidironero; 280.471, Dra 234, Skaloti; 280.544, Dra 237, Skaloti; 280.548, Dra 237, Skaloti; 280.556, Dra 237, Skaloti; 280.680, Dra 242, SW Walddorf Elatias; 280.717, Dra 243, SW Walddorf Elatias; 280.748, Dra 245, SW Walddorf Elatias; 280.960, Dra 255, Falakron; 281.024, Dra 258, Granitis; 281.649, Dra 279, Kato Vrondous; 281.766, Dra 281, OSO Granitis; 281.844, Dra 284, NNO Volakas; 282.211, Dra 300, SO Lefkogia; 282.216, Dra 300, SO Lefkogia; 282.487, Ser 282, Ori Vrondous; 
282.502, Ser 282, Ori Vrondous; 282.505, Ser 282, Ori Vrondous; 282.529 , Ser 283, Ori Vrondous; 282.547, Ser 285, Ori Vrondous; 282.554, Ser 285, Ori Vrondous; 282.702 , Ser 290, N Neo Petritsi; 282.914, Kil 297, WNW Kastaneri; 282.938, Kil 298, WNW Kastaneri; 283.050, Kil 305, SO Livadia; 283.114, Pel 340, SSO Archangelo; 283.229, Pel 345, Kerasia; 283.305, Pel 350, Kajmaktsalan; 283.797, Ima 174, S Ag. Pavlos; 283.802, Ima 174, S Ag.

Pavlos; 283.929, loa 1107, N Metsovo; 284.180, loa 1097, S Anilio

\section{Hieracium olympicum}

282.468, Ser 281, Ori Vrondous; 282.481, Ser 281, Ori Vrondous

\section{Hieracium pannosum}

283.004, Kil 301, Kastaneri; 283.237, Pel 346, Kajmaktsalan; 283.303, Pel 350, Kajmaktsalan

\section{Hieracium sparsum}

283.344, Pel 352, Kajmaktsalan; 283.345, Pel 352, Kajmaktsalan

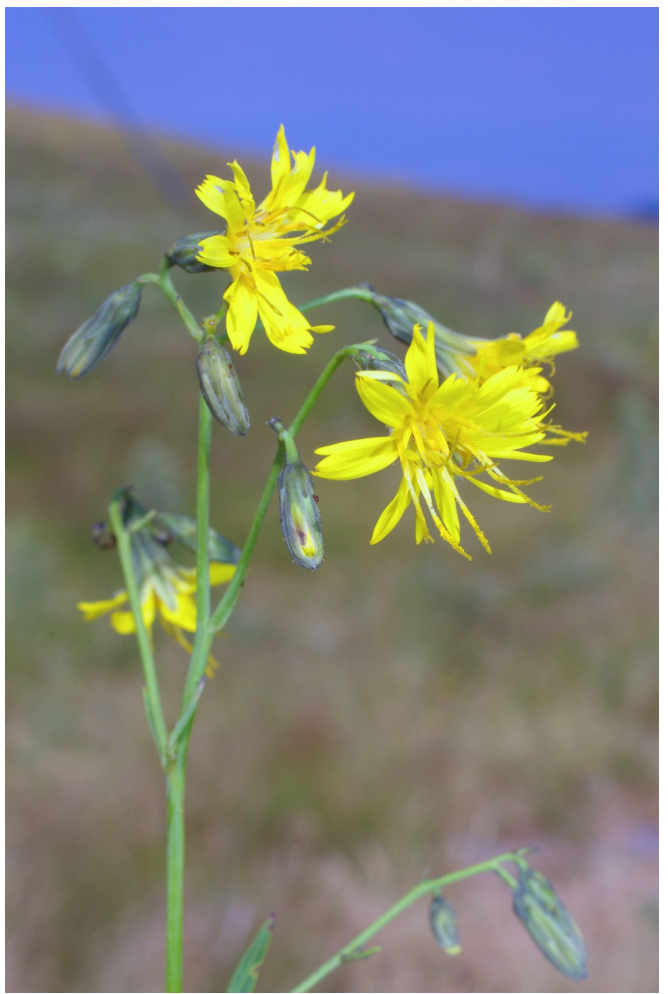

\section{Himantoglossum jankae}

281.452, Dra 274, Vathytopo; 282.037, Dra 293, Nevrokopi

\section{Hippocrepis ciliata}

280.193, Dra 223, NO Drama

\section{Hippocrepis emerus subsp. emeroides}

276.600, loa 1061, SSO Potamia; 277.314, loa 1092, NO Metsovo; 277.958, Koz 374, Vourinos; 280.061, Dra 218, Sidironero; -20.500, Ser 261, Proti

\section{Hirschfeldia incana}

277.776, Tri 462, N Chaliki; 278.491, Thes 215, Paralia Vrasna; 278.619, Thes 221, Arethousa; 278.695, Kav 107, Paralia Ofryniou; 278.790, Kav 112, NO Paralia Myrtofytou; 278.825, Kav 114, SO Folia; 279.044, Kav 120, Podochori; 279.113, Kav 90, SW Moustheni; 279.192, Ser 256, Paleokomi

\section{Holcus lanatus}

276.548, Ioa 1060, SSO Potamia; 277.417, loa 1094, S Anilio; 279.485, Kav 97, Pangaion; 279.892, Dra 209, Livadero; 280.008, Dra 216, Sidironero; 280.275, Dra 228, Livadero; 280.486, Dra 235, Skaloti; 281.225, Dra 265, NO Granitis; 281.319, Dra 268, W Nevrokopi; 281.386, Dra 272, Vathytopo; 281.555, Dra 277, Perithori; 281.721, Dra 280, Nevrokopi; 
281.902, Dra 287, O Mikroklisoura; 282.055, Dra 293, Nevrokopi; 282.116, Dra 296, NO Nevrokopi; 282.919, Kil 297, WNW Kastaneri; 283.116, Pel 340, SSO Archangelo; 283.159 , Pel 343, Kerasia; 283.416, Pel 355, NW Loutraki; 283.546, Pel 362, NW Promachi; 283.862, Ima 178, Seli; -20.511, Dra 236, Skaloti

\section{Hordelymus europaeus}

280.564, Dra 238, Skaloti; 281.062, Dra 259, Granitis; 282.590, Ser 287, Ori Vrondous; 283.019, Kil 302, Kastaneri; 284.227, Ser 287, Ori Vrondous

\section{Hordeum bulbosum}

277.701, Tri 460, N Chaliki; 278.281, Koz 379, Galatini

\section{Hordeum geniculatum}

276.624, loa 1062, SO Potamia; 276.814, loa 1070, NNW Metsovo; 277.142, loa 1084, N Metsovo; 277.283, loa 1090, NNO Metsovo

\section{Hordeum marinum}

277.068, loa 1080, SO Anilio; 284.018, loa 1115, N Metsovo

\section{Hordeum murinum}

277.469, loa 1096, S Anilio; 279.214, Ser 257, Nea Fili; 281.376, Dra 271, Vathytopo; 281.506, Dra 276, Perithori

\section{Hordeum murinum subsp. leporinum}

276.539, loa 1059, Ag. Nikolaos; 277.125, loa 1084, N Metsovo; 278.464, Thes 214, Paralia Vrasna; 278.509, Thes 217, Vrasna; 278.647, Kav 106, Paralia Ofryniou; 278.827, Kav 114, SO Folia; 279.174, Ser 256, Paleokomi; 279.302, Ser 259, Proti; 281.944, Dra 288, SW Potamia

\section{Hordeum vulgare subsp. spontaneum}

277.377, loa 1093, S Anilio

Hymenocarpus circinnatus

278.986, Kav 119, Podochori

\section{Hypericum barbatum}

277.269, loa 1090, NNO Metsovo; 277.650, loa 1105, Anilio; 277.718, Tri 460, N Chaliki

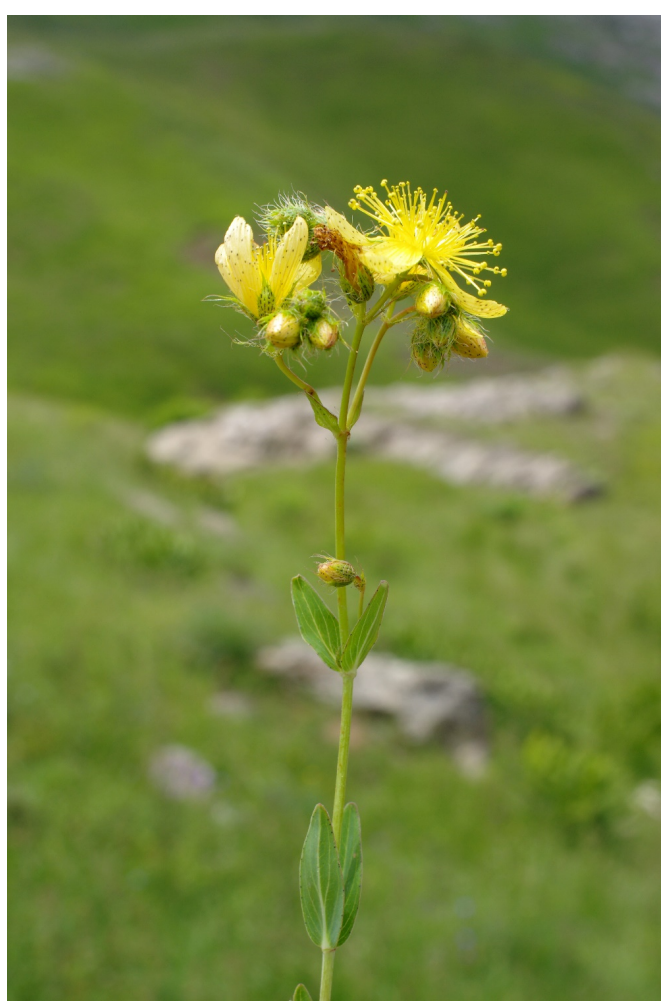


Hypericum linarioides

276.333, loa 1126, NO Dodoni; 280.877, Dra 249, Falakron

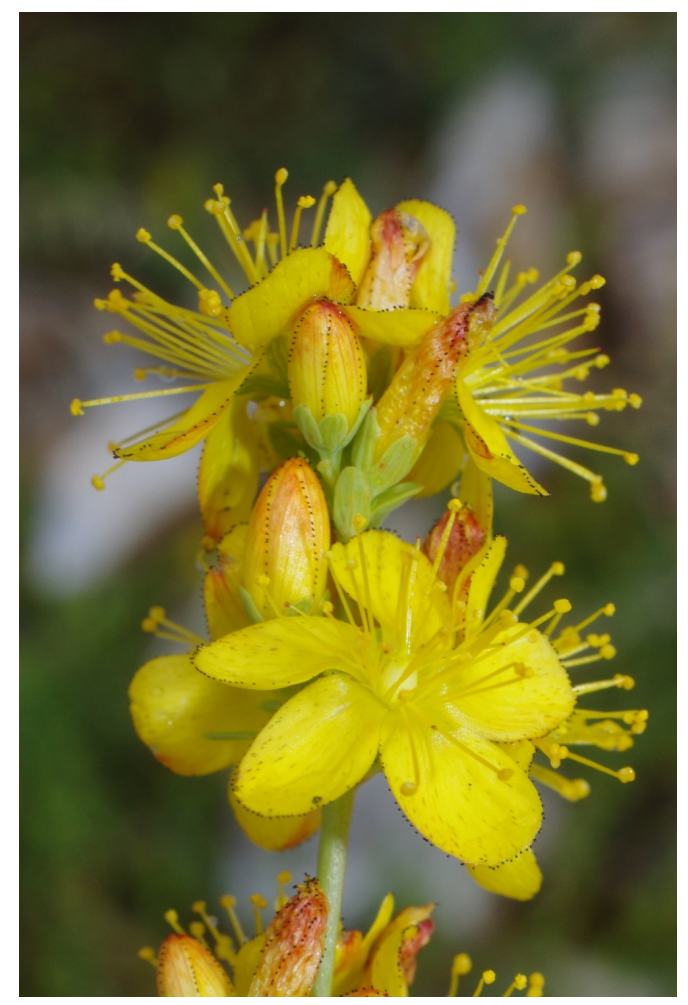

Hypericum maculatum

280.629, Dra 240, Elatias

\section{Hypericum montbretii}

279.389, Ser 262, Proti; 279.522, Kav 99, Pangaion

\section{Hypericum olympicum}

278.492, Thes 216, Vrasna; 278.516, Thes 217, Vrasna; 279.899, Dra 210, Livadero; 280.089, Dra 219, Sidironero; 280.128, Dra 221, NO Drama; 280.405, Dra 233, Sidironero; 280.890, Dra 250, Falakron; 281.224, Dra 265, NO Granitis; 281.347, Dra 270, W Nevrokopi; 281.573, Dra 277, Perithori; 281.597, Dra 278, Kato Vrondous; 282.100, Dra 295, NO Nevrokopi; 282.456, Ser 280, Orini; 282.671, Ser 290, N Neo Petritsi

\section{Hypericum perfoliatum}

283.752, Ima 172, S Ag. Pavlos

\section{Hypericum perforatum}

276.507, loa 1059, Ag. Nikolaos; 277.318, loa 1092, NO Metsovo; 277.325, loa 1093, S Anilio; 277.758, Tri 461, N Chaliki; 277.843, Koz 387, SO Siatista, Vourinos; 278.442, Thes 214, Paralia Vrasna; 278.520, Thes 217, Vrasna; 278.616, Thes 221, Arethousa; 278.651, Kav 106, Paralia Ofryniou; 278.755, Kav 109, Loutra Eleftherion; 278.782, Kav 112, NO Paralia Myrtofytou; 278.869, Kav 115, SW Folia; 278.886, Kav 116, S Ofrynia; 279.000, Kav 119, Podochori; 279.076, Kav 121, Platanotopos; 279.169, Ser 255, S Nea Mesolakkia; 279.225, Ser 257, Nea Fili; 279.245, Ser 258, Mikro Souli; 279.295, Ser 259, Proti; 279.410, Kav 92, Pangaion; 279.460, Kav 95, Pangaion; 279.620, Ser 263, Dravoskas; 279.686, Ser 266, SO Nea Zichni; 279.805, Ser 269, NO Skopia; 279.900, Dra 210, Livadero; 279.904, Dra 210, Livadero; 280.045, Dra 217, Sidironero; 280.132, Dra 221, NO Drama; 280.294 , Dra 228, Livadero; 280.358, Dra 232, Sidironero; 280.435, Dra 234, Skaloti; 280.937, Dra 253, Falakron; 281.026, Dra 258, Granitis; 281.171, Dra 264, NO Ochyro; 281.183, Dra 264, NO Ochyro; 281.448, Dra 274, Vathytopo; 281.531, Dra 276, Perithori; 281.586, Dra 278, 
Kato Vrondous; 281.809, Dra 283, NNO Volakas; 281.879, Dra 287, O Mikroklisoura; 281.993, Dra 291, W Prosotsani; 282.119, Dra 296, NO Nevrokopi; 282.232, Dra 300, SO Lefkogia; 282.316, Ser 274, SSW Ano Vrondous; 282.397, Ser 278, N Serres; 282.440 , Ser 280, Orini; 282.551, Ser 285, Ori Vrondous; 282.621, Ser 288, Ori Vrondous; 282.657 , Ser 290, N Neo Petritsi; 282.865, Kil 295, NW Griva; 282.944, Kil 298, WNW Kastaneri; 283.096, Pel 339, S Archangelo; 283.224, Pel 345, Kerasia; 283.426, Pel 355, NW Loutraki; 283.537, Pel 362, NW Promachi; 283.724, Pel 370, SW Foustani; 283.916, Ima 180, Seli; 20.455, loa 1052, NO Dodoni; -20.481, loa 1066, NO Karyes; -20.512, Dra 236, Skaloti

\section{Hypericum rochelii}

277.373, loa 1093, S Anilio

\section{Hypericum rumeliacum}

276.579, loa 1060, SSO Potamia; 277.799, Koz 385, SO Siatista; 277.921, Koz 391, SO Siatista, Vourinos; 278.112, Gre 492, NO Varis; 278.208, Koz 377, N Siatista; 278.216, Koz 378, N Siatista; 278.412, Koz 383, Galatini; 280.820, Dra 248, Falakron; 282.202, Dra 300, SO Lefkogia

\section{Hypericum tetrapterum}

283.519, Pel 361, SW Promachi; 283.937, loa 1108, N Metsovo

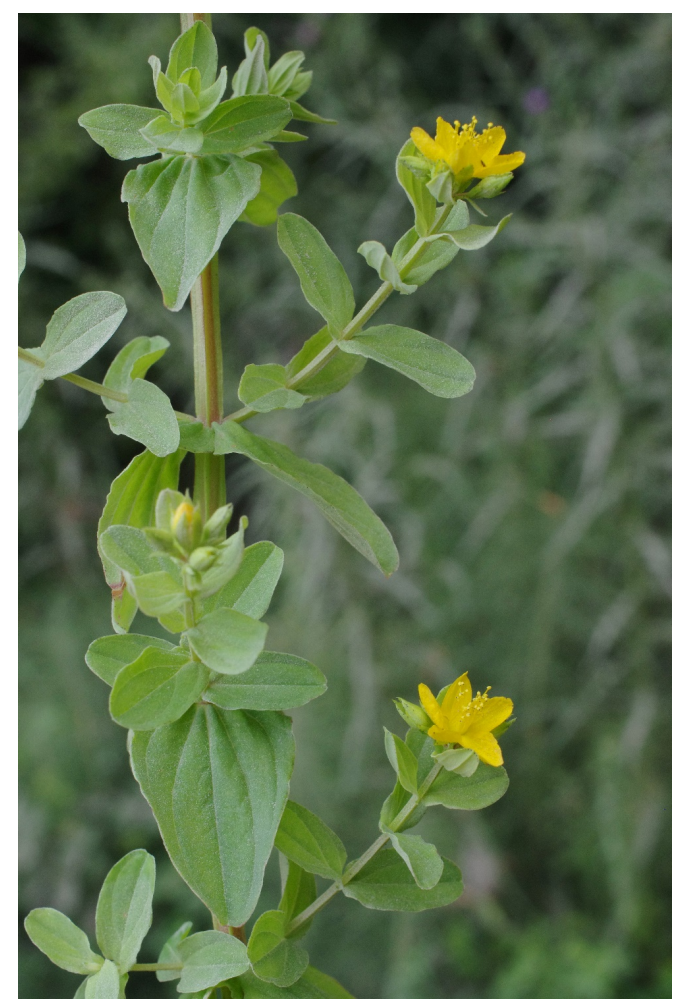

\section{Hypericum triquetrifolium}

280.780, Dra 247, Walddorf Elatias; 284.123, loa 1094 b, S Anilio

\section{Hypochaeris cretensis}

276.319, loa 1125, NO Dodoni; 276.512, loa 1059, Ag. Nikolaos; 276.524, loa 1059, Ag. Nikolaos; 276.685, loa 1065, NO Karyes; 276.843, loa 1071, NNW Metsovo; 277.444, loa 1095, S Anilio; 277.528, loa 1097, S Anilio; 277.720, Tri 460, N Chaliki; 277.849, Koz 387, SO Siatista, Vourinos; 278.848, Kav 115, SW Folia

\section{Hypochaeris glabra}

278.463, Thes 214, Paralia Vrasna

\section{Hypochaeris maculata}

276.513, loa 1059, Ag. Nikolaos; 278.820, Kav 114, SO Folia; 278.844, Kav 115, SW Folia; 279.982, Dra 214, Livadero; 280.580, Dra 238, Skaloti; 280.769, Dra 246, SW Walddorf Elatias; 280.974, Dra 256, Falakron; 281.552, Dra 277, Perithori; 282.414, Ser 279, Orini; 
282.444, Ser 280, Orini; 283.225, Pel 345, Kerasia; 283.480, Pel 358, N Loutraki; 283.564 , Pel 363, NW Promachi

\section{Hypochaeris radicata}

277.096, loa 1084, N Metsovo; 280.646, Dra 241, Elatias; 283.249, Pel 346, Kajmaktsalan

Hypopitys monotropa subsp. monotropa

279.519, Kav 99, Pangaion

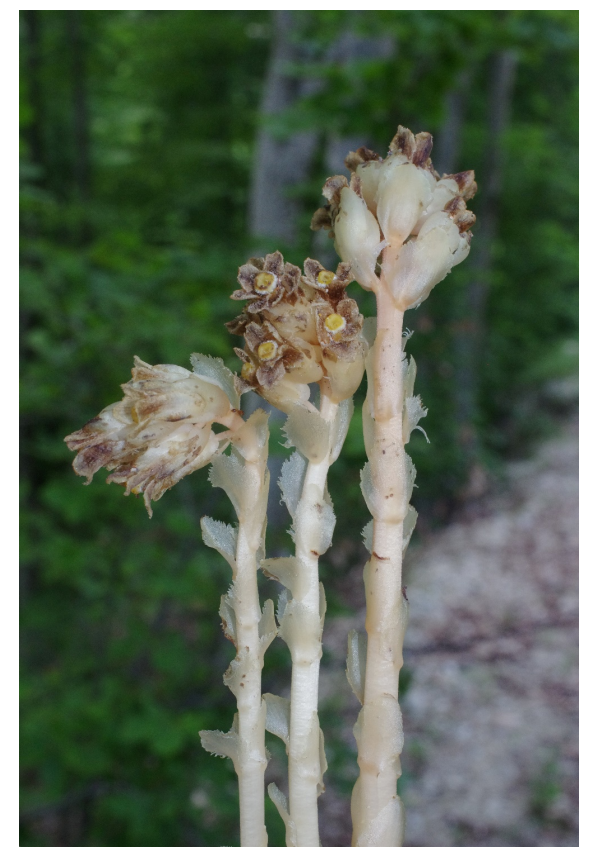

\section{Imperata cylindrica}

278.796, Kav 113, NO Paralia Myrtofytou; 279.850, Dra 208, NO Skopia

\section{Inula britannica}

281.792, Dra 282, NNO Volakas

\section{Inula conyzae}

279.820, Ser 269, NO Skopia; 283.552, Pel 362, NW Promachi; 283.811, Ima 176, TriaPende Pigadia

\section{Inula ensifolia}

281.159, Dra 262, NW Granitis

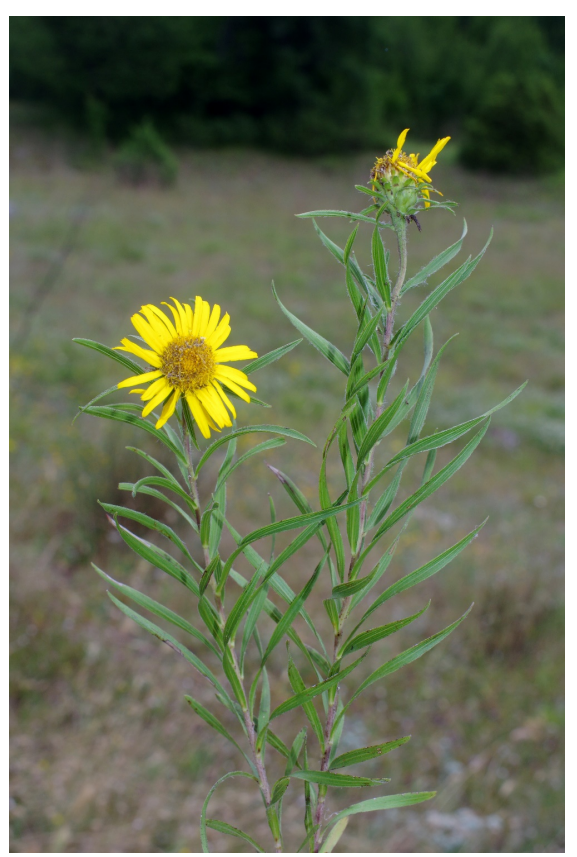




\section{Inula oculus-christi}

277.887, Koz 388, SO Siatista, Vourinos; 277.917, Koz 391, SO Siatista, Vourinos; 278.026, Koz 376, W Exarcho; 278.410, Koz 383, Galatini; 280.616, Dra 238, Skaloti; 280.819, Dra 248, Falakron; 280.951, Dra 255, Falakron; 281.134, Dra 262, NW Granitis; 281.236, Dra 265, NO Granitis; 281.486, Dra 275, Vathytopo; 282.031, Dra 292, Nevrokopi; 282.380, Ser 276, SSW Ano Vrondous; 282.955, Kil 299, Kastaneri; 283.442, Pel 356, NW Loutraki; 283.495, Pel 359, NW Loutraki; 283.813, Ima 176, Tria-Pende Pigadia; 283.886, Ima 179, Seli; 283.951, loa 1109, NW Metsovo; 283.971, loa 1056 b, NO Metsovo; 284.028, loa 1116, SW Milia; 284.064, Ioa 1118, Anilio

\section{Inula salicaria}

283.444, Pel 356, NW Loutraki

\section{Inula salicina}

278.482, Thes 215, Paralia Vrasna; 279.486, Kav 97, Pangaion; 280.248, Dra 227, Livadero; 282.933, Kil 298, WNW Kastaneri; 282.999, Kil 301, Kastaneri; 283.127, Pel 340, SSO

Archangelo; 283.158, Pel 343, Kerasia

Inula verbascifolia subsp. aschersoniana

279.027, Kav 120, Podochori; 279.802, Ser 269, NO Skopia; 282.846, Kil 294, NW Griva; 283.451, Pel 357, NW Loutraki

\section{Ipomoea purpurea}

281.415, Dra 273, Vathytopo; 282.818, Ser 296, NO Strymoniko

\section{Iris sintenisii}

277.956, Koz 374, Vourinos

Isatis tinctoria subsp. tinctoria

283.888, Ima 179, Seli

\section{Isolepis cernua}

277.403, loa 1094, S Anilio

\section{Jasione heldreichii}

278.715, Kav 108, Paralia Ofryniou; 279.953, Dra 212, Livadero; 281.223, Dra 265, NO Granitis; 281.351, Dra 270, W Nevrokopi; 281.591, Dra 278, Kato Vrondous; 282.123, Dra 296, NO Nevrokopi; 282.195, Dra 299, SO Lefkogia; 282.423, Ser 279, Orini; 282.672, Ser 290, N Neo Petritsi; 282.980, Kil 300, Kastaneri; 283.463, Pel 358, N Loutraki

\section{Jasione orbiculata}

283.336, Pel 351, Kajmaktsalan

\section{Juncus}

276.418, loa 1057, NO Metsovo; 277.620, loa 1104, S Anilio

\section{Juncus articulatus}

277.399, loa 1094, S Anilio; 277.759, Tri 461, N Chaliki; 278.564, Thes 219, Vrasna; 278.767, Kav 110, Loutra Eleftherion; 281.796, Dra 282, NNO Volakas; 283.289, Pel 349, Kajmaktsalan; 283.525, Pel 361, SW Promachi

\section{Juncus bufonius}

276.650, loa 1063, W Potamia; 277.404, loa 1094, S Anilio; 280.345, Dra 230, Livadero; 281.118, Dra 261, Granitis; 281.378, Dra 271, Vathytopo; 281.561, Dra 277, Perithori; 281.768, Dra 282, NNO Volakas; 282.971, Kil 299, Kastaneri

\section{Juncus compressus}

281.374, Dra 271, Vathytopo

\section{Juncus conglomeratus}




\section{Juncus effusus}

279.917, Dra 210, Livadero; 280.760, Dra 246, SW Walddorf Elatias; 281.367, Dra 271, Vathytopo; 282.246, Dra 301, Ano Vrondous

\section{Juncus inflexus}

276.674, loa 1064, ONO Karyes; 276.900, loa 1073, NNW Metsovo; 277.115, loa 1084, N Metsovo; 277.273, loa 1090, NNO Metsovo; 277.395, loa 1094, S Anilio; 277.691, Tri 460, N Chaliki; 279.134, Kav 91, Orfani; 281.885, Dra 287, O Mikroklisoura; 282.138, Dra 297, Lefkogia; 282.989, Kil 300, Kastaneri; 283.274, Pel 348, Kajmaktsalan; 283.543, Pel 362, NW Promachi; 283.739, Ima 172, S Ag. Pavlos; 283.830, Ima 176, Tria-Pende Pigadia

\section{Juncus minutulus}

284.213, Dra 261, Granitis

\section{Juncus tenuis}

276.603, loa 1061, SSO Potamia; 279.916, Dra 210, Livadero; 280.532, Dra 236, Skaloti

\section{Juncus thomasii}

283.323, Pel 351, Kajmaktsalan

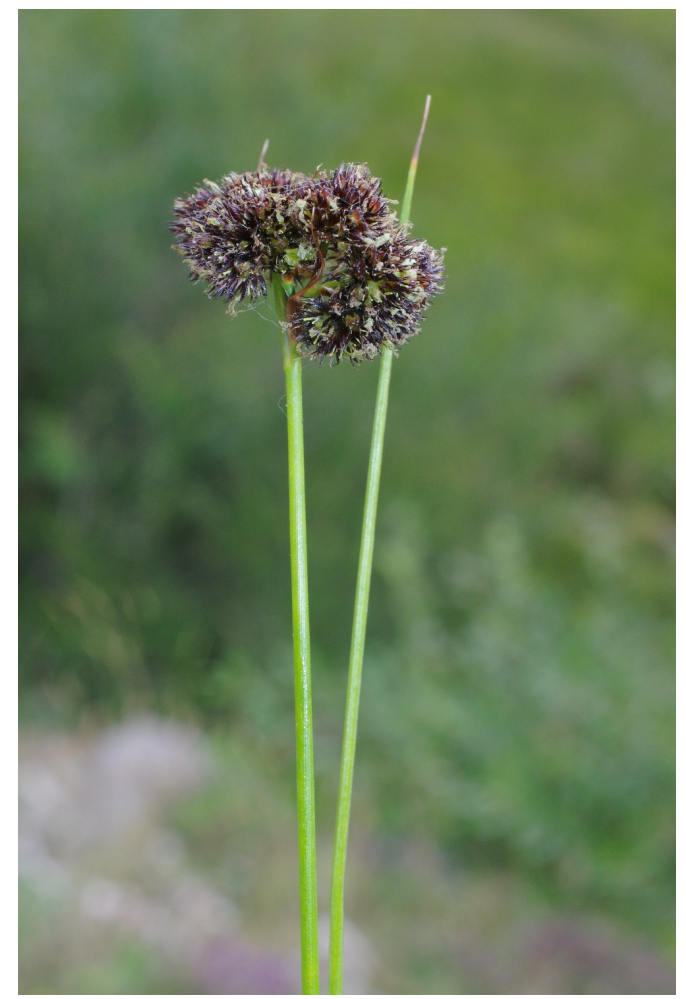

Jurinea mollis subsp. glycacantha

278.004, Koz 375, O Dafnero

Jurinea mollis subsp. mollis

280.617, Dra 238, Skaloti; 280.618, Dra 238, Skaloti; 280.817, Dra 248, Falakron; 281.154, Dra 262, NW Granitis

\section{Kickxia commutata subsp. commutata}

281.925, Dra 288, SW Potamia

Kickxia elatine subsp. crinita

278.156, Gre 494, SO Varis; 278.577, Thes 220, Arethousa

Kickxia elatine subsp. elatine

283.654, Pel 367, Vorino 
Kickxia spuria subsp. integrifolia

278.927, Kav 117, Galipsos

\section{Knautia ambigua}

280.442, Dra 234, Skaloti; 281.707, Dra 280, Nevrokopi

\section{Knautia arvensis}

279.518, Kav 99, Pangaion; 280.425, Dra 233, Sidironero; 280.958, Dra 255, Falakron; 280.962, Dra 255, Falakron; 281.034, Dra 258, Granitis; 281.312, Dra 268, W Nevrokopi; 281.784, Dra 282, NNO Volakas; 282.191, Dra 299, SO Lefkogia; 282.277, Ser 272, Ano Vrondous; 282.323, Ser 274, SSW Ano Vrondous; 282.458, Ser 280, Orini; 282.556, Ser 285, Ori Vrondous; 282.792, Ser 295, N Neo Petritsi; 284.232, Dra 292, Nevrokopi

\section{Knautia drymeia subsp. nympharum}

277.554, loa 1099, S Anilio; 279.435, Kav 94, Pangaion; 279.437, Kav 95, Pangaion; 280.249, Dra 227, Livadero; 280.511, Dra 236, Skaloti; 280.716, Dra 243, SW Walddorf Elatias; 281.635, Dra 279, Kato Vrondous; 281.843, Dra 284, NNO Volakas; 283.052, Kil 305, SO Livadia; 283.095, Pel 339, S Archangelo; 283.771, Ima 173, S Ag. Pavlos; 283.856, Ima 178, Seli; 283.934, loa 1108, N Metsovo; 284.069, loa 1118, Anilio; 284.154, loa 1122, Anilio; 284.201, Koz 385, SO Siatista

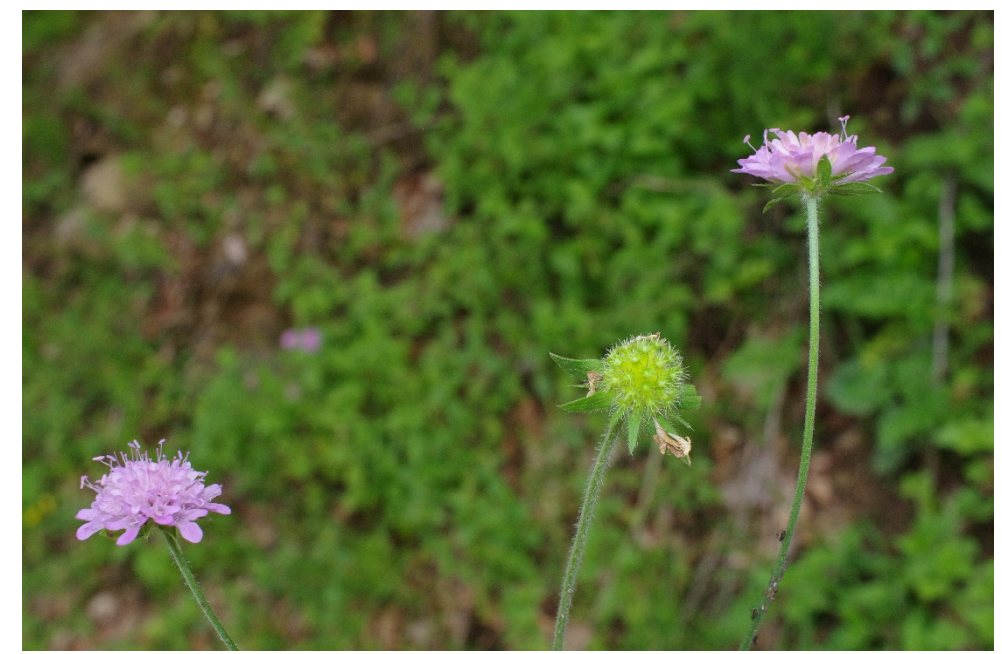

Knautia integrifolia

276.342, loa 1127, Dodonoupoli; 276.498, loa 1059, Ag. Nikolaos; 277.452, loa 1095, S Anilio; 277.788, Koz 385, SO Siatista; 278.245, Koz 378, N Siatista; 278.384, Koz 382, Galatini; 278.487, Thes 215, Paralia Vrasna; 279.082, Kav 89, Misoropi; 281.471, Dra 275, Vathytopo

\section{Knautia longifolia}

280.692, Dra 242, SW Walddorf Elatias

\section{Knautia macedonica}

278.053, Gre 491, NO Exarcho

\section{Knautia magnifica}

280.277, Dra 228, Livadero; 280.318, Dra 230, Livadero

\section{Knautia midzorensis}

277.556, loa 1099, S Anilio; 279.915, Dra 210, Livadero; 280.026, Dra 216, Sidironero; 280.782, Dra 247, Walddorf Elatias

\section{Knautia orientalis}

280.154, Dra 221, NO Drama

\section{Koeleria eriostachya}


280.900, Dra 250, Falakron

\section{Koeleria lobata}

276.932, loa 1074, NNW Metsovo

\section{Koeleria macrantha}

278.074, Gre 492, NO Varis; 281.976, Dra 290, NO Potamia

\section{Koeleria nitidula}

276.839, loa 1070, NNW Metsovo; 277.003, loa 1079, SO Anilio; 277.348, loa 1093, S Anilio; 277.684, Tri 460, N Chaliki; 277.801, Koz 385, SO Siatista; 277.882, Koz 388, SO Siatista, Vourinos; 277.978, Koz 374, Vourinos; 278.203, Koz 377, N Siatista; 278.343, Koz 381, Galatini; 278.948, Kav 118, Galipsos; 279.557, Kav 102, Pangaion; 280.000, Dra 215, Livadero; 280.067, Dra 218, Sidironero; 280.088, Dra 219, Sidironero; 280.161, Dra 221, NO Drama; 280.250, Dra 227, Livadero; 280.381, Dra 232, Sidironero; 280.470, Dra 234, Skaloti; 280.828, Dra 248, Falakron; 281.156, Dra 262, NW Granitis; 281.352, Dra 270, W Nevrokopi; 281.657, Ser 270, Kato Vrondous; 281.857, Dra 285, NNO Volakas; 282.169 , Dra 299, SO Lefkogia; 282.438, Ser 280, Orini; 282.463, Ser 281, Ori Vrondous; 282.469 , Ser 281, Ori Vrondous; 282.652, Ser 290, N Neo Petritsi; 282.916, Kil 297, WNW Kastaneri; 283.093, Pel 339, S Archangelo; 283.253, Pel 346, Kajmaktsalan; 283.312, Pel 350, Kajmaktsalan; 284.204, Koz 381, Galatini; 284.205, Dra 221, NO Drama; 284.206, Pel 350, Kajmaktsalan

\section{Koeleria pyramidata}

276.799, loa 1070, NNW Metsovo; 276.980, loa 1079, SO Anilio; 277.088, loa 1081, W Metsovo; 278.107, Gre 492, NO Varis; 278.232, Koz 378, N Siatista; 279.525, Kav 99, Pangaion; 279.605, Kav 105, Pangaion; 280.936, Dra 253, Falakron; 283.318, Pel 350, Kajmaktsalan

\section{Lactuca intricata}

277.006, loa 1079, SO Anilio

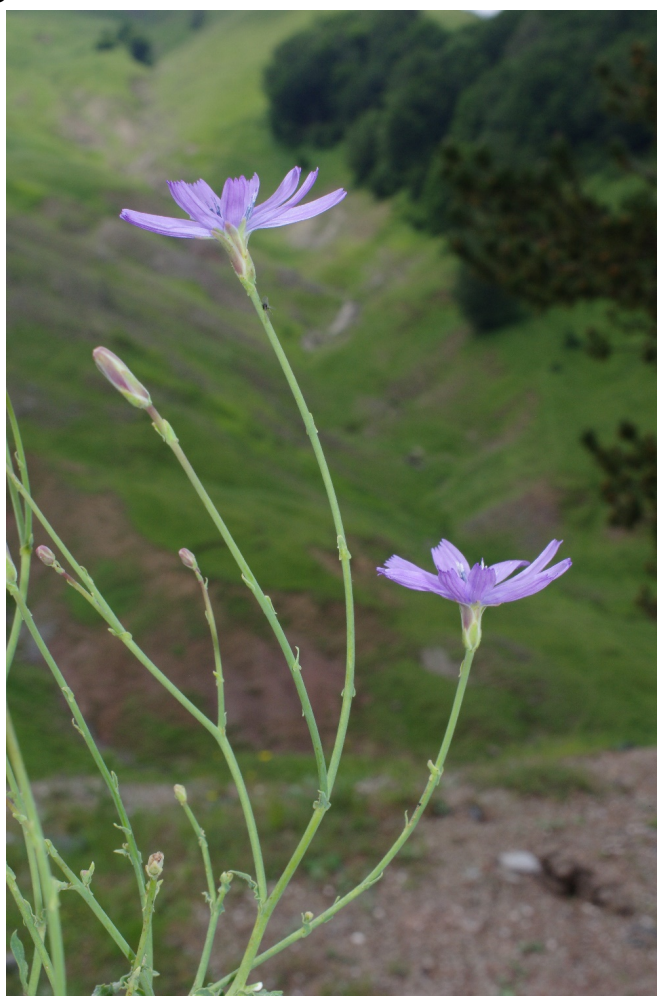

\section{Lactuca muralis}

277.509, loa 1097, S Anilio; 279.505, Kav 98, Pangaion; 279.534, Kav 100, Pangaion; 279.970, Dra 213, Livadero; 280.036, Dra 216, Sidironero; 280.502, Dra 236, Skaloti; 280.703, Dra 243, SW Walddorf Elatias; 280.950, Dra 255, Falakron; 281.628, Dra 279, Kato Vrondous; 282.238, Dra 301, Ano Vrondous; 282.507, Ser 282, Ori Vrondous; 282.698, Ser 
290, N Neo Petritsi; 282.885, Kil 295, NW Griva; 283.110, Pel 339, S Archangelo; 283.216, Pel 345, Kerasia; 283.425, Pel 355, NW Loutraki; 283.547, Pel 362, NW Promachi; 283.833, Ima 177, Tria-Pende Pigadia; 283.852, Ima 178, Seli; -20.520., Ser 285, NNO Volakas

\section{Lactuca saligna}

284.067, loa 1118, Anilio

\section{Lactuca serriola}

279.124, Kav 91, Orfani; 281.002, Dra 257, Granitis; 281.256, Dra 266, W Nevrokopi;

282.798, Ser 296, NO Strymoniko; 283.664, Pel 368, OSO Vorino

\section{Lactuca viminea subsp. ramosissima}

276.328, loa 1125, NO Dodoni; 284.126, loa 1094 b, S Anilio

\section{Lagoecia cuminoides}

279.319, Ser 260, Proti

\section{Lagurus ovatus}

278.456, Thes 214, Paralia Vrasna

\section{Lamium amplexicaule}

278.293, Koz 380, Galatini

\section{Lamium garganicum}

276.438, loa 1058, Katara-Pass; 277.964, Koz 374, Vourinos; 278.428, Koz 384, Galatini; 280.735, Dra 244, SW Walddorf Elatias; 284.203, Ser 282, Ori Vrondous

\section{Lamium maculatum}

282.291, Ser 272, Ano Vrondous; 282.511, Ser 282, Ori Vrondous; 282.603, Ser 288, Ori Vrondous; 282.696, Ser 290, N Neo Petritsi

\section{Lapsana communis subsp. adenophora}

276.625, loa 1062, SO Potamia; 276.906, loa 1073, NNW Metsovo; 277.255, loa 1089, NNO Metsovo; 277.458, loa 1096, S Anilio; 277.930, Koz 392, SO Siatista, Vourinos; 279.919, Dra 211, Livadero; 280.033, Dra 216, Sidironero; 280.474, Dra 234, Skaloti; 280.721, Dra 243, SW Walddorf Elatias; 280.995, Dra 257, Granitis; 281.473, Dra 275, Vathytopo; 281.617, Dra 278, Kato Vrondous; 282.237, Dra 301, Ano Vrondous; 282.312, Ser 274, SSW Ano Vrondous; 282.503, Ser 282, Ori Vrondous; 282.699, Ser 290, N Neo Petritsi; 282.901 , Kil 296, N Kastaneri; 283.092, Pel 339, S Archangelo; 283.182, Pel 343, Kerasia; 283.742, Ima 172, S Ag. Pavlos; 283.822, Ima 176, Tria-Pende Pigadia; 283.870, Ima 178, Seli; 20.507, Dra 236, Skaloti; -20.519, Ser 285, NNO Volakas

\section{Lathyrus aphaca}

277.471, loa 1096, S Anilio

\section{Lathyrus grandiflorus}

277.957, Koz 374, Vourinos; 283.204, Pel 344, Kerasia

\section{Lathyrus hirsutus}

278.273, Koz 379, Galatini

\section{Lathyrus latifolius}

281.082, Dra 260, Granitis

\section{Lathyrus laxiflorus}

276.520, loa 1059, Ag. Nikolaos; 276.711, loa 1065, NO Karyes; 277.227, loa 1089, NNO Metsovo; 277.498, loa 1097, S Anilio; 277.862, Koz 388, SO Siatista, Vourinos; 277.974, Koz 374, Vourinos; 278.866, Kav 115, SW Folia; 279.347, Ser 261, Proti; 279.541, Kav 101, Pangaion; 279.979, Dra 214, Livadero; 280.388, Dra 232, Sidironero; 281.469, Dra 275, Vathytopo; 282.354, Ser 275, SSW Ano Vrondous; 282.932, Kil 297, WNW Kastaneri; 
283.573, Pel 363, NW Promachi; 283.820, Ima 176, Tria-Pende Pigadia; 283.865, Ima 178, Seli

\section{Lathyrus nissolia}

276.396, loa 1056, NO Metsovo; 276.800, loa 1070, NNW Metsovo; 276.922, loa 1074, NNW Metsovo; 277.108, loa 1084, N Metsovo; 277.254, loa 1089, NNO Metsovo; 279.947, Dra 212, Livadero

\section{Lathyrus pratensis}

276.905, loa 1073, NNW Metsovo; 277.187, loa 1086, NNO Metsovo; 277.223, loa 1088, NNO Metsovo; 277.261, loa 1090, NNO Metsovo; 277.491, loa 1097, S Anilio; 280.509, Dra 236, Skaloti; 280.677, Dra 242, SW Walddorf Elatias; 280.913, Dra 251, Falakron; 281.091, Dra 260, Granitis; 282.861, Kil 295, NW Griva; 282.992, Kil 300, Kastaneri; 283.302, Pel 350, Kajmaktsalan; 283.826, Ima 176, Tria-Pende Pigadia

\section{Lathyrus setifolius}

276.725, loa 1066, NO Karyes; 278.418 , Koz 384, Galatini

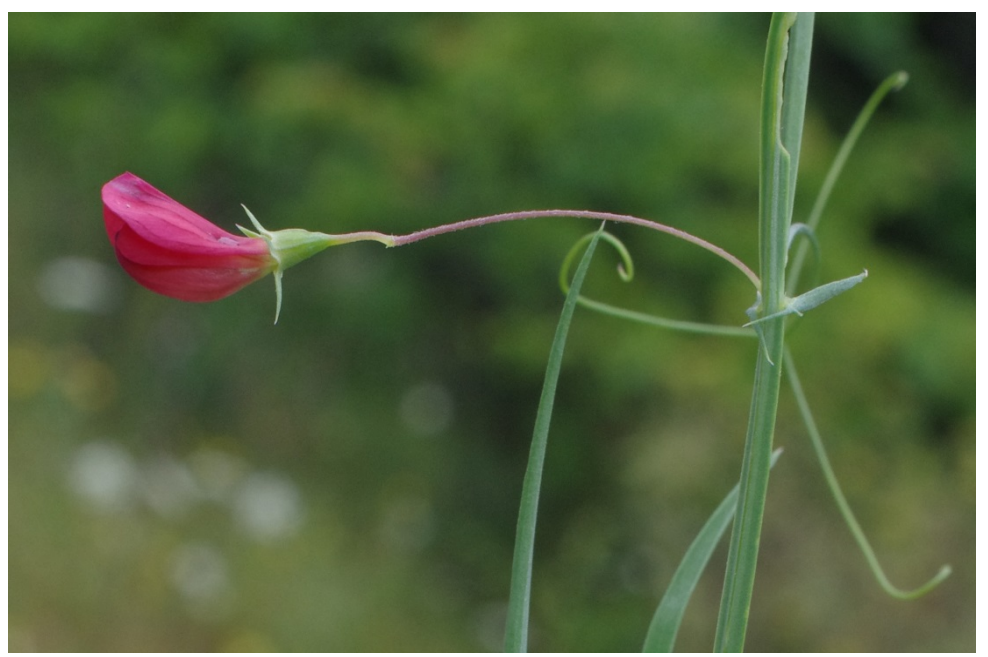

\section{Lathyrus sphaericus}

276.954, loa 1076, ONO Anilio

\section{Lathyrus venetus}

276.519, loa 1059, Ag. Nikolaos; 276.677, loa 1065, NO Karyes; 276.737, loa 1067, NW Metsovo; 277.217, loa 1088, NNO Metsovo

\section{Legousia speculum-veneris}

278.105, Gre 492, NO Varis; 278.375, Koz 382, Galatini; 281.320, Dra 268, W Nevrokopi

\section{Lembotropis nigricans}

276.505, loa 1059, Ag. Nikolaos

\section{Leontodon crispus subsp. asper}

276.605, loa 1061, SSO Potamia; 276.987, loa 1079, SO Anilio; 277.150 , loa 1085 , N Metsovo; 277.364, loa 1093, S Anilio; 277.565, loa 1099, S Anilio; 277.706, Tri 460, N Chaliki; 277.855, Koz 387, SO Siatista, Vourinos; 278.030, Koz 376, W Exarcho; 278.346, Koz 381, Galatini; 279.562, Kav 102, Pangaion; 279.891, Dra 209, Livadero; 280.121, Dra 220, Sidironero; 280.139, Dra 221, NO Drama; 280.384, Dra 232, Sidironero; 281.605, Dra 278, Kato Vrondous; 282.362, Ser 276, SSW Ano Vrondous; 282.421, Ser 279, Orini; 282.913, Kil 297, WNW Kastaneri; 283.909, Ima 180, Seli; 284.013, loa 1114, Katara-Paß

\section{Leontodon crispus subsp. crispus}

283.267, Pel 347, Kajmaktsalan

\section{Leontodon hispidus}

281.058, Dra 259, Granitis 


\section{Leontodon hispidus subsp. hispidus}

276.834, loa 1070, NNW Metsovo; 277.234, loa 1089, NNO Metsovo; 277.649, loa 1105, Anilio; 279.789, Ser 268, SW Skopia; 280.312, Dra 229, SO Dendrakia; 280.478, Dra 234, Skaloti; 280.643, Dra 241, Elatias; 280.670, Dra 242, SW Walddorf Elatias; 280.753, Dra 245, SW Walddorf Elatias; 280.805, Dra 247, Walddorf Elatias; 281.109, Dra 261, Granitis; 282.704, Ser 290, N Neo Petritsi; 283.014, Kil 302, Kastaneri; 283.829, Ima 176, Tria-Pende Pigadia; 283.968, loa 1056 b, NO Metsovo; 284.082, loa 1119, Anilio

\section{Leonurus cardiaca}

281.362, Dra 271, Vathytopo

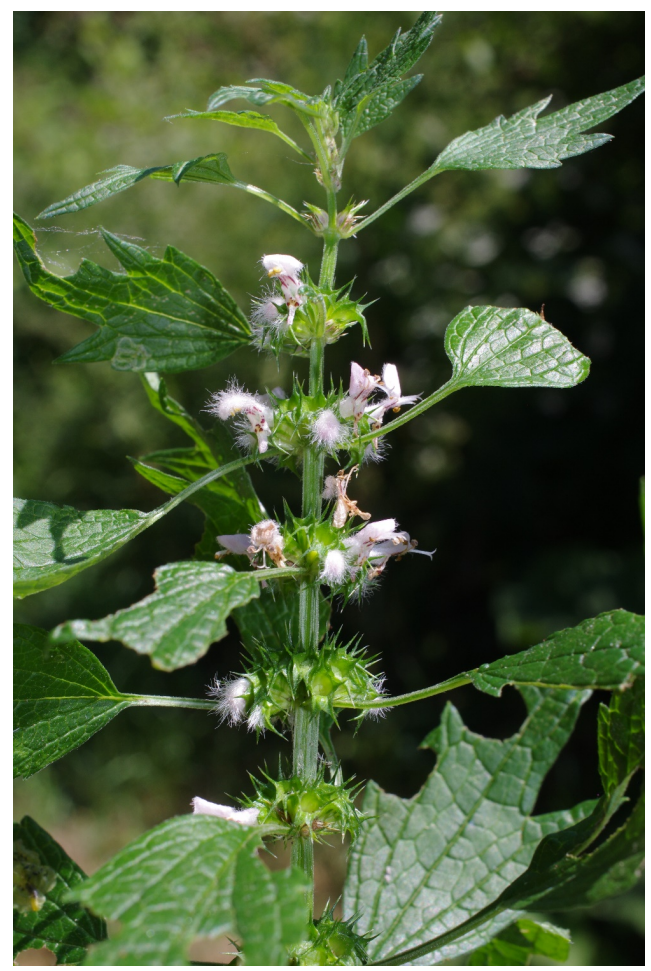

\section{Lepidium campestre}

277.173, loa 1086, NNO Metsovo; 277.941, Koz 392, SO Siatista, Vourinos; 278.173 , Gre 494, SO Varis; 280.472, Dra 234, Skaloti; 280.666, Dra 242, SW Walddorf Elatias; 282.713 , Ser 290, N Neo Petritsi

\section{Lepidium draba}

277.913, Koz 390, SO Siatista, Vourinos; 278.379, Koz 382, Galatini; 281.427, Dra 273, Vathytopo

\section{Lepidium virginicum}

276.313, loa 1125, NO Dodoni

\section{Leptoplax emarginata}

277.311, loa 1091, SW Milia; 278.134, Gre 492, NO Varis

\section{Leucanthemum chloroticum}

283.915, Ima 180, Seli

\section{Leucanthemum vulgare}

276.403, loa 1057, NO Metsovo; 276.822, loa 1070, NNW Metsovo; 277.497, loa 1097, S Anilio; 280.483, Dra 234, Skaloti; 280.527, Dra 236, Skaloti; 280.681, Dra 242, SW Walddorf Elatias; 281.102, Dra 261, Granitis; 281.485, Dra 275, Vathytopo; 281.864, Dra 286, SW Mikroklisoura; 281.888, Dra 287, O Mikroklisoura; 282.075, Dra 294, NO Nevrokopi; 283.931, loa 1107, N Metsovo; 283.982, loa 1111, NO Metsovo; 284.070, loa 1118, Anilio

\section{Ligusticum lucidum}

282.923, Kil 297, WNW Kastaneri 
Ligustrum vulgare

276.339, loa 1126, NO Dodoni; 281.948, Dra 289, NO Potamia

\section{Lilium martagon}

284.225, Ser 283, Ori Vrondous; -20.528, Ima 173, S Ag. Pavlos

\section{Linaria dalmatica}

276.937, loa 1075, NW Anilio; 277.408, loa 1094, S Anilio; 279.854, Dra 209, Livadero; 279.867, Dra 209, Livadero; 280.521, Dra 236, Skaloti; 280.567, Dra 238, Skaloti; 280.658, Dra 242, SW Walddorf Elatias; 280.667, Dra 242, SW Walddorf Elatias; 280.789, Dra 247, Walddorf Elatias; 281.053, Dra 259, Granitis; 281.246, Dra 266, W Nevrokopi; 281.394, Dra 272, Vathytopo; 281.606, Dra 278, Kato Vrondous; 281.915, Dra 287, O Mikroklisoura; 282.027, Dra 292, Nevrokopi; 282.149, Dra 297, Lefkogia; 282.198, Dra 299, SO Lefkogia; 282.300, Ser 273, Ano Vrondous; 282.527, Ser 283, Ori Vrondous; 283.568, Pel 363, NW Promachi

\section{Linaria genistifolia}

278.749, Kav 109, Loutra Eleftherion; 278.843, Kav 115, SW Folia; 278.914, Kav 117, Galipsos; 279.159, Ser 255, S Nea Mesolakkia; 279.660, Ser 265, Myrrini; 279.693, Ser 266, SO Nea Zichni; 279.714, Ser 266, SO Nea Zichni; 279.842, Dra 208, NO Skopia; 280.017, Dra 216, Sidironero; 280.023, Dra 216, Sidironero; 281.515, Dra 276, Perithori; 281.871, Dra 286, SW Mikroklisoura; 282.661, Ser 290, N Neo Petritsi

\section{Linaria pelisseriana}

276.635, loa 1063, W Potamia

\section{Linaria peloponnesiaca var. parnassica}

277.876, Koz 388, SO Siatista, Vourinos; 282.959, Kil 299, Kastaneri; 283.167, Pel 343, Kerasia; 283.257, Pel 347, Kajmaktsalan; 283.491, Pel 359, NW Loutraki; 283.769, Ima 172, S Ag. Pavlos

\section{Linaria simplex}

276.321, loa 1125, NO Dodoni; 277.780, Koz 385, SO Siatista; 278.319, Koz 381, Galatini

\section{Linaria vulgaris}

282.561, Ser 285, Ori Vrondous

\section{Linum bienne}

280.350, Dra 231, Sidironero; 281.824, Dra 283, NNO Volakas

\section{Linum catharticum}

276.358, loa 1128, N Metsovo; 276.859, loa 1071, NNW Metsovo; 276.901, loa 1073, NNW Metsovo; 277.029, loa 1080, SO Anilio; 277.494, loa 1097, S Anilio; 277.640, loa 1104, S Anilio; 277.737, Tri 460, N Chaliki; 279.469, Kav 96, Pangaion; 280.330, Dra 230, Livadero;

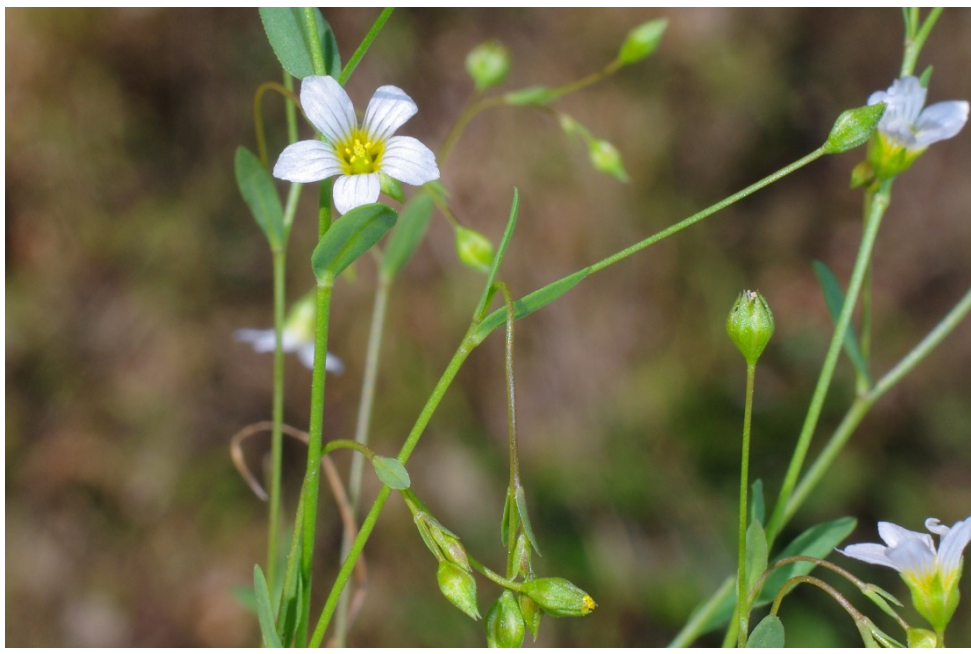


280.507, Dra 236, Skaloti; 280.752, Dra 245, SW Walddorf Elatias; 280.872, Dra 249, Falakron; 282.071, Dra 294, NO Nevrokopi; 283.300, Pel 350, Kajmaktsalan; 283.795, Ima 174, S Ag. Pavlos; 284.078, loa 1119, Anilio

\section{Linum corymbulosum}

277.346, loa 1093, S Anilio; 278.091, Gre 492, NO Varis; 280.168, Dra 221, NO Drama

\section{Linum elegans}

278.071, Gre 492, NO Varis; 278.771, Kav 111, Loutra Eleftherion; 279.052, Kav 121, Platanotopos; 279.411, Kav 92, Pangaion; 279.602, Kav 105, Pangaion; 279.823, Ser 269, NO Skopia; 280.832, Dra 248, Falakron; 281.969, Dra 290, NO Potamia; 284.207, Kav 111, Loutra Eleftherion

\section{Linum hirsutum subsp. hirsutum}

279.739, Ser 268, SW Skopia; 280.242, Dra 227, Livadero; 280.409, Dra 233, Sidironero; 281.237, Dra 265, NO Granitis; 281.439, Dra 274, Vathytopo; 283.132, Pel 341, S Archangelo; 283.410, Pel 355, NW Loutraki

Linum hirsutum subsp. spathulatum

277.812, Koz 387, SO Siatista, Vourinos; 280.212, Dra 224, NO Drama

\section{Linum hologynum}

276.347, loa 1128, N Metsovo; 276.841, loa 1071, NNW Metsovo; 277.036, loa 1080, SO Anilio; 277.270, loa 1090, NNO Metsovo; 284.086, loa 1120, Anilio

\section{Linum tenuifolium}

276.947, Ioa 1076, ONO Anilio; 277.875, Koz 388, SO Siatista, Vourinos; 277.988 , Koz 375, O Dafnero; 278.061, Gre 492, NO Varis; 278.353, Koz 381, Galatini; 279.738, Ser 268, SW Skopia; 280.492, Dra 235, Skaloti; 280.834, Dra 248, Falakron; 281.161, Dra 263, NW Granitis; 281.695, Dra 280, Nevrokopi; 281.973, Dra 290, NO Potamia; 282.054, Dra 293, Nevrokopi; 282.384, Ser 276, SSW Ano Vrondous

\section{Lolium multiflorum}

277.439, loa 1095, S Anilio; 278.171, Gre 494, SO Varis; 280.904, Dra 250, Falakron; 281.128, Dra 261, Granitis; 281.507, Dra 276, Perithori

\section{Lolium perenne}

276.292, loa 1125, NO Dodoni; 276.488, loa 1059, Ag. Nikolaos; 276.549, loa 1060, SSO Potamia; 276.589, loa 1061, SSO Potamia; 276.683, loa 1065, NO Karyes; 277.123, loa 1084, N Metsovo; 277.361, loa 1093, S Anilio; 277.749, Tri 461, N Chaliki; 277.935, Koz 392, SO Siatista, Vourinos; 278.099, Gre 492, NO Varis; 278.248, Koz 379, Galatini; 278.340, Koz 381, Galatini; 278.586, Thes 220, Arethousa; 278.674, Kav 107, Paralia Ofryniou; 278.999, Kav 119, Podochori; 279.186, Ser 256, Paleokomi; 279.280, Ser 258, Mikro Souli; 279.490, Kav 97, Pangaion; 279.839, Dra 207, NO Skopia; 280.267, Dra 228, Livadero; 280.391, Dra 232, Sidironero; 280.437, Dra 234, Skaloti; 280.792, Dra 247, Walddorf Elatias; 280.994, Dra 257, Granitis; 281.187, Dra 264, NO Ochyro; 281.219, Dra 265, NO Granitis; 281.325, Dra 268, W Nevrokopi; 281.416, Dra 273, Vathytopo; 281.503, Dra 276, Perithori; 282.251, Dra 301, Ano Vrondous; 282.694, Ser 290, N Neo Petritsi; 282.952, Kil 298, WNW Kastaneri; 283.560, Pel 362, NW Promachi; 283.891, Ima 179, Seli

\section{Lolium rigidum}

276.639, loa 1063, W Potamia; 278.532, Thes 218, Vrasna; 278.580, Thes 220, Arethousa; 278.821, Kav 114, SO Folia; 278.994, Kav 119, Podochori; 279.208, Ser 257, Nea Fili; 279.308, Ser 259, Proti; 279.750, Ser 268, SW Skopia; 282.183, Dra 299, SO Lefkogia

\section{Lolium subulatum}

283.175, Pel 343, Kerasia

\section{Lolium temulentum}

277.912, Koz 390, SO Siatista, Vourinos; 280.459, Dra 234, Skaloti; 282.636 , Ser 290, N Neo Petritsi; 283.309, Pel 350, Kajmaktsalan 


\section{Lomelosia argentea}

278.610, Thes 221, Arethousa; 281.227, Dra 265, NO Granitis; 281.813, Dra 283, NNO

Volakas; 281.916, Dra 287, O Mikroklisoura; 282.376, Ser 276, SSW Ano Vrondous

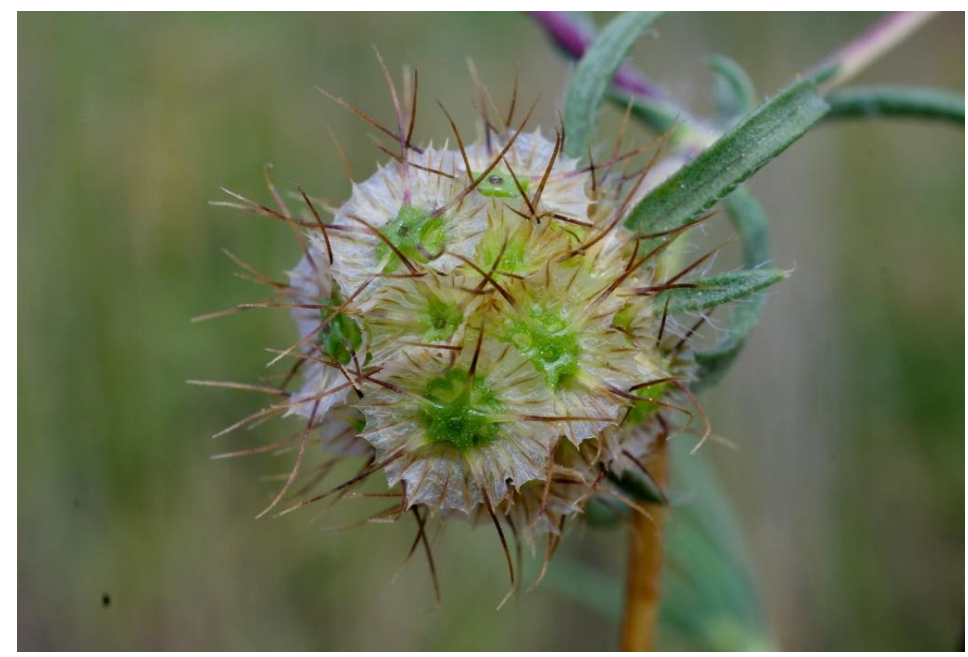

\section{Lomelosia brachiata}

276.574, loa 1060, SSO Potamia; 278.897, Kav 116, S Ofrynia; 278.998, Kav 119,

Podochori; 279.074, Kav 121, Platanotopos; 279.151, Ser 255, S Nea Mesolakkia; 279.264,

Ser 258, Mikro Souli; 279.422, Kav 93, Pangaion

\section{Lomelosia crenata subsp. crenata}

278.013, Koz 375, O Dafnero

\section{Lonicera implexa}

276.335, loa 1126, NO Dodoni

\section{Lotus}

279.054, Kav 121, Platanotopos

\section{Lotus angustissimus}

276.613, loa 1062, SO Potamia; 276.684, loa 1065, NO Karyes; 278.814 , Kav 114, SO Folia; 282.844, Ser 298, Parapotamos

\section{Lotus corniculatus}

276.294, loa 1125, NO Dodoni; 276.619, loa 1062, SO Potamia; 276.716, loa 1065, NO Karyes; 276.981, loa 1079, SO Anilio; 277.169, loa 1086, NNO Metsovo; 277.225, loa 1089, NNO Metsovo; 277.419, loa 1094, S Anilio; 277.865, Koz 388, SO Siatista, Vourinos; 278.178, Gre 495, Varis; 278.504, Thes 216, Vrasna; 278.507, Thes 217, Vrasna; 278.680, Kav 107, Paralia Ofryniou; 279.383, Ser 262, Proti; 279.475, Kav 96, Pangaion; 279.797 , Ser 269, NO Skopia; 279.871, Dra 209, Livadero; 280.280, Dra 228, Livadero; 280.403 , Dra 233, Sidironero; 280.439, Dra 234, Skaloti; 280.742, Dra 245, SW Walddorf Elatias; 281.022, Dra 258, Granitis; 281.536, Dra 276, Perithori; 281.960, Dra 289, NO Potamia; 282.072, Dra 294, NO Nevrokopi; 282.137, Dra 297, Lefkogia; 282.200, Dra 299, SO Lefkogia; 282.332, Ser 274, SSW Ano Vrondous; 282.570, Ser 286, Ori Vrondous; 282.637 , Ser 290, N Neo Petritsi; 283.037, Kil 303, SO Livadia; 283.467, Pel 358, N Loutraki; 283.828, Ima 176, Tria-Pende Pigadia; 283.868, Ima 178, Seli

\section{Lotus gebelia}

279.728, Ser 267, SW Skopia; 279.844, Dra 208, NO Skopia; 280.113, Dra 220, Sidironero; 280.235, Dra 226, N Taxiarches; 281.466, Dra 274, Vathytopo

\section{Lotus palustris}

283.192, Pel 343, Kerasia

\section{Lotus preslii}


276.835, loa 1070, NNW Metsovo; 283.374, Pel 353, Kajmaktsalan

\section{Luzula luzulina}

276.387, loa 1056, NO Metsovo; 276.763, loa 1069, NW Metsovo; 277.178, loa 1086, NNO Metsovo

\section{Luzula luzuloides}

280.626, Dra 240, Elatias; 280.663, Dra 242, SW Walddorf Elatias; 282.509 , Ser 282, Ori Vrondous; 282.575, Ser 286, Ori Vrondous; 282.761, Ser 293, N Neo Petritsi

\section{Lychnis coronaria}

276.614, loa 1062, SO Potamia; 276.708, loa 1065, NO Karyes; 277.286, loa 1091, SW Milia; 279.517, Kav 99, Pangaion; 279.870, Dra 209, Livadero; 280.006, Dra 216,

Sidironero; 280.044, Dra 217, Sidironero; 280.276, Dra 228, Livadero; 280.354, Dra 231, Sidironero; 280.416, Dra 233, Sidironero; 281.019, Dra 258, Granitis; 281.382, Dra 272, Vathytopo; 281.656, Ser 270, Kato Vrondous; 281.866, Dra 286, SW Mikroklisoura; 282.016, Dra 292, Nevrokopi; 282.626, Ser 289, Ori Vrondous; 282.639, Ser 290, N Neo Petritsi; 282.860, Kil 295, NW Griva; 283.208, Pel 344, Kerasia; 283.732, Ima 172, S Ag. Pavlos; 283.812, Ima 176, Tria-Pende Pigadia; 283.858, Ima 178, Seli; 284.037, loa 1116, SW Milia

\section{Lychnis subintegra}

276.756, loa 1068, NW Metsovo; 276.860, loa 1071, NNW Metsovo

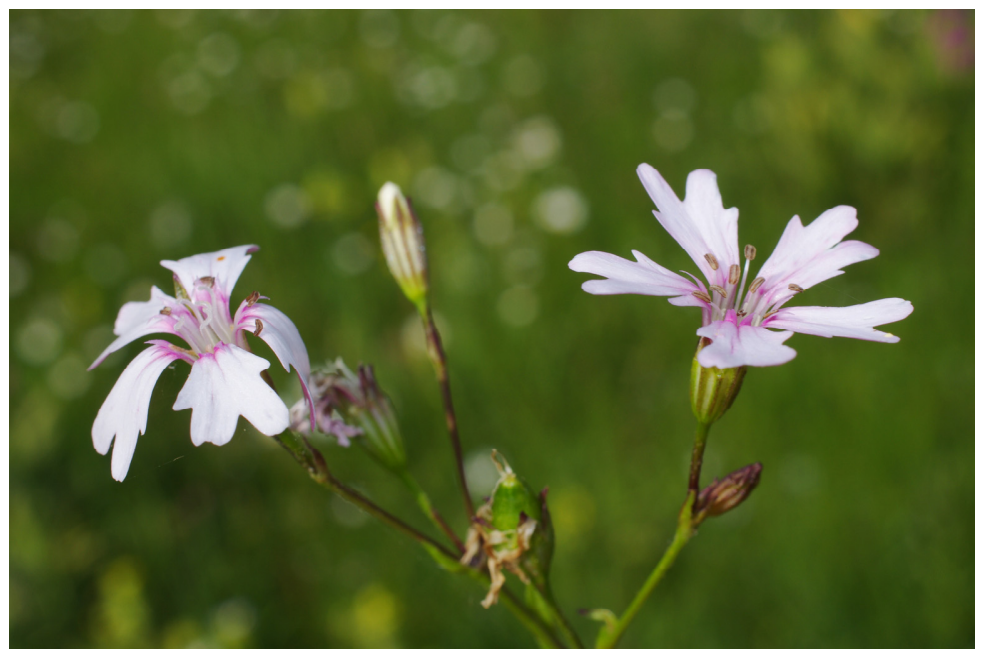

\section{Lycopus europaeus subsp. europaeus}

282.140, Dra 297, Lefkogia; 282.969, Kil 299, Kastaneri; 283.692, Pel 369, O Vorino

\section{Lysimachia dubia}

278.039, Gre 491, NO Exarcho

\section{Lysimachia nummularia}

281.881, Dra 287, O Mikroklisoura; 283.762, Ima 172, S Ag. Pavlos

\section{Lysimachia punctata}

279.533, Kav 100, Pangaion; 279.901, Dra 210, Livadero; 280.030, Dra 216, Sidironero; 280.476, Dra 234, Skaloti; 281.104, Dra 261, Granitis; 281.398, Dra 272, Vathytopo; 281.616, Dra 278, Kato Vrondous; 281.878, Dra 287, O Mikroklisoura; 282.242, Dra 301, Ano Vrondous; 282.578, Ser 286, Ori Vrondous; 282.644, Ser 290, N Neo Petritsi; 282.907, Kil 297, WNW Kastaneri; 282.998, Kil 301, Kastaneri; 283.190, Pel 343, Kerasia; 283.851, Ima 178 , Seli

\section{Lysimachia vulgaris}

281.089, Dra 260, Granitis 


\section{Lythrum salicaria}

278.768, Kav 110, Loutra Eleftherion; 279.116, Kav 91, Orfani; 279.914, Dra 210, Livadero; 281.092, Dra 260, Granitis; 281.311, Dra 268, W Nevrokopi; 281.556, Dra 277, Perithori; 281.680, Ser 271, Kato Vrondous; 281.708, Dra 280, Nevrokopi; 281.943, Dra 288, SW

Potamia; 282.059, Dra 294, NO Nevrokopi; 282.146. Dra 297, Lefkogia; 282.842, Ser 298, Parapotamos; 282.893, Kil 295, NW Griva; 283.386, Pel 354, NW Loutraki; 283.524, Pel 361, SW Promachi; 283.695, Pel 369, O Vorino

\section{Malabaila aurea}

279.267, Ser 258, Mikro Souli; 279.289, Ser 259, Proti

Malabaila involucrata

280.494, Dra 235, Skaloti; 280.634, Dra 240, Elatias

\section{Malva moschata}

283.998, loa 1112, Katara-Paß

\section{Malva neglecta}

283.872, Ima 179, Seli

\section{Malva nicaeensis}

278.699, Kav 107, Paralia Ofryniou; 279.129, Kav 91, Orfani

\section{Malva setigera}

277.435, loa 1095, S Anilio; 277.986, Koz 375, O Dafnero; 278.407, Koz 383, Galatini; 279.744, Ser 268, SW Skopia; 280.124, Dra 221, NO Drama; 281.138, Dra 262, NW Granitis

\section{Malva sylvestris}

276.623, loa 1062, SO Potamia; 277.462, loa 1096, S Anilio; 277.967, Koz 374, Vourinos; 278.143, Gre 492, NO Varis; 278.443, Thes 214, Paralia Vrasna; 278.552, Thes 219, Vrasna; 278.655, Kav 106, Paralia Ofryniou; 278.818, Kav 114, SO Folia; 278.913, Kav 117, Galipsos; 279.101, Kav 90, SW Moustheni; 279.141, Ser 255, S Nea Mesolakkia; 279.251, Ser 258, Mikro Souli; 279.296, Ser 259, Proti; 279.746, Ser 268, SW Skopia; 279.905, Dra 210, Livadero; 280.086, Dra 219, Sidironero; 280.087, Dra 219, Sidironero; 280.199, Dra 224, NO Drama; 280.434, Dra 234, Skaloti; 281.190, Dra 264, NO Ochyro; 281.377, Dra 271, Vathytopo; 281.999, Dra 291, W Prosotsani; 282.778, Ser 293, N Neo Petritsi; 282.828, Ser 298, Parapotamos; 283.592, Pel 364, Loutraki; 283.661, Pel 368, OSO Vorino; -20.463 , loa 1052, NO Dodoni

\section{Malva thuringiaca subsp. ambigua}

281.330, Dra 269, W Nevrokopi; 281.549, Dra 277, Perithori; 281.694, Dra 280, Nevrokopi; 281.842, Dra 284, NNO Volakas; 282.045, Dra 293, Nevrokopi; 282.309, Ser 273, Ano Vrondous; 282.341, Ser 274, SSW Ano Vrondous; 283.551, Pel 362, NW Promachi

\section{Marrubium peregrinum}

278.204, Koz 377, N Siatista; 278.290, Koz 380, Galatini; 278.518, Thes 217, Vrasna; 278.626, Thes 221, Arethousa; 279.247, Ser 258, Mikro Souli; 279.299, Ser 259, Proti; 279.697, Ser 266, SO Nea Zichni; 282.369, Ser 276, SSW Ano Vrondous; 282.770, Ser 293, N Neo Petritsi; 283.502, Pel 359, NW Loutraki

\section{Marrubium thessalum}

278.316, Koz 381, Galatini 


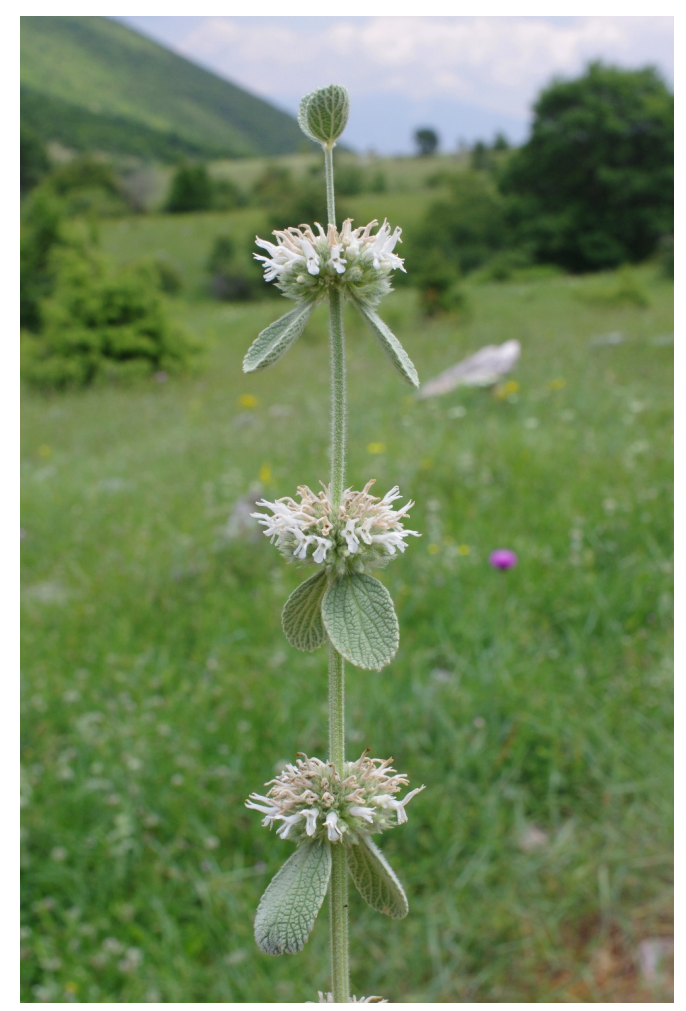

\section{Marrubium velutinum}

277.651, loa 1106, Anilio; 284.009, loa 1113, Katara-Paß; 284.170, loa 1124, N Charikli

\section{Marrubium vulgare}

280.838, Dra 248, Falakron

\section{Matricaria chamomilla}

276.572, loa 1060, SSO Potamia; 278.839, Kav 114, SO Folia; 278.977, Kav 119, Podochori; 279.743, Ser 268, SW Skopia

\section{Matricaria discoidea}

283.355, Pel 352, Kajmaktsalan

\section{Medicago disciformis}

278.984, Kav 119, Podochori

\section{Medicago lupulina}

276.552, loa 1060, SSO Potamia; 276.793, loa 1070, NNW Metsovo; 276.999, loa 1079, SO Anilio; 277.167, loa 1086, NNO Metsovo; 277.281, loa 1090, NNO Metsovo; 277.633, loa 1104, S Anilio; 277.831, Koz 387, SO Siatista, Vourinos; 278.369, Koz 382, Galatini; 278.539, Thes 219, Vrasna; 279.088, Kav 89, Misoropi; 279.498, Kav 98, Pangaion; 279.991, Dra 214, Livadero; 280.031, Dra 216, Sidironero; 280.263, Dra 228, Livadero; 280.686, Dra 242, SW Walddorf Elatias; 281.045, Dra 259, Granitis; 281.559, Dra 277, Perithori; 282.145, Dra 297, Lefkogia; 282.225, Dra 300, SO Lefkogia; 282.437, Ser 280, Orini; 282.743, Ser 292, N Neo Petritsi; 282.979, Kil 300, Kastaneri; 283.446, Pel 356, NW Loutraki; 283.558, Pel 362, NW Promachi; -20.503, Dra 234, Skaloti; -20.514, Dra 236, Skaloti; 20.517, Dra 238, Skaloti

\section{Medicago minima}

276.517, loa 1059, Ag. Nikolaos; 277.817, Koz 387, SO Siatista, Vourinos; 278.339, Koz 381, Galatini; 279.323, Ser 260, Proti; 280.236, Dra 226, N Taxiarches; 280.283, Dra 228, Livadero; 278.781, Kav 112, NO Paralia Myrtofytou; -20.464, loa 1052, NO Dodoni

\section{Medicago monspeliaca}

278.702, Kav 107, Paralia Ofryniou 


\section{Medicago orbicularis}

-20.462, loa 1052, NO Dodoni

\section{Medicago rigidula}

276.536, loa 1059, Ag. Nikolaos; 276.541, loa 1059, Ag. Nikolaos; 277.895, Koz 389, SO

Siatista, Vourinos; 278.322, Koz 381, Galatini; 278.989, Kav 119, Podochori; 279.011, Kav 119, Podochori; -20.465, loa 1052, NO Dodoni; -20.483, loa 1066, NO Karyes

\section{Medicago sativa subsp. falcata}

276.946, loa 1076, ONO Anilio; 277.351, loa 1093, S Anilio; 277.674, Tri 460, N Chaliki; 277.885, Koz 388, SO Siatista, Vourinos; 278.338, Koz 381, Galatini; 279.009, Kav 119, Podochori; 279.050, Kav 121, Platanotopos; 279.373, Ser 261, Proti; 279.751, Ser 268, SW Skopia; 279.824, Ser 269, NO Skopia; 280.955, Dra 255, Falakron; 280.984, Dra 257, Granitis; 281.594, Dra 278, Kato Vrondous; 281.702, Dra 280, Nevrokopi; 282.050, Dra 293, Nevrokopi; 282.327, Ser 274, SSW Ano Vrondous; 283.889, Ima 179, Seli

\section{Medicago sativa subsp. sativa}

276.576, loa 1060, SSO Potamia; 276.632, loa 1063, W Potamia; 278.160, Gre 494, SO Varis; 278.169, Gre 494, SO Varis; 278.497, Thes 216, Vrasna; 278.618, Thes 221, Arethousa; 278.675, Kav 107, Paralia Ofryniou; 278.835, Kav 114, SO Folia; 278.900, Kav 116, S Ofrynia; 279.097, Kav 90, SW Moustheni; 279.149, Ser 255, S Nea Mesolakkia; 279.798, Ser 269, NO Skopia; 280.218, Dra 225, NO Drama; 281.057, Dra 259, Granitis; 281.146, Dra 262, NW Granitis; 281.385, Dra 272, Vathytopo; 281.940, Dra 288, SW

Potamia; 282.808, Ser 296, NO Strymoniko; 283.452, Pel 357, NW Loutraki

\section{Medicago x varia}

280.436, Dra 234, Skaloti; -20.459, loa 1052, NO Dodoni

\section{Melampyrum arvense}

279.826, Dra 207, NO Skopia

Melampyrum ciliatum

281.395, Dra 272, Vathytopo; 282.051, Dra 293, Nevrokopi

\section{Melampyrum cristatum}

281.833, Dra 284, NNO Volakas; 282.007, Dra 292, Nevrokopi; 282.213, Dra 300, SO

Lefkogia; 284.243, Kav 92, Pangaion

\section{Melampyrum fimbriatum}

281.396, Dra 272, Vathytopo

\section{Melica ciliata subsp. ciliata}

276.497, loa 1059, Ag. Nikolaos; 276.723, loa 1066, NO Karyes; 277.381, loa 1093, S Anilio; 277.671, Tri 460, N Chaliki; 278.326, Koz 381, Galatini; 278.628, Thes 221, Arethousa; 281.436, Dra 274, Vathytopo

\section{Melica transsilvanica}

277.984, Koz 375, O Dafnero

\section{Melica uniflora}

276.627, loa 1062, SO Potamia; 276.960, loa 1078, O Anilio; 277.490 , loa 1097, S Anilio

\section{Melilotus albus}

278.587, Thes 220, Arethousa; 279.780, Ser 268, SW Skopia; 279.895, Dra 209, Livadero; 280.215, Dra 225, NO Drama; 280.382, Dra 232, Sidironero; 280.464, Dra 234, Skaloti; 281.035, Dra 258, Granitis; 281.194, Dra 264, NO Ochyro; 281.267, Dra 266, W Nevrokopi; 281.369, Dra 271, Vathytopo; 281.393, Dra 272, Vathytopo; 281.496, Dra 276, Perithori; 281.614, Dra 278, Kato Vrondous; 281.810, Dra 283, NNO Volakas; 281.897, Dra 287, O Mikroklisoura; 282.134, Dra 297, Lefkogia; 282.318, Ser 274, SSW Ano Vrondous; 282.662 , 
Ser 290, N Neo Petritsi; 282.797, Ser 296, NO Strymoniko; 283.152, Pel 342, S Archangelo; 283.177, Pel 343, Kerasia; 283.391, Pel 354, NW Loutraki; 283.703, Pel 369, O Vorino

\section{Melilotus neapolitanus}

276.577, loa 1060, SSO Potamia; 277.336, loa 1093, S Anilio; 277.772, Tri 462, N Chaliki; 278.700, Kav 107, Paralia Ofryniou; 280.164, Dra 221, NO Drama

\section{Melilotus officinalis}

276.322, loa 1125, NO Dodoni; 276.467, Tri 458, NO Katara-Pass; 276.564 , loa 1060, SSO Potamia; 277.172, loa 1086, NNO Metsovo; 277.405, loa 1094, S Anilio; 277.896, Koz 389, SO Siatista, Vourinos; 278.167, Gre 494, SO Varis; 278.275, Koz 379, Galatini; 278.494, Thes 216, Vrasna; 278.679, Kav 107, Paralia Ofryniou; 280.106, Dra 220, Sidironero; 281.195, Dra 264, NO Ochyro; 281.613, Dra 278, Kato Vrondous; 282.485, Ser 282, Ori Vrondous; 283.025, Kil 303, SO Livadia; 283.135, Pel 341, S Archangelo

\section{Melissa officinalis}

279.155, Ser 255, S Nea Mesolakkia; 279.814, Ser 269, NO Skopia; 282.877, Kil 295, NW Griva; 283.408, Pel 355, NW Loutraki; 283.512, Pel 360, SW Promachi; 283.696, Pel 369, O Vorino; 284.130 , ioa 1095 b, S Anilio

\section{Melittis melissophyllum}

276.622, loa 1062, SO Potamia; 279.545, Kav 99, Pangaion

\section{Mentha longifolia}

277.441, loa 1095, S Anilio; 277.792, Koz 385, SO Siatista; 279.380, Ser 262, Proti; 279.669, Ser 265, Myrrini; 279.811, Ser 269, NO Skopia; 280.187, Dra 223, NO Drama; 281.300, Dra 267, W Nevrokopi; 281.532, Dra 276, Perithori; 281.671, Ser 271, Kato Vrondous; 281.720, Dra 280, Nevrokopi; 281.740, Dra 281, OSO Granitis; 281.771, Dra 282, NNO Volakas; 281.846, Dra 284, NNO Volakas; 281.907, Dra 287, O Mikroklisoura; 282.041, Dra 293, Nevrokopi; 282.144, Dra 297, Lefkogia; 282.357, Ser 276, SSW Ano Vrondous; 282.756, Ser 292, N Neo Petritsi; 282.858, Kil 295, NW Griva; 282.905, Kil 297, WNW Kastaneri; 282.978, Kil 299, Kastaneri; 283.062, Kil 307, NW Livadia; 283.176, Pel 343, Kerasia; 283.407, Pel 355, NW Loutraki; 283.411, Pel 355, NW Loutraki; 283.423, Pel 355, NW Loutraki; 283.515, Pel 360, SW Promachi; 283.528, Pel 361, SW Promachi; 283.644, Pel 367, Vorino; 283.672, Pel 368, OSO Vorino; 283.690, Pel 369, O Vorino; 283.736, Ima 172, S Ag. Pavlos; 283.817, Ima 176, Tria-Pende Pigadia; 283.867, Ima 178, Seli; 283.874, Ima 179, Seli; 283.926, loa 1107, N Metsovo; 284.019, loa 1115, N Metsovo; 284.032, loa 1116, SW Milia; 284.075, loa 1118, Anilio; 284.115, loa 1121, Anilio; 284.156, Ioa 1103 b, S Anilio

\section{Mentha pulegium}

283.689, Pel 369, O Vorino

\section{Mentha spicata \\ 279.068, Kav 121, Platanotopos}

\section{Mercurialis annua}

279.030, Kav 120, Podochori

\section{Micromeria juliana}

277.350, loa 1093, S Anilio; 278.791, Kav 112, NO Paralia Myrtofytou; 278.878, Kav 116, S Ofrynia; 278.952, Kav 118, Galipsos; 279.017, Kav 119, Podochori; 279.326, Ser 260, Proti; 280.142, Dra 221, NO Drama; 284.113, loa 1121, Anilio

\section{Microthlaspi perfoliatum}

277.524, loa 1097, S Anilio

\section{Milium effusum}

282.591, Ser 287, Ori Vrondous 
Minuartia attica subsp. attica

276.367, loa 1128, N Metsovo; 277.044, loa 1080, SO Anilio; 277.626, loa 1104, S Anilio; 277.705, Tri 460, N Chaliki; 277.853, Koz 387, SO Siatista, Vourinos; 277.976, Koz 374, Vourinos; 278.124, Gre 493, NO Varis; 280.850, Dra 248, Falakron; 281.158, Dra 262, NW Granitis; 281.738, Dra 281, OSO Granitis; 282.984, Kil 300, Kastaneri; 283.348, Pel 352, Kajmaktsalan; 284.187, Dra 281, OSO Granitis

\section{Minuartia garckeana}

282.878, Kil 295, NW Griva

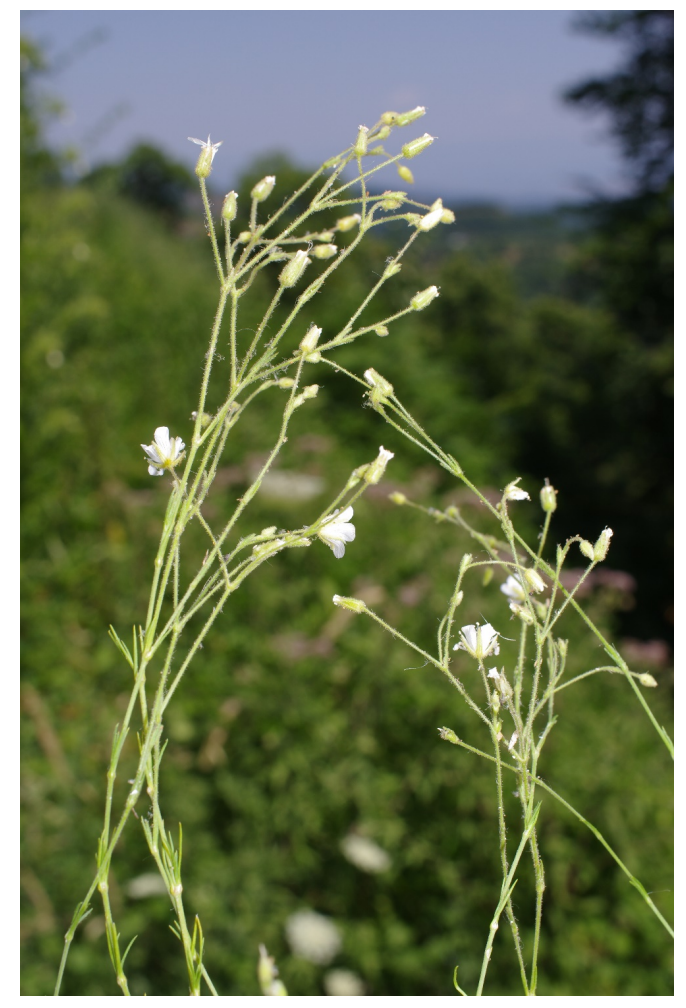

Minuartia glomerata subsp. glomerata

277.822, Koz 387, SO Siatista, Vourinos; 277.854, Koz 387, SO Siatista, Vourinos; 278.200, Koz 377, N Siatista; 278.313, Koz 381, Galatini; 278.393, Koz 383, Galatini; 278.431, Koz 384, Galatini; 280.847, Dra 248, Falakron; 281.241, Dra 265, NO Granitis; 281.445, Dra 274, Vathytopo; 281.723, Dra 280, Nevrokopi; 281.832, Dra 283, NNO Volakas

\section{Minuartia hamata}

278.392, Koz 383, Galatini

\section{Minuartia hirsuta}

276.788, loa 1070, NNW Metsovo; 277.155, loa 1086, NNO Metsovo

\section{Minuartia hybrida}

276.646, loa 1063, W Potamia; 278.318, Koz 381, Galatini; 279.449, Kav 95, Pangaion

\section{Minuartia mesogitana}

279.773, Ser 268, SW Skopia

\section{Minuartia verna subsp. verna}

283.337, Pel 351, Kajmaktsalan

\section{Moehringia trinervia}

276.419, loa 1057, NO Metsovo; 277.493, loa 1097, S Anilio; 279.974, Dra 213, Livadero; 280.328, Dra 230, Livadero; 280.720, Dra 243, SW Walddorf Elatias; 282.599, Ser 287, Ori Vrondous 


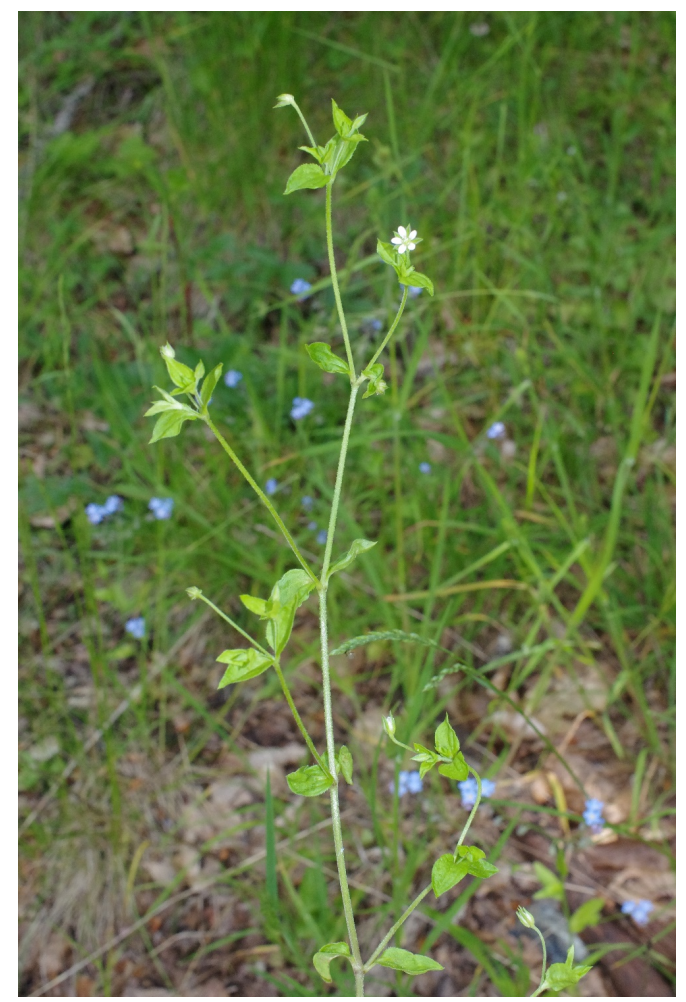

Moenchia mantica

276.750, loa 1068, NW Metsovo; 277.149, loa 1085, N Metsovo; 277.301, loa 1091, SW Milia

\section{Muscari comosum}

277.738, Tri 460, N Chaliki; 277.867, Koz 388, SO Siatista, Vourinos; 280.615, Dra 238, Skaloti

\section{Myosotis arvensis}

276.349, loa 1128, N Metsovo; 276.531, loa 1059, Ag. Nikolaos; 276.746, loa 1068, NW Metsovo; 276.892, loa 1072, NNW Metsovo; 277.677, Tri 460, N Chaliki; 277.954, Koz 374, Vourinos; 278.426, Koz 384, Galatini; 279.359, Ser 261, Proti; 280.419, Dra 233, Sidironero; 280.451, Dra 234, Skaloti; 280.529, Dra 236, Skaloti; 280.537, Dra 236, Skaloti; 280.649, Dra 242, SW Walddorf Elatias; 280.739, Dra 245, SW Walddorf Elatias; 282.288 , Ser 272, Ano Vrondous

\section{Myosotis nemorosa}

276.917, loa 1073, NNW Metsovo; 284.191, Ser 287, Ori Vrondous

\section{Myosotis ramosissima}

276.831, loa 1070, NNW Metsovo; 277.107, loa 1084, N Metsovo; 277.256, loa 1089, NNO Metsovo; 277.308, loa 1091, SW Milia; 277.468, loa 1096, S Anilio; 279.972, Dra 213, Livadero; 280.868, Dra 249, Falakron; 282.514, Ser 283, Ori Vrondous; 284.192, loa 1056, NO Metsovo

\section{Myosotis sicula}

282.139, Dra 297, Lefkogia; 283.286, Pel 349, Kajmaktsalan

\section{Myosotis sylvatica subsp. cyanea}

276.384, loa 1056, NO Metsovo; 276.437, loa 1058, Katara-Pass; 276.658, loa 1063, W Potamia; 276.776, loa 1069, NW Metsovo; 276.876, loa 1072, NNW Metsovo; 277.019, loa 1080, SO Anilio; 277.162, loa 1086, NNO Metsovo; 277.552, loa 1098, S Anilio; 279.539, Kav 101, Pangaion; 280.607, Dra 238, Skaloti; 280.804, Dra 247, Walddorf Elatias 
Nasturtium officinale

279.117, Kav 91, Orfani; 282.889, Kil 295, NW Griva

\section{Neotinea tridentata}

276.930, loa 1074, NNW Metsovo

\section{Neottia nidus-avis}

276.772, loa 1069, NW Metsovo; 277.517, loa 1097, S Anilio

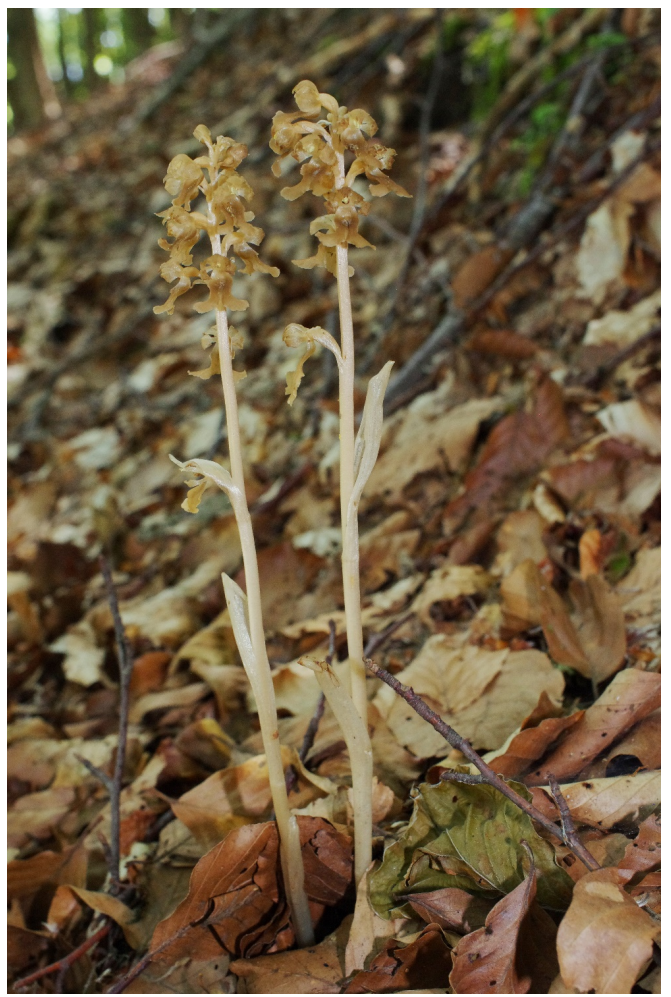

\section{Neottia ovata}

276.867, loa 1072, NNW Metsovo; 277.026, loa 1080, SO Anilio; -20.494, loa 1083, N Metsovo

Nepeta argolica subsp. argolica

276.724, loa 1066, NO Karyes

Nepeta argolica subsp. vourinensis

277.810, Koz 386, SO Siatista; 278.202, Koz 377, N Siatista; 278.231, Koz 378, N Siatista

\section{Nepeta nuda}

279.477, Kav 97, Pangaion; 279.478, Kav 97, Pangaion; 279.593, Kav 104, Pangaion; 279.936, Dra 211, Livadero; 280.584, Dra 238, Skaloti; 281.124, Dra 261, Granitis; 281.636 , Dra 279, Kato Vrondous; 282.264, Dra 301, Ano Vrondous; 282.320, Ser 274, SSW Ano Vrondous; 282.534, Ser 284, Ori Vrondous; 282.608, Ser 288, Ori Vrondous; 282.634, Ser 290, N Neo Petritsi; 283.048, Kil 304, SO Livadia; 283.075, Kil 307, NW Livadia; 283.755 , Ima 172, S Ag. Pavlos

\section{Nepeta spruneri}

280.813, Dra 248, Falakron; 281.696, Dra 280, Nevrokopi; 281.730, Dra 281, OSO Granitis

\section{Nigella arvensis subsp. aristata}

276.331, loa 1125, NO Dodoni; 278.185, Gre 495, Varis; 278.390, Koz 383, Galatini; 278.480, Thes 215, Paralia Vrasna; 278.727, Kav 108, Paralia Ofryniou; 278.950, Kav 118, Galipsos; 279.221, Ser 257, Nea Fili; 279.266, Ser 258, Mikro Souli; 279.331, Ser 260, Proti; 279.622, Ser 263, Dravoskas; 279.718, Ser 267, SW Skopia; 281.140, Dra 262, NW Granitis; 281.208, Dra 265, NO Granitis; 281.295, Dra 267, W Nevrokopi; 281.430, Dra 274, 
Vathytopo; 281.759, Dra 281, OSO Granitis; 281.802, Dra 282, NNO Volakas; 282.042 , Dra 293, Nevrokopi; 282.394, Ser 278, N Serres; 282.433, Ser 279, Orini; 283.609, Pel 365, N Vorino; 283.633, Pel 366, N Vorino; 283.712, Pel 370, SW Foustani

Nigella arvensis subsp. arvensis

278.199, Koz 377, N Siatista; 278.798, Kav 113, NO Paralia Myrtofytou; 279.002, Kav 119, Podochori; 279.110, Kav 90, SW Moustheni; 279.418, Kav 93, Pangaion; 281.575, Dra 277, Perithori; 281.825, Dra 283, NNO Volakas; 281.950, Dra 289, NO Potamia

Nigella damascena

276.305, Ioa 1125, NO Dodoni; 276.670, loa 1064, ONO Karyes; 277.453 , loa 1095, S Anilio

\section{Noccaea boeotica}

279.565, Kav 102, Pangaion

\section{Noccaea ochroleuca}

276.416, loa 1057, NO Metsovo; 276.607, loa 1061, SSO Potamia

\section{Oenanthe fistulosa}

276.617, loa 1062, SO Potamia; 277.642, loa 1104, S Anilio; 278.277, Koz 379, Galatini; 278.861, Kav 115, SW Folia

\section{Oenanthe pimpinelloides}

279.439, Kav 95, Pangaion

\section{Oenanthe silaifolia}

276.785, loa 1070, NNW Metsovo; 277.137, loa 1084, N Metsovo; 277.293, loa 1091, SW Milia; 284.085, loa 1120, Anilio

\section{Oenothera speciosa}

278.713, Kav 107, Paralia Ofryniou

\section{Onobrychis}

278.685, Kav 107, Paralia Ofryniou

\section{Onobrychis aequidentata}

278.970, Kav 119, Podochori

\section{Onobrychis alba subsp. alba}

278.198, Koz 377, N Siatista; 278.224, Koz 378, N Siatista

\section{Onobrychis alba subsp. calcarea}

278.409, Koz 383, Galatini; 280.823, Dra 248, Falakron; 282.056, Dra 293, Nevrokopi

\section{Onobrychis arenaria}

277.523, loa 1097, S Anilio; 277.682, Tri 460, N Chaliki; 278.411, Koz 383, Galatini;

279.828, Dra 207, NO Skopia; 281.808, Dra 283, NNO Volakas

\section{Onobrychis caput-galli}

276.534, loa 1059, Ag. Nikolaos; 276.719, loa 1066, NO Karyes

\section{Onobrychis montana subsp. scardica}

280.887, Dra 250, Falakron; 283.923, Ima 180, Seli

\section{Onobrychis pindicola}

276.359, loa 1128, N Metsovo; 276.722, loa 1066, NO Karyes; 277.347, loa 1093, S Anilio; 278.937, Kav 118, Galipsos; 278.991, Kav 119, Podochori; 279.330, Ser 260, Proti;

279.633, Ser 263, Dravoskas; 280.120, Dra 220, Sidironero; 281.464, Dra 274, Vathytopo

\section{Ononis pusilla}

277.352, loa 1093, S Anilio; 277.872, Koz 388, SO Siatista, Vourinos; 278.422, Koz 384, Galatini; 278.949, Kav 118, Galipsos; 280.929, Dra 253, Falakron; 281.157, Dra 262, NW Granitis; 283.221, Pel 345, Kerasia 
Ononis reclinata

278.673, Kav 107, Paralia Ofryniou

Ononis spinosa subsp. antiquorum

279.337, Ser 261, Proti; 279.755, Ser 268, SW Skopia; 282.117, Dra 296, NO Nevrokopi; 282.766, Ser 293, N Neo Petritsi; 283.139, Pel 341, S Archangelo; 283.383, Pel 354, NW Loutraki; 283.613, Pel 365, N Vorino

Ononis spinosa subsp. leiosperma

279.163, Ser 255, S Nea Mesolakkia; 279.262, Ser 258, Mikro Souli; 281.093, Dra 260,

Granitis; 281.648, Dra 279, Kato Vrondous; 281.951, Dra 289, NO Potamia

Ononis spinosa subsp. spinosa

277.082, loa 1081, W Metsovo; 277.521, loa 1097, S Anilio; 278.046, Gre 491, NO Exarcho; 279.708, Ser 266, SO Nea Zichni; 279.995, Dra 215, Livadero; 280.012, Dra 216, Sidironero; 280.255, Dra 227, Livadero; 280.400, Dra 233, Sidironero; 281.356, Dra 270, W Nevrokopi; 281.776, Dra 282, NNO Volakas; 282.265, Dra 301, Ano Vrondous; 283.202, Pel 344, Kerasia; 283.779, Ima 173, S Ag. Pavlos; 284.073, loa 1118, Anilio; 284.140, loa 1096 b, S Anilio

\section{Onopordum tauricum}

279.099, Kav 90, SW Moustheni; 279.801, Ser 269, NO Skopia; 281.271, Dra 266, W Nevrokopi; 281.364, Dra 271, Vathytopo; 281.533, Dra 276, Perithori; 281.814, Dra 283, NNO Volakas

\section{Onosma heterophylla}

276.661, loa 1064, ONO Karyes; 277.326, loa 1093, S Anilio; 280.180, Dra 223, NO Drama; 280.858, Dra 248, Falakron; 281.042, Dra 259, Granitis; 281.337, Dra 270, W Nevrokopi; 281.470, Dra 275, Vathytopo; 281.608, Dra 278, Kato Vrondous

\section{Onosma visianii}

277.990, Koz 375, O Dafnero; 277.992, Koz 375, O Dafnero; 281.458, Dra 274, Vathytopo

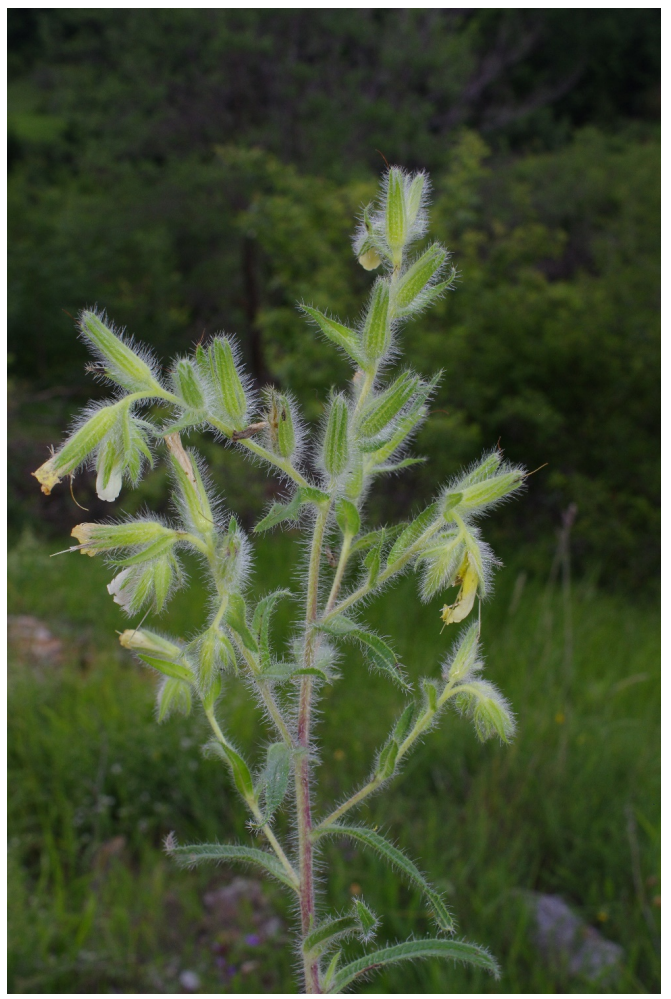

Ophrys apifera

276.934, loa 1074, NNW Metsovo; 277.152, loa 1085, N Metsovo; 277.553, loa 1098, S Anilio 
Ophrys scolopax subsp. cornuta

277.416, loa 1094, S Anilio

\section{Opopanax chironium}

284.050, loa 1117, Anilio; 284.131, ioa 1095 b, S Anilio

Opopanax hispidus

278.175, Gre 494, SO Varis

\section{Orchis mascula}

276.868, loa 1072, NNW Metsovo; 277.048, loa 1080, SO Anilio; -20.478, loa 1056, NO Metsovo

\section{Origanum vulgare subsp. hirtum}

278.631, Thes 221, Arethousa; 278.942, Kav 118, Galipsos; 279.025, Kav 120, Podochori; 279.072, Kav 121, Platanotopos; 279.152, Ser 255, S Nea Mesolakkia; 284.134, loa 1096 b, S Anilio

\section{Origanum vulgare subsp. viridulum}

282.769, Ser 293, N Neo Petritsi; 283.195, Pel 343, Kerasia; 283.402, Pel 354, NW Loutraki; 283.506, Pel 360, SW Promachi

\section{Origanum vulgare subsp. vulgare}

279.138, Ser 255, S Nea Mesolakkia; 279.781, Ser 268, SW Skopia; 280.136, Dra 221, NO Drama; 280.244, Dra 227, Livadero; 280.907, Dra 251, Falakron; 281.086, Dra 260,

Granitis; 281.380, Dra 272, Vathytopo; 281.654, Ser 270, Kato Vrondous; 281.715, Dra 280, Nevrokopi; 281.836, Dra 284, NNO Volakas; 281.909, Dra 287, O Mikroklisoura; 282.001, Dra 292, Nevrokopi; 282.177, Dra 299, SO Lefkogia; 282.683, Ser 290, N Neo Petritsi; 282.847, Kil 294, NW Griva; 283.091, Pel 339, S Archangelo; 283.206, Pel 344, Kerasia; 283.550, Pel 362, NW Promachi

\section{Orlaya daucorlaya}

276.327, loa 1125, NO Dodoni; 276.492, loa 1059, Ag. Nikolaos; 276.695, loa 1065, NO Karyes; 277.338, loa 1093, S Anilio; 277.451, loa 1095, S Anilio; 277.669, Tri 460, N Chaliki; 277.937, Koz 392, SO Siatista, Vourinos; 278.419, Koz 384, Galatini; 279.810, Ser 269, NO Skopia; 279.855, Dra 209, Livadero; 280.054, Dra 217, Sidironero; 280.135, Dra 221, NO Drama; 280.317, Dra 229, SO Dendrakia; 280.393, Dra 232, Sidironero; 281.020, Dra 258, Granitis; 281.162, Dra 263, NW Granitis; 281.214, Dra 265, NO Granitis; 281.338, Dra 270, W Nevrokopi; 281.433, Dra 274, Vathytopo; 281.572, Dra 277, Perithori; 281.662, Ser 270, Kato Vrondous; 281.703, Dra 280, Nevrokopi; 281.753, Dra 281, OSO Granitis; 281.827, Dra 283, NNO Volakas; 282.115, Dra 296, NO Nevrokopi; 282.366, Ser 276, SSW Ano Vrondous; 283.128, Pel 340, SSO Archangelo; 283.499, Pel 359, NW Loutraki; 283.884, Ima 179, Seli

\section{Ornithogalum montanum}

276.871, loa 1072, NNW Metsovo

\section{Ornithogalum pyrenaicum subsp. sphaerocarpum}

279.896, Dra 209, Livadero

\section{Orobanche}

277.089, loa 1081, W Metsovo; 277.950, Koz 374, Vourinos; 279.579, Kav 103, Pangaion; 280.647, Dra 241, Elatias; 280.853, Dra 248, Falakron; 280.860, Dra 249, Falakron; 280.927, Dra 253, Falakron; 282.364, Ser 276, SSW Ano Vrondous

Orobanche rechingeri

276.394, loa 1056, NO Metsovo

\section{Orthilia secunda}


280.771, Dra 246, SW Walddorf Elatias

\section{Ostrya carpinifolia}

276.945, loa 1075, NW Anilio; 277.415, loa 1094, S Anilio; 277.971, Koz 374, Vourinos; 280.039, Dra 216, Sidironero; 280.063, Dra 218, Sidironero; 282.482, Ser 281, Ori Vrondous; 282.763, Ser 293, N Neo Petritsi; 283.203, Pel 344, Kerasia; 283.456, Pel 357, NW Loutraki

\section{Osyris alba}

276.299, loa 1125, NO Dodoni

\section{Paliurus spina-christi}

279.286, Ser 259, Proti; 283.620, Pel 366, N Vorino; 284.210, Thes 221, Arethousa

\section{Pallenis spinosa}

278.717, Kav 108, Paralia Ofryniou; 279.020, Kav 119, Podochori; 279.153, Ser 255, S Nea Mesolakkia

\section{Papaver dubium}

277.449, loa 1095, S Anilio

\section{Papaver rhoeas}

276.808, loa 1070, NNW Metsovo; 277.422, loa 1095, S Anilio; 278.189, Gre 495, Varis; 278.253, Koz 379, Galatini; 278.363, Koz 382, Galatini; 278.470, Thes 215, Paralia Vrasna; 278.676, Kav 107, Paralia Ofryniou; 279.689, Ser 266, SO Nea Zichni; 279.775, Ser 268, SW Skopia; 279.841, Dra 207, NO Skopia; 280.999, Dra 257, Granitis; 281.175, Dra 264, NO Ochyro; 281.538, Dra 276, Perithori

\section{Parietaria judaica}

279.118, Kav 91, Orfani

\section{Parietaria lusitanica}

279.179, Ser 256, Paleokomi

\section{Parietaria officinalis}

281.013, Dra 257, Granitis; 281.425, Dra 273, Vathytopo; 283.131, Pel 340, SSO Archangelo

\section{Paronychia macedonica}

278.021, Koz 376, W Exarcho; 278.138, Gre 492, NO Varis; 278.219, Koz 378, N Siatista; 281.454, Dra 274, Vathytopo; 281.465, Dra 274, Vathytopo

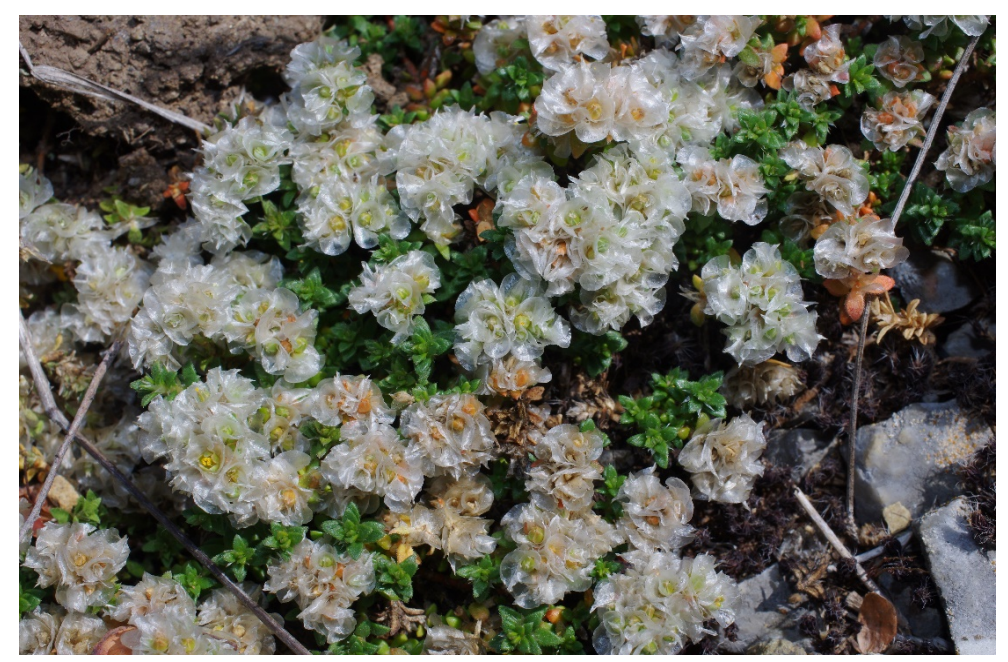

\section{Paronychia rechingeri}

279.604, Kav 105, Pangaion

\section{Paspalum dilatatum}

283.670, Pel 368, OSO Vorino 
Persicaria bistorta

283.321, Pel 351, Kajmaktsalan

Persicaria hydropiper

282.141, Dra 297, Lefkogia

Persicaria lapathifolia subsp. pallida

278.585, Thes 220, Arethousa

\section{Persicaria maculosa}

281.069, Dra 260, Granitis; 281.557, Dra 277, Perithori; 281.922, Dra 288, SW Potamia;

282.143, Dra 297, Lefkogia; 282.834, Ser 298, Parapotamos; 282.974, Kil 299, Kastaneri

\section{Petrorhagia dubia}

276.583, loa 1060, SSO Potamia

\section{Petrorhagia graminea}

278.871, Kav 115, SW Folia; 281.314, Dra 268, W Nevrokopi; 282.090, Dra 295, NO

Nevrokopi

\section{Petrorhagia illyrica subsp. haynaldiana}

277.750, Tri 461, N Chaliki; 278.033, Koz 376, W Exarcho; 279.725, Ser 267, SW Skopia; 280.138, Dra 221, NO Drama; 281.863, Dra 285, NNO Volakas; 282.039, Dra 293,

Nevrokopi; 282.439, Ser 280, Orini; 282.677, Ser 290, N Neo Petritsi

Petrorhagia illyrica subsp. illyrica

277.784, Koz 385, SO Siatista; 278.205, Koz 377, N Siatista; 278.349, Koz 381, Galatini; 282.120, Dra 296, NO Nevrokopi; 283.617, Pel 365, N Vorino; 284.014, loa 1114, Katara$\mathrm{Paß}$

\section{Petrorhagia obcordata}

276.297, loa 1125, NO Dodoni; 276.515, loa 1059, Ag. Nikolaos; 276.691, loa 1065, NO Karyes; 278.489, Thes 215, Paralia Vrasna

\section{Petrorhagia prolifera}

277.079, loa 1081, W Metsovo; 277.516, loa 1097, S Anilio; 277.806, Koz 386, SO Siatista; 278.102, Gre 492, NO Varis; 278.128, Gre 493, NO Varis; 278.271, Koz 379, Galatini; 278.332, Koz 381, Galatini; 278.433, Thes 214, Paralia Vrasna; 278.513, Thes 217, Vrasna; 278.536, Thes 218, Vrasna; 278.746, Kav 109, Loutra Eleftherion; 278.780, Kav 112, NO Paralia Myrtofytou; 278.894, Kav 116, S Ofrynia; 279.021, Kav 119, Podochori; 279.157, Ser 255, S Nea Mesolakkia; 279.201, Ser 257, Nea Fili; 279.244, Ser 258, Mikro Souli; 279.282, Ser 259, Proti; 279.406, Kav 92, Pangaion; 279.628, Ser 263, Dravoskas; 279.673, Ser 265, Myrrini; 279.865, Dra 209, Livadero; 280.047, Dra 217, Sidironero; 280.150, Dra 221, NO Drama; 281.135, Dra 262, NW Granitis; 281.346, Dra 270, W Nevrokopi; 281.774, Dra 282, NNO Volakas; 281.848, Dra 284, NNO Volakas; 282.776, Ser 293, N Neo Petritsi; 284.127, loa 1094 b, S Anilio

\section{Petrorhagia saxifraga}

277.785, Koz 385, SO Siatista; 281.457, Dra 274, Vathytopo

\section{Petrorhagia thessala}

283.477, Pel 358, N Loutraki

\section{Peucedanum austriacum}

277.940, Koz 392, SO Siatista, Vourinos; 283.788, Ima 173, S Ag. Pavlos

\section{Peucedanum oligophyllum}

283.351, Pel 352, Kajmaktsalan

\section{Phalaris minor}


278.737, Kav 108, Paralia Ofryniou; 279.131, Kav 91, Orfani

\section{Phleum}

279.537, Kav 100, Pangaion

\section{Phleum alpinum}

276.398, loa 1056, NO Metsovo; 276.775, loa 1069, NW Metsovo; 277.292, loa 1091, SW

Milia; 277.629, loa 1104, S Anilio; 283.358, Pel 352, Kajmaktsalan

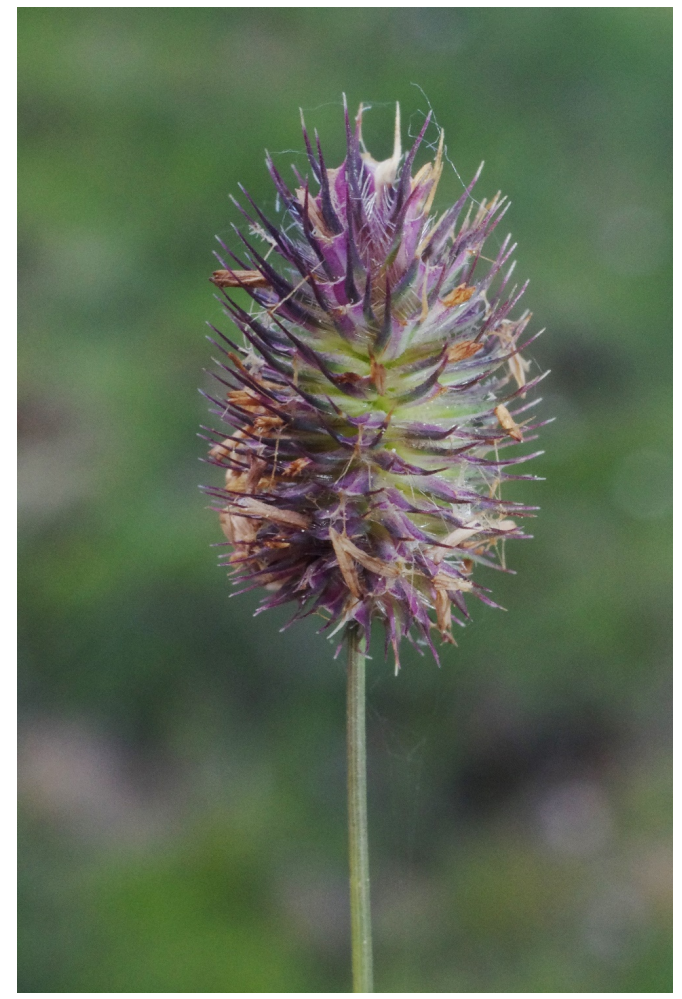

\section{Phleum echinatum}

276.878, loa 1072, NNW Metsovo; 277.014, loa 1080, SO Anilio; 277.607, loa 1103, S Anilio

\section{Phleum montanum}

277.564, loa 1099, S Anilio

\section{Phleum nodosum}

276.563, loa 1060, SSO Potamia; 276.668, loa 1064, ONO Karyes; 277.099 , loa 1084, N Metsovo; 277.525, loa 1097, S Anilio; 277.729, Tri 460, N Chaliki; 278.108, Gre 492, NO Varis; 278.282, Koz 379, Galatini; 280.050, Dra 217, Sidironero; 280.152, Dra 221, NO Drama; 280.396, Dra 232, Sidironero; 281.288, Dra 267, W Nevrokopi; 281.478, Dra 275, Vathytopo; 281.564, Dra 277, Perithori; 281.719, Dra 280, Nevrokopi; 281.775, Dra 282, NNO Volakas; 281.816, Dra 283, NNO Volakas; 281.906, Dra 287, O Mikroklisoura; 282.053, Dra 293, Nevrokopi; 282.091, Dra 295, NO Nevrokopi; 282.194, Dra 299, SO Lefkogia; 282.263, Dra 301, Ano Vrondous; 282.338, Ser 274, SSW Ano Vrondous; 282.909, Kil 297, WNW Kastaneri; 283.049, Kil 304, SO Livadia; 283.073, Kil 307, NW Livadia; 283.199, Pel 343, Kerasia; 283.436, Pel 355, NW Loutraki; 283.750, Ima 172, S Ag. Pavlos; 283.824, Ima 176, Tria-Pende Pigadia; 283.964, loa 1110, N Metsovo; 284.025, loa 1116, SW Milia; 284.142, loa 1096 b, S Anilio; 284.174, Tri 460, N Chaliki

\section{Phleum phleoides}

276.346, loa 1127, Dodonoupoli; 276.894, loa 1072, NNW Metsovo; 277.598, loa 1102, S Anilio; 277.746, Tri 461, N Chaliki; 277.794, Koz 385, SO Siatista; 279.885, Dra 209, Livadero; 281.043, Dra 259, Granitis; 281.342, Dra 270, W Nevrokopi; 281.641, Dra 279, Kato Vrondous; 282.128, Dra 296, NO Nevrokopi

\section{Phleum pratense}


276.317, loa 1125, NO Dodoni; 277.135, loa 1084, N Metsovo; 277.144, loa 1084, N Metsovo; 277.274, loa 1090, NNO Metsovo; 278.163, Gre 494, SO Varis; 280.252, Dra 227, Livadero; 282.065, Dra 294, NO Nevrokopi; 282.233, Dra 300, SO Lefkogia; 282.659, Ser 290, N Neo Petritsi; 282.910, Kil 297, WNW Kastaneri; 283.945, loa 1109, NW Metsovo

Phleum subulatum

279.291, Ser 259, Proti; 279.351, Ser 261, Proti

Phlomis herba-venti subsp. pungens

278.285, Koz 380, Galatini; 278.304, Koz 380, Galatini

\section{Phlomis samia}

279.436, Kav 94, Pangaion

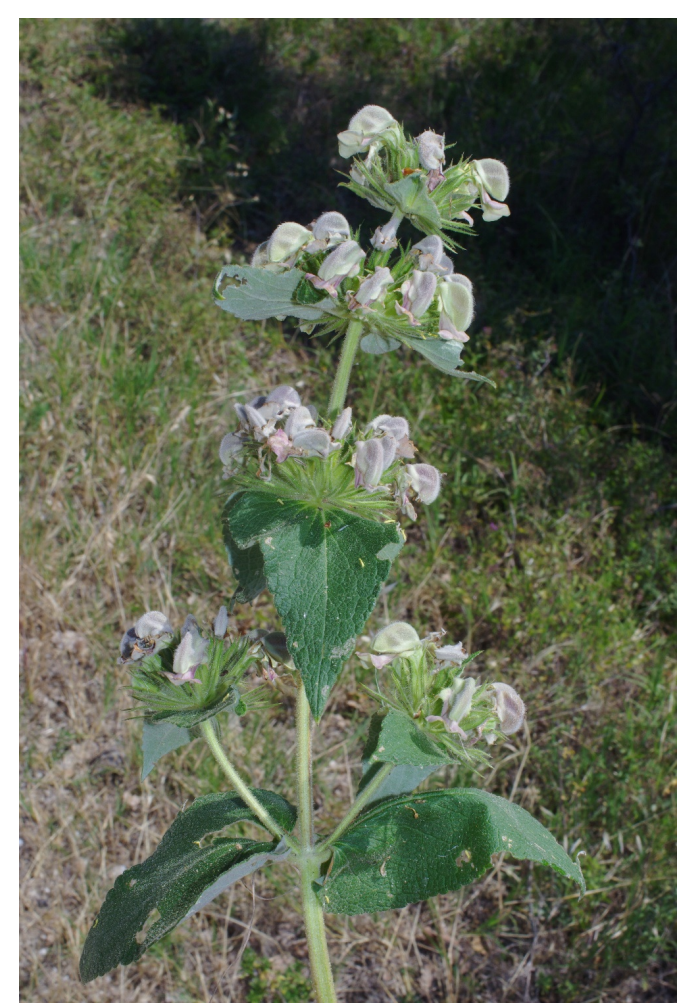

\section{Physalis angulata}

281.423, Dra 273, Vathytopo

\section{Physospermum cornubiense}

279.465, Kav 96, Pangaion; 279.561, Kav 102, Pangaion

\section{Phytolacca americana}

278.505, Thes 217, Vrasna

\section{Picea abies subsp. abies}

280.772, Dra 246, SW Walddorf Elatias

\section{Picnomon acarna}

276.318, loa 1125, NO Dodoni; 283.503, Pel 360, SW Promachi

\section{Picris hieracioides subsp. spinulosa}

279.786, Ser 268, SW Skopia; 279.795, Ser 269, NO Skopia; 281.302, Dra 267, W Nevrokopi; 281.600, Dra 278, Kato Vrondous; 281.837, Dra 284, NNO Volakas; 281.947, Dra 289, NO Potamia; 281.992, Dra 291, W Prosotsani; 282.179, Dra 299, SO Lefkogia; 282.804, Ser 296, NO Strymoniko; 282.851, Kil 294, NW Griva; 283.115, Pel 340, SSO Archangelo; 283.198, Pel 343, Kerasia; 283.384, Pel 354, NW Loutraki; 283.648, Pel 367 , 
Vorino; 283.751, Ima 172, S Ag. Pavlos; 283.955, loa 1110, N Metsovo; 284.055, loa 1117, Anilio; 284.139, loa 1096 b, S Anilio

\section{Picris pauciflora}

276.309, loa 1125, NO Dodoni; 277.367, loa 1093, S Anilio; 277.993, Koz 375, O Dafnero; 278.207, Koz 377, N Siatista; 278.221, Koz 378, N Siatista; 279.314, Ser 260, Proti;

279.412, Kav 92, Pangaion; 280.144, Dra 221, NO Drama

\section{Picris rhagadioloides}

278.758, Kav 109, Loutra Eleftherion; 279.146, Ser 255, S Nea Mesolakkia; 282.396, Ser 278, N Serres

\section{Pilosella alpicola}

283.361, Pel 352, Kajmaktsalan

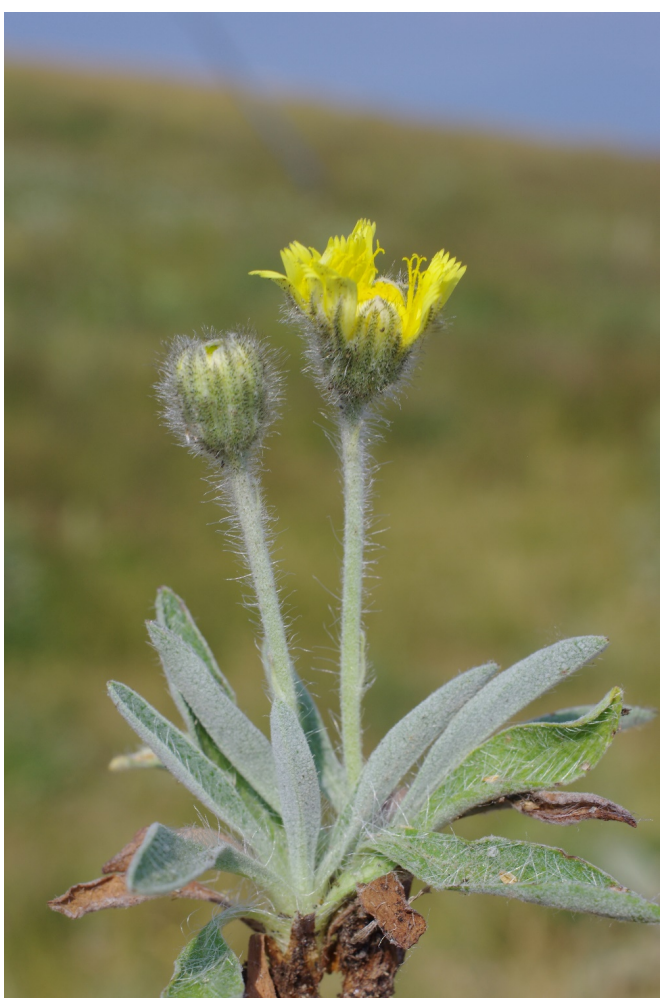

\section{Pilosella bauhini}

276.446, Tri 456, N Katara-Pass; 276.636, loa 1063, W Potamia; 276.825, loa 1070, NNW Metsovo; 276.849, loa 1071, NNW Metsovo; 277.148, loa 1085, N Metsovo; 277.208, loa 1088, NNO Metsovo; 277.307, loa 1091, SW Milia; 277.485, loa 1097, S Anilio; 277.740, Tri 461, N Chaliki; 277.845, Koz 387, SO Siatista, Vourinos; 277.916, Koz 391, SO Siatista, Vourinos; 277.943, Koz 392, SO Siatista, Vourinos; 278.214, Koz 377, N Siatista; 279.508 , Kav 98, Pangaion; 280.595, Dra 238, Skaloti; 280.640, Dra 241, Elatias; 280.697, Dra 242, SW Walddorf Elatias; 280.767, Dra 246, SW Walddorf Elatias; 281.218, Dra 265, NO Granitis; 282.214, Dra 300, SO Lefkogia; 282.586, Ser 287, Ori Vrondous; 283.963, loa 1110, N Metsovo; 283.981, loa 1111, NO Metsovo; 284.177, Tri 456, N Katara-Pass; 284.179, Dra 247, Walddorf Elatias

\section{Pilosella cymosa subsp. heldreichiana}

278.403, Koz 383, Galatini; 280.620, Dra 239, Skaloti; 280.779, Dra 247, Walddorf Elatias; 283.332, Pel 351, Kajmaktsalan

\section{Pilosella cymosa subsp. sabina}

276.388, loa 1056, NO Metsovo; 277.586, loa 1100, S Anilio; 278.873, Kav 115, SW Folia; 279.216, Ser 257, Nea Fili; 279.451, Kav 95, Pangaion; 280.002, Dra 215, Livadero; 280.829, Dra 248, Falakron; 282.165, Dra 298, SO Lefkogia; 283.220, Pel 345, Kerasia 
Pilosella leucopsilon subsp. pilisquama

277.693, Tri 460, N Chaliki; 279.793, Ser 268, SW Skopia; 280.641, Dra 241, Elatias; 280.755, Dra 245, SW Walddorf Elatias; 280.825, Dra 248, Falakron; 281.344, Dra 270, W Nevrokopi; 281.581, Dra 277, Perithori; 282.032, Dra 292, Nevrokopi; 282.129, Dra 296, NO Nevrokopi; 282.212, Dra 300, SO Lefkogia; 282.460, Ser 280, Orini; 282.588, Ser 287, Ori Vrondous; 282.674, Ser 290, N Neo Petritsi; 283.039, Kil 303, SO Livadia; 283.070, Kil 307, NW Livadia; 283.260, Pel 347, Kajmaktsalan; 283.488, Pel 359, NW Loutraki; 283.827, Ima 176, Tria-Pende Pigadia; 283.924, Ima 180, Seli; 283.974, loa 1056 b, NO Metsovo; 284.096, loa 1120, Anilio

\section{Pilosella piloselloides subsp. piloselloides}

276.522, loa 1059, Ag. Nikolaos; 277.660, loa 1106, Anilio; 277.663, Tri 459, N Chaliki; 278.050, Gre 491, NO Exarcho; 278.234, Koz 378, N Siatista; 279.378, Ser 262, Proti; 279.454, Kav 95, Pangaion; 279.526, Kav 99, Pangaion; 279.890, Dra 209, Livadero; 280.056, Dra 217, Sidironero; 280.299, Dra 229, SO Dendrakia; 280.477, Dra 234, Skaloti; 282.477, Ser 281, Ori Vrondous; 282.673, Ser 290, N Neo Petritsi; 282.758, Ser 293, N Neo Petritsi; 282.922, Kil 297, WNW Kastaneri; 282.994, Kil 301, Kastaneri; 283.120, Pel 340, SSO Archangelo; 283.218, Pel 345, Kerasia; 283.461, Pel 358, N Loutraki; 283.482, Pel 359, NW Loutraki; 283.549, Pel 362, NW Promachi; 283.920, Ima 180, Seli; 283.990, loa 1112, Katara-Paß; 284.027, loa 1116, SW Milia; 284.093, loa 1120, Anilio; 284.178, loa 1097, S Anilio

\section{Pimpinella peregrina}

278.845, Kav 115, SW Folia

\section{Pimpinella saxifraga}

282.262, Dra 301, Ano Vrondous; 283.121, Pel 340, SSO Archangelo

\section{Pimpinella tragium subsp. tragium}

276.465, Tri 458, NO Katara-Pass; 276.952, loa 1076, ONO Anilio; 277.561, loa 1099, S Anilio; 279.284, Ser 259, Proti; 280.225, Dra 225, NO Drama; 283.381, Pel 354, NW Loutraki; 283.513, Pel 360, SW Promachi; 283.614, Pel 365, N Vorino; 283.988, loa 1111, NO Metsovo; 284.034, loa 1116, SW Milia; 284.068, loa 1118, Anilio; 284.071, loa 1118, Anilio; 284.171, loa 1124, N Charikli; 284.229, Pel 360, SW Promachi

\section{Pinguicula crystallina subsp. hirtiflora}

276.447, Tri 456, N Katara-Pass; 277.394, loa 1094, S Anilio; 278.040, Gre 491, NO Exarcho

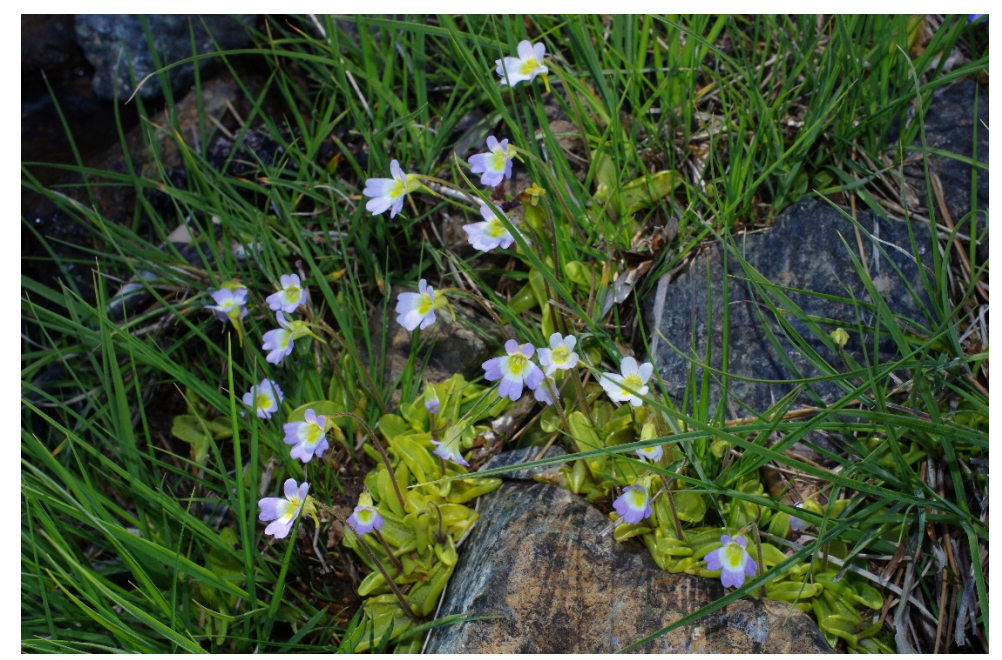

\section{Pinus sylvestris}

283.268, Pel 347, Kajmaktsalan

\section{Piptatherum miliaceum}


278.435, Thes 214, Paralia Vrasna; 278.684, Kav 107, Paralia Ofryniou; 278.756, Kav 109 , Loutra Eleftherion; 278.912, Kav 116, S Ofrynia; 278.956, Kav 119, Podochori; 279.093, Kav 89, Misoropi

\section{Plantago afra}

279.016, Kav 119, Podochori

\section{Plantago altissima}

278.639, Kav 106, Paralia Ofryniou; 279.382, Ser 262, Proti; 283.246, Pel 346, Kajmaktsalan

\section{Plantago coronopus}

276.482, loa 1059, Ag. Nikolaos; 278.447, Thes 214, Paralia Vrasna; 278.649, Kav 106, Paralia Ofryniou; 278.777, Kav 112, NO Paralia Myrtofytou; 279.413, Kav 92, Pangaion

\section{Plantago holosteum}

278.073, Gre 492, NO Varis; 279.923, Dra 211, Livadero; 280.095, Dra 219, Sidironero; 280.227, Dra 225, NO Drama; 280.599, Dra 238, Skaloti; 280.865, Dra 249, Falakron; 281.348, Dra 270, W Nevrokopi; 281.596, Dra 278, Kato Vrondous; 281.977, Dra 290, NO Potamia; 282.025, Dra 292, Nevrokopi; 282.113, Dra 296, NO Nevrokopi; 282.427, Ser 279, Orini; 282.681, Ser 290, N Neo Petritsi; 283.078, Kil 307, NW Livadia; 283.341, Pel 351, Kajmaktsalan; 283.356, Pel 352, Kajmaktsalan; 283.922, Ima 180, Seli; 283.967, loa 1056 b, NO Metsovo; 284.099, loa 1121, Anilio

\section{Plantago indica}

278.179, Gre 495, Varis; 278.455, Thes 214, Paralia Vrasna; 278.909, Kav 116, S Ofrynia; 280.230, Dra 226, N Taxiarches; 281.150, Dra 262, NW Granitis; 282.800, Ser 296, NO Strymoniko

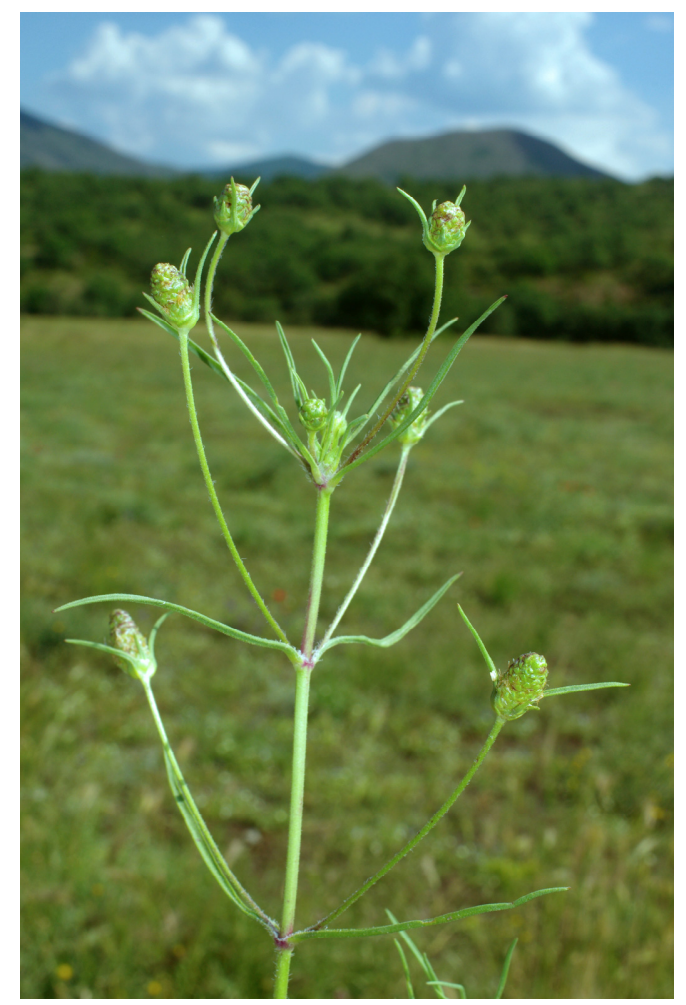

\section{Plantago lagopus}

278.659, Kav 106, Paralia Ofryniou; 278.772, Kav 112, NO Paralia Myrtofytou; 278.961, Kav 119, Podochori; 279.071, Kav 121, Platanotopos; 279.154, Ser 255, S Nea Mesolakkia; 279.206, Ser 257, Nea Fili; 279.285, Ser 259, Proti; 279.638, Ser 263, Dravoskas

\section{Plantago lanceolata}

276.587, loa 1060, SSO Potamia; 276.925, loa 1074, NNW Metsovo; 276.991, loa 1079, SO Anilio; 277.174, loa 1086, NNO Metsovo; 277.233, loa 1089, NNO Metsovo; 277.429, loa 
1095, S Anilio; 277.981, Koz 375, O Dafnero; 278.062, Gre 492, NO Varis; 278.408 , Koz 383, Galatini; 278.468, Thes 215, Paralia Vrasna; 278.606, Thes 221, Arethousa; 279.075, Kav 121, Platanotopos; 279.111, Kav 90, SW Moustheni; 279.312, Ser 259, Proti; 279.459, Kav 95, Pangaion; 279.690, Ser 266, SO Nea Zichni; 279.963, Dra 213, Livadero; 280.229, Dra 225, NO Drama; 280.453, Dra 234, Skaloti; 281.055, Dra 259, Granitis; 281.388, Dra 272, Vathytopo; 281.580, Dra 277, Perithori; 281.666, Ser 270, Kato Vrondous; 281.847 , Dra 284, NNO Volakas; 282.226, Dra 300, SO Lefkogia; 282.407, Ser 278, N Serres; 282.453, Ser 280, Orini; 282.643, Ser 290, N Neo Petritsi; 283.245, Pel 346, Kajmaktsalan; 283.472, Pel 358, N Loutraki; 283.557, Pel 362, NW Promachi; 283.599, Pel 364, Loutraki; 283.646, Pel 367, Vorino; -20.473, loa 1055, N Metsovo; -20.491, loa 1078, O Anilio; -20.510, Dra 236, Skaloti

\section{Plantago major subsp. intermedia}

277.578, loa 1100, S Anilio; 279.497, Kav 97, Pangaion; 280.465, Dra 234, Skaloti; 280.944, Dra 254, Falakron; 281.691, Ser 271, Kato Vrondous; 281.945, Dra 288, SW Potamia; 282.217, Dra 300, SO Lefkogia; 282.975, Kil 299, Kastaneri; 283.194, Pel 343, Kerasia; 283.370, Pel 353, Kajmaktsalan

\section{Plantago major subsp. major}

276.580, loa 1060, SSO Potamia; 276.968, loa 1078, O Anilio; 277.132, loa 1084, N Metsovo; 279.838, Dra 207, NO Skopia; 280.077, Dra 219, Sidironero; 280.340, Dra 230, Livadero; 280.664, Dra 242, SW Walddorf Elatias; 280.790, Dra 247, Walddorf Elatias; 280.989, Dra 257, Granitis; 281.357, Dra 270, W Nevrokopi; 281.794, Dra 282, NNO Volakas; 282.293, Ser 272, Ano Vrondous; 282.499, Ser 282, Ori Vrondous; 282.569, Ser 286, Ori Vrondous; 282.651, Ser 290, N Neo Petritsi; 283.581, Pel 363, NW Promachi; 283.869, Ima 178, Seli; -20.509, Dra 236, Skaloti; -20.515, Dra 238, Skaloti

\section{Plantago media}

280.587, Dra 238, Skaloti; 280.684, Dra 242, SW Walddorf Elatias; 280.943, Dra 254, Falakron

\section{Platanthera chlorantha}

277.181, loa 1086, NNO Metsovo; 277.243, loa 1089, NNO Metsovo; 277.568, loa 1099, S Anilio; -20.485, loa 1078, O Anilio

\section{Platanus orientalis}

283.621, Pel 366, N Vorino

\section{Plocama calabrica}

276.547, loa 1060, SSO Potamia; 278.014, Koz 375, O Dafnero

\section{Poa}

276.371, loa 1128, N Metsovo; 277.161, loa 1086, NNO Metsovo; 279.948, Dra 212, Livadero; 280.801, Dra 247, Walddorf Elatias

\section{Poa angustifolia}

276.543, loa 1059, Ag. Nikolaos; 277.087, loa 1081, W Metsovo; 279.387, Ser 262, Proti; 280.390, Dra 232, Sidironero; 280.524, Dra 236, Skaloti; 283.170, Pel 343, Kerasia

\section{Poa annua}

277.192, loa 1087, NNO Metsovo; 277.448, loa 1095, S Anilio; 278.904, Kav 116, S Ofrynia; 279.094, Kav 89, Misoropi; 279.543, Kav 101, Pangaion; 280.331, Dra 230, Livadero; 280.531, Dra 236, Skaloti; 280.628, Dra 240, Elatias; 281.113, Dra 261, Granitis; 282.613, Ser 288, Ori Vrondous; 282.966, Kil 299, Kastaneri; 283.106, Pel 339, S Archangelo

\section{Poa bulbosa}

276.884, loa 1072, NNW Metsovo; 277.239, loa 1089, NNO Metsovo

\section{Poa macedonica}


276.420, loa 1057, NO Metsovo; 276.436, loa 1058, Katara-Pass; 276.827, loa 1070, NNW Metsovo; 276.996, loa 1079, SO Anilio

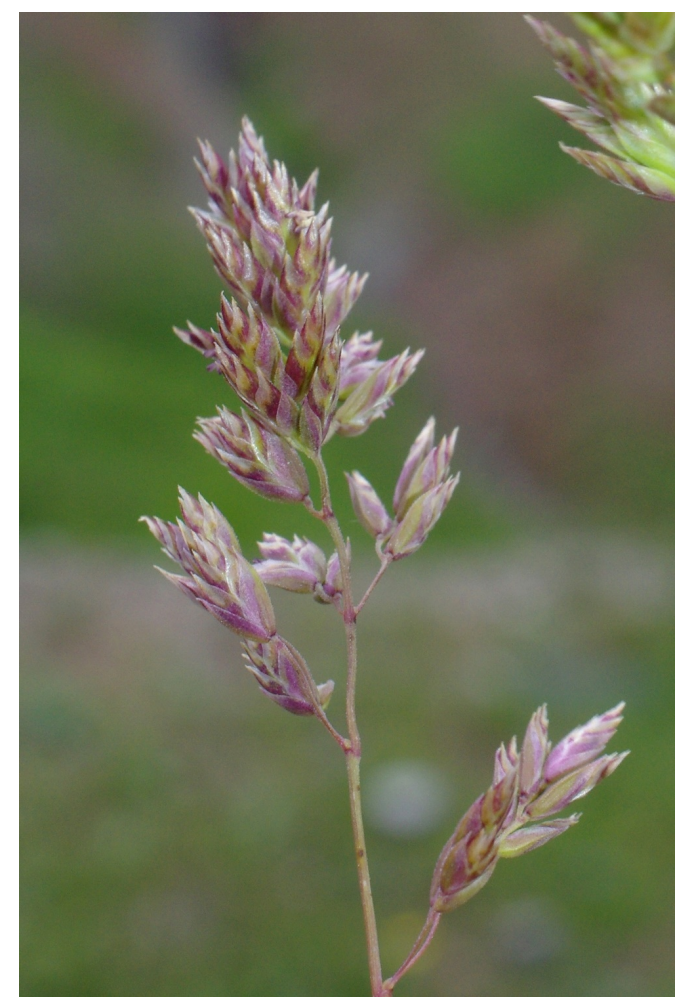

\section{Poa media}

278.187, Gre 495, Varis; 280.055, Dra 217, Sidironero; 283.261, Pel 347, Kajmaktsalan

\section{Poa molinerii}

277.659, loa 1106, Anilio

\section{Poa nemoralis}

276.459, Tri 457, NO Katara-Pass; 276.628, loa 1062, SO Potamia; 276.704 , loa 1065 , NO Karyes; 277.198, loa 1087, NNO Metsovo; 277.504, loa 1097, S Anilio; 279.513, Kav 99, Pangaion; 280.613, Dra 238, Skaloti; 280.699, Dra 242, SW Walddorf Elatias; 280.737, Dra 244, SW Walddorf Elatias; 281.117, Dra 261, Granitis; 281.652, Dra 279, Kato Vrondous; 282.512, Ser 282, Ori Vrondous; 282.577, Ser 286, Ori Vrondous; 283.485 , Pel 359, NW Loutraki; 283.850, Ima 178, Seli

\section{Poa ophiolithica}

276.389, loa 1056, NO Metsovo; 277.069, loa 1080, SO Anilio; 277.133, loa 1084, N Metsovo; 277.201, loa 1087, NNO Metsovo; 280.332, Dra 230, Livadero; 283.017, Kil 302, Kastaneri

\section{Poa pratensis}

276.424, loa 1057, NO Metsovo; 276.441, loa 1058, Katara-Pass; 276.545, loa 1060, SSO Potamia; 276.584, loa 1060, SSO Potamia; 277.245, loa 1089, NNO Metsovo; 277.320, loa 1092, NO Metsovo; 277.548, loa 1098, S Anilio; 279.476, Kav 96, Pangaion; 279.495, Kav 97, Pangaion; 280.268, Dra 228, Livadero; 280.399, Dra 232, Sidironero; 280.488, Dra 235, Skaloti; 280.633, Dra 240, Elatias; 280.636, Dra 241, Elatias; 280.665, Dra 242, SW Walddorf Elatias; 280.786, Dra 247, Walddorf Elatias; 282.218, Dra 300, SO Lefkogia; 282.461, Ser 280, Orini; 283.281, Pel 348, Kajmaktsalan

\section{Poa trivialis subsp. sylvicola}

276.350, loa 1128, N Metsovo; 277.496, loa 1097, S Anilio; 277.603, loa 1102, S Anilio; 277.712, Tri 460, N Chaliki; 277.770, Tri 462, N Chaliki; 278.247, Koz 379, Galatini; 280.321, Dra 230, Livadero; 280.475, Dra 234, Skaloti; 280.660, Dra 242, SW Walddorf 
Elatias; 280.977, Dra 256, Falakron; 281.072, Dra 260, Granitis; 283.283, Pel 349, Kajmaktsalan

Poa trivialis subsp. trivialis

276.813, loa 1070, NNW Metsovo

\section{Podospermum canum}

276.836, loa 1070, NNW Metsovo; 276.992, loa 1079, SO Anilio; 277.097, loa 1084, N Metsovo; 277.215, loa 1088, NNO Metsovo; 277.625, loa 1104, S Anilio; 277.708, Tri 460, N Chaliki; 277.884, Koz 388, SO Siatista, Vourinos; 278.195, Koz 377, N Siatista; 278.223, Koz 378, N Siatista; 278.287, Koz 380, Galatini; 278.324, Koz 381, Galatini; 279.584, Kav 104, Pangaion; 283.948, loa 1109, NW Metsovo; 284.104, loa 1121, Anilio

\section{Podospermum laciniatum}

276.364, loa 1128, N Metsovo; 277.157, loa 1086, NNO Metsovo; 278.935, Kav 118, Galipsos; 280.854, Dra 248, Falakron

\section{Polycarpon tetraphyllum}

278.465, Thes 214, Paralia Vrasna; 278.803, Kav 113, NO Paralia Myrtofytou; 279.078, Kav 89, Misoropi

\section{Polygala alpestris}

280.883, Dra 250, Falakron; 282.076, Dra 294, NO Nevrokopi; 283.254, Pel 346, Kajmaktsalan

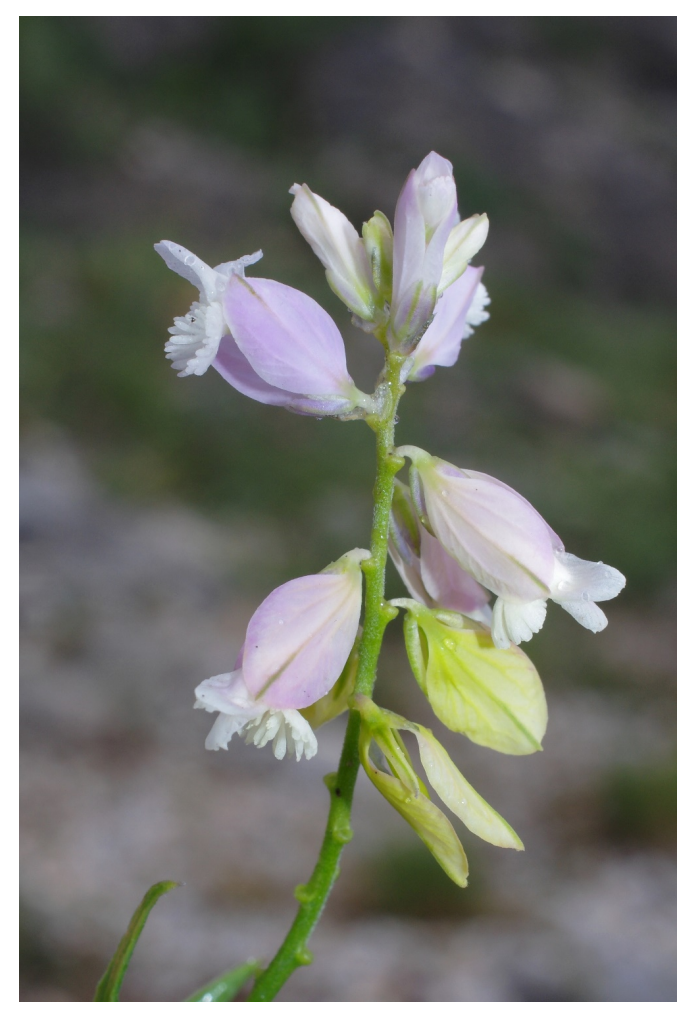

\section{Polygala comosa}

276.392, loa 1056, NO Metsovo; 278.235, Koz 378, N Siatista

\section{Polygala nicaeensis subsp. mediterranea}

276.353, loa 1128, N Metsovo; 276.383, loa 1056, NO Metsovo; 276.525, loa 1059, Ag. Nikolaos; 276.823, loa 1070, NNW Metsovo; 277.268, loa 1090, NNO Metsovo; 277.555, Ioa 1099, S Anilio; 277.762, Tri 462, N Chaliki; 277.878, Koz 388, SO Siatista, Vourinos; 277.879, Koz 388, SO Siatista, Vourinos; 278.070, Gre 492, NO Varis; 278.090, Gre 492, NO Varis; 279.551, Kav 101, Pangaion

\section{Polygonum arenarium}


278.051, Gre 491, NO Exarcho; 278.083, Gre 492, NO Varis; 279.003 , Kav 119, Podochori; 279.643, Ser 263, Dravoskas

\section{Polygonum arenastrum}

281.926, Dra 288, SW Potamia; 282.746, Ser 292, N Neo Petritsi

\section{Polygonum aviculare subsp. aviculare}

276.608, loa 1061, SSO Potamia; 277.437, loa 1095, S Anilio; 278.696, Kav 107, Paralia Ofryniou; 279.121, Kav 91, Orfani; 279.185, Ser 256, Paleokomi; 281.010, Dra 257,

Granitis; 282.615, Ser 288, Ori Vrondous; 282.967, Kil 299, Kastaneri; 283.555, Pel 362, NW Promachi

\section{Polygonum aviculare subsp. neglectum}

283.346, Pel 352, Kajmaktsalan

Polygonum aviculare subsp. rurivagum

283.761, Ima 172, S Ag. Pavlos

Polygonum bellardii

278.251, Koz 379, Galatini

\section{Polypogon viridis}

276.653, loa 1063, W Potamia; 277.396, loa 1094, S Anilio; 277.752, Tri 461, N Chaliki; 279.083, Kav 89, Misoropi; 279.133, Kav 91, Orfani; 281.368, Dra 271, Vathytopo

\section{Polystichum}

282.593, Ser 287, Ori Vrondous

\section{Populus alba}

279.651, Ser 264, Dravoskas; 282.926, Kil 297, WNW Kastaneri; 283.149, Pel 342, S

Archangelo; 283.578, Pel 363, NW Promachi

\section{Populus nigra}

279.997, Dra 215, Livadero; 280.700, Dra 242, SW Walddorf Elatias; 281.892, Dra 287, O Mikroklisoura; 282.034, Dra 292, Nevrokopi; 282.892, Kil 295, NW Griva

\section{Populus $x$ canadensis}

282.716, Ser 290, N Neo Petritsi

\section{Portulaca oleracea}

278.712, Kav 107, Paralia Ofryniou; 278.924, Kav 117, Galipsos; 279.680, Ser 265, Myrrini; 281.318, Dra 268, W Nevrokopi

\section{Potentilla}

281.815, Dra 283, NNO Volakas

\section{Potentilla argentea}

277.696, Tri 460, N Chaliki; 277.969, Koz 374, Vourinos; 279.875, Dra 209, Livadero; 279.983, Dra 214, Livadero; 280.287, Dra 228, Livadero; 280.496, Dra 235, Skaloti; 281.097, Dra 261, Granitis; 281.484, Dra 275, Vathytopo; 281.686, Ser 271, Kato Vrondous; 281.903, Dra 287, O Mikroklisoura; 282.098, Dra 295, NO Nevrokopi; 282.272, Ser 272, Ano Vrondous; 282.431, Ser 279, Orini; 282.767, Ser 293, N Neo Petritsi; 283.271, Pel 348, Kajmaktsalan

\section{Potentilla detommasii}

276.352, loa 1128, N Metsovo; 276.675, loa 1065, NO Karyes; 277.949, Koz 374, Vourinos; 278.164, Gre 494, SO Varis; 278.217, Koz 378, N Siatista; 278.238, Koz 378, N Siatista; 278.535, Thes 218, Vrasna; 279.440, Kav 95, Pangaion; 279.481, Kav 97, Pangaion; 280.691, Dra 242, SW Walddorf Elatias; 281.137, Dra 262, NW Granitis; 282.682, Ser 290, N Neo Petritsi; 283.295, Pel 349, Kajmaktsalan

\section{Potentilla inclinata}

277.724, Tri 460, N Chaliki; 279.530, Kav 99, Pangaion; 279.591, Kav 104, Pangaion; 280.534, Dra 236, Skaloti; 280.611, Dra 238, Skaloti; 280.788, Dra 247, Walddorf Elatias; 
280.914, Dra 251, Falakron; 282.541, Ser 284, Ori Vrondous; 282.563 , Ser 285, Ori Vrondous; 282.870, Kil 295, NW Griva

\section{Potentilla laciniosa}

276.332, loa 1125, NO Dodoni; 276.354, loa 1128, N Metsovo; 276.470, Tri 458, NO KataraPass; 276.637, loa 1063, W Potamia; 276.745, loa 1068, NW Metsovo; 276.778, loa 1069, NW Metsovo; 277.017, loa 1080, SO Anilio; 277.252, loa 1089, NNO Metsovo; 277.289, loa 1091, SW Milia; 277.557, loa 1099, S Anilio; 277.609, loa 1103, S Anilio; 277.900, Koz 389, SO Siatista, Vourinos; 278.037, Gre 491, NO Exarcho; 278.503, Thes 216, Vrasna; 278.857, Kav 115, SW Folia; 278.860, Kav 115, SW Folia; 278.997, Kav 119, Podochori; 279.263, Ser 258, Mikro Souli; 279.415, Kav 93, Pangaion; 279.619, Ser 263, Dravoskas; 279.719, Ser 267, SW Skopia; 279.752, Ser 268, SW Skopia; 280.042, Dra 217, Sidironero; 280.183, Dra 223, NO Drama; 280.624, Dra 239, Skaloti; 280.940, Dra 254, Falakron; 282.203, Dra 300, SO Lefkogia; 282.582, Ser 287, Ori Vrondous; 282.773 , Ser 293, N Neo Petritsi; 282.991, Kil 300, Kastaneri; 284.010, loa 1113, Katara-Paß

\section{Potentilla pedata}

277.725, Tri 460, N Chaliki; 277.846, Koz 387, SO Siatista, Vourinos; 278.072, Gre 492, NO Varis; 278.354, Koz 381, Galatini; 278.602, Thes 221, Arethousa; 279.091, Kav 89, Misoropi; 279.996, Dra 215, Livadero; 280.019, Dra 216, Sidironero; 280.112, Dra 220, Sidironero; 280.848, Dra 248, Falakron; 280.979, Dra 256, Falakron; 281.167, Dra 263, NW Granitis; 281.858, Dra 285, NNO Volakas; 281.910, Dra 287, O Mikroklisoura; 281.913, Dra 287, O Mikroklisoura; 282.314, Ser 274, SSW Ano Vrondous; 283.357, Pel 352,

Kajmaktsalan; 283.912, Ima 180, Seli

\section{Potentilla recta}

277.880, Koz 388, SO Siatista, Vourinos; 277.945, Koz 392, SO Siatista, Vourinos; 280.912, Dra 251, Falakron; 281.399, Dra 272, Vathytopo; 282.304, Ser 273, Ano Vrondous; 282.601, Ser 288, Ori Vrondous

\section{Potentilla reptans}

277.074, loa 1080, SO Anilio; 277.140, loa 1084, N Metsovo; 278.283, Koz 379, Galatini; 278.566, Thes 219, Vrasna; 278.812, Kav 114, SO Folia; 279.353, Ser 261, Proti; 279.456, Kav 95, Pangaion; 280.085, Dra 219, Sidironero; 280.462, Dra 234, Skaloti; 280.586, Dra 238, Skaloti; 281.110, Dra 261, Granitis; 281.682, Ser 271, Kato Vrondous; 282.228, Dra 300, SO Lefkogia; 283.104, Pel 339, S Archangelo; 283.207, Pel 344, Kerasia; 283.275, Pel 348, Kajmaktsalan; 284.157, loa 1103 b, S Anilio

\section{Prenanthes purpurea}

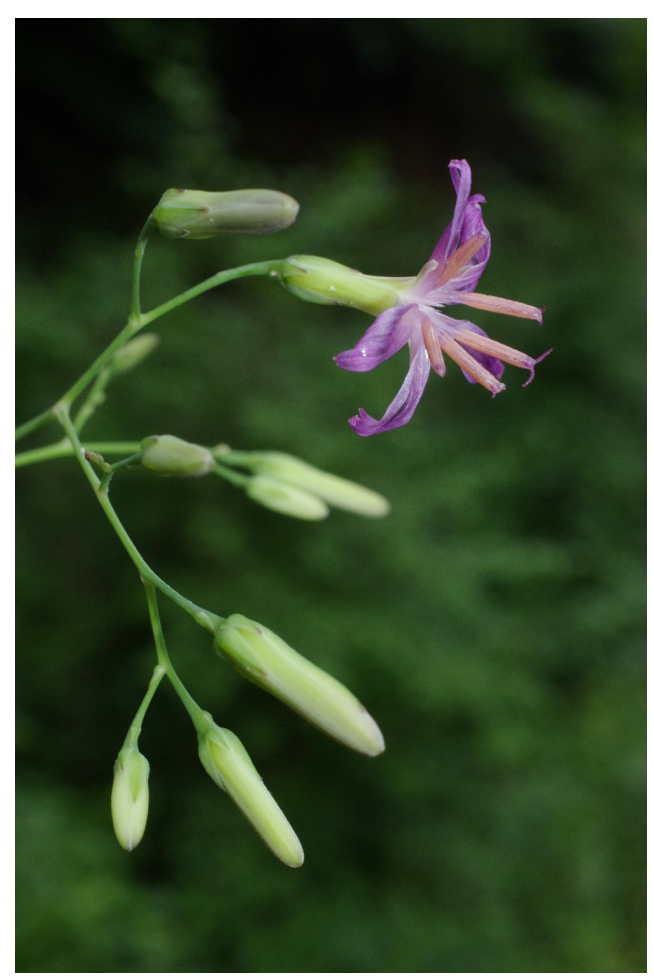




\section{Prunella grandiflora}

283.996, loa 1112, Katara-Paß; 284.088, loa 1120, Anilio

\section{Prunella laciniata}

276.293, loa 1125, NO Dodoni; 276.523, loa 1059, Ag. Nikolaos; 276.786, loa 1070, NNW Metsovo; 276.866, loa 1072, NNW Metsovo; 278.056, Gre 491, NO Exarcho; 278.068, Gre 492, NO Varis; 278.854, Kav 115, SW Folia; 279.366, Ser 261, Proti; 279.426, Kav 94, Pangaion; 279.880, Dra 209, Livadero; 280.082, Dra 219, Sidironero; 280.278, Dra 228, Livadero; 280.953, Dra 255, Falakron; 281.480, Dra 275, Vathytopo; 281.653, Dra 279, Kato Vrondous; 281.859, Dra 285, NNO Volakas; 281.919, Dra 287, O Mikroklisoura; 282.086, Dra 295, NO Nevrokopi; 283.464, Pel 358, N Loutraki; 283.906, Ima 180, Seli

\section{Prunella vulgaris}

276.540, loa 1059, Ag. Nikolaos; 276.712, loa 1065, NO Karyes; 276.857, loa 1071, NNW Metsovo; 277.168, loa 1086, NNO Metsovo; 277.237, loa 1089, NNO Metsovo; 277.424, loa 1095, S Anilio; 277.745, Tri 461, N Chaliki; 278.863, Kav 115, SW Folia; 279.367, Ser 261, Proti; 279.503, Kav 98, Pangaion; 279.566, Kav 102, Pangaion; 280.029, Dra 216, Sidironero; 280.342, Dra 230, Livadero; 280.695, Dra 242, SW Walddorf Elatias; 280.787 , Dra 247, Walddorf Elatias; 281.081, Dra 260, Granitis; 281.226, Dra 265, NO Granitis; 281.732, Dra 281, OSO Granitis; 281.777, Dra 282, NNO Volakas; 281.939, Dra 288, SW Potamia; 282.103, Dra 295, NO Nevrokopi; 282.530, Ser 284, Ori Vrondous; 282.715 , Ser 290, N Neo Petritsi; 283.005, Kil 302, Kastaneri; 283.428, Pel 355, NW Loutraki; 283.841 , Ima 177, Tria-Pende Pigadia; 283.848, Ima 178, Seli; -20.501, Dra 234, Proti; -20.513, Dra 236, Skaloti; -20.522, Ser 286, SW Mikroklisoura

\section{Prunella $\mathrm{x}$ intermedia}

276.944, loa 1075, NW Anilio; 277.383, loa 1093, S Anilio; 278.244, Koz 378, N Siatista; 279.791, Ser 268, SW Skopia; 280.080, Dra 219, Sidironero; 280.200, Dra 224, NO Drama; 280.315, Dra 229, SO Dendrakia; 281.165, Dra 263, NW Granitis

\section{Psilurus incurvus}

276.537, loa 1059, Ag. Nikolaos; 277.639, loa 1104, S Anilio

\section{Pteridium aquilinum}

276.701, loa 1065, NO Karyes; 277.047, loa 1080, SO Anilio; 279.474, Kav 96, Pangaion; 283.138, Pel 341, S Archangelo; 283.476, Pel 358, N Loutraki

\section{Pterocephalus plumosus}

278.243, Koz 378, N Siatista; 279.148, Ser 255, S Nea Mesolakkia; 279.624, Ser 263, Dravoskas

\section{Ptilostemon afer}

278.210, Koz 377, N Siatista; 280.932, Dra 253, Falakron; 282.462, Ser 280, Orini; 283.084, Kil 307, NW Livadia; 283.155, Pel 342, S Archangelo; 283.234, Pel 345, Kerasia; 283.805, Ima 175, S Ag. Pavlos; 284.026, loa 1116, SW Milia

\section{Puccinellia distans}

277.171, loa 1086, NNO Metsovo

\section{Pulicaria dysenterica}

281.112, Dra 261, Granitis; 282.898, Kil 296, N Kastaneri; 283.409, Pel 355, NW Loutraki; 283.516, Pel 360, SW Promachi; 283.643, Pel 367, Vorino; 284.047, loa 1117, Anilio; 284.125, loa 1094 b, S Anilio

\section{Pulmonaria rubra}

280.701, Dra 242, SW Walddorf Elatias 
Punica granatum

283.637, Pel 366, N Vorino

Pyrola minor

280.596, Dra 238, Skaloti

Quercus frainetto

276.696, loa 1065, NO Karyes; 282.015, Dra 292, Nevrokopi

Quercus ithaburensis subsp. macrolepis

276.593, Ioa 1061, SSO Potamia; 278.413, Koz 383, Galatini; 280.005, Dra 216, Sidironero; 282.653, Ser 290, N Neo Petritsi; 282.717, Ser 290, N Neo Petritsi; 283.508, Pel 360, SW Promachi

Quercus petraea

281.670, Ser 270, Kato Vrondous

\section{Quercus pubescens}

276.590, loa 1061, SSO Potamia; 277.920, Koz 391, SO Siatista, Vourinos; 278.240 , Koz

378, N Siatista; 282.880, Kil 295, NW Griva; 283.405, Pel 354, NW Loutraki

Quercus robur

280.296, Dra 228, Livadero

Ranunculus

277.637, loa 1104, S Anilio

\section{Ranunculus arvensis}

278.116, Gre 492, NO Varis; 278.250, Koz 379, Galatini; 278.378, Koz 382, Galatini;

278.574, Thes 220, Arethousa

\section{Ranunculus brutius}

277.176, loa 1086, NNO Metsovo; 280.729, Dra 244, SW Walddorf Elatias; 282.939, Kil 298, WNW Kastaneri; 283.011, Kil 302, Kastaneri; 283.836, Ima 177, Tria-Pende Pigadia

\section{Ranunculus fontanus}

282.583, Ser 287, Ori Vrondous

\section{Ranunculus marginatus}

276.602, loa 1061, SSO Potamia; 277.018, loa 1080, SO Anilio

\section{Ranunculus neapolitanus}

276.377, loa 1128, N Metsovo; 276.678, loa 1065, NO Karyes; 276.802, loa 1070, NNW Metsovo; 276.988, loa 1079, SO Anilio; 277.102, loa 1084, N Metsovo; 277.230, loa 1089, NNO Metsovo; 277.313, loa 1092, NO Metsovo; 277.500, loa 1097, S Anilio; 277.599, loa 1102, S Anilio; 279.345, Ser 261, Proti; 283.296, Pel 349, Kajmaktsalan

\section{Ranunculus pindicola}

276.421, loa 1057, NO Metsovo; 276.918, loa 1073, NNW Metsovo 


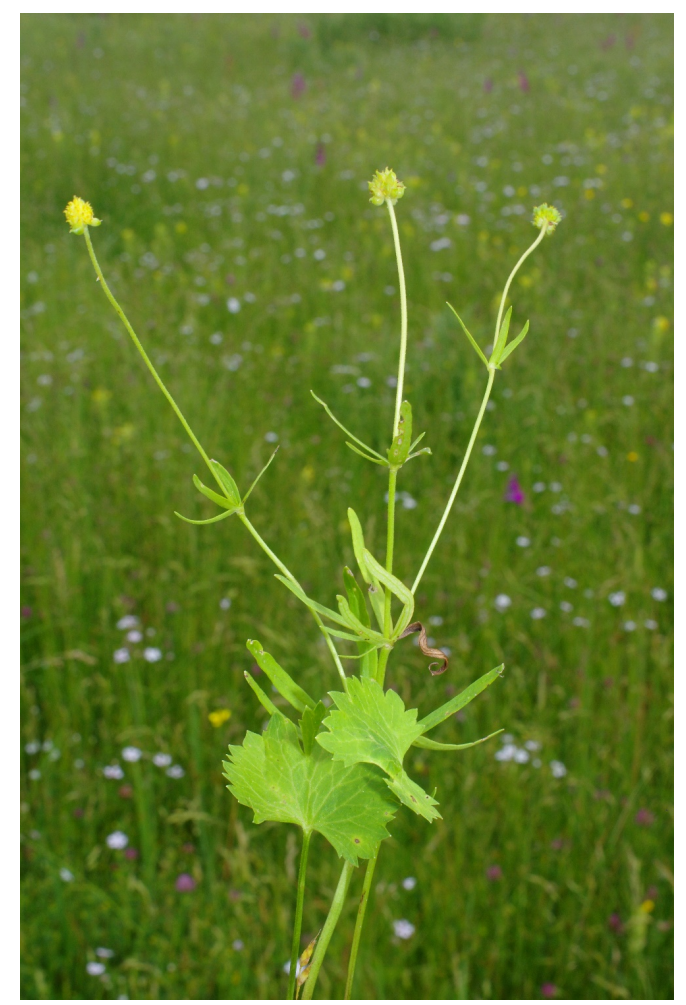

\section{Ranunculus polyanthemoides}

276.426, loa 1057, NO Metsovo; 276.733, loa 1067, NW Metsovo; 280.422, Dra 233, Sidironero; 280.506, Dra 236, Skaloti; 280.603, Dra 238, Skaloti; 280.746, Dra 245, SW Walddorf Elatias; 280.963, Dra 255, Falakron; 282.635, Ser 290, N Neo Petritsi

\section{Ranunculus polyanthemos}

276.374, loa 1128, N Metsovo; 276.869, loa 1072, NNW Metsovo; 276.874, loa 1072, NNW Metsovo; 276.964, loa 1078, O Anilio; 276.967, loa 1078, O Anilio; 277.051, loa 1080, SO Anilio; 277.056, loa 1080, SO Anilio; 277.197, loa 1087, NNO Metsovo; 277.617, loa 1103, S Anilio; 279.930, Dra 211, Livadero; 280.297, Dra 229, SO Dendrakia; 280.351, Dra 231, Sidironero; 280.479, Dra 234, Skaloti; 280.585, Dra 238, Skaloti; 280.631, Dra 240, Elatias; 280.659, Dra 242, SW Walddorf Elatias; 280.869, Dra 249, Falakron; 281.111, Dra 261, Granitis; 282.063, Dra 294, NO Nevrokopi; 282.085, Dra 295, NO Nevrokopi; 283.339, Pel 351, Kajmaktsalan; 283.366, Pel 353, Kajmaktsalan

\section{Ranunculus repens}

276.415, loa 1057, NO Metsovo; 276.731, loa 1067, NW Metsovo; 277.024, loa 1080, SO Anilio; 277.139, loa 1084, N Metsovo; 277.240, loa 1089, NNO Metsovo; 277.741, Tri 461, N Chaliki; 280.696, Dra 242, SW Walddorf Elatias; 280.730, Dra 244, SW Walddorf Elatias; 282.581, Ser 287, Ori Vrondous

\section{Ranunculus trichophyllus}

277.627, loa 1104, S Anilio

\section{Raphanus raphanistrum subsp. landra}

278.444, Thes 214, Paralia Vrasna; 278.481, Thes 215, Paralia Vrasna; 278.747, Kav 109, Loutra Eleftherion; 278.836, Kav 114, SO Folia

\section{Raphanus raphanistrum subsp. raphanistrum}

281.519, Dra 276, Perithori

\section{Rapistrum rugosum}

277.440, loa 1095, S Anilio; 278.918, Kav 117, Galipsos; 279.125, Kav 91, Orfani

\section{Reseda lutea}


277.434, loa 1095, S Anilio; 277.829, Koz 387, SO Siatista, Vourinos; 278.593, Thes 220 , Arethousa; 278.711, Kav 107, Paralia Ofryniou; 278.988, Kav 119, Podochori; 279.205, Ser 257, Nea Fili; 279.248, Ser 258, Mikro Souli; 279.640, Ser 263, Dravoskas; 279.665, Ser 265, Myrrini; 279.699, Ser 266, SO Nea Zichni; 279.762, Ser 268, SW Skopia; 280.523, Dra 236, Skaloti; 280.839, Dra 248, Falakron; 281.419, Dra 273, Vathytopo; 283.103, Pel 339, S Archangelo

\section{Rhagadiolus stellatus}

277.464, loa 1096, S Anilio

\section{Rhinanthus minor}

282.046, Dra 293, Nevrokopi; 283.026, Kil 303, SO Livadia; 283.067, Kil 307, NW Livadia; 283.758, Ima 172, S Ag. Pavlos

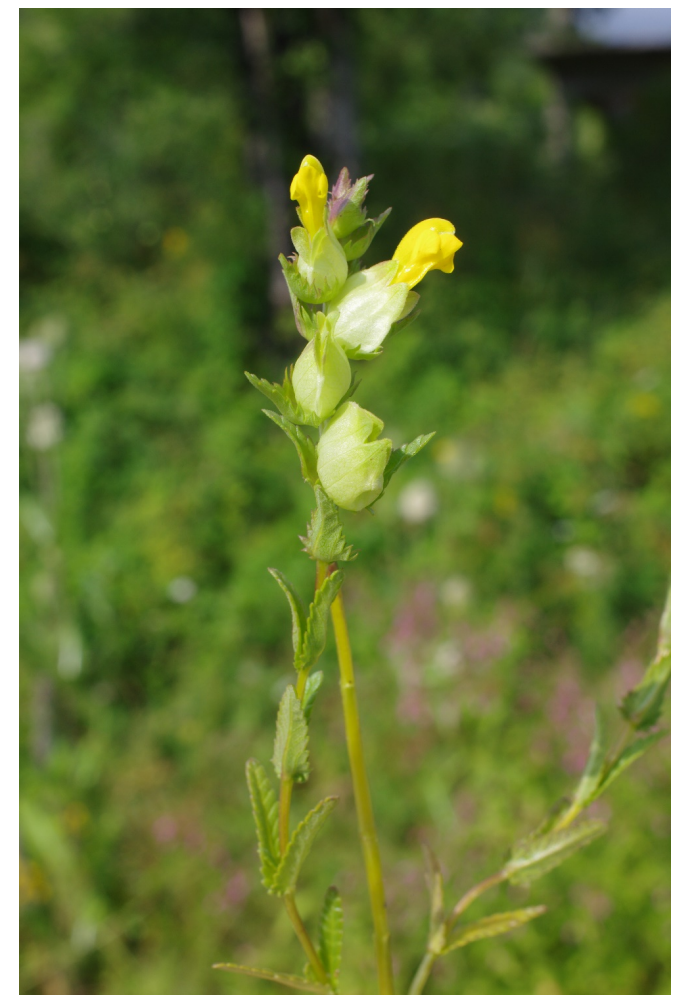

\section{Rhinanthus pindicus}

280.569, Dra 238, Skaloti; 283.845, Ima 178, Seli

\section{Rhinanthus pubescens}

283.952, loa 1109, NW Metsovo

\section{Rhinanthus rumelicus}

280.794, Dra 247, Walddorf Elatias; 280.903, Dra 250, Falakron; 282.322, Ser 274, SSW Ano Vrondous

\section{Rhinanthus sintenisii}

276.757, loa 1068, NW Metsovo; 276.794, loa 1070, NNW Metsovo; 277.092, loa 1083, NW Metsovo 


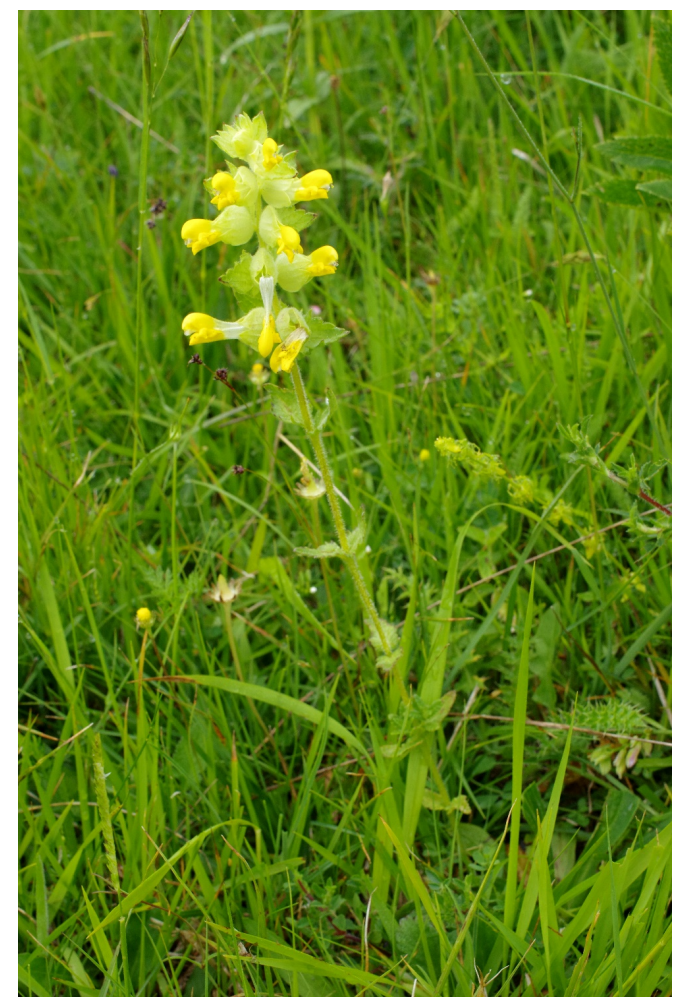

\section{Rhus coriaria}

283.406, Pel 354, NW Loutraki

\section{Robinia pseudoacacia}

278.496, Thes 216, Vrasna; 282.807, Ser 296, NO Strymoniko

\section{Rorippa pyrenaica}

276.761, loa 1069, NW Metsovo; 276.787, loa 1070, NNW Metsovo; 277.072, loa 1080, SO Anilio; 277.558, loa 1099, S Anilio

\section{Rorippa sylvestris}

278.269, Koz 379, Galatini; 278.794, Kav 113, NO Paralia Myrtofytou; 280.343, Dra 230, Livadero; 280.530, Dra 236, Skaloti; 280.597, Dra 238, Skaloti; 280.689, Dra 242, SW Walddorf Elatias; 280.778 , Dra 247, Walddorf Elatias; 281.422, Dra 273, Vathytopo; 282.552, Ser 285, Ori Vrondous; 283.556, Pel 362, NW Promachi; 283.684, Pel 369, O Vorino; 284.226, Dra 282, NNO Volakas

\section{Rosa}

279.590, Kav 104, Pangaion; 280.662, Dra 242, SW Walddorf Elatias; 284.220, Koz 392, SO Siatista, Vourinos

\section{Rosa canina}

276.754, loa 1068, NW Metsovo; 277.093, loa 1083, NW Metsovo; 277.584, loa 1100, S Anilio; 277.665, Tri 459, N Chaliki; 277.704, Tri 460, N Chaliki; 277.955, Koz 374, Vourinos; 279.555, Kav 102, Pangaion; 280.491, Dra 235, Skaloti; 282.629, Ser 289, Ori Vrondous

\section{Rosa pulverulenta}

276.366, loa 1128, N Metsovo; 276.910, loa 1073, NNW Metsovo; 277.299, loa 1091, SW Milia; 277.655, loa 1106, Anilio; 279.587, Kav 104, Pangaion; 280.592, Dra 238, Skaloti

\section{Rosa sempervirens}

276.948, loa 1076, ONO Anilio

\section{Rosa spinosissima}

277.703, Tri 460, N Chaliki

\section{Rosa stylosa}


276.672, loa 1064, ONO Karyes; 280.536, Dra 236, Skaloti; 276.777, loa 1069, NW Metsovo

\section{Rosa villosa}

277.926, Koz 392, SO Siatista, Vourinos

\section{Rostraria cristata}

276.494, loa 1059, Ag. Nikolaos; 277.825, Koz 387, SO Siatista, Vourinos; 278.252, Koz 379, Galatini; 278.397, Koz 383, Galatini; 278.474, Thes 215, Paralia Vrasna; 278.721, Kav 108, Paralia Ofryniou; 278.802, Kav 113, NO Paralia Myrtofytou; 279.311, Ser 259, Proti; 280.238, Dra 226, N Taxiarches; 283.107, Pel 339, S Archangelo

\section{Rubia peregrina}

278.853, Kav 115, SW Folia

\section{Rubus caesius}

281.629, Dra 279, Kato Vrondous; 283.183, Pel 343, Kerasia

\section{Rubus canescens}

277.317, loa 1092, NO Metsovo; 277.546, loa 1098, S Anilio; 277.891, Koz 389, SO Siatista, Vourinos; 277.979, Koz 374, Vourinos; 279.379, Ser 262, Proti; 279.467, Kav 96, Pangaion; 279.511, Kav 99, Pangaion; 279.984, Dra 214, Livadero; 280.293, Dra 228, Livadero; 280.372, Dra 232, Sidironero; 280.455, Dra 234, Skaloti; 280.978, Dra 256, Falakron; 281.491, Dra 275, Vathytopo; 281.725, Dra 280, Nevrokopi; 281.835, Dra 284, NNO Volakas; 282.104, Dra 295, NO Nevrokopi; 282.181, Dra 299, SO Lefkogia; 282.302, Ser 273, Ano Vrondous; 282.345, Ser 275, SSW Ano Vrondous; 282.520, Ser 283, Ori Vrondous; 282.549, Ser 285, Ori Vrondous; 282.712, Ser 290, N Neo Petritsi; 282.949, Kil 298, WNW Kastaneri; 283.089, Pel 339, S Archangelo; 283.784, Ima 173, S Ag. Pavlos; 283.849, Ima 178, Seli

\section{Rubus sanctus}

276.555, loa 1060, SSO Potamia; 276.693, loa 1065, NO Karyes; 278.554, Thes 219, Vrasna; 278.754, Kav 109, Loutra Eleftherion; 279.283, Ser 259, Proti; 279.444, Kav 95, Pangaion; 279.531, Kav 99, Pangaion; 279.794, Ser 269, NO Skopia; 279.911, Dra 210, Livadero; 280.115, Dra 220, Sidironero; 280.289, Dra 228, Livadero; 280.485, Dra 235, Skaloti; 281.085, Dra 260, Granitis; 281.957, Dra 289, NO Potamia; 282.786, Ser 295, N Neo Petritsi; 282.813, Ser 296, NO Strymoniko; 283.143, Pel 341, S Archangelo; 283.181, Pel 343, Kerasia; 283.554, Pel 362, NW Promachi; 283.666, Pel 368, OSO Vorino

\section{Rumex acetosella}

276.861, loa 1071, NNW Metsovo; 277.064, loa 1080, SO Anilio; 277.563, loa 1099, S Anilio; 277.723, Tri 460, N Chaliki; 277.914, Koz 390, SO Siatista, Vourinos; 278.038, Gre 491, NO Exarcho; 278.125, Gre 493, NO Varis; 278.469, Thes 215, Paralia Vrasna; 278.555, Thes 219, Vrasna; 278.874, Kav 115, SW Folia; 279.381, Ser 262, Proti; 279.580, Kav 103, Pangaion; 279.910, Dra 210, Livadero; 280.533, Dra 236, Skaloti; 280.651, Dra 242, SW Walddorf Elatias; 281.690, Ser 271, Kato Vrondous; 282.442, Ser 280, Orini; 282.732, Ser 291, N Neo Petritsi; 282.871, Kil 295, NW Griva; 283.171, Pel 343, Kerasia; 283.325, Pel 351 , Kajmaktsalan

\section{Rumex arifolius}

280.751, Dra 245, SW Walddorf Elatias; 281.281, Dra 267, W Nevrokopi; 282.287, Ser 272, Ano Vrondous

\section{Rumex conglomeratus}

278.558, Thes 219, Vrasna; 278.760, Kav 110, Loutra Eleftherion; 282.886, Kil 295, NW Griva

\section{Rumex crispus}

278.594, Thes 220, Arethousa; 280.460, Dra 234, Skaloti; 281.262, Dra 266, W Nevrokopi; 281.941, Dra 288, SW Potamia; 282.308, Ser 273, Ano Vrondous; 283.596, Pel 364, 
Loutraki; 283.667, Pel 368, OSO Vorino; 283.734, Ima 172, S Ag. Pavlos; 283.890, Ima 179, Seli; 284.036, loa 1116, SW Milia

Rumex obtusifolius subsp. obtusifolius

277.533, loa 1097, S Anilio; 281.785, Dra 282, NNO Volakas; 282.565, Ser 285, Ori Vrondous; 283.058, Kil 306, SO Livadia

\section{Rumex patientia}

276.738, loa 1068, NW Metsovo; 277.457, loa 1096, S Anilio; 277.899, Koz 389, SO Siatista, Vourinos; 278.153, Gre 494, SO Varis; 278.386, Koz 382, Galatini; 278.662, Kav 106, Paralia Ofryniou; 278.664, Kav 106, Paralia Ofryniou; 279.554, Kav 102, Pangaion; 281.513, Dra 276, Perithori; 282.835, Ser 298, Parapotamos; 283.682, Pel 369, O Vorino

\section{Rumex pulcher subsp. pulcher}

276.304, loa 1125, NO Dodoni; 276.476, loa 1059, Ag. Nikolaos; 277.438, loa 1095, S Anilio; 277.774, Tri 462, N Chaliki; 278.325, Koz 381, Galatini; 280.103, Dra 220, Sidironero

\section{Rumex thyrsiflorus}

276.907, loa 1073, NNW Metsovo; 281.584, Dra 277, Perithori

\section{Sagina apetala}

280.627, Dra 240, Elatias

\section{Sagina subulata}

277.619, loa 1103, S Anilio

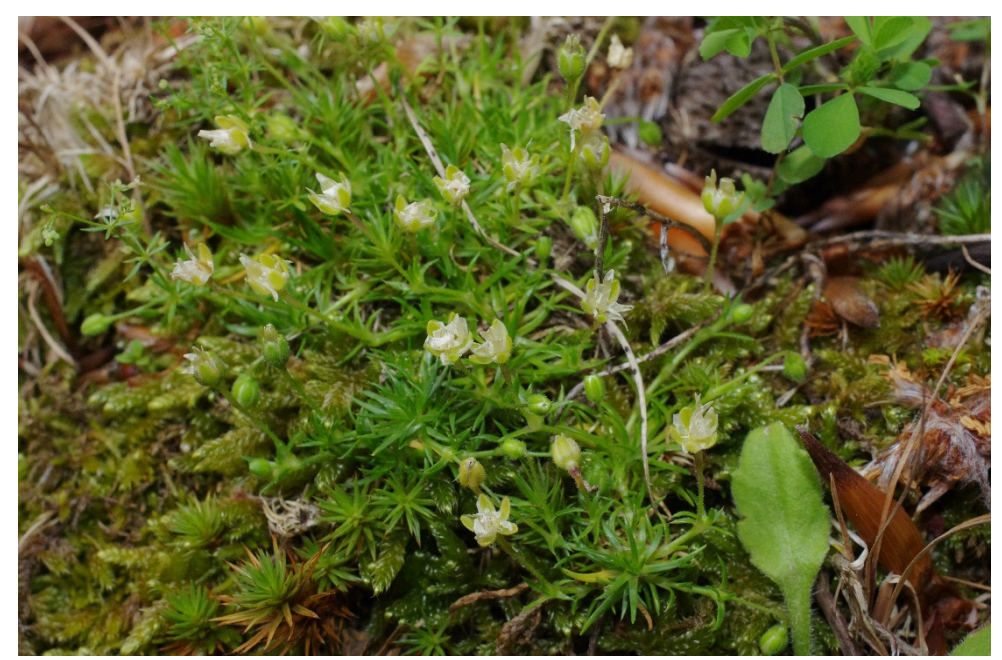

\section{Salix alba}

278.559, Thes 219, Vrasna; 279.857, Dra 209, Livadero; 281.890, Dra 287, O Mikroklisoura; 283.385, Pel 354, NW Loutraki

\section{Salix amplexicaulis}

279.959, Dra 213, Livadero; 282.028, Dra 292, Nevrokopi; 282.283, Ser 272, Ano Vrondous; 282.917, Kil 297, WNW Kastaneri; 283.445, Pel 356, NW Loutraki

\section{Salix caprea}

279.999, Dra 215, Livadero; 282.912, Kil 297, WNW Kastaneri; 283.165, Pel 343, Kerasia

\section{Salix cinerea}

282.483, Ser 282, Ori Vrondous; 282.700, Ser 290, N Neo Petritsi; 283.785, Ima 173, S Ag. Pavlos

\section{Salix elaeagnos}

282.908, Kil 297, WNW Kastaneri; 283.154, Pel 342, S Archangelo

\section{Salix fragilis}


281.545, Dra 276, Perithori; 282.018, Dra 292, Nevrokopi; 282.333, Ser 274, SSW Ano Vrondous

\section{Salix purpurea}

279.908, Dra 210, Livadero

\section{Salvia aethiopis}

278.154, Gre 494, SO Varis; 278.292, Koz 380, Galatini; 279.727, Ser 267, SW Skopia

\section{Salvia amplexicaulis}

276.950, loa 1076, ONO Anilio; 277.450, loa 1095, S Anilio; 277.795, Koz 385, SO Siatista; 278.286, Koz 380, Galatini; 278.358, Koz 382, Galatini; 278.527, Thes 218, Vrasna; 279.833, Dra 207, NO Skopia; 280.355, Dra 231, Sidironero; 281.166, Dra 263, NW Granitis; 281.449, Dra 274, Vathytopo; 281.770, Dra 282, NNO Volakas; 281.873, Dra 286, SW Mikroklisoura; 281.953, Dra 289, NO Potamia; 282.008, Dra 292, Nevrokopi; 282.379, Ser 276, SSW Ano Vrondous; 283.129, Pel 340, SSO Archangelo; 283.437, Pel 356, NW Loutraki; 284.063, loa 1118, Anilio

\section{Salvia argentea}

278.012, Koz 375, O Dafnero

Salvia officinalis

278.220, Koz 378, N Siatista

\section{Salvia ringens}

277.787, Koz 385, SO Siatista; 283.454, Pel 357, NW Loutraki

\section{Salvia sclarea}

276.606, loa 1061, SSO Potamia; 277.409, loa 1094, S Anilio; 279.301, Ser 259, Proti; 280.073, Dra 219, Sidironero; 280.192, Dra 223, NO Drama; 281.811, Dra 283, NNO Volakas

\section{Salvia verbenaca}

276.345, loa 1127, Dodonoupoli; 276.651, loa 1063, W Potamia

\section{Salvia verticillata}

280.440, Dra 234, Skaloti; 280.499, Dra 235, Skaloti; 281.018, Dra 257, Granitis; 281.173, Dra 264, NO Ochyro; 281.411, Dra 273, Vathytopo; 281.773, Dra 282, NNO Volakas; 282.003, Dra 292, Nevrokopi; 283.130, Pel 340, SSO Archangelo; 283.213, Pel 344, Kerasia; 283.492, Pel 359, NW Loutraki; 283.649, Pel 367, Vorino; 283.743, Ima 172, S Ag. Pavlos

\section{Salvia viridis}

276.528, loa 1059, Ag. Nikolaos

\section{Sambucus ebulus}

278.842, Kav 114, SO Folia; 279.123, Kav 91, Orfani; 279.178, Ser 256, Paleokomi; 279.659, Ser 265, Myrrini; 279.837, Dra 207, NO Skopia; 279.931, Dra 211, Livadero; 280.083, Dra 219, Sidironero; 281.014, Dra 257, Granitis; 281.199, Dra 264, NO Ochyro; 281.275, Dra 266, W Nevrokopi; 281.499, Dra 276, Perithori; 281.877, Dra 287, O Mikroklisoura; 282.131, Dra 297, Lefkogia; 282.711, Ser 290, N Neo Petritsi; 282.812, Ser 296, NO Strymoniko; 282.825, Ser 298, Parapotamos; 283.227, Pel 345, Kerasia; 283.651, Pel 367, Vorino; 283.757, Ima 172, S Ag. Pavlos; 283.831, Ima 176, Tria-Pende Pigadia

\section{Sambucus nigra}

280.583, Dra 238, Skaloti; 281.679, Ser 271, Kato Vrondous; 282.282, Ser 272, Ano

Vrondous; 282.521, Ser 283, Ori Vrondous

\section{Samolus valerandi}

277.391, loa 1094, S Anilio; 278.763, Kav 110, Loutra Eleftherion

\section{Sanguisorba minor subsp. balearica}


277.384, loa 1093, S Anilio; 277.886, Koz 388, SO Siatista, Vourinos; 278.398, Koz 383, Galatini; 278.537, Thes 218, Vrasna; 279.320, Ser 260, Proti; 280.239, Dra 226, N Taxiarches; 280.284, Dra 228, Livadero; 281.101, Dra 261, Granitis; 281.273, Dra 266, W Nevrokopi; 281.455, Dra 274, Vathytopo; 281.567, Dra 277, Perithori

\section{Sanguisorba minor subsp. minor}

276.578, loa 1060, SSO Potamia; 276.851, loa 1071, NNW Metsovo; 277.001, loa 1079, SO Anilio; 277.271, loa 1090, NNO Metsovo; 278.059, Gre 492, NO Varis; 279.227, Ser 257, Nea Fili; 279.419, Kav 93, Pangaion; 280.052, Dra 217, Sidironero; 280.369, Dra 232, Sidironero; 280.446, Dra 234, Skaloti; 282.020, Dra 292, Nevrokopi; 283.012, Kil 302, Kastaneri

\section{Sanguisorba officinalis}

276.753, loa 1068, NW Metsovo

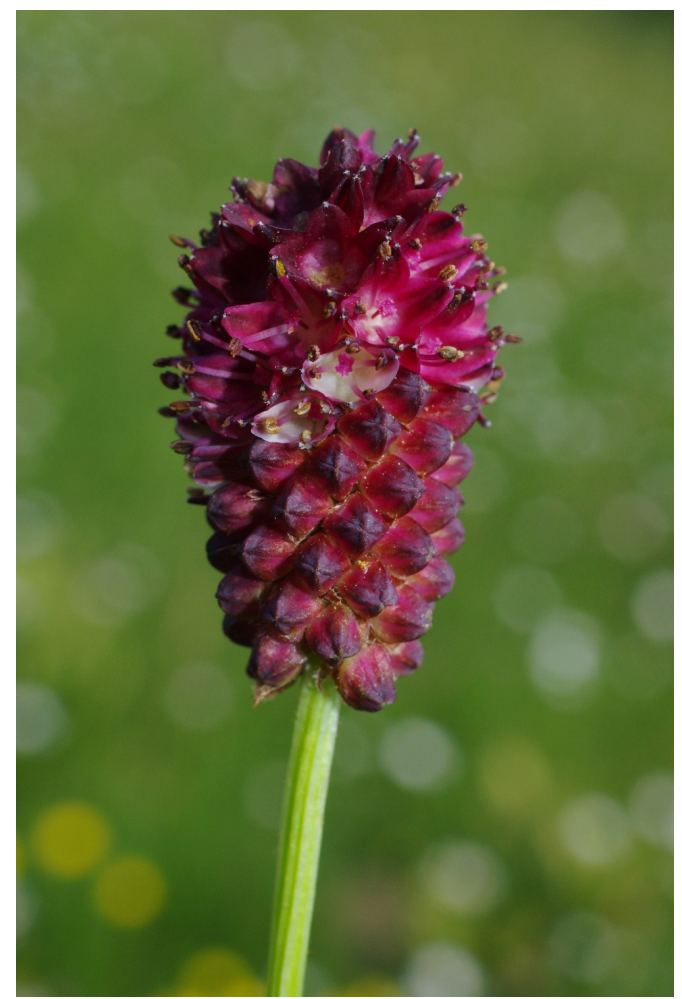

\section{Sanicula europaea}

276.758, loa 1069, NW Metsovo; 277.183, loa 1086, NNO Metsovo; 277.512, loa 1097, S Anilio; 280.516, Dra 236, Skaloti; 280.705, Dra 243, SW Walddorf Elatias; 281.099, Dra 261, Granitis; 281.624, Dra 279, Kato Vrondous; 283.794, Ima 174, S Ag. Pavlos; 284.145, Ioa 1096 b, S Anilio

\section{Saponaria calabrica}

276.573, loa 1060, SSO Potamia; 277.933, Koz 392, SO Siatista, Vourinos

\section{Saponaria intermedia}

276.953, Ioa 1076, ONO Anilio; 278.028, Koz 376, W Exarcho

\section{Saponaria officinalis}

278.486, Thes 215, Paralia Vrasna; 278.568, Thes 220, Arethousa; 279.089, Kav 89, Misoropi; 279.993, Dra 215, Livadero; 280.219, Dra 225, NO Drama; 280.307, Dra 229, SO Dendrakia; 280.431, Dra 234, Skaloti; 281.004, Dra 257, Granitis; 281.202, Dra 264, NO Ochyro; 281.249, Dra 266, W Nevrokopi; 281.495, Dra 276, Perithori; 281.946, Dra 289, NO Potamia; 282.152, Dra 297, Lefkogia; 282.257, Dra 301, Ano Vrondous; 282.326, Ser 274, SSW Ano Vrondous; 283.635, Pel 366, N Vorino

\section{Saxifraga rotundifolia subsp. rotundifolia}


276.412, loa 1057, NO Metsovo; 276.735, loa 1067, NW Metsovo; 277.510, loa 1097, S Anilio; 280.736, Dra 244, SW Walddorf Elatias; 283.055, Kil 306, SO Livadia

Saxifraga sempervivum

279.600, Kav 105, Pangaion; 279.603, Kav 105, Pangaion

Saxifraga stribrnyi

280.924, Dra 252, Falakron

Scabiosa balcanica

283.879, Ima 179, Seli

\section{Scabiosa ochroleuca}

278.222, Koz 378, N Siatista; 278.725, Kav 108, Paralia Ofryniou; 278.951, Kav 118,

Galipsos; 279.618, Ser 263, Dravoskas; 280.066, Dra 218, Sidironero; 280.247, Dra 227, Livadero; 280.282, Dra 228, Livadero; 281.239, Dra 265, NO Granitis; 281.293, Dra 267, W Nevrokopi; 281.547, Dra 277, Perithori; 283.418, Pel 355, NW Loutraki; 283.496, Pel 359, NW Loutraki; 283.816, Ima 176, Tria-Pende Pigadia

\section{Scabiosa tenuis}

276.326, loa 1125, NO Dodoni; 276.481, loa 1059, Ag. Nikolaos; 276.727, loa 1066, NO Karyes; 277.807, Koz 386, SO Siatista; 283.514, Pel 360, SW Promachi; 284.138, loa 1096 b, S Anilio

\section{Scabiosa triniifolia}

276.920, loa 1074, NNW Metsovo

\section{Scabiosa triniifolia}

278.006, Koz 375, O Dafnero

\section{Scabiosa triniifolia}

278.510, Thes 217, Vrasna; 278.851, Kav 115, SW Folia; 279.224, Ser 257, Nea Fili; 279.404, Kav 92, Pangaion; 279.612, Ser 263, Dravoskas; 279.731, Ser 267, SW Skopia; 280.881, Dra 250, Falakron; 281.044, Dra 259, Granitis; 281.152, Dra 262, NW Granitis; 281.611, Dra 278, Kato Vrondous; 281.704, Dra 280, Nevrokopi; 281.710, Dra 280, Nevrokopi; 282.044, Dra 293, Nevrokopi; 282.127, Dra 296, NO Nevrokopi; 282.188, Dra 299, SO Lefkogia; 282.391, Ser 277, ONO Serres; 282.432, Ser 279, Orini; 282.675, Ser 290, N Neo Petritsi; 282.986, Kil 300, Kastaneri; 283.098, Pel 339, S Archangelo

\section{Scabiosa webbiana}

278.078, Gre 492, NO Varis; 278.344, Koz 381, Galatini; 278.394, Koz 383, Galatini; 279.792, Ser 268, SW Skopia; 279.884, Dra 209, Livadero; 280.153, Dra 221, NO Drama; 281.453, Dra 274, Vathytopo; 281.829, Dra 283, NNO Volakas; 283.232, Pel 345, Kerasia; 283.942, loa 1108, N Metsovo; 283.997, loa 1112, Katara-Paß; 284.061, loa 1118, Anilio; 284.108, loa 1121, Anilio

\section{Scandix australis subsp. grandiflora}

276.829, loa 1070, NNW Metsovo; 277.484, loa 1097, S Anilio; 277.815, Koz 387, SO Siatista, Vourinos; 278.399, Koz 383, Galatini

\section{Scandix macrorhyncha}

278.225, Koz 378, N Siatista

\section{Scandix pecten-veneris}

277.425, loa 1095, S Anilio; 277.985, Koz 375, O Dafnero; 278.306, Koz 380, Galatini

\section{Scirpoides holoschoenus}

277.401, loa 1094, S Anilio; 280.223, Dra 225, NO Drama; 280.291, Dra 228, Livadero; 281.037, Dra 258, Granitis; 282.105, Dra 295, NO Nevrokopi; 282.178, Dra 299, SO Lefkogia; 282.965, Kil 299, Kastaneri; 283.389, Pel 354, NW Loutraki 


\section{Scirpus sylvaticus}

280.757, Dra 246, SW Walddorf Elatias

Scleranthus annuus subsp. polycarpos

277.635, loa 1104, S Anilio; 279.569, Kav 103, Pangaion; 282.680, Ser 290, N Neo Petritsi

\section{Scleranthus perennis}

Der in Flora Hellenica Vol 2 pg.218 gegebene Bestimmungsschlüssel ist meines Erachtens für eine eindeutige Bestimmung von Belegen aus N-Griechenland weniger geeignet. Vielleicht ist die Art in dieser Region aber auch nicht eindeutig in Unterarten aufzuteilen.

\section{Scleranthus perennis subsp. dichotomus}

276.805, loa 1070, NNW Metsovo; 279.926, Dra 211, Livadero; 280.598, Dra 238, Skaloti; 281.048, Dra 259, Granitis; 281.349, Dra 270, W Nevrokopi; 281.488, Dra 275, Vathytopo; 282.087, Dra 295, NO Nevrokopi; 282.622, Ser 288, Ori Vrondous

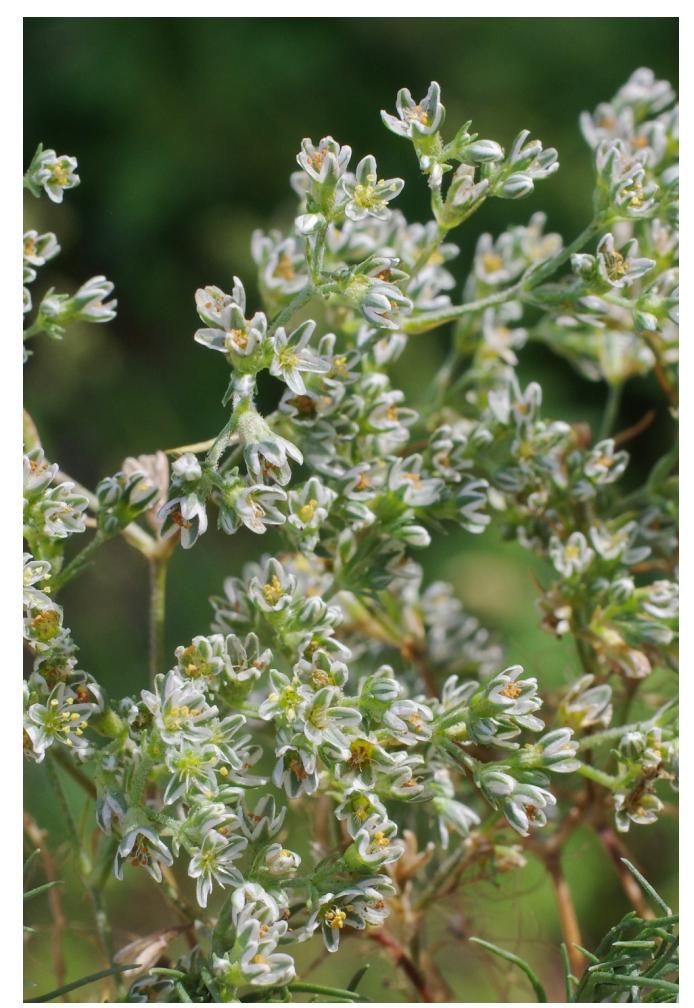

Scleranthus perennis subsp. marginatus

280.871, Dra 249, Falakron; 281.675, Ser 271, Kato Vrondous; 282.452, Ser 280, Orini; 283.350, Pel 352, Kajmaktsalan; 283.371, Pel 353, Kajmaktsalan

\section{Scleranthus perennis subsp. perennis}

276.439, loa 1058, Katara-Pass; 280.694, Dra 242, SW Walddorf Elatias; 280.776, Dra 247, Walddorf Elatias; 281.587, Dra 278, Kato Vrondous

\section{Scolymus hispanicus}

276.473, loa 1059, Ag. Nikolaos; 278.493, Thes 216, Vrasna; 279.243, Ser 258, Mikro Souli; 279.653, Ser 264, Dravoskas; 283.627, Pel 366, N Vorino; 283.708, Pel 369, O Vorino

\section{Scorzonera hispanica}

277.214, loa 1088, NNO Metsovo; 278.077, Gre 492, NO Varis

\section{Scorzonera purpurea}

276.372, loa 1128, N Metsovo

\section{Scorzoneroides cichoriacea}

276.870, loa 1072, NNW Metsovo; 277.020, loa 1080, SO Anilio; 277.648, loa 1105, Anilio 
Scrophularia canina subsp. bicolor

276.828, loa 1070, NNW Metsovo; 276.924, loa 1074, NNW Metsovo; 276.974, loa 1079, SO Anilio; 277.114, loa 1084, N Metsovo; 277.630, loa 1104, S Anilio; 277.769, Tri 462, N

Chaliki; 277.881, Koz 388, SO Siatista, Vourinos; -20.475, loa 1055, N Metsovo

\section{Scrophularia laciniata}

279.606, Kav 105, Pangaion

\section{Scrophularia lucida}

276.428, loa 1058, Katara-Pass; 276.667, loa 1064, ONO Karyes

\section{Scrophularia nodosa}

277.218, loa 1088, NNO Metsovo; 277.580, loa 1100, S Anilio; 282.727, Ser 291, N Neo Petritsi; 283.759, Ima 172, S Ag. Pavlos

\section{Scrophularia scopolii}

280.325, Dra 230, Livadero; 280.588, Dra 238, Skaloti; 280.723, Dra 243, SW Walddorf

Elatias

\section{Scutellaria albida}

278.479, Thes 215, Paralia Vrasna; 278.506, Thes 217, Vrasna; 278.741, Kav 109, Loutra Eleftherion; 278.974, Kav 119, Podochori; 279.144, Ser 255, S Nea Mesolakkia; 279.328, Ser 260, Proti; 284.202, Dra 209, Livadero

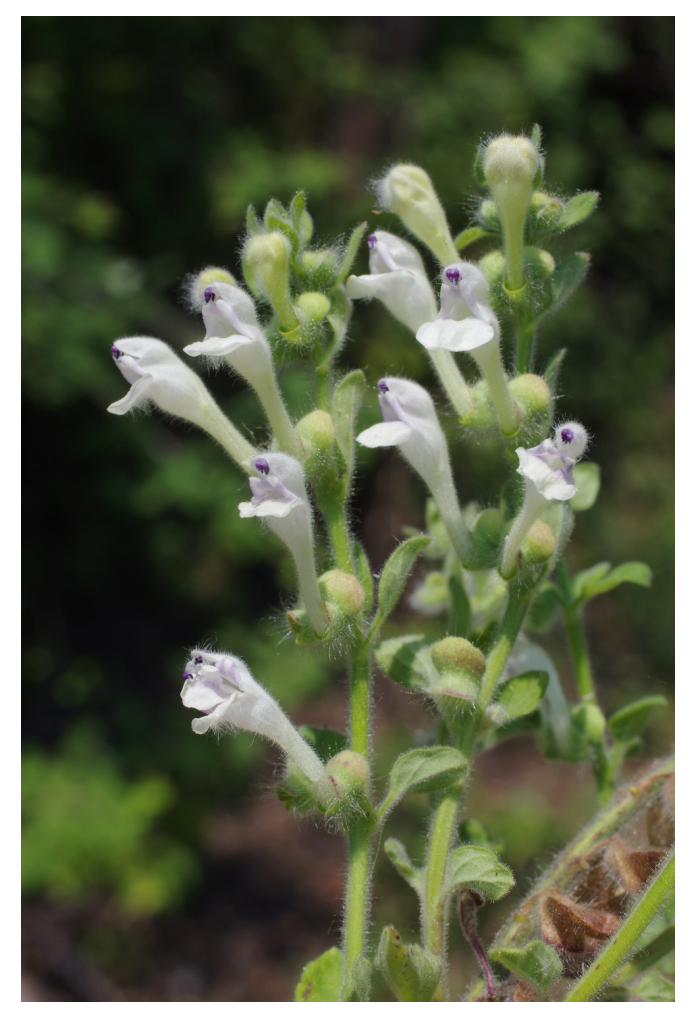

\section{Scutellaria columnae}

277.470, loa 1096, S Anilio; 282.745, Ser 292, N Neo Petritsi; 282.894, Kil 296, N Kastaneri

\section{Scutellaria galericulata}

281.366, Dra 271, Vathytopo

\section{Secale montanum}

282.471, Ser 281, Ori Vrondous

\section{Securigera cretica}

278.966, Kav 119, Podochori; 279.303, Ser 259, Proti 


\section{Securigera varia}

276.464, Tri 458, NO Katara-Pass; 277.755, Tri 461, N Chaliki; 277.842, Koz 387, SO Siatista, Vourinos; 278.165, Gre 494, SO Varis; 278.380, Koz 382, Galatini; 278.545, Thes 219, Vrasna; 280.025, Dra 216, Sidironero; 280.130, Dra 221, NO Drama; 280.272, Dra 228, Livadero; 280.367, Dra 232, Sidironero; 280.480, Dra 234, Skaloti; 280.923, Dra 252, Falakron; 281.276, Dra 266, W Nevrokopi; 281.463, Dra 274, Vathytopo; 281.701, Dra 280, Nevrokopi; 281.970, Dra 290, NO Potamia; 282.319, Ser 274, SSW Ano Vrondous; 282.519, Ser 283, Ori Vrondous; 282.706, Ser 290, N Neo Petritsi; 282.931, Kil 297, WNW Kastaneri; 283.126, Pel 340, SSO Archangelo; 283.259, Pel 347, Kajmaktsalan; 283.776, Ima 173, S Ag. Pavlos; 283.854, Ima 178, Seli

\section{Sedum acre}

276.325, loa 1125, NO Dodoni; 277.768, Tri 462, N Chaliki; 278.206, Koz 377, N Siatista; 281.467, Dra 274, Vathytopo; 283.917, Ima 180, Seli

\section{Sedum album}

277.386, loa 1093, S Anilio; 279.046, Kav 120, Podochori; 280.171, Dra 222, NO Drama; 280.916, Dra 251, Falakron; 281.144, Dra 262, NW Granitis; 281.758, Dra 281, OSO Granitis; 282.476, Ser 281, Ori Vrondous; 283.985, loa 1111, NO Metsovo; 284.094, loa 1120, Anilio

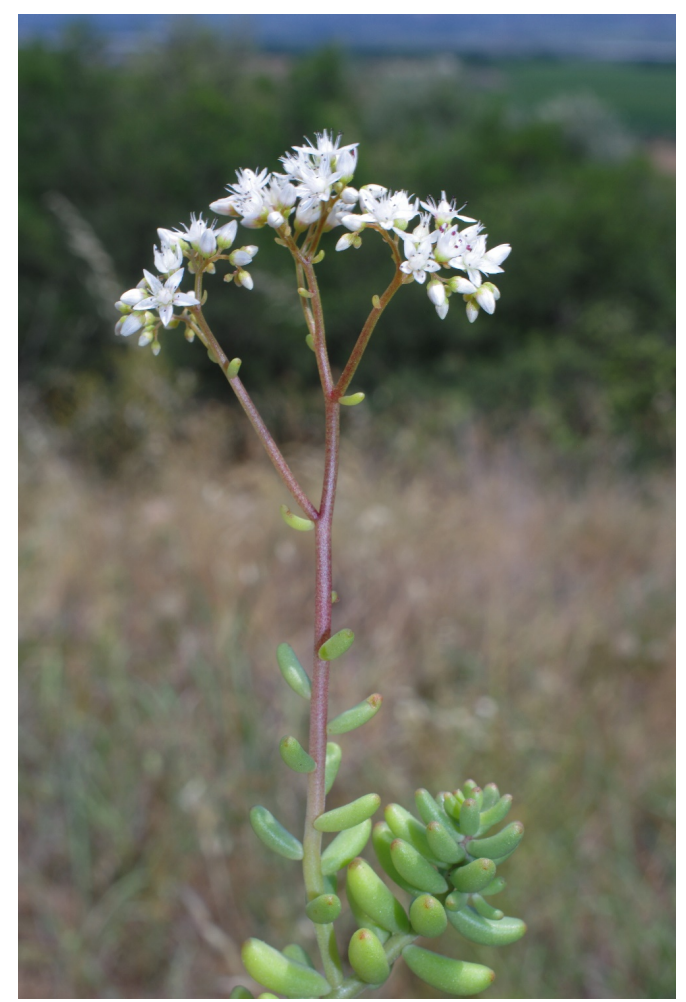

\section{Sedum amplexicaule subsp. tenuifolium}

276.642, loa 1063, W Potamia; 277.778, Tri 462, N Chaliki; 283.077, Kil 307, NW Livadia; 283.288, Pel 349, Kajmaktsalan; 283.950, loa 1109, NW Metsovo; 284.043, loa 1116, SW Milia

\section{Sedum cepaea}

278.868, Kav 115, SW Folia; 280.347, Dra 230, Livadero; 281.638, Dra 279, Kato Vrondous; 282.235, Dra 301, Ano Vrondous; 282.720, Ser 290, N Neo Petritsi; 282.897, Kil 296, N Kastaneri; 283.478, Pel 358, N Loutraki; 284.128, loa 1094 b, S Anilio 


\section{Sedum eriocarpum subsp. apertiflorum}

278.023, Koz 376, W Exarcho; 278.544, Thes 219, Vrasna; 279.047, Kav 120, Podochori; 280.197, Dra 224, NO Drama; 281.862, Dra 285, NNO Volakas; 282.409, Ser 279, Orini

\section{Sedum grisebachii}

277.387, loa 1093, S Anilio

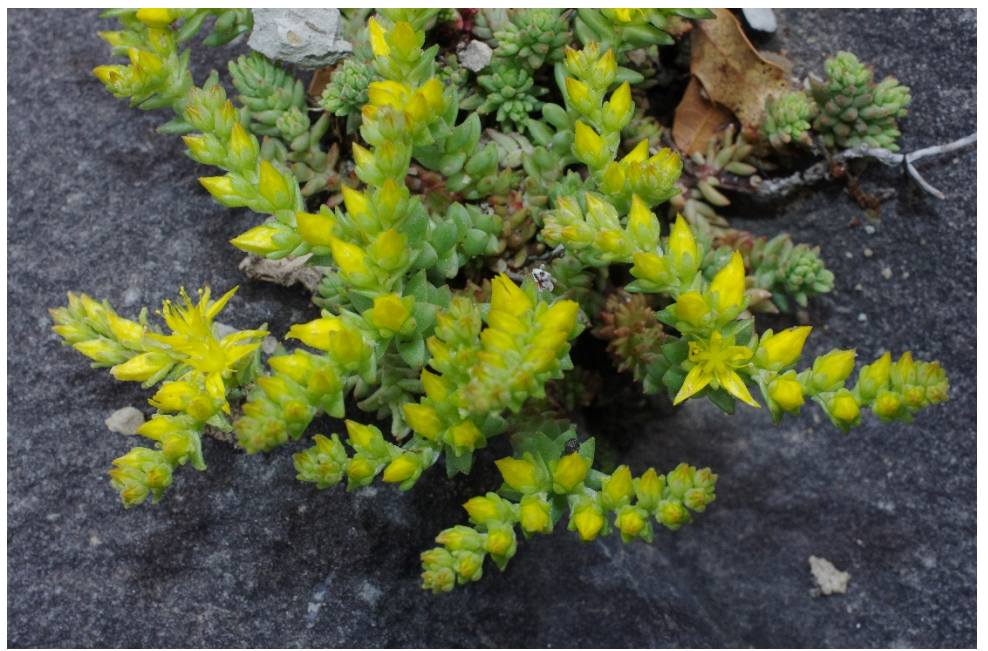

\section{Sedum hispanicum}

278.563, Thes 219, Vrasna; 279.322, Ser 260, Proti; 279.368, Ser 261, Proti; 279.897, Dra 209, Livadero; 280.053, Dra 217, Sidironero; 280.116, Dra 220, Sidironero; 280.310, Dra 229, SO Dendrakia; 280.407, Dra 233, Sidironero; 280.452, Dra 234, Skaloti; 280.844, Dra 248, Falakron; 280.945, Dra 254, Falakron; 281.051, Dra 259, Granitis; 281.729, Dra 281, OSO Granitis; 281.896, Dra 287, O Mikroklisoura; 282.526, Ser 283, Ori Vrondous; 282.646, Ser 290, N Neo Petritsi; 284.211, Thes 219, Vrasna

\section{Sedum ochroleucum}

277.077, loa 1081, W Metsovo; 278.226, Koz 378, N Siatista; 281.762, Dra 281, OSO Granitis; 283.999, loa 1113, Katara-Paß

\section{Sedum rubens}

276.306, loa 1125, NO Dodoni; 276.644, loa 1063, W Potamia

\section{Sedum sexangulare}

283.378, Pel 353, Kajmaktsalan

\section{Sedum urvillei subsp. urvillei}

277.811, Koz 386, SO Siatista; 278.034, Koz 376, W Exarcho; 278.095, Gre 492, NO Varis; 278.229, Koz 378, N Siatista; 278.350, Koz 381, Galatini; 279.034, Kav 120, Podochori; 280.070, Dra 218, Sidironero; 280.119, Dra 220, Sidironero; 280.165, Dra 221, NO Drama; 280.642, Dra 241, Elatias; 280.830, Dra 248, Falakron; 281.030, Dra 258, Granitis; 281.151, Dra 262, NW Granitis; 281.228, Dra 265, NO Granitis; 281.294, Dra 267, W Nevrokopi; 281.760, Dra 281, OSO Granitis; 282.435, Ser 279, Orini; 282.961, Kil 299, Kastaneri; 283.294, Pel 349, Kajmaktsalan; 283.808, Ima 175, S Ag. Pavlos; 283.986, loa 1111, NO Metsovo; 284.000, loa 1113, Katara-Paß; 284.097, loa 1120, Anilio

\section{Selinum silaifolium}

276.526, loa 1059, Ag. Nikolaos; 276.660, loa 1063, W Potamia; 277.589, loa 1100, S Anilio; 283.146, Pel 342, S Archangelo; 284.153, loa 1122, Anilio

\section{Senecio hercynicus}

284.162, loa 1123, N Charikli 


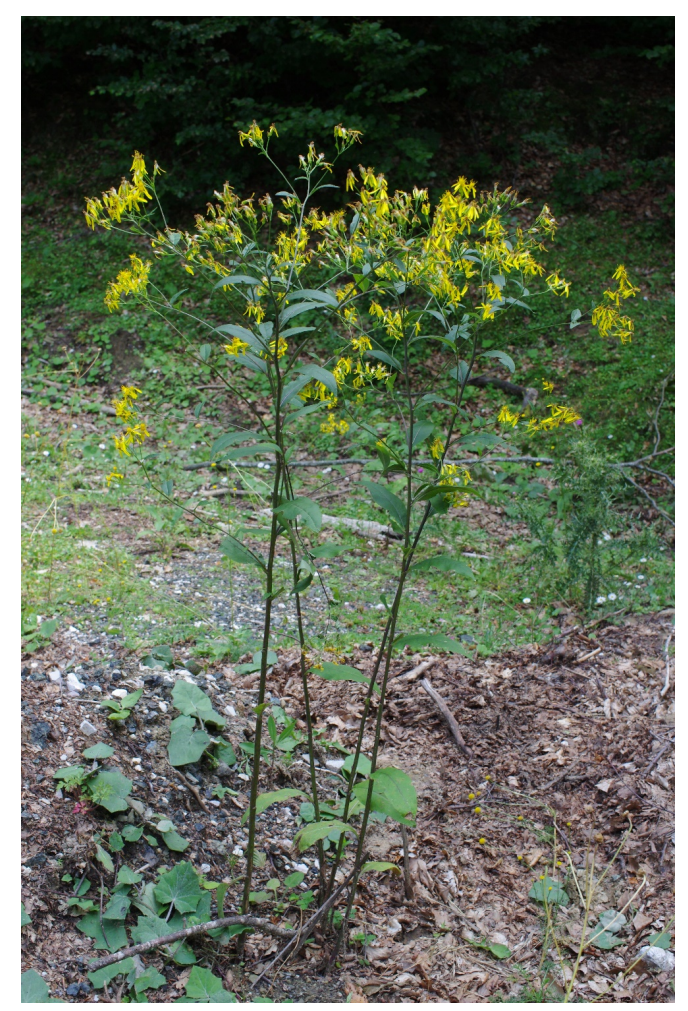

\section{Senecio jacobaea}

282.035, Dra 293, Nevrokopi

\section{Senecio macedonicus} 280.815, Dra 248, Falakron

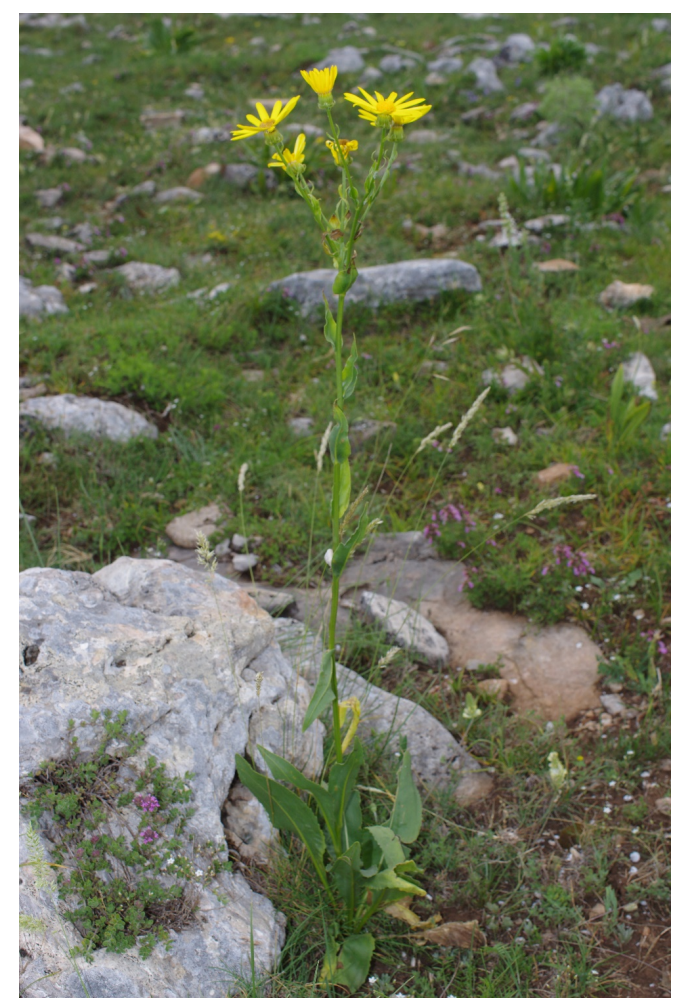

\section{Senecio papposus}

282.632, Ser 290, N Neo Petritsi

\section{Senecio rupestris}

280.861, Dra 249, Falakron; 282.572, Ser 286, Ori Vrondous; 283.343, Pel 352, Kajmaktsalan 
Senecio squalidus

282.531, Ser 284, Ori Vrondous

\section{Senecio thapsoides}

277.045, loa 1080, SO Anilio

\section{Senecio vernalis}

277.420, loa 1094, S Anilio

\section{Senecio viscosus}

284.002, loa 1113, Katara-Paß

\section{Senecio vulgaris}

277.446, loa 1095, S Anilio; 278.841, Kav 114, SO Folia; 278.990, Kav 119, Podochori; 279.270, Ser 258, Mikro Souli; 281.178, Dra 264, NO Ochyro; 281.405, Dra 273, Vathytopo; 283.881, Ima 179, Seli

\section{Seseli peucedanoides}

277.007, loa 1079, SO Anilio; 280.965, Dra 255, Falakron; 282.515, Ser 283, Ori Vrondous; 283.902, Ima 180, Seli

\section{Seseli rigidum}

280.758, Dra 246, SW Walddorf Elatias

\section{Setaria italica}

283.653, Pel 367, Vorino

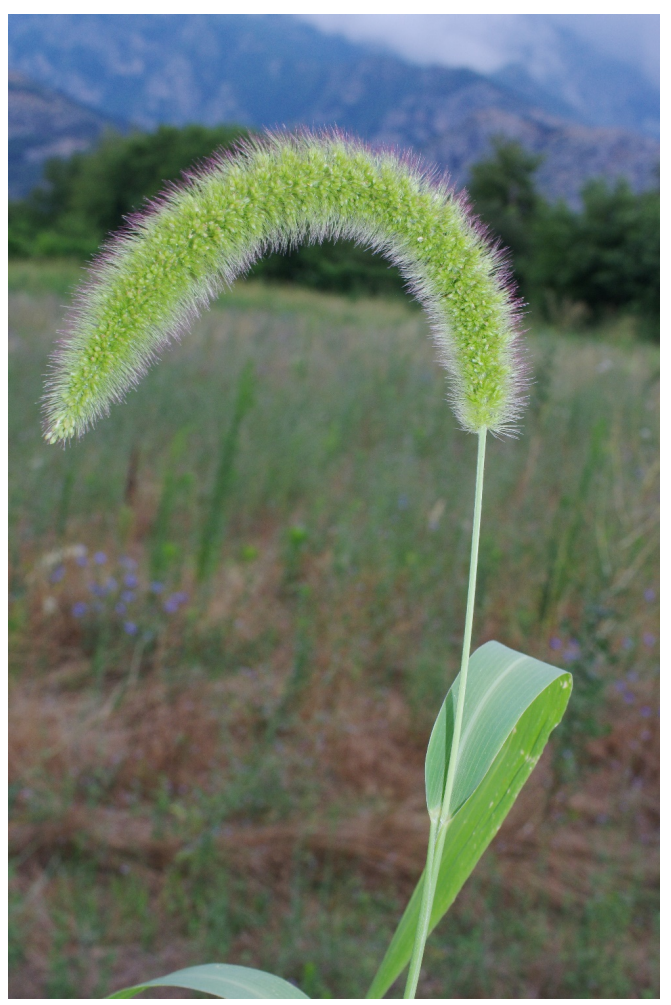

\section{Setaria pumila}

278.573, Thes 220, Arethousa; 281.933, Dra 288, SW Potamia; 282.838, Ser 298,

Parapotamos; 282.843, Ser 298, Parapotamos; 283.534, Pel 361, SW Promachi; 283.631, Pel 366, N Vorino; 283.694, Pel 369, O Vorino; 283.718, Pel 370, SW Foustani

\section{Setaria verticillata}

278.720, Kav 108, Paralia Ofryniou; 278.957, Kav 119, Podochori; 279.127, Kav 91, Orfani; 279.172, Ser 256, Paleokomi; 279.617, Ser 263, Dravoskas; 281.404, Dra 273, Vathytopo 


\section{Setaria viridis}

278.159, Gre 494, SO Varis; 279.107, Kav 90, SW Moustheni; 279.236, Ser 258, Mikro Souli; 279.706, Ser 266, SO Nea Zichni; 279.766, Ser 268, SW Skopia; 280.048, Dra 217,

Sidironero; 280.097, Dra 220, Sidironero; 280.392, Dra 232, Sidironero; 280.987, Dra 257, Granitis; 281.177, Dra 264, NO Ochyro; 281.261, Dra 266, W Nevrokopi; 281.421, Dra 273, Vathytopo; 281.539, Dra 276, Perithori; 281.932, Dra 288, SW Potamia; 281.986, Dra 291, W Prosotsani; 282.186, Dra 299, SO Lefkogia; 282.329, Ser 274, SSW Ano Vrondous; 282.403, Ser 278, N Serres; 282.689, Ser 290, N Neo Petritsi; 282.801, Ser 296, NO Strymoniko; 282.866, Kil 295, NW Griva; 282.983, Kil 300, Kastaneri; 283.421, Pel 355, NW Loutraki; 283.470, Pel 358, N Loutraki; 283.583, Pel 364, Loutraki; 283.706, Pel 369, O Vorino; 283.723, Pel 370, SW Foustani; 284.058, loa 1117, Anilio; 284.173, Ser 268, SW Skopia

\section{Sideritis curvidens}

278.691, Kav 107, Paralia Ofryniou; 279.713, Ser 266, SO Nea Zichni; 280.137, Dra 221, NO Drama

\section{Sideritis montana}

277.329, loa 1093, S Anilio; 277.808, Koz 386, SO Siatista; 278.115, Gre 492, NO Varis; 278.196, Koz 377, N Siatista; 278.342, Koz 381, Galatini; 278.396, Koz 383, Galatini; 278.896, Kav 116, S Ofrynia; 278.955, Kav 119, Podochori; 279.627, Ser 263, Dravoskas; 279.721, Ser 267, SW Skopia; 280.072, Dra 218, Sidironero; 280.842, Dra 248, Falakron; 281.148, Dra 262, NW Granitis; 281.461, Dra 274, Vathytopo; 281.727, Dra 281, OSO Granitis; 281.799, Dra 282, NNO Volakas; 282.006, Dra 292, Nevrokopi; 282.175, Dra 299, SO Lefkogia; 282.367, Ser 276, SSW Ano Vrondous

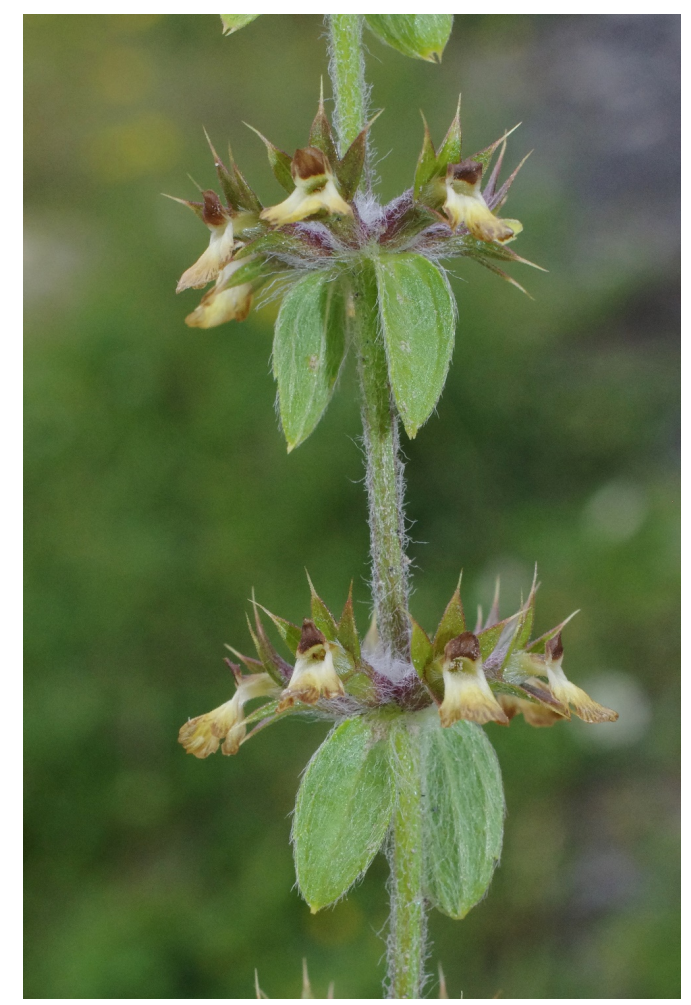

\section{Sideritis scardica}

279.582, Kav 104, Pangaion; 280.897, Dra 250, Falakron

\section{Silene armeria}

279.853, Dra 209, Livadero; 279.962, Dra 213, Livadero; 280.985, Dra 257, Granitis; 281.593, Dra 278, Kato Vrondous; 282.093, Dra 295, NO Nevrokopi; 282.215, Dra 300, SO Lefkogia; 282.479, Ser 281, Ori Vrondous; 282.627, Ser 289, Ori Vrondous

\section{Silene baccifera}

281.074, Dra 260, Granitis 


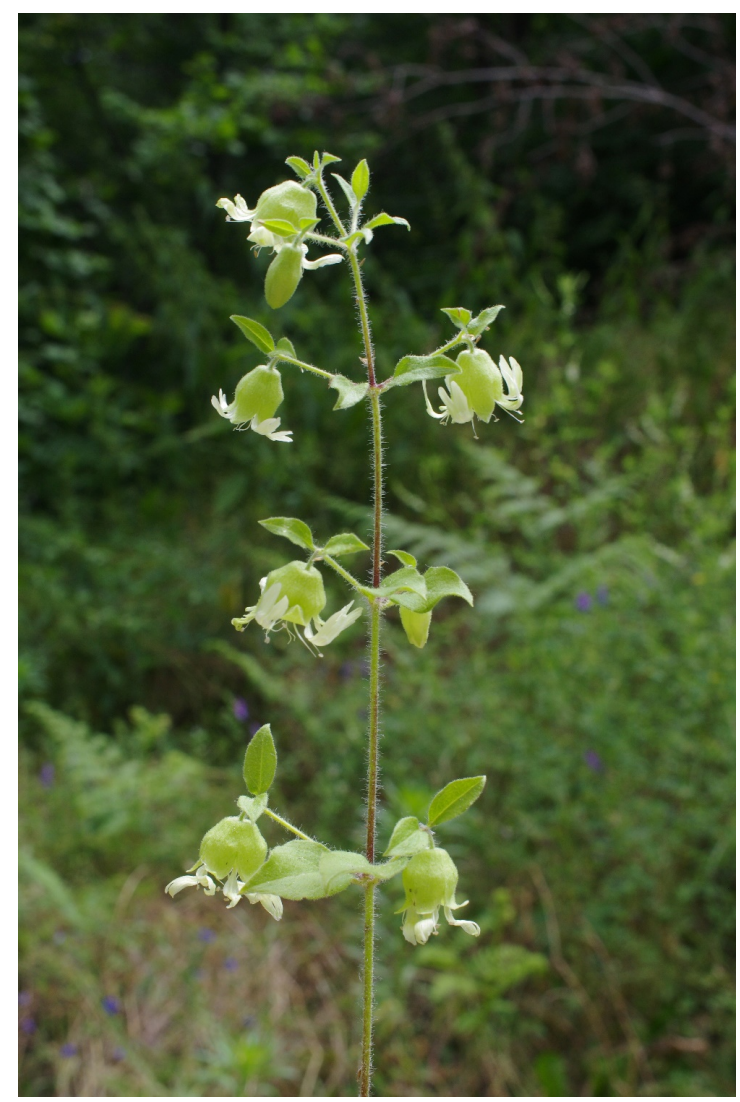

Silene bupleuroides subsp. staticifolia

280.812, Dra 248, Falakron; 280.840, Dra 248, Falakron; 282.470, Ser 281, Ori Vrondous; 282.658, Ser 290, N Neo Petritsi

\section{Silene conica}

278.347, Koz 381, Galatini

\section{Silene exaltata}

277.800, Koz 385, SO Siatista; 279.647, Ser 264, Dravoskas; 279.709 , Ser 266, SO Nea Zichni

\section{Silene fabarioides}

276.442, Tri 456, N Katara-Pass; 277.323, loa 1092, NO Metsovo; 277.678, Tri 460, N Chaliki; 277.939, Koz 392, SO Siatista, Vourinos

Silene flavescens subsp. flavescens

280.362, Dra 232, Sidironero; 280.855, Dra 248, Falakron 


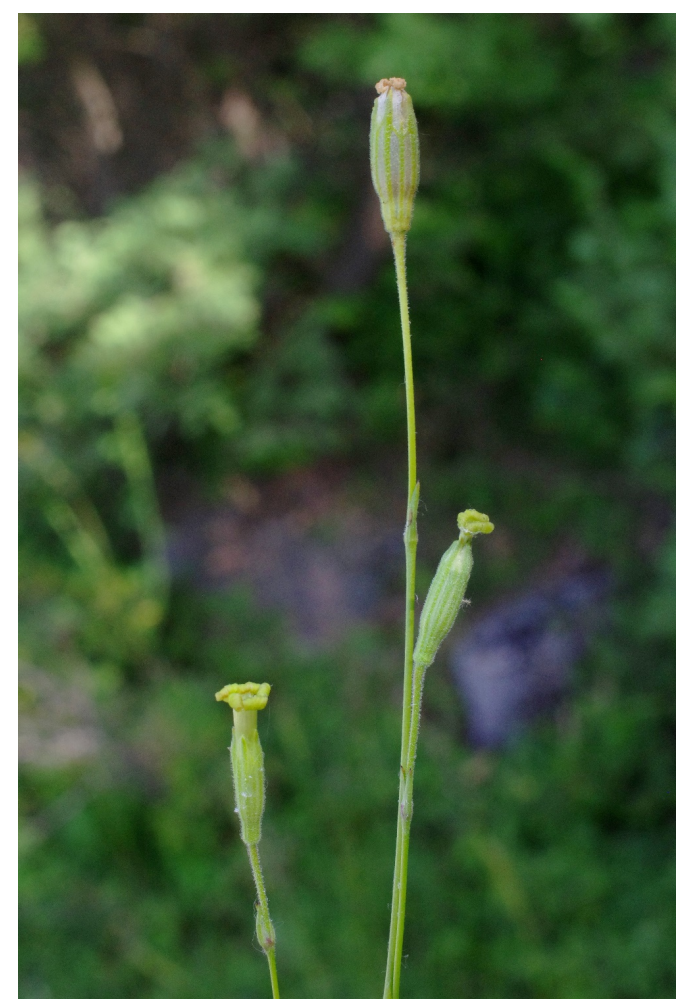

\section{Silene gallinyi}

278.485, Thes 215, Paralia Vrasna; 280.231, Dra 226, N Taxiarches; 281.569, Dra 277, Perithori; 281.872, Dra 286, SW Mikroklisoura; 281.956, Dra 289, NO Potamia; 282.092, Dra 295, NO Nevrokopi; 282.148, Dra 297, Lefkogia; 282.774, Ser 293, N Neo Petritsi; 283.493, Pel 359, NW Loutraki

\section{Silene graeca}

277.998, Koz 375, O Dafnero

\section{Silene haussknechtii}

276.450, Tri 456, N Katara-Pass; 276.984, loa 1079, SO Anilio; 284.091, loa 1120, Anilio

\section{Silene italica subsp. italica}

277.316, loa 1092, NO Metsovo; 277.570, loa 1099, S Anilio; 277.575, loa 1100, S Anilio; 283.387, Pel 354, NW Loutraki

\section{Silene latifolia}

277.459, loa 1096, S Anilio; 278.357, Koz 382, Galatini; 278.434, Thes 214, Paralia Vrasna; 281.000, Dra 257, Granitis; 282.271, Ser 272, Ano Vrondous; 282.829, Ser 298,

Parapotamos; 283.429, Pel 355, NW Loutraki; 283.729, Pel 370, SW Foustani

\section{Silene multicaulis}

283.342, Pel 351, Kajmaktsalan; 283.919, Ima 180, Seli; 284.004, loa 1113, Katara-Paß

\section{Silene oligantha}

284.100, loa 1121, Anilio

\section{Silene otites}

278.570, Thes 220, Arethousa; 280.639, Dra 241, Elatias; 280.806, Dra 247, Walddorf Elatias; 282.790, Ser 295, N Neo Petritsi

\section{Silene paradoxa}

279.724, Ser 267, SW Skopia; 281.459, Dra 274, Vathytopo; 283.775, Ima 173, S Ag. Pavlos

\section{Silene roemeri subsp. macrocarpa}

277.052, loa 1080, SO Anilio; 277.615, loa 1103, S Anilio; 284.218, Pel 351, Kajmaktsalan 


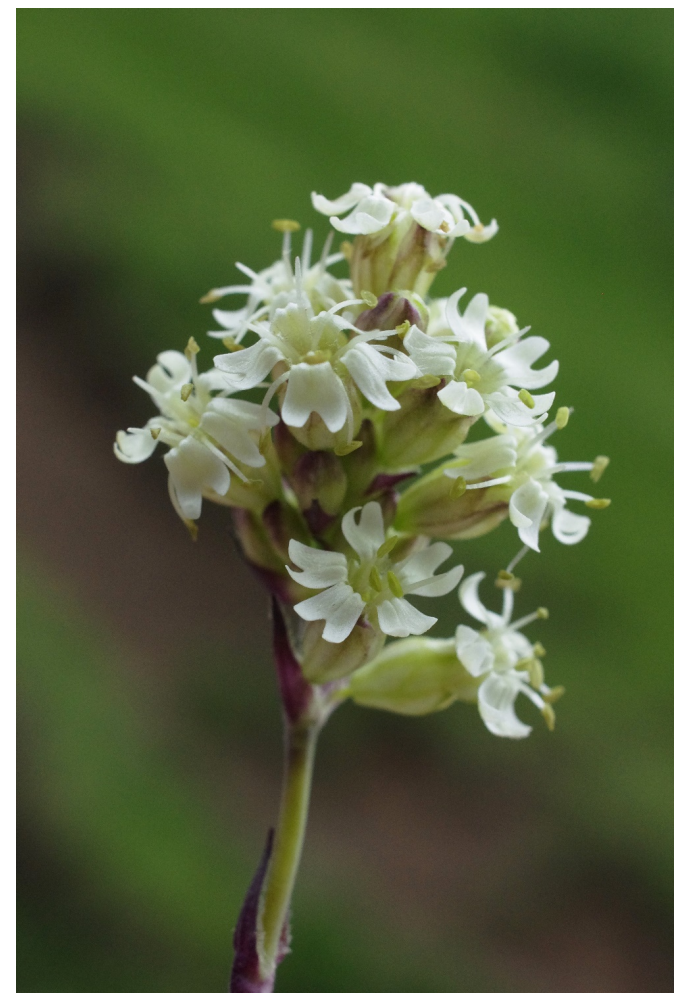

Silene sendtneri subsp. balcanica

280.920, Dra 252, Falakron; 281.359, Dra 270, W Nevrokopi; 282.030, Dra 292, Nevrokopi; 282.094, Dra 295, NO Nevrokopi; 283.774, Ima 173, S Ag. Pavlos

\section{Silene subconica}

280.814, Dra 248, Falakron

\section{Silene supina}

278.734, Kav 108, Paralia Ofryniou

\section{Silene viridiflora}

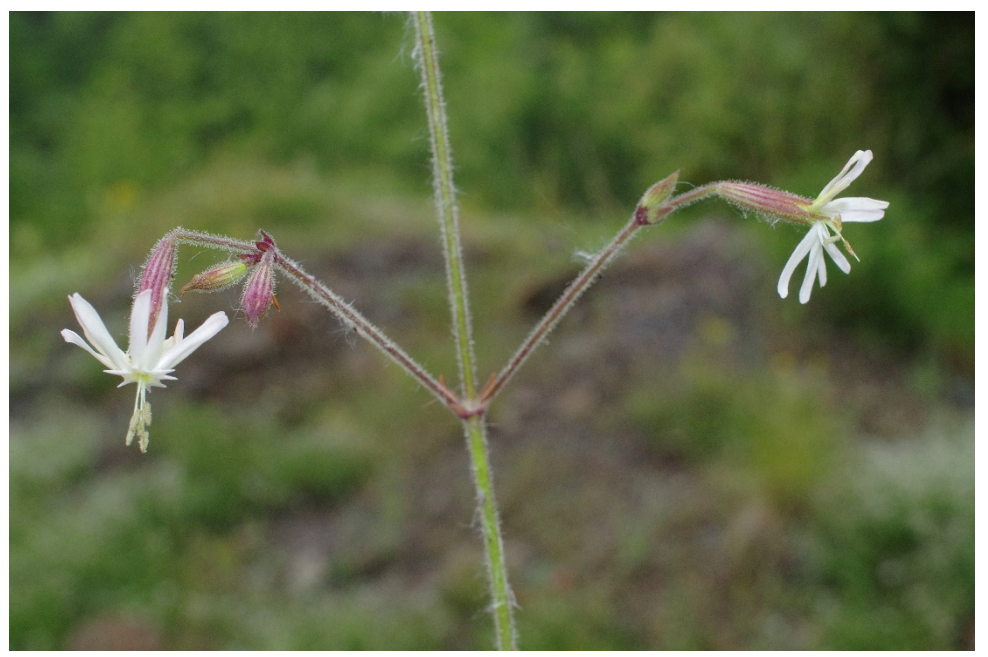

276.330, loa 1125, NO Dodoni; 276.941, loa 1075, NW Anilio; 278.748, Kav 109, Loutra Eleftherion; 278.941, Kav 118, Galipsos; 279.161, Ser 255, S Nea Mesolakkia; 279.386, Ser 262, Proti; 279.945, Dra 212, Livadero; 280.170, Dra 222, NO Drama; 280.303, Dra 229, SO Dendrakia; 281.025, Dra 258, Granitis; 281.039, Dra 258, Granitis; 281.119, Dra 261, Granitis; 281.663, Ser 270, Kato Vrondous; 281.852, Dra 284, NNO Volakas; 282.210, Dra 300, SO Lefkogia; 283.044, Kil 304, SO Livadia; 283.117, Pel 340, SSO Archangelo; 283.215, Pel 345, Kerasia; 283.792, Ima 173, S Ag. Pavlos

\section{Silene vulgaris}


Eine Zuordnung der Belege zu den in Flora Hellenica aufgeführten Unterarten ist kaum möglich. Dies gilt besonders für Pflanzen aus dem Falakron, den W-Rodopen, den Ori Vrondous und dem Voras.

277.572, loa 1100, S Anilio; 277.906, Koz 390, SO Siatista, Vourinos; 278.381, Koz 382, Galatini; 279.307, Ser 259, Proti; 279.535, Kav 100, Pangaion; 279.951, Dra 212, Livadero; 280.650, Dra 242, SW Walddorf Elatias; 280.919, Dra 251, Falakron; 281.114, Dra 261, Granitis; 281.633, Dra 279, Kato Vrondous; 282.205, Dra 300, SO Lefkogia; 282.495, Ser 282, Ori Vrondous; 282.555, Ser 285, Ori Vrondous; 282.697, Ser 290, N Neo Petritsi; 282.895, Kil 296, N Kastaneri; 283.000, Kil 301, Kastaneri; 283.124, Pel 340, SSO Archangelo; 283.239, Pel 346, Kajmaktsalan; 283.317, Pel 350, Kajmaktsalan; 283.658, Pel 367, Vorino

Silene vulgaris subsp. commutata

276.769, loa 1069, NW Metsovo

Silene vulgaris subsp. prostrata

276.434, loa 1058, Katara-Pass

Sinapis alba subsp. mairei

279.232, Ser 258, Mikro Souli

\section{Sinapis arvensis}

276.815, loa 1070, NNW Metsovo; 278.172, Gre 494, SO Varis; 278.589, Thes 220,

Arethousa; 278.661, Kav 106, Paralia Ofryniou; 281.406, Dra 273, Vathytopo; 281.931, Dra 288, SW Potamia

\section{Sisymbrium altissimum}

278.579, Thes 220, Arethousa; 282.678, Ser 290, N Neo Petritsi

\section{Sisymbrium officinale}

276.812, loa 1070, NNW Metsovo; 277.016, loa 1080, SO Anilio; 277.423, loa 1095, S Anilio; 277.901, Koz 389, SO Siatista, Vourinos; 278.834, Kav 114, SO Folia; 279.007, Kav 119, Podochori; 279.649, Ser 264, Dravoskas; 280.327, Dra 230, Livadero; 281.363, Dra 271, Vathytopo

\section{Sisymbrium orientale}

278.377, Koz 382, Galatini; 278.752, Kav 109, Loutra Eleftherion; 279.305, Ser 259, Proti; 279.688, Ser 266, SO Nea Zichni; 279.705, Ser 266, SO Nea Zichni; 280.964, Dra 255,

Falakron

\section{Solanum decipiens}

278.672, Kav 107, Paralia Ofryniou; 278.800, Kav 113, NO Paralia Myrtofytou; 278.926, Kav 117, Galipsos; 278.962, Kav 119, Podochori; 281.414, Dra 273, Vathytopo; 282.863, Kil 295, NW Griva; 283.432, Pel 355, NW Loutraki; 283.585, Pel 364, Loutraki; 283.727, Pel 370, SW Foustani

\section{Solanum dulcamara}

279.976, Dra 213, Livadero; 282.887, Kil 295, NW Griva

\section{Solanum elaeagnifolium}

278.687, Kav 107, Paralia Ofryniou; 278.773, Kav 112, NO Paralia Myrtofytou; 278.786, Kav 112, NO Paralia Myrtofytou; 278.816, Kav 114, SO Folia; 278.881, Kav 116, S Ofrynia; 279.028, Kav 120, Podochori; 279.175, Ser 256, Paleokomi; 279.238, Ser 258, Mikro Souli; 279.309, Ser 259, Proti; 280.214, Dra 225, NO Drama; 281.990, Dra 291, W Prosotsani; 282.811, Ser 296, NO Strymoniko

\section{Solanum luteum}

281.309, Dra 268, W Nevrokopi

\section{Solanum nigrum}


282.810, Ser 296, NO Strymoniko

\section{Solidago virgaurea}

283.241, Pel 346, Kajmaktsalan; 283.800, Ima 174, S Ag. Pavlos

\section{Sonchus asper subsp. asper}

276.546, loa 1060, SSO Potamia; 277.322, loa 1092, NO Metsovo; 277.380, loa 1093, S Anilio; 277.904, Koz 390, SO Siatista, Vourinos; 278.653, Kav 106, Paralia Ofryniou; 278.905, Kav 116, S Ofrynia; 279.001, Kav 119, Podochori; 279.287, Ser 259, Proti; 279.632, Ser 263, Dravoskas; 279.685, Ser 266, SO Nea Zichni; 280.322, Dra 230, Livadero; 281.279, Dra 266, W Nevrokopi; 282.223, Dra 300, SO Lefkogia; 282.737, Ser 291, N Neo Petritsi; 282.862, Kil 295, NW Griva; 283.572, Pel 363, NW Promachi; 283.725, Pel 370, SW Foustani; 283.767, Ima 172, S Ag. Pavlos

\section{Sonchus oleraceus}

277.407, Ioa 1094, S Anilio; 278.792, Kav 113, NO Paralia Myrtofytou; 279.061, Kav 121, Platanotopos; 279.507, Kav 98, Pangaion; 280.501, Dra 235, Skaloti; 281.009, Dra 257, Granitis; 283.594, Pel 364, Loutraki; 283.678, Pel 368, OSO Vorino

\section{Sonchus tenerrimus}

282.802, Ser 296, NO Strymoniko

\section{Sorghum halepense}

278.681, Kav 107, Paralia Ofryniou; 278.703, Kav 107, Paralia Ofryniou; 278.880, Kav 116, S Ofrynia; 278.890, Kav 116, S Ofrynia; 278.992, Kav 119, Podochori; 279.060, Kav 121, Platanotopos; 279.150, Ser 255, S Nea Mesolakkia; 279.635, Ser 263, Dravoskas; 279.658, Ser 265, Myrrini; 279.846, Dra 208, NO Skopia; 280.202, Dra 224, NO Drama; 282.805, Ser 296, NO Strymoniko; 283.392, Pel 354, NW Loutraki; 283.590, Pel 364, Loutraki; 283.669, Pel 368, OSO Vorino; 283.717, Pel 370, SW Foustani

\section{Spartium junceum}

-20.457 , loa 1052, NO Dodoni

\section{Spergularia rubra}

276.826, loa 1070, NNW Metsovo; 278.449, Thes 214, Paralia Vrasna; 278.774, Kav 112, NO Paralia Myrtofytou; 279.035, Kav 120, Podochori; 279.516, Kav 99, Pangaion; 280.645 , Dra 241, Elatias; 281.693, Ser 271, Kato Vrondous; 284.105, loa 1121, Anilio

\section{Spinacia oleracea}

282.600, Ser 288, Ori Vrondous; 283.134, Pel 341, S Archangelo

\section{Sporobolus indicus}

283.693, Pel 369, O Vorino

\section{Stachys alpina}

282.349, Ser 275, SSW Ano Vrondous

\section{Stachys angustifolia}

281.354, Dra 270, W Nevrokopi; 281.566, Dra 277, Perithori; 282.079, Dra 295, NO Nevrokopi; 282.189, Dra 299, SO Lefkogia 


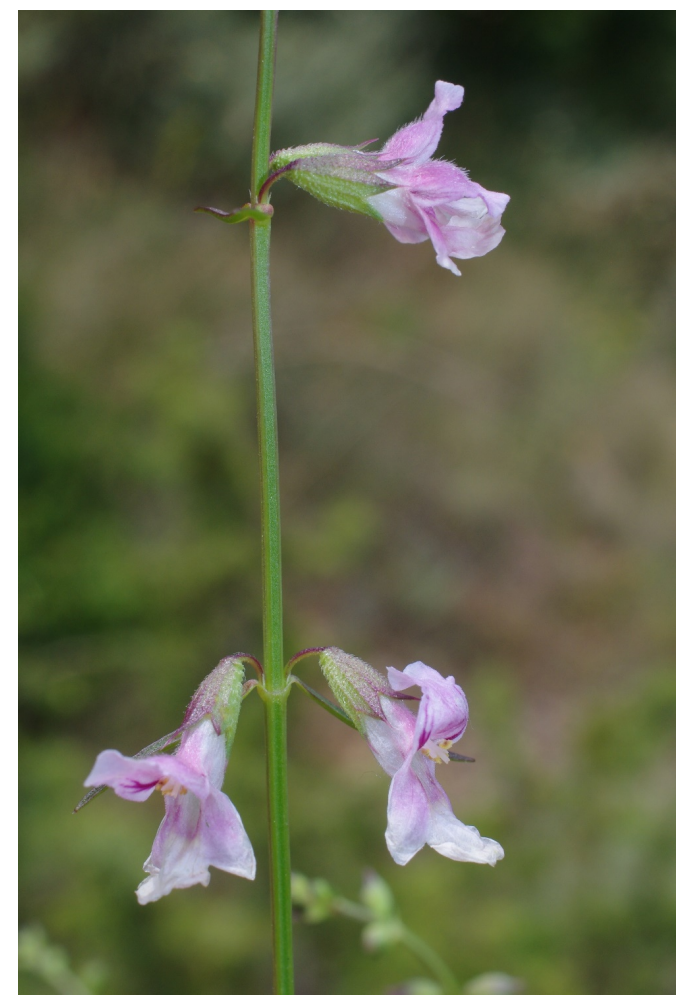

Stachys cretica subsp. bulgarica

281.291, Dra 267, W Nevrokopi; 282.005, Dra 292, Nevrokopi

\section{Stachys cretica subsp. salviifolia}

281.767, Dra 282, NNO Volakas

\section{Stachys germanica subsp. germanica}

283.088, Pel 339, S Archangelo

\section{Stachys germanica subsp. heldreichii}

276.375, loa 1128, N Metsovo; 276.741, loa 1068, NW Metsovo; 276.846, loa 1071, NNW Metsovo; 276.858, loa 1071, NNW Metsovo; 277.113, loa 1084, N Metsovo; 277.285, loa 1091, SW Milia; 277.518, loa 1097, S Anilio; 277.742, Tri 461, N Chaliki; 278.158, Gre 494, SO Varis; 278.320, Koz 381, Galatini; 278.414, Koz 383, Galatini; 278.608, Thes 221, Arethousa; 282.940, Kil 298, WNW Kastaneri; 283.034, Kil 303, SO Livadia; 283.838, Ima 177, Tria-Pende Pigadia; 283.885, Ima 179, Seli; 283.900, Ima 180, Seli; 283.976, loa 1056 b, NO Metsovo; 283.989, loa 1111, NO Metsovo

\section{Stachys plumosa}

277.031, loa 1080, SO Anilio; 277.870, Koz 388, SO Siatista, Vourinos; 278.066, Gre 492, NO Varis; 279.558, Kav 102, Pangaion; 279.598, Kav 105, Pangaion; 283.123, Pel 340, SSO Archangelo; 283.148, Pel 342, S Archangelo; 283.273, Pel 348, Kajmaktsalan; 283.307, Pel 350, Kajmaktsalan; 283.377, Pel 353, Kajmaktsalan

\section{Stachys recta subsp. baldaccii}

277.455, loa 1095, S Anilio; 277.756, Tri 461, N Chaliki; 277.779, Koz 385, SO Siatista; 278.667, Kav 107, Paralia Ofryniou; 278.925, Kav 117, Galipsos; 279.845, Dra 208, NO Skopia; 280.576, Dra 238, Skaloti; 281.185, Dra 264, NO Ochyro; 281.409, Dra 273, Vathytopo; 281.791, Dra 282, NNO Volakas; 282.038, Dra 293, Nevrokopi; 282.224, Dra 300, SO Lefkogia

\section{Stachys recta subsp. recta} 280.970, Dra 256, Falakron

\section{Stachys scardica}


277.820, Koz 387, SO Siatista, Vourinos; 283.994, loa 1112, Katara-Paß; 284.168, loa 1124, N Charikli

\section{Stachys sylvatica}

277.613, loa 1103, S Anilio; 280.512, Dra 236, Skaloti; 280.734, Dra 244, SW Walddorf Elatias; 282.236, Dra 301, Ano Vrondous; 282.559, Ser 285, Ori Vrondous; 283.018, Kil 302, Kastaneri; 283.748, Ima 172, S Ag. Pavlos; 283.818, Ima 176, Tria-Pende Pigadia

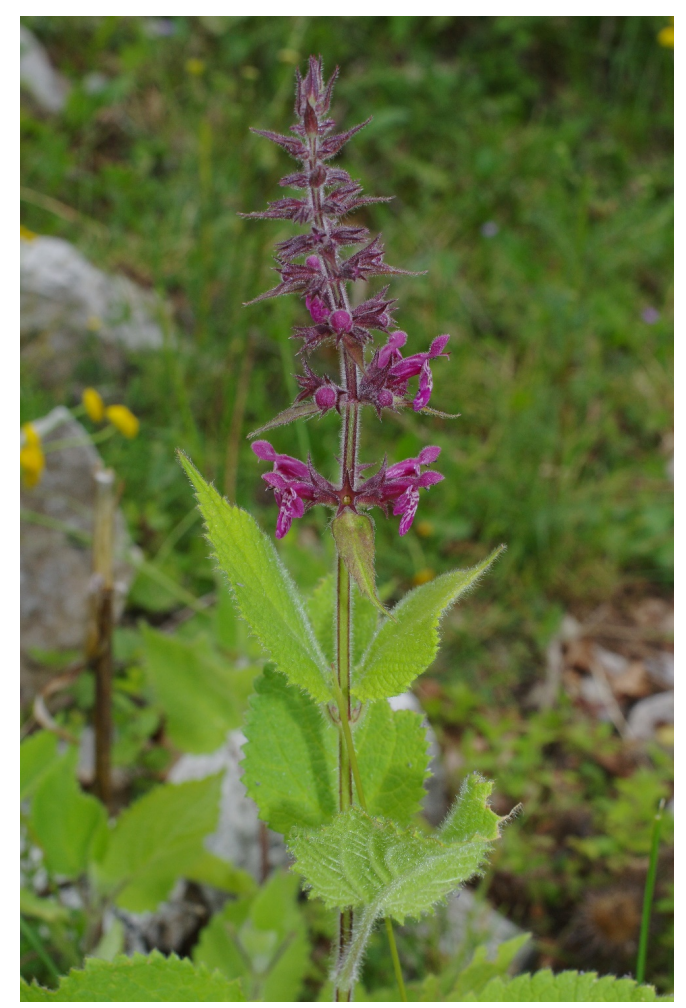

\section{Stachys tymphaea}

276.493, loa 1059, Ag. Nikolaos; 276.615, loa 1062, SO Potamia; 277.612, loa 1103, S Anilio; 280.525, Dra 236, Skaloti; 280.908, Dra 251, Falakron; 282.685, Ser 290, N Neo Petritsi; 283.306, Pel 350, Kajmaktsalan

\section{Staehelina uniflosculosa}

284.109, loa 1121, Anilio

\section{Stellaria apetala}

277.430, loa 1095, S Anilio; 279.515, Kav 99, Pangaion

\section{Stellaria aquatica}

281.071, Dra 260, Granitis

\section{Stellaria cupaniana}

281.370, Dra 271, Vathytopo

\section{Stellaria graminea}

276.740, loa 1068, NW Metsovo; 276.895, loa 1072, NNW Metsovo; 277.041, loa 1080, SO Anilio; 277.127, loa 1084, N Metsovo; 277.265, loa 1090, NNO Metsovo; 277.305, loa 1091, SW Milia; 277.616, Ioa 1103, S Anilio; 277.730, Tri 460, N Chaliki; 279.902, Dra 210, Livadero; 280.505, Dra 236, Skaloti; 280.612, Dra 238, Skaloti; 280.713, Dra 243, SW Walddorf Elatias; 281.098, Dra 261, Granitis; 282.097, Dra 295, NO Nevrokopi; 282.279, Ser 272, Ano Vrondous; 282.330, Ser 274, SSW Ano Vrondous; 282.544 , Ser 284, Ori Vrondous; 282.605, Ser 288, Ori Vrondous; 283.015, Kil 302, Kastaneri; 283.292, Pel 349, Kajmaktsalan 


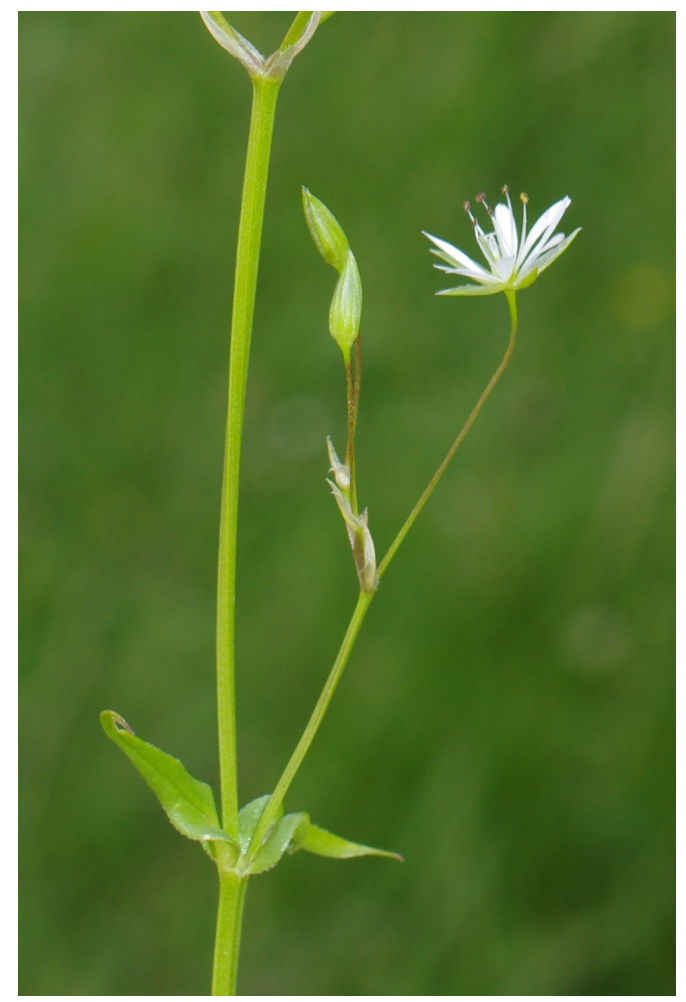

\section{Stellaria media}

277.551, loa 1098, S Anilio; 280.335, Dra 230, Livadero; 280.574, Dra 238, Skaloti

\section{Stellaria montana}

276.760, loa 1069, NW Metsovo; 277.579, loa 1100, S Anilio

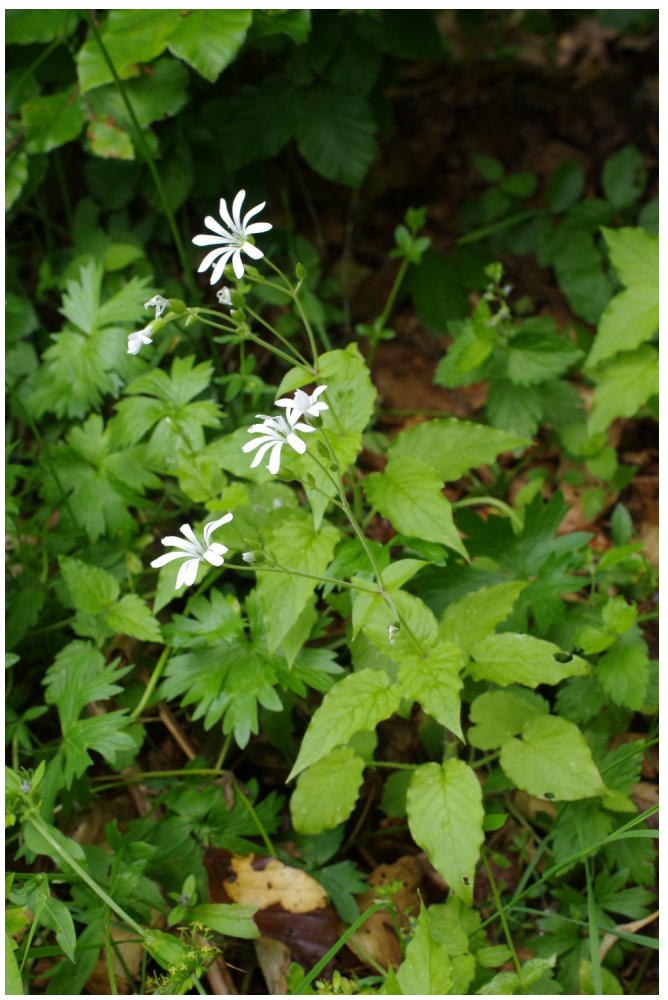

\section{Stipa bromoides}

279.006, Kav 119, Podochori; 279.220, Ser 257, Nea Fili; 279.333, Ser 260, Proti; 279.813 , Ser 269, NO Skopia; 282.988, Kil 300, Kastaneri

\section{Stipa capensis}

278.939, Kav 118, Galipsos; 278.982, Kav 119, Podochori 
Stipa pulcherrima

278.351, Koz 381, Galatini; 279.596, Kav 104, Pangaion

Stipa rechingeri

277.643, loa 1105, Anilio; 278.132, Gre 492, NO Varis

\section{Symphytum bulbosum}

276.882, loa 1072, NNW Metsovo; 277.482, loa 1097, S Anilio

\section{Symphytum ottomanum}

279.514, Kav 99, Pangaion; 280.339, Dra 230, Livadero; 280.750, Dra 245, SW Walddorf Elatias; 282.290, Ser 272, Ano Vrondous; 282.528, Ser 283, Ori Vrondous; 282.568 , Ser 286, Ori Vrondous

\section{Taeniatherum caput-medusae}

278.025, Koz 376, W Exarcho; 278.089, Gre 492, NO Varis; 282.448, Ser 280, Orini

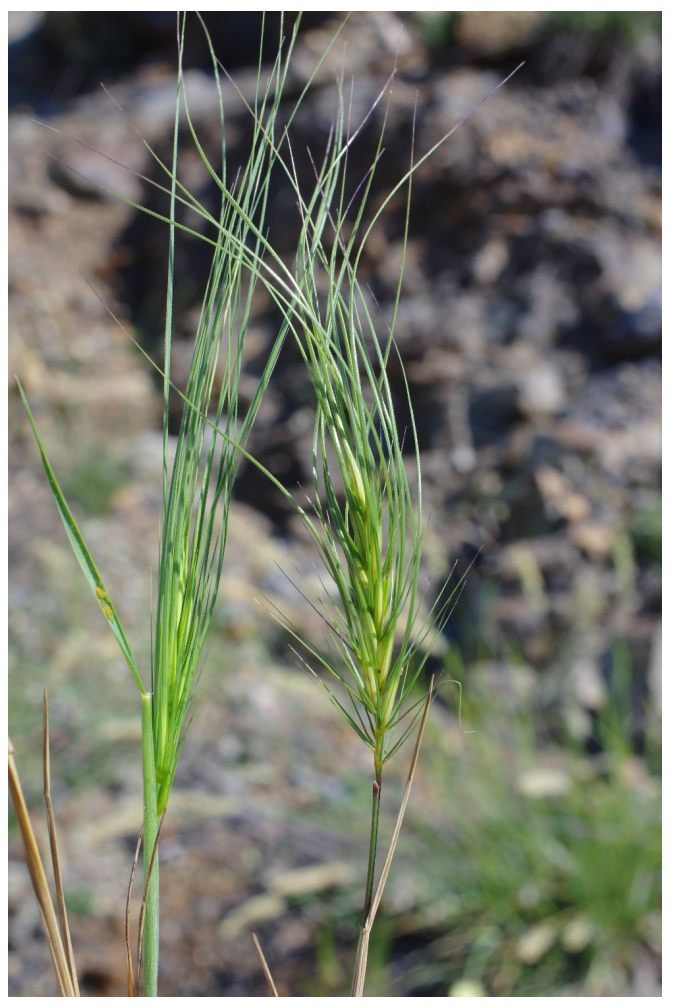

\section{Tanacetum corymbosum}

276.503, loa 1059, Ag. Nikolaos; 277.987, Koz 375, O Dafnero; 280.035, Dra 216,

Sidironero; 280.256, Dra 227, Livadero; 280.563, Dra 237, Skaloti; 280.969, Dra 256,

Falakron; 281.622, Dra 279, Kato Vrondous; 281.853, Dra 284, NNO Volakas; 282.517 , Ser 283, Ori Vrondous; 283.043, Kil 304, SO Livadia; 283.932, loa 1107, N Metsovo

\section{Tanacetum macrophyllum}

280.034, Dra 216, Sidironero; 282.587, Ser 287, Ori Vrondous; 282.730 , Ser 291, N Neo

Petritsi; 282.915, Kil 297, WNW Kastaneri; 283.570, Pel 363, NW Promachi

\section{Tanacetum parthenium}

277.456, loa 1096, S Anilio; 279.506, Kav 98, Pangaion; 283.243, Pel 346, Kajmaktsalan; 284.133, ioa 1095 b, S Anilio; 284.160, loa 1123, N Charikli

\section{Tanacetum vulgare}

279.883, Dra 209, Livadero; 280.996, Dra 257, Granitis; 281.200, Dra 264, NO Ochyro; 281.250, Dra 266, W Nevrokopi; 281.390, Dra 272, Vathytopo; 281.508, Dra 276, Perithori; 281.724, Dra 280, Nevrokopi; 281.921, Dra 288, SW Potamia; 282.150, Dra 297, Lefkogia; 
282.342, Ser 274, SSW Ano Vrondous; 282.642, Ser 290, N Neo Petritsi; 283.210, Pel 344, Kerasia; 283.733, Ima 172, S Ag. Pavlos

\section{Taraxacum}

276.928, loa 1074, NNW Metsovo; 277.002, loa 1079, SO Anilio; 277.442, loa 1095, S Anilio; 277.443, loa 1095, S Anilio; 277.634, loa 1104, S Anilio; 278.045, Gre 491, NO Exarcho; 278.640, Kav 106, Paralia Ofryniou; 278.641, Kav 106, Paralia Ofryniou; 278.660, Kav 106, Paralia Ofryniou; 280.395, Dra 232, Sidironero; 280.466, Dra 234, Skaloti; 280.864, Dra 249, Falakron; 281.358, Dra 270, W Nevrokopi; 281.782, Dra 282, NNO Volakas; 282.281, Ser 272, Ano Vrondous; 282.823, Ser 297, W Kerkini-See; 283.598, Pel 364, Loutraki; 283.680, Pel 369, O Vorino; 283.681, Pel 369, O Vorino

\section{Taraxacum haussknechtii}

278.044, Gre 491, NO Exarcho; 282.417, Ser 279, Orini; 283.235, Pel 346, Kajmaktsalan; 283.892, Ima 179, Seli

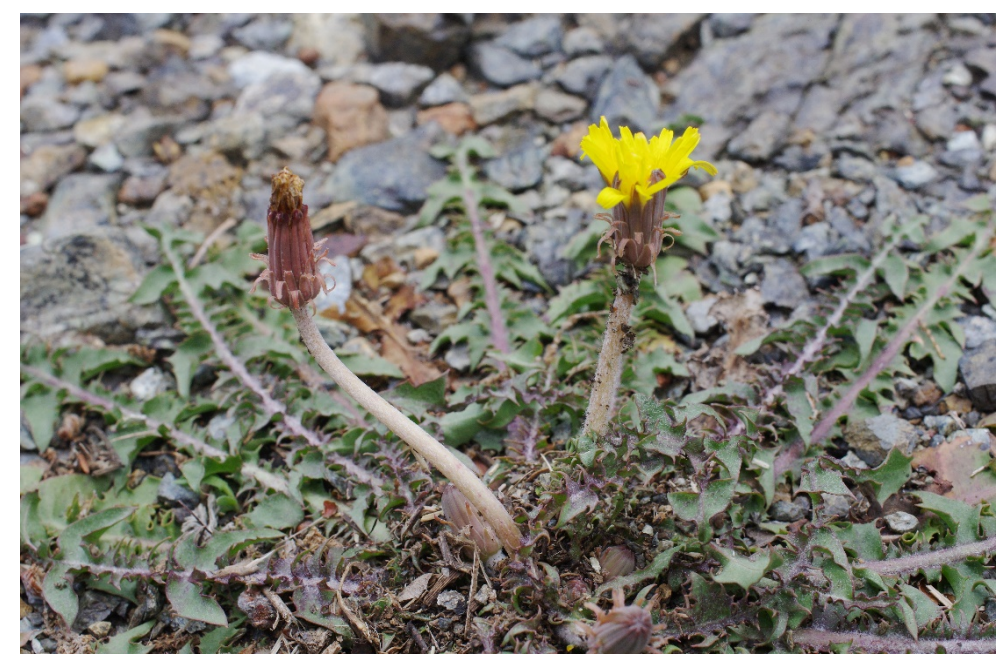

\section{Telekia speciosa}

280.756, Dra 245, SW Walddorf Elatias

\section{Teucrium capitatum}

276.506, loa 1059, Ag. Nikolaos; 277.388, loa 1093, S Anilio; 278.211, Koz 377, N Siatista; 278.600, Thes 221, Arethousa; 278.729, Kav 108, Paralia Ofryniou; 278.895, Kav 116, S Ofrynia; 278.947, Kav 118, Galipsos; 279.070, Kav 121, Platanotopos; 279.160, Ser 255, S Nea Mesolakkia; 279.229, Ser 257, Nea Fili; 279.261, Ser 258, Mikro Souli; 279.316, Ser 260, Proti; 279.402, Kav 92, Pangaion; 279.623, Ser 263, Dravoskas; 279.695, Ser 266, SO Nea Zichni; 280.141, Dra 221, NO Drama; 281.141, Dra 262, NW Granitis; 281.209, Dra 265, NO Granitis; 281.298, Dra 267, W Nevrokopi; 281.456, Dra 274, Vathytopo; 281.743, Dra 281, OSO Granitis; 281.912, Dra 287, O Mikroklisoura; 282.014, Dra 292, Nevrokopi; 282.383, Ser 276, SSW Ano Vrondous; 282.389, Ser 277, ONO Serres; 282.775 , Ser 293, N Neo Petritsi; 283.140, Pel 341, S Archangelo; 283.397, Pel 354, NW Loutraki; 283.608, Pel 365, N Vorino; 284.111, loa 1121, Anilio

\section{Teucrium chamaedrys}

276.343, loa 1127, Dodonoupoli; 276.599, loa 1061, SSO Potamia; 276.680 , loa 1065, NO Karyes; 277.078, loa 1081, W Metsovo; 277.328, loa 1093, S Anilio; 277.763, Tri 462, N Chaliki; 277.819, Koz 387, SO Siatista, Vourinos; 278.130, Gre 493, NO Varis; 278.209, Koz 377, N Siatista; 278.336, Koz 381, Galatini; 278.526, Thes 217, Vrasna; 278.867, Kav 115, SW Folia; 279.008, Kav 119, Podochori; 279.218, Ser 257, Nea Fili; 279.336, Ser 260, Proti; 279.452, Kav 95, Pangaion; 279.737, Ser 267, SW Skopia; 279.754, Ser 268, SW Skopia; 279.894, Dra 209, Livadero; 280.024, Dra 216, Sidironero; 280.271, Dra 228, Livadero; 280.349, Dra 231, Sidironero; 280.852, Dra 248, Falakron; 281.050, Dra 259, Granitis; 281.292, Dra 267, W Nevrokopi; 281.548, Dra 277, Perithori; 281.609, Dra 278, Kato Vrondous; 281.755, Dra 281, OSO Granitis; 281.821, Dra 283, NNO Volakas; 281.938 , Dra 288, SW Potamia; 282.160, Dra 298, SO Lefkogia; 282.343, Ser 274, SSW Ano 
Vrondous; 282.854, Kil 294, NW Griva; 283.030, Kil 303, SO Livadia; 283.076, Kil 307, NW Livadia; 283.262, Pel 347, Kajmaktsalan; 283.893, Ima 179, Seli

Teucrium montanum subsp. helianthemoides

278.065, Gre 492, NO Varis; 283.903, Ima 180, Seli

Teucrium montanum subsp. montanum

279.585, Kav 104, Pangaion; 280.158, Dra 221, NO Drama; 280.824, Dra 248, Falakron; 284.006, loa 1113, Katara-Paß; 284.092, loa 1120, Anilio

\section{Teucrium scordium subsp. scordioides}

284.141, loa 1096 b, S Anilio

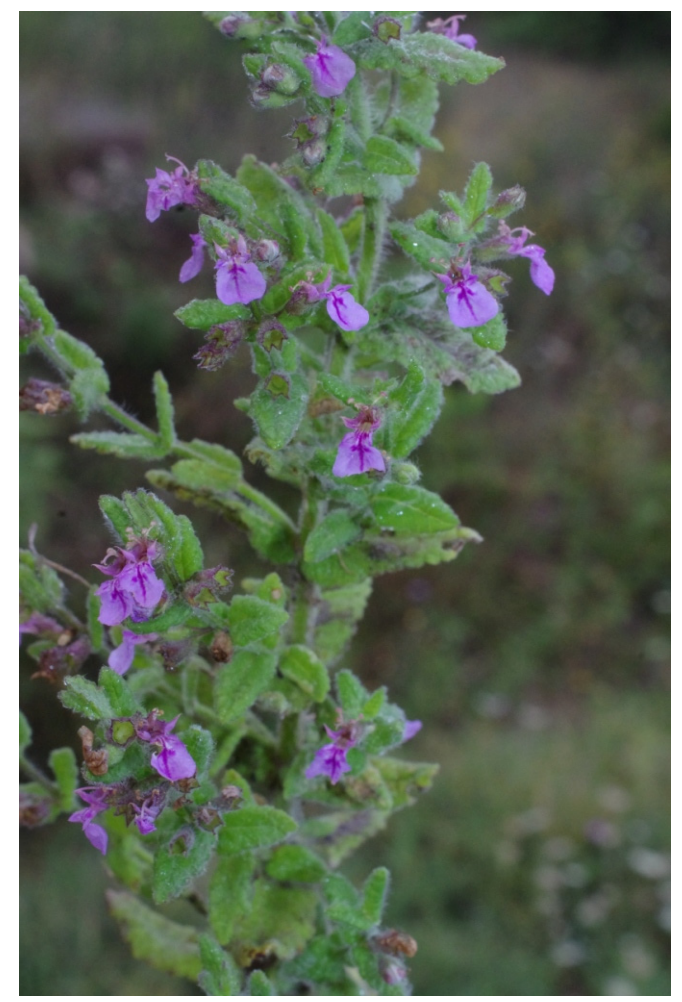

Thalictrum simplex

282.096, Dra 295, NO Nevrokopi

\section{Thesium divaricatum}

279.774, Ser 268, SW Skopia; 281.216, Dra 265, NO Granitis; 281.905, Dra 287, O

Mikroklisoura

\section{Thesium humile}

278.938, Kav 118, Galipsos; 279.712, Ser 266, SO Nea Zichni; 280.241, Dra 226, N Taxiarches; 281.215, Dra 265, NO Granitis

\section{Thesium macedonicum}

278.228, Koz 378, N Siatista; 278.416, Koz 384, Galatini; 281.440, Dra 274, Vathytopo; 283.144, Pel 341, S Archangelo

\section{Thlaspi arvense}

278.276, Koz 379, Galatini; 282.691, Ser 290, N Neo Petritsi

\section{Thymbra spicata}

278.769, Kav 111, Loutra Eleftherion 


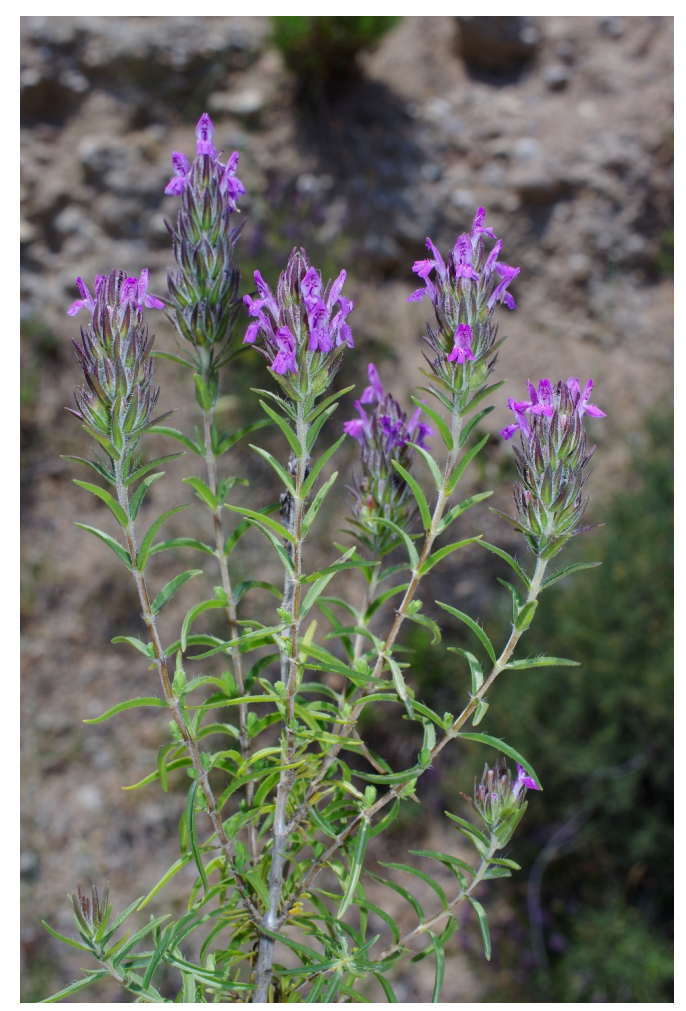

\section{Thymus atticus}

279.335, Ser 260, Proti; 279.733, Ser 267, SW Skopia; 279.769, Ser 268, SW Skopia;

280.182, Dra 223, NO Drama; 281.240, Dra 265, NO Granitis; 281.444, Dra 274, Vathytopo; 281.974, Dra 290, NO Potamia

\section{Thymus boissieri}

277.389, loa 1093, S Anilio; 282.201, Dra 299, SO Lefkogia

\section{Thymus cherlerioides}

278.983, Kav 119, Podochori

\section{Thymus degenii}

282.415, Ser 279, Orini; 282.419, Ser 279, Orini

\section{Thymus leucospermus}

277.782, Koz 385, SO Siatista; 278.724, Kav 108, Paralia Ofryniou; 279.277, Ser 258, Mikro Souli; 283.122, Pel 340, SSO Archangelo

\section{Thymus longicaulis}

276.902, loa 1073, NNW Metsovo; 276.973, loa 1079, SO Anilio; 277.610, loa 1103, S Anilio; 277.689, Tri 460, N Chaliki; 277.857, Koz 387, SO Siatista, Vourinos; 278.525, Thes 217, Vrasna; 278.787, Kav 112, NO Paralia Myrtofytou; 279.532, Kav 100, Pangaion; 279.860, Dra 209, Livadero; 280.412, Dra 233, Sidironero; 280.468, Dra 234, Skaloti; 280.635, Dra 241, Elatias; 280.754, Dra 245, SW Walddorf Elatias; 280.966, Dra 255, Falakron; 281.028, Dra 258, Granitis; 281.343, Dra 270, W Nevrokopi; 281.568, Dra 277, Perithori; 281.595, Dra 278, Kato Vrondous; 282.102, Dra 295, NO Nevrokopi; 282.612, Ser 288, Ori Vrondous; 282.654, Ser 290, N Neo Petritsi; 283.265, Pel 347, Kajmaktsalan

\section{Thymus longicaulis subsp. longicaulis}

276.791, loa 1070, NNW Metsovo

\section{Thymus praecox subsp. jankae}

280.859, Dra 249, Falakron; 283.308, Pel 350, Kajmaktsalan

\section{Thymus sibthorpii}

283.364, Pel 352, Kajmaktsalan 


\section{Thymus teucrioides}

276.433, loa 1058, Katara-Pass; 278.183, Gre 495, Varis; 284.103, loa 1121, Anilio

Thymus teucrioides subsp. alpinus

284.003, loa 1113, Katara-Paß

\section{Thymus thracicus}

279.487, Kav 97, Pangaion; 279.589, Kav 104, Pangaion; 282.365, Ser 276, SSW Ano Vrondous

\section{Tolpis umbellata \\ 278.460, Thes 214, Paralia Vrasna \\ Tordylium apulum \\ 278.488, Thes 215, Paralia Vrasna}

\section{Tordylium maximum}

276.594, loa 1061, SSO Potamia; 277.670, Tri 460, N Chaliki; 277.907, Koz 390, SO Siatista, Vourinos; 278.376, Koz 382, Galatini; 278.387, Koz 382, Galatini; 281.565, Dra 277,

Perithori; 282.750, Ser 292, N Neo Petritsi

\section{Tordylium officinale}

279.246, Ser 258, Mikro Souli; 279.281, Ser 259, Proti; 279.371, Ser 261, Proti

\section{Torilis africana}

276.612, loa 1062, SO Potamia; 276.893, loa 1072, NNW Metsovo; 277.076, loa 1081, W Metsovo; 277.143, loa 1084, N Metsovo; 277.310, loa 1091, SW Milia; 277.535, loa 1097, S Anilio; 277.683, Tri 460, N Chaliki; 277.893, Koz 389, SO Siatista, Vourinos; 279.441, Kav 95, Pangaion; 282.475, Ser 281, Ori Vrondous; 283.097, Pel 339, S Archangelo; 283.866, Ima 178, Seli; 284.021, Ioa 1116, SW Milia

\section{Torilis arvensis subsp. neglecta}

276.500, loa 1059, Ag. Nikolaos; 276.544, loa 1059, Ag. Nikolaos; 277.385, loa 1093, S Anilio; 277.858, Koz 387, SO Siatista, Vourinos; 278.137, Gre 492, NO Varis; 278.265, Koz 379, Galatini; 278.829, Kav 114, SO Folia; 279.038, Kav 120, Podochori; 279.662, Ser 265, Myrrini; 280.298, Dra 229, SO Dendrakia; 281.172, Dra 264, NO Ochyro; 281.258, Dra 266, W Nevrokopi; 281.389, Dra 272, Vathytopo; 281.598, Dra 278, Kato Vrondous; 281.752 , Dra 281, OSO Granitis; 281.787, Dra 282, NNO Volakas; 281.823, Dra 283, NNO Volakas; 281.928, Dra 288, SW Potamia; 282.142, Dra 297, Lefkogia; 282.405, Ser 278, N Serres; 282.424, Ser 279, Orini; 283.629, Pel 366, N Vorino; 283.731, Ima 172, S Ag. Pavlos; 284.193, Dra 280, Nevrokopi

\section{Torilis elongata}

278.477, Thes 215, Paralia Vrasna; 278.528, Thes 218, Vrasna; 278.592, Thes 220, Arethousa; 278.666, Kav 106, Paralia Ofryniou; 278.742, Kav 109, Loutra Eleftherion; 278.884, Kav 116, S Ofrynia; 278.915, Kav 117, Galipsos; 279.085, Kav 89, Misoropi; 279.180, Ser 256, Paleokomi; 279.300, Ser 259, Proti; 279.414, Kav 93, Pangaion; 279.704 , Ser 266, SO Nea Zichni; 280.224, Dra 225, NO Drama; 281.012, Dra 257, Granitis; 282.000, Dra 291, W Prosotsani; 282.830, Ser 298, Parapotamos; 283.222, Pel 345, Kerasia; 283.677, Pel 368, OSO Vorino; 284.048, loa 1117, Anilio

\section{Torilis japonica}

277.191, loa 1087, NNO Metsovo; 282.953, Kil 298, WNW Kastaneri; 283.396, Pel 354, NW Loutraki; 283.435, Pel 355, NW Loutraki; 283.518, Pel 360, SW Promachi; 283.580, Pel 363, NW Promachi

\section{Torilis leptophylla}

277.679, Tri 460, N Chaliki; 278.201, Koz 377, N Siatista; 278.272, Koz 379, Galatini; 278.365, Koz 382, Galatini 
Torilis nodosa

277.436, loa 1095, S Anilio; 279.012, Kav 119, Podochori

Torilis ucranica

282.784, Ser 294, N Neo Petritsi

Tragopogon porrifolius

278.126, Gre 493, NO Varis; 278.658, Kav 106, Paralia Ofryniou; 279.735, Ser 267, SW

Skopia

\section{Tragopogon pratensis}

276.361, loa 1128, N Metsovo; 276.939, loa 1075, NW Anilio; 276.990, loa 1079, SO Anilio; 277.131, loa 1084, N Metsovo; 277.353, loa 1093, S Anilio; 279.881, Dra 209, Livadero; 280.520, Dra 236, Skaloti

\section{Tragopogon samaritani}

276.986, loa 1079, SO Anilio; 277.636, loa 1104, S Anilio; 278.213, Koz 377, N Siatista; 283.987, loa 1111, NO Metsovo

\section{Tragus racemosus}

278.140, Gre 492, NO Varis; 278.706, Kav 107, Paralia Ofryniou; 278.901, Kav 116, S Ofrynia; 279.140, Ser 255, S Nea Mesolakkia; 279.777, Ser 268, SW Skopia; 281.054, Dra 259, Granitis; 281.179, Dra 264, NO Ochyro

\section{Tribulus terrestris}

278.019, Koz 376, W Exarcho; 278.476, Thes 215, Paralia Vrasna; 278.648, Kav 106, Paralia Ofryniou; 278.903, Kav 116, S Ofrynia; 279.181, Ser 256, Paleokomi; 282.816, Ser 296, NO Strymoniko

\section{Trifolium alpestre}

276.365, loa 1128, N Metsovo; 276.912, loa 1073, NNW Metsovo; 277.094, loa 1083, NW Metsovo; 277.569, loa 1099, S Anilio; 277.716, Tri 460, N Chaliki; 277.863, Koz 388, SO Siatista, Vourinos; 280.265, Dra 228, Livadero; 280.764, Dra 246, SW Walddorf Elatias; 280.959, Dra 255, Falakron; 282.492, Ser 282, Ori Vrondous; 282.504, Ser 282, Ori Vrondous; 282.707, Ser 290, N Neo Petritsi; 283.905, Ima 180, Seli; 283.946, loa 1109, NW Metsovo; 284.209, Dra 233, Sidironero; 284.216, Ser 282, Ori Vrondous

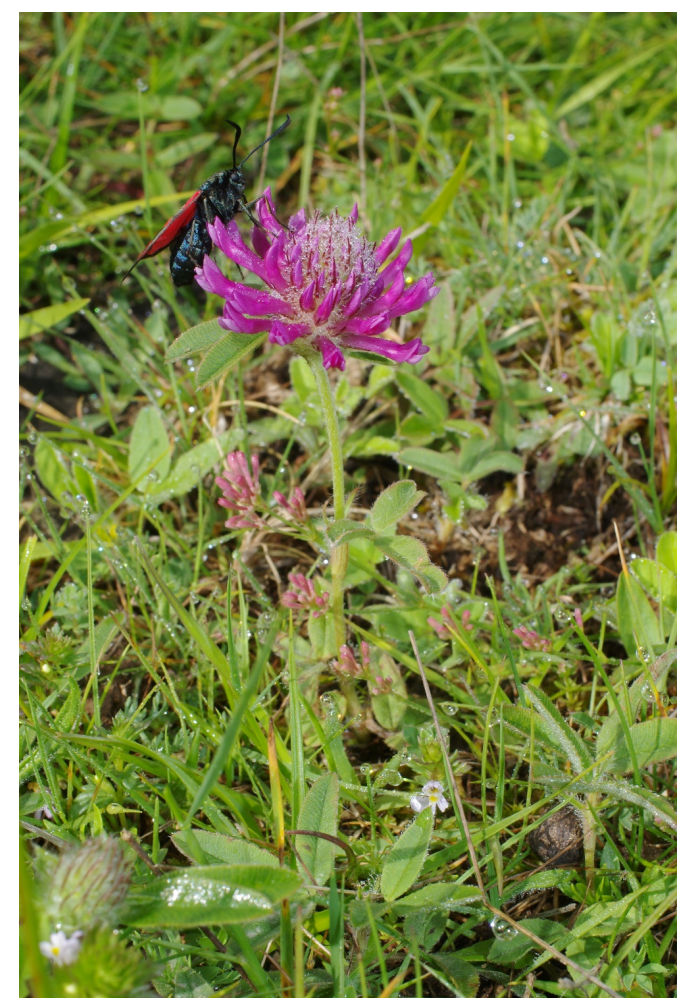




\section{Trifolium angustifolium}

276.499, loa 1059, Ag. Nikolaos; 277.080, loa 1081, W Metsovo; 277.375, loa 1093, S Anilio; 278.147, Gre 492, NO Varis; 278.471, Thes 215, Paralia Vrasna; 278.542, Thes 219, Vrasna; 278.846, Kav 115, SW Folia; 278.972, Kav 119, Podochori; 279.364, Ser 261, Proti; 279.396, Kav 92, Pangaion; 279.806, Ser 269, NO Skopia; 279.869, Dra 209, Livadero; 279.990, Dra 214, Livadero; 280.194, Dra 223, NO Drama; 281.577, Dra 277, Perithori; 281.645, Dra 279, Kato Vrondous

\section{Trifolium angustifolium}

282.416, Ser 279, Orini

\section{Trifolium arvense}

276.559, loa 1060, SSO Potamia; 277.410, loa 1094, S Anilio; 277.963, Koz 374, Vourinos; 278.104, Gre 492, NO Varis; 278.192, Gre 495, Varis; 278.450, Thes 214, Paralia Vrasna; 278.541, Thes 219, Vrasna; 278.783, Kav 112, NO Paralia Myrtofytou; 278.823, Kav 114, SO Folia; 278.864, Kav 115, SW Folia; 279.058, Kav 121, Platanotopos; 279.375, Ser 262, Proti; 279.398, Kav 92, Pangaion; 279.872, Dra 209, Livadero; 280.081, Dra 219, Sidironero; 280.186, Dra 223, NO Drama; 280.413, Dra 233, Sidironero; 281.036, Dra 258, Granitis; 281.332, Dra 269, W Nevrokopi; 281.537, Dra 276, Perithori; 281.590, Dra 278, Kato Vrondous; 281.895, Dra 287, O Mikroklisoura; 282.107, Dra 296, NO Nevrokopi; 282.172, Dra 299, SO Lefkogia; 282.305, Ser 273, Ano Vrondous; 282.408 , Ser 278, N Serres; 282.443, Ser 280, Orini; 282.647, Ser 290, N Neo Petritsi; 282.845, Ser 298, Parapotamos; 283.174, Pel 343, Kerasia; 283.471, Pel 358, N Loutraki

\section{Trifolium aureum}

279.975, Dra 213, Livadero; 280.582, Dra 238, Skaloti; 280.674, Dra 242, SW Walddorf Elatias; 280.775, Dra 247, Walddorf Elatias; 280.799, Dra 247, Walddorf Elatias; 282.535 , Ser 284, Ori Vrondous; 282.624, Ser 288, Ori Vrondous; 283.060, Kil 306, SO Livadia; 283.082, Kil 307, NW Livadia; 283.315, Pel 350, Kajmaktsalan

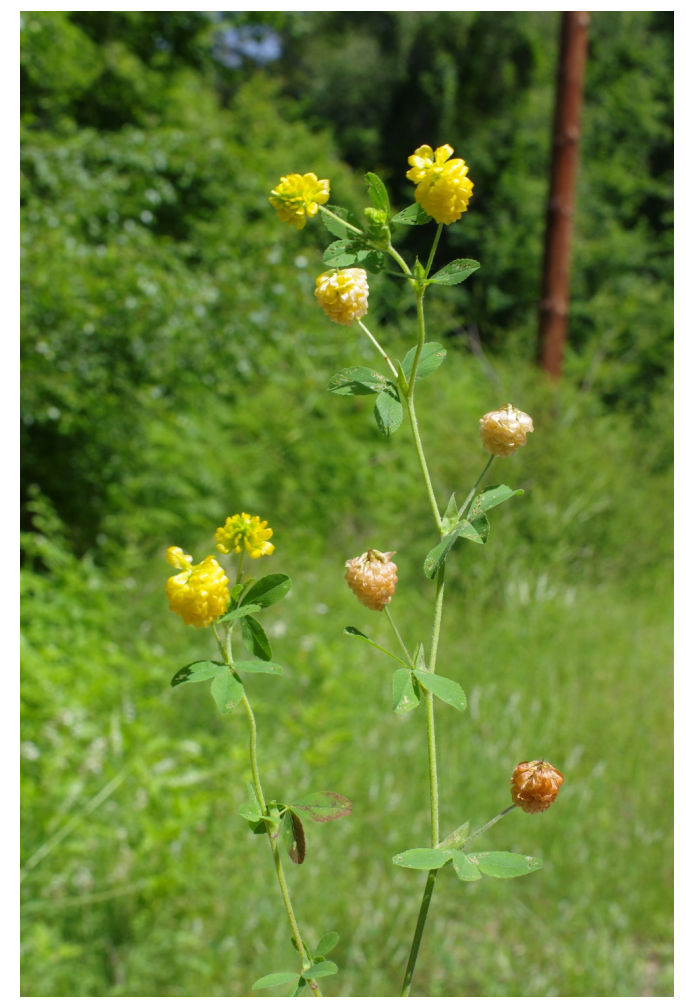

\section{Trifolium campestre}

276.491, loa 1059, Ag. Nikolaos; 276.838, loa 1070, NNW Metsovo; 277.166, loa 1086, NNO Metsovo; 277.341, loa 1093, S Anilio; 277.722, Tri 460, N Chaliki; 277.851, Koz 387, SO Siatista, Vourinos; 278.117, Gre 492, NO Varis; 278.348, Koz 381, Galatini; 278.832, Kav 114, SO Folia; 279.343, Ser 261, Proti; 279.455, Kav 95, Pangaion; 279.776, Ser 268, SW 
Skopia; 279.928, Dra 211, Livadero; 280.014, Dra 216, Sidironero; 280.169, Dra 222, NO Drama; 280.261, Dra 227, Livadero; 280.281, Dra 228, Livadero; 280.411, Dra 233, Sidironero; 281.023, Dra 258, Granitis; 281.121, Dra 261, Granitis; 281.462, Dra 274, Vathytopo; 281.560, Dra 277, Perithori; 281.757, Dra 281, OSO Granitis; 282.083, Dra 295, NO Nevrokopi; 282.446, Ser 280, Orini; 283.172, Pel 343, Kerasia; 283.576, Pel 363, NW Promachi

\section{Trifolium cherleri}

276.495, loa 1059, Ag. Nikolaos; 278.978, Kav 119, Podochori

\section{Trifolium dalmaticum}

276.302, loa 1125, NO Dodoni; 276.518, loa 1059, Ag. Nikolaos; 276.618, loa 1062, SO Potamia; 277.754, Tri 461, N Chaliki; 278.534, Thes 218, Vrasna

\section{Trifolium dubium}

277.400, loa 1094, S Anilio

\section{Trifolium echinatum}

279.808, Ser 269, NO Skopia; 280.111, Dra 220, Sidironero; 281.828 , Dra 283, NNO Volakas; 281.904, Dra 287, O Mikroklisoura

\section{Trifolium heldreichianum}

280.851, Dra 248, Falakron; 280.918, Dra 251, Falakron

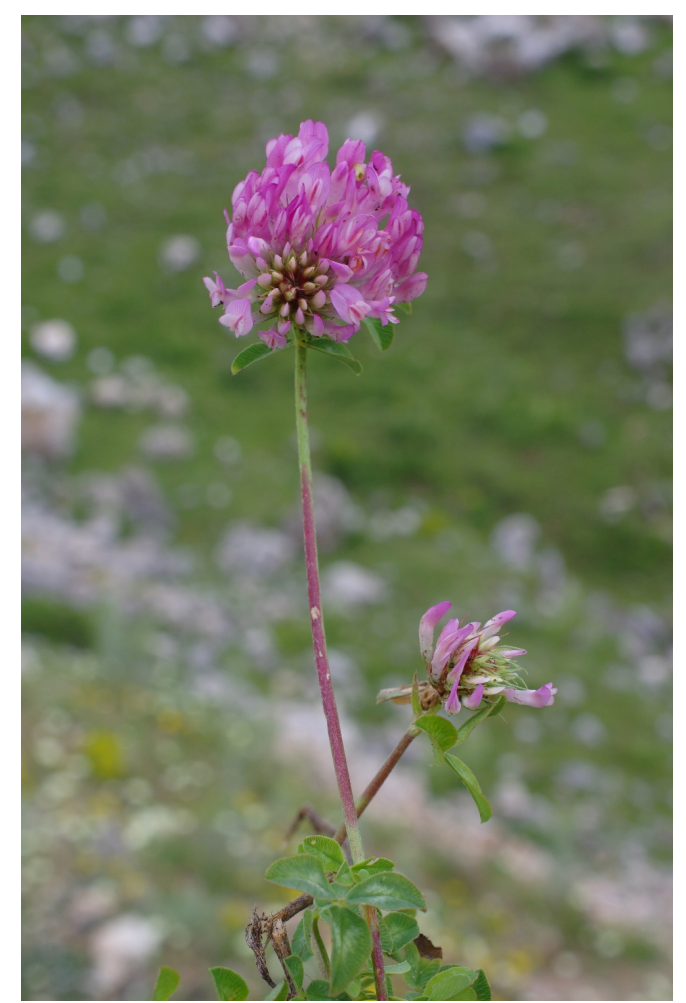

\section{Trifolium hirtum}

276.562, loa 1060, SSO Potamia; 282.451, Ser 280, Orini

\section{Trifolium hybridum subsp. hybridum}

276.405, loa 1057, NO Metsovo; 276.935, loa 1074, NNW Metsovo; 277.158, loa 1086, NNO Metsovo; 277.282, loa 1090, NNO Metsovo; 280.535, Dra 236, Skaloti; 280.766, Dra 246, SW Walddorf Elatias; 280.795, Dra 247, Walddorf Elatias; 281.096, Dra 261, Granitis; 281.481, Dra 275, Vathytopo; 281.527, Dra 276, Perithori; 282.064, Dra 294, NO Nevrokopi; 283.299, Pel 349, Kajmaktsalan; 283.367, Pel 353, Kajmaktsalan

\section{Trifolium lucanicum}

276.298, loa 1125, NO Dodoni; 277.359, loa 1093, S Anilio 
Trifolium medium subsp. balcanicum

277.258, loa 1089, NNO Metsovo; 279.393, Ser 262, Proti; 279.578, Kav 103, Pangaion; 280.498, Dra 235, Skaloti; 280.559, Dra 237, Skaloti; 280.967, Dra 255, Falakron; 284.038, Ioa 1116, SW Milia

\section{Trifolium medium subsp. medium}

276.444, Tri 456, N Katara-Pass; 277.212, loa 1088, NNO Metsovo; 281.620, Dra 278, Kato Vrondous; 283.061, Kil 306, SO Livadia

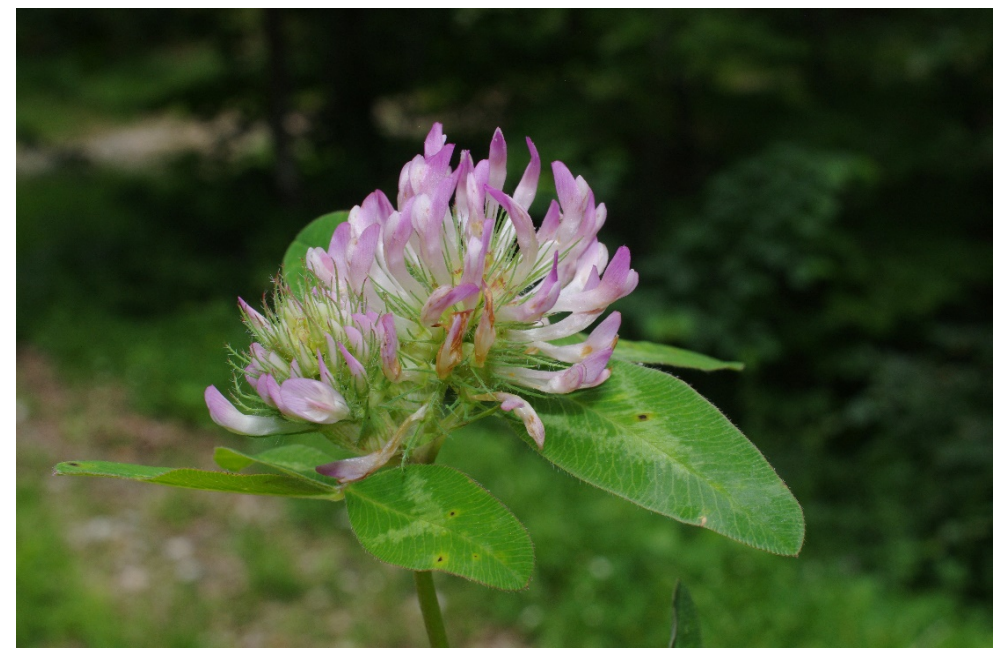

Trifolium nigrescens

279.346, Ser 261, Proti; 279.927, Dra 211, Livadero; 280.320, Dra 230, Livadero; 282.755, Ser 292, N Neo Petritsi

\section{Trifolium noricum}

279.362, Ser 261, Proti; 279.370, Ser 261, Proti; 279.889, Dra 209, Livadero

\section{Trifolium ochroleucon subsp. ochroleucon}

276.703, loa 1065, NO Karyes; 277.343, loa 1093, S Anilio; 277.413, loa 1094, S Anilio; 277.532, loa 1097, S Anilio; 277.869, Koz 388, SO Siatista, Vourinos; 278.274, Koz 379, Galatini; 278.405, Koz 383, Galatini; 279.520, Kav 99, Pangaion; 280.010, Dra 216, Sidironero; 280.057, Dra 217, Sidironero; 280.243, Dra 227, Livadero; 280.279, Dra 228, Livadero; 280.379, Dra 232, Sidironero; 280.956, Dra 255, Falakron; 281.475, Dra 275 , Vathytopo; 282.089, Dra 295, NO Nevrokopi; 282.321, Ser 274, SSW Ano Vrondous; 283.032, Kil 303, SO Livadia; 283.066, Kil 307, NW Livadia; 283.196, Pel 343, Kerasia; 283.972, loa 1056 b, NO Metsovo; 284.165, loa 1124, N Charikli

\section{Trifolium ochroleucon subsp. roseum}

279.405, Kav 92, Pangaion; 279.572, Kav 103, Pangaion; 281.747, Dra 281, OSO Granitis; 282.496, Ser 282, Ori Vrondous; 283.674, Pel 368, OSO Vorino; 284.024, loa 1116, SW Milia

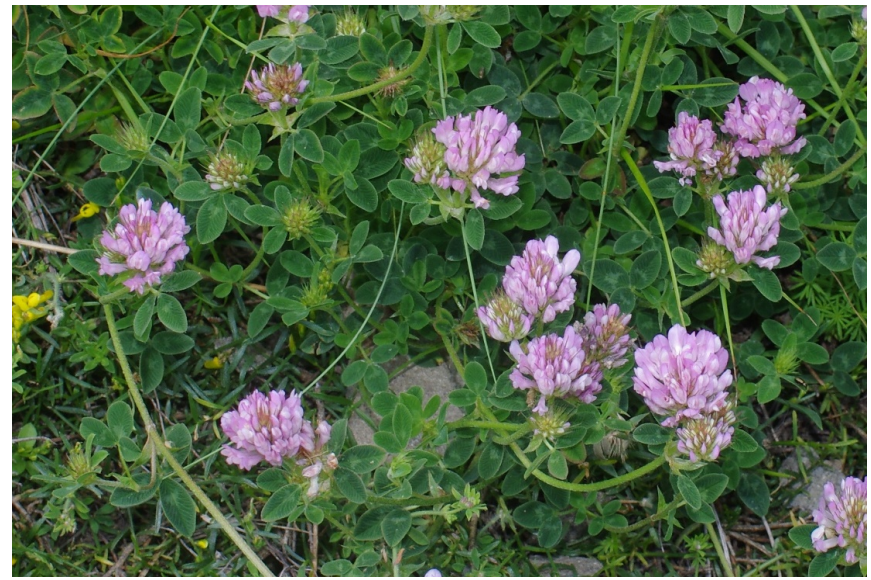


Trifolium pallidum

276.679, loa 1065, NO Karyes

Trifolium patulum

276.542, loa 1059, Ag. Nikolaos; 277.257, loa 1089, NNO Metsovo

Trifolium phleoides

277.697, Tri 460, N Chaliki; 277.791, Koz 385, SO Siatista; 277.961, Koz 374, Vourinos

\section{Trifolium physodes}

276.351, loa 1128, N Metsovo; 276.833, loa 1070, NNW Metsovo; 276.887, loa 1072, NNW

Metsovo; 277.647, loa 1105, Anilio; 277.719, Tri 460, N Chaliki; 277.962, Koz 374,

Vourinos; -20.466, loa 1053, NO Dodoni

\section{Trifolium pignantii}

276.457, Tri 457, NO Katara-Pass; 277.228, loa 1089, NNO Metsovo

\section{Trifolium pratense}

276.817, loa 1070, NNW Metsovo; 276.847, loa 1071, NNW Metsovo; 276.979, loa 1079, SO Anilio; 277.012, loa 1080, SO Anilio; 277.156, loa 1086, NNO Metsovo; 279.446, Kav 95, Pangaion; 279.528, Kav 99, Pangaion; 279.964, Dra 213, Livadero; 280.253, Dra 227, Livadero; 280.418, Dra 233, Sidironero; 280.445, Dra 234, Skaloti; 280.675, Dra 242, SW Walddorf Elatias; 280.685, Dra 242, SW Walddorf Elatias; 280.762, Dra 246, SW Walddorf Elatias; 281.075, Dra 260, Granitis; 281.375, Dra 271, Vathytopo; 281.667, Ser 270, Kato Vrondous; 281.812, Dra 283, NNO Volakas; 281.899, Dra 287, O Mikroklisoura; 282.193, Dra 299, SO Lefkogia; 282.315, Ser 274, SSW Ano Vrondous; 282.665, Ser 290, N Neo Petritsi; 282.667, Ser 290, N Neo Petritsi; 282.947, Kil 298, WNW Kastaneri; 283.193, Pel 343, Kerasia; 283.297, Pel 349, Kajmaktsalan; 283.433, Pel 355, NW Loutraki; 283.579, Pel 363, NW Promachi; 283.839, Ima 177, Tria-Pende Pigadia; 283.843, Ima 178, Seli; 284.035, loa 1116, SW Milia; 284.208, Dra 216, Sidironero; -20.489, loa 1078, O Anilio; 20.523, Ser 286, SW Mikroklisoura

\section{Trifolium purpureum}

278.016, Koz 376, W Exarcho; 278.176, Gre 495, Varis; 278.766, Kav 110, Loutra Eleftherion; 278.893, Kav 116, S Ofrynia; 279.019, Kav 119, Podochori; 279.156, Ser 255, S Nea Mesolakkia; 279.292, Ser 259, Proti; 279.817, Ser 269, NO Skopia; 280.071, Dra 218, Sidironero; 280.127, Dra 221, NO Drama; 280.246, Dra 227, Livadero; 281.874, Dra 286, SW Mikroklisoura; 281.952, Dra 289, NO Potamia

\section{Trifolium repens}

276.553, loa 1060, SSO Potamia; 276.698, loa 1065, NO Karyes; 276.977, loa 1079, SO Anilio; 277.340, loa 1093, S Anilio; 277.934, Koz 392, SO Siatista, Vourinos; 279.761, Ser 268, SW Skopia; 279.992, Dra 214, Livadero; 280.561, Dra 237, Skaloti; 280.676, Dra 242, SW Walddorf Elatias; 280.870, Dra 249, Falakron; 282.243, Dra 301, Ano Vrondous; 282.539, Ser 284, Ori Vrondous; 283.022, Kil 302, Kastaneri; 284.083, loa 1119, Anilio; 20.525, Ser 286, SW Mikroklisoura

Trifolium resupinatum subsp. resupinatum

276.641, loa 1063, W Potamia; 277.889, Koz 389, SO Siatista, Vourinos

\section{Trifolium scabrum}

277.824, Koz 387, SO Siatista, Vourinos; 277.915, Koz 390, SO Siatista, Vourinos; 278.317 , Koz 381, Galatini; 278.395, Koz 383, Galatini; 279.324, Ser 260, Proti

\section{Trifolium squamosum}

278.632, Thes 221, Arethousa; 278.887, Kav 116, S Ofrynia; 279.644, Ser 264, Dravoskas; 280.131, Dra 221, NO Drama; 281.966, Dra 289, NO Potamia

\section{Trifolium striatum}

278.131, Gre 493, NO Varis; 278.309, Koz 380, Galatini; 279.940, Dra 212, Livadero;

281.487, Dra 275, Vathytopo; 282.219, Dra 300, SO Lefkogia 
Trifolium tenuifolium

282.837, Ser 298, Parapotamos

Trifolium xanthinum

276.692, loa 1065, NO Karyes

Trigonella corniculata subsp. corniculata

278.370, Koz 382, Galatini; 283.722, Pel 370, SW Foustani

Trinia glauca subsp. pindica

276.391, loa 1056, NO Metsovo; 277.287, loa 1091, SW Milia; 283.333, Pel 351,

Kajmaktsalan

\section{Tripleurospermum tempskyanum}

276.401, loa 1056, NO Metsovo; 276.809, loa 1070, NNW Metsovo; 277.071, loa 1080, SO Anilio; 277.126, loa 1084, N Metsovo; 277.302, loa 1091, SW Milia; 277.600, loa 1102, S Anilio; 284.098, loa 1120, Anilio

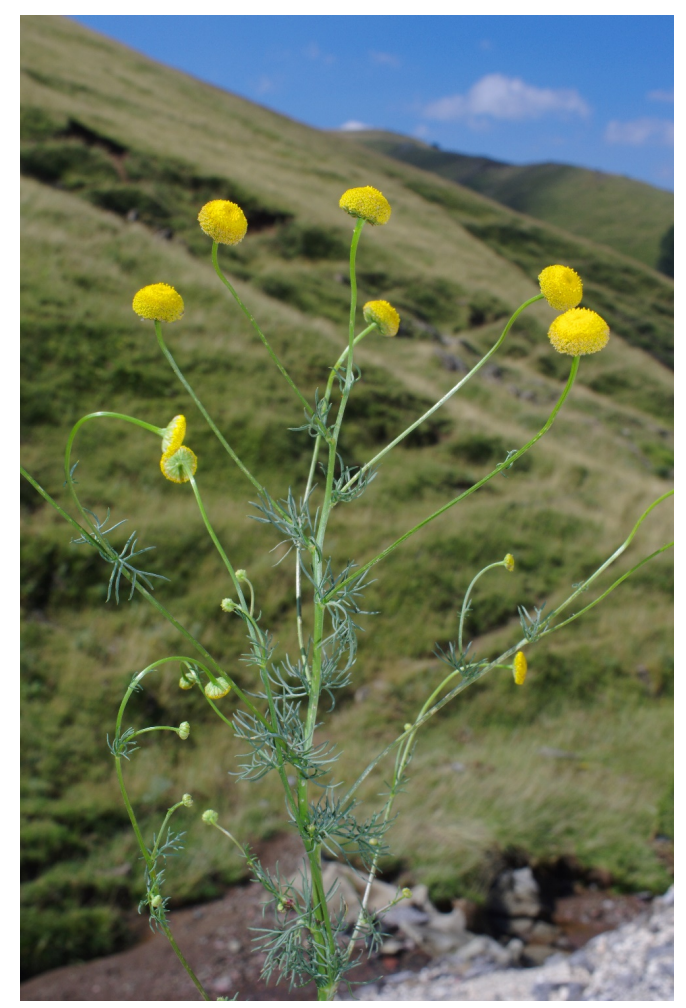

\section{Tripleurospermum tenuifolium}

280.565, Dra 238, Skaloti; 281.073, Dra 260, Granitis; 281.301, Dra 267, W Nevrokopi; 281.514, Dra 276, Perithori; 281.681, Ser 271, Kato Vrondous; 282.049, Dra 293, Nevrokopi; 282.133, Dra 297, Lefkogia; 282.156, Dra 298, SO Lefkogia; 282.298, Ser 273, Ano Vrondous; 282.942, Kil 298, WNW Kastaneri; 282.970, Kil 299, Kastaneri; 283.080, Kil 307, NW Livadia; 283.360, Pel 352, Kajmaktsalan; 283.652, Pel 367, Vorino

Trisetum flavescens subsp. flavescens

277.121, loa 1084, N Metsovo; 277.632, loa 1104, S Anilio

Trisetum flavescens subsp. splendens

276.807, loa 1070, NNW Metsovo; 277.122, loa 1084, N Metsovo; 277.864, Koz 388, SO Siatista, Vourinos

Triticum monococcum subsp. aegilopoides

278.111, Gre 492, NO Varis

Turgenia latifolia

278.254, Koz 379, Galatini

Tussilago farfara

-20.472, loa 1055, N Metsovo 


\section{Ulmus laevis}

283.535, Pel 361, SW Promachi

\section{Ulmus minor subsp. minor}

281.955, Dra 289, NO Potamia

\section{Urospermum picroides}

279.682, Ser 265, Myrrini

\section{Urtica dioica}

276.682, loa 1065, NO Karyes; 277.010, loa 1080, SO Anilio; 277.574, loa 1100, S Anilio; 279.173, Ser 256, Paleokomi; 279.536, Kav 100, Pangaion; 280.346, Dra 230, Livadero; 281.011, Dra 257, Granitis; 283.365, Pel 353, Kajmaktsalan; -20.526, Ser 286, SW Mikroklisoura

\section{Vaccaria hispanica}

277.805, Koz 385, SO Siatista

\section{Valeriana officinalis subsp. officinalis} 282.254, Dra 301, Ano Vrondous

Valeriana pratensis subsp. angustifolia 276.743, loa 1068, NW Metsovo; 276.853, loa 1071, NNW Metsovo; 280.704, Dra 243, SW Walddorf Elatias; 282.935, Kil 298, WNW Kastaneri; 283.042, Kil 304, SO Livadia

\section{Valerianella coronata}

277.827, Koz 387, SO Siatista, Vourinos; 278.321, Koz 381, Galatini; 281.780, Dra 282, NNO Volakas

\section{Valerianella muricata}

276.747, loa 1068, NW Metsovo; 284.221, loa 1068, NW Metsovo

\section{Velezia rigida}

276.295, loa 1125, NO Dodoni; 278.417, Koz 384, Galatini; 278.501, Thes 216, Vrasna; 278.615, Thes 221, Arethousa; 279.203, Ser 257, Nea Fili; 279.254, Ser 258, Mikro Souli; 280.162, Dra 221, NO Drama

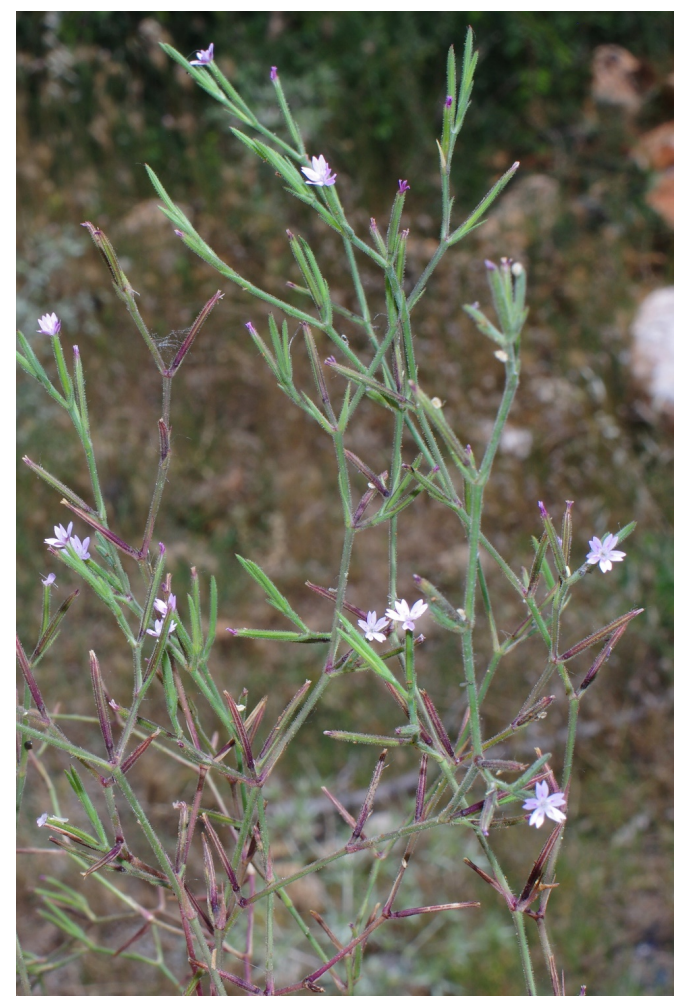


Ventenata dubia

276.926, loa 1074, NNW Metsovo

Veratrum lobelianum

283.311, Pel 350, Kajmaktsalan; 283.949, loa 1109, NW Metsovo

Verbascum chaixii subsp. chaixii

279.942, Dra 212, Livadero; 280.915, Dra 251, Falakron

Verbascum densiflorum

282.628, Ser 289, Ori Vrondous

Verbascum dieckianum

281.268, Dra 266, W Nevrokopi

Verbascum glabratum

278.466, Thes 214, Paralia Vrasna

Verbascum graecum

276.474, loa 1059, Ag. Nikolaos

Verbascum longifolium

277.662, Tri 459, N Chaliki

Verbascum nigrum subsp. abietinum

282.633, Ser 290, N Neo Petritsi

Verbascum phlomoides

283.020, Kil 302, Kastaneri; 283.494, Pel 359, NW Loutraki

Verbascum sinuatum

277.995, Koz 375, O Dafnero

Verbascum undulatum

277.813, Koz 387, SO Siatista, Vourinos

\section{Verbena officinalis}

277.433, loa 1095, S Anilio; 278.533, Thes 218, Vrasna; 278.636, Kav 106, Paralia Ofryniou; 278.776, Kav 112, NO Paralia Myrtofytou; 279.062, Kav 121, Platanotopos; 279.105, Kav 90, SW Moustheni; 279.256, Ser 258, Mikro Souli; 279.400, Kav 92, Pangaion; 279.730, Ser 267, SW Skopia; 279.760, Ser 268, SW Skopia; 279.888, Dra 209, Livadero; 280.075, Dra 219, Sidironero; 280.198, Dra 224, NO Drama; 280.290, Dra 228, Livadero; 281.115, Dra 261, Granitis; 281.229, Dra 265, NO Granitis; 281.251, Dra 266, W Nevrokopi; 281.403, Dra 273, Vathytopo; 281.935, Dra 288, SW Potamia; 282.109, Dra 296, NO Nevrokopi; 282.401, Ser 278, N Serres; 282.749, Ser 292, N Neo Petritsi; 283.142, Pel 341, S Archangelo; 283.414, Pel 355, NW Loutraki; 283.507, Pel 360, SW Promachi; 283.587, Pel 364, Loutraki; 283.630, Pel 366, N Vorino; 283.688, Pel 369, O Vorino; 283.719, Pel 370, SW Foustani; 20.468, loa 1053, NO Dodoni; -20.502, Dra 234, Skaloti

\section{Veronica acinifolia}

276.423, loa 1057, NO Metsovo

\section{Veronica anagallis-aquatica}

276.610, loa 1062, SO Potamia; 276.649, loa 1063, W Potamia; 276.652, loa 1063, W Potamia; 276.819, loa 1070, NNW Metsovo; 277.128, loa 1084, N Metsovo; 277.266, loa 1090, NNO Metsovo; 277.393, loa 1094, S Anilio; 277.898, Koz 389, SO Siatista, Vourinos; 279.090, Kav 89, Misoropi; 279.120, Kav 91, Orfani; 281.783, Dra 282, NNO Volakas; 282.153, Dra 297, Lefkogia; 283.272, Pel 348, Kajmaktsalan; 283.520, Pel 361, SW Promachi 


\section{Veronica arvensis}

276.431, loa 1058, Katara-Pass; 276.842, loa 1071, NNW Metsovo; 277.009, loa 1080, SO Anilio; 277.124, loa 1084, N Metsovo; 277.232, loa 1089, NNO Metsovo; 277.476, loa 1097, S Anilio; 280.352, Dra 231, Sidironero; 280.621, Dra 239, Skaloti; 280.800, Dra 247,

Walddorf Elatias; -20.518, Dra 238, Skaloti

\section{Veronica beccabunga}

276.425, loa 1057, NO Metsovo; 277.039, loa 1080, SO Anilio; 277.221, loa 1088, NNO Metsovo; 277.622, loa 1104, S Anilio

\section{Veronica bozakmanii}

276.407, loa 1057, NO Metsovo; 276.811, loa 1070, NNW Metsovo; 276.970, loa 1079, SO Anilio; 277.042, loa 1080, SO Anilio; 277.235, loa 1089, NNO Metsovo; 277.529, loa 1097, S Anilio

Veronica chamaedrys subsp. chamaedryoides

277.204, loa 1087, NNO Metsovo; 277.544, loa 1098, S Anilio

Veronica chamaedrys subsp. chamaedrys

276.880, loa 1072, NNW Metsovo; 277.040, loa 1080, SO Anilio

Veronica jacquinii

280.911, Dra 251, Falakron; 281.749, Dra 281, OSO Granitis

\section{Veronica officinalis}

276.962, loa 1078, O Anilio; 277.188, loa 1086, NNO Metsovo; 280.601, Dra 238, Skaloti; 282.500, Ser 282, Ori Vrondous

\section{Veronica orsiniana subsp. orsiniana}

276.393, loa 1056, NO Metsovo; 283.353, Pel 352, Kajmaktsalan

\section{Veronica persica}

276.475, loa 1059, Ag. Nikolaos; 276.816, loa 1070, NNW Metsovo; 277.426, loa 1095, S Anilio; 278.279, Koz 379, Galatini; 278.831, Kav 114, SO Folia; 279.470, Kav 96, Pangaion; 281.068, Dra 260, Granitis; 282.693, Ser 290, N Neo Petritsi

\section{Veronica scardica}

278.052, Gre 491, NO Exarcho

\section{Veronica serpyllifolia}

276.455, Tri 457, NO Katara-Pass; 277.043, loa 1080, SO Anilio; 277.141, loa 1084, N Metsovo; 280.526, Dra 236, Skaloti; 280.673, Dra 242, SW Walddorf Elatias

\section{Veronica triphyllos}

283.349, Pel 352, Kajmaktsalan

\section{Veronica urticifolia}

282.937, Kil 298, WNW Kastaneri; 283.046, Kil 304, SO Livadia

\section{Veronica vindobonensis}

280.653, Dra 242, SW Walddorf Elatias

\section{Vicia angustifolia}

276.820, loa 1070, NNW Metsovo; 277.309, loa 1091, SW Milia; 277.392, loa 1094, S Anilio; 277.702, Tri 460, N Chaliki; 277.927, Koz 392, SO Siatista, Vourinos; 278.430, Koz 384, Galatini; 281.684, Ser 271, Kato Vrondous; -20.508, Dra 236, Skaloti

Vicia cassubica

279.527, Kav 99, Pangaion; 280.300, Dra 229, SO Dendrakia; 283.191, Pel 343, Kerasia 


\section{Vicia cracca}

276.458, Tri 457, NO Katara-Pass; 277.249, loa 1089, NNO Metsovo; 278.617, Thes 221, Arethousa; 279.501, Kav 98, Pangaion; 279.981, Dra 214, Livadero; 280.326, Dra 230, Livadero; 280.406, Dra 233, Sidironero; 280.482, Dra 234, Skaloti; 280.540, Dra 236, Skaloti; 280.719, Dra 243, SW Walddorf Elatias; 281.078, Dra 260, Granitis; 281.198, Dra 264, NO Ochyro; 281.494, Dra 276, Perithori; 282.918, Kil 297, WNW Kastaneri; 283.781, Ima 173, S Ag. Pavlos

\section{Vicia grandiflora}

276.616, loa 1062, SO Potamia; 276.699, loa 1065, NO Karyes; 277.467, loa 1096, S Anilio; 279.442, Kav 95, Pangaion; 280.020, Dra 216, Sidironero; 281.006, Dra 257, Granitis; 281.090, Dra 260, Granitis; 281.520, Dra 276, Perithori; 281.659, Ser 270, Kato Vrondous; 282.276, Ser 272, Ano Vrondous

Vicia hirsuta

279.944, Dra 212, Livadero; -20.470, loa 1053, NO Dodoni

Vicia hybrida

-20.467, loa 1053, NO Dodoni

Vicia lathyroides

277.714, Tri 460, N Chaliki

Vicia pannonica subsp. striata

277.909, Koz 390, SO Siatista, Vourinos; 278.109, Gre 492, NO Varis; 278.182, Gre 495, Varis; 278.266, Koz 379, Galatini; 278.366, Koz 382, Galatini

\section{Vicia parviflora}

278.560, Thes 219, Vrasna; 280.301, Dra 229, SO Dendrakia

Vicia sativa subsp. sativa

278.576, Thes 220, Arethousa; 279.361, Ser 261, Proti; 279.924, Dra 211, Livadero; 281.095, Dra 261, Granitis; 282.348, Ser 275, SSW Ano Vrondous; 282.351, Ser 275, SSW Ano Vrondous

Vicia tenuifolia subsp. tenuifolia

277.210, loa 1088, NNO Metsovo

\section{Vicia tetrasperma}

276.923, loa 1074, NNW Metsovo; 279.376, Ser 262, Proti; 280.051, Dra 217, Sidironero; 280.578, Dra 238, Skaloti; 281.880, Dra 287, O Mikroklisoura

Vicia villosa subsp. varia

276.310, loa 1125, NO Dodoni; 276.510, loa 1059, Ag. Nikolaos; 277.466, loa 1096, S Anilio; 277.839, Koz 387, SO Siatista, Vourinos; 277.905, Koz 390, SO Siatista, Vourinos; 278.166, Gre 494, SO Varis; 278.261, Koz 379, Galatini; 278.367, Koz 382, Galatini; 278.565, Thes 219, Vrasna; 279.073, Kav 121, Platanotopos; 279.103, Kav 90, SW Moustheni; 281.798, Dra 282, NNO Volakas; 281.819, Dra 283, NNO Volakas; 283.083, Kil 307, NW Livadia; 283.125, Pel 340, SSO Archangelo; 283.710, Pel 370, SW Foustani

\section{Vicia villosa subsp. villosa}

278.440, Thes 214, Paralia Vrasna; 278.657, Kav 106, Paralia Ofryniou; 278.916, Kav 117 , Galipsos; 279.921, Dra 211, Livadero; 279.985, Dra 214, Livadero; 281.664, Ser 270, Kato Vrondous; 282.261, Dra 301, Ano Vrondous; 282.325, Ser 274, SSW Ano Vrondous;

282.618, Ser 288, Ori Vrondous

\section{Vincetoxicum hirundinaria}

278.761, Kav 110, Loutra Eleftherion; 280.886, Dra 250, Falakron

\section{Vincetoxicum hirundinaria subsp. nivale}

283.623, Pel 366, N Vorino 
Viola

279.392, Ser 262, Proti; 281.307, Dra 268, W Nevrokopi; 281.779, Dra 282, NNO Volakas;

282.473, Ser 281, Ori Vrondous; 284.223, Ser 281, Ori Vrondous

\section{Viola aetolica}

276.844, loa 1071, NNW Metsovo

Viola arvensis

276.454, Tri 457, NO Katara-Pass; 281.540, Dra 276, Perithori

Viola graeca

276.397, loa 1056, NO Metsovo

Viola hymettia

280.930, Dra 253, Falakron

\section{Viola macedonica}

277.460, loa 1096, S Anilio; 277.890, Koz 389, SO Siatista, Vourinos; 278.142, Gre 492, NO Varis; 279.546, Kav 99, Pangaion; 279.929, Dra 211, Livadero; 280.619, Dra 239, Skaloti; 280.668, Dra 242, SW Walddorf Elatias; 280.802, Dra 247, Walddorf Elatias; 280.866 , Dra 249, Falakron; 280.933, Dra 253, Falakron; 282.498, Ser 282, Ori Vrondous; 282.606, Ser 288, Ori Vrondous; 282.695, Ser 290, N Neo Petritsi; 282.708, Ser 290, N Neo Petritsi; 284.222, Ser 282, Ori Vrondous

Viola orphanidis

283.269, Pel 348, Kajmaktsalan; 283.277, Pel 348, Kajmaktsalan

Viola riviniana

277.170, loa 1086, NNO Metsovo; 282.724, Ser 291, N Neo Petritsi; 284.079, loa 1119, Anilio

Viola tricolor

279.980, Dra 214, Livadero

\section{Viscaria atropurpurea}

276.784, loa 1069, NW Metsovo; 277.549, loa 1098, S Anilio

Vitis vinifera subsp. sylvestris

282.303, Ser 273, Ano Vrondous

Vulpia ciliata

276.575, loa 1060, SSO Potamia; 277.836, Koz 387, SO Siatista, Vourinos

Vulpia muralis

278.323, Koz 381, Galatini; 279.463, Kav 95, Pangaion; 279.989, Dra 214, Livadero

Vulpia myuros

277.972, Koz 374, Vourinos; 279.568, Kav 103, Pangaion; 280.497, Dra 235, Skaloti

Xanthium orientale subsp. italicum

278.141, Gre 492, NO Varis; 283.656, Pel 367, Vorino

\section{Xanthium spinosum}

278.150, Gre 492, NO Varis; 278.291, Koz 380, Galatini; 278.654, Kav 106, Paralia Ofryniou; 278.920, Kav 117, Galipsos; 278.963, Kav 119, Podochori; 279.193, Ser 256, Paleokomi; 279.239, Ser 258, Mikro Souli; 281.793, Dra 282, NNO Volakas

\section{Xeranthemum annuum}

277.809, Koz 386, SO Siatista; 278.018, Koz 376, W Exarcho; 278.730, Kav 108, Paralia Ofryniou; 278.879, Kav 116, S Ofrynia; 279.037, Kav 120, Podochori; 279.167, Ser 255, S 
Nea Mesolakkia; 279.321, Ser 260, Proti; 279.631, Ser 263, Dravoskas; 279.703, Ser 266, SO Nea Zichni; 279.849, Dra 208, NO Skopia; 280.125, Dra 221, NO Drama; 281.139, Dra 262, NW Granitis; 281.336, Dra 270, W Nevrokopi; 281.437, Dra 274, Vathytopo; 281.801, Dra 282, NNO Volakas; 281.818, Dra 283, NNO Volakas; 281.968, Dra 289, NO Potamia; 282.017, Dra 292, Nevrokopi; 282.036, Dra 293, Nevrokopi; 282.040, Dra 293, Nevrokopi; 282.126, Dra 296, NO Nevrokopi; 282.377, Ser 276, SSW Ano Vrondous; 282.393 , Ser 278 , N Serres; 282.788, Ser 295, N Neo Petritsi; 283.468, Pel 358, N Loutraki; 283.607, Pel 365, N Vorino

\section{Xeranthemum cylindraceum}

278.057, Gre 492, NO Varis; 281.546, Dra 277, Perithori; 281.959, Dra 289, NO Potamia; 282.047, Dra 293, Nevrokopi

\section{Ziziphora capitata}

276.582, loa 1060, SSO Potamia; 278.000, Koz 375, O Dafnero

\section{Literatur}

Dimopoulos, P. et al. (2013): Vascular Plants of Greece. An annotated checklist, Englera 31 Jahn, R. \& P.Schönfelder (1995): Exkursionsflora für Kreta

Strid,A. (2016): Atlas of the Aegean Flora, Part 1 \& 2, Englera 33

Strid, A. \& K.Tan (1997): Flora Hellenica, Vol.1

Strid, A. \& K.Tan (2002): Flora Hellenica, Vol.2 